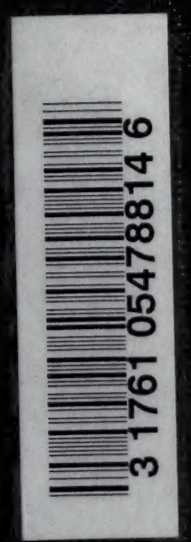


Digitized by the Internet Archive in 2007 with funding from Microsoft Corporation 



\title{
THE ELEMENTS OF
}

\section{ELECTRICAL ENGINEERING}

\author{
VOLUME II.
}

ALTERNATING CURRENTS. 



\section{THE ELEMENTS \\ OF \\ ELECTRICAL ENGINEERING \\ A TEXT BOOK FOR}

TECHNICAL SCHOOLS AND COLLEGES

BY

WILLIAM SUDDARDS FRANKLIN

PROFESSOR OF PHYSICS IN LEHIGH UNIVERSITY

AND

WILLIAM ESTY

PROYRSSOR OF ELECTRICAL ENGINERRING IN LEHIGH UNIVERSITY

VOLUME II.

ALTERNATING CURRENTS

SECONd Edition. REVISEd and ENLARged.

FIFTH THOUSAND.

此elo gor

THE MACMILLAN COMPANY

LONDON : MACMILLAN \& CO., LTD.

1908

All rights reserved 


\section{Copyright, 1907}

BY THE MACMILLAN COMPANY

$$
\text { Copyright, I908 }
$$

BY THE MACMILLAN COMPANY

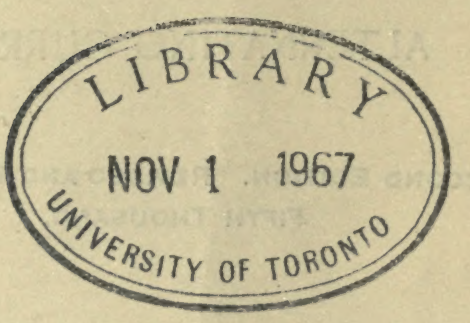




\section{TABLE OF CONTENTS.}

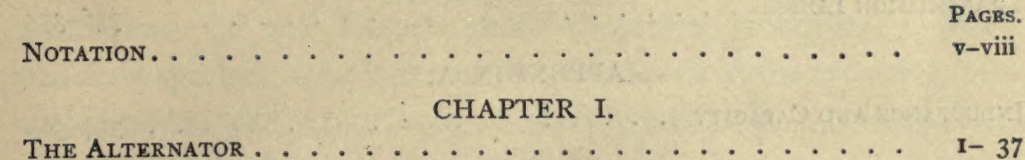

CHAPTER II.

- Alternating Current Measurements........... . $3^{8-} 5^{1}$

CHAPTER III.

Harmonic Electromotive Force and Current . . . . . . $5 \mathbf{5 2} 65$

CHAPTER IV.

Fundamental Problems . . . . . . . . . . . . . 66-87

CHAPTER V.

The Use of Complex Quantity . . . . . . . . . . . . . . 88- 97

CHAPTER VI.

The Polyphase System ............... 98-II6

CHAPTER VII.

Voltage and Voltage Regulation of Alternators . . . . . . . II7-I40

CHAPTER VIII.

The Synchronous Motor. Alternators in Parallel . . . . . . . I4I-I69

CHAPTER IX.

Conversion of Alternating Current into Direct Current . . . . I7O-i93

CHAPTER $\mathrm{X}$.

The Transformer.................. I94-232

CHAPTER XI.

General Theory of the Transformer ........... 233-250

CHAPTER XII.

THE INDUCTION MOTOR. . . . . . . . . . . . . 251-274

CHAPTER XIII.

General Theory of the Induction Motor . . . . . . . 275-300 
CONTENTS.

CHAPTER $\cdot$ XIV.

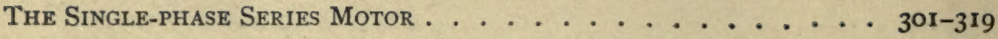

CHAPTER XV.

Switchboards ANd SWITChboard Appliances . . . . . . . 320-345

CHAPTER XVI.

TRANSMISSION LINES . . . . . . . . . . . . . . . 346-353

APPENDIX A.

Inductance And CAPACITy . . . . . . . . . . . 354-369

APPENDIX B. . . . . 370-378

APPENDIX C.

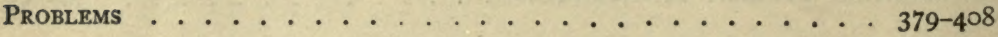

INDEX ........ 409-414 


\section{NOTATION.}

The notation used in this volume follows in the main the recommendations of the International Electrical Congress. Nonstandard symbols which are used but once or twice are not given in this table; the significance of such symbols is fully explained whenever and wherever they are used.

$a, \beta, \phi$ and $\theta$ are frequently used for angle.

$b$ susceptance of an alternating-current circuit

$$
\left[=-x /\left(r^{2}+x^{2}\right)\right] \text {. }
$$

$\mathscr{B}$ magnetic flux density.

$C$ condenser capacity.

E maximum value of harmonic alternating electromotive force.

$E$ effective value of alternating electromotive force.

$e$ instantaneous value of alternating electromotive force.

$f$ frequency in cycles per second.

$g$ conductance of an alternating-current circuit

$$
\left[=r /\left(r^{2}+x^{2}\right)\right] \text {. }
$$

of magnetic field intensity.

I maximum value of harmonic alternating current.

$I$ effective value of alternating current.

$i$ instantaneous value of alternating current.

$j$ square root of minus one, $\sqrt{-1}$.

$L$ inductance.

$l$ length.

$M$ magnetizing current of a transformer or induction motor.

$M_{p}$ power component of $M$.

$M_{w}$ wattless component of $M$.

$\mu$ magnetic permeability.

$n$ speed generally expressed in revolutions per second. This letter is frequently used where a number of things, $n$, is to be represented in a formula. 
$N^{\prime}, N^{\prime \prime}$ number of turns of wire in the primary and secondary coils of a transformer.

$n^{\prime}$. rotor speed of an induction motor.

$P$ power. Also used for leakage inductance of a transformer.

$P_{h}$ hysteresis loss in a laminated iron core.

$P$. eddy current loss in a laminated iron core.

$P_{c}$ copper loss in a transformer.

$p$ number of field magnet poles, or number of polar regions as in the induction motor. This letter is also used for power factor, and sometimes for instantaneous power.

$p^{\prime}$ number of current paths in an armature winding.

$\Phi$ maximum value of harmonic alternating magnetic flux. Sometimes used for value of a steady flux.

$\phi$ instantaneous value of magnetic flux. Also used for angle.

$Q, q$ quantity of electric charge.

$R, r$ resistance. Also used for radius.

$\mathscr{R}$ magnetic reluctance.

$s$ sectional area. Also used for slip of an induction motor.

$t$ time. Also used for temperature.

$T$ torque.

$W$ work.

$\omega$ angular velocity, frequency of a harmonic alternating electromotive force or current in radians per second. $X, x$ reactance in ohms. The letters $X$ and $x$ are also used as coördinates.

$Y, y$ admittance of a circuit $\left(=\sqrt{g^{2}+b^{2}}\right.$ or $\left.g-j b\right)$. The letters $Y$ and $y$ are also used as coördinates.

$Z, z$ impedance of a circuit $\left(=\sqrt{r^{2}+x^{2}}\right.$ or $\left.r+j x\right)$. The letter $Z$ is also used for the number of conductors in an armature winding, or the number of conductors in a stator or rotor winding of an induction motor, or the number of turns of wire in a coil. 


\section{ELEMENTS OF}

\section{ELECTRICAL ENGINEERING.}

\section{CHAPTER I.}

THE ALTERNATOR. ALTERNATING ELECTROMOTIVE FORCE AND ALTERNATING CURRENT.

1. The alternator. - The simplest form of the alternator is shown in Fig. I. A wire $W$, perpendicular to the plane of the paper, is moved sidewise along the dotted line so as to cut the

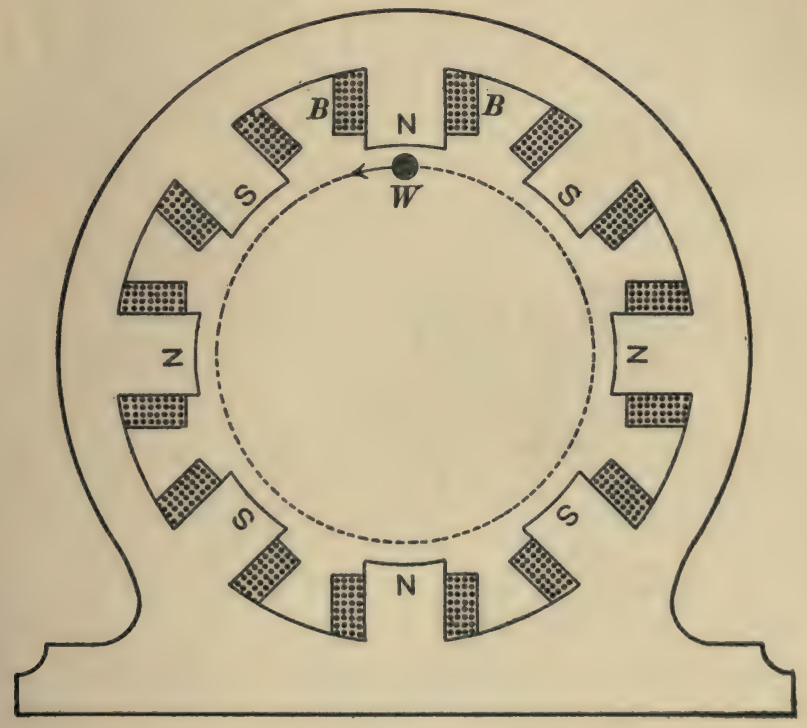

Fig. 1.

magnetic lines of force which emanate from the inwardly projecting poles NSNS of a field magnet. While the wire is sweep- 
ing across a north pole an electromotive force is induced in the wire in one direction, and while the wire is sweeping across a south pole an electromotive force is induced in the wire in the opposite direction. This repeatedly reversed electromotive force is called an alternating electromotive force and it produces an alternating current in the wire and in an outside circuit to which the ends of the wire are connected.

In commercial alternators a large number of wires is used instead of the single wire $W$ shown in Fig. I, and these wires

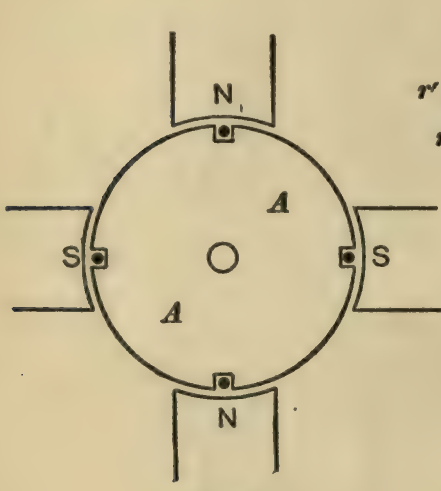

Flg. $2 a$.

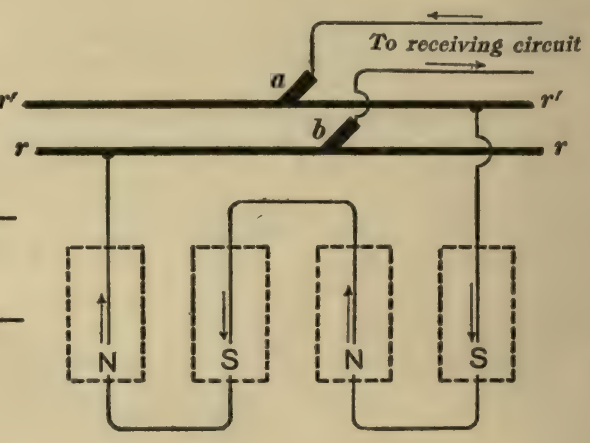

Fig. 28

are placed in slots in the periphery of a rotating mass of laminated iron. Thus Fig. 2 shows four wires placed in four slots, and Fig. 3 shows sixteen wires placed in sixteen slots.

Figures $2 b$ and $3 b$ are what are called developed diagrams which show how the wires are connected together and how they are connected to two insulated metal rings $r$ and $r^{\prime}$ upon which two brushes $a$ and $b$ rub and keep the moving wires connected to an outside receiving circuit.

The laminated structure $A A$ with its winding of wire is called the armature of the alternator, and the metal rings $r$ and $r^{\prime}$ are called collecting rings.

The field magnet of an alternator is generally an electromagnet which is excited by direct current from a small auxiliary direct- 
current generator called the exciter. The field windings, $B B$, Fig. I, of an alternator are arranged exactly like the field windings of a direct-current dynamo.

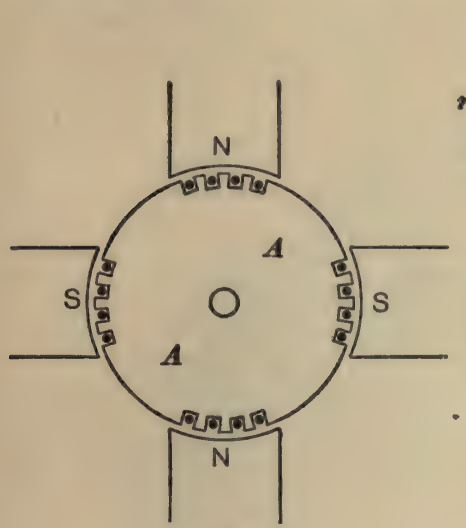

Fig. $3 a$.

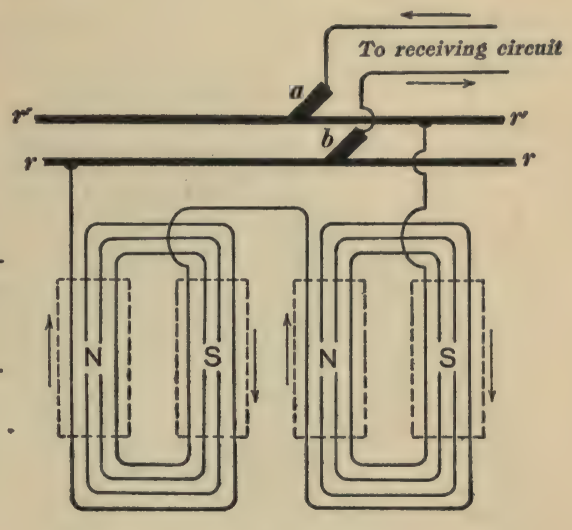

Fig. $3 b$.

It is the relative motion, only, of armature and field magnet which is essential in any dynamo, and alternators are frequently made with a revolving field magnet, the outwardly projecting poles of which NSNS, Fig. 4, sweep past the stationary armature

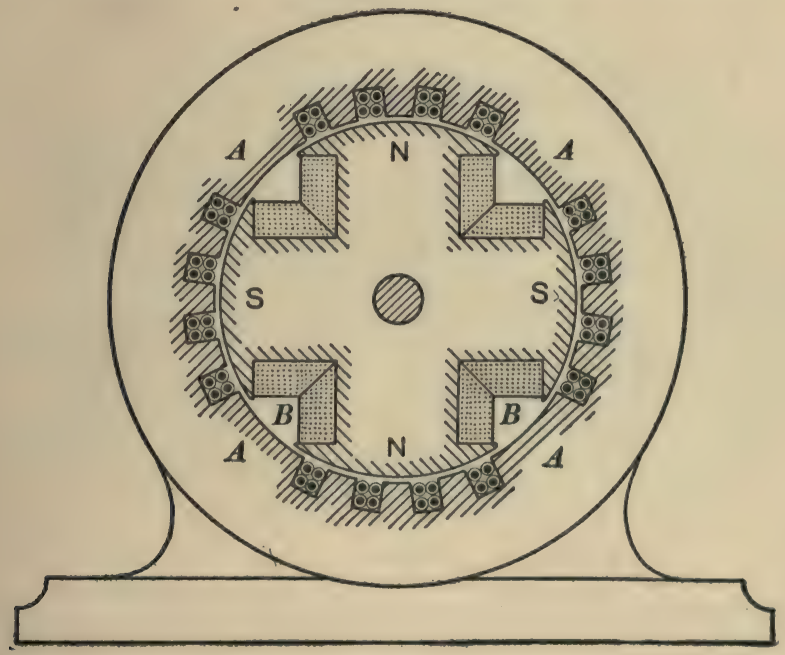

Fig. 4. 
wires which are placed in slots around the inner periphery of a ring-shaped structure of laminated iron $A A$. Figure 4 shows sixteen armature slots in each of which four wires are placed.

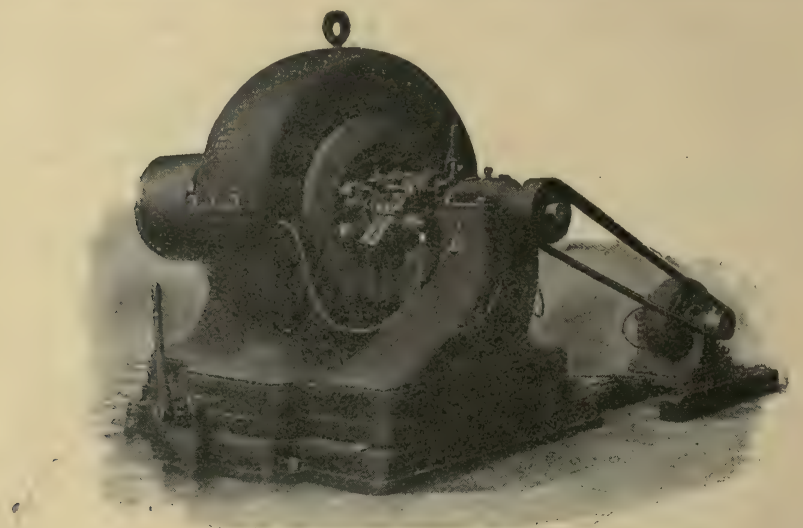

Flg. 5. Revolving-armature alternator with belted exciter.

In the revolving field type of alternator, the direct current for exciting the revolving field magnet is delivered to the rotating structure by brushes which rub on two insulated metal rings; and

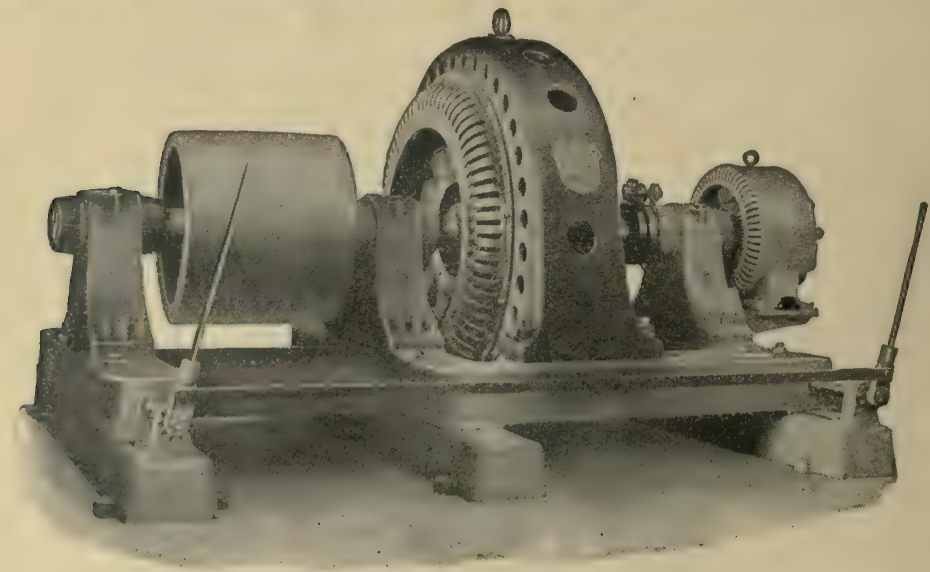

Fig. 6. Revolving-field alternator with direct-connected exciter.

the terminals of the stationary armature winding are connected directly to the receiving circuit. 
The revolving-armature type of alternator is generally used in small electric lighting stations. Figure 5 shows an alternator of this type manufactured by the Fort Wayne Electric Works. The figure shows the small belt-driven exciter, and it shows, although somewhat indistinctly, the rectifying commutator through which

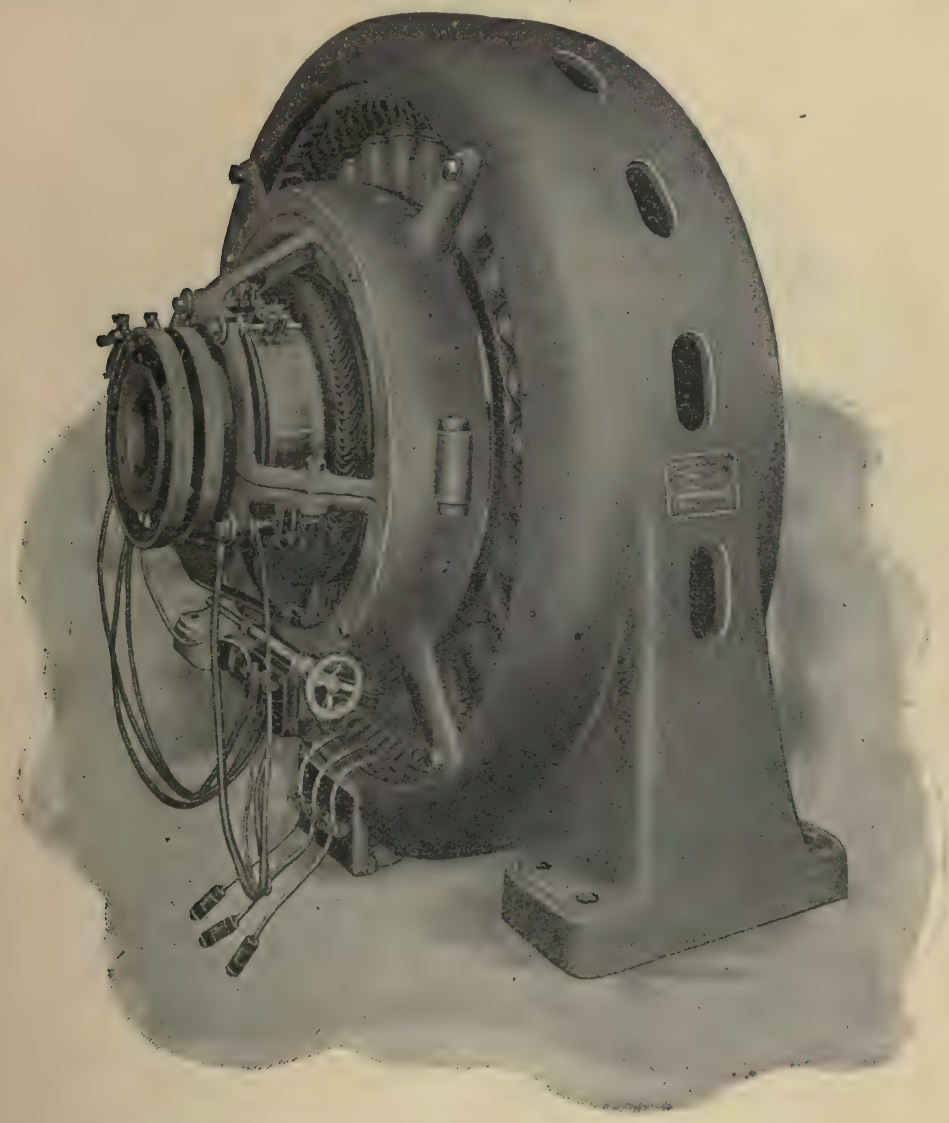

Fig. 7. Revolving-field alternator with direct-connected exciter.

a series field winding is connected in the main circuit of the machine as explained in Art. 2.

Very large alternators are usually of the revolving-field type. A small belt-driven machine of this type, built by the Allis- 
Chalmers Company, is shown in Fig. 6. A small machine of this type manufactured by the General Electric Company and arranged for mounting on the shaft of the driving engine or water-wheel, is shown in Fig. 7. Figure 8 shows the revolving field magnet of this machine, and Fig. 9 shows one of the laminated pole pieces and one of the field coils. This field coil is made of heavy copper strap wound edgewise and insulated by a

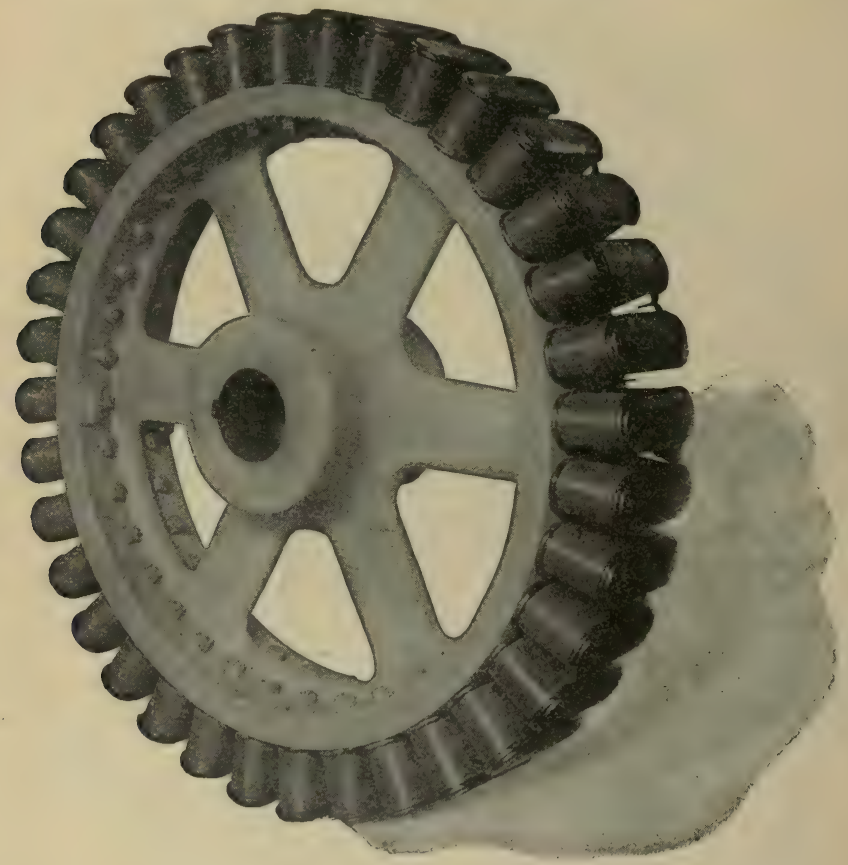

Fig. 8.

paper strip laid between the turns of the copper strap. The two collector rings shown in Figs. 6 and 7 serve for the delivery of direct current to the revolving field magnet, and the stationary armature terminals are connected directly to the receiving circuits in each case.

Figure 10 is a view of a portion of the revolving field magnet, and Fig. I I shows a portion of the inner face of the stationary 
armature of a 5,000 kilowatt alternator manufactured by the Westinghouse Electric and Manufacturing Company. This alternator is mounted on the crank shaft of a 7,000 horse-power steam engine having a speed of 75 revolutions per minute.

Alternators of very large capacity, but comparatively small in size on account of the high speed of driving, are now extensively used in conjunction with steam turbines. These alternators are always of the revolving-field type and their peculiar features are the small number of field magnet poles, the wide gap space between armature core and field poles, and the extreme solidity of the field magnet structure. Thus Fig. I 2 shows, to a scale of

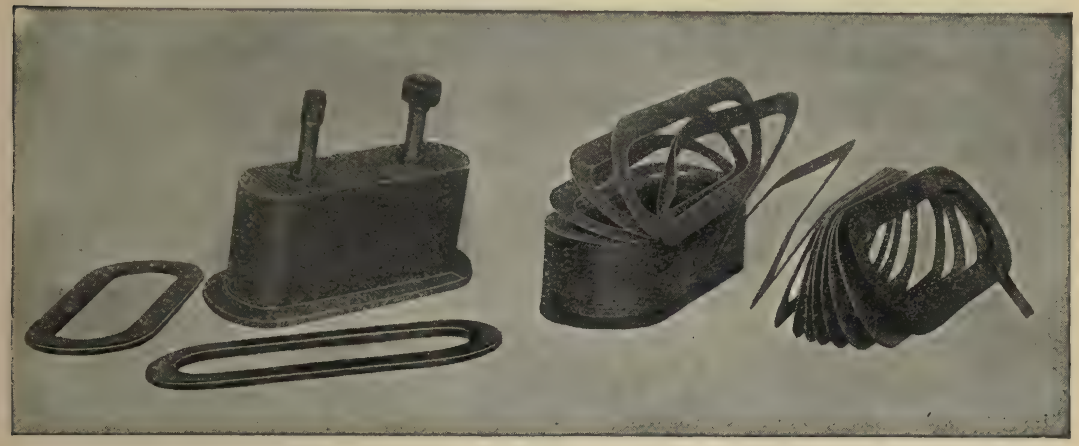

Fig. 9.

about I to 20 , the revolving field magnet of a 5,000 kilowatt alternator which is mounted on the shaft of a 7,500 horse-power steam turbine and driven at a speed of 500 revolutions per minute.

Besides the revolving armature type and revolving field type, there is a third type of alternator, called the inductor alternator in which the iron field structure alone rotates, both the field winding and the armature being stationary. The rotating iron structure NNSS, Fig. I 3, is called the inductor, and it is magnetized by a large stationary coil $B B$ which encircles it. The magnetic flux produced by this coil passes from the polar projections $N N N N$ into a laminated structure $A A$, through the iron rods $R R$ to the other laminated structure $A A$, and thence to the polar 
projections SSSS as shown by the dotted lines in Fig. I 3. The laminated structures $A A$ and $A A$ are slotted on their inner faces to receive the armature wires across which the lines of force sweep when the inductor is rotated.

Single-phase and polyphase alternators. - The details shown in

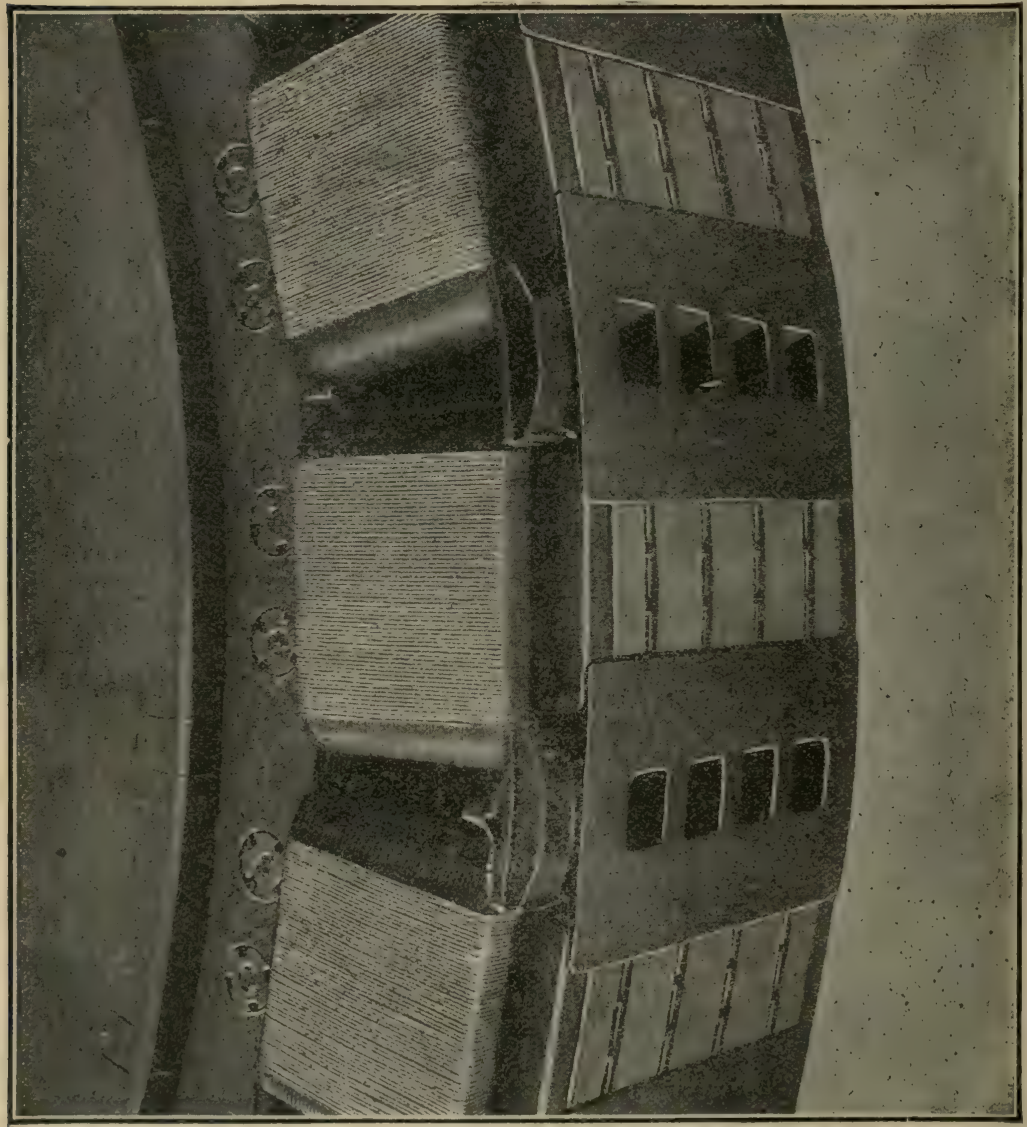

Fig. 10.

Figs. I, 2, 3 and 4 refer to what is called a single-phase alternator. A polyphase alternator is an alternator upon the armature of which two or more entirely distinct windings are placed, each 
winding being arranged to deliver current to a separate receiving circuit. Thus the armature winding shown in Fig. I I is a twophase winding. The coils which are square on the ends to the right constitute one winding and the coils which bend backwards and pass behind the square ends constitute the other winding. Polyphase alternators are discussed in Chapter VII.

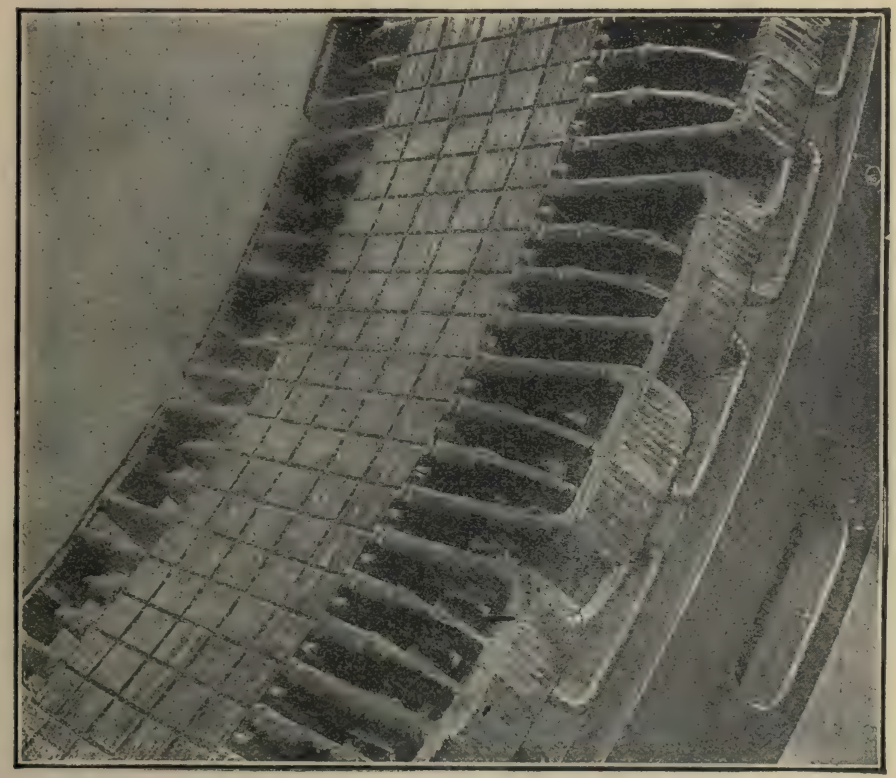

Fig. 11.

Concentrated and distributed armature windings. - The type of armature winding in which all of the wires on the armature are grouped in $p$ slots, where $p$ is the number of field magnet poles, is called a concentrated armature winding. The slots for a concentrated armature winding are usually rather large and each slot contains a bundle of wires. Figure 2 shows a concentrated armature winding with one wire per slot.

The type of armature winding shown in Figs. 3 and 4 , in which the wires on the armature are spread out in $p$ similar groups of slots, where $p$ is the number of field magnet poles, is 
called a distributed winding. Thus each of the armature windings of the two-phase alternator shown in Fig. I I is distributed in $2 p$ slots, that is in two slots per field pole.

In the concentrated winding, the electromotive force rises suddenly in value as the slots come under the pole tips, and falls suddeniy in value as the slots pass out from under the pole pieces,

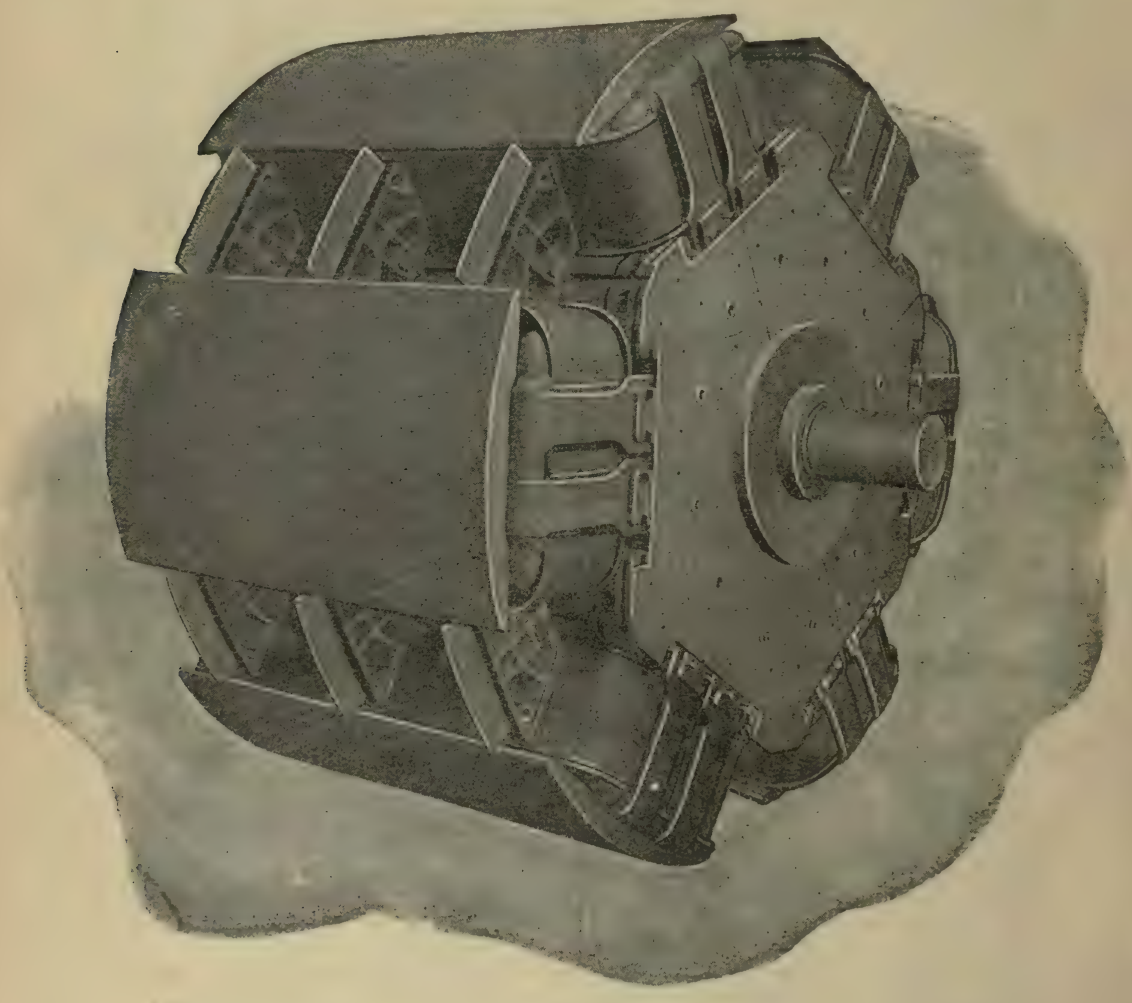

Fig. 12.

whereas in the distributed winding the electromotive force rises and falls more gradually.

2. Field excitation of the alternator. - Usually the field magnet of an alternator is excited by direct current obtained from a small auxiliary direct-current generator called the exciter. When 
the field magnet of an alternator is so excited, there is a tendency for the electromotive force between the terminals of the alternator to fall off with increase of current output, so that it is necessary to have a switch-board attendant to adjust the exciting current with changes of load on the alternator in order to keep the terminal voltage of the alternator constant.

In the case of a direct-current shunt generator, the variations of terminal voltage with change of current output may be counteracted by using an auxiliary (series) field winding through which the current delivered by the generator flows, thus provid-
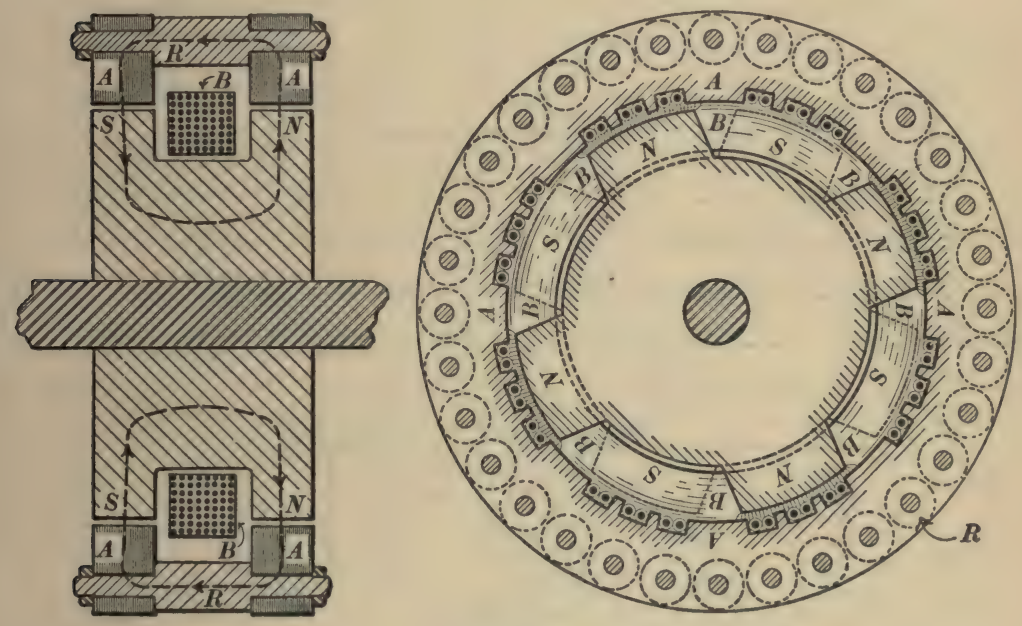

Fig. 13.

ing for an automatic increase of field excitation with increase of load. A direct-current generator provided with both shunt and series field windings is called a compound generator.

In order to make use of the current delivered by an alternator for automatically increasing its field excitation with increase of current output, it is necessary to interpose a device for reversing the connections of the series field windings at each reversal of the alternating current so that the current may flow always in the same direction in the series field coil and thus have the desired 
magnetizing action. This reversing device is called a rectifier; and an alternator provided with a rectifier and a series field winding is called a composite or compound alternator.

The essential features of the connections of a compound alter-

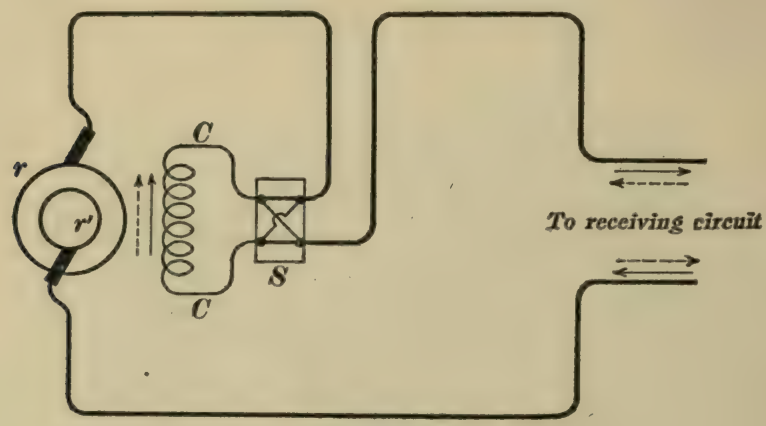

Fig. 14.

nator are shown in Fig. I4. The series field winding $C C$ is connected into the main alternating-current circuit through the rectifier, $S$, which is essentially a reversing switch, and the cur-

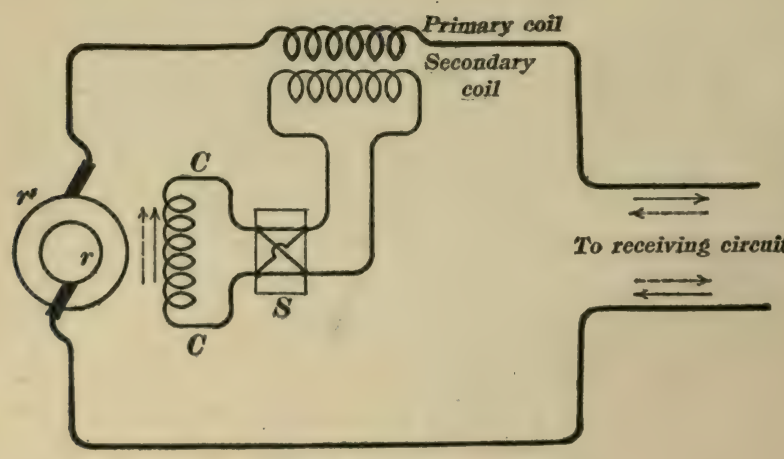

Fig. 15.

rent in $C C$ flows always in one direction although the current in the main circuit is an alternating current.

Instead of tapping the coil $C C$ into the main circuit, it is usually connected to the secondary coil of a small transformer 
whose primary coil is in the main circuit, as shown in Fig. I5. This is equivalent in its effect to the insertion of the coil $C C$ directly into the main circuit as shown in Fig. I4.

Figure I6 shows the actual arrangement of the compound alternator. The small transformer, the collecting rings $r r^{\prime}$, and the rectifier $S$ are all mounted together on the armature shaft. The small transformer is in fact usually wound upon a laminated spoke of the armature core. The alternating current flows through the primary coil of the small transformer before it reaches the collecting ring $r$.

The rectifier consists of a commutator having $p$ insulated segments, where $p$ is the number of field magnet poles of the alternator. The odd numbered segments are connected together and to one terminal of the secondary coil of the small transformer, and the even numbered segments are connected together and to the other terminal of the second-

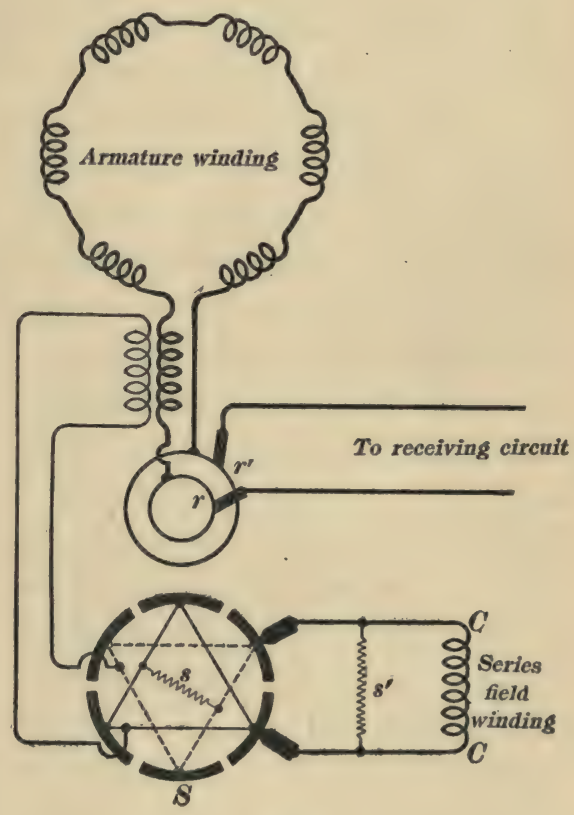

Fig. 16. ary coil of the small transformer. Two brushes, at a distance apart equal to the distance ${ }^{*}$ from center to center of adjacent commutator segments, rub on the commutator and deliver rectified current to the series field winding as shown.

It is necessary to shift the rectifier brushes back and forth until the position is found which gives minimum sparking. Then the

* Or any odd multiple of this distance. 
brushes will be passing from one segment to the next at the instants when the alternating current is passing through the zero value. The sparking, however, is serious at best, and in order to reduce this sparking, it is necessary to connect resistances $s$ and $s^{\prime}$ as shown. Sometimes one only of these resistances, generally $s^{\prime}$, is used. Thus, in the compound alternator which is shown in Fig. 5, the brushes of the rectifier are connected to the series field winding and also to a resistance which is mounted inside of the bearing pedestal of the machine.

The Tirrell field regulator. - The complications involved in the compounding of an alternator, as above explained, add to the cost of the machine, the compounding cannot be adjusted to compensate completely for variations of the terminal voltage, especially when the alternator supplies current to inductive circuits, and the rectifying commutator becomes very troublesome when it is attempted to use it to rectify the large current output of a large alternator. It is therefore not usual to compound large alternators.

Voltage control of large alternators is usually accomplished by a switchboard attendant, although an automatic regulator, known as the Tirrell regulator,* is now coming into use, especially for alternators of medium size. This regulator is essentially an automatic field rheostat which is adjusted, not by varying the amount of the resistance in circuit, but by repeatedly short-circuiting a fixed resistance, the duration of each short-circuit being varied automatically. Thus if a resistance of Io ohms is in circuit with a field coil for one-third of the time, the effect is the same as if $3 \mathrm{I} / 3 \mathrm{ohms}$ were in circuit continuously, provided that the $10 \mathrm{ohms}$ is short-circuited repeatedly during very short intervals of time.

3. Advantages and disadvantages of alternating current. - The electrical transmission of a given amount of power may be accomplished by a large current at low voltage or by a small current at high voltage. In the first case very large and expensive transmission wires must be used or the loss of power in the trans-

* See General Eléctric Company's Bulletin, No. 4,351, July, 1906, for a detailed description of the Tirrell regulator. 
mission line will be excessive. In the second case comparatively small and inexpensive transmission wires may be used without excessive loss of power. Therefore it is necessary to use high voltages in the long-distance transmission of power.

High voltages, however, are dangerous under the conditions which ordinarily exist among users of electric power, and many kinds of apparatus, such, for example, as incandescent lamps, require low voltages for satisfactory operation. Therefore power which is transmitted at high voltage must be transformed at the receiving station from high-voltage-and-small-current to lowvoltage-and-large-current if long-distance transmission is to be successful, and the advantage of alternating current over direct current lies almost wholly in the cheapness of construction, cheapness of operation, and high efficiency of the alternating current apparatus that is required for this transformation.

In the step-down transformation of direct current, a motor takes a small current from the high voltage transmission lines and drives a generator which delivers a large current at low voltage to the service mains. This apparatus is expensive to construct, it requires constant attention, and its efficiency is never greater than about 90 per cent.

The step-down transformation of alternating current is accomplished by means of the alternating current transformer, which is very much cheaper to construct than a motor-generator of the same capacity, it requires no attention, and its efficiency under full load is usually greater than 97 per cent. The alternating current transformer is described in Chapter X.

The alternating-current system has a slight advantage over the direct current system because the alternator, having no commutator, is somewhat simpler than the direct-current generator, and in the alternating-current system one may use the inductor alternator and the induction motor which have no sliding electrical contacts whatever.

Another advantage of the alternating-current system is that the current passes through zero value many times per second so 
that a circuit breaker operates more effectively on an alternatingcurrent circuit than it does on a direct-current circuit. Indeed, it is claimed that large generating stations, such as those at Niagara Falls, could not be operated at all if it were not for this peculiar effectiveness of the circuit breaker on an alternating current circuit.

These advantages of the alternating current are to some extent balanced by certain disadvantages which are outlined in the following paragraphs. Where power is to be transmitted over short distances, the one great advantage of the alternating current system vanishes, and the direct current system is distinctly the better of the two, especially if a central station is to supply current, not only for lighting, but also for driving motors and for charging storage batteries.

Alternating-current lighting. The electric lamp depends fundamentally upon the heating effect of the electric current, and insofar as the mere heating effect is concerned the alternating current is as satisfactory as the direct current. There is a tendency, however, for an alternating-current lamp to give an intermittent light because of the cooling of the luminous element between the successive pulses of current. The intermittent character of the light is especially marked in alternating-current arc lamps, and glow lamps give a perceptibly intermittent light at low frequencies, especially if the lamp filament is very fine.

The electric arc, too, is peculiarly unstable when it is operated by alternating current, and certain types of arc lamps, some of the luminous-arc lamps for example, cannot be operated by alternating current. Furthermore the tantalum filament glow lamp cannot be operated satisfactorily by alternating current.

On the other hand the Nernst lamp operates very much better with alternating current than with direct current.

Alternating-current motors. The simple alternating current is less satisfactory for motor driving than direct current. The ordinary direct-current series motor can, however, be driven by alternating current if it is properly designed. When so designed 
it is called a single-phase commutator motor. This motor is described in Chapter XIV.

The alternator can be operated as a motor if it is started and brought up to exactly the proper speed by an independent source of power. When so used the alternator is called a synchronous motor. The synchronous motor is described in Chapter VIII.

The induction motor, which is described in Chapters XII and XIII, must be arranged to operate as a commutator motor at starting, or it must be supplied with two or more distinct alternating currents transmitted to the motor over distinct transmission lines. The latter arrangement is called the polyphase system of transmission and it is described in Chapter VI.

Conversion of alternating current into direct current. For many electrochemical processes, direct current only can be used. Thus, storage batteries cannot be charged by alternating current. Furthermore, direct current is quite generally used for operating street railways. When power is transmitted by the alternatingcurrent system and utilized in the form of direct current, the conversion from alternating current to direct current is usually accomplished on a large scale by means of the synchronous converter, or rotary converter, as it is often called. The synchronous converter is described in Chapter IX.

The conversion from alternating current to direct current is sometimes accomplished on a large scale by an alternating-current motor driving a direct-current generator.

The conversion of alternating current into direct current can be accomplished on a small scale by the mechanical rectifier or by the mercury-arc rectifier. The mechanical rectifier is described in Art. 2 and the mercury-arc rectifier is described in Chapter IX.

4. Characteristics of alternating current motors. A general idea of the mechanical characteristics of the various kinds of alternating-current motors is necessary for a clear appreciation of the advantages and disadvantages of alternating currents, inas- 
much as the greatest disadvantages of the alternating-current system relate to motor operation, and especially to the problem of starting and the problem of speed control.

(a) The synchronous motor requires an auxiliary source of mechanical power to start it, or indeed it may be started and brought up to speed by inherent but very weak induction motor action. The mechanical load of a synchronous motor must usually be connected to the motor after the motor is in operation at full speed.

Once in operation, however, the synchronous motor runs at absolutely constant speed, frequency of alternating-current supply being constant, its efficiency is quite high, and its power-factor may be adjusted at will by varying its field excitation. The synchronous motor is often used on account of its adjustable powerfactor in order to compensate for lagging current taken by induction motors.

The synchronous motor is not suitable for variable speed service, and it is used only where frequent starting is not required.

(b) The polyphase induction motor starts readily under load and it runs at nearly constant speed, very much like a direct-current shunt motor which is driven from a constant voltage supply. The speed of an induction motor cannot be varied except by completely altering the arrangement of its stator winding, or by inserting resistance in circuit with its rotor windings. The first method requires a specially designed motor with an elaborate arrangement of switches for quickly altering the winding connections, and, under practical conditions, it gives but two speeds. The second method is unsatisfactory, because it means a considerable loss of power, because it gives a speed which varies greatly with the motor load, and because it necessitates the use of collector rings on the rotor. The induction motor is not suitable, therefore, for variable speed service.

(c) The single-phase induction motor to be self-starting must be arranged to start either as a repulsion motor or as a polyphase induction motor. In the former case the motor may develop 
sufficient torque to start under load, in the latter case the motor is usually supplied by two phases of current by splitting the single-phase supply as described in Chapter XII, and the starting torque is very small. The single-phase motor of the Wagner Electric Company is a single-phase induction motor which starts as a repulsion motor.

The single-phase induction motor is not suitable for variable speed service.

(d) The single-phase commutator motor has the same characteristics as the series motor operated by direct current, and it is the only alternating-current motor which operates satisfactorily throughout a wide range of speed. The single-phase commutator motor is beginning to be used extensively in railway service where a large starting torque and fairly efficient operation throughout a wide range of speed are required.

5. The physical basis of the differences between direct-current calculations and alternating-current calculations. - Figure I 7 represents a valveless pump, $P$, the piston of which oscillates up and down causing a current of water to surge back and forth through a circuit of pipe, and Fig. I 8 represents an alternator $A$ of which the alternating electromotive force causes an electric current to surge back and forth through a circuit of wire.

The alternating pressure generated by the pump in Fig. I7 must not only overcome the resistance which the current of water encounters in the pipe, but a large part of the pressure developed by the pump must be used to overcome the inertia of the water in the pipe, first in getting the current of water started and then in stopping the current and starting it again in the opposite direction. Similarly, the alternating electromotive force generated by the alternator in Fig. I 8 must not only overcome the electrical resistance of the wire, but a large part of the electromotive force developed by the alternator must be used to overcome the electrical inertia, or inductance, ${ }^{*}$ of the circuit of wire, first in starting the

* It is assumed in this text that the student is thoroughly familiar with the ideas of inductance and capacity as outlined in Appendix A. 
electric current and then in stopping it and starting it again in the opposite direction.

The alternating pressure generated by the pump in Fig. I9 must not only overcome the resistance of the pipe and the inertia

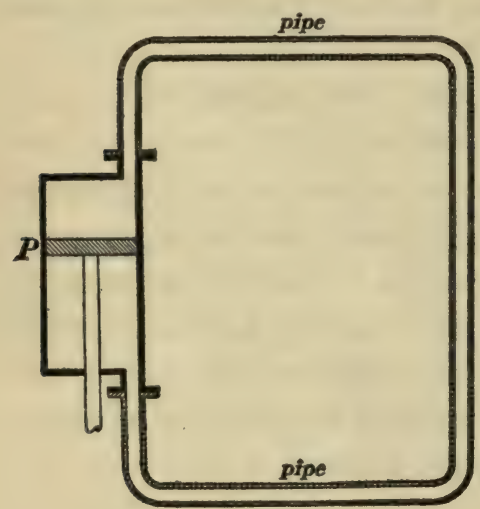

Fig. 17.

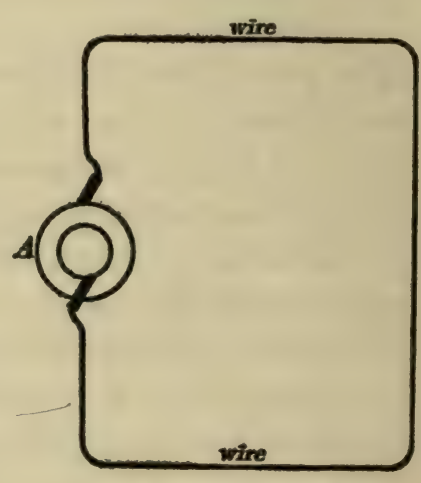

Fig. 18.

of the water in the pipe, but a portion of the pressure developed by the pump must be used to distort the elastic diaphragm $D D$ which bridges across the chamber $C C$. Similarly, the alternating

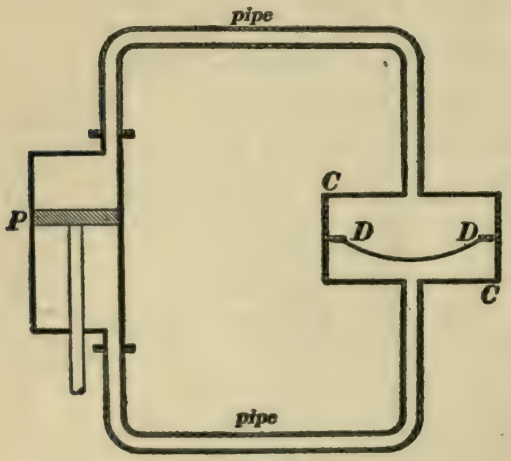

Fig. 19.

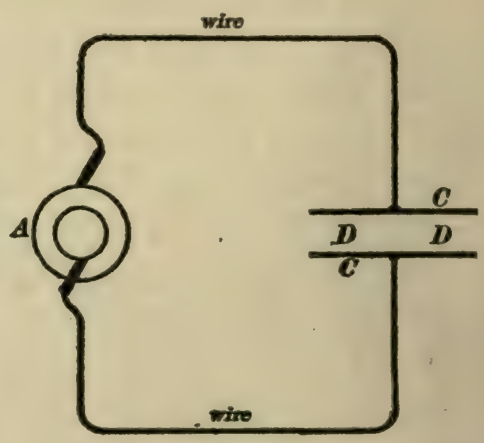

Fig. 20.

electromotive force generated by the alternator in Fig. 20 must not only overcome the electrical resistance of the wire and the electrical inertia, or inductance, of the circuit, but a portion of the 
electromotive force developed by the alternator must be used to produce the electrical stress which is created in the insulating material $D D$ between the metal plates $C C$ as these plates are electrically charged first in one direction (upper plate positive and lower plate negative) and then in the reverse direction (upper plate negative and lower plate positive).

The metal plates $C C$ Fig. 20 with the insulating material $D D$ between them constitute what is called a condenser, and the action of this condenser is exactly analogous to the action of the chamber $C C$, Fig. 19, with its elastic diaphragm $D D$. The elastic diaphragm permits an alternating current of water to surge back and forth through the circuit of pipe, but it does not permit the flow of a steady current of water. Similarly, the condenser $C C$, Fig. 20 , permits an alternating electric current to surge back and forth through the circuit of wire, but it does not permit the flow of a steady electric current.

Concentrated capacity and distributed capacity. - Figure 2 I represents a valveless pump which causes an alternating current of water to surge back and forth through a circuit of distensible rubber tube. When the piston starts upwards the rapidly increasing pressure in the portion $a$ of the rubber tube causes this portion of the tube to swell, and the rapidly decreasing pressure in the portion $b$ of the rubber tube causes this portion of the rubber tube to shrink. The swelling and the shrinking of the rubber tube extends with decreasing intensity to the middle point $c$, as shown by the tapering of the tube, and as indicated by the positive and negative signs of decreasing size. One result of the elasticity of the tube is that the whole of the water current which enters the tube at $a$ does not flow around to $b$, and the whole of the water current that enters the pump at $b$ does not flow around from $a$, but the value of the current (amount of water passing a point of the tube per second) decreases from $a$ to $c$ and increases from $c$ to $b$. The current of water does not have the same value all along the circuit of tube. Similarly, when the alternator in Fig. 22 starts a pulse of electric current in the direction of the 
arrow, a large current enters the wire at $a$, part of this current goes to charge the wire positively, and the current decreases in value towards $c$; and the current which enters the alternator at $b$ does not flow all the way around from $a$ but part of it comes from the wire and leaves the wire negatively charged. The action is analogous in every detail to the action which takes place in Fig. 2 I.

In Fig. I9 the only appreciably elastic element is the diaphragm $D D$, whereas in Fig. $2 \mathrm{I}$ the entire circuit of pipe is elastic. In Fig. 20 the only place where an appreciable amount

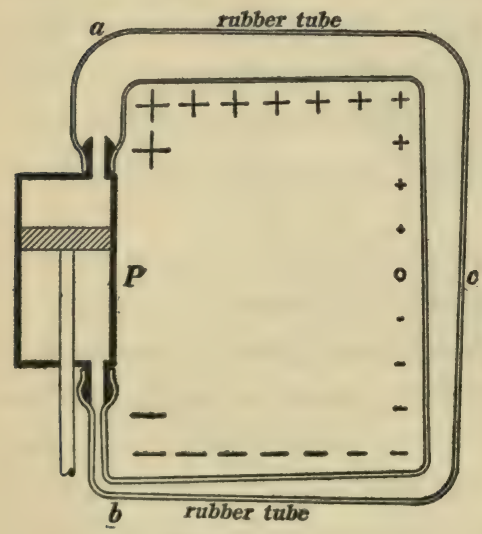

Fig. 21.

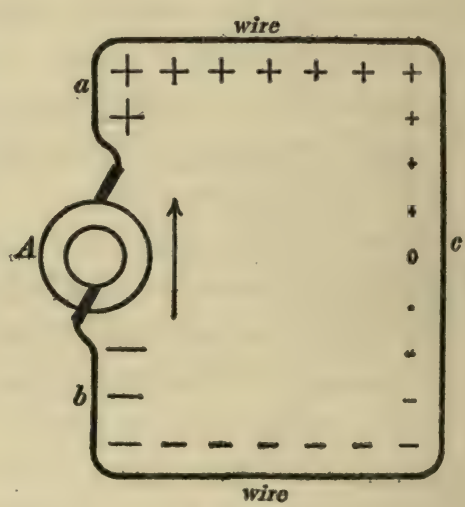

Fig. 22.

of electric charge accumulates is on the two metal plates $C C$, the amount of charge that accumulates on the wire being negligible. In Fig. 22 the amount of charge that accumulates on the wire is supposed to be appreciable; this is true only when the circuit of wire is very long, as in a long transmission line, and in this case an appreciable charge accumulates all along the wires. The metal plates in Fig. 20 constitute what is called a concentrated capacity, and the wire in Fig. 22 constitutes what is called a distributed capacity.

The effects of distributed capacity are exceedingly complicated and they are always ignored in ordinary alternating-current prob- 
lems. The effects of distributed capacity cannot be ignored, however, in long transmission lines, and therefore the mathematical treatment of the transmission-line problem stands by itself in the theory of alternating currents.

Direct-current problems and alternating-current problems contrasted. Direct-current problems are generally the finding of one or two of the quantities, voltage, current and power, when the other or others are given, and when the circuit conditions are known. Alternating-current problems also are generally the finding of one or two of the quantities, voltage, current and power, when the other or others are given, and when the circuit conditions are known. The difference between direct-current problems and alternating-current problems grows out of the fact that in an alternating-current circuit the relation between voltage, current and power, depends upon the resistance, the inductance and the capacity in the circuit, whereas, in a direct-current circuit, the relation between current and voltage is independent of inductance and capacity.

The relation between voltage and current in an alternatingcurrent circuit can be simply formulated only for the ideal case in which the voltage and current are of the type which is represented by a curve of sines and when the capacity is concentrated. Therefore in most alternating-current problems the voltage and current are assumed to be of this type, which is called harmonic, and the effects of distributed capacity are ignored. The conception of harmonic voltage and current is developed in Chapter III, the voltage-current relation (harmonic) is discussed in Chapter $\mathrm{IV}$, and this ideal voltage-current relation is the basis for all of the theoretical developments in Chapters $\mathrm{V}$ to $\mathrm{XV}$.

A discussion of non-harmonic voltages and currents is given in Appendix B, and a discussion of the phenomena of distributed capacity is given in Appendix C.

6. Speed and frequency. The electromotive force of an alternator passes through a set of positive values while a given group 
of armature wires is passing a north pole of the field magnet, and through a set of negative values while the given group of armature wires is passing a south pole of the field magnet. The complete set of values, including positive and negative values, is called a cycle, the duration of a cycle is called a period and the number of cycles per second is called the frequency. If the field magnet of an alternator has $p$ poles $(p / 2$ north poles and $p / 2$ south poles), then

$$
f=\frac{p n}{2}
$$

in which $f$ is the frequency in cycles per second of the electromotive force which is developed by the alternator, and $n$ is the speed of the alternator in revolutions per second. This is evident when we consider that a complete cycle corresponds to the passage of a given group of armature wires across two field poles, a north pole and a south pole, so that there are $p / 2$ cycles in one revolution.

When north poles and south poles are similar in shape and size, with similar air gaps, which is usually the case in commercial alternators, the successive sets of positive and negative values of the electromotive force are alike. That is, a cycle is made up of a set of positive values of electromotive force and a similar set of negative values of electromotive force.

Synchronism. Two alternating electromotive forces or currents are said to be in synchronism when they have the same frequency. Two alternators are said to run in synchronism when their electromotive forces are in synchronism.*

7. Electromotive force and current curves. The successive instantaneous values of the electromotive force of an alternator may be represented by the ordinates of points on a curve, the corresponding abscissas representing elapsed time reckoned from some

* The term " to synchronize," as used by power-station men, has a double meaning. Thus, to synchronize an alternator preparatory to connecting it in parallel with another alternator, means not only to bring it into synchronism with the other alternator but also to bring about a certain phase relation between the two. 
chosen instant. Such a curve is called the electromotive force curve of the alternator. In a similar manner the successive instantaneous values of an alternating current may be represented by ordinates, and elapsed times by abscissas, giving a current curve. These curves are determined by means of the oscillograph, or by means of the contact maker as explained in Art. IO.

The shape of the electromotive force curve of an alternator depends upon the shape and distance apart of the pole pieces of the field magnet, and upon the distribution of the armature winding. This is exemplified in Figs. 23, 24 and 25. Figures 23 and 24 are given in duplicate to show the effect of the fringe of magnetic flux beyond the pole tips.

Figure 23 represents a single armature wire $a$ cutting the uniformly distributed flux under the pole pieces, and the resulting electromotive force curve is shown in the lower part of the figure. Figure $23 a$ represents the magnetic flux as stopping short at the pole tips, and Fig. $23 b$ shows the fringe of magnetic field which always exists beyond the pole tips.

Figure 24 represents a band of armature wires $a b$ cutting the uniformly distributed flux under the pole pieces, and the resulting electromotive force curve is shown in the lower part of the figure. Figure $24 a$ represents the magnetic flux as stopping short at the pole tips, and Fig. $24 b$ shows the fringe of magnetic field which always exists beyond the pole tips.

It is important to note that a certain armature wire must be chosen as an index point in Figs. 23, 24, and 25, and that the ordinate of the electromotive force curve which is directly beneath this chosen wire at a given instant as the wire moves to the right represents the value of the electromotive force at that instant. Thus the wire $a$ is chosen as the index point in Fig. 24, and wherever this wire may be at a given instant, that ordinate of the electromotive force curve which is immediately beneath $a$ represents the electromotive force at the given instant.

Fig. 25 is a more or less fanciful drawing which is intended to show how the shape and width of the pole pieces affect the shape 
of the electromotive force curve, and to show how the positive and negative sets of electromotive force values are unlike when the north poles of the field magnet are not similar to the south poles.

The electromotive force curves in Fig. 23 are of the shape that

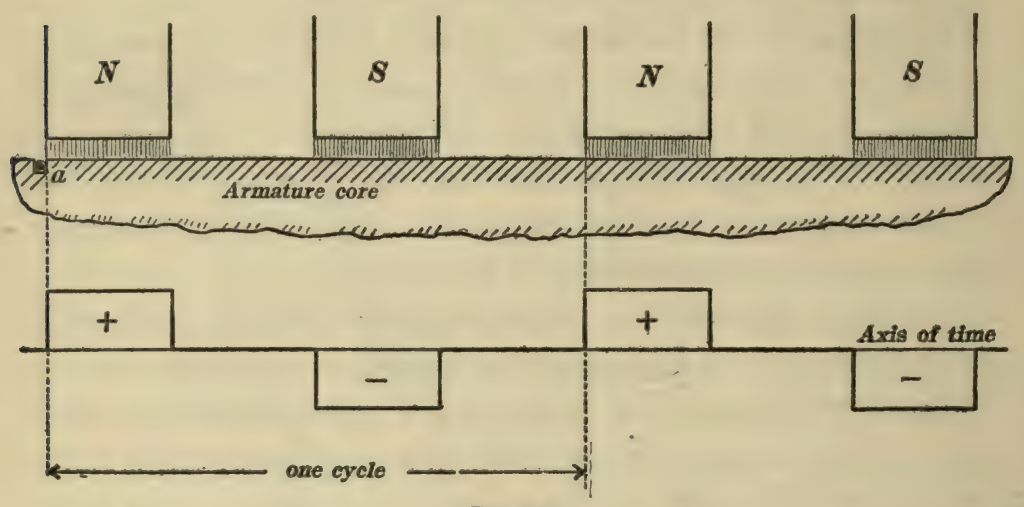

Fig. 23a.

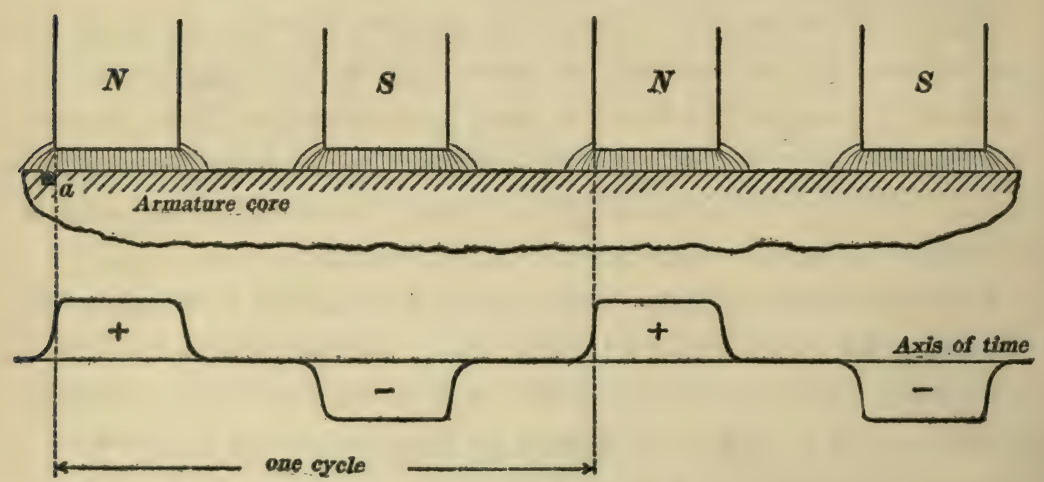

Fig. $23 b$.

is generally given by alternators with concentrated armature windings, and the curves in Fig. 24 are of the shape that is generally given by alternators with distributed armature windings. When, however, a concentrated armature winding is placed in very large slots, a very considerable reduction of total field flux occurs 
when these large slots come under the pole pieces, and in this case the pulsations of flux tend to modify the shape of the electromotive force curve, making it like the curve $e$ of Fig. 26.

Examples of current curves. - When an alternating electromotive force acts upon a circuit which contains resistance only, that is, a circuit which has a negligibly small inductance and which

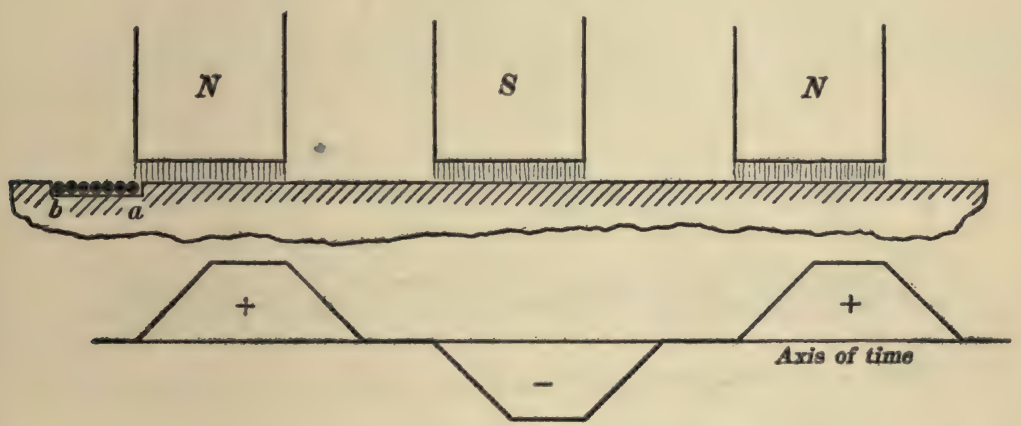

Fig. 24a.

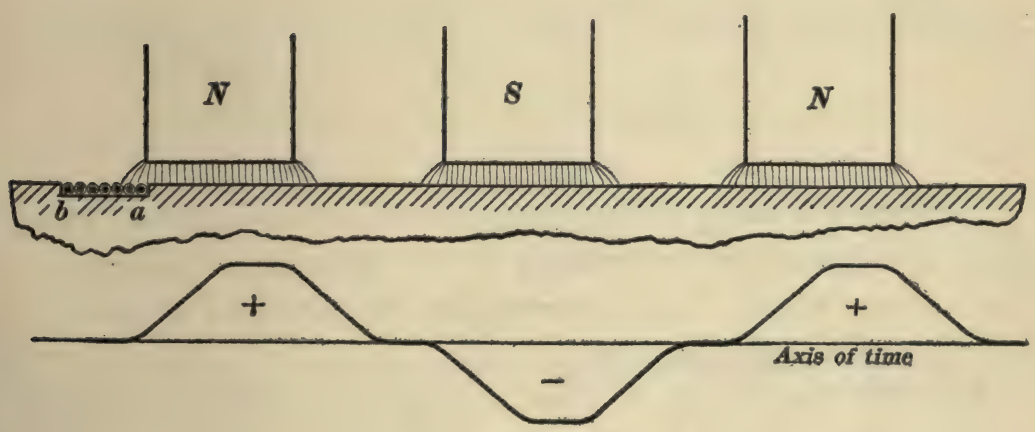

Fig.24b.

does not include a condenser, the current curve is of exactly the same shape as the electromotive force curve, inasmuch as the current is in this case equal at each instant to the electromotive force divided by the resistance of the circuit.

When an alternating-current circuit has an appreciable inductance (ironless), or when it contains a condenser, the current curve 
has the same shape as the electromotive force curve if the electromotive force curve is a curve of sines, but not otherwise ; and even when the electromotive force curve is a curve of sines, the current curve is displaced (shifted along the axis of time) with reference to the electromotive force curve. These voltage-current relations are discussed in detail in Chapter IV.

When an electromotive force or current curve is a curve of sines the electromotive force or current is said to be harmonic, otherwise the electromotive force or current is non-harmonic.
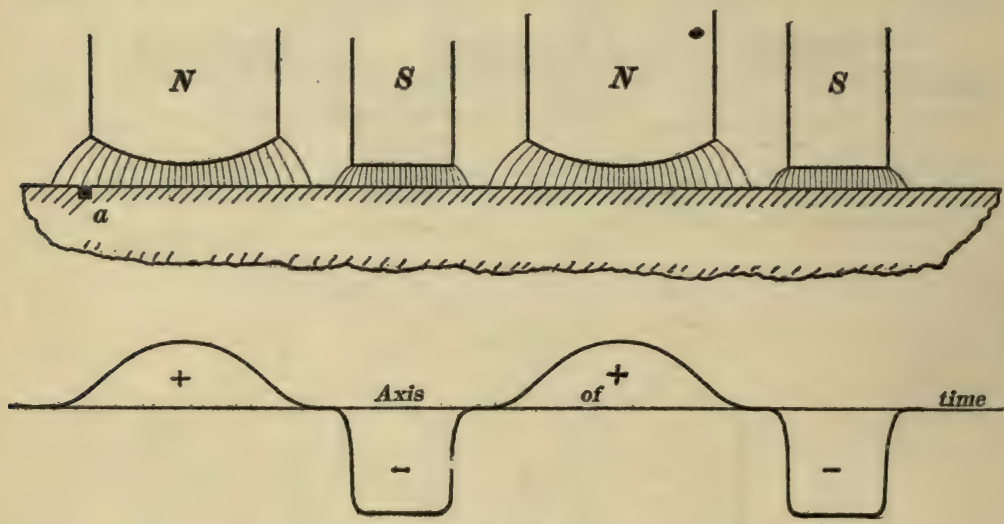

Fig. 25.

The full line curve in Fig. 26 represents a non-harmonic electromotive force, and the similarly shaped dotted curve represents the current which the electromotive force produces in a circuit which contains resistance only.

The full line curves in Figs. 27 and 28 represent harmonic electromotive forces; the dotted curve in Fig. 27 represents the harmonic current which the electromotive force produces in a circuit having resistance and inductance; and the dotted curve in Fig. 28 represents the harmonic current which the electromotive force produces in a circuit having resistance and containing a condenser.

It is to be noted that the maximum positive value of a current occurs after the maximum positive value of the electromotive 
force in Fig. 27, and before the maximum positive value of the electromotive force in Fig. 28. This is usually expressed by saying that the current lags behind the electromotive force in a circuit having resistance and inductance, and that the current leads

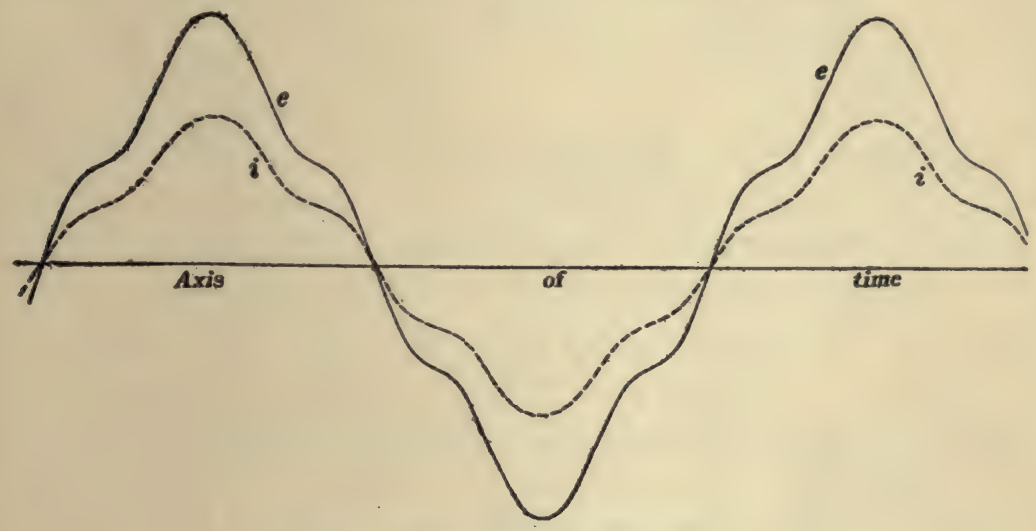

Fig. 26. Circuit containing resistance only.

or is ahead of the electromotive force in a circuit having resistance and containing a condenser.

A harmonic electromotive force does not always, however, pro-

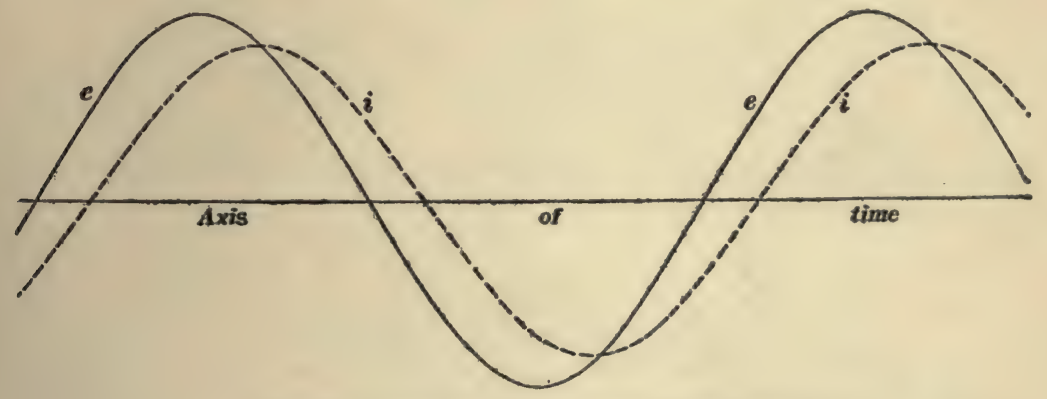

Fig. 27. Circuit having resistance and inductance.

duce a harmonic current. Thus Fig. 29 shows a harmonic electromotive force, and the current which this electromotive force produces in a coil of wire wound on an iron core. 
8. Average values and effective values of alternating electromotive force and current. - It is customary to speak of an alternating electromotive force as so many volts and of an alternating current as so many amperes, although a given alternating electromotive force or current is a rapidly varying quantity. Thus, an alternat-

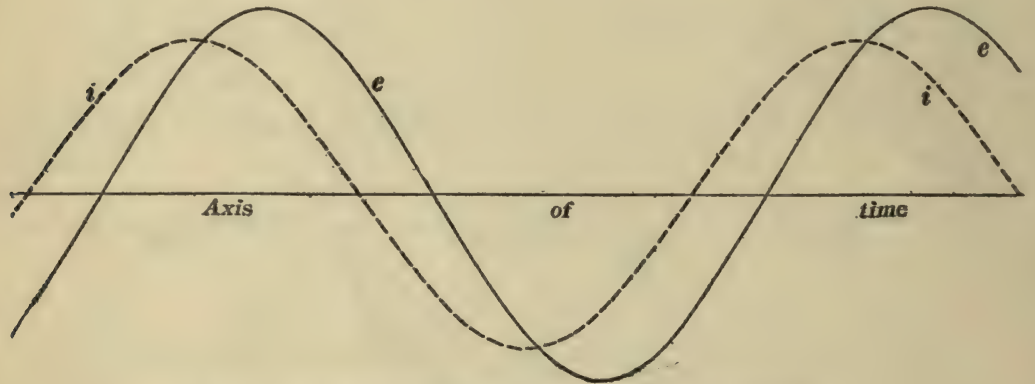

Fig. 28. Circuit having resistance and containing a condenser.

ing current which would be specified as Io amperes is, of course, a current which is alternating rapidly in direction and varying rapidly in value from zero to a more or less definite maximum. The specified value of an alternating electromotive force or current always refers to the square-root-of-the-average-value-of-the-square

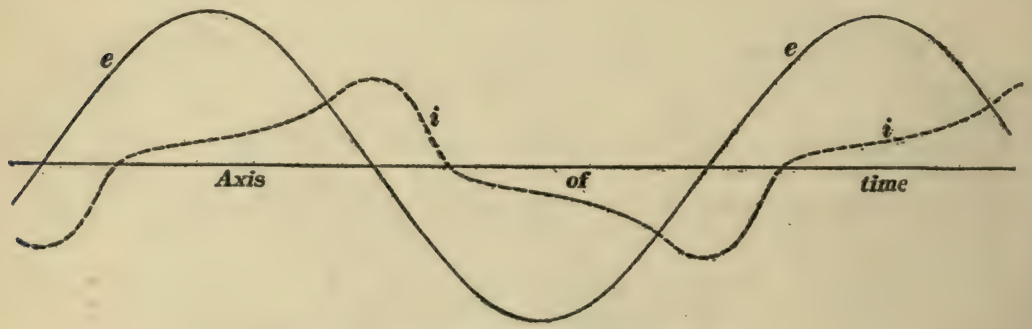

Fig. 29. Coil of wire wound on an iron core.

of the electromotive force or current, the so-called effective value, as explained below.

The simple average of the successive values of an alternating electromotive force or current is always zero. This is true even in a case like that shown in Fig. 25, and the only exception is in 
the case of the current which is produced by an alternating electromotive force through a circuit which allows current to pass in one direction but not in the other direction. The mercury-arc and the aluminum-valve rectifiers are examples of such circuits.

The simple average of the positive (or negative) values of an aiternating electromotive force or current during half a cycle is of course not zero.

Effective values. - Consider an alternating current of which the value at a given instant is $i$. During a cycle, $i$ passes through a set of positive values and a similar set of negative values, whereas $i^{2}$ is always positive. Therefore the average value of $i^{2}$ is not zero. The square root of the average value of $i^{2}$, which is called the effective value of the alternating current, is always used in specifying an alternating current in amperes. Thus an alternating current of 10 amperes is an alternating current of which the average value of $i^{2}$ is 100 amperes $^{2}$. An alternating electromotive force is also specified by giving its effective value. Thus an alternating electromotive force of $\mathrm{r}, 000$ volts is an alternating electromotive force of which the average value of the square is $\mathrm{I}, 000,000$ volts $^{2}$.

The principal reason for using effective values in the specification of alternating electromotive forces and currents is that the voltmeters and ammeters used in alternating-current measurements always give effective values, as explained in Chapter II. The term "effective value" originated as follows: Consider an alternating current of which the instantaneous value is $i$. The rate at which heat is generated at a given instant in a circuit through which this current flows is $R i^{2}$, where $R$ is the resistance of the circuit, and the average rate at which heat is generated in the circuit is $R$ times the average value of $i^{2}$, or $R I^{2}$, where $I^{2}$ is equal to the average value of $i^{2}$, or where $I$ is equal to the square-root-of-the-average-value-of $-i^{2}$. That is, to calculate the rate at which heat is generated in a circuit by an alternating current, the resistance is multiplied by the square of 
the effective value of the current, and it is for this reason primarily that the term "effective" value came into use.

Example. - The successive instantaneous values of an alternating electromotive force at equal intervals during a half-cycle are : $0,30,60,80,90,95,90,80,60$ and 30 volts. Dividing the sum of these voltages by their number, 10 , gives 61.5 volts which is the average value of the alternating electromotive force during the half-cycle. Squaring each of these voltages, adding these squares and dividing by their number gives the average value of their squares, namely, 4,702.5 volts ${ }^{2}$, and the square root of this average square is the effective value of the alternating electromotive force, namely, 68.6 volts.

9. Instantaneous and average power delivered by an alternator. Let $e$ be the value, at a given instant, of the electromotive force of an alternator and $i$ the value at the same instant of the current delivered by the alternator; then $e i$ is the power in watts which is delivered by the alternator at the given instant, and the average value of $e i$ is the average power delivered by the alternator.

When the alternator delivers current to a circuit which has resistance only, the average value of $e i$ is equal to the effective value of the alternating electromotive force multiplied by the effective value of the alternating current ; but in general the average power delivered by an alternator (average value of $e i$ ) is less than the product of effective voltage and effective current. This matter is discussed in Chapters II and III.

Examples. The full-line curve in Fig. 30 is the electromotive force curve of an alternator, the dotted curve is the current curve for the case in which the alternator delivers current to a circuit which has resistance only, and the ordinates of the dot-dash curve represent the successive instantaneous values of the power $e i$ delivered by the alternator. In this case the values of $e i$ are all positive but they vary from zero to a certain maximum value. That is to say, the alternator delivers power in pulses, one for each half-cycle of electromotive force and current. 
The full-line curve in Fig. $3 \mathrm{I}$ is the electromotive force curve of an alternator, the dotted curve is the current curve for the case in which the alternator delivers current to a circuit which has both resistance and inductance, and the ordinates of the dot-

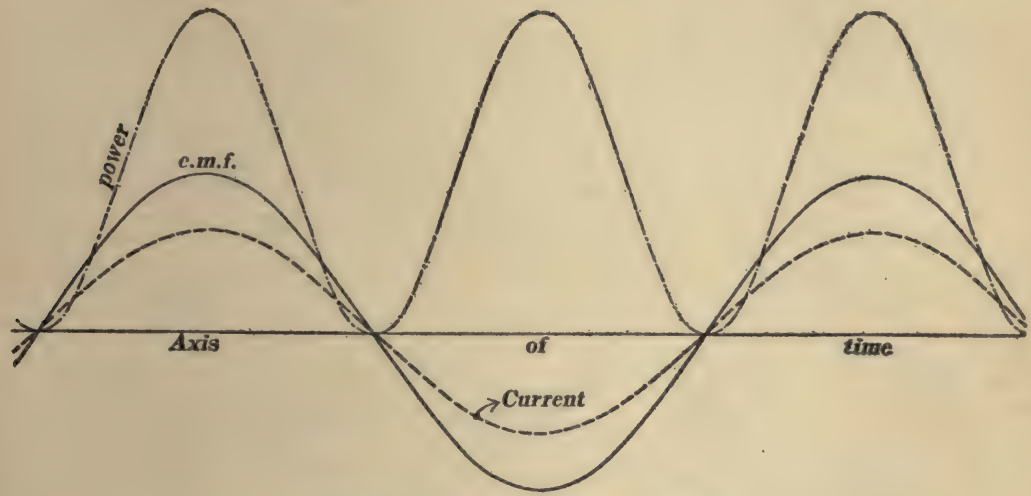

Fig. 30. Current in phase with voltage.

dash curve represent the successive instantaneous values of the power $\epsilon i$ delivered by the alternator. In this case there are two pulses of power delivered by the alternator to the receiving

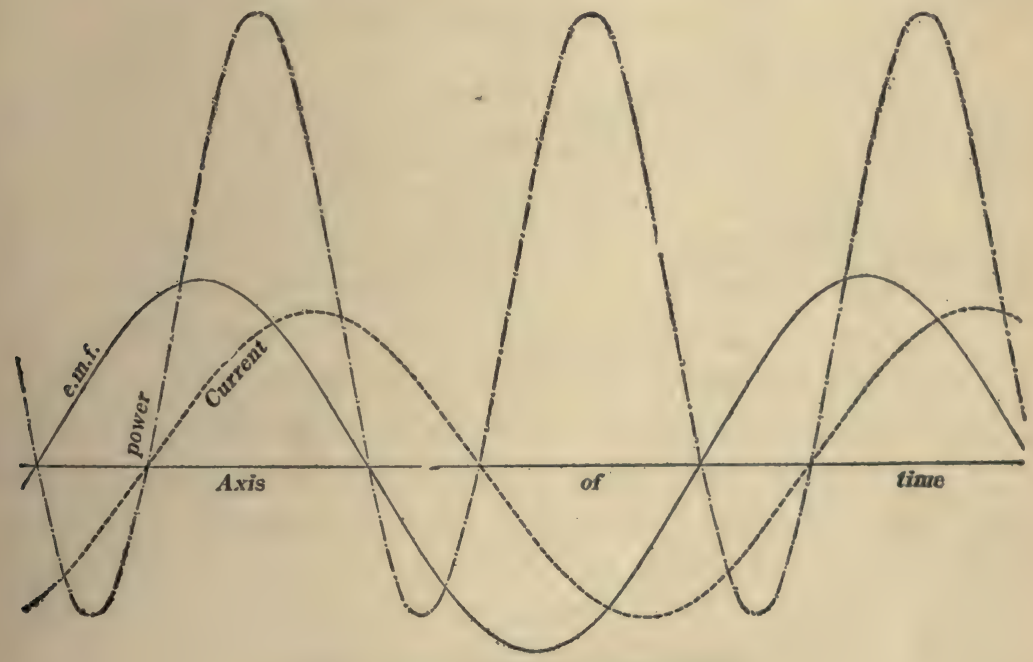

Fig. 31. Current lagging $60^{\circ}$ behind voltage. 
circuit ( $i$ positive), and two smaller pulses of power taken back by the alternator from the receiving circuit ( $e i$ negative) during each complete cycle of electromotive force and current.

The full-line curve in Fig. 32 is the electromotive force curve of an alternator, the dotted curve is the current curve for the case in which the alternator delivers current to a circuit which has inductance but no appreciable resistance, and the ordinates of the dot-dash curve represent the successive instantaneous values of $e i$. In this case there are two pulses of power delivered by

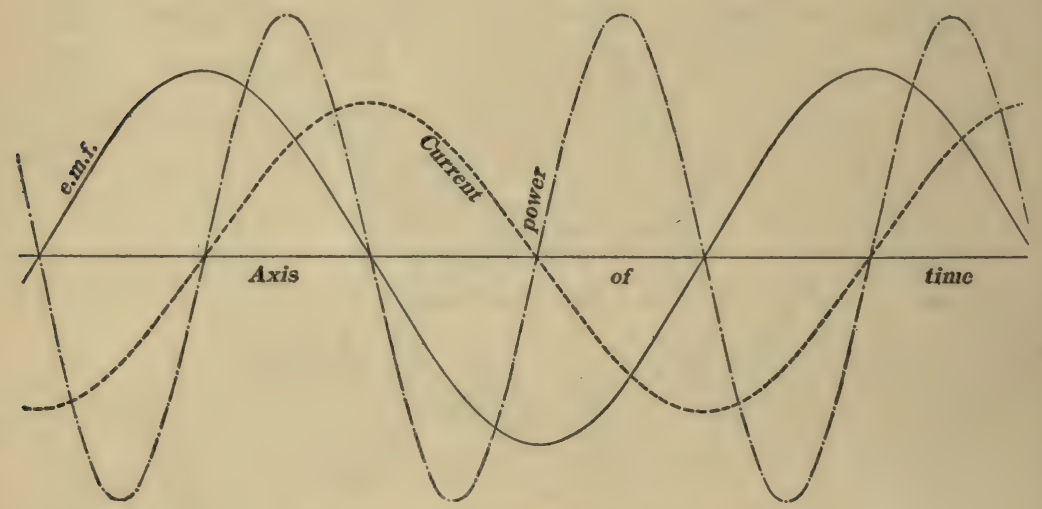

Fig. 32. Current lagging $90^{\circ}$ behind voltage.

the alternator and two similar pulses of power taken back by the alternator from the receiving circuit during each complete cycle. In this case, therefore, the average value of $e i$ is zero. A mechanical analogue of this third case is afforded by the movement of the balance wheel of a watch, assuming it to be frictionless. The hair spring does work upon the wheel setting it in motion, and the spring takes all of the energy back again in stopping the wheel. Then work is delivered to the wheel once more in reversing its motion, and this energy is all taken back by the spring as the wheel returns to its starting point, thus completing one cycle of the motion during which two pulses of power have been delivered to the wheel by the hair spring, and two similar pulses of power have been taken back from the wheel. 
10. The determination of electromotive force and current curves. - (a) By the contact maker. The contact maker, whose essential features are shown in Fig. 33, is a device for repeatedly connecting a large condenser, $C$, to the terminals of an alternator at a certain instant in an electromotive force cycle, thus keeping the condenser charged up to the voltage $e$ that exists at the given instant in the cycle, in order that the value of $e$ may be measured by a voltmeter $V$.

A disk of insulating material is fixed to the armature shaft of the alternator of which the electromotive force curve is to be de-

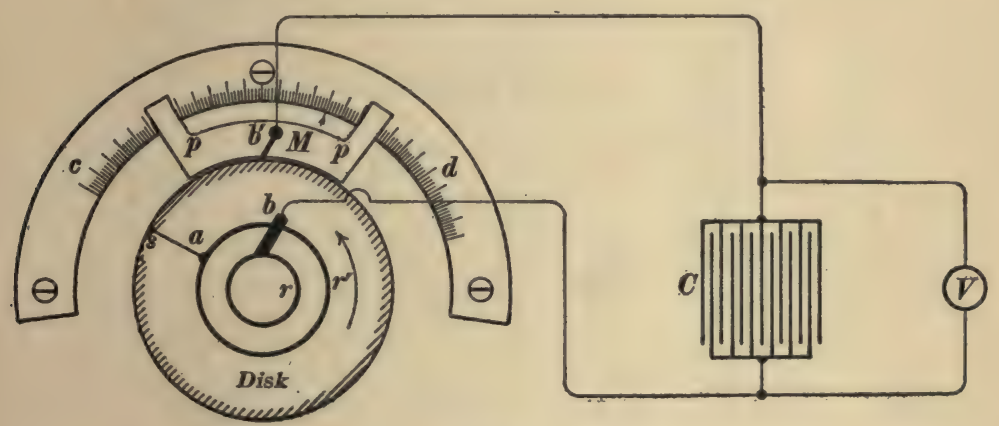

Fig. 33.

termined. A thin metal brush $b^{\prime}$ rubs on the edge of this disk and makes momentary contact once per revolution with a narrow metal strip $s$ which is set in the edge of the disk. The brush $b^{\prime}$ is supported on a sector $p p$ which slides around on the inner edge of the divided circle $c d$, and the reading on the divided circle of the mark $M$ indicates the position of the brush $b^{\prime}$.

One terminal of the condenser $C$ is permanently connected to one collecting ring $r$ of the alternator by means of the brush $b$, and the other terminal of $C$ is connected to the brush $b^{\prime}$ and thence through the strip $s$ and wire $a$ to the other collecting ring $r^{\prime}$.

The voltmeter $V$ must take a very small current in order that the condenser may be able to supply the current during the 
intervals between contacts of $b^{\prime}$ and $s$ without perceptible decrease of voltage across the condenser terminals.

The sector $p p$ is shifted step by step around the divided circle, and the circle readings and voltmeter readings are taken. A complete cycle of electromotive force values is obtained by shifting the sector $p p$ over the angular distance between two adjacent north poles (or south poles) of the field magnet, and in plotting the electromotive force curve the circle readings are laid off as abscissas and the voltmeter readings as ordinates.

To determine a current curve by means of the contact maker, the device shown in Fig. 33 is mounted on the armature shaft of

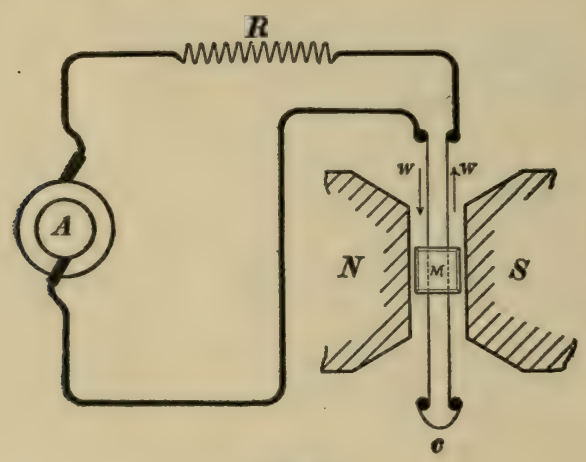

Fig. 34 .

the alternator which supplies the current, the current is made to flow through a non-inductive resistance $R$, the curve of the electromotive force across the non-inductive resistance is determined, and the ordinates of this electromotive force curve are divided by $R$ to give the points on the current curve.

(b) By the oscillograph. - In order that a galvanometer needle may indicate the successive instantaneous values of a rapidly changing current, such as an alternating current, the galvanometer needle must be extremely light and the period of free vibration of the needle must be extremely short. The oscillograph is a galvanometer constructed to meet these requirements.

The Duddell oscillograph consists of two very fine wires, $W W$, 
Fig. 34, stretched very close together between the poles NS of a strong magnet. A very light mirror $M$ is attached to the wires, and the current to be measured flows down one wire, across the connection $c$ and up the other wire, so that one wire is pushed forwards and the other wire is pushed backwards by the magnet, thus deflecting the mirror. A beam of bright light falls on the mirror and is reflected to a moving photographic plate upon which is left a permanent trace of the movements of the mirror. The alternator $A$, of which the electromotive force

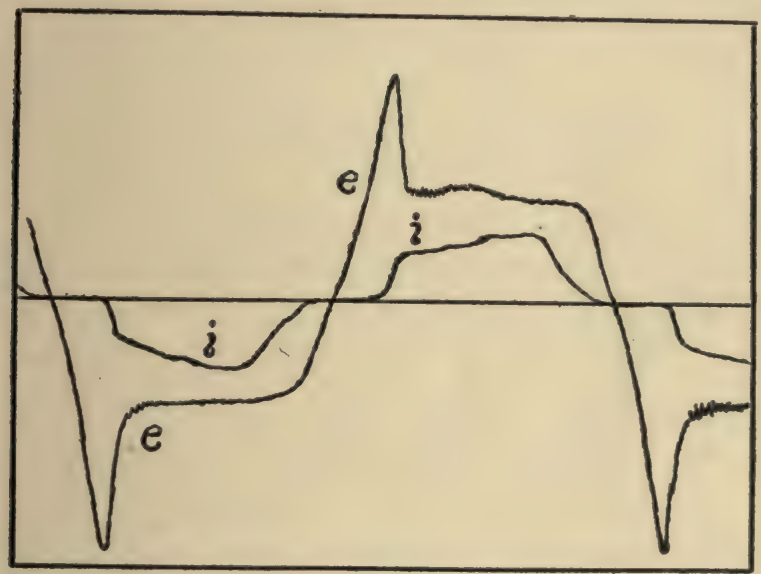

Fig. 35.

curve is to be determined, is connected through a non-inductive resistance $R$ to the oscillograph as shown in Fig. 34 .

To determine a current curve, the oscillograph is connected to the terminals of a non-inductive resistance through which the current flows.

An example of electromotive force and current curves taken simultaneously by two oscillographs is shown in Fig. $35{ }^{*}$ The curve $e$ shows the electromotive force across the terminals of an alternating arc, and the curve $i$ shows the current flowing through the arc. The fine waves in the electromotive force curve are due to the free oscillations of the needle.

* Reproduced from a paper On Arc Lamps by André Blondel, Transactions of the International Electrical Congress, St. Louis, I904, Vol. II, pages 731-767. 


\section{CHAPTER II.}

\section{ALTERNATING CURRENT MEASUREMENTS.}

\section{The difference between direct-current and alternating-current}

ammeters and voltmeters. * - In direct-current ammeters and voltmeters the force which acts upon the pointer and produces the deflection may be any function whatever of the current or electromotive force, provided only that the force has a perfectly definite value for a given current or electromotive force, thus ensuring that the given current or electromotive force will produce 2 definite deflection.

In alternating-current ammeters and voltmeters, on the other hand, the force tending at any instant to move the pointer is proportional $\uparrow$ to the square of the current or electromotive force at that instant, so that the average force, upon which the steady deflection depends, is proportional to the average square of the current or electromotive force. Alternating-current ammeters and voltmeters really measure, therefore, the average value of the square of an alternating current or electromotive force.

The standard method of calibrating an alternating-current ammeter or voltmeter is by using direct currents or direct electromotive forces, and the most reliable alternating-current ammeters and voltmeters are those which indicate effective values of alternating current or electromotive force correctly when so calibrated, irrespective of frequency and wave form.

Consider, for example, an ammeter in which the deflecting

* All voltmeters, with the exception of the electrostatic voltmeter, are essentially ammeters. That is, the electromotive force to be measured produces a current, which actuates the pointer; and the scale, instead of being arranged to indicate the value of the current, is arranged to indicate the value of the electromotive force between the terminals of the instrument.

† This is intended only as a general statement and the qualification necessary to make it exact would tend to make it unintelligible. See Art. 15. 
force is proportional to the square of the current. The constant deflecting force, due to a direct current $C$, is equal to $k C^{2}$, the instantaneous force due to an alternating current is equal to $k i^{2}$, where $i$ is the value of the alternating current at a given instant, and the average force due to the alternating current is equal to $k \times$ average $i^{2}$; but, if the alternating current gives the same deflection as the direct current, the constant force, $k C^{2}$, must be equal to the average force, $k \times$ average $i^{2}$, so that average $i^{2}$ must be equal to $C^{2}$, or $\sqrt{\text { average }} i^{2}$ must be equal to $C$. That is to say, although the ammeter really measures the average square of any current flowing through it, the scale numbers (values of $C$ ) determined by direct-current calibration, are effective values of alternating current $\left(\sqrt{\text { average } i^{2}}=C\right)$.

12. The hot-wire ammeter and voltmeter. In these instruments the current flows through a stretched wire, and the wire, heated by the current, lengthens and actuates a pointer which plays over a divided scale.

The hot-wire instrument, when calibrated by direct current, indicates effective values of alternating current, and when calibrated by direct electromotive force it indicates effective values of alternating electromotive force. This is evident in the case of the ammeter when we consider that a direct current $C$ and an alternating current $i$ which give the same deflection must generate heat in the stretched wire at the same average rate. That is, $R C^{2}$ must be equal to $R \times$ average $i^{2}$, or $C^{2}=$ average $i^{2}$, or $C=\sqrt{\text { average } i^{2}}$.

The hot-wire instrument has not been considered very satisfactory hitherto on account of the tendency of the heated wire to take a permanent set, that is, to become permanently elongated.

13. The electrodynamometer. - The electrodynamometer when used as an ammeter or as a voltmeter consists of a fixed coil and a movable coil connected in series. The current flows through both coils, and the fixed coil exerts a force upon the movable coil which deflects it and actuates a pointer which plays over a divided scale. In some instruments the force action between the 
coils is balanced by moving a weight or by twisting a helical spring which is attached to the movable coil. In this case the reading of the instrument is the distance that the weight is moved, or the angle through which the spring is twisted.

The electrodynamometer when standardized by direct current indicates effective valucs of alternating current. This is evident when we consider that the force action between the coils is proportional to the square of the current, or equal to a constant, $k$, times the square of the current, and that the force action, $k C^{2}$, due to a direct current $C$ must be equal to the average force action, $k \times$ average $i^{2}$, due to an alternating current $i$; if $C$ and $i$ produce the same deflection, $k C^{2}$ must be equal to $k \times$ average $i^{2}$, or $C=\sqrt{\text { average } i^{2}}$.

The electrodynamometer is the most reliable form of alternating-current ammeter.

When the electrodynamometer is used as a voltmeter, its coils are made of fine wire, and an auxiliary non-inductive resistance is connected in series with the coils.

When the inductance of the electrodynamometer coils is small* the instrument indicates effective values of alternating electromotive force accurately when it has been calibrated by direct electromotive force. This is evident when we consider that the current $i$ in a non-inductive circuit is at each instant equal to $\ell / R$, where $R$ is the resistance of the circuit, and $e$ is the value at the given instant of the electromotive force across the terminals of the circuit; and when we consider, furthermore, that the average deflecting force due to an alternating electromotive force, namely, $k / R^{2} \times$ average $e^{2} \quad\left(=k \times\right.$ average $i^{2}$ if the circuit is non-inductive) must be equal to the constant deflecting force $k / R^{2} \times \mathcal{E}^{2}$ $\left(=k \times\right.$ current $\left.^{2}\right)$ due to a direct electromotive force $\mathcal{E}$ which produces the same deflection.

The effect of the inductance of a voltmeter is to make an instrument which has been calibrated by direct electromotive force

\footnotetext{
* That is, when the reactance $\omega L$ is small compared with the resistance. See chapter IV.
} 
indicate less than the effective value of an alternating electromotive force, the error being the greater the higher the frequency. Nevertheless the electrodynamometer type of voltmeter is the most generally satisfactory and reliable type of alternating-current voltmeter.

A disadvantage of the electrodynamometer (ammeter or voltmeter) as compared with the plunger type of instrument is its greater cost.

Inductance crror of the electrodynamometer when used as a voltmeter. - The following discussion is limited to the case of harmonic electromotive force and harmonic current, and it presupposes a knowledge of Chapters III and IV. Let $\mathcal{E}$ be a direct electromotive force which gives the same deflection as an alternating electromotive force whose effective value is $E$, and whose frequency is $f$ cycles per second, or $\omega(=2 \pi f)$ radians per second. Since the deflections are the same, the square of the direct current produced by $\mathcal{E},\left(\mathcal{E}^{2} / R^{2}\right)$, must be equal to the average square of the alternating current $\left[E^{2} /\left(R^{2}+\omega^{2} L^{2}\right)\right]$ produced by $E$, that is,

whence we find

$$
\frac{\mathcal{E}^{2}}{R^{2}}=\frac{E^{2}}{R^{2}+\omega^{2} L^{2}}
$$

$$
E=\frac{\sqrt{R^{2}+\omega^{2} L^{2}}}{R} \cdot \mathcal{E}
$$

in which $E$ is the true effective value of a harmonic alternating electromotive force of a frequency of $\omega$ radians per second, $\mathcal{S}$ is the voltmeter reading produced thereby, $R$ is the resistance of the voltmeter, and $L$ is the inductance of the electrodynameter coils.

14. The electrostatic voltmeter. - Two insulated metal plates connected to the terminals of a battery, or to any source of electromotive force, attract each other with a force which is exactly proportional to the square of the electromotive force. This principle is applied in the electrostatic voltmeter which consists essentially of a fixed metal plate and a delicately poised or suspended metal plate which carries a pointer, the two plates being connected to the terminals of the source of the electromotive force to be measured.

Such an instrument, when calibrated by direct electromotive force, indicates effective values of alternating electromotive force. This is evident when we consider that the steady force action, $k \mathcal{E}^{2}$, due to a direct electromotive force $\mathcal{E}$ must be equal to the 
average force action, $k \times$ average $e^{2}$, due to the alternating electromotive force $e$, which gives the same deflection as $\mathcal{E}$, that is, $k \mathcal{E}^{2}=k \times$ average $e^{2}$, or $\mathcal{E}=\sqrt{\text { average } e^{2}}$.

The great difficulty with the electrostatic voltmeter arises from the excessively weak forces of attraction between two metal plates with electromotive forces less than several hundred volts, so that a low-reading electrostatic voltmeter must be very delicate, and therefore unsuited to the conditions of practical use. For high electromotive forces, however, the electrostatic voltmeter is very satisfactory.

One form of electrostatic voltmeter, the so-called absolute electrometer, ${ }^{*}$ does not need to be calibrated, inasmuch as it gives the value of the electromotive force directly in terms of purely mechanical measurements.

15. Plunger type ammeters and voltmeters. - In instruments of this type the current flows through a coil of wire which magnetizes and moves a pivoted or suspended piece of soft iron to which the pointer is attached.

Plunger ammeters. - In this type of instrument the force tending to move the pivoted piece of iron is not proportional to the square of the current, and therefore such an instrument does not indicate effective values of alternating current when it has been calibrated with direct current. It is necessary therefore to calibrate a plunger type ammeter with alternating current which, for the purpose of the calibration, is measured by an electro-dynamometer ammeter.

Plunger type voltmeters. - An instrument which will indicate effective alternating current correctly when calibrated as an ammeter with direct current, will also indicate effective alternating electromotive force correctly when calibrated as a voltmeter with direct electromotive force, provided the inductance of the instrument is negligible. This is exemplified by the use of the electrodynamometer as a voltmeter as explained in Art. I 3. The plunger type instrument, however, does not indicate effective alternating

* See Elements of Physics, Nichols and Franklin, Vol. II, pages 183-184. 
current correctly unless specially calibrated as explained above, and, moreover, the inductance of the plunger type instrument is always fairly large. Therefore, for two reasons, a plunger type voltmeter should be calibrated with alternating electromotive force which, for the purpose of the calibration, is measured by an electro-dynamometer-voltmeter of negligible inductance, or by an electrostatic voltmeter.

The Thomson inclined coil instrument of the General Electric Company is a good example of the plunger type. The essential parts of the instrument are shown in Fig. 36. The coil $A$,

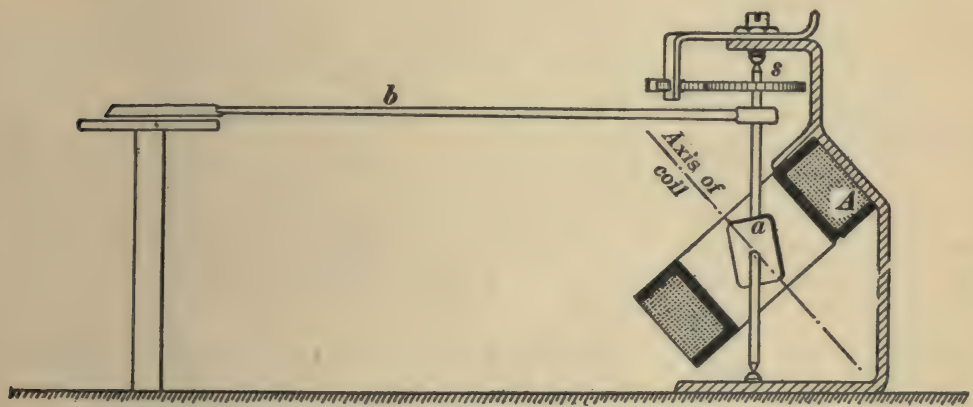

Fig. 36.

through which the current flows, is mounted with its axis inclined as shown. A vertical spindle mounted in jewelled bearings and controlled by a hair spring $s$, passes through the coil.

A vane of thin sheet iron $a$ is mounted obliquely on the spindle so that when a current flows through the coil the tendency is for the vane to set itself parallel to the axis of the coil, thus moving the pointer.

16. The spark gauge. The high electromotive forces used in "break-down" tests of insulators are usually measured by means of the spark gauge which consists of an adjustable spark gap between needle points. This spark gap is varied until the electromotive force to be measured is barely able to strike across it in the form of a spark. The length of the spark gap is then 
measured and the value of the electromotive force is taken from the following table.*

\section{TABLE.}

SPARKING DISTANCES IN AIR BETWEEN OPPOSED SHARP NEEDLE POINTS FOR VARIOUS EFFECTIVE HARMONIC VOLTAGES.

\begin{tabular}{|c|c|c|c|}
\hline Dis & & Dis & \\
\hline Inches & Cms. & Inches & Cms \\
\hline 0.225 & 0.57 & .....13.95 & $5 \cdot 4$ \\
\hline .. 0.47 & 1. 19 & $\ldots 15.0$ & $3^{8.1}$ \\
\hline .. 0.725 & 1.84 & $\ldots 16.05$ & 40.7 \\
\hline$\therefore x .0$ & 2.54 & ...17.10 & $43 \cdot 4$ \\
\hline ... 1.3 & $3 \cdot 3$ & $\ldots 18.15$ & 46.1 \\
\hline ... 1.625 & 4. I & $\ldots 19.20$ & 48.8 \\
\hline ........ 2.0 & 5.1 &. .20 .25 & 51.4 \\
\hline ....... 2.45 & 6.2 & $\ldots 21.30$ & 54.1 \\
\hline .......... 2.95 & $7 \cdot 5$ &. .22 .35 & 56.8 \\
\hline …..... $3 \cdot 55$ & 9.0 & $\ldots 23.40$ & $59 \cdot 4$ \\
\hline .................... 4.65 & II. 8 & .24 .45 & 62.1 \\
\hline ….............. 5.85 & .9 &. .25 .50 & $64 \cdot 7$ \\
\hline 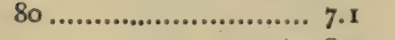 & 18.0 & $260 .$. & $67 \cdot 3$ \\
\hline $90 \ldots \ldots \ldots . . .$. & 21.2 & $\ldots \ldots . .27 \cdot 50$ & 69.8 \\
\hline n................. 9.6 & 24.4 &. .28 .50 & 72.4 \\
\hline ..................... 10.75 & $27 \cdot 3$ & $290 \ldots \ldots \ldots \ldots \ldots \ldots \ldots \ldots$ & 74.9 \\
\hline $120 \ldots \ldots \ldots$. & 30. 1 & .30 .50 & 77.4 \\
\hline $30 \ldots$ & 32.8 & & \\
\hline
\end{tabular}

Figure 37 shows a testing transformer of the General Electric Company. On the top of the containing case is a spark gauge which is adjusted by means of a micrometer screw, the reading of which gives the length of the spark gap. The transformer is arranged to give electromotive forces (alternating) up to I0,000 volts.

The indications of a spark gauge are subject to very considerable error on account of melting of needle points by the discharge (needle points must be very sharp to give best results), on account of drafts of air, on account of departure of electromotive force curve from a curve of sines, and on account of what appears to be the inherently erratic character of a spark discharge. It is, however, by far the most convenient method for measuring high

* Based on experiments of C. P. Steinmetz on the Dielectric Strength of Air, Trans. American Institute of Electrical Engineers, Vol. XV, pages 281-230, 1898. 
voltages, and with care the unavoidable errors may be reduced to one or two per cent. or even less. See a paper by H. W. Fisher, Transactions of International Electrical Congress, Vol. II, pages 294-3 I 2, St. Louis, I904.

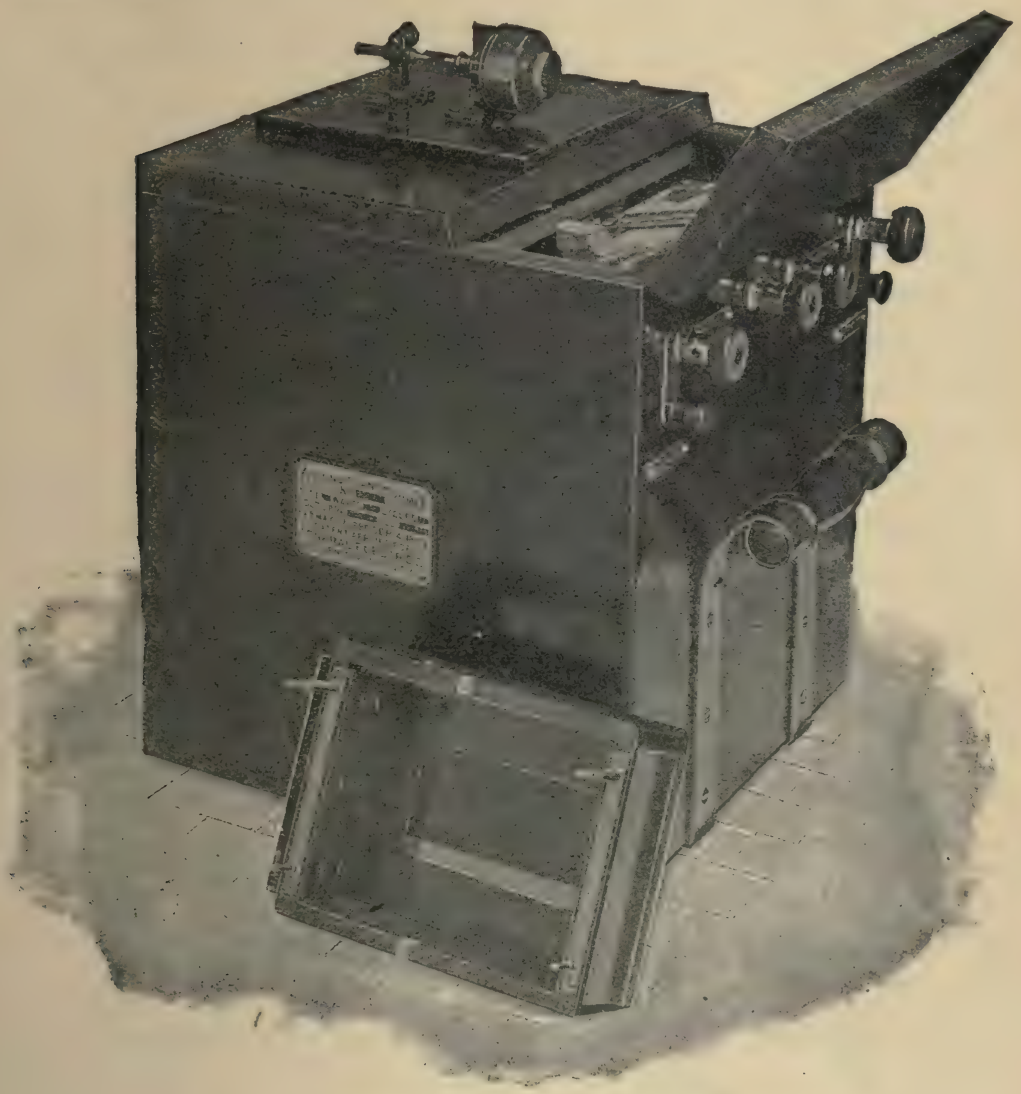

Fig. 37.

17. Transformer multipliers for alternating current instruments. - A given direct-current voltmeter may be adapted to measure a high voltage by connecting a high resistance in series with the instrument. Such a resistance is called a multiplier; it may be used with an alternating-current voltmeter (except the electrostatic voltmeter). 
A given direct-current ammeter may be adapted to read the value of a large current by connecting a low resistance shunt across the terminals of the instrument. The use of such a shunt with an alternating-current ammeter, however, is open to the objection that the multiplying value of the shunt varies with the frequency, so that a shunted alternating-current ammeter should be calibrated by alternating current of the same frequency as that for which the instrument is to be used.

The so-called "potential transformer" is a small transformer for stepping down a high voltage in a known ratio so that the high voltage may be measured by a low reading voltmeter. Most switch-board voltmeters in alternating-current generating stations are connected to the mains through step-down " potential " transformers.

The so-called "current transformer" is a small transformer through whose primary coil the current to be measured flows, and whose secondary coil is short-circuited through an ammeter. The ratio of primary to secondary current is known, so that the value of the primary current may be inferred from the ammeter reading. Most switch-board ammeters in alternating-current generating stations are connected through " current" transformers in this way.

The use of the potential transformer and of the current transformer makes it possible to completely insulate the switch-board instruments from high-voltage mains.

18. Measurement of power in alternating current circuits. Definition of power factor. - The power delivered to a circuit by a direct-current generator is equal to the product of the voltage and the current, so that the power delivered by a direct-current generator can be determined by a voltmeter and an ammeter. The power delivered by an alternating-current generator, on the other hand, is generally less, and often very much less, than the product of the effective electromotive force of the generator and the effective current, so that the power delivered by an alternating- 
current generator cannot be determined by a voltmeter and an ammeter.

The wattmeter is almost universally employed for measuring power in alternating-current circuits.

Power factor. - Let the effective value of the electromotive force of an alternator as measured by a voltmeter be $E$, let the effective value of the current delivered by the alternator as measured by an ammeter be $I$, and let the actual power delivered by the alternator as measured by a wattmeter be $P$. The product EI is called the apparent power, and it is customary to specify the value of EI in volt-amperes in order to avoid the suggestion of actual power which would be conveyed by specifying the value of $E I$ in watts. The ratio of the actual power $P$ to the apparent power $E I$ is called the pozver factor of the receiving circuit; representing the power factor of the receiving circuit by $p$, we have

$$
P=E I p
$$

The power factor of a receiving circuit depends not only upon the nature of the receiving circuit, but also upon the frequency and wave shape of the electromotive force of the alternator. Thus, the power factor of an electric arc between carbon rods is usually about 0.85 , that is, the actual power delivered to the arc is only about 85 per cent. of the apparent power EI.

19. The wattmeter. - The standard method for measuring the power delivered by an alternator is by means of the wattmeter. The wattmeter is a special form of electrodynamometer, the connections of which are shown in Fig. 38. A fixed coil of coarse wire $B$ is connected in series with the receiving circuit to which the power to be measured is delivered, and a suspended or pivoted coil $A$ of fine wire is connected across the supply mains in series with a non-inductive resistance $R$. The total current which is delivered to the receiving circuit flows through the fixed coil $B$, a current proportional to the supply voltage flows through the pivoted coil $A$, and the force action between the 
coils causes coil $A$ to move and carry a pointer over a divided scale. The compensated wattmeter is described in Vol. I., Art. 91 .

The wattmeter, when calibrated with direct current and direct clectromotive force, indicates power accurately when used with alternating current, provided the inductance of the voltage coil, $A$, Fig. 38 , is negligibly small.

Proof. - Consider a wattmeter which gives a certain deflection with a steady electromotive force $\mathcal{E}$ across its voltage coil and a steady current $C$ flowing through its coarse wire coil. The deflecting force is proportional to the product of the currents, $\mathcal{E} / R$ and $C$, in the two coils, that is, it is equal to $k \times \mathcal{E} / R \times C$.

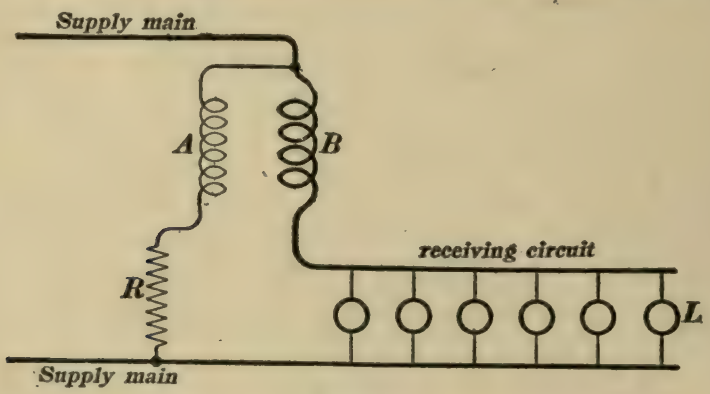

Fig. 38.

Suppose that the instrument gives the same deflection as above when an alternating current $i$ flows through its current coil and an alternating electromotive force $e$ acts across its voltage coil, $i$ and $e$ both referring to the instantaneous values; then the current in the voltage coil is $e / R$, if the circuit $A R$, Fig. 38 , is non-inductive, the instantaneous force action between the coils is $k \times e / R \times i$ or $k / R \times e i$, and the average force action is equal to $k / R \times$ average $c i$; but, since the deflection is assumed to be the same as before, this average force action is equal to the constant force action $k \times \mathcal{E} / R \times C$, so that average $e i=\mathcal{E} C$. Now, average $e i$ is the power delivered by the alternating current, and $\mathcal{E} C$ is the power delivered by the direct current, and since average $e i$ is equal to $\mathcal{E} C$, it is evident that, if the instrument indi- 
cates watts correctly on a direct-current circuit, it will indicate watts correctly on an alternating-current circuit.

Inductance error of the wattmeter. - This discussion applies only to harmonic electromotive force and current, and it presupposes a knowledge of Chapters III and IV. Let the line $O E$ in the clock diagram, Fig. 39, represent the electromotive

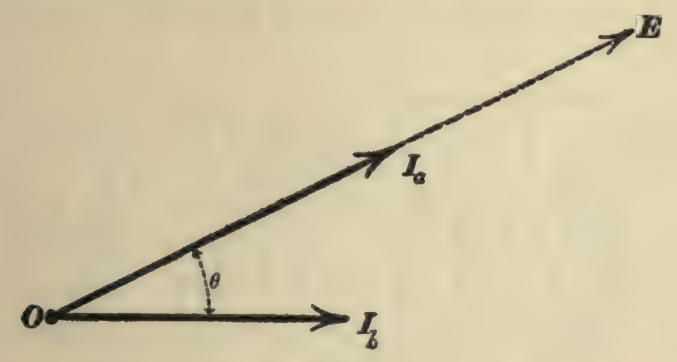

Fig. 39.

force between the supply mains, the line $O I_{b}$ the current delivered to the receiving circuit, and the line $O I_{a}$ the current (generally very small) in the circuit $A R$, Fig. 38. The diagram shows the current $I_{a}$ in phase with $E$ which would be the case if the circuit $A R$, Fig. 38 , were absolutely non-inductive. The effect of a small amount of inductance in the circuit $A R$ is to turn $I_{a}$ very slightly towards $I_{b}$, thus lessening the angle $\theta$ between $I_{a}$ and $I_{b}$ by the small amount $\Delta \theta$, but without producing an appreciable decrease in the value of $I_{a}$.

The average value of the product of the currents in the two wattmeter coils is $I_{a} I_{b} \cos \theta$, the force action between the coils is proportional to this average product, and the deflection $D$ is proportional to the average force action. Therefore we may write

$$
D=k I_{a} I_{b} \cos \theta
$$

in which $k$ is a constant. The change of reading $\Delta D$ due to the decrease, $\Delta \theta$, in the angle $\theta$ is

$$
\Delta D=k I_{a} I_{b} \sin \theta \cdot \Delta \theta
$$

Therefore, the actual wattmeter error, $\triangle D$, due to inductance in the circuit $A R$ is zero when the receiving circuit is non-inductive ( $\sin \theta=0$ ), and it increases with $\theta$ and reaches a maximum when the receiving circuit is very highly inductive $(\sin \theta=\mathbf{1})$; and the percentage error of the instrument, namely, $\Delta D / D(=\tan \theta \cdot \Delta \theta)$ is equal to zero when the receiving circuit is non-inductive, and it becomes indefinitely large when the receiving circuit is highly inductive, that is when $\theta$ approaches $90^{\circ}$.

20. The watt-hour meter is an instrument for summing up the total work or energy delivered to a circuit. There are two types of watt-hour meter in general use, namely, the commutator-motor type and the induction-motor type. The commutator-motor type 
of watt-hour meter is exemplified by the Thomson meter which is described in Art. 92 of the first volume of this text. This meter can be used on either direct-current circuits or alternatingcurrent circuits, but the induction meter is suitable for alternatingcurrent circuits, only. The induction meter is generally used on. alternating-current circuits because of its simplicity and cheapness.
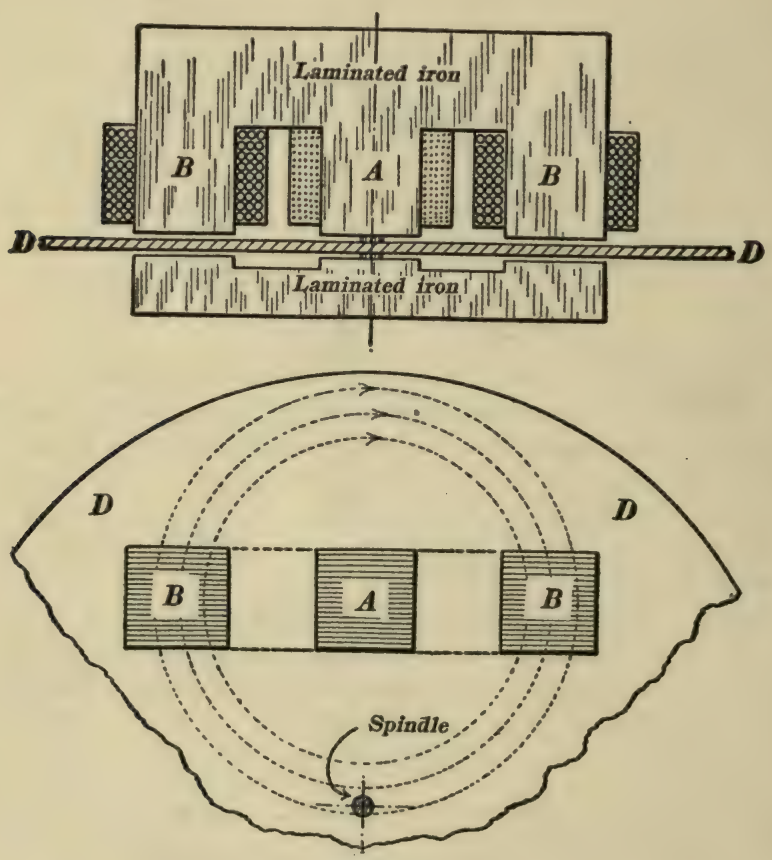

Fig. 40.

In the induction watt-hour meter a thin disk of aluminum or copper is mounted on a delicately pivoted spindle and driven by an electromagnetic device at a speed proportional to the power delivered to the receiving circuit. The total number of revolutions of the spindle is therefore proportional to the work delivered to the receiving circuit, and this is indicated directly in watt-hours by dials which are driven by the rotating spindle.

The essential features of the electromagnetic device for driving 
the metal disk are shown in Fig. 40. The disk $D D$ rotates in front of the three magnet poles $A B B$ of a laminated iron structure as shown. The lugs $B B$ are wound with coarse wire through which flows the total current $i$ that is delivered to the receiving circuit, and the lug $A$ is wound with fine wire which is connected across the supply mains.

The driving torque exerted by this device would be exactly proportional at each instant to $e i$ if the following two conditions were exactly satisfied, namely: (a) If the magnetic flux in the lugs $B$ and $B$ were exactly proportional at each instant to $i$, and $(b)$ if the electromotive force $e$ across the supply mains were exactly balanced at each instant by the electromotive force induced in coil $A$ by the changing flux through the lug $A$, which would be the case if the resistance of coil $A$ were negligibly small. The action of the device will therefore be explained on the assumption that these two conditions are satisfied; in fact they are approximately satisfied in a well designed induction meter.

The changing flux * through lug $A$ produces not only an electromotive force in coil $A$ which is equal to $e$ at each instant, but this flux also induces a proportional electromotive force in the disk along the dotted circles, and this electromotive force produces a proportional current in the disk along the dotted circles. This current in the disk, which is proportional to $e$, in flowing under the lugs $B B$, causes the flux under $B B$ to push on the disk with a force which is proportional to the current $(k e)$, in the disk and proportional to the flux $\left(k^{\prime} i\right)$, under $B B$. The force acting on the disk is therefore proportional to $e i$ at each instant. The average driving torque is therefore proportional to average $e i$, and, by using permanent magnets for damping the motion of the disk, the disk is made to run at a speed proportional to the driving torque.

* The changing flux in lugs $B B$ produces current in the disk, which current flows under lug $A$. The average force due to this combination is also proportional to the average power delivered to the receiving circuit. 


\section{CHAPTER III.}

HARMONIC ELECTROMOTIVE FORCE AND CURRENT.

21. Definition of harmonic electromotive force and current. - A harmonic electromotive force (or current) is an electromotive force (or current) which is at each instant proportional to the sine or cosine of an angle which increases at a constant rate. Thus, $\omega t$ is an angle which increases at a constant rate, $t$ being elapsed time reckoned from some chosen instant, and $\omega$ being a constant, so that a harmonic electromotive force, $e$, may be represented by the equation :

$$
e=\mathbf{E} \sin \omega t
$$

A harmonic electromotive force (or current) is represented, in rectangular coordinates, by a curve of sines of which the abscissas represent elapsed times, and the ordinates represent successive values of electromotive force (or current).

The clock diagram. - A line $O P$, Fig. $4 \mathrm{I}$, rotates at a uniform angular velocity of $f$ revolutions per second, or $\omega(=2 \pi f)$

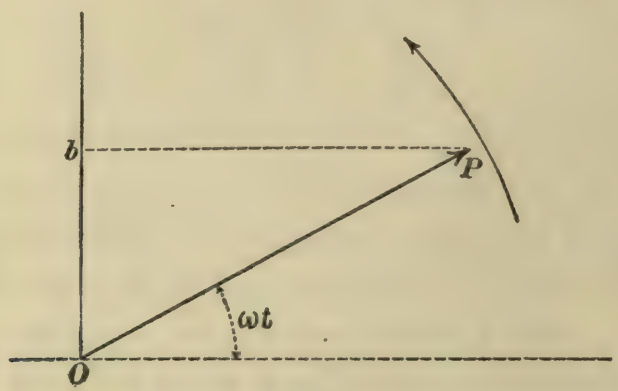

FIg. 41.

radians per second, about the point $O$ in the direction of the curved arrow, and the vertical projection of $O P$ is at each instant equal to $O P$ times the sine of the angle $\omega t$. Therefore, if the 
length of $O P$ represents the maximum value $\mathbf{E}$ (or $\mathbf{I}$ ) of a harmonic electromotive force (or current), then the vertical projection $O b$ will represent at each instant the actual value of the harmonic electromotive force $e$ (or current $i$ ).

Consider, for example, the two lines E and I, Fig. 42, which are imagined to be rotating $f$ revolutions per second in the direction of the curved arrow, so that their vertical projections may represent successive instantaneous values of a harmonic electromotive force and of a harmonic current, respectively. Under these conditions the lines $\mathbf{E}$ and $\mathbf{I}$ are said to represent the harmonic electromotive force and current, and the diagram, Fig. 42, is called a clock diagram.

The definitions of cycle,

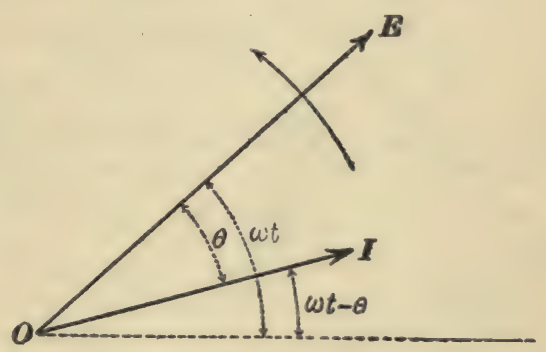

Fig. 42. period and frequency given in Art. 6 apply also to harmonic electromotive forces and currents. In case of harmonic electromotive force and current, however, the frequency, $f$, which is represented by the number of revolutions per second of the lines $\mathbf{E}$ and $\mathbf{I}$ in Fig. 42, can also be expressed in radians per second, $\omega$; and, since there are $2 \pi$ radians in a revolution, we have

$$
\omega=2 \pi f
$$

22. Phase difference. - Consider a harmonic electromotive force $e$ and a harmonic current $i$ of the same frequency. It often happens that the current reaches its maximum value after the electromotive force has passed its maximum value, as shown in Fig. 43. In this case the current is said to lag behind the electromotive force in phase. In some cases the current reaches its maximum value before the electromotive force reaches its maximum value; and in such a case the current is said to be ahead of the electromotive force in phase. When a current is behind an 
electromotive force in phase, it is called a lagging current; and when a current is ahead of an electromotive force in phase, it is called a leading current.

The phase difference between a harmonic electromotive force

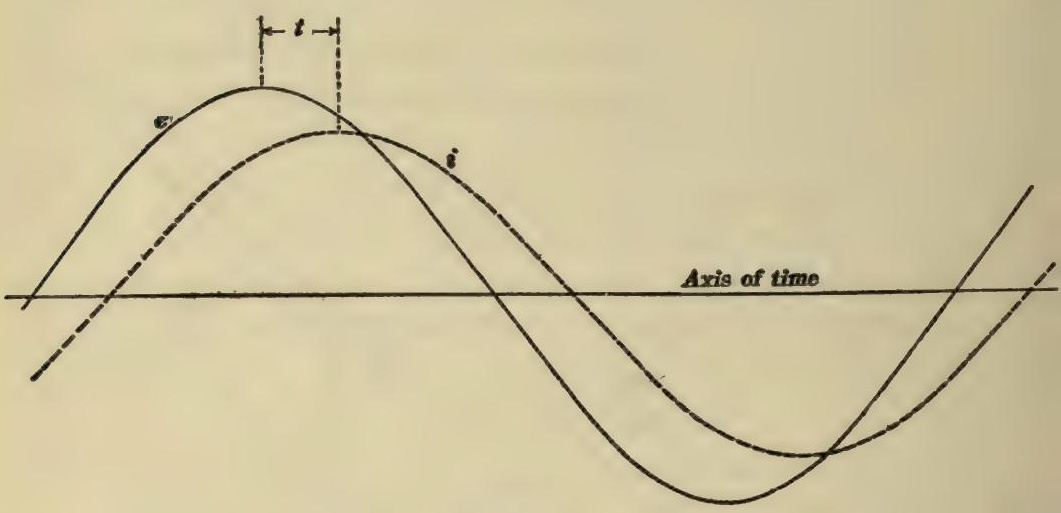

Fig. 43.

and a harmonic current is, properly speaking, a time interval, namely, the time interval $t$ in Fig. 43 ; but it is most convenient to express phase difference as an angle. Thus, Fig. 44 is a clock diagram in which the lines $\mathbf{E}$ and $\mathbf{I}$ represent the har-

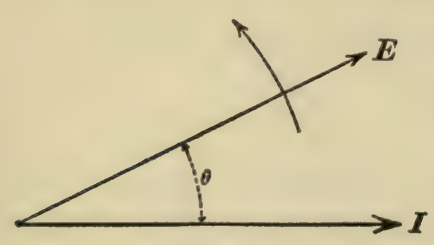

Fig. 44. monic electromotive force $e$ and the harmonic current $i$ which are shown in Fig. 43, and the phase difference is represented by the angle $\theta$.

The electromotive force and current represented in Fig. 42 differ in phase, and the algebraic expressions for the vertical projections of the lines $\mathbf{E}$ and $\mathbf{I}$ are

$$
e=\mathbf{E} \sin \omega t
$$

and

$$
i=\mathbf{I} \sin (\omega t-\theta)
$$


When the angle $\theta$ is zero, $e$ and $i$ are said to be in phase with each other. In this case $e$ and $i$ reach their maximum values together, they pass through zero together, and so on.

When the angle $\theta$ is $90^{\circ}, e$ and $i$ are said to be in quadrature with each other. In this case $e$ is zero when $i$ is at its maximum value, and vice versa.

When the angle $\theta$ is $180^{\circ}, e$ and $i$ are said to be in opposition. In this case $e$ and $i$ pass through zero together, but when one is positive the other is negative.

23. Composition and resolution of harmonic electromotive forces and currents. - It often happens that two or more harmonic electromotive forces (or currents) are added together. Thus, when two alternators are connected in series their electromotive forces are added, and when two alternators are connected in parallel their currents are added. Again, it often happens that a given harmonic electromotive force (or current) is divided into parts. Thus, when two coils are connected in series between supply mains, the supply voltage is divided between them, and when two coils are connected in parallel between supply mains, the total current is divided between them. The adding of harmonic electromotive forces (or currents) is called composition, and the dividing of a harmonic electromotive force (or current) into parts is called resolution.

Composition. - Consider two harmonic electromotive forces $e_{1}$ and $e_{2}$

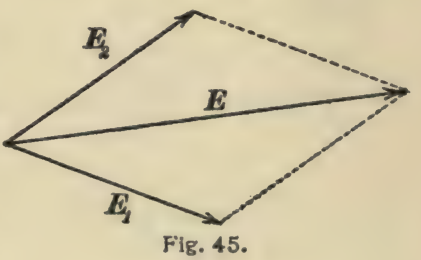
of the same frequency. Let $e_{1}$ and $e_{2}$ be represented by the lines $\mathbf{E}_{1}$ and $\mathbf{E}_{2}$ in the clock diagram, Fig. 45. The sum $e_{1}+e_{2}$ is a harmonic electromotive force of the same frequency as $e_{1}$ and $e_{2}$, and this sum is represented by the line E, Fig. 45, which is the geometric or vector sum of the lines $\mathbf{E}_{1}$ and $\mathbf{E}_{2}$. This is evident when we consider that the projection, on any line, of the diagonal of a parallelogram is equal to the sum of the projections of the two adjacent sides of the parallelogram. 
Resolution. - A given harmonic electromotive force (or current) may be resolved into a number of parts each of which is a harmonic electromotive force (or current) of the same frequency as the given harmonic electromotive force (or current). Thus the line E, Fig. 45, represents a given harmonic electromotive force, and the lines $E_{1}$ and $E_{2}$ represent two parts into which the given electromotive force may be resolved.

Examples of composition and resolution. - (a) Two alternatingcurrent generators, $A$ and $B$, running in synchronism, are connected in series between mains as shown in Fig. $46 a$, and the total electromotive force between the mains is related to the electromotive forces of the respective machines as shown in

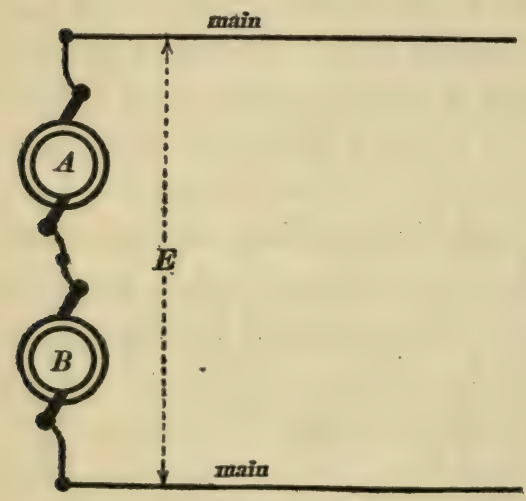

Fig. 46a.

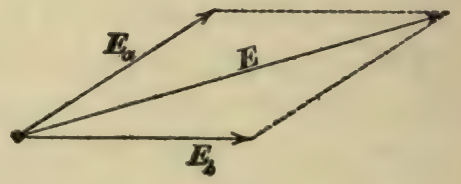

Fig. 46b. The phase difference between $\mathbf{E}_{a}$ and $\mathbf{E}_{b}$ may have any value whatever.

(b) Two alternating-current generators, $A$ and $B$, running in synchronism are connected in parallel between mains as shown in Fig. $47 a$, and the total current delivered to the mains is related to the currents delivered by the respective machines as shown in Fig. $47 b$. The phase difference $\theta$ may have any value whatever.

(c) Figure $48 a$ shows two coils (or receiving units of any kind) $A$ and $B$, connected in series between alternating-current supply mains. The voltage between the mains is represented by the 
line $\mathbf{E}$ in Fig. $48 b$, and the voltages across $A$ and across $B$ are represented by the lines $\mathbf{E}_{a}$ and $\mathbf{E}_{b}$ respectively. The phase difference $\theta$ may have any value between zero and $\mathrm{I} 80^{\circ}$.

(d) Figure $49 a$ shows two coils (or receiving units of any kind)

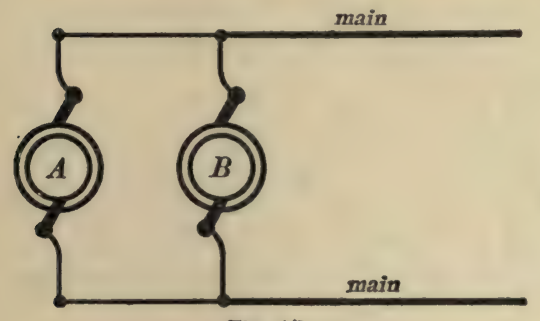

Flg. $47 a$.

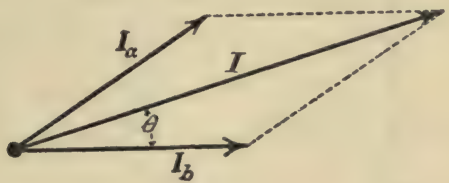

Fig. 476 .

$A$ and $B$, connected in parallel between alternating-current supply mains. The total current delivered to $A$ and $B$ is represented by the line I in Fig. 49b, and the currents in $A$ and $B$ are represented by the lines $I_{a}$ and $I_{b}$ respectively.

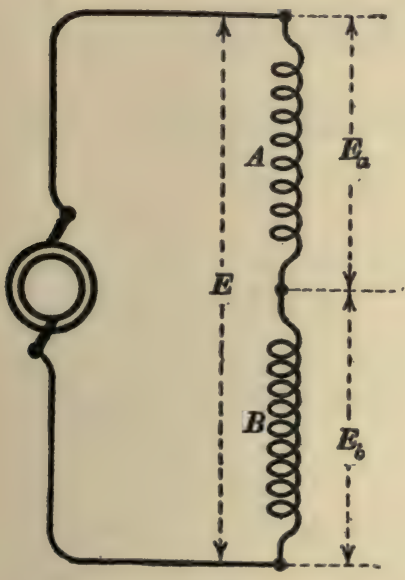

Fig. $48 a$.

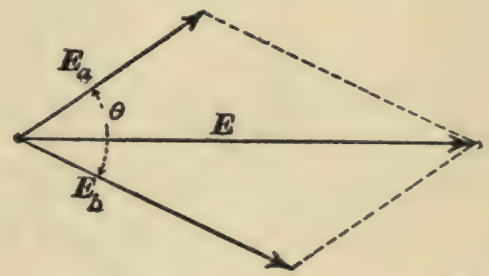

Fig. $48 b$.

The phase difference $\theta$ may have any value between zero and $180^{\circ}$.

24. Rate of change of harmonic current. - When the current in a circuit changes, a portion of the electromotive force which 
acts on the circuit is used to cause the current to change. The portion of the electromotive force so used is equal to the product of the inductance of the circuit and the rate of change of the current.* Therefore a discussion of the relation between current and

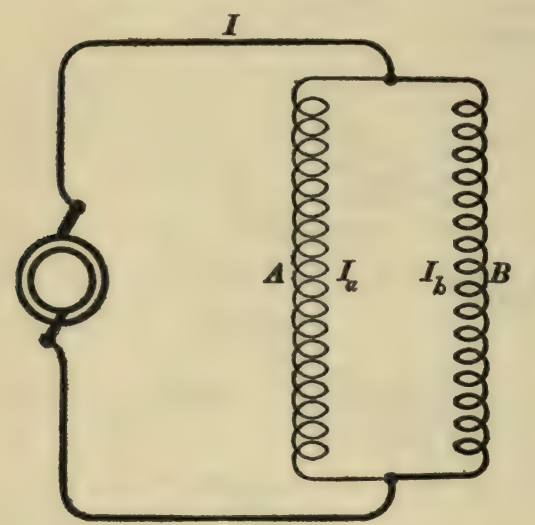

Fig. 49a.

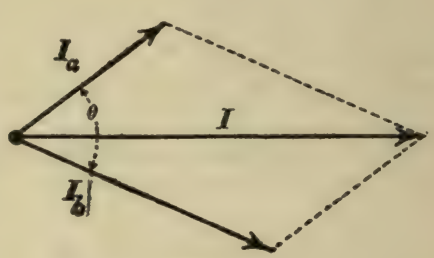

Fig. 496.

voltage in an alternating-current circuit depends upon a knowledge of the rate of change of the current.

The rate of change, di/dt, of a harmonic current $i$ is a har-

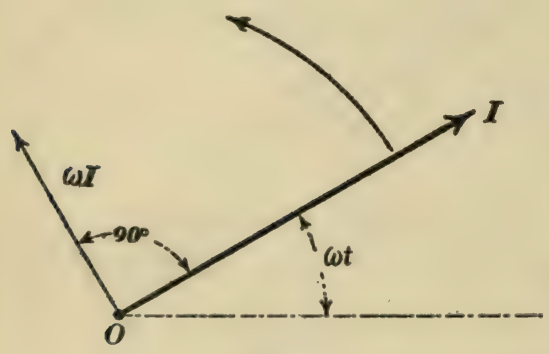

Fig. 50.

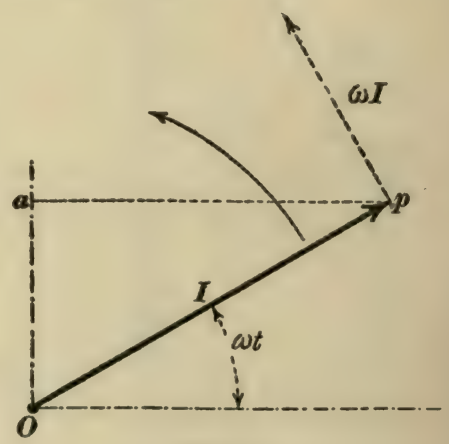

Fig. 51 .

monically varying quantity, that is, dildt is proportional at each instant to the sine or cosine of a uniformly increasing angle $\omega t$, the

* The student who is not familiar with the phenomena of inductance should read Appendix A. 
maximum value of $d i / d t$ is equal to $\omega$ times the maximum value of $i$, and dildt is $90^{\circ}$ ahead of $i$ in phase.

The meaning of this proposition may be made clear by a clock diagram. Thus the vertical projection of the rotating line I, Fig. 50 , represents the value of $i$ at each instant, and the vertical projection of the line $\omega \mathrm{I}$ represents the value of $d i / d t$ at each instant.

The truth of this proposition is evident from the following considerations: The length of the rotating line OI, Fig. $5 \mathrm{I}$, is I, its angular velocity is $\omega$, and the actual velocity of the end $p$ of the line is $\omega \mathrm{I}$, as shown by the dotted arrow in Fig. $5 \mathrm{I}$. Furthermore, the vertical projection of this velocity is the velocity

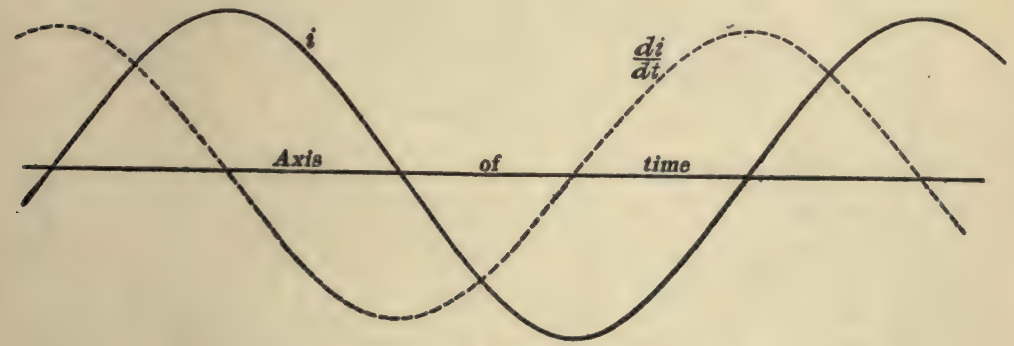

Fig. 52 .

of the point $a$, the velocity of $a$ is the rate of change of $0 a$, and $O a$ is the value of $i$.

The truth of the above proposition may also be shown by differentiating the expression for $i$, namely,

which gives

$$
i=\mathbf{I} \sin \omega t
$$

$$
\frac{d i}{d t}=\omega I \cos \omega t
$$

or

$$
\frac{d i}{d t}=\omega \mathrm{I} \sin \left(\omega t+90^{\circ}\right)
$$

The relation between a harmonic alternating current $i$ and its rate of change, $d i / d t$, is so important in the discussion of the 
relation of voltage and current, that it is worth while to show this relation in every possible way. Thus, the ordinates of the full-line curve in Fig. 52 represent the successive values of an alternating current $i$, and the steepness of this curve represents the value of $d i / d t$ at each instant. To show the relation between $i$ and $d i / d t$ still more clearly, a curve may be drawn, the dotted curve in Fig. 52, of which the ordinates at each point represent the steepness of the current curve. This dotted curve has its maximum value where the current curve crosses the axis ; that is the maximum positive value of $d i / d t$ occurs one quarter of a cycle $\left(=90^{\circ}\right.$ of angle) before the maximum positive value of $i$.

Example. - A harmonic alternating current has a frequency of 60 cycles per second or $2 \pi \times 60$ radians per second $(=\omega)$, and its maximum value is 100 amperes. The maximum rate of change of this current occurs at the instant that the current passes through zero, and it is equal to $2 \pi \times 60 \times 100$ amperes per second, or 37,699 amperes per second. To force this current through a circuit having an inductance of o. I henry, but having no resistance at all, would require a harmonic electromotive force whose maximum value would be o. I $\times 37,699$ volts, or 3769.9 volts, and this electromotive force would be $90^{\circ}$ ahead of the current in phase.

25. Average values and effective values of harmonic electromotive forces and currents. (a). Definition of average value. - The average value of any varying quantity $y$ during an interval of time from $t^{\prime}$ to $t^{\prime \prime}$ is by definition equal to $\Sigma y \cdot \Delta t$ divided by the duration of the interval, the summation being extended over the whole interval. That is

$$
A v \cdot y=\frac{1}{t^{\prime \prime}-t^{\prime}} \int_{t^{\prime}}^{t^{\prime \prime}} y d t
$$

If the successive values of $y$ be represented by the ordinates of the curve $a b$, Fig. 53 , then $\int_{t^{\prime}}^{t^{\prime \prime}} y d t$ is equal to the shaded 
area, and $\frac{\mathrm{I}}{t^{\prime \prime}-t^{\prime}} \int_{t^{\prime}}^{t^{\prime \prime}} y d t$ is the height of the rectangle $t^{\prime} c d t^{\prime \prime}$ which has an area equal to the shaded area, the average value of $y$ during the interval $t^{\prime}$ to $t^{\prime \prime}$ is the height of this rec-

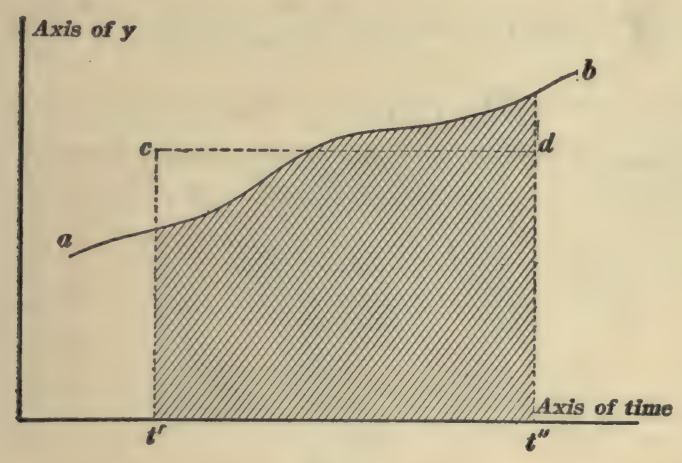

Fig. 53.

tangle, and the height of this rectangle is equal to the area under the curve divided by the length of base.

(b) Average value of the sum of a number of variable quantities. - Let $x, y, z \cdots$ be a number of variable quantities. Then the average value of $(x+y+z+\cdots)$ is equal to Av. $x+$ Av. $y+$ Av. $z+\cdots$. This is evident when we consider that, Av. $(x+y+z \cdots)$ is by definition equal to $\frac{\mathrm{I}}{t^{\prime \prime}-t^{\prime}} \int_{t^{\prime}}^{t^{\prime \prime}}(x+y+z+\cdots) d t$, which is equal to $\frac{\mathrm{I}}{t^{\prime \prime}-t^{\prime}} \int_{t^{\prime}}^{t^{\prime \prime}} x d t+\frac{\mathrm{I}}{t^{\prime \prime}-t^{\prime}} \int_{t^{\prime}}^{t^{\prime \prime}} y d t+\frac{\mathrm{I}}{t^{\prime \prime}-t^{\prime}} \int_{t^{\prime}}^{t^{\prime \prime}} z d t+\cdots$, but this latter expression is by definition equal to Av. $x+$ Av. $y+$ Av. $z+\cdots$.

Proposition. The average value of a harmonic electromotive force (or current) during a half cycle is equal to two times the maximum value $\mathbf{E}$ (or $\mathbf{I})$ divided by $\pi$.

The average value during one or more complete cycles is of course equal to zero.

Proof. Let $e=\mathbf{E} \sin \omega t$ be a given harmonic electromotive force. The average value of $e$ is by definition equal to $\frac{\mathrm{I}}{t^{\prime \prime}-t^{\prime}} \int_{t^{\prime}}^{t^{\prime \prime}} e d t$, or $\frac{\mathbf{E}}{t^{\prime \prime}-t^{\prime}} \int_{t^{\prime}}^{t^{\prime \prime}} \sin \omega t d t$. A 
half cycle (positive values of $e$ ) lies between the limits $\omega t=0$, and $\omega t=\pi$, so that $t^{\prime}=0$ and $t^{\prime \prime}=\pi / \omega$. Therefore the average value of $e$ during a half cycle is equal to $\frac{\omega \mathbf{E}}{\pi} \int_{t=0}^{t=\pi / \omega} \sin \omega t d t$. Let $x=\omega t$, then $d x=\omega d t$; also when $t=0$, $x=0$, and when $t=\pi / \omega, x=\pi$. Therefore

$$
\text { average value of } e=\frac{\mathbf{E}}{\pi} \int_{x=0}^{x=\pi} \sin x d x=\frac{2 \mathbf{E}}{\pi}
$$

Proposition. The square root of the average square of a harmonic electromotive force (or current) during one or more whole cycles is equal to the maximum value $\mathbf{E}$ (or $\mathbf{I}$ ) divided by the square root of two. That is

$$
\begin{aligned}
& E=\frac{\mathrm{E}}{\sqrt{2}} \\
& I=\frac{\mathrm{I}}{\sqrt{2}}
\end{aligned}
$$

These relations may be established by reducing the expression $\frac{\mathrm{I}}{t^{\prime \prime}-t^{\prime}} \int_{t^{\prime}}^{t^{\prime \prime}} e^{2} d t$; but the following considerations lead to the desired result more easily. Let $e=\mathbf{E} \sin \omega t$, then $e^{2}=\mathbf{E}^{2} \sin ^{2} \omega t$, and Av. $e^{2}=\mathrm{Av} .\left(\mathbf{E}^{2} \sin ^{2} \omega t\right)=\mathbf{E}^{2} \mathrm{Av} \cdot\left(\sin ^{2} \omega t\right)$; but we have the general relation:

so that

$$
\sin ^{2} \omega t+\cos ^{2} \omega t=\mathrm{I}
$$

$$
\text { Av. }\left(\sin ^{2} \omega t\right)+\text { Av. }\left(\cos ^{2} \omega t\right)=\mathbf{I}
$$

and, since the cosine of a uniformly variable angle passes similarly through the same set of values during a cycle as the sine, we have

$$
\text { Av. }\left(\sin ^{2} \omega t\right)=A v,\left(\cos ^{2} \omega t\right)
$$

Hence, from equation $(b)$ we have

or

$$
2 \text { Av. }\left(\sin ^{2} \omega t\right)=\mathbf{I}
$$

Therefore

$$
\text { Av. }\left(\sin ^{2} \omega t\right)=\frac{1}{2}
$$

$$
\text { Av. } e^{2}=\mathbf{E}^{2} \mathbf{A v} \cdot\left(\sin ^{2} \omega t\right)=\frac{\mathbf{E}^{2}}{2}
$$

or

$$
\sqrt{\text { Av. } e^{2}}=\frac{\mathbf{E}}{\sqrt{2}}
$$

The use of effective values of electromotive force and current in the clock diagram. According to Art. 2 I the lines in a clock diagram are supposed to represent maximum values of electro- 
motive force and current, but, since the ratio of maximum values to effective values is a constant according to equations (5) and (6), it is permissible and customary to consider that the lines in a clock diagram represent effective values.

26. Expression for power in the case of harmonic electromotive force and current. The power developed by an alternating electromotive force pulsates as explained in Art. 9, and the average power (average value of $e i$ ) is the important consideration.

The average power $P$ developed by a harmonic electromotive force $e(=\mathbf{E} \sin \omega t)$ in maintaining a harmonic current $i$ $[=\mathbf{I} \sin (\omega t-\theta)]$ is :

$$
P=\frac{\mathrm{EI}}{2} \cos \theta
$$

or

$$
P=E I \cos \theta
$$

in which $E$ and $I$ are the effective values of the electromotive

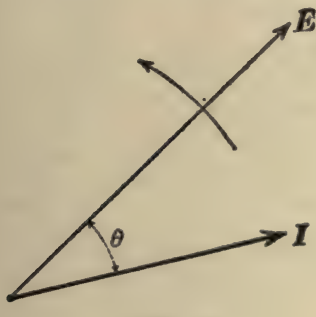

Fig. 54.

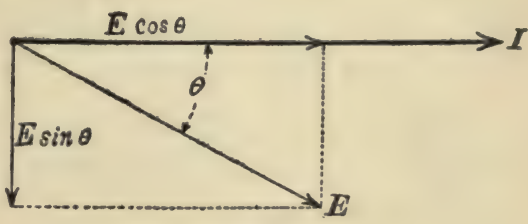

Fig. 55 .

force and current respectively, and $\theta$ is their phase difference, as shown in Fig. 54.

To establish equation $(7)$ it is necessary to find the average value of $e i$. Using $\mathbf{E} \sin \omega t$ for $e$, and $\mathbf{I} \sin (\omega t-\theta)$ for $i$, we have :

$$
e i=\mathbf{E I} \sin \omega t \sin (\omega t-\theta)
$$

or, since $\sin (\omega t-\theta)=\sin \omega t \cos \theta-\cos \omega t \sin \theta$, we have :

$$
e i=\mathbf{E I} \cos \theta \cdot \sin ^{2} \omega t-\mathbf{E I} \sin \theta \cdot \sin \omega t \cos \omega t
$$


so that

Av. $e i=\mathbf{E I} \cos \theta \cdot \mathrm{Av} \cdot\left(\sin ^{2} \omega t\right)-\mathbf{E I} \sin \theta \cdot \mathrm{Av} \cdot(\sin \omega t \cos \omega t)$

But the average value of $\sin ^{2} \omega t$ is $1 / 2$, and the average value of $\sin \omega t \cos \omega t$ is zero. Therefore

$$
\text { Av. } e i=\frac{E I}{2} \cos \theta
$$

or, using equations (5) and (6), we have :

$$
\text { Av. } e i=E I \cos \theta
$$

Power factor. - By comparing equation (7) with equation (2), it is evident that the power factor of a receiving circuit is equal to the cosine of the angle of phase difference between the harmonic electromotive force which acts on the circuit and the harmonic current which is maintained in the circuit.

Power-component and wattless-component of electromotive force. - Let the clock diagram, Fig. 55, represent a given harmonic electromotive force $E$ and the harmonic current $I$ which is maintained by $E$, let the line $I$ be chosen as the reference axis, and let us consider the two components of $E$, namely, $E \cos \theta$ and $E \sin \theta$, as shown. The component, $E \cos \theta$, which is parallel to $I$ is called the power-component of $E$, and the component, $E \sin \theta$, which is at right angles to $I$ is called the wattless-component of $E$.

So-called power-component and wattless-component of current.Let the clock diagram, Fig. 56, represent a given harmonic elec-

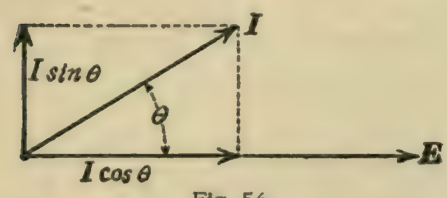

Fig. 56. tromotive force, $E$, and the harmonic current, $I$, which is maintained by $E$, let the line $E$ be chosen as the reference axis, and let us consider the two components of $I$, namely, $I \cos \theta$ and $I$ $\sin \theta$, as shown. The component, $I \cos \theta$, which is parallel to $E$ is sometimes called the power component of the current, and 
HARMONIC ELECTROMOTIVE FORCE AND CURRENT. 65

the component, $I \sin \theta$, which is at right angles to $E$ is sometimes called the wattless component of the current.

It is more legitimate, physically, to speak of a wattless electromotive force than it is to speak of a wattless current. Thus, the rate at which heat is generated in a circuit by a harmonic current is $R I^{2}$, and this is equal to $R \times$ (power component of $\left.I\right)^{2}$ plus $R \times$ (wattless component of $I)^{2}$. That is, there is no such thing, physically, as a wattless current in a circuit which has resistance, although a given current may be resolved into two components as shown in Fig. 56 , and the component $I \sin \theta$ does not receive power from the generator. 


\section{CHAPTER IV}

\section{FUNDAMENTAL PROBLEMS}

27. The current-voltage relation. - To determine the electromotive force required to maintain a given harmonic current in a circuit having resistance and inductance and containing a condenser.

A coil of resistance $R$ and inductance $L$, and a condenser of capacity $C$ are connected in series across alternating current mains as shown in Fig. 57. An alternating current flows back

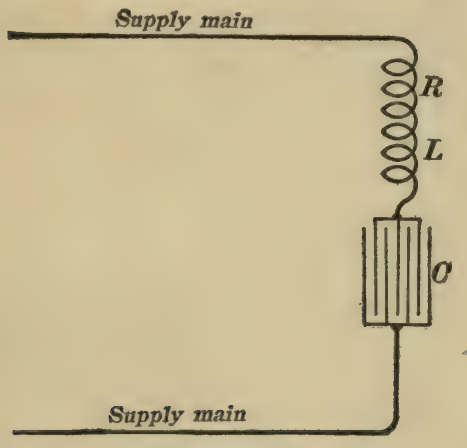

Fig. 57.

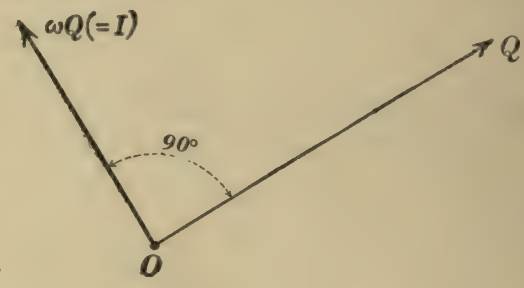

Fig. 58.

and forth through the coil and charges the condenser first in one direction and then in the reverse direction repeatedly. The problem of finding the relation between the current in the coil and the electromotive force between the mains is reduced to its simplest form as follows :

To determine the electromotive force necessary to make the charge $q$ on the condenser vary harmonically, so that

$$
q=Q \sin \omega t
$$

in which $t$ is the elapsed time, $\omega t$ is an angle increasing at a constant rate, and $\mathbf{Q}$ is the maximum charge on the condenser. This varying charge may be represented by the rotating line $\mathbf{Q}$ 
in the clock diagram Fig. 58. The current in the circuit is the rate of change of $q$, that is

$$
i=\frac{d q}{d t}
$$

Now, in Art. 24 it is shown that the rate of change of a harmonically varying quantity is another harmonically varying quantity of the same frequency, that its maximum value is $\omega$ times the maximum value of the given harmonically varying quantity, and that it is $90^{\circ}$ ahead of the given harmonically varying quantity in phase. That is, $d q i d t(=i)$ is $90^{\circ}$ ahead of $q$ in phase, and its maximum value is $\omega Q(=I)$ as shown in Fig. 58 .

The total electromotive force required to cause the charge on: the condenser to vary according to equation $(a)$ under the conditions shown in Fig. 57, may be considered in three parts :

I. The part required to overcome the resistance $R$. This part is equal at each instant to $R i$, and it is therefore a harmonic electromotive force in phase with $i$, and its maximum value is $R I$.

2. The part required to cause the current $i$ to increase and decrease, or, in other words, the part required to overcome the reaction of the inductance $L$. This part is equal to $L(d i / d t)$ at each instant, and it is therefore (see Art. 24) a harmonic electromotive force which is $90^{\circ}$ ahead of $i$ in phase, and its maximum value is $\omega L I$.

3. The part required at each instant to hold the charge $q$ on the condenser, or, in other words, the part that is required to overcome the reaction of the condenser. Now, the charge $q$ on a condenser is equal to $e C$, where $e$ is the electromotive force across the condenser terminals, and $C$ is the capacity of the condenser, so that $e=q / C$. Therefore the electromotive force required to overcome the reaction of the condenser is equal to $q / C$, that is, it is in phase with $q$ (or $90^{\circ}$ behind 2 ), and its maximum value is $\mathrm{Q} / C$ (or $\mathrm{I} / \omega C$, since $\mathrm{I}=\omega \mathrm{Q}$ ).

Solution of the given problem by the clock diagram. Let the line I, Fig. 59, represent the given harmonic current (the same 
as the line $\omega Q$ in Fig. 58$)$, then the three parts of the total electromotive force are represented by the lines $R I, \omega L I$, and $\mathrm{I} / \omega C$, respectively, as shown, and the total electromotive force is represented by the line $\mathbf{E}$ which is the vector sum of $R \mathbf{I}, \omega L I$, and $I / \omega C$. Therefore, from the right triangle whose sides are $(R \mathbf{I}),(\omega L \mathbf{I}-\mathbf{I} / \omega C)$, and $\mathbf{E}$, we have :

$$
\mathbf{E}=\mathbf{I} \sqrt{R^{2}+\left(\omega L-\frac{\mathbf{I}}{\omega C}\right)^{2}}
$$

or, using equations (5) and (6) of Art. 25, we have

$$
E=I \sqrt{R^{2}+\left(\omega L-\frac{\mathrm{I}}{\omega C}\right)^{2}}
$$

and

$$
\tan \theta=\frac{\omega L-\frac{\mathrm{I}}{\omega C}}{R}
$$

These two equations determine the effective value and phase of the harmonic electromotive force required to maintain a given

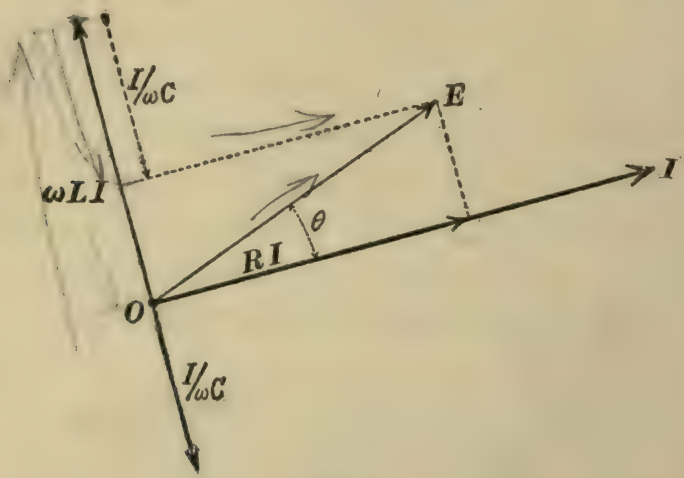

Fig. 59.

harmonic current, or they determine the effective value and phase of the harmonic current that is maintained by a given harmonic electromotive force under the conditions represented in Fig. 57. 
28. Resistance, reactance, and impedance. By inspection of Fig. 59 it is seen that the component of $E$ parallel to $I$ is equal to $R I$ ** The resistance of an alternating current circuit is in general defined as that factor which multiplied by $I$ gives the component of $E$ parallel to $I$. In the case discussed above, the resistance of the circuit is the ordinary resistance, $R$, of the coil shown in Fig. 57. This simple case is the only one that need now be kept in mind, although the definition here given applies to cases to be discussed later, in which the so-called equivalent resistance of an alternating-current circuit is very different from the resistance of the circuit as ordinarily defined. $\dagger$

By inspection of Fig. 59 it is seen that the component of $E$, which is $90^{\circ}$ ahead of $I$ in phase, is equal to $(\omega L-1 / \omega C) I$. The factor $X(=\omega L-\mathrm{I} / \omega C)$, by which an alternating current is to be multiplied to give the component of $E$ which is $90^{\circ}$ ahead of $I$ in phase, is called the reactance of the alternating current circuit. According to this definition the reactance of the circuit is to be considered as negative if $E$ is behind $I$ in phase. In the case discussed above, the reactance is equal to $(\omega L-\mathrm{I} / \omega C)$, and this simple case is the only one that need now be kept in mind, although the definition here given applies to cases to be discussed later, in which the so-called equvalent reactance of an alternatingcurrent circuit is not equal, simply, to $(\omega L-\mathrm{I} / \omega C$. $)$.

By inspecting equation ( 8 ) it is seen that the effective value of an alternating electromotive force is equal to the effective value of the current multiplied by the square root of the sum of the squares of the resistance and the reactance of the circuit. The square root of $\left(R^{2}+X^{2}\right)$ is called the impedance of the circuit.

By substituting the value

$$
X=\omega L-\frac{\mathbf{I}}{\omega C}
$$

* See Art. 25, page 62, for a statement as to the representation of effective values in the clock diagram.

tSee the topic "equivalent resistance" in the index.

$\ddagger$ See the topic " equivalent reactance" in the index. 
in equations (8) and (9) we have :

or

and

$$
\left.\begin{array}{l}
E=I \sqrt{R^{2}+X^{2}} \\
I=\frac{E}{\sqrt{R^{2}+X^{2}}}
\end{array}\right\}
$$

$$
\tan \theta=\frac{X}{R}
$$

Resistance, reactance, and impedance are all expressed in ohms. It is to be remembered however that reactance, and of course impedance also, depend upon frequency as well as upon the conditions actually existent in the circuit.

29. Conductance, susceptance, and admittance. The factor $g$ by which the electromotive force $E$ is multiplied to give the component of $I$ parallel to $E$ is called the conductance of an alternating current circuit.

The factor $b$ by which the electromotive force $E$ is multiplied to give the component of $I$ which is $90^{\circ}$ behind $E$, is called the susceptance of an alternating current circuit, and

The factor $\sqrt{g^{2}+b^{2}}$ by which the value of the electromotive force must be multiplied to give the value of $I$ is called the admittance of an alternating current circuit.

The quantities conductance, susceptance, and admittance are related to resistance, reactance, and impedance as follows :

$$
\begin{aligned}
& g=\frac{R}{R^{2}+X^{8}} \\
& b=\frac{X}{R^{2}+X^{2}} \\
& y=\sqrt{g^{2}+b^{2}}=\frac{I}{z} \\
& z=\sqrt{R^{2}+X^{2}}=\frac{I}{y}
\end{aligned}
$$

in which $y$ is the admittance, and $z$ is the impedance.

The use of the quantities conductance, susceptance, and admittance simplifies the algebraic treatment of the problem of coils in parallel as exemplified in Art. 46.

30. Special cases of the problem of Art. 27. (a) Non-inductive circuit. In this case the electromotive force must overcome resistance only, and therefore the electromotive force is equal to $R i$ at each instant, that is the electromotive force is in phase with $i$ and its effective value is equal to $R I$. 
(b) Circuit containing resistance and inductance. In this case the component of $E$ parallel to $I$ is equal to $R I$, the reactance of the circuit is equal to $\omega L$, and the component of $E$ which is $90^{\circ}$ ahead of $I$ is equal to $\omega L I$, as shown in Fig. 6o. In

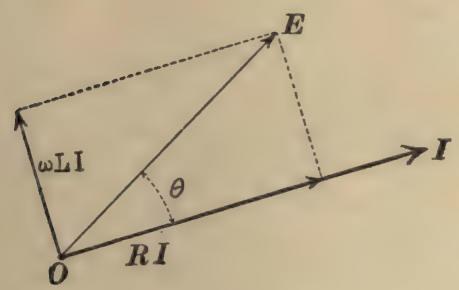

Fig. 60 .

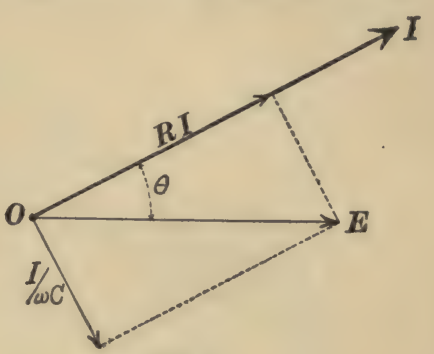

Fig. 61.

this case the current lags behind $E$ in phase by the angle whose tangent is $\omega L / R$, an angle which approaches $90^{\circ}$ when $\omega L$ is very large compared with $R$.

(c) Circuit containing resistance and a condenser. In this case the component of $E$ parallel to $I$ is equal to $R I$, the reactance of the circuit is equal to minus $\mathrm{I} / \omega C$, and the component of $E$ which is $90^{\circ}$ behind $I$ is equal to $I \times \mathrm{I} / \omega C$ as shown in Fig. $6 \mathrm{I}$. In this case the current is ahead of $E$ in phase by the angle whose tangent is $\mathrm{I} / \omega C$ divided by $R$, an angle which approaches $90^{\circ}$ when $\mathrm{I} / \omega C$ is very large compared with $R$.

31. Growth and decay of current in an inductive circuit. (a) When a constant electromotive force $\mathcal{E}$ is applied at a given instant to a circuit containing resistance and inductance, the current gradually rises from its initial value of zero to a final steady value equal to $\mathcal{E} / R$. During the time that the current is growing in value, part of the impressed electromotive force acts to overcome resistance and the part so used is equal to $R i$; and part of the impressed electromotive force acts to cause the current to increase and the part so used is equal to $L \cdot d i / d t$. Therefore we have :

$$
\mathcal{E}=R i+L \frac{d i}{d t}
$$

The integration of this differential equation under the condition that $i=0$ when $t=0$ (that is $i=0$ at the instant when the electromotive force $\mathcal{E}$ is applied), gives the equation of the growing current, namely :

in which $\varepsilon$ is the Napierian base.

$$
i=\frac{\mathcal{E}}{R}-\frac{\mathcal{E}}{R} \cdot e^{-\frac{R}{L} \cdot t}
$$


A graphical representation of the growing current in an inductive circuit immediately after a constant electromotive force $\mathcal{E}$ is applied to the circuit is shown in Fig. 62 .

(b) If a given current is established in an inductive circuit and the circuit is then closed and left to itself, the current dies away or decays. In this case no electromotive force acts on the circuit from without, and equation (i) becomes :
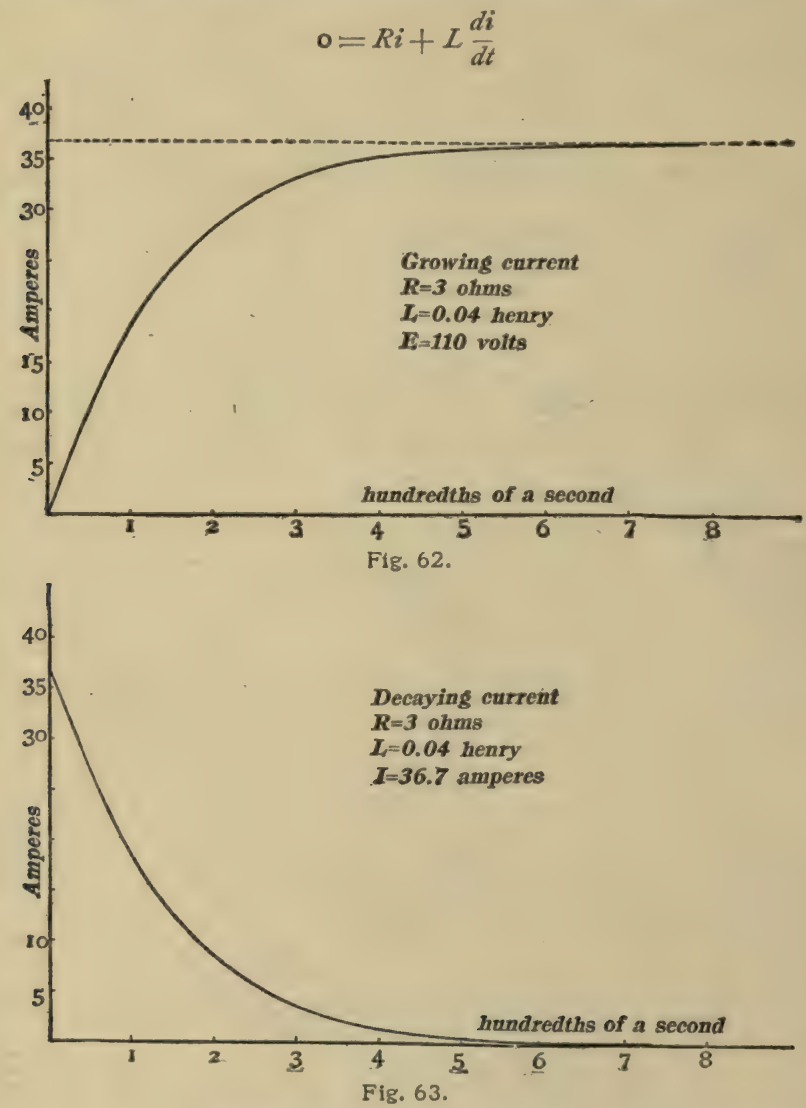

The integration of this differential equation under the condition that $i=$ a given value, $\mathfrak{J}$, when $t=0$, gives the equation of the decaying current, namely ;

$$
i=g \varepsilon^{-\frac{R}{L} \cdot t}
$$

in which $\varepsilon$ is the Napierian base, and $\mathcal{g}$ is the given value of the current when $\boldsymbol{t}=\mathrm{o}$.

A graphical representation of the decaying current in an inductive circuit is shown in Fig. 63 . 
32. The decaying oscillatory current. When a charged condenser is shortcircuited by a circuit containing resistance and inductance, a current surges back and forth through the circuit, each surge of current being less in value than the preceding surge, until the surges cease. Such a decreasing or decaying oscillatory current is represented graphically in Fig. 64.*

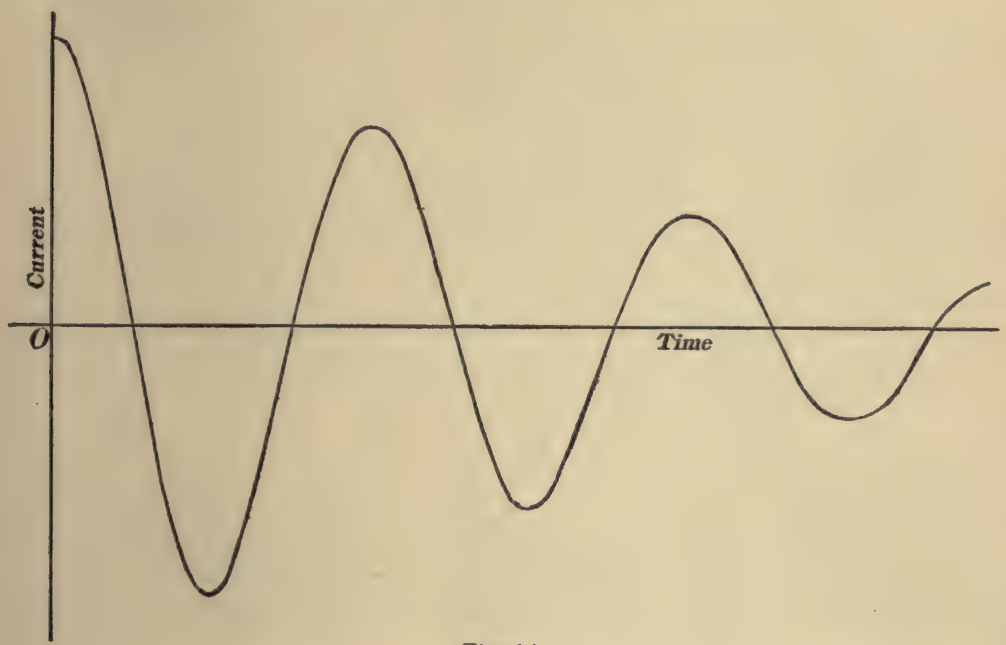

Fig. 64.

33. The effects which are produced immediately after a harmonic alternating electromotive force is connected to a circuit. (a) When the circuit contains resistance and inductance. The dotted curve e Fig. 65 represents a given harmonic alternating electromotive force, and the curve $m$ represents the harmonic current which would be maintained by $e$ in a given inductive circuit. The relation between $e$ and $m$ is given by equations (II) and (I2) Art. 28. If the harmonic electromotive force is connected to the circuit at any instant $t$ at which the maintained current $m$ would be zero, then the maintained current starts off at once without any complications. . If, however, the harmonic electromotive force is connected to the circuit at any instant $t^{\prime}$ at which the maintained $m$ would not be zero, then, since the actual current must be zero at the instant that $e$ is connected to the circuit, the result is complicated. The current which starts is the maintained current $m$ plus a decaying current $d$, the decaying current being such that $(m+d)$ is zero at the instant $t^{\prime}$. After a very short time the decaying current $d$ disappears, and the maintained current $m$ alone exists.

* The differential equation of the decaying oscillatory current is

$$
L \frac{d^{2} q}{d t^{2}}+R \frac{d q}{d t}+\frac{q}{C}=0
$$

in which $q$ is the varying charge on the condenser. The integration of this equation is discussed in Bedell and Crehore's Alternating Currents, 2d edition, Chap. VI, pages 105-108. 
(b) When the circuit has resistance and inductance and contains a condenser. In this case the current which starts to flow is the maintained current $m$ plus a decaying oscillatory current, and after a very short time the decaying oscillatory current disappears and the maintained current $m$ alone exists.

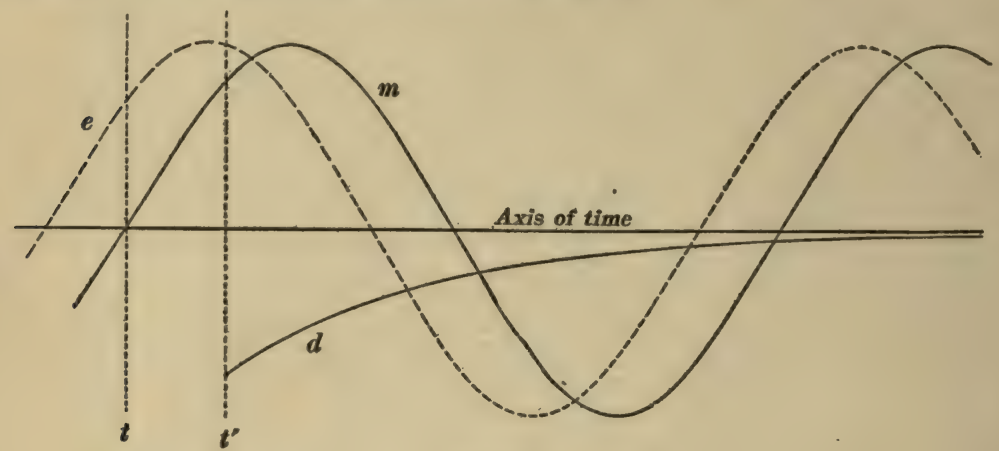

Fig. 65.

34. Electric resonance. A harmonic electromotive force of given effective value will produce the greatest possible value of alternating current in a given circuit (given resistance, given inductance, and given capacity arranged in series as shown in Fig. 57 ), if the frequency of the electromotive force is such as to make

$$
\omega L-\frac{\mathbf{I}}{\omega C}=0
$$

This is at once evident from equation (8) of Art. 27. This production of a maximum current in a given circuit by an electromotive force of a given effective value at a certain critical frequency is called resonance. The critical frequency in radians per second $(\omega)$ is determined by equation (i), namely :

$$
\omega=\sqrt{\frac{\mathrm{I}}{L C}}
$$

or since the frequency, $f$, in cycles per second is equal to $\omega / 2 \pi$, we have :

$$
f=\frac{\mathrm{I}}{2 \pi} \sqrt{\frac{\mathrm{I}}{L C}}
$$


At the critical frequency the reactance $(\omega L-\mathrm{I} / \omega C)$ is equal to zero, equation (8) of Art. 27 reduces to $I=E / R$, and equation (9) of Art. 27 shows that $\theta$ is zero. That is, at the critical frequency the reaction of the condenser annuls the reaction of the inductance, and, after the current becomes established, the circuit behaves as if it contained resistance only. The annulling of inductive reaction by capacity reaction means simply that the electromotive force, $\omega L I$, Fig. 59, which overcomes inductance is equal and opposite to the electromotive force, $I / \omega C$, which overcomes the reaction of the condenser, so that the electromotive force which acts on the circuit has to overcome resistance only.

The very sharply defined frequency at which resonance occurs is shown in Fig. 66. The ordinates of the curve represent the

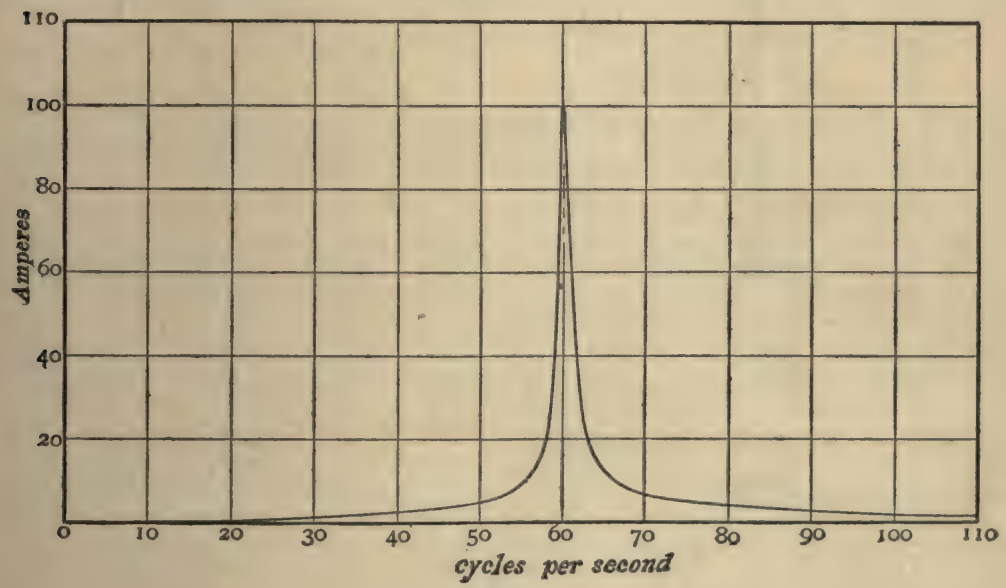

Fig. 66 .

effective values of the current produced by a harmonic electromotive force of 200 volts (effective) in a circuit like Fig. 57 in which $R=2$ ohms, $L=0.352$ henry, and $C=20 \times 10^{-6}$ farad; and the abscissas represent various frequencies. At very low frequencies the value of $I / \omega C$ is very great, and the current is limited by the condenser reaction; at the critical frequency of 60 cycles per second, the current is equal to $E / R(=$ IOO amperes effective); and at very high frequencies the value 
of $\omega L$ is very great, and the current is limited by the inductance reaction. It is interesting to note that at the critical frequency of 60 cycles per second, with a current of Ioo amperes, the electromotive force across the condenser terminals is 13,270 volts effective $(=I / \omega C)$ although the total electromotive force acting on the circuit is only 200 volts.

Mechanical resonance, analog of electric resonance. A weight $M$, Fig. 67, is attached to the end of a flat spring which is clamped in a vise, and the weight $M$ is caused to oscillate back

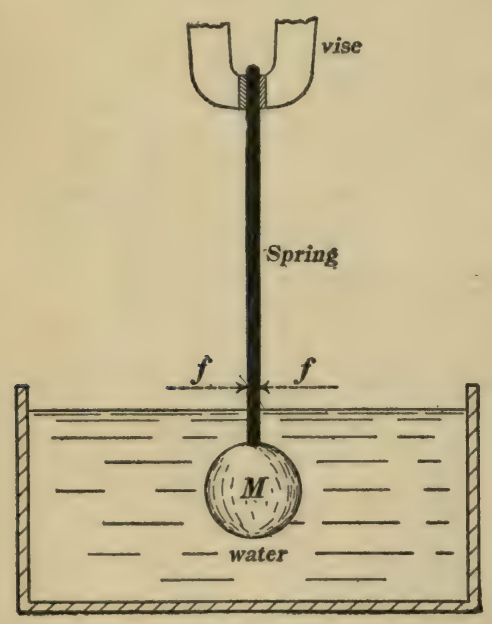

Fig. 67. and forth in a vessel of water by: an alternating force $f$ of definite frequency. In general the force $f$ has to do three things, namely : (a) Overcome the resistance with which the water opposes the to-and-fro motion of $M ;(b)$ overcome the inertia of the weight $M$ as it gains and loses velocity; and (c) overcome the elastic reaction of the spring. These three parts of $f$ are related to each other and to the changing velocity $v$ of $M$ in exactly the same way that the three parts, $R i, L \cdot d i j d$, and $q / C$ (see Art. 27), of an impressed electromotive force are related to each other and to the changing current $i$ in the circuit shown in Fig. 57. The weight $M$ when mounted as shown in Fig. 67 has a definite frequency of free oscillation, and at this frequency the elasticity of the spring takes care of the inertia reaction of $M$, and the inertia reaction of $M$ provides the force necessary to bend the spring. Therefore, if the frequency of the force $f$, Fig. 67 , is the same as the frequency of the free oscillations of $M$, then, after the oscillatory motion of $M$.is fully established, the force $f$ is used only to overcome resistance. 
A careful consideration of this mechanical analog will serve to elucidate one of the most important aspects of electrical resonance. Suppose that the mechanical resistance of the water in Fig. 67 is not very large, then a very small alternating force $f$ applied to the system shown in Fig. 67 will, if it continues to act for some time, build up a very violent oscillatory motion, and the spring will be subjected to bending forces vastly greater than the maximum value of the force $f$. In fact the spring may be broken by the continued application of a comparatively weak alternating force of the proper frequency. Similarly, a weak alternating electromotive force of the critical frequency applied to the circuit shown in Fig. 57 will, if it continues to act for some time, build up a very large alternating current, and the electromotive force across the condenser terminals $(=I / \omega C)$ may become so large as to break through the insulation of the condenser.

35. Multiplication of electromotive force by resonance. When resonance exists in a circuit containing a condenser and an inductance in series, the effective electromotive force, $\omega L I$, across the terminals of the inductance and the effective electromotive force $I / \omega C$ across the terminals of the condenser may both be much greater than the effective electromotive force $R I$ which acts on the circuit. This fact is easily understood by referring to the mechanical analog. Thus, if a periodic force acts on a weight which is suspended by a helical spring, the weight will be set into very violent oscillation even if the periodic force is weak, provided the frequency of the force is the same as the frequency of free oscillation of the weight. Under these conditions the force which alternately stretches and compresses the spring, and the force which accelerates the weight up and down may both be enorm. ously greater than the periodic force which acts on the system from outside.

Example. - Under the conditions specified in connection with Fig. 66 , namely, $R=2$ ohms, $L=0.352$ henry, and $C=20$ $\times 10^{-6}$ farad, the electromotive force of 200 volts at the critical 
frequency produces IOo amperes, and $\omega L I$ and $I / \omega C$ are both equal to 13,270 volts.

36. Multiplication of current by resonance. - When an inductance and a condenser are connected in parallel,* as shown in Fig.

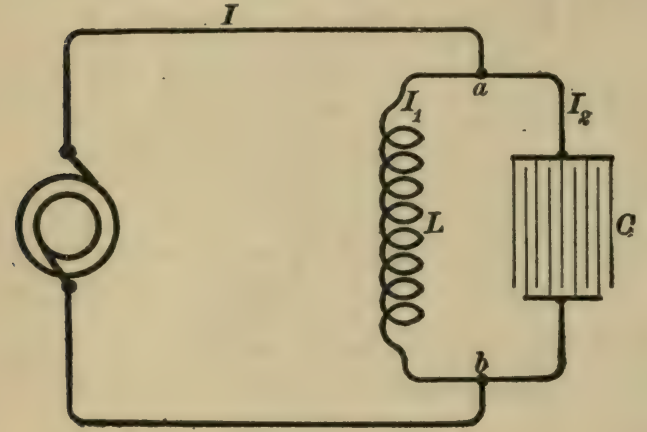

Fig. 68.

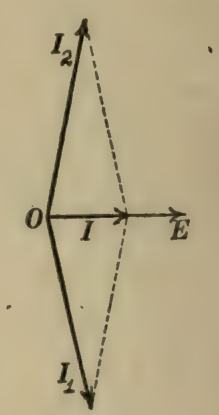

Fig. 69.

68 , the total current delivered to the two divides, and the current in each may greatly exceed the total current. The current relations are shown in the clock diagram Fig. 69. In this figure the line $O E$ represents the electromotive force between the branch points $a$ and $b$, the line $O I_{1}$ represents the current that flows through the inductance, the line $\mathrm{OI}_{2}$ represents the current that flows through the condenser, and the line $O I$ represents the total current delivered by the alternator.

The multiplication of current by resonance may be easily understood by referring to the mechanical analog. A lever $l l$, Fig. 70, is suspended at its center by a bar $b$ which oscillates up and down through a small amplitude. One end of the lever carries a weight $L$, and the other end is held down by a helical spring $C$. If the up and clown oscillations of the bar $b$ take place at the proper frequency, the lever will be set into a violent see-saw motion, and the velocities $I_{1}$ and $I_{2}$ of the ends of the lever will greatly exceed the

* Although the inductance and the condenser in Fig. 68 are in parallel with each other with reference to the alternator, they are in series with each other in the short circuit $\mathrm{LaCb}$, and multiplication of current by resonance is due to the surging of cur. rent to and fro around this short circuit so that the phvsical action of the inductance and condenser during resonance is exactly the same in Fig. 68 as it is in Fig. 57. In both cases the condition of resonance is given by equation (i), Art. 34 . 
velocity $I$ of the center of the lever, although (half) the sum of the velocities of the ends of the lever is at each instant equal to

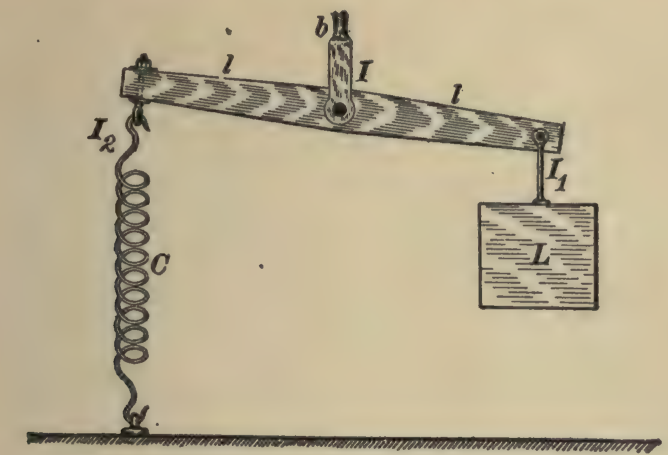

Flg. 70.

the velocity of the center of the lever, just as the sum of the currents in $L$ and $C$ Fig. 68 is at each instant equal to the current delivered by the alternator.

37. The influence of inductive reaction and of capacity reaction upon the shape of the current curve (non-harmonic electromotive

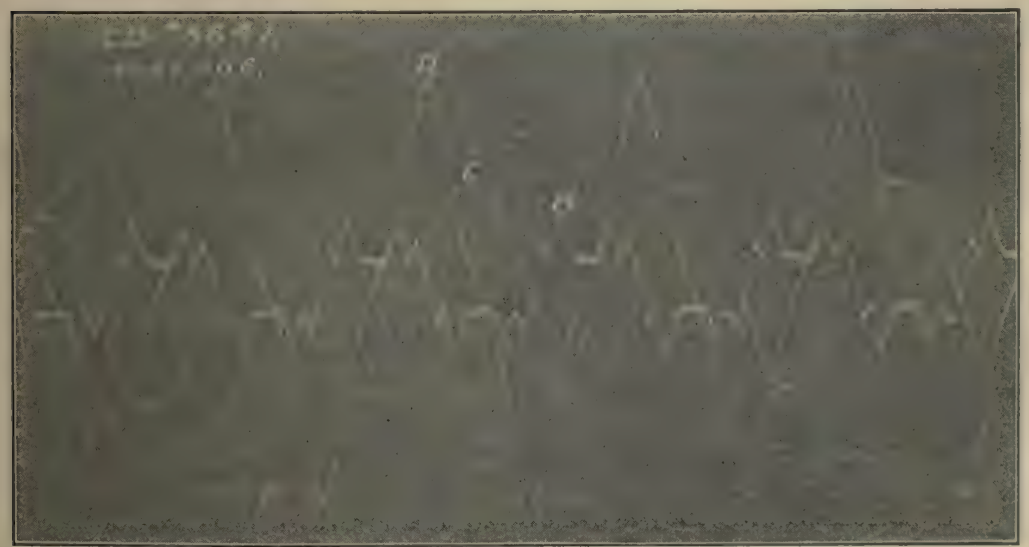

Fig. 71.

force). As has been stated in Art. 7, the alternating current which is produced by any alternating electromotive force in a cir- 
cuit which has resistance only, has the same wave shape as the electromotive force, inasmuch as the current is at each instant equal to $e / R$, that is, the current curve may be considered as representing the electromotive force curve. Thus the oscillograph curve $A$ in Fig. $7 \mathrm{I}$ is the electromotive force curve of an alternator whose armature winding is placed in slots, the effect of the slots being to introduce slight fluctuations in the the electromotive force wave, as shown by the curve $A$.

The effects of inductance. A circuit having inductance is a circuit having electrical inertia, and very rapid variations of electromotive force produce but little variation in the current on account of the inertia. This is shown by the oscillograph curve $C$ in Fig. $7 \mathrm{I}$, which is the curve of the current which is produced in an inductance coil (without an iron core) by the electromotive force $A$. Any non-harmonic alternating electromotive force produces an approximately harmonic current in a highly inductive circuit (with no iron core).

The effects of a condenser. Any quick change of electromotive force produces a sudden rush of current into or out of a condenser, and therefore rapid variations of electromotive force produce large variations of current in a circuit which contains a condenser. This is shown by the oscillograph curve $B$ in Fig. $7 \mathrm{I}$, which is the curve of current produced by the electromotive force $A A$ in a circuit containing a condenser.

The effect of inductance in giving a smooth current curve and the effect of capacity in giving a current in which the irregularities of the electromotive force are greatly exaggerated are very strikingly shown by Figs. 72 and 73 . Fig. 72 shows a peaked electromotive force curve produced by an alternator having a few very large armature slots, the curve $A A$, Fig. 73 , is the same as the curve of Fig. 72 , the curve $C C$ is the fairly smooth curve of current produced in an iron-less inductance by the electromotive force $A A$, and the extremely irregular curve $B B$ is the curve of current produced in a condenser by the electromotive force $A A$. 
FUNDAMENTAL PROBLEMS.

Effects of inductance and capacity (each without the other) at the instant of connecting an alternating electromotive force to a circuit. The current in a non-inductive circuit starts out at once

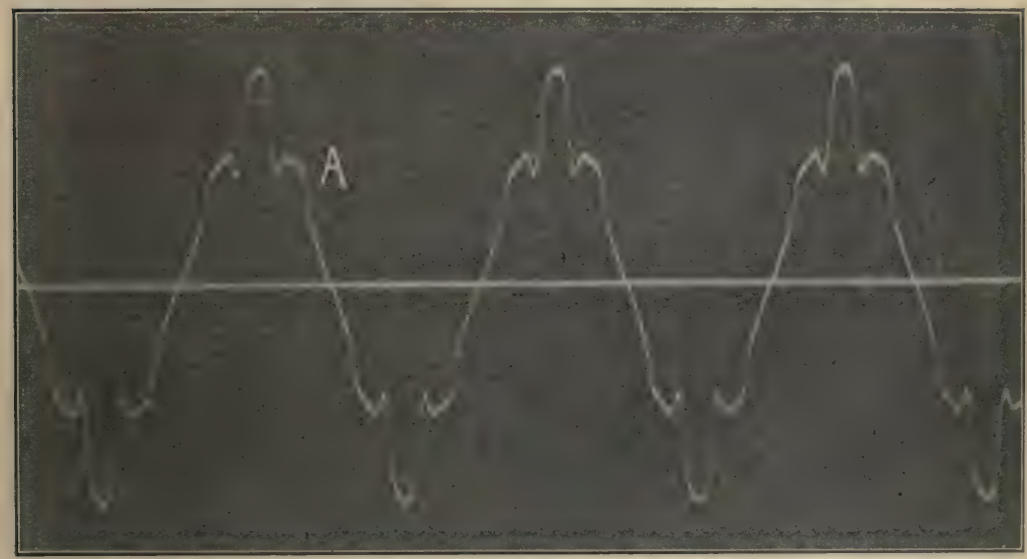

Fig. 72.

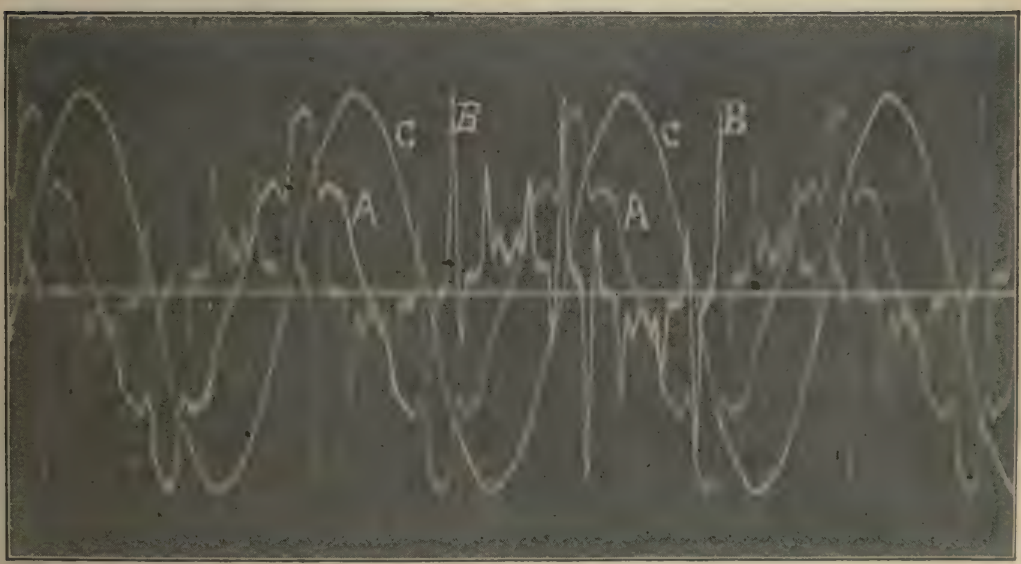

Fig. 73.

as the steady maintained current, at whatever instant during the cycle the electromotive force may begin to act on the circuit. The current in an inductive circuit starts out in a manner which is explained in Art. 33, and the current in a circuit containing a 
condenser starts out with the value $e / R$ at the very instant at which the electromotive force begins to act. These effects are shown in Fig. 74; $A A$ is the electromotive force curve (current curve in a non-inductive circuit), $C C$ is the current produced by $A A$ in an iron-less inductance, and $B B$ is the current produced by $A A$ in a condenser. The inequalities of the first two or

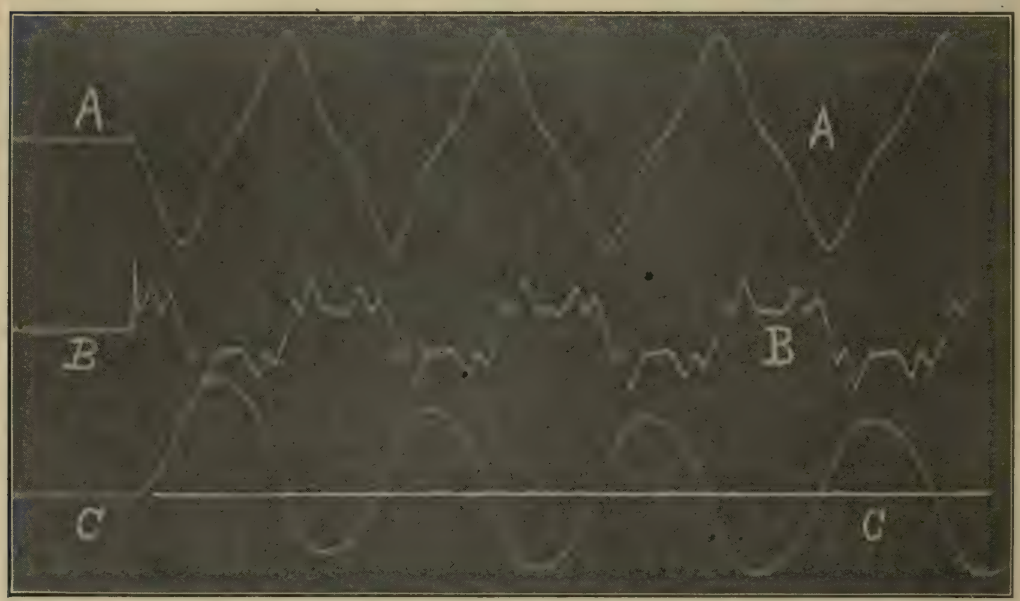

Fig. 74.*

three positive and negative half-waves in curve $C C$ illustrate the effects described. in Art. 33 (Fig. 65), and the extremely sharp peak in curve $B B$ at the instant of connecting illustrates the effect of a condenser. The curve $A A$ is upside down, that is, the oscillograph which traced curve $A A$ should have had its connections reversed.

38. Current loci. In some practical problems, for example in the analysis of the action of the transformer and of the induction motor, it is necessary to consider the manner in which the current varies in value and in phase: (a) When the resistance of the circuit is constant and the reactance is changed slowly from zero to an indefinitely large value, and $(b)$ When the reactance of the circuit is constant and the resistance is changed slowly from zero to an indefinitely large value; the electromotive force having a constant (effective) value and a constant frequency in each case.

* The photographs from which Figs. $71,72,73$ and 74 are reproduced were kindly furnished to the authors by the General Electric Company. 
(a) Current iocus with constant resistance and variable reactance. A given electromotive force $O E$, Fig. 75, acts on a circuit whose resistance $R$ is constant and whose reactance $X$ varies. Let the line $O I$, Fig. 75, represent a possible value of the current. Then $R I$ is the component of $E$ parallel to $I$, and $X I$ is the component of $E 90^{\circ}$ ahead of $I$. Therefore the triangle $O P E$ is a right triangle with a constant hypothenuse $O E$, so that the point $P$ must lie on the circle of which $O E$ is the diameter. That is, the end of the line $R I$ describes a circle as $X$ varies, therefore, since $R$ is constant, the end of the line $O I$ must also describe a circle. The circle described by the end of the line $O I$ is called the current locus under the given conditions, namely, $E$ and $R$ constant, and $X$ variable. The diameter $O a$ of the circular current locus is equal to $E / R$ and it represents the value of $I$ which corresponds to $X=0$.

It is especially noteworthy that the current at first decreases imperceptibly in value when, starting from $X=0$, the value of $X$ is increased. That is to say, the effect

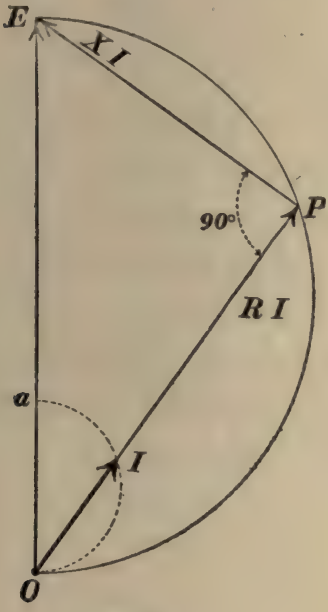

Fig. 75.

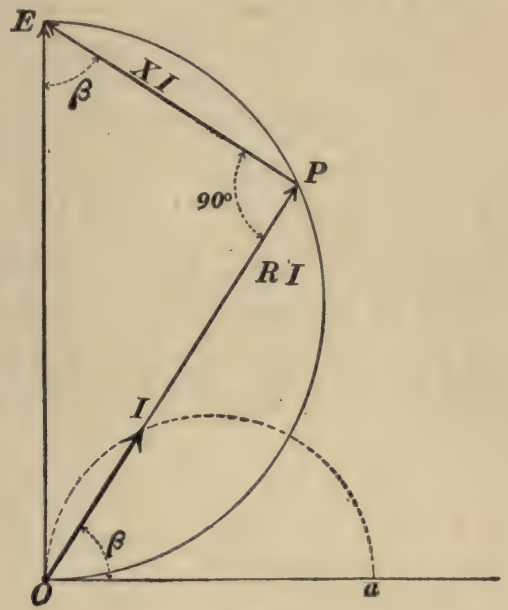

Fig. 76.

of a small value of $X$ introduced into a circuit of given resistance is to turn $I$ very perceptibly behind $E$ in phase, but not to produce any appreciable decrease in the value of $I$.

(b) Current locus with constant reactance and variable resistance. A given electromotive force, $O E$ Fig. 76, acts on a circuit whose reactance $X$ is constant, and whose resistance $R$ varies. Let the line $O I$ represent a possible value of the current. Then $R I$ is the component of $E$ parallel to $I$, and $X I$ is the component of $E 90^{\circ}$ ahead of $I$. Therefore the triangle $O P E$ is a right triangle with a constant hypothenuse $O E$, so that the point $P$ must lie on the circle of which $O E$ is the diameter. Now, the two angles $\beta$ are equal, and $X I$ is proportional to $I$ since $X$ is constant. Therefore the point $I$ lies on a circle (the required current locus), of which the diameter $O a$ is at right angles to $O E$, and equal to the current $E / X$, which corresponds to $R=0$. 
39. Voltage drop in transmission lines. A transmission line is of course in series with the circuit to which it delivers current, and the complete solution of the problem of the loss of voltage in a transmission line depends upon the complete solution of the problem of coils in series. This general problem is discussed in detail in Chapter V.

(a) Voltage drop in a transmission line which delivers current to a non-inductive receiving circuit. Let $O I$, Fig. 77 , represent

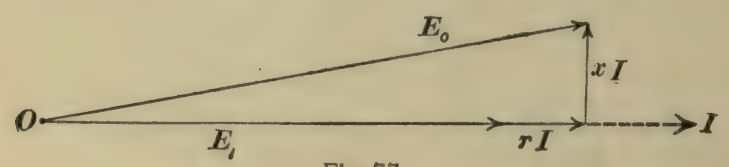

Fig. 77.

the current flowing through the transmission line and the receiving circuit. The voltage $E_{1}$ at the terminals of the receiving circuit is in phase with $I$, and the generator voltage $E_{0}$ is the vector sum of $E_{1}, r I$, and $x I$; where $r I$ is the electromotive force used to overcome the line resistance, and $x I$ is the electromotive force used to overcome the line reactance. An examina-

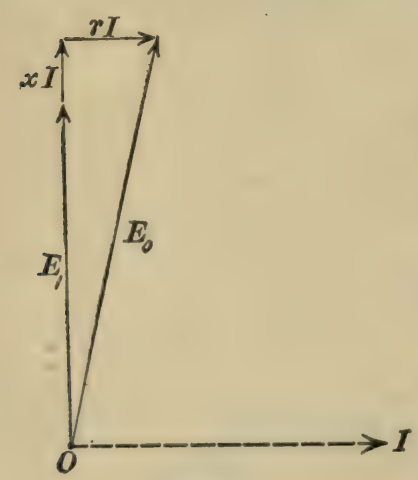

Fig. 78 . tion of the figure shows that when the receiving circuit is non-inductive the line resistance produces a difference in value between $E_{0}$ and $E_{1}$, whereas the line reactance produces a difference in phase between $E_{0}$ and $E_{1} *$

(b) Voltage drop in a transmission line which delivers current to a highly inductive receiving circuit of small resistance. Let $O I$, Fig. 78, represent the current flowing through the transmission line and the receiving circuit. The voltage $E_{1}$ at the terminals of the receiving circuit is nearly $90^{\circ}$ ahead of $I$ in phase as shown, and the generator voltage $E_{0}$ is the vector sum of $E_{1}$,

\footnotetext{
* This statement is made on the assumption that $x I$ is small as compared with $E_{1}$.
} 
$r I$, and $x I$ as before. In the present case, however, the line resistance produces a difference in phase between $E_{0}$ and $E_{1}$, and the line reactance produces a difference in value between $E_{0}$ and $E_{1}$ *

(c) Voltage drop in a transmission line which delivers current to a condenser or any receiving circuit having negative reactance, but having small resistance. This case is shown in Fig. 79, and $E_{0}$ is the vector sum of $E_{1}, r I$, and $x I$ as before; but, $E_{1}$ and $x I$ are nearly opposite in direction, inasmuch as $E_{1}$ is nearly $90^{\circ}$ behind $I$ under the assumed conditions. Therefore in this case the line resistance produces a difference in phase between $E_{0}$ and $E_{1}$ and the line reactance produces a difference in value between $E_{0}$ and $E_{1}$, making $E_{1}$ greater than $E_{0} *$

(a) Voltage drop in a transmission line which delivers current to a receiving circuit whlose power factor, $(\cos \theta)$,

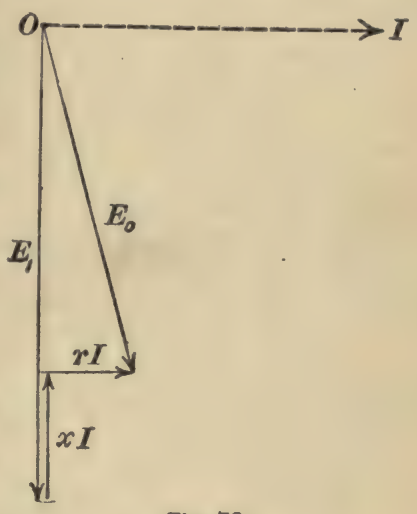

Fig. 79. has any given value. Let the line $O I$, Fig. 80, represent the current flowing through the transmission line and the receiving circuit. Let the line $E_{1}$ represent the voltage across the receiving circuit, $\theta$ being the phase difference between $I$ and $E_{1}$ as shown.

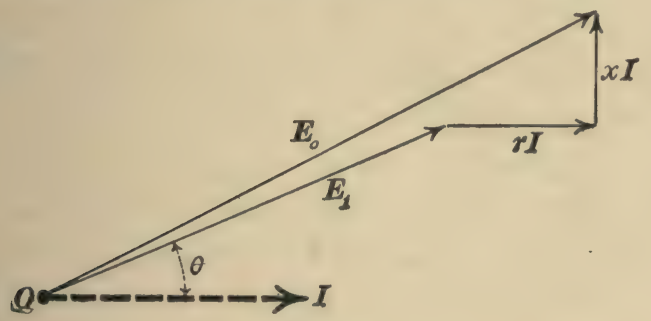

Fig. 80 .

Let $r$ be the resistance and $x$ the reactance of the transmission line, and let $E_{0}$ be the voltage at the generator. Lay off $r I$ parallel to $O I$, and $x I$ at right angles to $O I$. Then the difference

*This statement is made on the assumption that $r I$ is small as compared with $E_{1}$. 
between the values of $E_{1}$ and $E_{0}$ is the required transmission line drop.

The value of $\sqrt{r^{2} I^{2}+x^{2} I^{2}}$ is called the impedance drop, and the difference between the numerical values of $E_{0}$ and $E_{1}$ is called simply the (voltage) drop in the line.

40. Circuits in parallel. The general problem of circuits in parallel is discussed in Chapter V. Two simple cases of this problem are, however, of sufficient interest to be treated in this chapter on fundamental problems, namely, $(a)$ the problem of finding the power factor of two receiving circuits in parallel, the power factor of each and the current delivered to each being given, and $(b)$ the problem of compensating for lagging currents.

(a) Power factor of receiving circuits in parallel. Let the line $O E$, Fig. $8 \mathrm{I}$, represent the voltage across the two receiving

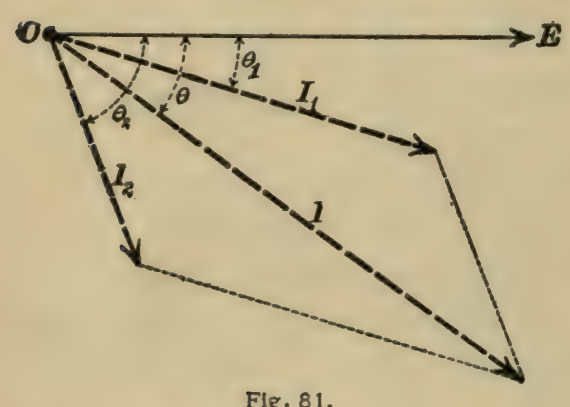

Fig. 81. circuits. Let $I_{1}$ be the current delivered to receiving circuit number $\mathrm{I}$ and $\cos \theta_{1}$ its power factor, and let $I_{2}$ be the current delivered to receiving circuit number 2 and $\cos$ $\theta_{2}$ its power factor. Lay off the clock diagram in Fig. 8I carefully to scale, and draw the diagonal $I$ as shown. The angle $\theta$ between $E$ and $I$ is then the angle whose cosine is the required power factor of the two receiving circuits in parallel.

(b) Compensation for lagging currents. - When a transmission line delivers current to an inductive receiving circuit, power is delivered over the line to the receiving circuit during the time that the product $e i$ is positive, and power is delivered back over the line from the receiving circuit to the generator during the time that the product $e i$ is negative (compare Art. 9). This backward flow of energy from receiving circuit to generator represents an essentially unnecessary service of the transmission line, and it is 
desirable, and in some cases feasible, to reduce this backward flow of energy by connecting a condenser or something which is equivalent to a condenser (such as an over-excited synchronous motor) in parallel with the receiving circuit.

Let $O E$, Fig. 82 , represent the voltage at the terminals of the

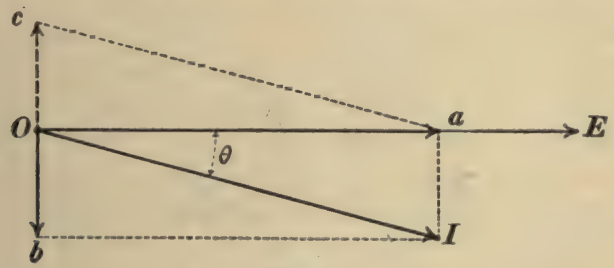

Fig. 82.

receiving circuit, and let $O I$ represent the current delivered to the receiving circuit. Let $R$ be the resistance of the receiving circuit and $X$ its reactance. Then

$$
\tan \theta=\frac{X}{R}
$$

and

$$
\sin \theta=\frac{X}{\sqrt{R^{2}+X^{2}}}
$$

The component $O b$ of the current $I$ is equal to $I \sin \theta$ or $I X / \sqrt{R^{2}+X^{2}}$. But $I$ is equal to $E / \sqrt{R^{2}+X^{2}}$, so that the component $O b$ is equal to $E X /\left(R^{2}+X^{2}\right)$. If a condenser of capacity $C$ is connected across the transmission line at the receiver end, then the current flowing into the condenser will be $90^{\circ}$ ahead of $E$, or parallel to $O c$, Fig. 82 , and its value will be equal to $E$ divided by the condenser reactance $\mathrm{I} / \omega C$, or to $E \omega C$; and if this current is numerically equal to $O b$, the sum of $I$ and $O c$ will be in phase with $E$. That is, the receiving circuit and the condenser together will take current in phase with $E$, and the instantaneous value of power delivered over the transmission line will never be negative. The capacity of the condenser required to produce this result is given by the relation above mentioned, namely, $E \omega C=E X /\left(R^{2}+X^{2}\right)$, which gives

$$
C=\frac{X}{\omega\left(R^{2}+X^{2}\right)}
$$




\section{CHAPTER V}

\section{THE USE OF COMPLEX QUANTITY}

41. Methods in alternating currents. The current-voltage relations in any network of circuits, or in any generator, motor, or transformer, may be completely represented by the geometric relations of the lines in a clock diagram, on the assumption that all voltages and currents are harmonic. Therefore any problem in alternating currents may be solved in either of two ways, namely, (a) Graphically, by drawing the clock diagram carefully, so as to represent the given data to scale, and measuring off the desired result, or $(b)$ Trigonometrically, by establishing the trigonometric equations which express the conditions represented in the clock diagram, and solving these equations so as to express the desired results in terms of the given data.

The difficulty with the trigonometric method is that the equations are often very complicated and the necessary transformations are often very tedious. This difficulty

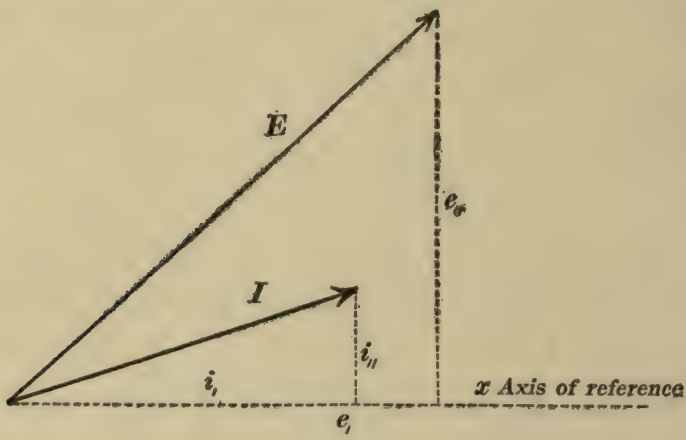

Fig. 83.

is greatly reduced by the use of complex quantity, inasmuch as in this case the initial equations for the solution of a problem can be written out with the utmost ease, especially if a rough sketch of the clock diagram is used as a guide, and all trigonometric transformations are greatly simplified. It is to be remembered, however, that the use of complex quantity in alternating-current problems does not constitute a distinct method; it is merely a systematic scheme for carrying out method $(b)$ above.

42. The use of complex quantity in alternating-current problems involves two distinct ideas, namely, $(a)$ The specification of a vector in the clock diagram by giving its components in the directions of chosen axes of reference, and $(b)$ The idea of impedance as an algebraic operator.

(a) Specification of current and electromotive force vectors by giving their components. Consider the clock diagram Fig. 83, in which an axis of reference is indicated. This axis of reference is supposed to rotate with $E$ and $I$ so that $E$ 
and $I$ are stationary with respect to the reference axis. Let $i_{1}$ and $i_{11}$ be the components of $I$, and let $e_{1}$ and $e_{11}$ be the components of $E$, then we may write:

and

$$
I=i_{1}+j i_{11}
$$

$$
E=e_{1}+j e_{11}
$$

in which the letter $j$ is merely a mark used to distinguish the $y$-component, and the plus sign means no more than to say, for example, that $I$ consists of an $x$-component $i_{1}$ and a $y$-component $i_{11}$.

The utility of equations (i) and (ii) can be at once exemplified by considering the composition and resolution (addition and subtraction) of currents or electromotive forces. The algebraic meaning of the index letter $j$ will be completely determined when we consider the idea of impedance as an algebraic operator.

Example 1. Addition. Given three harmonic alternating electromotive forces, of the same frequency, which are to be added together. If these three electromotive forces be represented by three lines $A, B, C$, in a clock diagram, then their sum will be represented by the line which is the vector sum or resultant of $A, B$ and $C$; the $x$-component of this resultant will be equal to the sum of the $x$-components of $A, B$ and $C$; and its $y$-component will be equal to the sum of the $y$-components of $A, B$ and $C$. That is to say, electromotive forces (or currents) are added by adding their $x$-components to give the $x$-component of the sum, and by adding their $y$-components to give the $y$-component of the sum. Thus $\left(e_{1}+j e_{11}\right)+\left(f_{1}+j f_{11}\right)=$ $e_{1}+f_{1}+j\left(e_{11}+f_{11}\right)$.

Example 2. Subtraction. Given two harmonic electromotive forces, of the same frequency, one of which is to be subtracted from the other. If these electromotive forces be represented by lines $A$ and $B$ in a clock diagram, then their difference will be represented by the line which is the vector difference of $A$ and $B$, the $x$-component of this line will be the difference of the $x$-components of $A$ and $B$, and its $y$-component will be the difference of the $y$-compo-

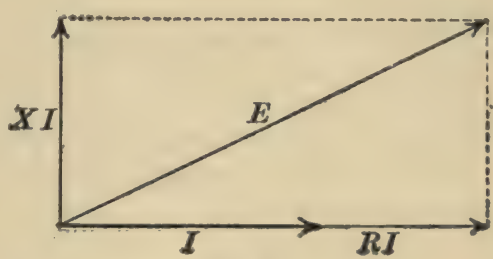

Fig. 84. nents of $A$ and $B$. That is to say, electromotive forces (or currents) are subtracted by subtracting their $x$-components to give the $x$-component of their difference, and by subtracting their $y$-components to give the $y$-component of their difference. Thus $\left(e_{1}+j e_{11}\right)-\left(f_{1}+j f_{11}\right)$ $=e_{1}-f_{1}+j\left(e_{11}-f_{11}\right)$.

(b) The idea of impedance as an algebraic operator. Let the current vector $I$, Fig. 84 , be chosen as the reference axis, then the $x$-component (parallel to $I$ ) of $E$ is equal to $R I$, and the $y$-component ( $90^{\circ}$ ahead of $l$ ) of $E$ is $X I$. Therefore, marking the $y$-component with the index letter $j$, we have

or

$$
E=R I+j X I
$$

$$
E=(R+j X) I
$$


If $I$ is not the reference axis we have, from equation $(i)$ :

$$
I=i_{1}+j i_{11}
$$

which, substituted in equation (15) gives

$$
E=(R+j X)\left(i_{1}+j \ddot{i}_{11}\right)
$$

If we are to build up a consistent system of algebra on equations (i), (ii), and (15), it is necessary to assign to the letter $j$ a meaning which will not only enable it to serve as a distinguishing mark for the $y$-component in such expressions as (i), (ii), and (15), but which will make equation (iii) give the correct expressions for the components of $E$. That is, we must have :

$$
E=e_{1}+j e_{11}=R i_{1}+j^{2} X i_{11}+j X i_{1}+j R i_{11}
$$

which comes from equation (iii) by substituting $e_{1}+j e_{11}$ for $E$ and carrying out the multiplication indicated in the right hand member of (iii). It can be shown, however, by ordinary trigonometry that the components of a line whose length is $I V \overline{R^{2}+X^{q}}$ and which is $\theta$ degrees ahead of $I$, where $\theta$ is the angle whose tangent is $X / R$, are

and

$$
\left.\begin{array}{r}
e_{1}=R i_{1}-X i_{11} \\
e_{11}=X i_{1}+R i_{11}
\end{array}\right\}
$$

Therefore the term $j^{2} X i_{11}$ in the above expression (iv) must be equal to $-X i_{11}$, or, in other words, we must have :

$$
j^{2}=-\mathbf{I}
$$

It should be stated in explanation of the derivation of equation (vi) from (iv) and (v) that a complex equation is always equivalent to two simple equations; those parts of the two members which do not involve the unit $j$ are equal to each other, and those parts which contain $j$ as a factor are equal to each other.

In equation (15) the expression $R+j X$ stands as a multiplier and it is called a direct operator. If, however, we solve equation (15) for $I$ we have :

$$
I=\frac{E}{R+j X}
$$

In this equation the expression $R+j X$ stands as a divisor and in this case it is called an inverse operator. If we multiply numerator and denominator of the right hand member of equation (vii) by $R-j X$, remembering that $j^{2}=-I$ we have:

or

$$
I=\frac{R-j X}{R^{2}+X^{2}} \cdot E
$$

$$
I=\left(\frac{R}{R^{2}+X^{2}}-j \frac{X}{R^{2}+X^{2}}\right) E
$$

According to this equation $E \times R /\left(R^{2}+X^{2}\right)$ should be the component of $I$ parallel to $E$, and $-E \times X /\left(R^{2}+X^{2}\right)$ should be the component of $I 90^{\circ}$ ahead of $E$, or $E \times X /\left(R^{2}+X^{2}\right)$ should be the component of $I 90^{\circ}$ behind $E$. In fact these are the correct expressions for the components of $I$. 
Admittance, conductance, and susceptance. By comparing equations (vii) and (viii) it is evident that impedance $R+j X$ used as an inverse operator is equivalent to the expression $R /\left(R^{2}+X^{2}\right)-j X /\left(R^{2}+X^{2}\right)$ used as a direct operator. This operator is called the admittance of the circuit; the factor $R /\left(R^{2}+X^{2}\right)$ which, multiplied by $E$, gives the component of $I$ parallel to $E$, is called the conductance of the circuit ; and the factor $X /\left(R^{2}+X^{2}\right)$ which, multiplied by $E$ gives the component of $I 90^{\circ}$ behind $E$ in phase, is called the susceptance of the circuit. Therefore, using $g$ for conductance and $b$ for susceptance we have

$$
\begin{aligned}
& g=\frac{R}{R^{2}+X^{2}} \\
& b=\frac{X}{R^{2}+X^{2}}
\end{aligned}
$$

and, of course

$$
I=(g-j b) E
$$

It is usual to represent the impedance of a circuit as a complex quantity by the letter $Z$ and to represent the numerical value of impedance by the letter $z$; and it is also usual to represent the admittance of a circuit as a complex quantity by the letter $Y$, and to represent the numerical value of admittance by the letter $y$. Thus

$$
\begin{aligned}
& Z=R+j X \\
& z=\sqrt{R^{2}+X^{2}} \\
& Y=g-j b \\
& y=\sqrt{g^{2}+b^{2}}
\end{aligned}
$$

Further practical information concerning the use of complex quantity in alternating-current problems is given in Arts. 44-47

43. DeMoivre's Theorem. The use of complex quantity in trigonometric transformations and the correctness of the formulas given in the foregoing article may be most elegantly shown by means of the relation :

$$
\varepsilon^{j \theta}=\cos \theta+j \sin \theta
$$

which was first pointed out by DeMoivre, $\varepsilon$ being the Napierian base. This relation may be established by writing $j \beta$ for $x$ in the infinite series for $\varepsilon^{x}$ and separating the terms which do not contain $j$ as a factor ( $=$ series for $\cos \theta$ ) from the terms which do contain $j$ as a factor (= series for $\sin \theta$ ).

(a) Regarding trigonometric transformations. Examples. Squaring both members of equation (i) we have :

$$
\varepsilon^{j \cdot 2 \theta}=\cos ^{2} \theta-\sin ^{2} \theta+2 j(\sin \theta \cos \theta)
$$

but, according to equation (i) we must have :

$$
e^{j \cdot 2 \theta}=\cos 2 \theta+j \sin 2 \theta \text {. }
$$

Therefore, comparing equations (ii) and (iii), we have :

$$
\cos 2 \theta=\cos ^{2} \theta-\sin ^{2} \theta
$$

and

$$
\sin 2 \theta=2 \sin \theta \cos \theta
$$


As a second example divide $\varepsilon^{j \theta}=\cos \theta+j \sin \theta$ by $\varepsilon^{j \psi}=\cos \psi+j \sin \psi$, member by member, and we have :

$$
\varepsilon^{j(\theta-\psi)}=\frac{\cos \theta+j \sin \theta}{\cos \psi+j \sin \psi}
$$

or, multiplying numerator and denominator of the right hand member of equation (iv) by $\cos \psi-j \sin \psi$, we have :

$$
\varepsilon^{j(\theta-\psi)}=\cos \theta \cos \psi+\sin \theta \sin \psi+j(\sin \theta \cos \psi-\cos \theta \sin \psi)
$$

but, according to equation (i) we have :

$$
\varepsilon^{j(\theta-\psi)}=\cos (\theta-\psi)+j \sin (\theta-\psi)
$$

so that, comparing equations $(v)$ and (vi), we have

and

$$
\cos (\theta-\psi)=\cos \theta \cos \psi+\sin \theta \sin \psi
$$

$$
\sin (\theta-\psi)=\sin \theta \cos \psi-\cos \theta \sin \psi
$$

As a third example the reader may find the expressions for sine and cosine of $3^{A}$ by cubing both members of equation (i), or he may find the expressions for sine and cosine of $(\theta+\psi+\phi)$ by multiplying together the expressions for $\varepsilon^{j \theta}$, $\varepsilon^{j \psi}$ and $\varepsilon^{j \phi}$.

(b) Regarding the formulas of Art. 42. - Let $z$ be the numerical value of the impedance of an alternating current circuit, that is, let $z=\sqrt{R^{2}+X^{2}}$, and let $\theta$ be the angle whose tangent is $X / R$, then

$$
z \varepsilon^{j \theta}=R+j X
$$

Furthermore, let $I$ be the numerical value of the current and let $\alpha$ be the angle between the current and the $x$-axis of reference, then

$$
I \varepsilon^{j a}=i_{1}+j i_{11}
$$

The expression for the electromotive force is then found by multiplying (vii) by (viii), member by member, and we have :

$$
E=2 I \varepsilon^{j(a+\theta)}=(R+j X)\left(i_{1}+j i_{11}\right)
$$

That is, the numerical value of $E$ is $2 I$, and $E$ is $\theta^{\circ}$ ahead of the current in phase. On the other hand, if we divide equation (ix) by equation (vii) member by member, we get the correct expression for the current.

(c) Regarding the products of current by current, and current by voltage. If the impedance of a circuit $\left(z \varepsilon^{j \theta}=R+j X\right)$ be looked upon as a vector it must be considered as a stationary vector making the angle $\theta$ with a fixed axis of reference; whereas the angle between the electromotive force vector and a fixed axis is $\omega t$, and the angle between the current vector and a fixed axis is $(\omega t-\theta)$. Therefore, we may write, with reference to a fixed axis :

and

$$
\begin{aligned}
& \text { Impedance }=2 \varepsilon^{j \theta} \\
& \text { Voltage }=\mathscr{E} \varepsilon^{j \omega t} \\
& \text { Current }=g \varepsilon^{j(\omega t-\theta)}
\end{aligned}
$$


and these expressions satisfy the fundamental relations:-current is equal to electromotive force divided by impedance, and electromotive force is equal to current multiplied by impedance. On the other hand, the product of current by voltage gives $\mathcal{E} \mathscr{J}_{\varepsilon}^{j(2 \omega t-\theta)}$ which is a vector which rotates at the angular velocity $2 \omega$. Evidently the impedance vector which is stationary cannot be properly shown in the clock diagram, inasmuch as the clock diagram is supposed to rotate at angular velocity $\omega$, and the vector $\mathcal{E} g e^{j(2 \omega t-\theta)}$ cannot be properly shown in the clock diagram because it rotates at angular velocity $2 \omega$. The use of complex quantity does not lead to any graphical representation of power because the vector $\mathcal{E} \mathcal{E}^{j(2 \omega t-\theta)}$ cannot be interpreted as a representation of power. Products of current by current and of current by voltage are foreign to the clock diagram, and these products as physical facts do not enter into the complex quantity method of representation.

44. Coils in series. Two coils are connected in series between alternating current mains as shown in Fig. 85. The total electromotive force $E$, the resistances

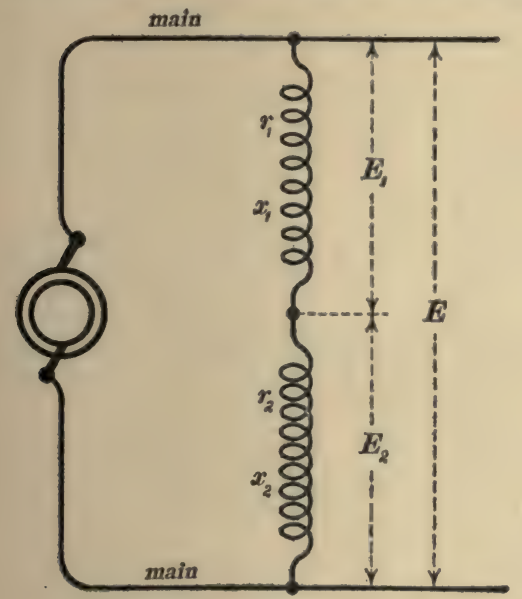

Flg. 85 .

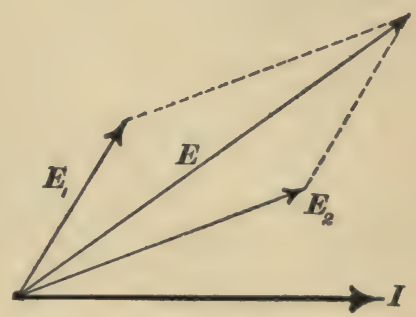

Fig. 86 .

$r_{1}$ and $r_{2}$ and the reactances $x_{1}$ and $x_{2}$ of the respective coils are given, and it is required to find $E_{1}$ and $E_{2}$. Let $I$ be the current flowing through the two coils. The general relation between $E, E_{1}, E_{2}$, and $I$ is shown in the clock diagram Fig. 86. This clock diagram is given in order that the following equations (i), (ii) and (iii) may be easily understood. According to equation (15) Art. 42 we have :

and

$$
E_{1}=\left(r_{1}+j x_{1}\right) I
$$

$$
E_{2}=\left(r_{2}+j x_{2}\right) I
$$

Furthermore, the electromotive force $E$ is the vector sum of $E_{1}$ and $E_{2}$ as shown in Fig. 86 so that, remembering that $E, E_{1}$ and $E_{2}$ are complex quantities, we have :

$$
E_{1}+E_{\mathrm{g}}=E
$$


Substituting the values of $E_{1}$ and $E_{3}$ from (i) and (ii) in (iii) and solving for $I$, we have:

$$
I=\frac{E}{r_{1}+r_{2}+j\left(x_{1}+x_{2}\right)}
$$

Substituting this value in (i) and (ii) we have :

and

$$
E_{1}=\frac{\left(r_{1}+j x_{1}\right) E}{r_{1}+r_{2}+j\left(x_{1}+x_{2}\right)}
$$

$$
E_{2}=\frac{\left(r_{2}+j x_{2}\right) E}{r_{1}+r_{2}+j\left(x_{1}+x_{2}\right)}
$$

These equations (v) and (vi) express the electromotive forces $E_{1}$ and $E_{2}$ in terms of the known quantities $E, r_{1}, r_{2}, x_{1}$ and $x_{2}$.

In order to make actual numerical calculations, of $E_{1}$ for example, the second member of $(v)$ must be separated into its components, that is, into real and imaginary parts, and then the numerical value of $E_{1}$ is found by taking the square root of the sum of the squares of these components, and the tangent of the angle between $E$ and $E_{1}$ (the angle by which $E_{1}$ leads $E$ in phase) is equal to the $j$-component of $E_{1}$ divided by the real component of $E_{1}$.

Thus, multiplying numerator and denominator of $(v)$ by $\left[r_{1}+r_{2}-j\left(x_{1}+x_{2}\right)\right]$, $j$ is removed from the denominator, and we have :

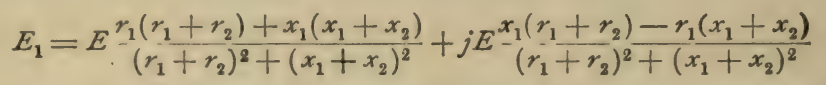

The first term of this expression is the component of $E_{1}$ parallel to $E$ and the second term, dropping $j$, is the component of $E_{1}$ perpendicular to $E$. Taking the square root of the sum of the squares of these components we have :

$$
\text { Numerical value of } E_{1}=\frac{\sqrt{r_{1}^{2}+x_{1}^{2}}}{\sqrt{\left(r_{1}+r_{2}\right)^{2}+\left(x_{1}+x_{2}\right)^{2}}} \times E
$$

Taking the ratio of the two components we have :

$$
\text { Tangent of angle from } E \text { forwards to } E_{1}=\frac{x_{1}\left(r_{1}+r_{2}\right)-r_{1}\left(x_{1}+x_{2}\right)}{r_{1}\left(r_{1}+r_{2}\right)+x_{1}\left(x_{1}+x_{2}\right)}
$$

A negative value of this tangent indicates that $E_{1}$ is behind $E$ in phase.

45. General outline of complex quantity method. The above discussion illustrates the general use of complex quantity in alternating-current problems, and the procedure is always essentially the same, namely: First, the formulation of the problem by equations expressing the relation between current and voltage in elementary circuits, and by equations expressing the known sum of certain voltages when elementary circuits are in series, or the known sum of certain currents when elementary circuits are in parallel; Second, the transformation of these complex equations so as to express the unknown quantities in terms of their components as in equation (vii) Art. 44 ; Third, the derivation of equations (not involving complex quantity) expressing numerical values and phase angles in terms of the given data; and, Fourth, the actual calculation of numerical results. 
A fifth step is necessary when it is desired to calculate power, which is equal to the numerical value (effective) of voltage times the numerical value (effective) of current times the cosine of phase difference. When current and voltage are found in terms of their components, for example, when :

and

$$
I=i_{1}+j i_{11}
$$

$$
E=e_{1}+j e_{11}
$$

then the power may be easily calculated by the formula :

$$
P=e_{1} i_{1}+e_{11} i_{11}
$$

This equation does not of course involve $j$ in any way, but in using this equation especial care must be taken to give to each component its proper algebraic sign.

46. Coils in parallel. A given alternating current $I$ divides between two coils connected in parallel as shown in Fig. 87. It is required to find $I_{1}$ and $I_{2}$, each in terms of $I, r_{1}, r_{2}, x_{1}$ and $x_{2}$. The general relation between $I, I_{1}, I_{2}$, and the voltage $E$ between the branch points is shown in the clock diagram Fig. 88. The

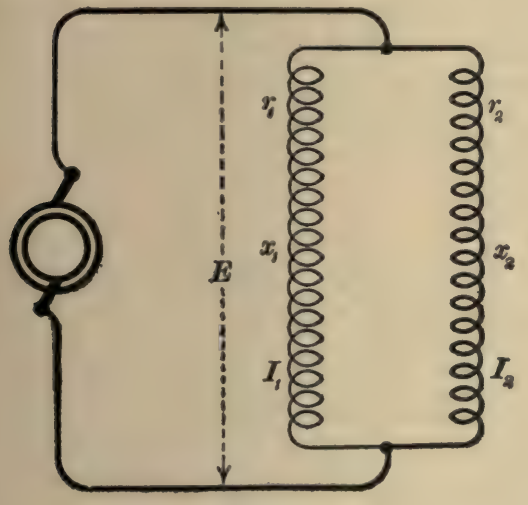

Fig. 87.

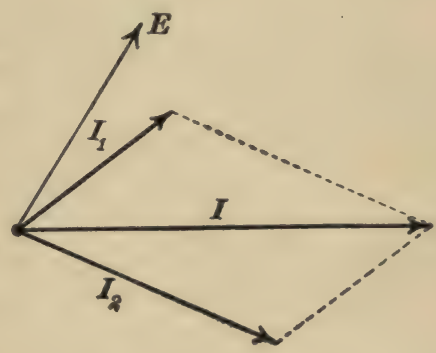

Fig. 88.

equations are somewhat simplified if we introduce the conductance $g$ and the susceptance $b$ of each circuit according to the definitions given in Art. 42, namely:

$$
\begin{aligned}
& g_{1}=\frac{r_{1}}{r_{1}^{2}+x_{1}^{2}} \\
& b_{1}=\frac{x_{1}}{r_{1}^{2}+x_{1}^{2}} \\
& g_{2}=\frac{r_{2}}{r_{2}^{2}+x_{2}^{2}} \\
& b_{2}=\frac{x_{2}}{r_{2}^{2}+x_{2}^{2}}
\end{aligned}
$$


Then

$$
\begin{aligned}
& I_{1}=\left(g_{1}-j b_{1}\right) E \\
& I_{2}=\left(g_{2}-j b_{2}\right) E
\end{aligned}
$$

and, since the current $I$ is the vector sum of $I_{1}$ and $I_{2}$, we have:

$$
I_{1}+I_{2}=I
$$

The problem is solved from these three equations in exactly the same way that the problem of coils in series is solved from equations (i), (ii) and (iii) of Art. 44.

47. The transformer. The use of complex quantity may be further illustrated by a discussion of the problem of the transformer. The discussion here given of this problem is unnecessarily complicated and it is therefore useless for practical purposes, and it is all the more useless in that it takes no account of the effects of magnetic saturation. One must not, however, conclude that the use of complex quantity is useless in the development of the practical theory of the transformer.

The difference between the practical theory of the transformer and the general theory here outlined is that the practical theory is based on the assumption that the magnetic flux which passes through one coil and does not pass through the other, that is the leakage flux, is small in comparison with the flux which passes through both coils. This assumption greatly simplifies the practical theory of the transformer, it avoids any explicit use of the idea of the mutual inductance coefficient of the two coils, and it gives rise to an idea of self-inductance depending upon leakage flux only.

Two separate coils of wire $A$ and $B$, Fig. 89, are wound on an iron core, or

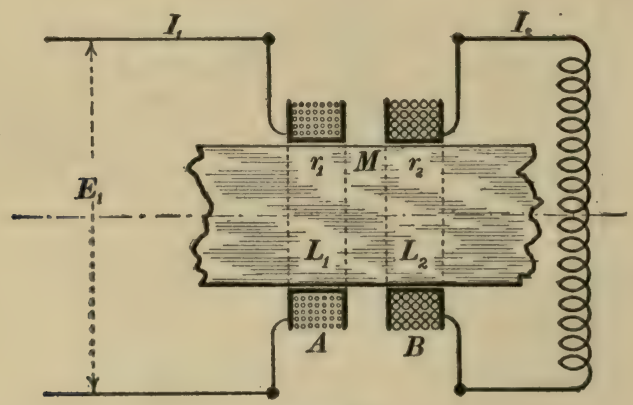

Fig. 89.

merely placed near together. The coil $A$, called the primary coil, receives alternating current from supply mains, its resistance is $r_{1}$ and its inductance is $L_{1}$. The coil $B$, called the secondary coil, is connected to a receiving circuit. The total resistance of the coil $B$ and its receiving circuit is $r_{2}$ and the total inductance is $L_{2}$. It is required to find the electromotive force, $E_{1}$, which must act on coil $A$ to maintain in it a given harmonic current $I_{1}$. The coefficient of mutual inductance $*$ of the two coils is $M$.

* See Nichols and Franklin's Elements of Physics, Vol. II, Chapter VII, for a definition of mutual inductance. 
Determination of the secondary current $I_{2^{\circ}}$ - The given primary current induces in the secondary coil an electromotive force which is at each instant equal to $-M \cdot d i_{1} / d t$.

This electromotive force is $90^{\circ}$ behind $I_{1}$, its effective value is $\omega M I_{1}$, and its symbolic expression is $-j \omega M I_{1}$ and this electromotive force produces in the secondary coil a current

$$
I_{2}=-\frac{j \omega M I_{1}}{r_{2}+j \omega L_{2}}
$$

Reaction of the secondary current upon the primary coil. - The secondary current $I_{2}$ induces in the primary coil an electromotive force which is at each instant equal to $-M \cdot d i_{2} / d t$. This electromotive force is $90^{\circ}$ behind $I_{2}$ in phase, its effective value is $\omega M I_{2}$, and its complex expression is $-j \omega M I_{2}$. The electromotive force induced in the primary by the secondary current must be overcome by the electromotive force which acts upon the primary. The portion of the acting electromotive force which thus balances the reaction of the secondary current is equal to this reaction and opposite to it in sign and is, therefore, equal to $+\jmath \omega M I_{2}$.

Determination of tolal electromotive force acting on primary. - This total electromotive force consists of three parts as follows:

I. The part described above which balances the reaction of the secondary current. This part is equal to $+j \omega M I_{2}$, or using the value of $I_{2}$ from equation $(i)$, we have for this part of the total electromotive force:

$$
+\frac{\omega^{2} M^{2} I_{1}}{r_{2}+j \omega L_{2}}
$$

2. The part used to overcome the resistance of the primary coil. This is at each instant equal to $r_{1} i_{1}$, its effective value is $r_{1} I_{1}$, and its complex expression is $r_{1} I_{1}$ since it is in phase with $I_{1}$.

3. The part used to overcome the inductance of the primary coil. This is at each instant equal to $L_{1} \cdot d i_{1} / d t$, it is $90^{\circ}$ ahead of $I_{1}$, its effective value is $\omega L_{1} I_{1}$, and its complex expression is $j \omega L_{1} I_{1}$. Therefore the total electromotive force required to maintain the given primary current is :

or, separating components ;

$$
E_{1}=r_{1} I_{1}+j \omega L_{1} I_{1}+\frac{\omega^{2} M^{2} I_{1}}{r_{2}+j \omega L_{2}}
$$

$$
E_{1}=\left[\left(r_{1}+\frac{r_{2} \omega^{2} M^{2}}{r_{2}^{2}+\omega^{2} L_{2}^{2}}\right)+j\left(\omega L_{1}-\frac{\omega^{3} L_{2} M^{2}}{r_{2}^{2}+\omega^{2} L_{2}^{2}}\right)\right] I_{1}
$$

This equation shows that the effect of the secondary coil is to make the primary coil behave as if its resistance were :

and its inductance were :

$$
r_{1}+\frac{r_{2} \omega^{2} M^{2}}{r_{2}^{2}+\omega^{2} L_{2}^{2}}
$$

$$
L_{1}-\frac{\omega^{2} L_{2} M^{2}}{r_{2}^{2}+\omega^{2} L_{2}^{2}}
$$

and these expressions are called the equivalent resistance and the equivalent inductance, respectively, of the system represented in Fig. 89. Compare Art. 28. 


\section{CHAPTER VI}

\section{THE POLYPHASE SYSTEM}

48. The single-phase system and the polyphase system. A brief reference was made in Chapter I to the distinction between the single-phase alternator and the polyphase alternator, and, although the importance of the polyphase system cannot be fully appreciated until the induction motor and the rotary converter are discussed, still it is necessary to outline here some of the general features of the polyphase system inasmuch as this system enters into every division of our subject, alternators, transmission lines, transformers, synchronous motors, rotary converters, and induction motors.

The single-phase system consists essentially of a simple alternator delivering an alternating current to a single circuit.

The polyphase system consists essentially of two or more electrically distinct (but not mechanically distinct) alternators delivering distinct alternating currents over distinct transmission lines to electrically distinct receiving circuits.

49. The two-phase alternator. A two-phase alternator is an alternator upon whose armature two distinct windings are placed each with its own pair of collector rings, the two windings being arranged so that the electromotive force generated in one winding is in quadrature with the electromotive force generated in the other winding. A clear idea of the essential features of the twophase alternator may be obtained from Fig. 90 which represents two distinct armatures $A$ and $B$ mounted on one shaft and revolving inside of the same crown of field magnet poles. The armature $B$ is shown smaller than the armature $A$ for the sake of clearness. The two armatures are arranged so that the groups of winding slots in one armature are midway between the field magnet poles when the groups of winding slots in the other 
armature are midway under the field magnet poles. This brings the electromotive forces of the two armature windings into quadrature with each other. In practice a two-phase alternator is made by placing all the slots shown in Fig. 90 on one armature body, the two windings being however kept as distinct as if they were on separate armature bodies. Thus the armature winding shown in Fig. I I is a two-phase winding; the coils which are square on the ends to the right constitute one armature winding which delivers current to one receiving circuit or set of receiving circuits, and the coils which bend backwards and pass behind the square

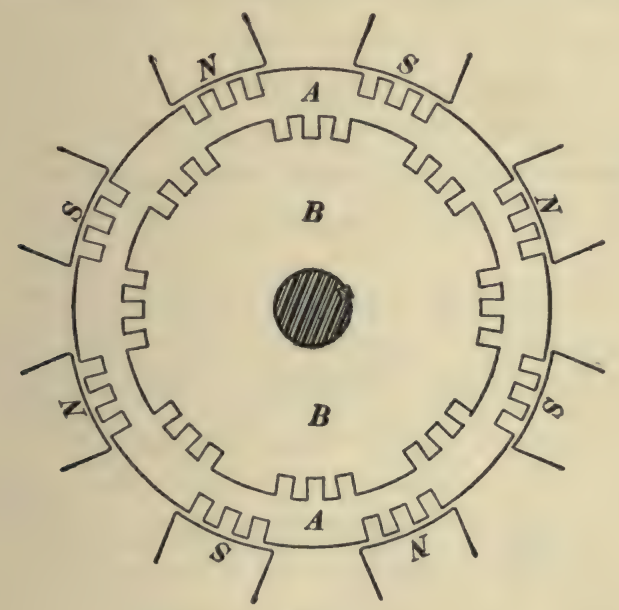

Fig. 90 .

ends constitute the other armature winding which delivers current to another receiving circuit or set of receiving circuits.

A two-phase alternator is usually provided with four collector rings, two for each armature winding; one ring may, however, be made to serve as a common terminal for the two windings $A$ and $B$ as shown in Fig. 9I. In this case three transmission wires are used and the two receiving circuits $x$ and $y$ are connected as shown in the figure. The objections to the arrangement shown in Fig. 91 are: (a) that the voltage drop in the middle main may cause the voltages across $x$ and $y$ to become 
unequal in value and more or less than $90^{\circ}$ apart in phase, and (b) that a two-phase (4-ring) rotary converter cannot be connected to three-wire mains.

50. Tw0-phase electromotive forces and currents. The two

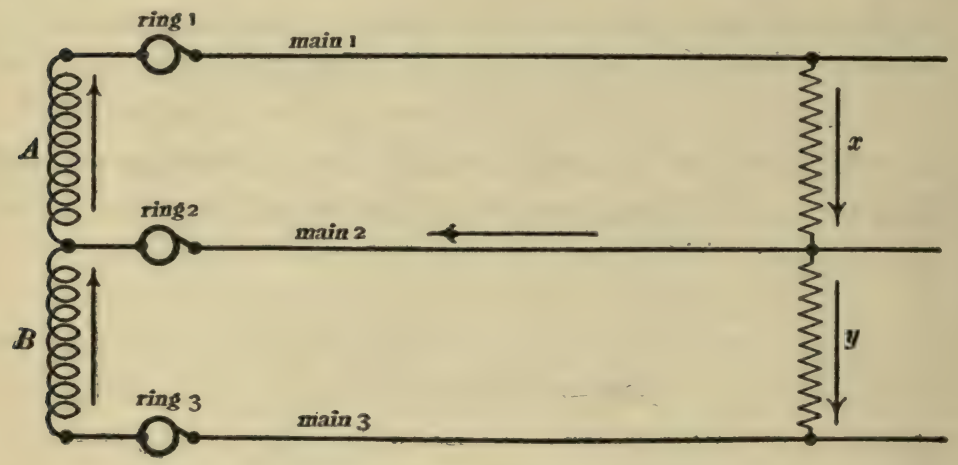

Fig. 91.

lines $A$ and $B$ in the clock diagram, Fig. 92, represent the electromotive forces of the $A$ and $B$ windings respectively of a twophase alternator. If the circuit which receives current from the

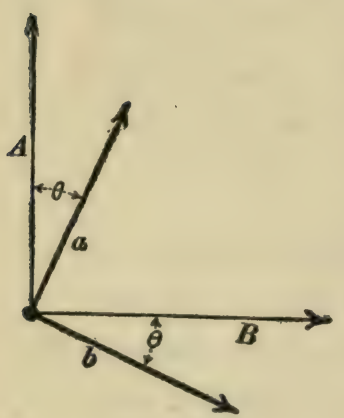

Fig. 92. $A$ winding has the same resistance and reactance as the circuit which receives current from the $B$ winding, the system is said to be balanced. In this case the currents delivered by $A$ and $B$ are equal to each other in value, and they lag in phase equally behind $A$ and $B$ respectively, as shown by the two lines $a$ and $b$.

It is worthy of note that it is not possible to say whether $A$ is $90^{\circ}$ behind $B$, or $B$ is $90^{\circ}$ behind $A$, even when the physical facts concerning a two-phase alternator are completely established. Complete definiteness of phase relation depends upon an arbitrary choice of the direction in which the electromotive force and current of each phase is to be considered as positive. Conversely, a clock diagram like Fig. 92 cannot completely represent the physical facts concerning a two-phase alternator until positive directions are chosen. Let the arrows in Fig. 91 represent the arbitrarily chosen positive directions in the windings, in the middle main, and in the receiving circuits, and suppose that, on the 
basis of this choice of signs, the clock diagram Fig. 92 represents the facts. Then the vector sum $A+B$ represents the electromotive force from main one across to main two, and the vector difference, $a-b$, of the currents in $x$ and $y$ represents the current in the middle main. If, however, the positive directions in winding $B$ and receiving circuit $y$ be chosen opposite to the directions shown in Fig. 9I, then, if the physical facts remain unchanged, the lines $B$ and $b$ in the clock diagram must be reversed, the electromotive force from main one to main two will be represented by the vector difference $A-B$, and the current in the middle main will be represented by the vector sum $a+b$. If in the first case $A$ is $90^{\circ}$ ahead of $B$, then in the second case $A$ must be considered as $90^{\circ}$ behind $B$ because of the change in the choice of signs.*

51. The three-phase alternator. A three-phase alternator is an alternator upon whose armature three distinct windings $A, C$ and $B$ are placed, each having, in the most general case, a separate pair of collector rings, and the windings are arranged so that the electromotive forces $A, C$ and $B$ are $60^{\circ}$ apart in phase. $\dagger$

The four-ring arrangement of the three-phase alternator. A number of batteries can supply currents to a number of distinct bell circuits, and all of the bell circuits can have a common re-

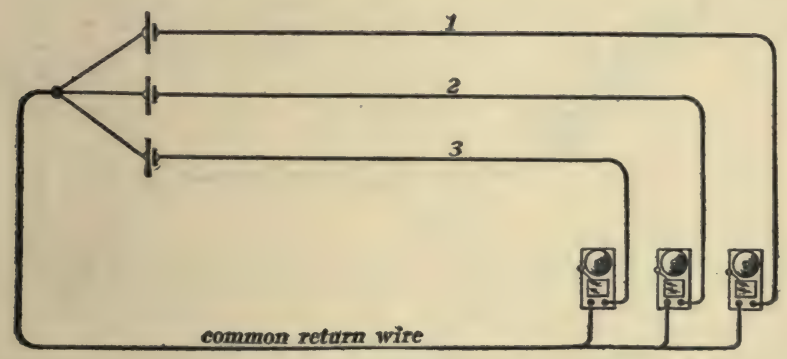

Fig. 93.

turn wire as shown in Fig. 93. In the same way the three armature windings, $A, B$ and $C$, of a three-phase alternator can deliver currents to three receiving circuits $x, y$ and $z$, a common return wire being used as indicated in Fig. 94. In this case one

* See A Discussion of some Points in Alternating-current Theory [Section (b) On the necessity of choosing signs in complicated networks], by W. S. Franklin, Tranactions of the American Institute of Electrical Engineers, Vol. 21, page 589, I903.

+ It will be found convenient later to consider the three electromotive forces $A, B$ and $C$ to be $120^{\circ}$ apart in phase, the change being merely a matter of the choice of signs. See Art. 53 . 
of the collector rings, No. 4 in Fig. 94, forms a common terminal for all three armature windings.

The essential features of the windings of a three-phase armature are shown in Fig. 95. The figure shows each winding distributed in two slots per field pole (the $A$ winding only is shown complete, windings $B$ and $C$ are exactly like $A$ ), and it represents the simple case in which each slot contains but one conductor. The straight radial lines represent the conductors lying in the slots, the curved lines outside the circular row of radial lines represent the end-connections at one end of the armature,

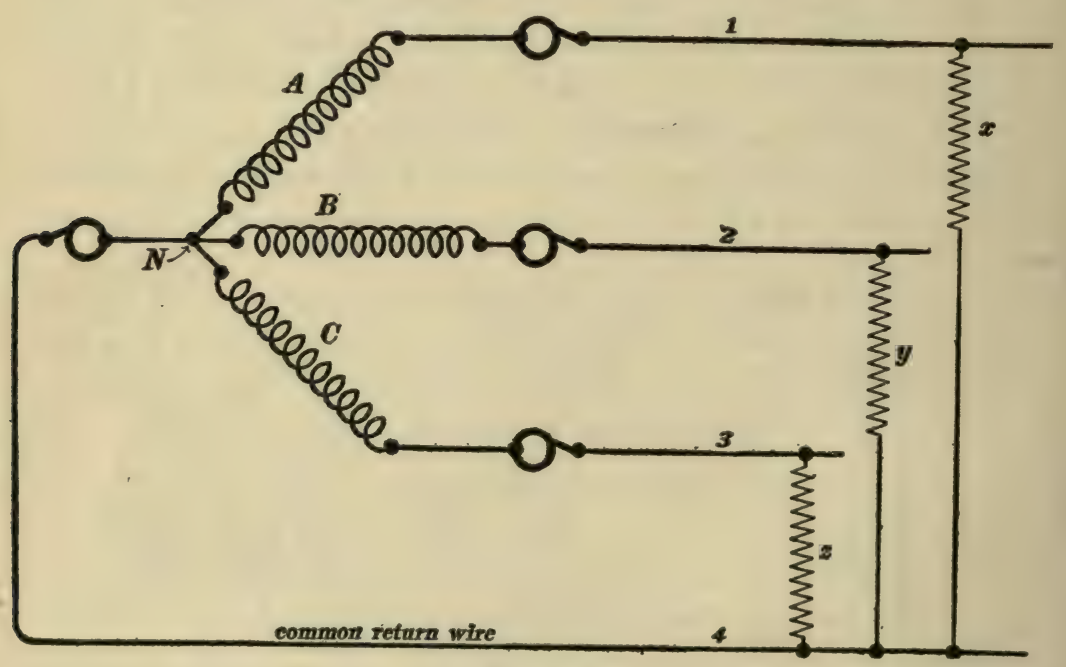

Fig. 94.

and the curved lines inside of the circular row of radial lines represent the end-connections at the other end of the armature. One terminal of each winding is connected to ring 4 (or to a neutral connection $N$, Fig. 94 , if ring 4 is dispensed with), and the other terminals of windings of $A, B$ and $C$ are connected to rings 1,2 and 3 respectively.

52. The three-ring three-wire arrangement of the three-phase alternator. (a) The $Y$-connection. Let the three lines $a, b$ and 
c, Fig. 96, represent the three equal currents, $60^{\circ}$ apart in phase, which are delivered to three similar receiving circuits $x, y$ and $z$

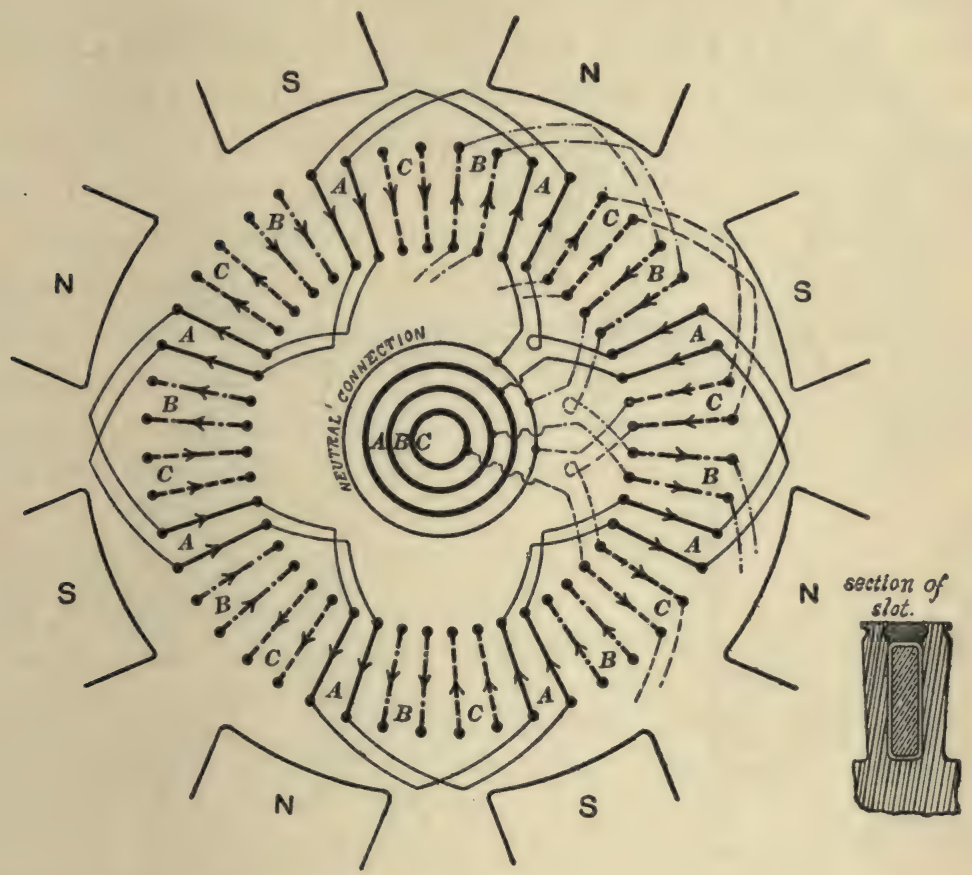

Fig. 95.

connected as shown in Fig. 94. It is evident that the current $c$ is equal to the vector sum of $a$ and $b$, or, in other words, the

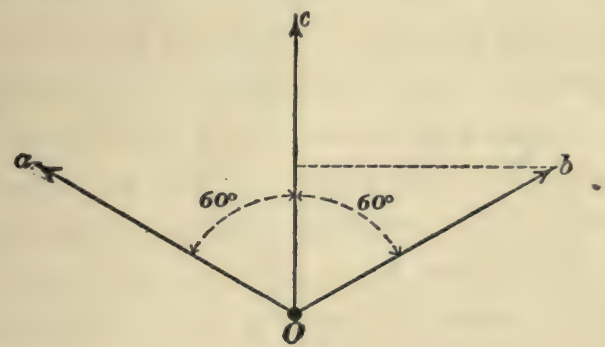

Fig. 96.

current $c$ is at each instant equal to the sum of the two currents $a$ and $b$. Therefore, if the connections of the winding $C$, Fig. 
94, are arranged so that the current $c$ flows towards the generator in main No. 3 when $a$ and $b$ flow away from the generator in mains No. I and No. 2, then the current in main No. 4 will be zero, since $a+b=c$ at each instant. Therefore, when the receiving circuits $x, y$ and $z$ are similar, main No. 4 may be dispensed with if the windings are properly connected. The proper connections are shown in Fig. 95. This arrangement of the windings of a three-phase armature is called the $Y$-connection.

(b) The $\Delta$-connection. Suppose the three armature windings of a three-phase alternator to be connected so as to form a closed circuit, and let the three lines $A, C$ and $B$ in the clock diagram, Fig. 97, represent the electromotive forces of the respective windings. The electromotive

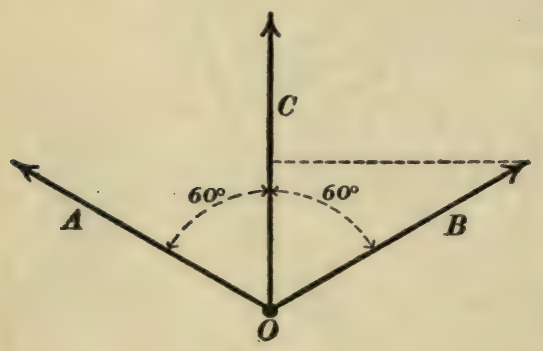

Fig 97. force $C$ is equal to $A+B$, and therefore, if the windings are properly connected, the electromotive force around the closed circuit of the three windings will be zero. Each junction of two windings may then be connected to a collector ring so that the three windings can deliver current to three-wire mains. This arrangement of the windings of a three-phase armature is called the $\Delta$-connection.

53. Conventional diagrams. Choice of signs to give symmetrical clock diagrams. (a) $Y$-connection. The three armature windings of a three-phase armature branch out from a common junction, the so-called neutral point $N$, Fig. 94, when the windings are $\mathrm{Y}$-connected. The essential features of this scheme of connections are best shown by a symmetrical diagram like Fig. 98. It is desirable to consider the current (and voltage) in each winding as positive when it flows away from the neutral point and the arrows in Fig. 98 show these positive directions.* On the basis of this

* It must not be imagined that the arrows in Figs. 98 and 100 represent the actual directions of the currents at any given instant. 
choice of signs, the clock diagram which shows the currents $a$, $b$ and $c$ and the electromotive forces $A, B$ and $C$ in the respective windings, becomes symmetrical as shown in Fig. 99.

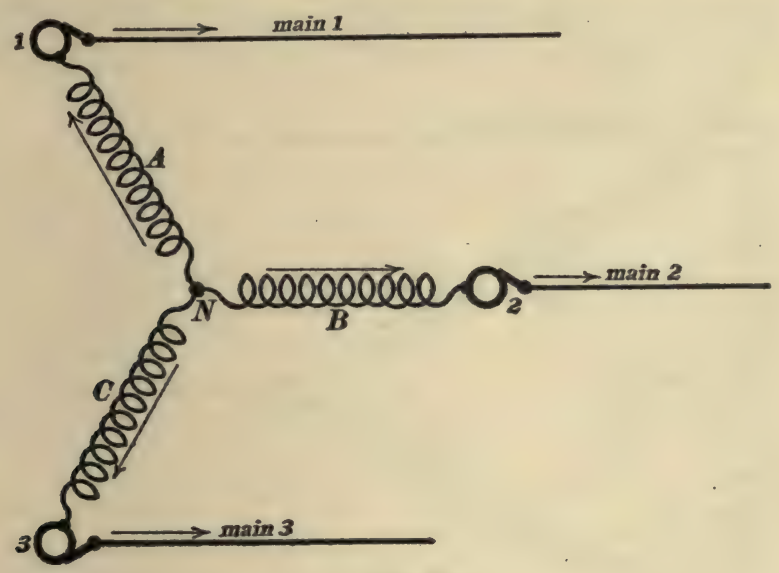

Fig. 98.

(b) $\Delta$-Connection. The three armature windings of a three phase alternator, when $\Delta$-connected, form a complete circuit or

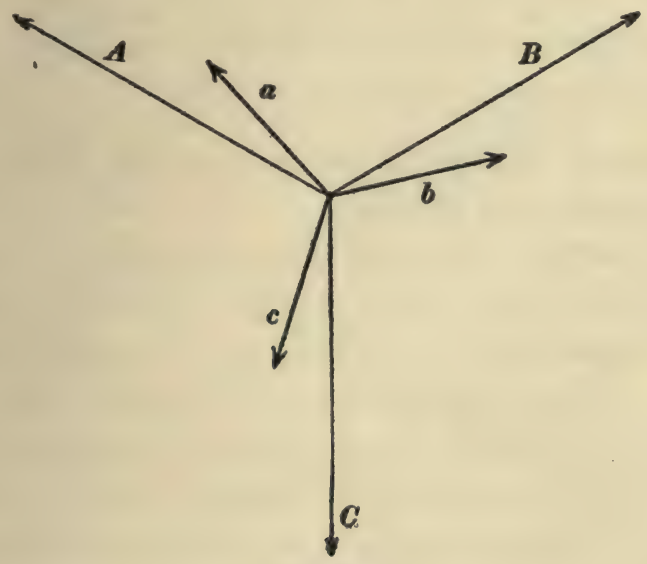

Fig. 99.

mesh, and the essential features of this scheme of connections are best shown by a symmetrical diagram like Fig. IOO. It is 
desirable to consider the current (and voltage) in each winding as positive when it flows in a given direction around the mesh, and the arrowes in Fig. 100 show these positive directions. On the basis of this choice of signs, the clock diagram of the currents and electromotive forces in the respective armature windings becomes

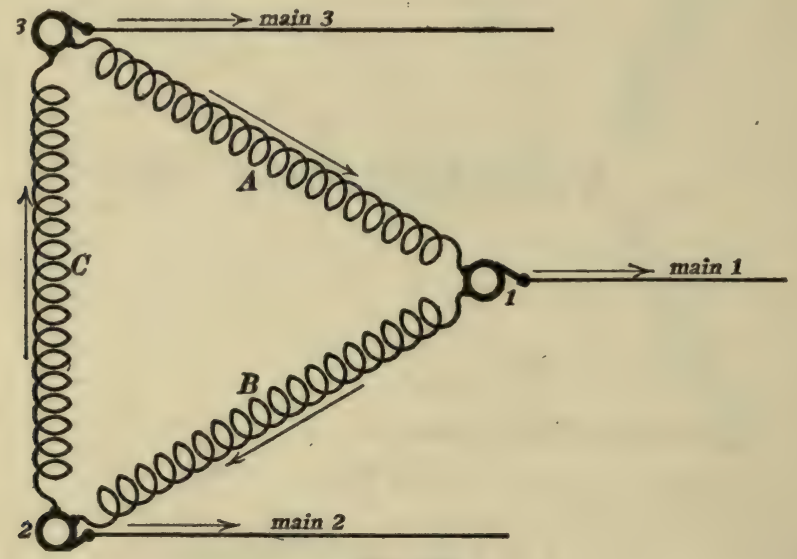

Fig. 100 .

symmetrical. The diagram of Fig. 99 represents the electromotive forces and currents in the armature windings, whether the windings be $\mathrm{Y}$-connected or $\Delta$-connected.

54. Manner of connecting receiving circuits to the three-wire mains of a three-phase system.

(a) For dissimilar receiving circuits (unbalanced system). In this case the four-wire system should be employed as shown in Fig. 94. This requires of course four collector rings on the generator. A polyphase generator is, however, used primarily for supplying currents to rotary converters and induction motors, such machines always involve similar receiving circuits, one receiving circuit for each phase, and when current is delivered from a polyphase system to individual (single-phase) receiving circuits, such as lamps, it is generally possible to distribute these individual receiving circuits among the several phases so as to 
give an approximately balanced system. Polyphase systems are in fact nearly always arranged with the idea of delivering currents to balanced receiving circuits.

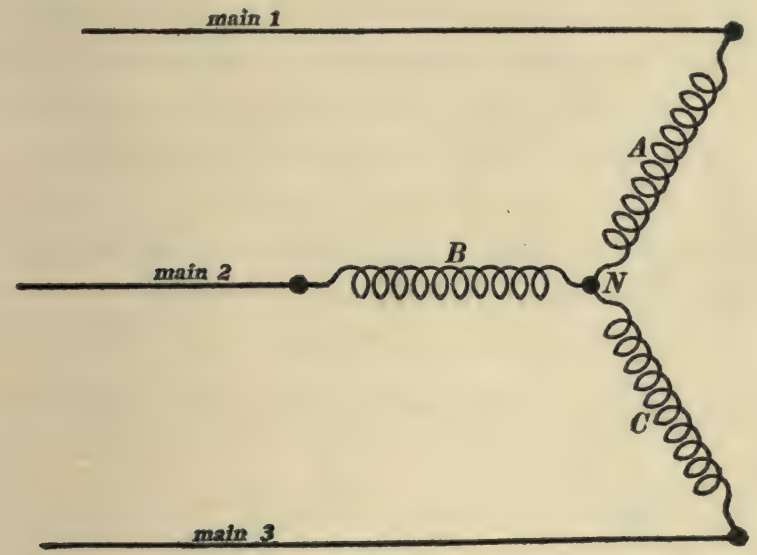

Fig. 101.

(b) For similar receiving circuits (balanced system). In this case the receiving circuits may be connected to three-wire threephase mains according to the $\mathrm{Y}$-scheme as shown by Fig. IOI,

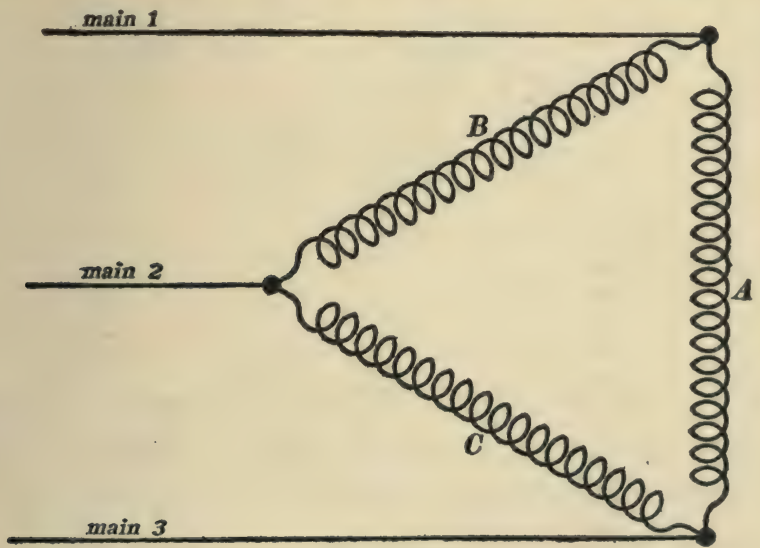

Fig. 102.

or according to the $\Delta$-scheme as shown by Fig. 102, and either scheme may be employed irrespective of the manner in which the armature windings of the generator are connected. 
55. Electromotive force and current relations in the $\mathrm{Y}$-scheme of connections.

(I) Referring to the generator. (a) Electromotive force relations. Let $E_{1}, E_{2}$ and $E_{3}$ be the electromotive forces from main I to main 2, from main 2 to main 3, and from main 3 to main I, respectively, see Fig. 98. From Fig. 98 it is evident that a positive electromotive force in winding $A$ produces an electromotive force from main I to main 2 (positive), and that a positive electromotive force in winding $B$ produces an electromotive force from main 2 to main I (negative). Therefore $E_{1}$ is equal to $A-B$ (vector difference). Similarly, $E_{2}$ is equal to $B-C$,

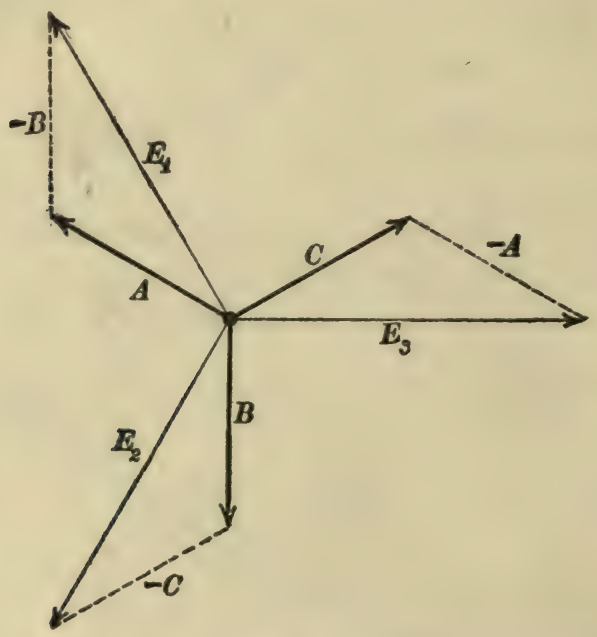

Fig. 103.

and $E_{3}$ is equal to $C-A$. These relations are shown in Fig. IO3, and from this figure we see at once that the value of the electromotive force between any pair of mains is $\sqrt{3}$ times the electromotive force in each $Y$-connected armature winding.

(b) Current relations. In the $\mathrm{Y}$-scheme of connection the currents in the mains are identical with the currents in the respective windings. This is evident from Fig. 98. 
2. Referring to the receiving circuits. (a) Electromotive force relations. Given the electromotive forces $E_{1}, E_{2}$ and $E_{3}$ between the mains as shown in the clock diagram Fig. IO3, then the electromotive forces acting on the respective $\mathrm{Y}$-connected receiving circuits are represented by the lines $A, B$ and $C$. Therefore the value of the electromotive force acting on each $Y$-connected receiving circuit is equal to the electromotive force between mains divided by $\sqrt{3}$.

(b) Current relations. In the $\mathrm{Y}$-scheme of connection the current in each main is identical with the current in each receiving circuit.

56. Electromotive force and current relations in the $\Delta$-scheme of connections.

(I) Referring to the generator. (a) Electromotive force relations. In the $\Delta$-scheme of connections the electromotive forces

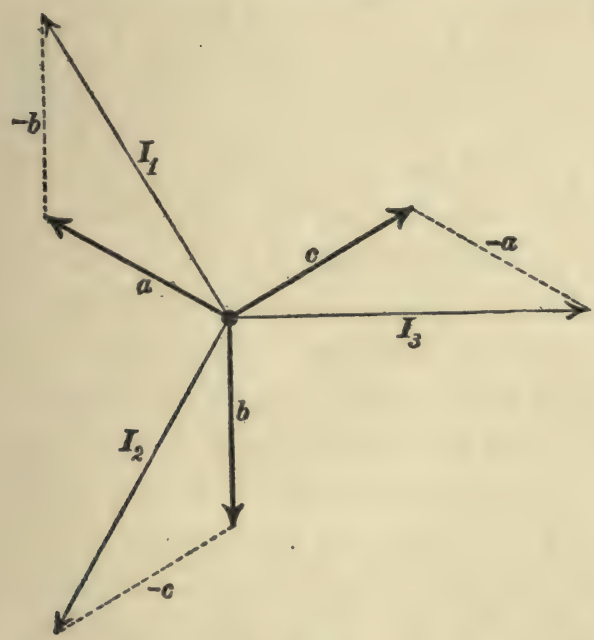

Fig. 104.

between mains are identical with the electromotive forces developed by the respective armature windings.

(b) Current relations. Let $I_{1}, I_{2}$ and $I_{3}$ be the currents in mains $\mathrm{I}, 2$ and 3 respectively, each being considered positive 
when it flows away from the generator as shown by the arrows $i_{n}$ Fig. IOO. From this figure it is evident that a positive current in winding $A$ produces a positive current in main $\mathbf{I}$, and that a positive current in winding $B$ produces a negative current in main I. Therefore $I_{1}$ is equal to $a-b$ (vector difference). Similarly, $I_{2}$ is equal to $b-c$, and $I_{3}$ is equal to $c-a$. These relations are shown in Fig. 104, and from this figure we see at once that the value of the current in each main is $\sqrt{3}$ times the current in each $\Delta$-connected armature winding.

2. Referring to the receiving circuits. (a) Electromotive force relations. In the $\Delta$-scheme of connection the electromotive force acting on each receiving circuit is identical with the electromotive force between each pair of mains.

(b) Current relations. Given the currents, $a, b$ and $c$ in the respective receiving circuits (see Fig. 104), then the currents in the respective mains are represented by the lines $I_{1}, I_{2}$ and $I_{3}$. Therefore, the value of the current in each $\Delta$-connected receiving circuit is equal to the current in each main divided by $\sqrt{3}$.

57. Power in polyphase systems. When the receiving circuits are unlike (system unbalanced) the amounts of power delivered to each are in general different, and the system must be treated as independent single-phase systems. The only case in which the power relations in a polyphase system can be treated on a simpler basis than that corresponding to independent single-phase systems, is the case in which the system is balanced. In this case there are two important matters to be considered: (a) The constancy of power, and $(b)$ the value of the power.

(a) Constancy of power in balanced polyphase system. In the single-phase alternator the instantaneous value, $e i$, of the power pulsates as explained in Art. 8, whereas a polyphase generator delivers a perfectly steady flow of energy, that is, a steady power, to balanced receiving circuits. In this case the power delivered to each receiving circuit of course pulsates, but the pulsations are so related that the sum does not pulsate. An 
interesting consequence of this steady flow of power in a balanced polyphase system is that, whereas, the driving torque of a singlephase alternator must pulsate except in so far as the pulsations of torque are averaged out by the fly-wheel effect of the rotating armature, the driving torque of a polyphase generator is perfectly steady when it delivers current to balanced receiving circuits. Moreover a single-phase motor of any kind is driven by a pulsating torque, whereas a polyphase motor of any kind is driven by a steady torque.

Discussion for a two-phase alternator. - Consider a single-phase alternator of which the electromotive force is:

$$
e=\mathbf{E} \sin \omega t
$$

and which gives a current:

$$
i=\mathbf{I} \sin (\omega t-\theta)
$$

or

$$
i=\mathbf{I} \sin \omega t \cos \theta-\mathbf{I} \cos \omega t \sin \theta
$$

The instantaneous power is :

$$
e i=\mathbf{E I} \cos \theta \sin ^{2} \omega t-\mathbf{E I} \sin \theta \sin \omega t \cos \omega t
$$

which pulsates with a frequency twice as great as the frequency of $e$ and $i$.

Let equations $(a)$ and $(b)$ express the electromotive force and current of one phase of a (balanced) two-phase alternator, then the electromotive force and current of the other phase are :

$$
\begin{aligned}
& e^{\prime}=\mathbf{E} \cos \omega t \\
& t^{\prime}=\mathbf{I} \cos (\omega t-\theta)=\mathbf{I} \cos \omega t \cos \theta+\mathbf{I} \sin \omega t \sin \theta
\end{aligned}
$$

The instantaneous power output of this phase is

$$
e^{\prime} i^{\prime}=\mathbf{E I} \cos \theta \cos ^{2} \omega t+\mathbf{E I} \sin \theta \sin \omega t \cos \omega t
$$

Therefore the total power output of the two-phase machine is

$$
\begin{aligned}
e i+e^{\prime} i^{\prime} & =\mathbf{E I} \cos \theta\left(\sin ^{2} \omega t+\cos ^{2} \omega t\right) \\
& =\mathbf{E I} \cos \theta
\end{aligned}
$$

which is constant.

(b) Value of power in balanced polyphase system. If $A$ is the value of the electromotive force across one of the receiving circuits of an $n$-phase system, $a$ the value of the current in the receiving circuit, and $\cos \theta$ the power factor; then $A a \cos \theta$ is the power delivered to the receiving circuit and the total power delivered to the $n$ similar receiving circuits is :

$$
P=n A a \cos \theta
$$


If $E$ is the electromotive force between mains of a three-wire three-phase system, and if $I$ is the current in each main, then the total power delivered by the mains is :

$$
P=\sqrt{3} E I \cos \theta
$$

in which $\cos \theta$ is the power factor of each receiving circuit. This form of the equation is confusing for the reason that the voltage $E$, the current $I$, and the power factor do not all refer to a given circuit. The form $(a)$ of the equation is therefore to be preferred.

58. Measurement of power in polyphase systems. When a polyphase system is unbalanced, the power delivered by each phase must be measured exactly as in the case of an ordinary single-phase system (see Arts. I8, I9 and 20). When a polyphase system is balanced, the measured power delivered by one phase may be multiplied by the number of phases to give the total power, but exact similarity of the various receiving circuits is never realized in practice and therefore a considerable error may be made if the total power is assumed to be $n P$, where $P$ is the measured power delivered by one phase and $n$ is the number of phases. Therefore it is usually considered necessary to measure the actual total power.

Best method for measuring power approximately by means of a single wattmeter. It is usually very inconvenient to disconnect the current coil of a wattmeter and transfer it quickly from one main to another, but it is always very easy to change the connections of the voltage coil. The best method therefore for using a single wattmeter for measuring the power delivered to approximately balanced receiving circuits by a three-wire system (three-phase) is to connect the current coil in a given main and take the wattmeter readings with the voltage coil connected from the given main first to one and then to the other of the two other mains. The sum of these two wattmeter readings gives a fairly accurate value for the total power delivered. 
59. Use of two wattmeters for measuring accurately the power delivered by any three-wire system. The total power delivered by any three-wire system may be measured by two wattmeters with their current coils placed in two of the mains, and their voltage coils connected from each of these two mains to the third main. Thus Fig. IO5 shows two wattmeters connected for

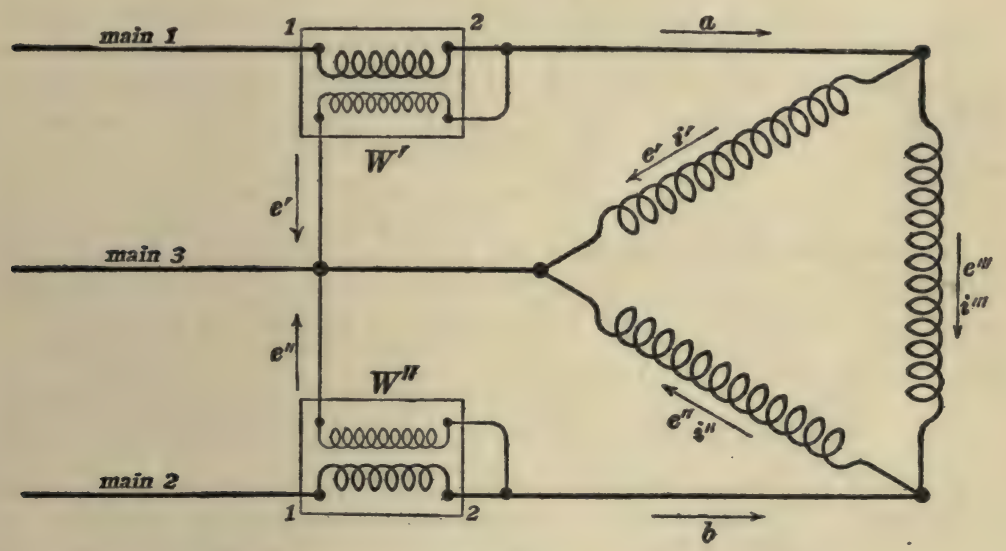

Fig. 105.

measuring the power delivered by a three-wire three-phase system. The receiving circuits may be either $\Delta$-connected or Y-connected.

In some cases the two wattmeter readings are to be added, and in other cases the lesser reading must be subtracted from the greater to give the total power delivered. To determine whether the readings are to be added or subtracted, disconnect one wattmeter and put the other in its place, leaving the same terminal of the voltage coil connected to the middle main and connecting terminals I and 2 of the one current coil in place of terminals I and 2 respectively of the other current coil. If the deflection of the transferred wattmeter is reversed, the readings are to be subtracted.

To show that the sum of the two wattmeter readings gives the total power. Let the positive directions in the mains $I$ and 2 and in the three receiving circuits be chosen as shown by the arrows in Fig. 105. These directions are chosen symmetric- 
ally with reference to the two wattmeters in order that each wattmeter reading may be considered as positive when it represents delivered power. Let $e^{\prime}, e^{\prime \prime}$ and $e^{\prime \prime \prime}$ represent the instantaneous values of the voltages, let $i^{\prime}, i^{\prime \prime}$ and $i^{\prime \prime \prime}$ represent the instantaneous values of the currents in the receiving circuits, and let $a$ and $b$ represent the instantaneous values of the currents in mains I and 2 as shown. Then, taking proper account of signs we have :

$$
\begin{aligned}
& a=i^{\prime}+i^{\prime \prime \prime} \\
& b=i^{\prime \prime}-i^{\prime \prime \prime}
\end{aligned}
$$

The reading $W^{\prime}$ of the upper wattmeter is equal to the average value of the product of the current $a$ in its current coil and the voltage $e^{\prime}$ across its voltage coil. That is :

and similarly

$$
W^{\prime}=\text { average } a e^{\prime}
$$

$$
W^{\prime \prime}=\text { average } b e^{\prime \prime}
$$

Substituting the above values of $a$ and $b$ in the expression for $W^{\prime}+W^{\prime \prime}$ we have :

$$
W^{\prime}+W^{\prime \prime}=\text { average } e^{\prime} i^{\prime}+\text { average } e^{\prime \prime} i^{\prime \prime}+\text { average }\left(e^{\prime}-e^{\prime \prime}\right) i^{\prime \prime \prime}
$$

but $e^{\prime}-e^{\prime \prime}$ is equal to $e^{\prime \prime \prime}$ so that :

$$
W^{\prime}+W^{\prime \prime}=\text { average } e^{\prime} i^{\prime}+\text { average } e^{\prime \prime} i^{\prime \prime}+\text { average } e^{\prime \prime \prime} i^{\prime \prime \prime}
$$

Discussion of arrangement shown in Fig. 105 for the case of harmonic voltages and currents. The sum of the two wattmeter readings, having due regard to the possible necessity of considering the reading of one of the wattmeters as essentially negative, is equal to the total power delivered, whether the voltages and currents be harmonic or not, and whether the system be balanced or not; but a clear understanding of the necessity for considering one of the readings as negative in certain cases may be most easily attained for the case of a balanced system with harmonic electromotive forces and currents. In order to discuss this case it is necessary to determine the correct clock diagram for representing the facts with due reference to the choice of signs indicated in Fig. 105. To determine this clock diagram consider first that if the positive directions of $e^{\prime}, e^{\prime \prime}$ and $e^{\prime \prime \prime}$ (and of course of $i^{\prime}, i^{\prime \prime}$ and $i^{\prime \prime \prime}$ also) are chosen symmetrically with respect to the receiving circuits (in a given direction around the $\Delta$-mesh), then the clock diagram is symmetrical like Fig. 99 (the letters $a, b$ and $c$ in Fig. 99 correspond to $i^{\prime}, i^{\prime \prime}$ and $i^{\prime \prime \prime}$ in Fig. 105), and that to reverse the chosen positive direction of $e^{\prime}$ and $i^{\prime}$, as shown in Fig. 105, is to reverse the lines in the clock diagram which represent $e^{\prime}$ and $i^{\prime}$. Therefore the clock diagram which correctly represents the facts in Fig. 105 is that which is shown in Fig. I06. By carefully considering the signs as represented by the arrows in Fig. I05 we see that $a=i^{\prime}+i^{\prime \prime \prime}$, and $b=i^{\prime \prime}-i^{\prime \prime \prime}$, as shown by the heavy dotted lines in Fig. I06.

Having thus constructed the correct clock diagram we find that the phase difference between $a$ and $e^{\prime}$ is $30+\theta$ and that the phase difference between $b$ and $e^{\prime \prime}$ is $30^{\circ}-\theta$, so that :

$$
\begin{aligned}
& W^{\prime}=a e^{\prime} \cos \left(30^{\circ}+\theta\right) \\
& W^{\prime \prime}=b e^{\prime \prime} \cos \left(30^{\circ}-\theta\right)
\end{aligned}
$$


in which $W^{\prime}$ and $W^{\prime \prime}$ are the respective wattmeter readings and $a, b, e_{1}^{\prime}$ and $e^{\prime \prime}$ represent the effective values of the respective voltages and currents. * Now when $\mathscr{E}$ is $60^{\circ}$, that is when the power factor of each receiving circuit is $1 / 2$, the angle $30^{\circ}+\theta$ is $90^{\circ}$, and $W^{\prime}$ reduces to zero; and when $\theta$ is greater than $60^{\circ}$ the angle $30^{\circ}+\theta$ is greater than $90^{\circ}$, and $W^{\prime}$ becomes negative. That is, when the power factor of the similar receiving circuits is greater than $1 / 2$, both wattmeter readings are positive, when the power factor is equal to $1 / 2$ one wattmeter reading becomes zero, and the other indicates the total power, and when the power factor is less than $1 / 2$, one wattmeter reading becomes negative and the other reading is greater than the total power.

\section{Relative copper economy} of two-phase and three-phase transmission lines. As a basis of comparison of the copper economy let us consider a fourwire two-phase transmission line and a three-wire three-

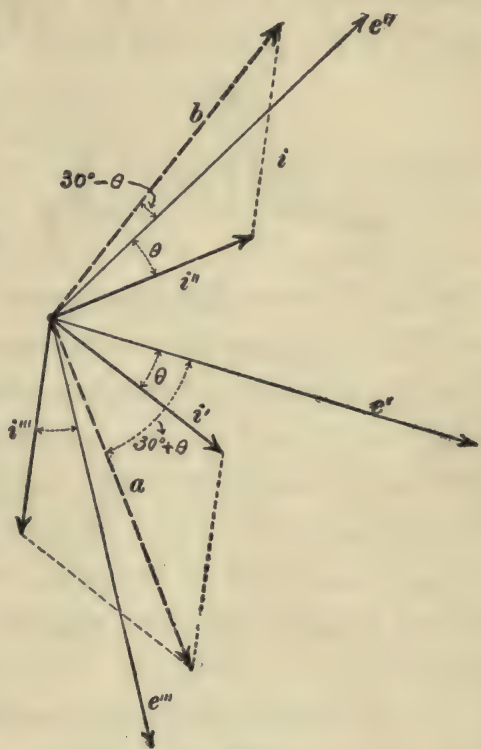

Fig. 106. phase transmission line of the same length with the same voltages between mains in each case. Let $E$ be the common value of the voltages at the receiving end, and let it be required to find the relative amounts of copper required to transmit the same amount of power (unity power factor) with the same loss of power in the mains in each case. Let $R_{2}$ be the resistance of each of the four wires of the two-phase line, let $R_{3}$ be the resistance of each of the three wires of the three-phase line, let $I_{2}$ be the current in each of the four wires of the two-phase line, and let $I_{3}$ be the current in each of the three wires of the three-phase line. Then $2 E I_{2}$ is the power delivered by the two-phase line and $\sqrt{3} E I_{3}$ is the power delivered by the three-phase line, and, since the total power is assumed to be the same in each case, we have $2 E I_{2}=\sqrt{3} E I_{3}$ or

* It is contrary to established conventions to use small letters to represent effective values, and it is done here for the sake of brevity. Small letters stand for instantaneous values throughout this article except in the above two equations for $W^{\prime}$ and $W^{\prime \prime}$. 


$$
I_{2}=\frac{\sqrt{3}}{2} \cdot I_{3}
$$

Furthermore, $4 R_{2} I_{2}^{2}$ is the power lost in the two-phase line, and ${ }_{3} R_{3} I_{3}^{2}$ is the power lost in the three-phase line, so that

$$
{ }_{4} R_{2} I_{2}^{2}={ }_{3} R_{3} I_{3}^{2}
$$

Therefore, using equation $(a)$, we have

$$
R_{2}=R_{3}
$$

That is, the wires have the same resistance and therefore the same weight, and, since four wires are used in the two-phase line and only three wires in the three-phase line, it follows that the total weight of copper in the two-phase line is $\frac{4}{3}$ times as great as in the three-phase line. The same result is reached on the basis of equal percentage drops of electromotive forces in the two systems. The result is independent of the power factor of the receiving circuits provided the power factor is the same for both systems.

The three-wire two-phase transmission line requires less copper than the three-wire three-phase line in the ratio 2.914 to 3 , but the three-wire two-phase arrangement is not desirable for reasons explained in Art. 49, and, furthermore, a three-wire two-phase line with $E$-volts between the middle wire and each outside wire has $\sqrt{2} E$-volts between the outside wires, whereas, in a threewire three-phase line with $E$-volts between any two wires, there is no higher voltage than $E$ anywhere in the system. Therefore, it is unfair to compare the three-phase system with the three-wire two-phase system on the basis of equal voltages $E$.

On account of the greater copper economy and on account of the use of three wires instead of four, the three-phase system is nearly always used for long distance transmission. 


\section{CHAPTER VII.}

VOLTAGE AND VOLTAGE REGULATION OF THE ALTERNATOR.

61. Electromotive force of the alternator, its dependence upon speed and flux. - The electromotive force of a direct current generator depends in a very simple way upon the magnetic flux $\Phi$ per pole, upon the number of armature conductors $Z^{*}$, and upon the armature speed $n$. The electromotive force (effective) of an alternator, however, depends not only upon $\Phi, Z$, and $n$, but also upon the width and shape $\dagger$ of the pole pieces of the field magnet, and upon the extent to which the windings are spread over the surface of the armature.

The average value $\ddagger$ of the electromotive force induced in one armature conductor is always equal to $p \Phi n$ abvolts, where $p$ is the number of field magnet poles, $\Phi$ is the magnetic flux from one pole, and $n$ is the armature speed in revolutions per second. This is evident when we consider that a given armature conductor cuts $\Phi$ lines of force in I/p of a revolution or in I/pn of a second, so that the average rate of cutting lines of force is $\Phi$ divided by $\mathrm{I} / p n$ which is equal to $p \Phi n$.

Concentrated versus distributed armature windings. - When the armature conductors are grouped in $p$ equidistant slots, $p$ being the number of field magnet poles, the armature winding is said to be concentrated; when the armature conductors are spread out in $p$ broad bands or in $p$ groups of slots, the winding is said to be distributed.

In a concentrated winding the same electromotive force $e$ is

* Number of armature conductors per path.

$\dagger$ This is intended to include everything which affects the distribution of flux around the armature.

$\ddagger$ Whenever the average value of an alternating electromotive force or current is spoken of, the average value during a half-cycle is always meant. 
induced in every armature conductor at any given instant; therefore the electromotive force between the collector rings at the given instant is equal to $Z e, Z$ being the number of armature conductors in series between the collector rings; the average electromotive force between the collector rings is equal to $Z$ times the average electromotive force, $p \Phi n$, in a single conductor; and the effective electromotive force between the collector rings is equal to $Z$ times the effective electromotive force in a single conductor.

In a distributed winding, on the other hand, the same electromotive force is not induced in all of the armature conductors at any given instant, because the conductors cannot be all similarly situated under the respective field magnet poles. Or, in other words, there is a phase difference between the electromotive forces induced in the different conductors. On account of this phase difference the average electromotive force between the collector rings is not equal to $Z$ times the average electromotive force in a single conductor, and the effective electromotive force between the collector rings is not equal to $Z$ times the effective electromotive force in a single conductor.

In the case of a concentrated armature winding the average electromotive force between the collector rings is equal to $p \Phi Z n$, but the effective value of the electromotive force cannot be completely expressed in terms of $p, \Phi, Z$ and $n$, because it depends upon the size and shape of the pole pieces, as well as upon $p$, $\Phi, Z$ and $n$.

In the case of a distributed armature winding neither the average value nor the effective value of the electromotive force between the collector rings can be completely expressed in terms of $p, \Phi, Z$ and $n$, because they both depend upon the size and shape of the pole pieces and upon the exact mode of distribution of the windings.

A complete discussion of the dependence of average values and effective values of electromotive force upon the size and shape of pole pieces and upon the manner of distribution of the armature 
windings would be of little practical value, and the following discussion is limited therefore to a few simple special cases.

Uniform distribution of flux under pole faces. - Suppose that the flux $\Phi$ is evenly distributed under the pole faces, as shown in Fig. 107, the fringe of flux beyond the pole tips being ignored for the sake of simplicity. Let $w$ be the width of the pole faces in degrees, and let $s$ be the distance between the tips of adjacent poles, in degrees. Then $w$ may be taken as the number of units of time that a given armature conductor is under a pole face, $s$ as the number of units of time that the conductor is between two pole tips, and $w+s$ as the number of units of time in a half-cycle. Under these conditions let us consider two different armature windings, namely: (a) A concentrated winding; and $(b)$ a particular distributed winding.

(a) Concentrated armature winding. - In this case the average value (during half-a-cycle) of the electromotive force of the alternator is equal to $p \Phi Z n$ abvolts as stated above, and to find the effective value, we must find the value of the ratio:* effective value divided by average value. With this object in view let $\mathcal{E}$ be the actual value of the electromotive force of the alternator during the $w$ units of time that the armature conductors are under the pole faces. Then, since the actual electromotive is zero during the $s$ units of time that the windings are between the pole tips on the assumption that there is no flux beyond the pole tips, it is evident that the average electromotive force of the alternator during half-a-cycle is $\mathcal{E} w /(w+s)$, and that the effective electromotive force of the alternator is $\varepsilon \sqrt{w /(w+s)}$, so that the ratio, effective value divided by average value, is equal to $\sqrt{(w+s) / w}$. Therefore the effective value of the electromotive force of the alternator is equal to $p \Phi Z n \sqrt{[(w+s) / w]}$ abvolts.

(b) Distributed armature winaing. - Let us consider an armature winding in which the armature conductors are grouped in

* This ratio depends upon the shape of the electromotive force curve, and it is called the form factor of the electromotive force curve. 
$2 p$ slots as shown in Fig. I07. In this figure the breadth of the pole faces is 6 units, the distance between pole tips is 4 units, and the distance from center to center of slots $a$ and $b$ is $2 \frac{1}{2}$ units.

To determine either the average value (during half-a-cycle), or the effective value of the electromotive force of an alternator from a given distribution of flux and a given arrangement of the windings, it is in general necessary to calculate the instantaneous values of the electromotive force for a series of successive positions of the armature, and then find the average, or the squareroot-of-the-average-square of these instantaneous values.

The ordinates of the rectangular "curve" $b b b b$, Fig. 107, rep-
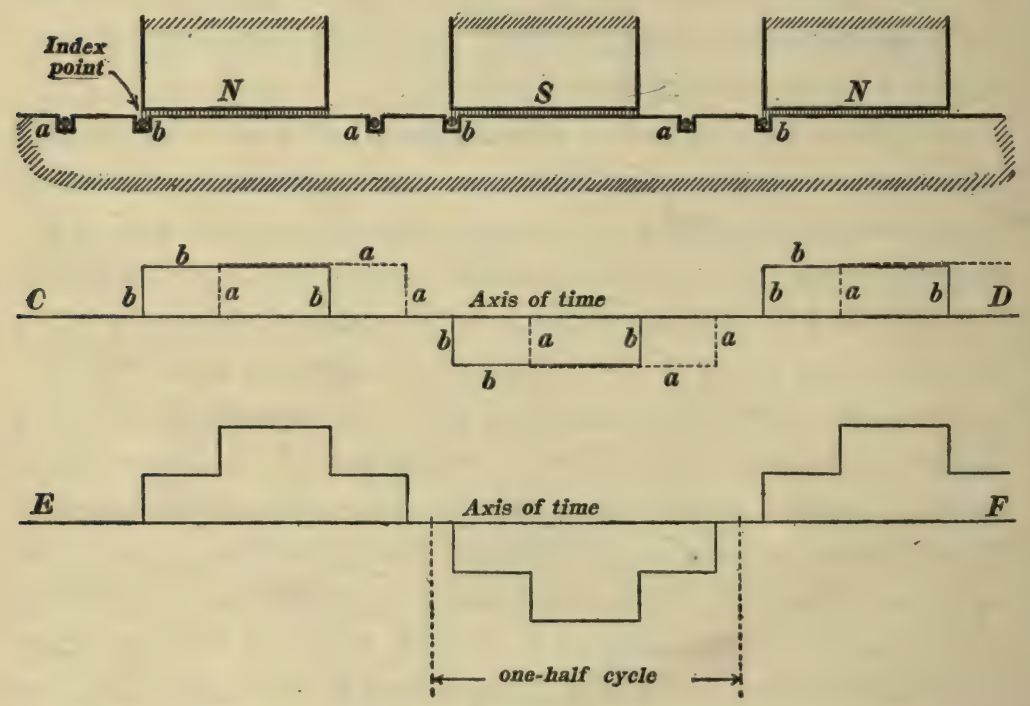

Fig. 107.

resent the successive values of the electromotive force of the windings which lie in the $b$ slots, the ordinates of the "curve" aaaa represent the successive values of the electromotive force of the windings which lie in the $a$ slots, and the ordinates of the curve $E F$ represent the successive values of the electromotive force of the complete winding. Knowing the successive values of the electromotive force of the complete winding as represented by the 
curve $E F$, the average value (during half-a-cycle) and the effective value are easily found.

In Fig. 107 it is assumed that there is no fringe of flux beyond the pole tips, and the distance apart of each pair of slots $a$ and $b$ is less than the distance between pole tips. Under these conditions there is never an instant when the induced electromotive forces in the different parts of the given armature winding oppose each other, and, such being the case, the average value (during half-a-cycle) of the electromotive force of the alternator is equal to $Z$ times the average value (during halfa-cycle) of the electromotive force in a single conductor; and when this relation holds, the effective electromotive force of the alternator can be expressed as $p \Phi Z n$ times the form factor of the electromotive force curve. In general, however, the form factor can be used in this way only in case of a concentrated armature winding.

Harmonic distribution of flux. - Figure ro8 shows an armature between two nearly flat pole-pieces, so that the flux density in the gap space is greatest at the points $a a$. If the pole faces

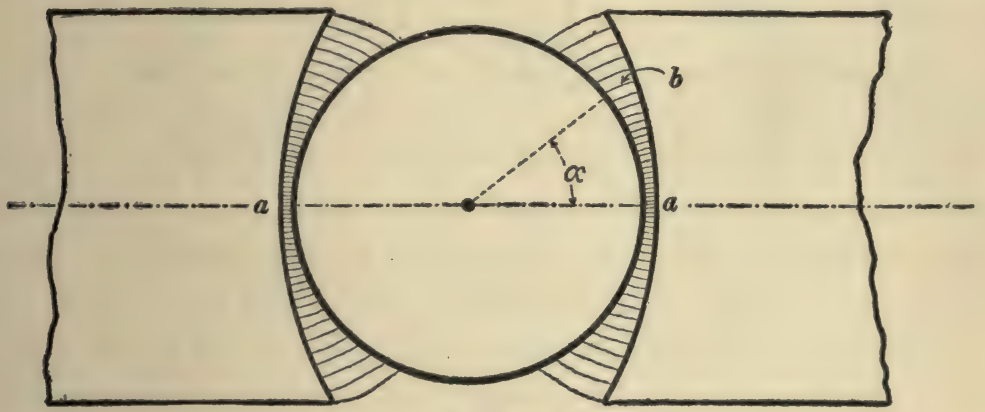

Fig. 108.

were so shaped that the flux density at any point $b$ in the gap space would be proportional to the cosine of the angle $\alpha$, we would have what is called a harmonic distribution of flux, and the electromotive force induced in each armature conductor would be a harmonic electromotive force capable of being represented by a line in a clock diagram.

(a) Concentrated armature winding. - In the case of a concentrated winding, the average value (during half-a-cycle) of the electromotive force of the alternator is equal to $p \Phi Z n$ abvolts as stated above, and if the electromotive force is harmonic (harmonic distribution of flux) the ratio, effective value divided by average 
value, is equal to $\pi /(2 \sqrt{2})$. Therefore the effective value of the electromotive force of an alternator having a concentrated armature winding, and having a harmonically distributed flux, is equal to

$$
\pi /(2 \sqrt{2}) \times p \Phi Z n \text { abvolts. }
$$

(b) Distributed armature winding. - Consider an armature winding in which the conductors are grouped in $4 p$ slots, that is in $p$ groups of slots each group having four slots as shown in Fig. I09. Let $q$ be the angular distance between centers of ad-

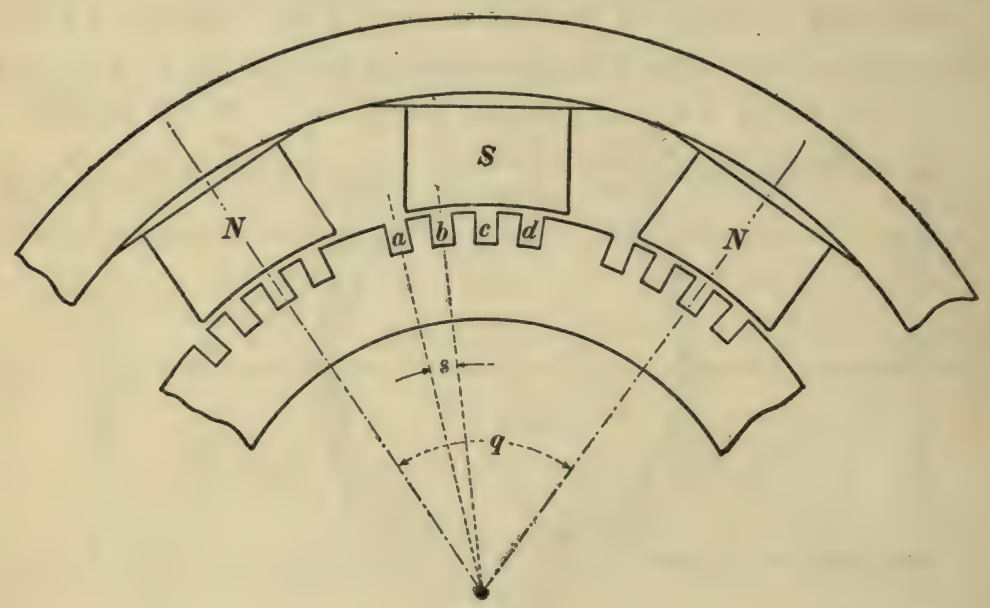

Fig. 109.

jacent north poles of the field magnet, and let $s$ be the angular distance from center to center of adjacent slots.

Ail of the conductors in the $a$ slots constitute a concentrated winding, all of the conductors in the $b$ slots constitute a concentrated winding, all of the conductors in the $c$ slots constitute a concentrated winding, and so on. These concentrated windings are all alike, the respective electromotive forces $A, B, C$ and $D$ which they develop are all the same in value, but they differ in phase, and the phase difference $A$ to $B, B$ to $C$, and $C$ to $D$ is $s / q \times 360^{\circ}$. Therefore the four electromotive forces $A, B$, 
$C$ and $D$ may be represented by the four equidistant vectors $A$, $B, C$ and $D$ in the clock diagram Fig. I Io.

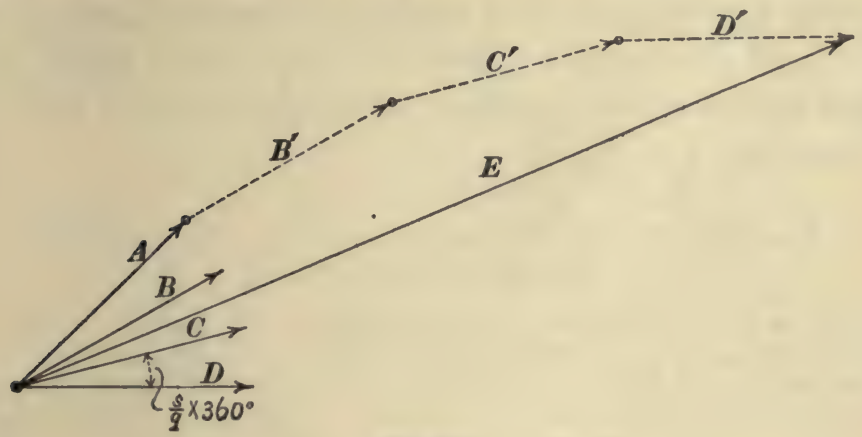

Fig. 110.

If the windings in all of the slots in Fig. I09 are connected in series as a single distributed winding, the effective value of the electromotive force produced by the combined winding will be the vector sum of $A, B, C$ and $D$, as shown by the line $E$ in Fig. IIO, in which the lines $A, B^{\prime}, C^{\prime}$ and $D^{\prime}$ form a portion of a regular polygon of which $E$ is the chord. If the angular distance between $A$ and $B, B$ and $C$ and so on, is small, the lines $A, B^{\prime}, C^{\prime}$ and $D^{\prime}$ form sensibly the arc of a circle of which $E$ is the chord, and the angle subtended by the arc is the phase difference between $A$ and $D$.

If the electromotive forces $A, B, C$ and $D$ were in phase with each other, that is if all of the armature conductors were concentrated in $p$ slots, the effective value of $E$ could be calculated from the formula $\pi /(2 \sqrt{2}) \times p \Phi Z n$ abvolts, and it would be equal to the arithmetical sum (not the vector sum) of $A, B, C$ and $D$; that is, the value of $E$ would be represented by the length of the arc $A B^{\prime} C^{\prime} D^{\prime}$ in Fig. I Io. In fact, however, $E$ is represented by the length of the chord, so that $\pi /(2 \sqrt{2}) \times p \Phi Z n$ must be multiplied by the ratio chord/arc to give the correct value of $E$, the chord and arc being such as to subtend an angle equal to the phase difference between the electromotive forces induced in those armature conductors which lie in the most distant slots of a 
group. This ratio chord/arc, as here defined, is sometimes called the phase constant of a distributed winding. The following table gives the values of this phase constant based not upon the length of circular arc but upon the actual length of the proper portion of the periphery of the electromotive force polygon, see Fig. I 10.

\section{VALUES OF PHASE CONSTANTS FOR DISTRIBUTED ARMATURE WINDINGS.}

\begin{tabular}{c|c|c|c|c|c}
\hline \hline \multirow{2}{*}{$\begin{array}{c}\text { Number of slots in } \\
\text { each group. }\end{array}$} & \multicolumn{5}{|c}{ Widths of groups of slots in fractional parts of $N$ to $S$. } \\
\cline { 2 - 6 } & $1 / 4$ & $3 / 3$ & $1 / 2$ & $3 / 4$ & Whole. \\
\hline 2 & .980 & .966 & .924 & .831 & .707 \\
3 & .977 & .960 & .912 & .805 & .666 \\
4 & .976 & .958 & .908 & .795 & .653 \\
Infinity. & .975 & .955 & .901 & .784 & .637 \\
\hline
\end{tabular}

The slots for a given winding are always arranged in $p$ similar groups, $p$ being the number of field magnet poles. The slots in a group are always equidistant, and the width of a group is understood to mean $n s$, where $n$ is the number of slots in a group and $s$ is the distance from center to center of adjacent slots. It is evident that if the width of the groups of slots is $1 / 3$ of the distance from $N$ to $S$, center to center, then three such windings can be placed on the armature giving a three-phase alternator, and if the width of the groups of slots is $1 / 2$ of $N$ to $S$, center to center, then two such windings can be placed on the armature giving a two-phase alternator. Figure I I shows the outline of an 8-pole alternator with its armature slotted for a winding distributed in $3 p$ slots, the width $n s$ of each group of slots being $1 / 3$ of the distance $N$ to $S$, center to center, so that there is room on this armature for two additional windings of the same type.

It is undesirable to spread the winding of a single-phase alternator over the whole armature surface. Thus the phase constant of a winding spread over the whole surface is 0.637 and the phase constant of a winding spread over $3 / 4$ of the armature surface is 0.784 . The number of armature conductors in the first 
case being taken as 100 , the number in the second case would be 75 , and the electromotive forces would be in the ratio of

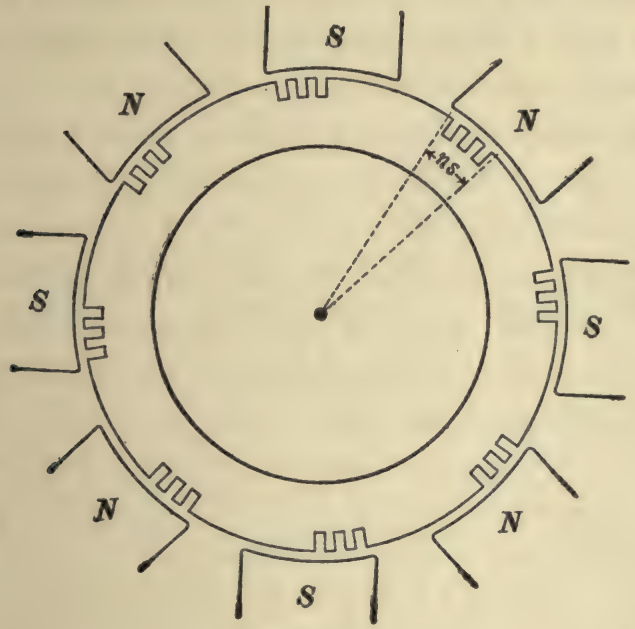

Fig. 111.

$100 \times 0.637$ to $75 \times 0.784$, that is in the ratio of 63.7 to 58.8 . It is therefore evident that only a slight increase of electromotive force would be obtained by filling up the whole armature surface of a single-phase alternator with windings, whereas the armature resistance, the loss of power due to armature resistance, and the cost of copper would all be increased in the ratio of 75 to 100 in the case cited. A single-phase alternator giving, say, I, ooo volts and having an armature wound with wire large enough to carry IOO amperes, and therefore able to deliver Ioo kilowatts to a noninductive receiving circuit, must be larger and more costly than a two-phase or three-phase alternator capable of delivering the same total amount of power.

62. Voltage regulation of an alternator. - An alternator, driven at a constant speed and having a constant field excitation, gives a certain terminal voltage when its current output is zero, and its terminal voltage generally falls off in value with increase of current output; or, if the machine is adjusted to give a certain ter- 
minal voltage at full load, its terminal voltage generally rises with decrease of current output. The increase of terminal voltage from full load to zero load expressed in per cent. of normal fullload voltage, field excitation and speed both being constant, is called the voltage regulation of the machine.

The voltage regulation of an alternator varies greatly with the power factor of the receiving circuit. For example, a certain alternator delivers its full-load current to a non-inductive receiving circuit (power factor equal to unity) at its normal full-load voltage of I IOO volts, and the terminal voltage rises to II 88 volts when the load is reduced to zero by opening the main switch. The same alternator has its field excitation adjusted so that it gives its normal full-load voltage of I IOO volts when it delivers its full-load current to an inductive receiving circuit of which the power factor is 0.80 , and the terminal voltage rises to I 386 volts when the load is reduced to zero. That is, the given alternator has a regulation of 8 per cent. when it delivers current to a receiving circuit having unity power factor, and it has a regulation of 26 per cent. when it delivers current to an inductive receiving circuit having a power factor equal to 0.80 . When an alternator delivers current to a receiving circuit like a condenser, in which the current is ahead of the electromotive force in phase, the regulation of the alternator is better (smaller) than when the receiving circuit is non-inductive. Thus, the above alternator has a regulation of zero per cent. when it delivers current to a condenser circuit having a power factor of 0.97 , and a regulation of minus 14 per cent. when it delivers current to a condenser circuit having a power factor equal to 0.80 . A zero per cent. regulation means no rise of terminal voltage from full load to zero load, and a negative regulation means a decrease of terminal voltage when the current output is reduced from full load to zero load.

Example. - The two curves in Fig. I 2 show the regulation of a I 35 kilowatt, 2-phase, 60 cycle inductor alternator of the Stanley Electric Manufacturing Company. The abscissa of a 
point on one of the curves represents a given current output per phase, and the ordinate of the point represents the terminal voltage observed when the given current output is reduced to zero, the field current having been adjusted to give the rated voltage of 2400 volts with the given current output. Thus when the field excitation is adjusted to give 2400 volts across the terminals of the alternator with full-load current output of 28. I amperes per phase, and unity power factor, the terminal voltage rises to 2625 volts at zero load. That is, the full-load regulation of the machine with unity power factor is $(2625-2400) \div 2400=0.094$

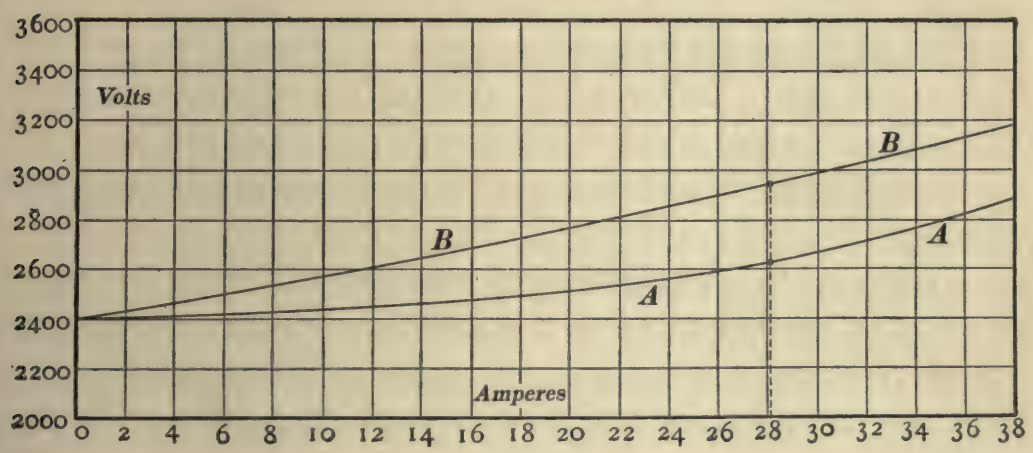

Fig. 112.

Curve $A$ refers to unity power factor and curve $B$ refers to 0.70 power factor.

or 9.4 per cent. With half-load current of 14.05 amperes per phase and unity power factor, the terminal voltage rises from 2400 volts at half-load to 2450 volts at zero load. That is, the half-load regulation of the machine with unity power factor is $(2450-2400) \div 2400=0.02$ I or 2 . I per cent. With a power factor of 0.70 the full-load regulation is 22.5 per cent. and the half-load regulation is IO.O per cent.

63. Theory of voltage regulation of the alternator.-The decrease of terminal voltage of an alternator with increase of current output is due to two effects, namely, (a) the resistance of the armature windings, and $(b)$ the magnetizing action of the armature current or currents. In discussing the effect of magnetizing 
action of the armature current, or currents, it is convenient to think of the field windings as producing a certain constant flux $\Phi$, and of the armature current or currents as producing a certain additional flux $\Phi^{\prime}$; for brevity we will call $\Phi$ the "field flux" and $\Phi^{\prime}$ the "armature flux," although of course both $\Phi$ and $\Phi^{\prime}$ pass through the armature.

The field flux $\Phi$ may be thought of as producing a certain "total induced electromotive force" in each armature winding; a portion of this total induced electromotive force is lost in balancing the electromotive force induced by the armature flux $\Phi^{\prime}$, another portion is lost in overcoming the resistance of the armature winding, and the remainder appears as the terminal voltage of the machine. Therefore, to calculate the voltage regulation of an alternator it is necessary to consider the value and phase of the electromotive force induced in the armature windings by the flux $\Phi^{\prime}$.

In the polyphase alternator with a balanced load the armature flux $\Phi^{\prime}$ is constant in value (for a given value of the armature currents) and fixed in space, and electromotive forces are induced in the moving armature conductors as they cut this flux. In the single-phase alternator, on the other hand, the armature flux $\Phi^{\prime}$ is neither constant in value nor fixed in space, and the electromotive forces induced in the armature conductors by $\Phi^{\prime}$ are therefore very complicated in the single phase alternator. The following discussion applies primarily to the polyphase alternator with a balanced load, and the discussion is made to refer explicitly to a two-pole machine for the sake of simplicity.

When a polyphase alternator delivers current to balanced noninductive receiving circuits, the axis of the armature-magnetizingaction (axis of $\Phi^{\prime}$ ) is at right angles to the axis of the field as shown in Fig. I I $3 a$, in which $n$ and $s$ represent the magnetic poles on the armature core due to the armature magnetizing action. The fine lines in Fig. I I $3 a$ represent the actual distribution of the armature flux $\Phi^{\prime}$, and the fine lines in Fig. ${ }^{1} 1_{3} b$ represent the distribution of $\Phi^{\prime}$ on the assumption that the iron 
of the pole pieces completely and uniformly surrounds the armature.

When a polyphase alternator delivers current to balanced inductive receiving circuits, the axis of armature-magnetizing-

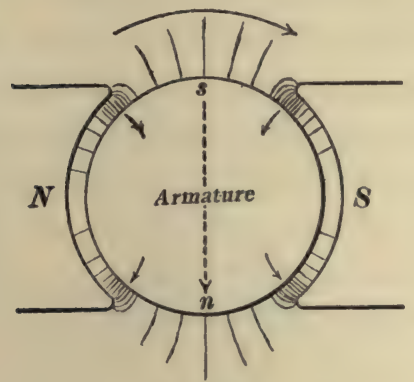

Fig. $113 a$.

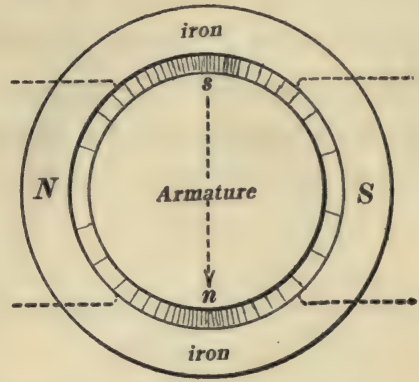

Fig. $113 b$.

Actual and assumed distribution of $\Phi^{\prime}$, unity power factor.

action (axis of $\Phi^{\prime}$ ) is shifted forwards $\theta^{\circ}$ as shown in Figs. I I $4 a$ and $\operatorname{II}_{4} b$, where $\theta^{\circ}$ is the phase angle between voltage and current in each receiving circuit. When the currents in the receiving circuits lead the electromotive forces in phase by the angle

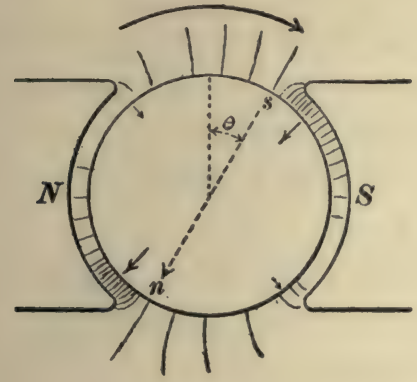

Fig. $114 a$.

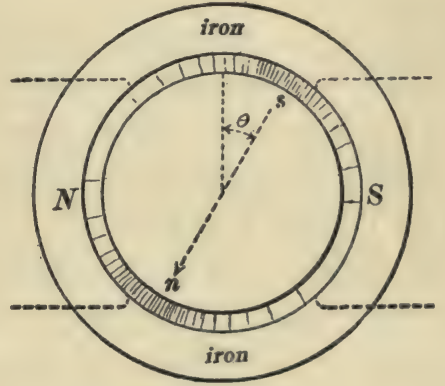

Fig. $114 b$.

Actual and assumed distribution of $\phi^{\prime}$, lagging currents.

$\theta$, then the axis of $\Phi^{\prime}$ is shifted backwards as shown in Figs. I I $5 a$ and I I $5 b$.

Several important things are at once evident from Figs. I I3, I 4 and I I 5 as follows: (a) The armature flux $\Phi^{\prime}$ is to a certain extent opposed to the field flux $\Phi$ in Fig. II4, (b) The 
armature flux $\Phi^{\prime}$ is to a certain extent in the direction of the field flux $\Phi^{\prime}$ in Fig. I I 5, and (c) The armature flux $\Phi^{\prime}$ neither helps nor opposes the field flux $\Phi$ in Fig. I I3. This matter is discussed in Art. 65.

(d) The distribution of $\Phi^{\prime}$ is far from being harmonic (like Fig. 107) and therefore the electromotive forces induced by $\Phi^{\prime}$ in the moving armature conductors are far from being harmonic. This is the most serious obstacle to the formulation of a simple method for calculating the voltage regulation of an alternator,

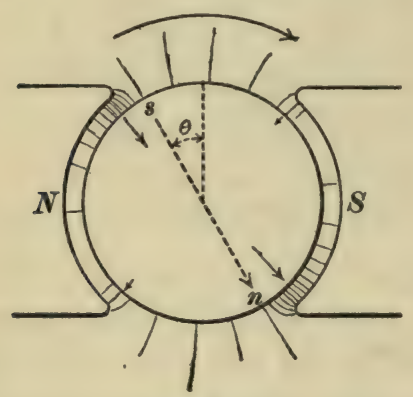

Fig. $115 a$.

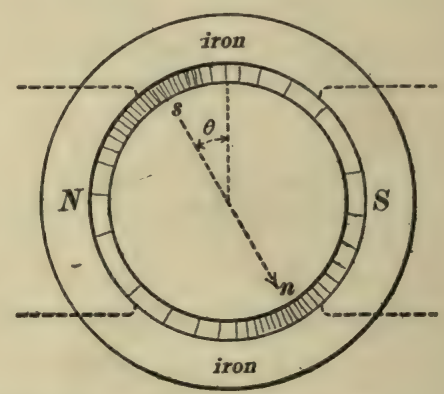

Flg. $115 b$.

Actual and assumed distribution of $\Phi^{\prime}$, leading currents.

and indeed a simple method must be based on the assumption that $\Phi^{\prime}$ is harmonically distributed as shown in Figs. I I $3 b$, I I $4 b$ and I $5 b$, and this means that the field magnet is assumed not to have polar projections but to surround the armature uniformly on all sides as indicated.

(e) It is evident from a careful consideration of Fig. I I $3 b$ that the electromotive force induced in each armature winding by the flux $\Phi^{\prime}$ is $90^{\circ}$ behind the electromotive force induced by the field flux $\Phi$, so that the portion of the "total induced electromotive force" (due to $\Phi$ ) which is used to overcome the electromotive force produced by $\Phi^{\prime}$ is $90^{\circ}$ ahead of the total induced electromotive force, or $90^{\circ}$ ahead of the current in the given armature winding, since, in Fig. I I 3, current and voltage are supposed to be in phase with each other. A careful consideration of Figs. I $4 b$ and I I $5 b$ will make it evident that in every case the portion 
of the total induced electromotive force which is lost in overcoming the electromotive force produced by $\Phi^{\prime}$ is $90^{\circ}$ ahead of the current in phase. This lost electromotive force is, furthermore, proportional to the armature current $I$ so that it may be expressed as $X I$ where $X$ is a reactance exactly like an inductance-reactance. This quantity $X$ is called the "synchronous reactance" of the armature.

The general relation between the total induced voltage (due to field flux $\Phi$ ) and the terminal voltage of an alternator is shown in Fig. I 16. The line $O I$ represents the current in one of the armature windings, the line $O E$ represents the total induced electromotive force, the line $E_{x}$ represents the terminal voltage, $R I$ represents the electromotive

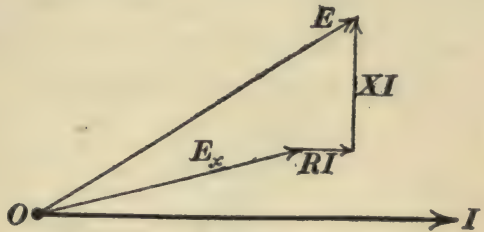

Fig. 116. force lost in overcoming armature resistance, and the line $X I$ represents the electromotive force which is lost on account of armature magnetizing action.

64. Calculation of voltage regulation of an alternator. - Nearly all alternating current generating stations deliver current at an approximately constant voltage, and the various lamps and motors are connected in parallel across the mains. It would be desirable therefore if an alternating current generator could be made to give an exactly constant voltage, however its current output might vary, and in practice alternators are constructed to give as small a variation of voltage as possible from zero load to full load. Purchasers generally require of a manufacturer a certain guarantee as to the voltage regulation of an alternator. In view of this guarantee, it is always necessary for the manufacturer to determine the voltage regulation of an alternator by test, so as to be sure that it comes within the guarantee. In the case of a small machine, this test can be made directly by driving the machine, adjusting its field excitation to give normal voltage at full load, and observing the rise of voltage when the load is thrown off, speed and field excitation being unchanged. In the case of large 
machines, however, this direct method is not usually feasible and it is therefore customary to calculate the voltage regulation of large machines in terms of certain easily obtained data. The data which are used for this purpose are included in what are called the saturation curve and the synchronous reactance curve of the machine.

The saturation curve. - The saturation curve of an alternator is a curve of which the abscissas represent the values of the field current and the ordinates represent the corresponding observed values of the terminal voltage of the machine at zero load. Thus, Fig. I I shows the saturation curve of a certain 2,000 kilowatt,

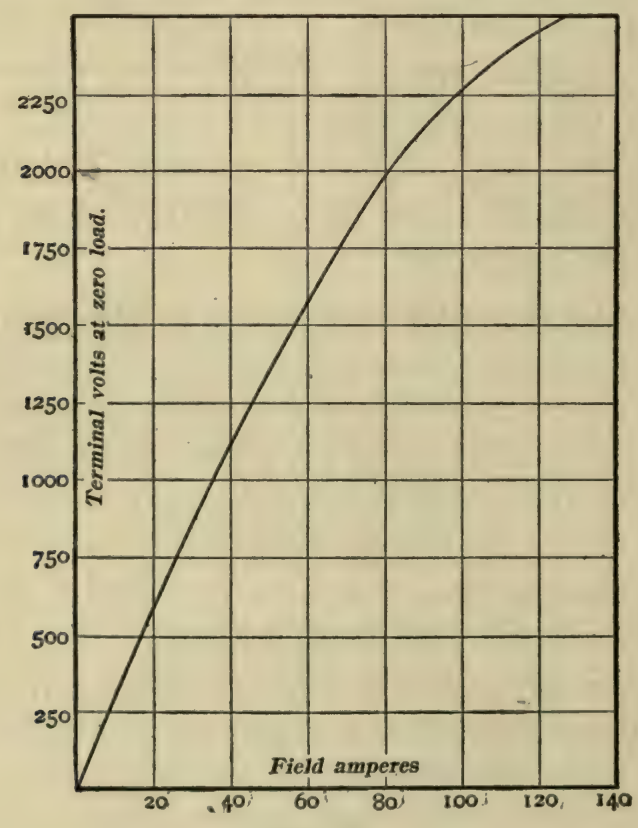

Fig. 117.

$\Delta$-connected, 3-phase alternator. The ordinates of this curve represent the observed voltages across any two collector rings of the machine, and the abscissas represent the corresponding values of field current in amperes. 
The synchronous reactance curve is determined as follows: The armature is short-circuited $*$ through an ammeter, the machine is driven at normal speed, the field excitation is adjusted until fullload current (or any desired fraction of full-load current) flows through the ammeter and the ammeter reading is taken. The short-circuit is then opened, and, with unchanged speed and field excitation, the voltage across the alternator terminals is measured. The ammeter readings are then plotted as abscissas and the corresponding open-circuit voltages are plotted as ordinates. Figure I 18 is the synchronous reactance curve of the 2,000-kilowatt,

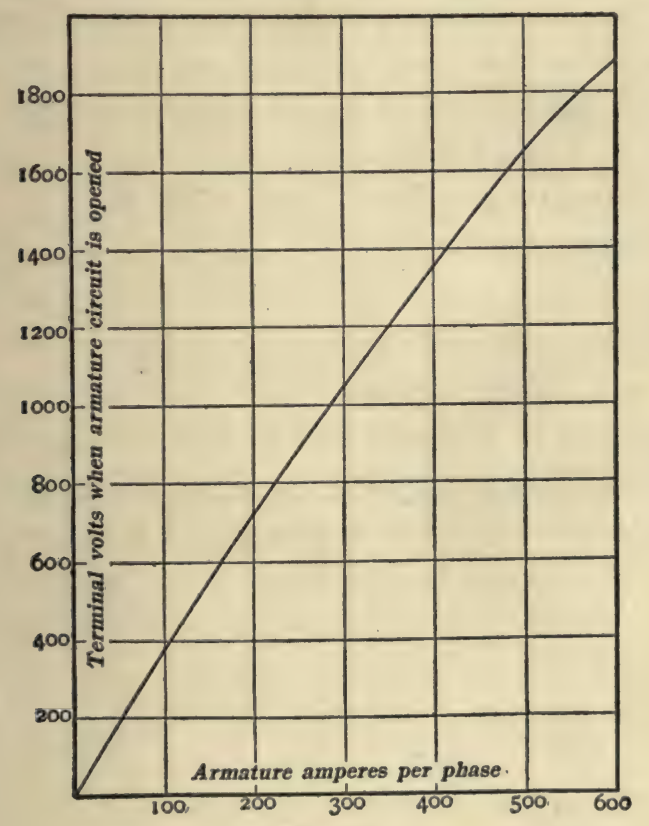

Fig. 118.

3-phase alternator which is referred to in connection with Fig. I I 7 above. In Fig. I 8 the abscissas represent amperes in each armature winding (ammeter readings divided by $\sqrt{3}$ ).

The reason for calling this curve the synchronous reactance curve is that the voltage corresponding to any given abscissa

* In a polyphase armature all of the phases must be simultaneously short-circuited. 
(current) is the "total induced electromotive force" required to force the given current through the short-circuited armature, and, since the resistance of the short-circuited armature is negligibly small, it is evident that the only thing which opposes the flow of this short-circuited current is the armature inductance or magnetizing action, whichever one may wish to call it. The reactance value of this inductance depends of course upon the frequency, and the term synchronous reactance refers to the reactance value of the armature inductance or magnetizing action at the normal speed of the machine.

\section{Electromotive force method of calculating voltage regulation. -} This method may be best explained by giving numerical examples. Let it be required to calculate the voltage regulation (full load to zero load) of the 2,000-volt, 2,000-kilowatt, 3-phase alternator referred to in connection with Figs. II 7 and I 8 $(a)$ for receiving circuits of unity power factor and $(b)$ for receiving circuits of 0.85 power factor, the resistance of one of the armature windings (one phase) being $0.07 \mathrm{ohm}$.

(a) For unity power factor. - The full-load current in one armature winding is 333 amperes, so that the $R I$ drop in the armature at full load is 23.3 volts. This $R I$ drop is in phase with the current and therefore in phase with the terminal voltage,

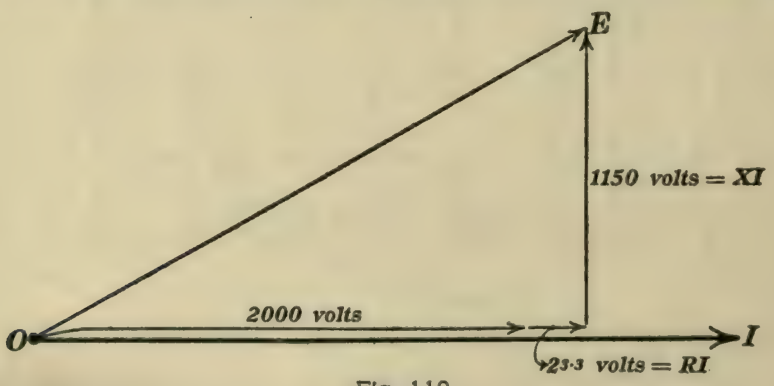

Fig. 119.

since the power factor of the receiving circuit is assumed to be unity, so that the sum of the $R I$ drop and the terminal voltage is 2023.3 volts. From Fig. I I 8 we find that I I 50 volts correspond to 333 amperes, that is to say, the value of $X I$ in Fig. I 9 
is equal to I I 50 volts. Therefore the "total induced electromotive force," that is the electromotive force which is induced by the "field flux" $\Phi$, is equal to $\sqrt{(2023 \cdot 3)^{2}+(\text { I I 50 })^{2}}$ or 2327 volts, which is of course the value to which the terminal voltage would rise if the current output were reduced to zero. Therefore the regulation of the machine for unity power factor is $(2327-2000) / 2000=0.163$ or 16.3 per cent.

(b) For a pozerer factor equal to 0.85 . - Choose the current vector $O I$, Fig. I2O, as the reference axis, lay off the full-load

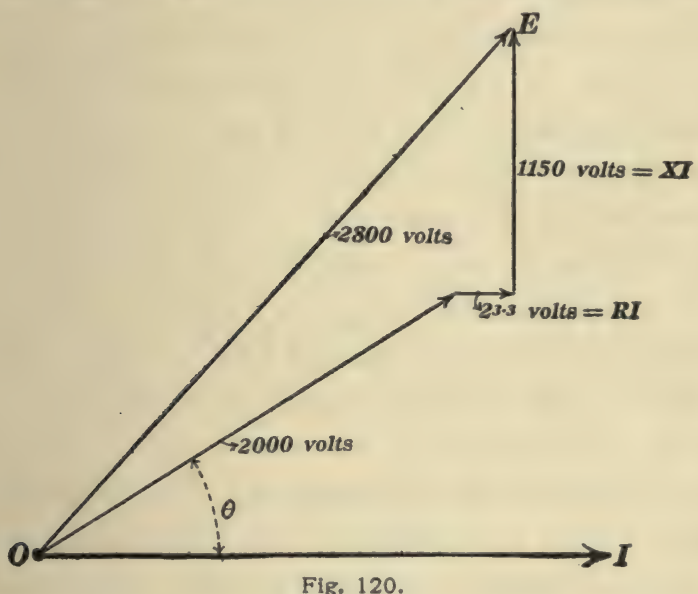

terminal voltage of 2000 volts in its proper phase relation to $I(\cos \theta=0.85)$, and lay off $R I$ (23.3 volts) and $X I$ (I I 50 volts) as shown; the line $O E$ will then represent the total induced electromotive force, and it is, of course, the value to which the terminal voltage of the machine would rise if the current output were reduced to zero. The value of the zero-load voltage (correresponding to 2000 volts terminal voltage at full load and 0.85 power factor) is thus found to be 2800 volts, so that the regulation is $(2800-2000) / 2000=0.40$ or 40 per cent.

The magnetomotive force method.* - If the magnetizing action of the field winding and of the armature were not complicated by

* This is called the American Institute Method, inasmuch as it is recommended by the Institute Committee on Standardization. See page 255 of volume 16 of the Transactions. A new report is now (1907) in preparation. 
the polar projections of the field magnet, that is, if the armature were completely and uniformly surrounded with iron as shown in Figs. I $13 b, I_{4} b$ and $I_{1} b$, then the combined effect of two magnetizing actions $A$ and $B$ would be as follows: Consider the flux $\Phi$ across the armature due to $A$ and the flux $\Phi^{\prime}$ due to $B$. Draw lines in the directions of $\Phi$ and $\Phi^{\prime}$ and let the lengths of these lines represent the magnetomotive force values (ampereturns) of $A$ and $B$ respectively. Then the vector sum of these lines will represent the direction and value (in ampere-turns) of the single magnetizing action which is equivalent to the combined action of $A$ and $B$. This is the fundamental idea of the magnetomotive force method for calculating voltage regulation, and the method is best explained by considering numerical examples. Let it be required to calculate the voltage regulation (full load to zero load) of the 2000-volt, 2000-kilowatt, 3-phase alternator referred to in connection with Figs. I I 7 and I I 8, (a) for receiving circuits of unity power factor and $(b)$ for receiving circuits of 0.85 power factor, the resistance of one of the armature windings (one phase) being 0.07 ohms.

(a) Unity power factor. - When the alternator delivers fullload current of 333 amperes from each of its armature windings to noninductive receiving circuits, the two components of the total induced voltage parallel to and at right angles to the current are 2023.3 volts and I I 50 volts as explained above under the electromotive force method. From Fig. I 7 we find that a field current of 84 amperes is required to give 2023 volts total induced electromotive force; and a field current of 42 amperes is required to give a total induced electromotive force of I I 50 volts. To give these two quadrature electromotive forces simultaneously a field current equal to 94 amperes $\left(=\sqrt{84^{2}+42^{2}}\right)$ would be required; and, according to Fig. I I 7 , this field current of 94 amperes would give a total induced electromotive force of 2200 volts, which is the value to which the terminal voltage of the machine would rise at zero load. Therefore the regulation of the machine is $(2200-2000) / 2000=0.10$ or 10 per cent. 
(b) For pozver factor equal to 0.85.- From Fig. I 20 the two components of the total induced electromotive force, parallel to and perpendicular to the current, are 1723.3 volts and 2207 volts respectively. The field currents required to produce total induced voltages of 1723.3 and 2207 are found from Fig. II 7 to be 68 amperes and 94 amperes respectively, and to give these two quadrature electromotive forces simultaneously would require I 6 amperes $\left(=\sqrt{68^{2}+94^{2}}\right)$. The zero-load voltage corresponding to I 6 amperes of field current is found from Fig. I I 7 to be 2400 volts. Therefore the regulation of the alternator is $(2400-2000) / 2000=0.20$ or 20 per cent.

\section{Comparison of the two methods for calculating voltage regula-} tion. - The two methods for calculating voltage regulation are based on assumptions that depart very widely indeed from the conditions which actually exist in an alternator (compare Figs. II $3 a$, I $4 a$ and II $5 a$, with Figs. I $3 b$, I $4 b$ and I $5 b$ ) and a theoretical comparison of the two methods would depend upon a complete analysis of the actual conditions represented in Figs. I I $3 a$, I I $4 a$ and II $5 a$. The only satisfactory way to compare the two methods is to compare the results of calculations with actually observed values of voltage regulation. The electromotive force method generally gives a larger (poorer) regulation than is found by actual test, and the magnetomotive force method generally gives a smaller (better) regulation than is found by actual test. The former method has therefore been called the "pessimistic method" and the latter the "optimistic method." *

65. Armature demagnetizing action. - The discussion of the magnetizing action of the armature current, or currents, of an alternator which is given in Arts. $6_{3}$ and 64 covers the ground as completely as it can be covered without entering into the theory

* See "The Experimental Basis for the Theory of Regulation of Alternators," by B. A. Behrend, Transactions of the American Institute of Electrical Engineers, Vol. 21, pages 497-517, 1903; and "The Regulation of Alternators," by D. B. Rushmore, Transactions of the International Electrical Congress, Vol. I., pages 720-76r, St. Louis, 1904 . 
of non-harmonic electromotive forces, and to do this would lead to impractical mathematical complications. There are, however, two fairly simple aspects of armature magnetizing action which, although they are involved by implication in the discussion of Arts. $6_{3}$ and 64 , should be brought out more distinctly. In the first place the flux under the pole pieces is crowded to one side in the direction of rotation, and in the second place the armature currents oppose the field winding in forcing flux through the armature when the receiving circuit is inductive and they help the field winding in forcing flux through the armature when the receiving circuit has negative reactance like a condenser.

Discussion of demagnetizing action. - Consider the conductors TT, Fig. 121, which constitute the armature winding of a 2-pole single-phase alternator armature. The electromotive force of the machine may be written

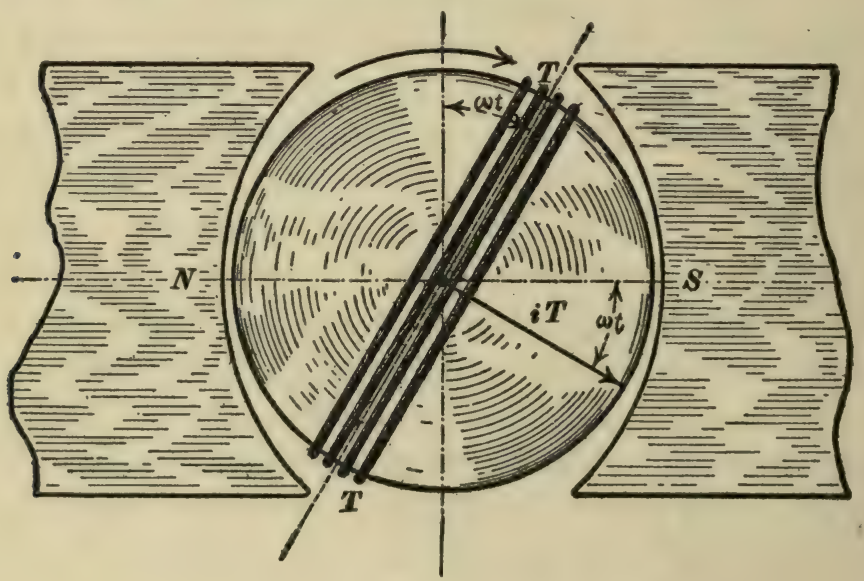

Fig. 121.

$$
e=\mathbf{E} \sin \omega t
$$

where $\omega t$ is the angle shown in Fig. I2I, and the current delivered by the alternator may be expressed thus

$$
i=\mathbf{I} \sin (\omega t-\theta)
$$

where $\theta$ is the angle of lag of the current behind the electromotive force, which angle of lag depends of course upon the character of the receiving circuit.

Let $T$ be the number of turns of wire on the armature (four turns are shown in Fig. 12I), then $i T$ is the ampere-turns due to the winding at a given instant, and this action is in the direction of the arrow $i T$, Fig. I2I. The component of $i T$ in the direction of the line $N S$ is equal to $i T \cos \omega t$, and this is a measure, in ampere- 
turns, of the amount that the armature current is helping the field winding at the given instant. Let this be represented by $m$, so that

$$
m=i T \cos \omega t
$$

or, substituting the value of $i$ from equation (ii) we have

or

$$
m=\mathrm{I} T \cos \omega t \sin (\omega t-\theta)
$$

$$
m=\mathrm{I} T \cos \omega t \sin \omega t \cos \theta-\mathrm{I} T \cos ^{2} \omega t \sin \theta
$$

The average value of $m$ is the average amount in ampere-turns that the field winding is helped by the armature current, and the average value of $m$ is equal to the sum of the average values of the two terms of the right-hand member of equation (iv), but the average value of the first term is zero and the average value of the second term is $-\frac{1}{2} I T \sin \theta$, inasmuch as the average value of $\cos ^{2} \omega t$ is equal to $\frac{1}{2}$. Therefore

$$
\text { average value of } m=-\frac{1}{2} \mathrm{I} T \sin \theta
$$

or, substituting the effective value $I=\mathbf{I} / \sqrt{\mathbf{2}}$, we have

$$
\text { average value of } m=-\frac{1}{\sqrt{2}} I T \sin \theta
$$

The meaning of this equation is as follows : $(a)$ When $\theta$ is zero the armature current neither helps nor opposes the passage of flux, on the average; $(b)$ when $\theta$ is positive, that is when the current lags behind the voltage in phase, the armature current opposes the passage of flux, on the average; and (c) when $\theta$ is negative, that is when the current is ahead of the voltage in phase, the armature current helps the passage of flux, on the average.

The constancy of the magnetizing action of the armature currents in a two-phase armature, when the receiving circuits are balanced, is easily shown. Let $m$, equation (iv), be the instantaneous value of the magnetizing action of phase $A$ of a two-phase armature. The instantaneous value of the magnetizing action $m^{\prime}$ of phase $B$ is obtained by substituting $\omega t \pm 90^{\circ}$ for $\omega t$ in equation (iv) which gives :

$$
m^{\prime}=-I T \sin \omega t \cos \omega t \cos \theta-I T \sin ^{2} \omega t \sin \theta .
$$

Therefore the total magnetizing action of the two phases is

or

$$
m+m^{\prime}=-I T\left(\sin ^{2} \omega t+\cos ^{2} \omega t\right) \sin \theta
$$

which is constant.

$$
m+m^{\prime}=-\mathbf{I} T \sin A
$$

66. Power rating of alternators. - The current delivered by an alternator generates heat in the armature which heat, together with the heat generated by eddy currents and hysteresis, causes the temperature of the armature to rise until it gives off heat as fast as heat is generated in it. Therefore, to increase the current output of an alternator causes an increased rise of temperature of the machine. This heating effect usually determines the current rating of an alternator, and the power rating of an alternator is 
always understood to be the power that it can deliver steadily to a non-inductive receiving circuit (power factor unity) without undue rise of temperature. That is, the power rating of an alternator is understood to be the product of its rated voltage and its rated current. This power rating of an alternator is sometimes specified in terms of volt-amperes or kilovolt-amperes, to distinguish it from the actual power the alternator can deliver, for the actual power depends upon the power-factor of the receiving circuit. Thus an alternator rated at 2000 volts and 100 amperes is called a 200 kilowatt or a 200 kilovolt-ampere alternator. Such an alternator could in fact deliver 200 kilowatts to a noninductive receiving circuit, but with the same rise of temperature it could deliver only 150 kilowatts to a receiving circuit of 0.75 power factor.

The recommendations of the Committee on Standardization, of the American Institute of Electrical Engineers, concerning overroad capacities, are given on page I 7 I of the first volume of this treatise.

67. Frequencies. - The standard frequencies employed in American practice* are 25 cycles per second, 60 cycles per second, and in older installations 125 or 133 cycles per second.

Very low frequencies are not suitable for lighting on account of the tendency of low frequency to produce flickering of the lights. A frequency of about 60 cycles per second seems to be the lower limit for satisfactory lighting.

High frequencies are not well adapted for the operation of induction motors, synchronous motors, or rotary converters, because high frequencies necessitate either great speed or a great number of field poles, and in single-phase commutator motors high frequencies involve excessive sparking and low power factors. For driving motors and rotary converters a frequency of 25 cycles per second is the standard in American practice.

When an alternator is to supply current for lighting and also for driving motors a frequency of 60 cycles per second is generally used in American practice.

* See page 170 of the first volume of this treatise. 


\section{CHAPTER VIII.}

THE SYNCHRONOUS MOTOR. THE OPERATION OF ALTERNATORS IN PARALLEL.

68. The synchronous motor. The operation of alternators in parallel - Fig. 122 shows the essential connections for operating two alternators in parallel so that the combined output of the two alternators may be delivered to a single receiving circuit; and Fig. I 23 shows the essential connections for operating one alter-

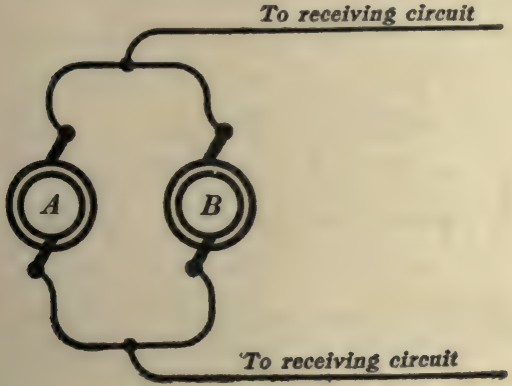

Fig. 122.

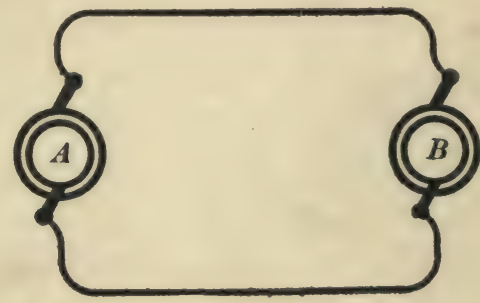

Fig. 123.

nator, say, $A$, as a generator and the other, $B$, as a synchronous motor. In the operation of an alternator as a synchronous motor it is usually at a distance from the alternating current generator which drives it, so that the generator $A$ and the motor $B$, Fig. I 23, require separate sources of direct current for field excitation.

In Fig. I 22 the two alternators $A$ and $B$ form a closed circuit independently of the receiving circuit exactly as in Fig. I23. In fact, the interaction of two alternators which are connected in parallel as generators is in many respects the same as the interaction of two alternators one of which drives the other as a synchronous motor. Therefore the two cases are here treated under one general heading; in fact, the parallel operation of alternators as generators is best treated as a special case of syrichronous motor operation 
The two machines, $A$ and $B$, in Figs. 122 and 123 are in series with each other in regard to the circuit formed by the two machines, and this circuit formed by the two machines is the only circuit at present to be considered. The electromotive force which tends to produce current in this circuit is at each instant equal to the algebraic sum* of the electromotive forces of the respective machines. Therefore, if the machines are in synchronism, that is, if their electromotive forces have the same frequency, the total electromotive force which tends to produce current in the circuit may be represented in the clock diagram as the resultant $E$ of the two lines $A$ and $B$ which represent the electromotive forces

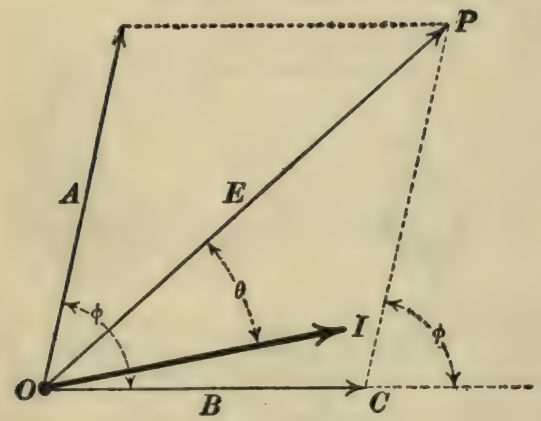

FIg. 124. of the respective machines, as shown in Fig. 124. In this diagram $\phi$ is the phase difference between $A$, and $B$, and this angle remains constant in value so long as the two machines $A$ and $B$ run exactly in synchronism. If, however, one machine runs momentarily faster than the other, the value of $\phi$ will change. It is evident that $\phi$ can have any value whatever, except in so far as it may be kept within certain limits by the action of the two machines on each other. For the present we will consider that the angle $\phi$ can have any value from zero to $360^{\circ}$.

The resultant electromotive force $E$ produces in the circuit of the two machines a current $I$ of which the value is

$$
I=\frac{E}{\sqrt{R^{2}+X^{2}}}
$$

and which lags $\theta$ degrees behind $E$ in phase where $\theta$ is the angle whose tangent is $X / R$, that is,

* The electromotive force of each machine is here considered to be positive when it acts in a chosen direction around the circuit formed by the two machines. 


$$
\tan \theta=\frac{X}{R}
$$

$R$ being the resistance and $X(=\omega L)$ the reactance of the circuit. The power $P^{\prime}$ delivered to the circuit by machine $A$ is

$$
P^{\prime}=A I \cos (A I)
$$

where $(A I)$ is the angle between $A$ and $I$ in Fig. I24, and the power $P^{\prime \prime}$ delivered to the circuit by machine $B$ is

$$
P^{\prime \prime}=B I \cos (B I)
$$

where $(B I)$ is the angle between $B$ and $I$ in Fig. I24.

For certain values of $\phi$ the angle $(A I)$ may be less than $90^{\circ}$ and the angle $(B I)$ greater than $90^{\circ}$, and if this condition occurs, then $P^{\prime}$ will be positive and $P^{\prime \prime}$ will be negative. That is, machine $A$ will give out power and machine $B$ will take in power, of in other words, machine $A$ will act as a generator and machine $B$ will act as a motor.

For certain values of $\phi$ the angle $(A I)$ may be greater than $90^{\circ}$ and the angle $(B I)$ less than $90^{\circ}$, and if this condition occurs, machine $A$ will act as a motor and machine $B$ will act as a generator.

69. Variation of $\mathrm{P}^{\prime}$ and of $\mathrm{P}^{\prime \prime}$ with the phase angle $\phi .-$ Before the physical aspects of either of the above conditions can be discussed, it is necessary to examine carefully into the geometrical relations involved in Fig. I 24 on the assumption that $\phi$ may have any value whatever. It is sufficient for our purpose however to consider the power output (or intake) of one machine, say machine $B$. For this purpose it is convenient to take the line $B$ in Fig. I 24 as a fixed reference axis. Then, as the angle $\phi$ changes, the point $P$ will describe a circle about the point $C$, for the line $C P$ is of constant length, being equal to $A$ [the electromotive forces (effective) of machines $A$ and $B$ are of course supposed to be constant and their phase difference $\phi$, only, is supposed to change]. The triangle $O C P$ in Fig. I 25 is the triangle $O C P$ of Fig. I 24, and the circle in Fig. I 25 is the above mentioned locus of the point $P$. 
Draw the line ef, Fig. I25, through the point $O$ making with $O C$ the angle $\theta$ as shown, then $O Q$, the projection of $O P$ on the line ef, represents the power output $P^{\prime \prime}$ of machine $B$. This is shown as follows: The angle between $B$ and $E$ is equal to $\theta+(B I)$ and it is also equal to $\theta$ plus the angle

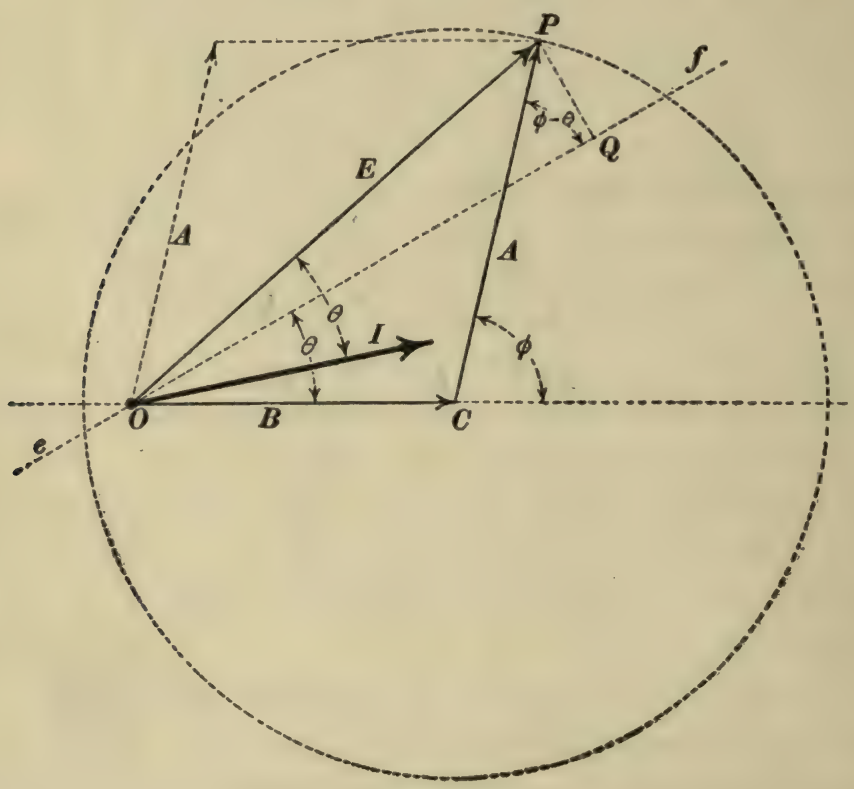

Fig. 125.

$P O f$, so that the angle $P O f$ is the same as the angle $(B I)$. Therefore

$$
\overline{O Q}=\overline{O P} \cos (B I)=E \cos (B I)=\sqrt{R^{2}+X^{2}} \cdot I \cos (B I)
$$

or

$$
I \cos (B I)=\frac{\overline{O Q}}{\sqrt{R^{2}+X^{2}}}
$$

whence, substituting this value of $I \cos (B I)$ in equation (iv), we have

$$
P^{\prime \prime}=\frac{B \cdot \overline{O Q}}{\sqrt{R^{2}+X^{2}}}
$$


That is, the power output of machine $B$ is proportional to $\overline{O Q}$ and the proportionality factor is the constant $B / \sqrt{R^{2}+} X^{2}$.

Equation (v) may be put into a more convenient form as follows: The projection of $A$, Fig. I25, on the line of is equal to $A \cos (\phi-\theta)$; the projection of $B$ on ef is $B \cos \theta$; and $\overline{O Q}$ is the sum of these two projections. Therefore, substituting $A \cos (\phi-\theta)+B \cos \theta$ for $\overline{O Q}$ in equation (v), we have

$$
P^{\prime \prime}=\frac{A B}{\sqrt{R^{2}+X^{2}}} \cdot \cos (\phi-\theta)+\frac{B^{2}}{\sqrt{R^{2}+X^{2}}} \cdot \cos \theta
$$

Figure $\mathrm{I} 26$ is a construction essentially similar to Fig. I 25 , but with the electromotive force $A$ taken as the fixed reference axis;

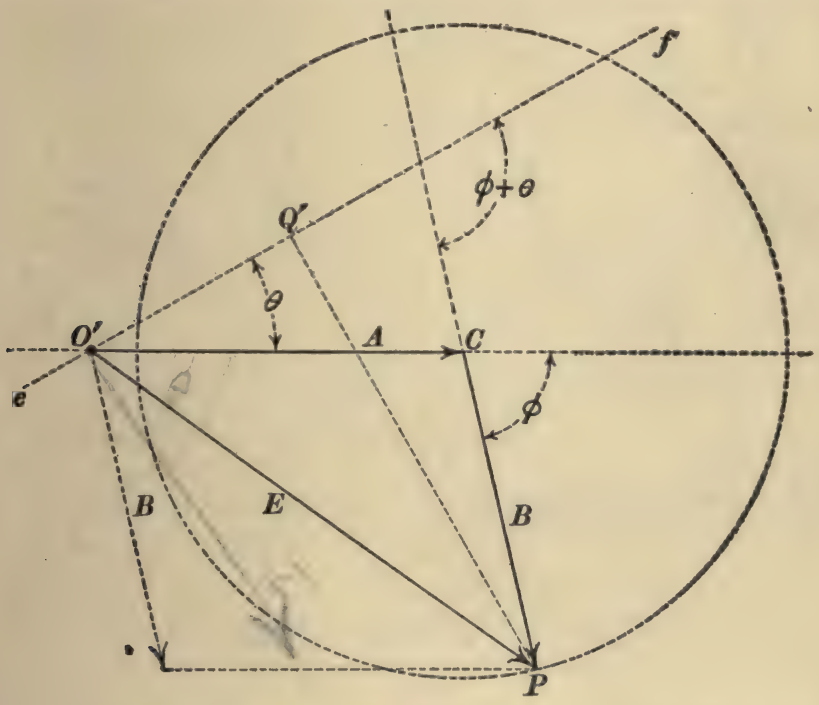

Fig. 126.

both figures, 125 and 126 , being constructed for the same value of $\phi$. From Fig. I 26 it may be shown that

$$
F^{\prime \prime}=\frac{A \cdot \overline{O^{\prime} Q^{\prime}}}{\sqrt{R^{2}+X^{2}}}
$$

from which an expression for $P^{\prime}$ may be derived corresponding to equation (1 5 ) namely : 


$$
P^{\prime}=\frac{A B}{\sqrt{R^{2}+X^{2}}} \cdot \cos (\phi+\theta)+\frac{A^{2}}{\sqrt{K^{2}+X^{2}}} \cdot \cos \theta
$$

These equations, (I 5) and (16), are the fundamental equations of the synchronous motor. Of course the algebraic sum of $P^{\prime}$ and $P^{\prime \prime}$ is equal to $R I^{2}$.

A very clear idea of the variations of $P^{\prime \prime}$ with $\phi$ is given by the variation of $\overline{O Q}$, Fig. 125, and a clear idea of the variation of $P^{\prime}$ with $\phi$ is given by the variation of $\overline{O^{\prime} Q^{\prime}}$, Fig. 126. This variation of $P^{\prime}$ and and $P^{\prime \prime}$ with $\phi$ is extremely important in the following physical discussion, and a clearer insight into this variation may be obtained with the help of Figs. I 27 and I 28 ,

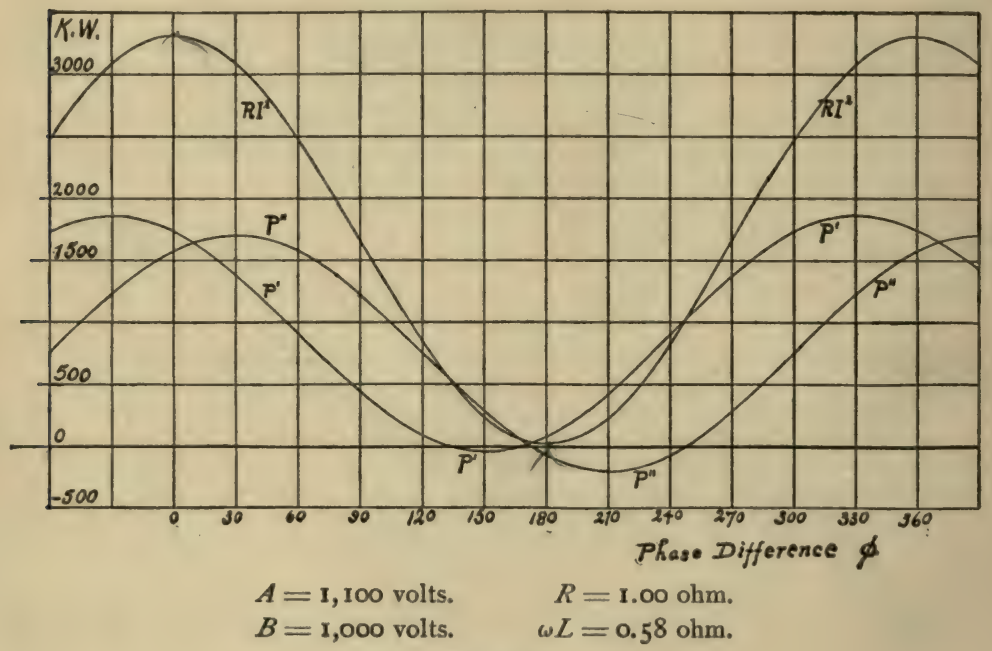

Fig. 127.

in which the values of $\phi$ are represented as abscissas, and the values of $P^{\prime}, P^{\prime \prime}$, and $R I^{2}\left(=P^{\prime}+P^{\prime \prime}\right)$ as ordinates. Figure I 28 is an enlargement of the middle portion of Fig. I 27 , and the ordinates of the curve $\eta$ represent the values of the ratio $P^{\prime \prime} / P^{\prime}$, disregarding sign. This ratio, when $P^{\prime \prime}$ is an intake of power by machine $B\left(P^{\prime \prime}\right.$ negative) and $P^{\prime}$ an output of power by machine $A$ ( $P^{\prime}$ positive) is the efficiency, ignoring all losses except the $R I^{2}$ loss in the circuit. 
The variation of $P^{\prime \prime}$ with $\phi$ may be shown in greater detail as follows: Draw a line $s s^{\prime}$ through $O$, Fig. I25, at right angles to ef, as shown in Fig. I 29. Consider that if machine $A$ runs momentarily faster than $B$ or, in other words, if machine $B$ runs momentarily slower than $A$, then the angle $\phi$ increases, and the point $P$, Fig. I 29, moves around the circle in the direction of the curved arrow. So long as $\overline{O Q}$ is towards $f$ the

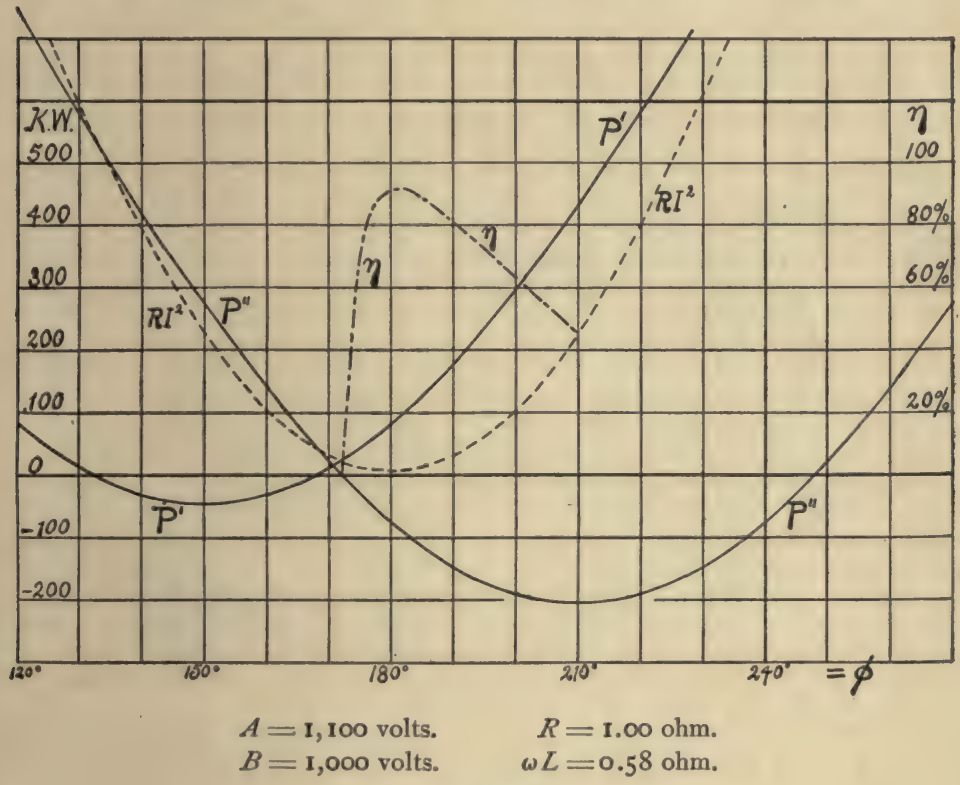

Fig. 128.

value of $P^{\prime \prime}$ is positive and machine $B$ gives out power; when the point $P$, Fig. I29, is at $s$ or $s^{\prime}$, however, the value of $\overline{O Q}$ is zero and machine $B$ neither gives out nor takes in power; and when the point $P$ is anywhere in the region $s M s^{\prime}$ the value of $\overline{O Q}$ is negative, and machine $B$ takes in power. This region in which $\overline{O Q}$, Fig. I 29, is negative is the region in which $P^{\prime \prime}$ is negative in Figs. 127 and 128.

It is to be particularly noted that when the value of $\phi$ is nearly zero, that is, when the electromotive forces $A$ and $B$ are nearly in phase with each other, the power output of both machines is excessively large. Thus, in the neighborhood of 
of $\phi=0^{\circ}$ or $360^{\circ}$ in Fig. I 27, $P^{\prime}$ and $P^{\prime \prime}$ are both positive and each is more than 1500 kilowatts, although the machines to which Figs. 127 and 128 refer, are each of about 100 kilowatts rating. The excessive values of $P^{\prime}$ and $P^{\prime \prime}$ in the neighbor-

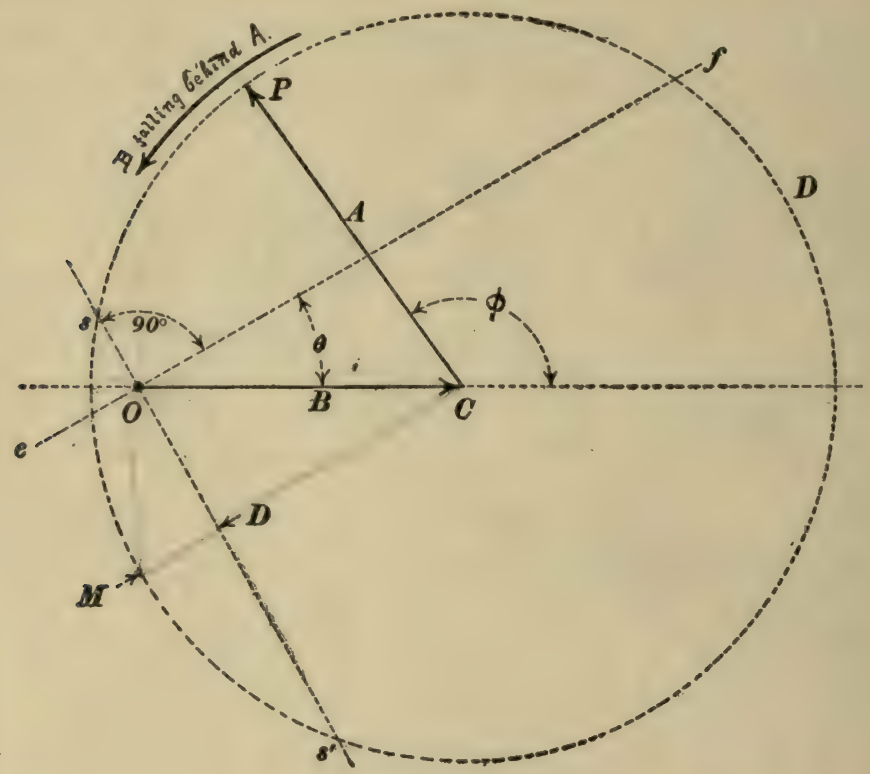

Fig. 129.

hood of $\phi=0^{\circ}$ or $360^{\circ}$ is due to the fact that when $\phi$ is zero each machine forms a short-circuit for the other, whereas in the neighborhood of $\phi=180^{\circ}$ the two electromotive forces $A$ and $B$ oppose each other and the current is small.

70. Necessity of exact synchronism for the operation of an alternator as a motor. - Suppose that machine $B$ runs continuously a little slower or a little faster than machine $A$, then the value of the angle $\phi$ will change continuously, and the point $P$, Figs. 125 and 126 , will move steadily around the circle in one direction or the other. Under these conditions the power outputs $P^{\prime}$ and $P^{\prime \prime}$ of the two machines pass repeatedly through all of the values shown in Fig. 127, and, on the average, 
both $P^{\prime}$ and $P^{\prime \prime}$ will be positive, that is, both machines will on the average give out power.

If, however, machines $A$ and $B$ are in exact synchronism the value of $\phi$ remains constant, and either $P^{\prime}$ or $P^{\prime \prime}$ may be negative, that is, either machine $A$ or machine $B$ may take in power steadily (motor action), according to the value of the angle $\phi$.

Therefore, if an alternator (motor) is to take power steadily from another alternator (generator) the two machines must run in exact synchronism, and it is for this reason that an alternator when used as a motor is called a synchronous motor.

71. Stability of running of a synchronous motor. - The necessity of exact synchronism of running of an alternator which is to be used as a motor may seem to be an impracticable condition; but if an alternator is once started to operate as a motor, exact synchronism is automatically maintained, unless the load on the motor becomes excessive. This stability of operation may be shown by the help of Fig. I 29, remembering that any kind of a motor whatever, steam or electric, will show stability of running if a momentary decrease in its speed causes it to take more power from boiler or electric generator, whereas the running will be unstable if a momentary decrease in its speed causes it to take less power from boiler, or electric generator.

Suppose that machine $A$ is driven at constant speed by a governed engine or water wheel, and suppose that machine $B$ is in synchronism with $A$ with the point $P$, Fig. 129, at any given position on the circle. If the point $P$ is anywhere between $s$ and $M$ a momentary decrease of speed of $B$ will cause the point $P$ to move towards $M$ ( $B$ falling behind $A$ in phase), and the power intake of machine $B$ will increase, and thus counteract the momentary decrease of speed due for example to a sudden increase of motor load on machine $B$. If, on the other hand, machine $B$ runs momentarily faster than $A$, then the point $P$, Fig. I 29, will move away from $M$ and towards $s$ ( $B$ getting ahead of $A$ in phase), and the power intake of machine $B$ 
will decrease and thus counteract the momentary increase of speed due for example to a sudden decrease of motor load on machine $B$. That is to say, the region between $s$ and $M$ is the region of stable running of machine $B$ as a synchronous motor.

The region between $M$ and $s^{\prime}$, Fig. I 29, is a region of unstable running, for, when the point $P$ is in this region, a momentary decrease of speed of machine $B$, due for example to a sudden increase of its motor load, causes machine $B$ to take in a decreased amount of power so that the decrease of speed will not only persist but it will become more and more pronounced until the point $P$, Fig. I 29 is carried beyond $s^{\prime}$; then machine $B$ not only has a belt load as a motor but it actually gives out power as a generator, so that its speed will fall very rapidly indeed and carry the point $P$ round and round the circle until the momentum of the rotating armature of machine $B$ is exhausted and the machine comes to a dead stop.

If machine $B$ is operating steadily with the point $P$ in the stable region $s M$, then any increase of load which carries the point $P$ beyond $M$ will cause the machine to come to a dead stop as above described.

From Fig. I 29 it is evident that there are two positions of the point $P$ for a given value of motor intake, one position is in the region $s M$ of stable running and the other position is in the region $M s^{\prime}$ of unstable running. The position for stable running has the smaller value of $E$ and therefore the smaller value of $I$.

If machine $B$ is operating steadily with the point $P$ in the stable region $s M$, then if the motor load on $B$ were reduced to zero, the speed of $B$ would be momentarily increased and the point $P$ would move to $s$. When $P$ is at $s, \overline{O s}$ is the resultant electromotive force $E$, the current vector $I$, which is $\theta^{\circ}$ behind $E$ in phase, is vertically upwards, the angle $(B I)$ is $90^{\circ}$, and $\mathrm{P}^{\prime \prime}$ is zero according to equation (iv) Art. 68.

72. Hunting action of the synchronous motor. - When the load on a synchronous motor is suddenly increased, the motor speed 
decreases momentarily and the motor falls behind the generator in phase. By the time the motor has fallen behind the generator sufficiently to take in power enough to enable it to carry its load, its speed has become a minimum because up to this instant it has been taking less than enough power to carry its load. Therefore the motor continues to fall behind the generator, taking in more than enough power to carry its load, and this excess of power quickly brings the motor up to the generator speed. At this instant the motor is farthest behind the generator in phase because up to this instant its speed has been less than the speed of the generator. Then, the motor as it continues to take in an excess of power begins to exceed the generator in speed and gains on the generator until it takes in less than enough power to carry its load, when its speed again falls below that of generator and so on. This oscillation of a synchronous motor backwards and forwards through a certain mean position is called hunting and it is similar to the hunting of a badly governed steam engine.

The hunting oscillations of a synchronous motor are always accompanied by oscillations of the driving generator, the motor and generator oscillations being of the same rhythm and related to each other like the oscillations of the two prongs of a tuning fork.

The hunting of a synchronous motor (or rotary converter) is often very troublesome, making it impossible to operate the motor even with a fairly light load, on account of the carrying of the point $P$, Fig. I 29, so far into the region $M s^{\prime}$ of unstable running that it cannot return (motor intake being less than motor load), but goes on beyond $s^{\prime}$, and the motor comes to a dead stop as described in Art. $7 \mathrm{I}$.

In general the hunting oscillations of a synchronous motor when once started tend to go on increasing in amplitude, and the prevention of troublesome hunting depends upon some action which tends to decrease the amplitude of the oscillations. Two such actions are available, namely, $(a)$ The use of a fly-wheel loosely attached to the synchronous motor shaft so as to slip as 
the speed of the synchronous motor increases and decreases. This device sometimes takes the form of a hollow fly-wheel rigidly fixed to the motor shaft and filled with liquid, and (b) The use of damping frames which consist of copper bars set into the pole faces of the synchronous motor parallel to the armature shaft, the ends of these bars being short-circuited by end-bars of copper.

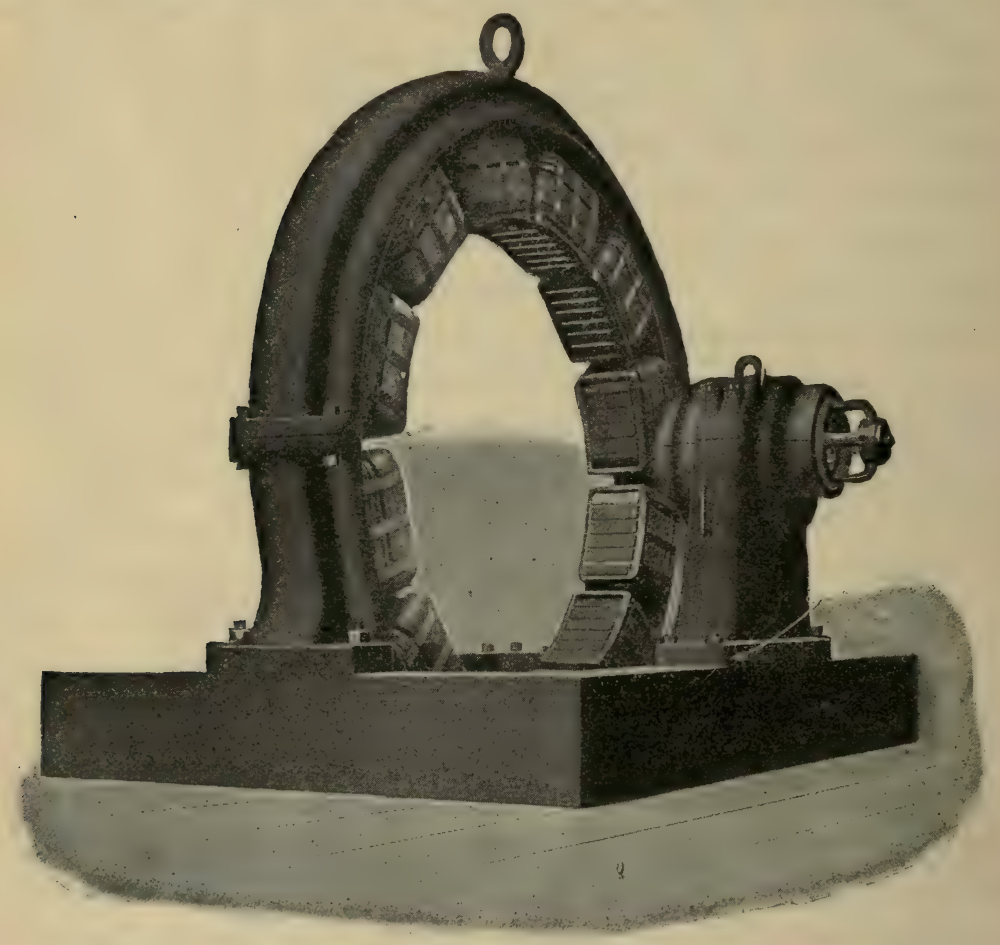

Fig. 130.

Figure I30 shows the field magnet of a synchronous motor, or rotary converter, the pole faces of which are provided with copper damping frames or grids. This arrangement is seldom used on belted synchronous motors, it is however very frequently used on rotary converters and upon synchronous motors which are coupled to direct-current generators. The action of these damping frames is as follows: As the speed of a hunting syn- 
chronous motor increases and decreases, the torque exerted on the armature by the field magnet increases and decreases, this increase and decrease of torque is accompanied by a shifting of the magnetic flux backwards and forwards over the pole faces, and this shifting flux induces current in the short-circuited bars of the damping frame, which current opposes the shifting of the flux and thereby dampens the hunting oscillations.

A synchronous motor with a belt load is much less prone to hunting oscillations than a rotary converter or a synchronous motor directly connected to a generator, for the reason that the slipping of the belt dampens the hunting oscillations like a loose fly-wheel.

When the generator which supplies current to a synchronous motor is driven by an engine the slight pulsations of speed of the engine tend to set up hunting oscillations, especially if the frequency of the hunting oscillations is the same as the frequency of pulsations of the engine speed. In such a case troublesome hunting may be avoided by increasing the moment of inertia of the armature of the synchronous motor by attaching a flywheel to it. The effect of moment of inertia upon the hunting frequency is shown in the following discussion.

Theory of the hunting of the synchronous motor. - When a synchronous motor is running steadily it takes in power steadily from the mains and gives out power steadily on its belt (or from its direct-current commutator in case of the rotary converter). The pulsations of power intake due to the alternations of electromotive force and current are extremely rapid in comparison with hunting oscillations and need not be considered, indeed these pulsations do not exist in case of polyphase machines.

The mean position, at a given instant, of the armature of a synchronous motor which is hunting is the position it would have at that instant if it were turning at a constant angular velocity. When the motor hunts its armature oscillates forwards and backwards through its mean position.

When the armature is in its mean position the power intake of the motor and its belt load (including friction losses and the like) are equal, and no unbalanced torque acts on the armature.

When the armature gets ahead of its mean position its intake is lessened, the belt bad of the machine, which is assumed to be constant, exceeds the intake, and an unbalanced retarding torque acts on the armature.

When the armature falls behind its mean position its intake exceeds its belt load, and an unbalanced accelerating torque acts on the armature. 
Let $\psi$ be the angle between the mean position of the armature and its actual position at a given instant, and let $T$ be the unbalanced torque acting on the armature at this instant. Our problem is to find the relation between $\psi$ and $T$. This relation, when $\psi$ is small, is

$$
T=-b \psi
$$

where $b$ is a constant. Therefore, from the laws of harmonic motion, we have

$$
\frac{4 \pi^{2} K}{t^{2}}-b
$$

in which $K$ is the moment of inertia of the rotating part of the machine, and $t$ is the period of the hunting oscillations.

Derivation of equation (i). - We shall derive equation (i) for a special case, namely, for the case in which the moments of inertia of the armatures (or rotating parts) of generator and synchronous motor are equal, and for the particular phase angle $\phi=180^{\circ}$, see Figs. 125 and 128 . In this case, namely, when $\phi=180^{\circ}$, a small change of $P^{\prime}$ is accompanied by an equal and opposite change of $P^{\prime \prime}$, so that equal unbalanced torques act at each instant on the armatures of machines $A$ and $B$, and their moments of inertia being equal the ranges of the oscillations of the armatures of both machines are equal. That is, the armature of machine $A$ is as much ahead of its mean position at each instant as the armature of machine $B$ is behind its mean position at the same instant and vice versa. Therefore the change of the phase angle $\phi$ is equal to $2 p \psi, 2 \psi$ being the angular displacement of one armature referred to the other and $p$ being the number of pairs of field magnet poles in each machine.

Differentiating equation (15) with respect to $\phi$, writing $2 p \psi$ for $d \phi$, and after the differentiation is performed, putting $\phi=180^{\circ}$, we have

$$
d P^{\prime \prime}=-\frac{2 p \psi A B}{\sqrt{R^{2}+X^{2}}} \cdot \sin \theta
$$

Now $d P^{\prime \prime}$ equals $2 \pi n T$ where $n$ is the speed of the machine and $T$ is the unbalanced torque. Therefore

$$
T=-\frac{p \psi A B}{\pi n \sqrt{R^{2}+X^{2}}} \cdot \sin \theta
$$

The value of $b$, equation (i), is therefore

$$
b=\frac{p A B}{\pi n} \frac{X^{2}}{R^{2}+X^{2}} \cdot \sin \theta
$$

Substituting this value of $b$ in equation (ii) and solving for $t^{2}$, we have

or, since

$$
t^{2}=\frac{4 \pi^{3} n K \sqrt{R^{2}+X^{2}}}{p A B \sin \theta}
$$

we have

$$
\sin \theta=\frac{\omega L}{\sqrt{R^{2}+X^{2}}} \text { and } \omega=2 \pi n
$$

$$
t^{2}=\frac{2 \pi^{2} K\left(R^{2}+X^{2}\right)}{p A B L}
$$


73. Starting of the synchronous motor. - Let the machine $A$ which is to operate as a generator be running with its field fully excited; then to put the machine $B$ into operation as a synchronous motor it has to be driven by some independent source of power, its field has to be properly excited, and its speed has to be carefully adjusted until (a) it is exactly in synchronism with machine $A$, and $(b)$ its electromotive force is nearly opposite in phase to the electromotive force of $A$; and when these two conditions are realized the circuit of the two machines $A$ and $B$ may be closed, and the independent source of power which was used to start $B$ may be disconnected.

In bringing about the two conditions $(a)$ and $(b)$ previous to the closing of the circuit of the two machines, it is necessary to use some sort of a device for indicating when the two machines are in synchronism and for indicating their phase relation. Such a device is called a synchronizing device or a synchronizer.

The simplest synchronizer is an ordinary incandescent lamp connected in the circuit of the two machines as shown in Fig. I 3 I.

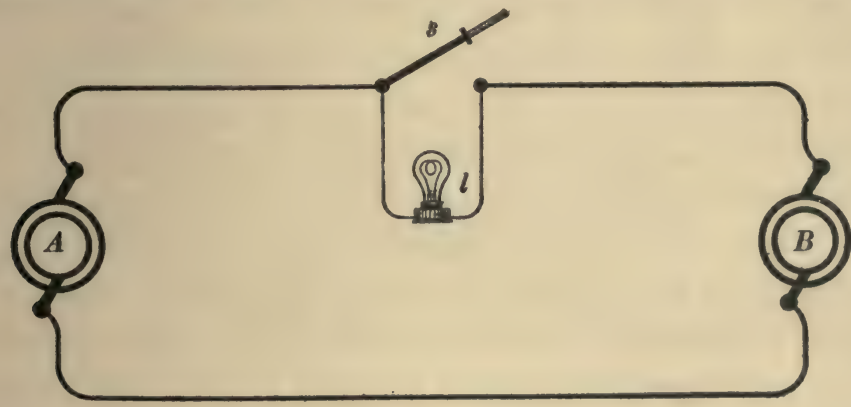

Fig. 131.

This lamp pulsates in brightness as machine $B$ is speeded up, and the pulsations of brightness become slower and slower as the frequency of $B$ approaches the frequency of $A$. When the lamp is at its maximum of brightness the phase difference $\phi$ of $A$ and $B$, see Fig. I25, is zero, and when the lamp is at its minimum of brightness the phase difference of $A$ and $B$ is $180^{\circ}$. When the pulsations of brightness become very slow the 
machines $A$ and $B$ are practically in synchronism [condition (a)] and the switch s, Fig. I $3 \mathrm{I}$, is closed when the lamp is at its minimum of brightness [condition $(b)$ ].

In practice the voltages of machines $A$ and $B$ are much too large to permit of the use of a lamp as shown in Fig. I3 I, and two step-down transformers $T$ and $T^{\prime}$ are used as shown in Fig. 132, the lamp $l$ being connected in circuit with the two

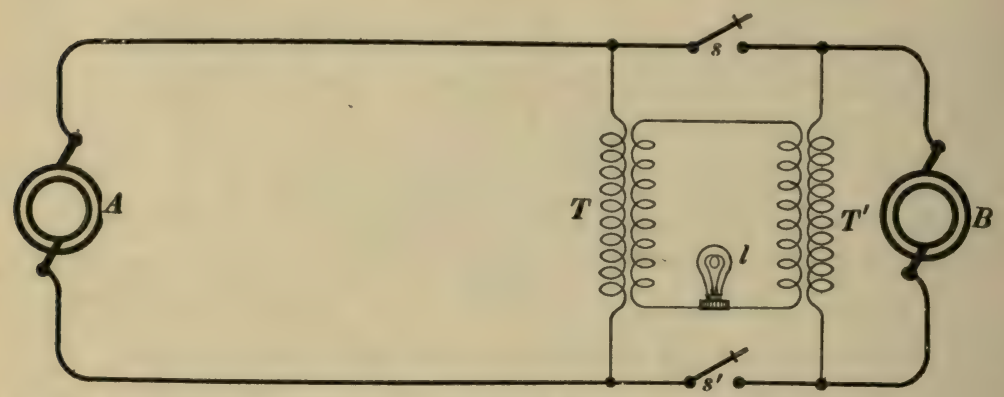

Fig. 132 .

secondary coils of the two transformers. With this arrangement the connections of one of the transformers may be reversed so that the lamp may be at its maximum brightness when the electromotive forces of machines $A$ and $B$ are opposed, that is when condition $(b)$ is right for closing the double-pole switch $s s^{\prime}$.

The dial synchronizer is a device in which a pointer is caused to rotate at a speed which is equal to the difference of the frequencies of machines $A$ and $B$, Fig. 132. The dial synchronizer is now extensively used and one form of this synchronizer is described in Chapter XVI. The dial indicates approximate synchronism (condition $a$ ) by slowness of speed of the pointer, and it indicates the proper phase relation (condition $b$ ) by the position of the pointer on the dial. The chief advantage of the dial synchronizer is that it shows whether the speed of machine $B$ is too great or too small, whereas the sychronizing lamp behaves in exactly the same way whether the speed of machine $B$ be too great or too small. 
74. The polyphase synchronous motor. - The foregoing articles (and also the remainder of this chapter) refer explicitly to the single-phase synchronous motor, that is to a single-phase alternator taking power as a motor from a single-phase generator. The entire discussion applies equally well, however, to the polyphase synchronous motor, that is, to a polyphase alternator taking power as a motor from a polyphase generator. In such a case each armature winding of the motor takes current from one armature winding of the generator and the total power intake is $n P^{\prime \prime}$, where $P^{\prime \prime}$ is the intake of each armature winding or phase and $n$ is the number of phases. To apply any portion of the discussion given in this chapter to a polyphase synchronous motor it is only necessary to remember that the quantities $A, B, R$, $X, P^{\prime}, P^{\prime \prime}, E$, and $I$ refer to one phase only of the system.

The single-phase synchronous motor will run in either direction, but the polyphase synchronous motor with given connections to the polyphase supply mains will run in a certain direction only.

75. The rotary converter. - The rotary converter, or, as it is frequently called the synchronous converter, is described in the next chapter. This machine, as ordinarily used, is at once a synchronous alternating-current motor and a direct-current generator, it is usually polyphase, and it exhibits all of the features of the synchronous motor as to stability of running, as to hunting, and as to starting. The next chapter is devoted chiefly to those aspects of the rotary converter in which it differs from a synchronous motor developing mechanical power, such, for example, as the relation between its voltages, its armature reaction, and its armature heating.

76. The over-excited synchronous motor. - A synchronous motor of which the electromotive force (effective) is greater than the electromotive force of the generator or supply mains from which it takes current is called an over-excited synchronous motor. In all of the foregoing discussion the machine $B$ which acts as a synchronous motor is supposed to have a smaller voltage than the machine $A$ which acts as the generator. It is, evident, how- 
ever, from Figs. 127 and 128 that the higher voltage machine can operate as a motor and take power from the lower voltage machine acting as a generator, and two things concerning the motor action of the higher voltage machine are at once apparent from Figs. 127 and 128 , namely, (a) The larger voltage machine cannot take in as a motor as much power as the lower voltage machine, and $(b)$ the $R I^{2}$ loss in the circuit is very large when the higher voltage machine acts as a motor. It is therefore undesirable to operate $\mathrm{as}^{\cdot}$ a synchronous motor an alternator which has a voltage higher than the generator or supply mains from which it takes current, except when the above mentioned disadvantages are off-set by an advantage depending on the following peculiarity.

An over-excited synchronous motor takes from its source of supply a current which is ahead of the supply voltage in phase, that is to say, an over-excited synchronous motor acts like a condenser; and inasmuch as the reactance $(\mathrm{I} / \omega C)$ of a condenser is considered as a negative reactance, so also an over-excited synchronous motor is equivalent to a negative reactance. The possible use of a condenser (or of an over-excited synchronous motor) for supplying the wattless current taken by an inductive receiving circuit is discussed in Art. 40, and in fact the over-excited synchronous motor is sometimes used for this purpose. In this connection it is important not to confuse the inductive reactance $\omega L$ of the synchronous motor armature, which is what is represented by the letter $X^{*}$ in this chapter, with what is called the negative reactance of the over-excited machine in operation. The negative reactance of a condenser circuit is equal to the wattless component of the impressed voltage divided by

* The letter $X$ in this chapter represents the value of $\omega L$, where $L$ is the entire inductance of the circuit including machines $A$ and $B$. If a synchronous motor is operated from supply mains and if $A$ represents the voltage across the supply mains then there is no need to consider anything back of the point to which $A$ refers. $\mathrm{Al}^{\prime}$ of the discussion of this chapter applies to this case if $R$ is taken as the resistance of the synchronous motor and its connecting wires, and if $X$ is taken as $\omega$ times the inductance of the synchronous motor armature and its connecting wires. 
the current, and the negative reactance of an over-excited synchronous motor in operation is equal to the wattless component of the supply voltage divided by the current flowing through the machine. Thus, Fig. I 33 is a clock diagram exactly similar to Fig. I 29

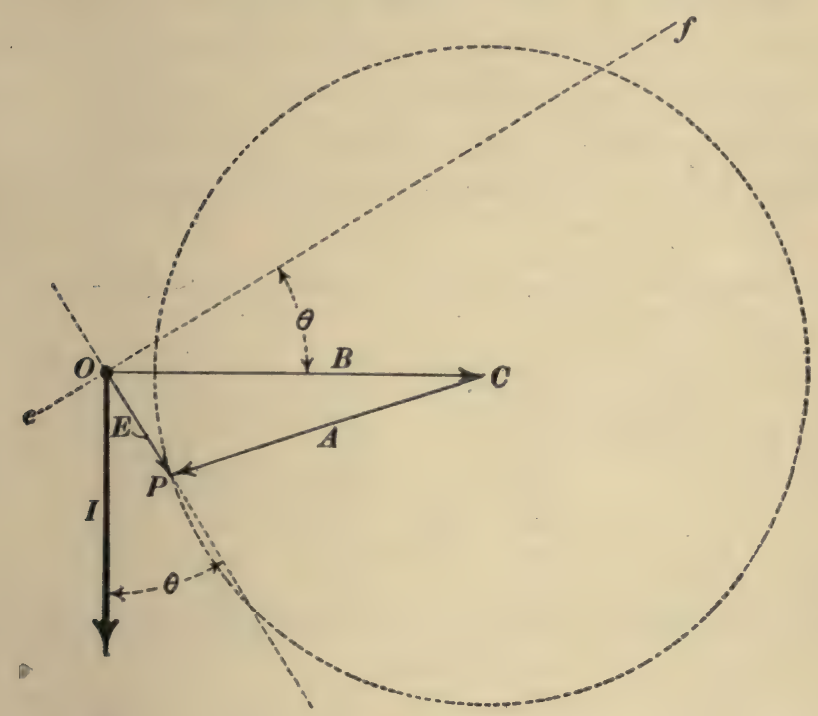

Fig. 133.

except that the motor voltage $B$ is greater than the supply voltage $A$, and the point $P$ is shown in the position corresponding to zero motor load. The resultant voltage $E$ and the current $I$ are shown, and it may be seen that $I$ is far ahead of the supply voltage $A$ in phase. The negative reactance of the machine is equal to the component of $A$ at right angles to $I$ divided by the value of $I$.

77. The operation of alternating-current generators in parallel. Consider an alternator $A$, Fig. I34, driven by an engine and delivering current to a receiving circuit, and consider what must be done to start up another alternator $B$ and connect it in parallel with $A$ so that the receiving circuit may be supplied from both generators. The procedure is exactly the same as would 
be followed in getting machine $B$ into operation as a synchronous motor. Machine $A$ being in operation, machine $B$ is started up by starting its driving engine, its field is excited, and its speed is adjusted until the synchronizing lamp shows (I) that $B$ is in synchronism with $A$ and (2) that the electromotive force of $B$ is opposite (opposite, that is, with reference to the circuit formed by the two machines) to the electromotive force of $A$, and then the double-pole switch $s s^{\prime}$ is closed. If machine $B$ is to be operated as a synchronous motor its voltage should be somewhat

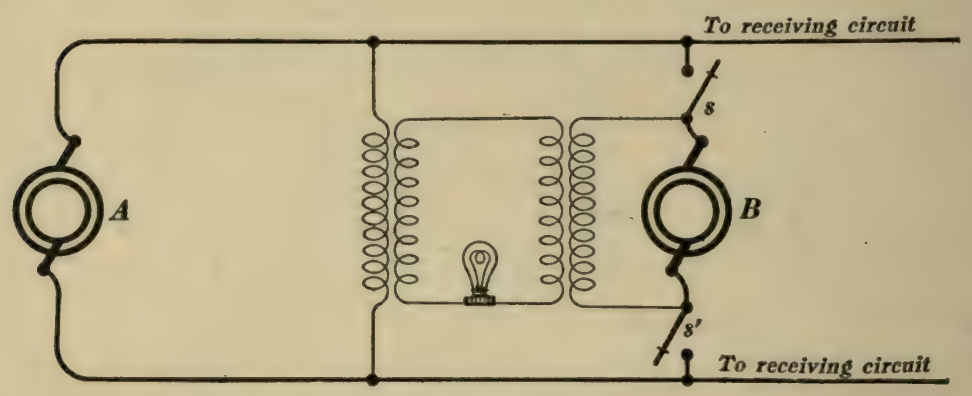

Fig. 134.

less than the voltage of $A$, but if $B$ is to be operated as a generator in parallel with $A$ its voltage should be nearly the same as the voltage of $A$.

A number of alternating-current generators operating in parallel must run at absolutely the same speed, or rather, at absolutely the same frequency, and the possibility of operating a number of alternating-current generators in parallel depends upon the fact that when one generator runs momentarily slower than the others its consequent change of phase relative to the other machines causes its share of the station load to be greatly reduced thus counteracting its tendency to run at a reduced speed.

The necessity of absolute equality of speed of a number of alternating-current generators operating in parallel makes it impossible for the governors of the several engines to operate independently of each other, inasmuch as the independent opera- 
tion of the governors requires the speed of each engine to vary with its load.

Sharing of load between two engine-driven alternating-current generators operating in parallel. (a) When one engine only is governed, the other engine being set at a fixed cut-off. - In this case the power output of the alternator which is driven by the engine with the fixed cut-off is constant, and the variations of the power output of the station are met by the governed engine. If the station output falls below the constant output of the alternator which is driven by the fixed cut-off engine, the other alternator takes in power as a synchronous motor, it may even take in enough power to drive its engine; and if the station output falls too low the fixed cutoff engine may cause the entire system to race. This arrangement is seldom used in practice.

(b) When both engines are governed the distribution of load between them is approximately as follows: Let $a$ be the zero-load-speed of engine $A$, and let $b$ be the zero-load-speed of engine $B$. Let $s$ be the common speed of both engines when they are driving the two alternators. Let $P^{\prime}$ be the power delivered by engine $A$ to its alternator, and $P^{\prime \prime}$ the power delivered by engine $B$ to its alternator. Then, approximately:

and

$$
P^{\prime}=m(a-s)
$$

$$
p^{\prime \prime}=n(b-s)
$$

These equations are based on the assumption that the speed of a governed engine falls off in proportion to its output. The quantity $m$ is obtained by dividing the fullload output of engine $A$ by its full-load drop in speed. The quantity $n$ is found by dividing the full-load output of engine $B$ by its full-load drop in speed.

The total station output determines the combined output $P^{\prime}+P^{\prime \prime}$ of the two engines, and equations (i) and (ii) determine $P^{\prime}, P^{\prime \prime}$ and $s$.

The engines share the load equally only when their zero-load speeds are equal and when their full-load drop in speed is the same.

78. The efficiency of transmission of power by means of an alternating-current generator and a synchronous motor. - The ratio $P^{\prime \prime} \mid P^{\prime}$ of the motor intake to the generator output is called the efficiency of transmission. This is not the net efficiency of the system inasmuch as power consumed in field excitation of the two machines and power lost by friction and by eddy currents and hysteresis are not considered. The conditions necessary for maximum efficiency of transmission depend upon which of the quantities $A, B, X, R$ and $P^{\prime \prime}$ are open to choice or capable of adjustment. The quantities $X$ and $R$ are ordinarily fixed in value, while $A$ and $B$ may be changed more or less by varying the field excitation of the respective machines, and $P^{\prime \prime}$ may be changed by varying the load on the motor.

1. When the electromotive force $A$ of the generating alternator is adjustable, maximum efficiency is obtained when the current (and also $R I^{2}$ ) is a minimum; values of $B, X, R$ and $P^{\prime \prime}$ being given. This minimum current is obtained when $A$ is adjusted until $B$ and $I$ are opposite to each other in phase.

Proof. - The motor intake is $P^{\prime \prime}=B I \cos (B I)$, according to equation (iv), Art. 69. Therefore, since $P^{\prime \prime}$ and $B$ are given, the minimum value of $I$ corresponds 
to maximum value of $\cos (B I)$. But the maximum (negative) value of $\cos (B I)$ is - I, and the corresponding value of the angle $(B I)$ is $180^{\circ}$.

To calculate the value of $A$ which will bring $B$ and $I$ opposite to each other in phase, for the given values of $B, X, R$ and $P^{\prime \prime}$, consider Fig. 135 , in which $l$

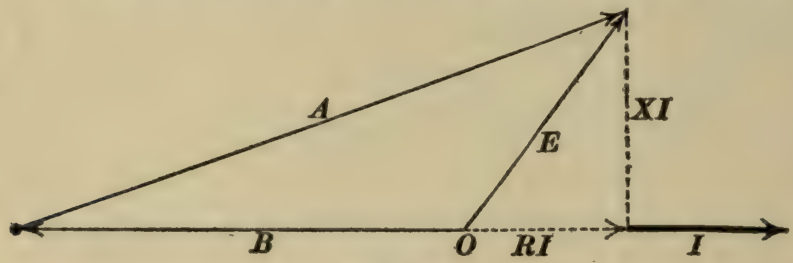

Fig. 135.

represents the current, and $E$ the resultant electromotive force of machines $A$ and $B$. From this figure we have

$$
A^{2}=(B+R I)^{2}+X^{2} I^{2}
$$

from which $A$ may be calculated when $I$ is known. The value of $I$ may be determined from the equation $P^{\prime \prime}=B I \cos (B I)$.

2. When the electromotive force $B$ of the synchronous motor is adjustable, maximum efficiency is obtained when the current (and also $R I^{2}$ ) is a minimum; values of $A, X, R$ and $P^{\prime \prime}$ being given. This minimum current is obtained when $B$ is adjusted until $A$ and $I$ are in phase with each other. The proof of this proposition is given below.

To calculate the value of $B$ which will bring $A$ and $I$ into phase with each other for the given values of $A, X, R$ and $P^{\prime \prime}$, consider Fig. 136, in which $A$ rep-

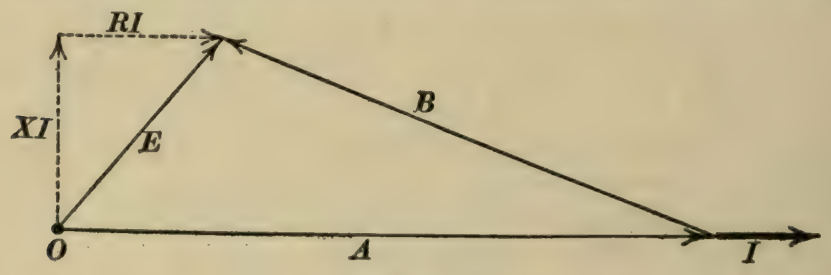

Fig. 136.

resents the value of the electromotive force of machine $A$, and $E$ represents the resultant electromotive force of machines $A$ and $B$. From this figure we have

$$
(A-R I)^{2}+X^{2} I^{2}=B^{2}
$$

from which $B$ may be calculated when $I$ is known. The value of $I \mathrm{~m}_{\text {u. be cal- }}$ culated from the relation

$$
A I \cos (A I)=P^{\prime \prime}+R I^{2}
$$

Proof that $I$ is a minimum when $B$ is adjusted until $A$ and $I$ are in phase with each other; $A, X, R$ and $P^{\prime \prime}$ being given. - Plot the curve of which the ordinates represent values of $(X I)$ and the abscissas represent values of $[B \cos (B I)]$. 
This curve is a hyperbola inasmuch as $B \cos (B I) \times I=P^{\prime \prime}$, or $B \cos (B I)$ $X X I=X P^{\prime \prime}=a$ constant. This curve is plotted in Fig. 137 with the values of $B \cos$ $(B I)$ laid off to the left inasmuch as $P^{\prime \prime}$, being an intake, is negative. The $x$-axis of reference $O I$, represents the current in the circuit; and the line $O E$, making with

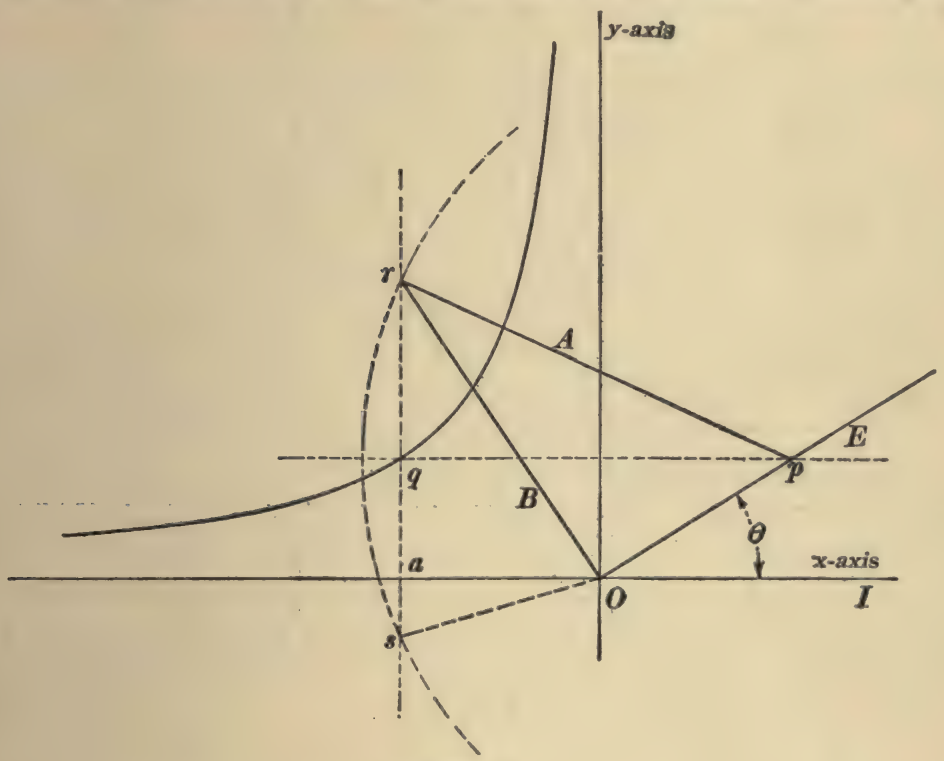

Fig. 137.

$O I$ the known angle $\theta$, but of unknown length, represents the resultant electromotive force of machines $A$ and $B$.

Draw a horizontal line cutting $O E$ in $p$ and cutting the hyperbola in $q$. Then, for that particular value of the current which corresponds to the chosen ordinate $a q$ $(=X I)$ the line $O p$ represents the actual resultant electromotive force inasmuch as the vertical component of $E$ is equal to $X I$. Describe about the point $p$ a circle of which the radius represents $A$. Then the lines $O r$ and $O s$ represent the two possible values of $B$ for the chosen value of $X I$. From this diagram it is evident that for the shortest possible value of $O P$ (smallest possible current) the circle described about $p$ just touches the ordinate $a q$, in which case the line $A$ is horizontal and parallel to $O I$, as shown in Fig. 138. For any shorter value of $O P$ (smaller value of $E$ or $I$ ) the circle does not reach to the ordinate $a q$, which means that for so small a value of the current the given value of $A$ is too small to supply the line losses $R I^{2}$ and the given motor intake $P^{\prime \prime}$.

3. When $P^{\prime \prime}$ is adjustable, maximum efficiency occurs when the differential coefficient of $P^{\prime \prime} \mid P^{\prime}$ with respect to $\phi$ is zero. From equations (15) and (16) we have

$$
\frac{P^{\prime \prime}}{P^{\prime}}=\frac{A B \cos (\phi-\theta)+B^{2} \cos \theta}{A B \cos (\phi+\theta)+A^{2} \cos \theta}
$$

whence, applying the condition 
I64 ELEMENTS OF ELECTRICAL ENGINEERING.

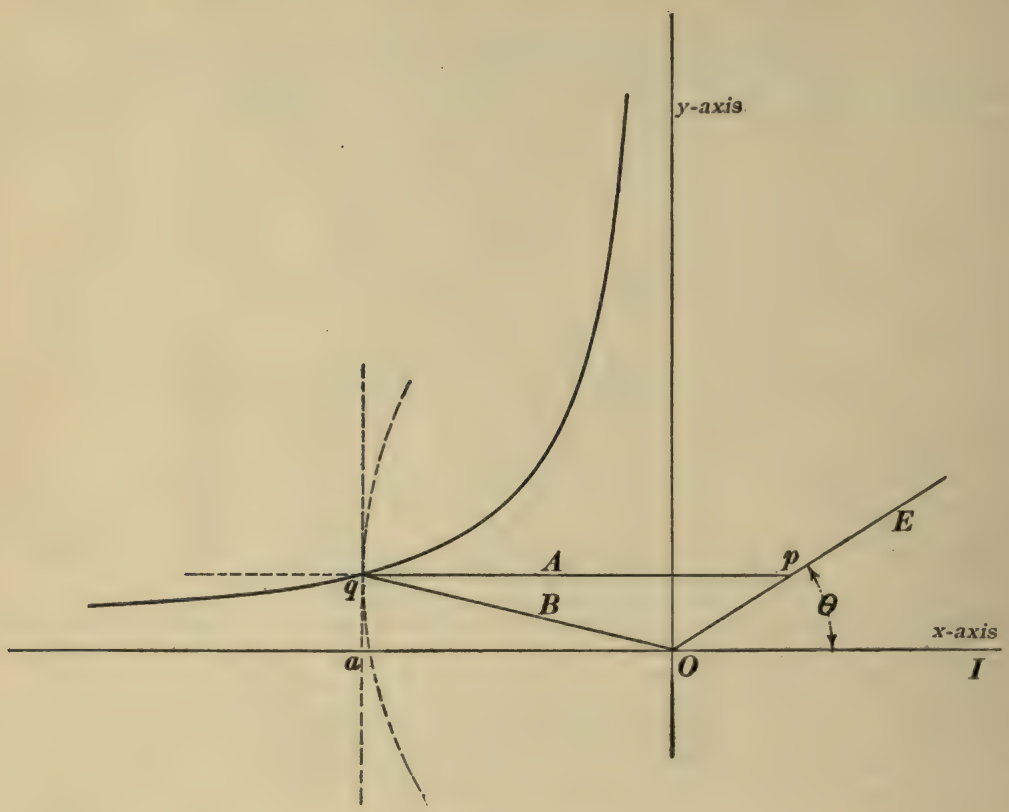

Fig. 138.

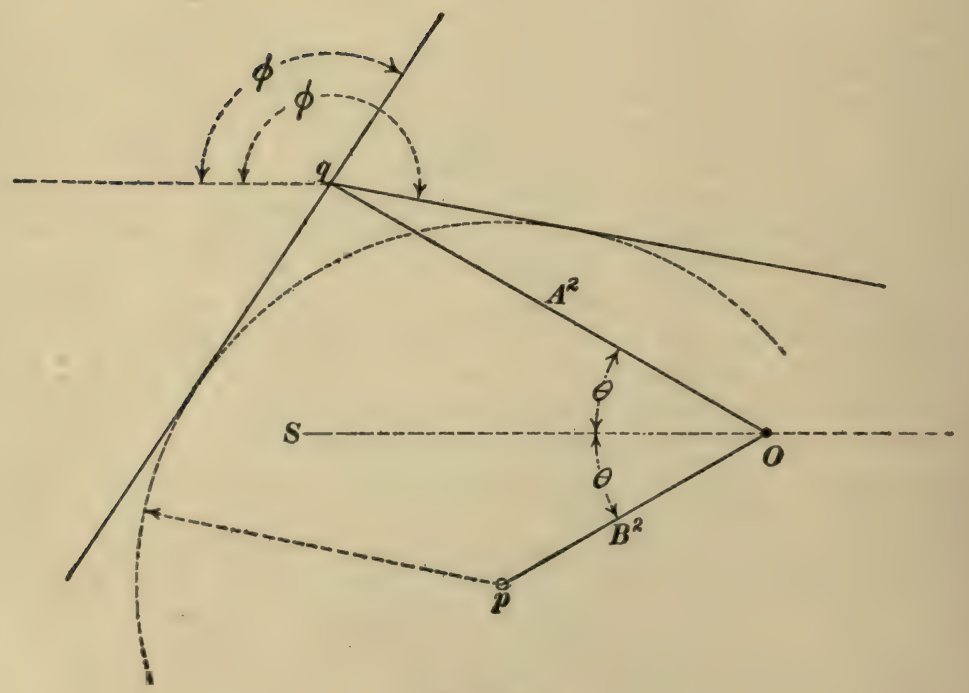

Fig. 139. 


$$
\frac{d\left(\frac{P^{\prime \prime}}{P^{\prime}}\right)}{d \phi}=0
$$

we have

$$
A^{2} \sin (\phi-\theta)-B^{2} \sin (\phi+\theta)=2 A B \sin \theta
$$

which determines the value of $\phi$ tor which the efficiency of transmission is a maximum.

Geometrical construction of equation (i). - Draw lines, Fig. 139, representing $A^{2}$ and $B^{2}$ to scale, the angle between them being $2 \theta$. About the point $p$ as a center describe a circle of which the radius represents $2 A B \sin \theta$. From the point $q$ draw a tangent to this circle. The angle between the line $O S$ and this tangent is the required value of $\phi$. Two tangents can be drawn from the point $q$. One of these tangents determines the value of $\phi$ (less than $180^{\circ}$ ) for which the efficiency of transmission is a maximum with machine $A$ acting as a synchronous motor, and the other tangent determines the value of $\phi$ (greater than $180^{\circ}$ ) for which the efficiency of transmission is a maximum with machine $B$ acting as a synchronous motor. The machine $A$ is distinguished as having the greater electromotive force. The angle $\phi$ is the lag of $B$ behind $A$.

79. Value of $B$ to give maximum intake of machine $B$ with given current; $A, X$ and $R$ being given. Let 1 , Fig. 140, be the given current and $E\left(=I \sqrt{R^{2}+X^{2}}\right)$ the resultant electromotive force. In order that the intake of $B$ may be a maximum, $B I \cos (B I)$ or $B \cos (B I)$ must be a maximum. Now $B \cos (B I)$ is the projection of $B$ on the current line $O I$. Describe a circle of radius $A$ about the point $P$, Fig. 140 ; then $O x$ is the greatest possible value of

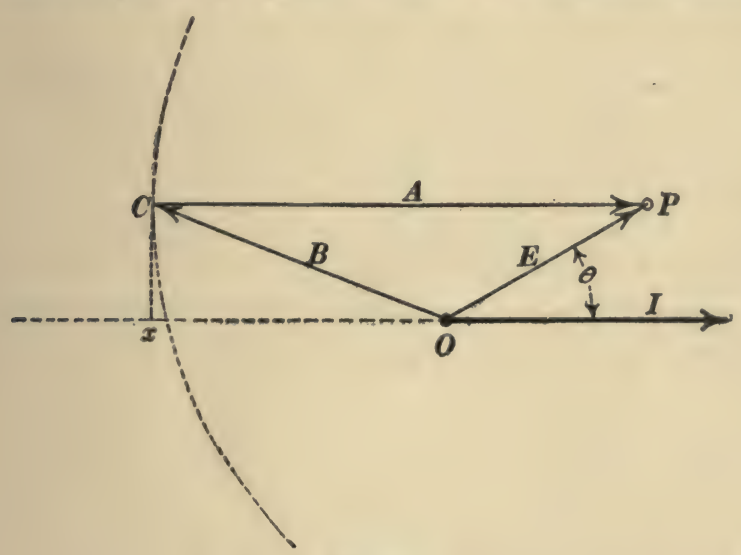

Fig. 140.

$B \cos (B I)$ for the given current, and $O C$ is the required value of $B$. From the triangle $O P C^{\circ}$ we have as the required value of $B$

$$
B=\sqrt{A^{2}+E^{2}-2 A E \cos \theta}
$$

Remark. - From Fig. 140 it is evident that $A$ is in phase with $I$ when $B$ is adjusted to give maximum $P^{\prime \prime}$ with given $I$. It was shown in Article 78 that $A$ 
is also in phase with $I$ when $B$ is adjusted to give minimum $I$ with given $P^{\prime}$ (or with given $P^{\prime \prime}$ ). This correspondence is by no means self-evident.

80. Maximum intake of machine $B ; A, B, X$ and $R$ being given. $-P^{\prime \prime}$ has its maximum negative value when $\cos (\phi-\theta)=-\mathbf{I}$, and equation (15) becomes

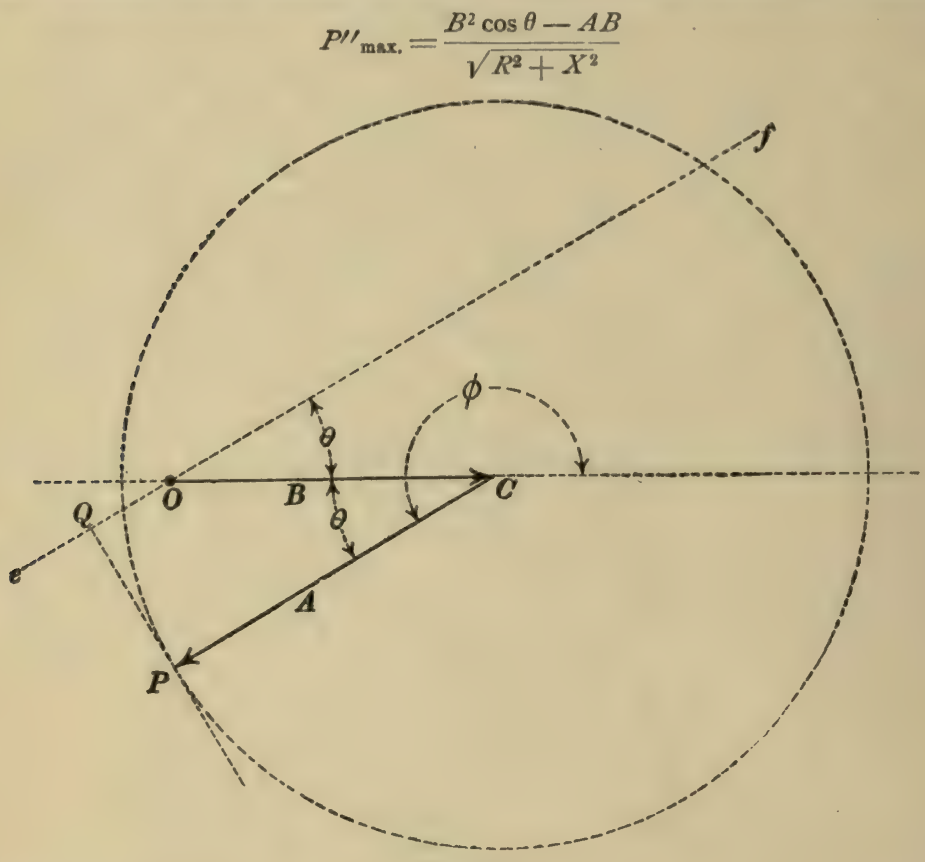

Fig. 141.

Figure $14 \mathrm{I}$ shows the state of affairs when intake of $B(=O Q)$ is at its greatest.

81. Greatest value of the electromotive force $B$ for which machine $B$ can act as a motor; $A, X$ and $R$ being given. - So long as

is greater than

$$
\frac{A B}{\sqrt{R^{2}+X^{2}}}
$$

$$
\frac{B^{2}}{\sqrt{R^{2}+X^{2}}} \cdot \cos \theta
$$

then $P^{\prime \prime}$ can have negative values according to equation (I5). Therefore the limiting case is where

or

$$
\frac{A B}{\sqrt{R^{2}+X^{2}}}=\frac{B^{2}}{\sqrt{R^{2}+X^{2}}} \cdot \cos \theta
$$

$$
B=\frac{A}{\cos \theta}
$$

This limiting case is shown in Fig. 142. 


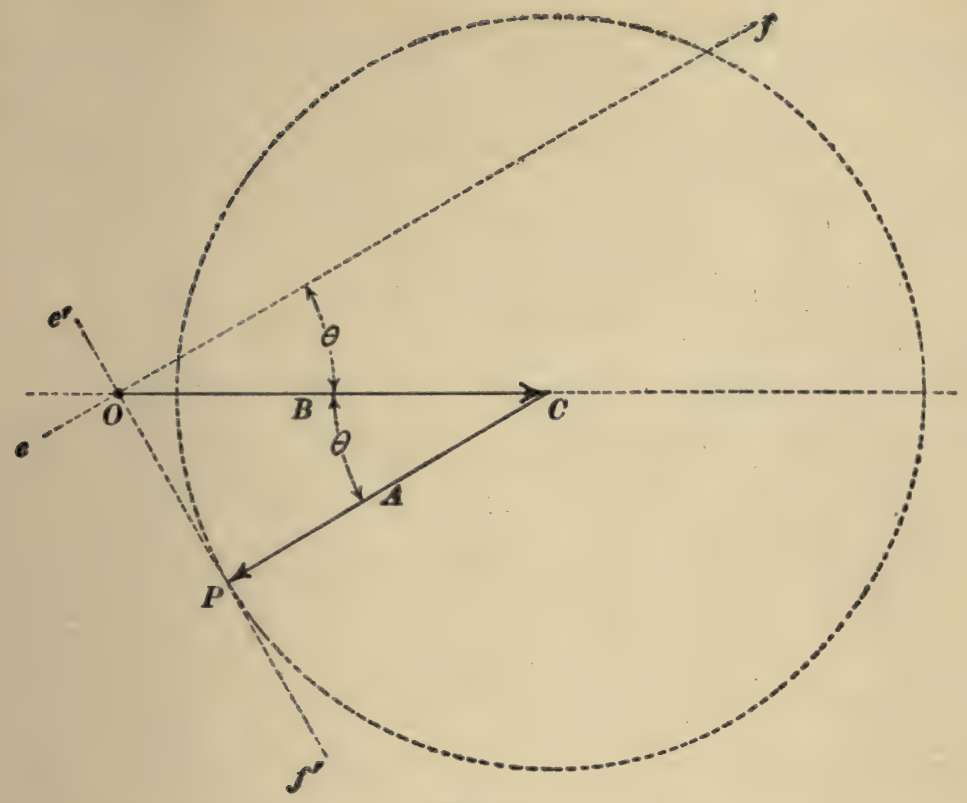

Fig. 142.

82. To find value of $B$ for which the machine $B$ may take in the greatest possible power from $A ; A, X$ and $R$ being given.-Equation(i), Art. 8o, expresses the greatest intake of $B$ for given values of $A, B, X$ and $R$. It is required to find the value of $B$ which will make this greatest intake a maximum. This value of $B$ must render $B^{2} \cos \theta-A B$ [the numerator of right-hand member of equation (i), Art. 80], a maximum. Differentiating this expression with respect to $B$ and placing the differential coefficient equal to zero we have

or

$$
2 B \cos \theta-A=0
$$

$$
B=\frac{A}{2 \cos \theta}
$$

Remark. - A comparison of equations (i), Art. 8I and (i) of this article shows that the value of $B$ for greatest possible intake of machine $B$ is half the greatest value of $B$ for which machine $B$ can act as a motor at all. This is also the case with a direct-current motor. The greatest electromotive force such a motor can have is the electromotive force of the dynamo which drives it, and the value of its electromotive force to permit the greatest possible intake is one-half the electromotive force of the dynamo which drives it.

83. Excitation characteristics. - With given load on a synchronous motor (given value of $P^{\prime \prime}$ ) its electromotive force $B$ may be changed by varying its field excitation, and for each value of $B$ there is a definite value of the current $I$. Thus the abscissas of the curves, Fig. 143, represent values of $I$, and ordinates represent 
values of $B$ for loads of zero, roo kilowatts and 200 kilowatts respectively. These curves are called the excitation characteristics of the motor. Fig. I43 is based on the values $A=\mathrm{I}, \mathrm{I00}$ volts, $R=\mathrm{I} \mathrm{ohm}$ and $X=0.58 \mathrm{ohm}$. For the greatest pos-

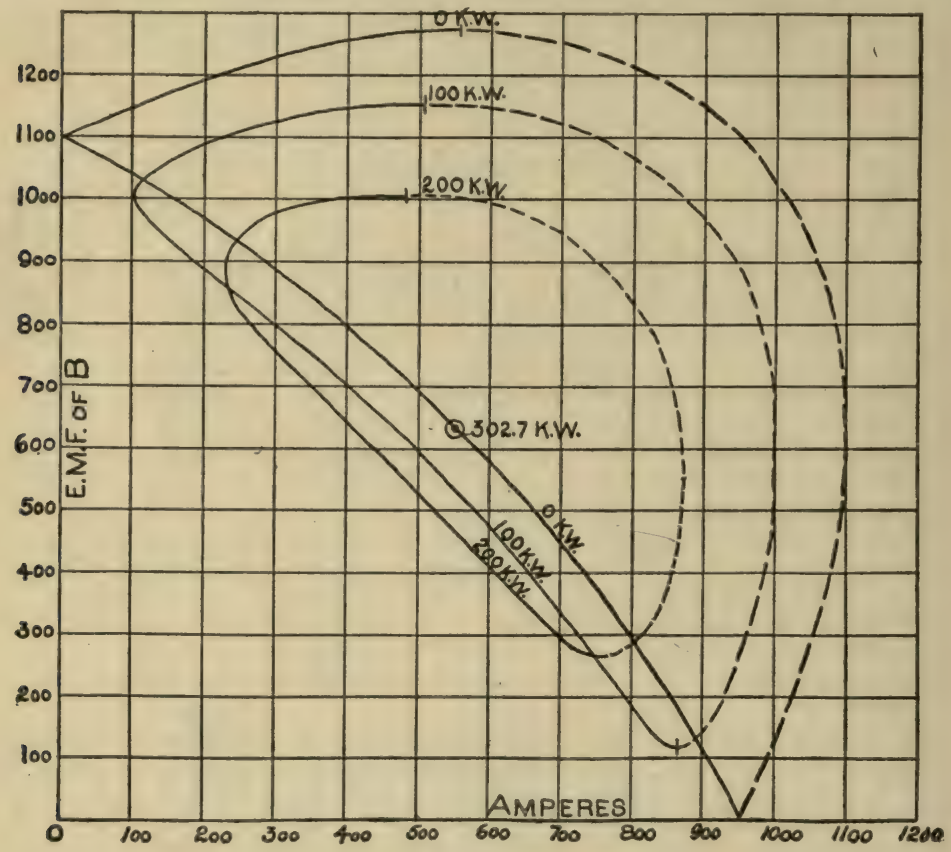

Fig. 143.

sible intake, 302.7 kilowatts, the characteristic reduces to the point enclosed in the small circle. It was pointed out in Art. $7 \mathrm{I}$ that with given load there are two values of $I$ for each value of $B$, and that the larger value of $I$ corresponds to unstable

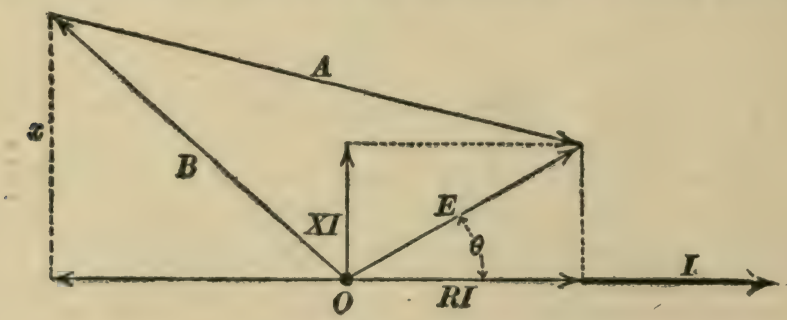

Fig. 144.

and the smaller value to stable running. The dotted portions of the curves, Fig. 143, correspond to the larger values of $I$. These dotted portions cannot, of course, be determined by experiment, on account of the instability of running. 
The equation to the excitation characteristics may be derived as follows - Let I, Fig. 144, represent the current and $E$ the resultant electromotive force; the components of $E$ are $R I$ and $X I$. The electromotive force $E$ is the vector sum of $A$ and $B$, as shown, and the component of $B$ parallel to $I$ is $P^{\prime \prime} / I$. From the right-angled triangles of the figure we have

$$
B^{2}=x^{2}+\left(\frac{P^{\prime \prime}}{I}\right)^{2}
$$

and

$$
A^{2}=\left(R I+\frac{P^{\prime \prime}}{I}\right)^{2}+(x-X I)^{2}
$$

By eliminating $x$ from these equations we have the required relation between $B$ and $I ; P^{\prime \prime}, A, R$ and $X$ being given. The curves, Fig. 143, were calculated graphically by means of the clock diagram, Fig. 125. 


\section{CHAPTER IX.}

\section{THE CONVERSION OF ALTERNATING CURRENT INTO DIRECT CURRENT.}

\section{The conversion of alternating current into direct current. -}

It is frequently necessary to utilize in the form of direct current the power which is delivered by an alternator, or, in other words, to convert alternating current into direct current. Thus, if the field of an alternator is to be compounded, it is necessary to rectify the alternating current in the series field winding of the machine, as explained in Art. 2. If the power delivered by an alternator is to be used for charging storage batteries it must be used in the form of direct current. If power, which is transmitted to a distance in the form of alternating current, is to be used to drive direct-current motors, such as are extensively employed in electric railway operation; it must be converted from alternating current to direct current. This conversion from alternating current to direct current is accomplished in practice in four ways; namely, (a) by the rectifying commutator, (b) by the motor-generator, $(c)$ by the synchronous converter and $(d)$ by the mercury-arc rectifier.

(a) The rectifying commutator. - The ordinary direct-current generator is essentially an alternator with a commutator arranged to properly reverse the connections between the armature windings and the receiving circuit so that, although the current in a given armature conductor is an alternating current, the current in the receiving circuit is always in one direction. The commutator of a direct-current generator, however, reverses the connections of successive small portions of the armature windings, whereas the rectifying commutator, strictly so-called, which is described in Art. 2, reverses the connections of the armature winding of an alternator as a whole. The rectifying commutator 
is not suited to heavy service, that is to say, it cannot rectify a large alternating current at a moderately high voltage because of its excessive tendency to spark.

(b) The motor-generator. - Fig. 145 shows a 500 kilowatt compound wound direct-current generator directly coupled to a three-phase synchronous motor with a small direct-current ex-

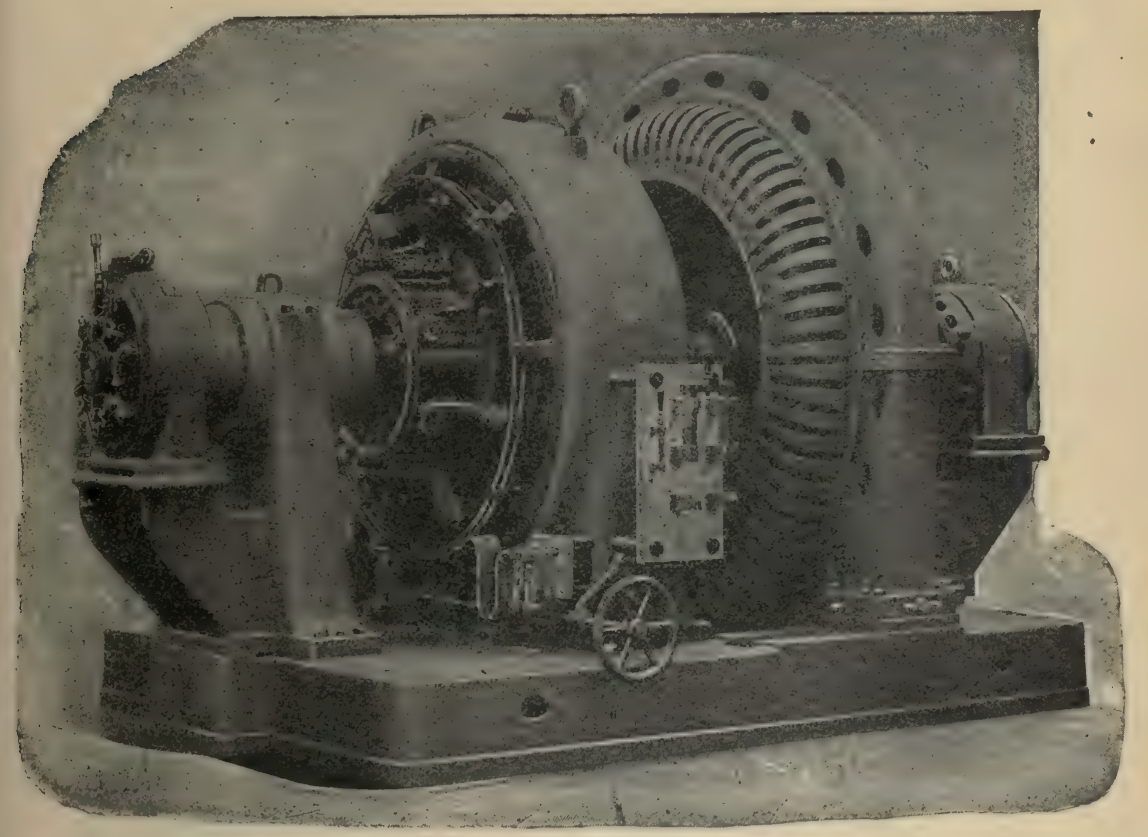

$500 \mathrm{Kw} ., 575$ Volt, Compound W̧ound Generator and xroo Volt, Three-Phase, 3000 Alternation, Synchronous Motor; 375 R.P.M., with.A.C. Starting Motor and D.C. Exciter. (Westinghouse Company.)

Fig 145 .

citer on one end of the main shaft, and a small induction startingmotor on the other end of the main shaft. The synchronous motor takes power from a three-phase alternating-current supply, and drives the direct-current generator which delivers direct current. Generally the field excitation of the synchronous motor is supplied from the main direct-current. generator without the use of a small auxiliary exciter; and whenever direct current is 
available for starting, the machine is brought up to speed by operating the main direct-current generator as a motor, in which case the small induction motor is not required for starting.

(c) The synchronous converter. - The device which is most frequently used for converting alternating current into direct current is the synchronous converter, which is described in Art. 85 .

(d) The mercury-arc rectifier* consists essentially of a highly

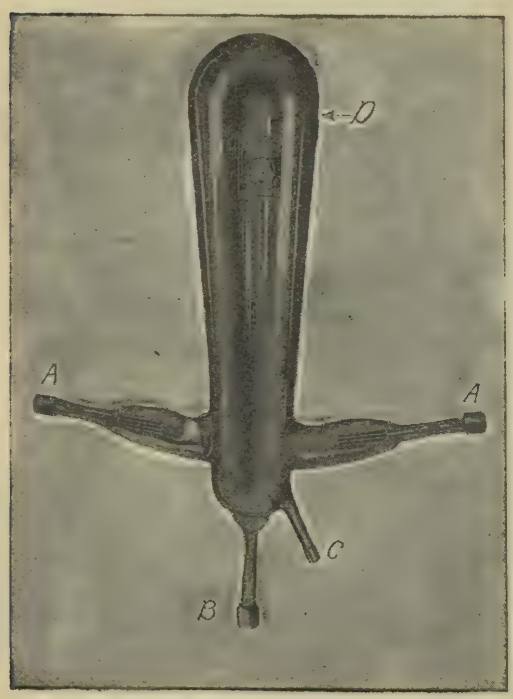

Mercury Arc Rectifier Tube.

Fig. 146. exhausted glass bulb, Fig. I46, with two iron or graphite electrodes connecting to terminals $A A$, and the mercurypool electrode connecting to terminal $B$. The terminal $C$ connects to an auxiliary pool of mercury very near to the pool which connects with terminal $B$. The arrangement of the mercury-arc rectifier is shown in Fig. 147, in which $J$ represents a storage battery which is being charged. The full-line arrows represent the flow of current during one half of a cycle, and the dotted arrows represent the flow of current during the other half of a cycle. A slight shake of the bulb causes the mercury to bridge over from $B$ to $C$, and the current starts to flow, making the mercury pool $B$ a cathode. Then current can flow readily from either of the terminals $A$ or $A^{\prime}$ to $B$, but it cannot flow out

* See a paper on "The Electric Arc," by C. P. Steinmetz, Transactions of the International Elcctrical Congress, Vol. 2, pages 710 to 730, St. Louis, 1904; also a paper on "The Mercury Arc Rectifier," by C. P. Steinmetz, Transactions of the American Institute of Electrical Engineers, Vol. 24, pages 371 to 393, 1905; and a paper by P. D. Wagoner, read before the National Electric Light Association at Denver, Colo., in June, 1905. 
of the tube at either of the electrodes $A$ or $A^{\prime}$, that is to say, the two electrodes $A$ and $A^{\prime}$ act like valves. Two inductance (reactance) coils, $E$ and $F$ are inserted as shown in the figure. The effect of these coils is as follows: Consider the flow of current represented by the full-line arrows. This current passes through coil $E$. As this half-wave of alternating current dies away, the momentum of the current in the inductance coil $E$ causes the current to continue to circulate in the short circuit $D E A^{\prime} B$, and thus maintain an outflowing current at $B$ until the reverse wave of alternating current begins to flow into the tube at $A^{\prime}$. The continued operation of the rectifier requires that the outflowing current at $B$ never become zero.
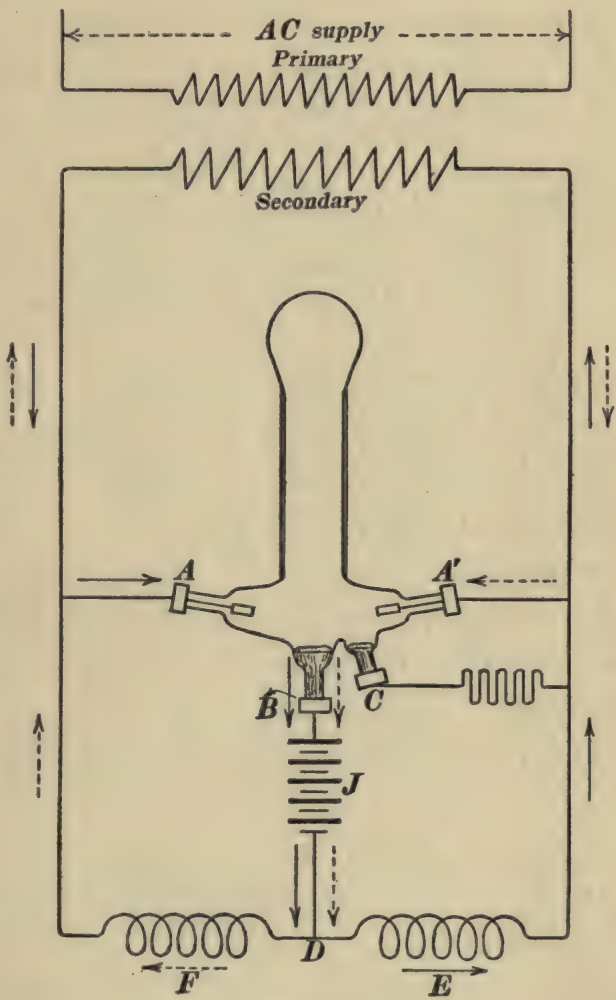

Flg. 147.

Comparison of methods $(b)$ and $(c)$. - The motor-generator and the synchronous converter are the only devices which are available for heavy service in the conversion of alternating current into direct current.* The synchronous converter has a higher

* The practical possibilities of the mercury-arc rectifier are not as yet fully established. At the present time this rectifyer is built in sizes large enough to deliver thirty or forty amperes of direct current with a loss of about $\mathbf{1 4}$ volts in the mercury arc. The mercury-arc rectifier is suitable for any voltage up to 10,000 volts or more. 
efficiency than the motor-generator and its cost is very much less, but there is a nearly fixed relation between the effective value of the voltage of the alternating-current supplied to the synchronous converter and the steady voltage of the direct current delivered by the machine, so that the voltage of the direct-current side of the machine cannot be changed through a wide range without changing the effective voltage of the alternating-current supply. See Art. 90. The motor-generator, on the other hand, is very flexible in this respect, the voltage of the direct-current generator can be varied independently of the voltage of the alternating-current supply by varying the field excitation of the generator.

85. The synchronous converter. - Consider two opposite commutator bars on an ordinary two-pole direct-current dynamo. The electromotive force between these two bars alternates as the armature rotates, and if these two commutator bars are connected to two collector rings the direct-current dynamo may be used to deliver alternating current; the machine becomes an alternator and, its commutator being undisturbed, it remains a direct-current dynamo. Such a machine may be belt driven and deliver direct current, or alternating current or both; it may be driven as a direct-current motor and deliver mechanical power, or alternating current, or both; or it may be driven as a synchronous alternating-current motor and deliver mechanical power, or direct current or both. The most important use of such a machine is to convert alternating current into direct current, for which purpose it is driven as a synchronous alternating-current motor and loaded by delivering direct current; and the machine is called a syncironous converter * when used in this way.

Single-phase and polyphase synchronous converters. - The machine above described, having two collector-rings, is called a tworing or single-phase converter. The three-ring or three-phase converter has three collector rings connected to three commutator bars $120^{\circ}$ apart (on a two-pole machine), the four-ring, or so-called

* Also often called a rotary converter. 
two-phase converter, has four collector rings connected to four commutator bars $90^{\circ}$ apart (on a two-pole machine) and so on.

In the case of a multipolar machine the $n$ collector rings of an $n$-ring converter are connected as follows : Ring No. I is connected to all the commutator bars* which for a given position of the armature lie midway under the north poles of the field magnet. Let $l$ be the distance between adjacent commutator bars of this first set. Then ring No. 2 is connected to all commutator bars I/ $n$th of $l$ ahead of the first set; ring No. 3 is connected to all commutator bars

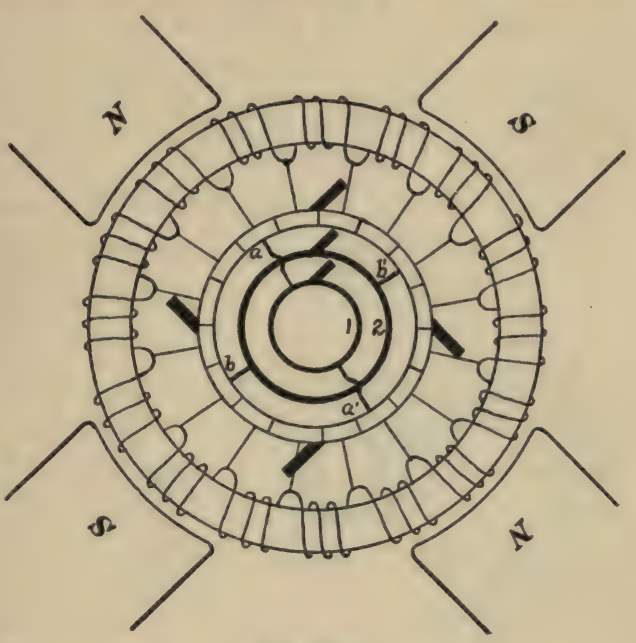

Fig. 148. $2 / n$ ths of $l$ ahead of the first set; and so on. Thus Fig. I48 shows a four-pole two-ring converter, each ring being connected to two commutator bars.

86. The internal actions of a synchronous converter. - In its relation to the alternating-current supply a synchronous converter is like a synchronous motor, and in its relation to the direct-current receiving circuit a synchronous converter is like a direct-current generator; but in regard to $(a)$ the actual motor and generator actions in the machine, $(b)$ the magnetizing action of the armature current, and $(c)$ the heating effect of the armature current, the machine is essentially unlike a synchronous motor and essentially unlike a direct-current generator.

* This statement applies to that type of armature winding which is called the simplex lap winding. In the simplex wave winding each collector ring is connected to but one commutator bar whatever the number of field magnet poles may be. The multiplex winding is not well adapted to the synchronous converter. 
The details of motor and generator action are of little practical importance and they are described in the following paragraphs of this article, but the magnetizing action and heating effects of the armature current are important, inasmuch as they determine the power rating of the machine.

Motor and generator action in the synchronous converter.* - Let $a$ and $b$, Fig. I49, be the direct-current brushes of a synchronous converter. Let the negative brush

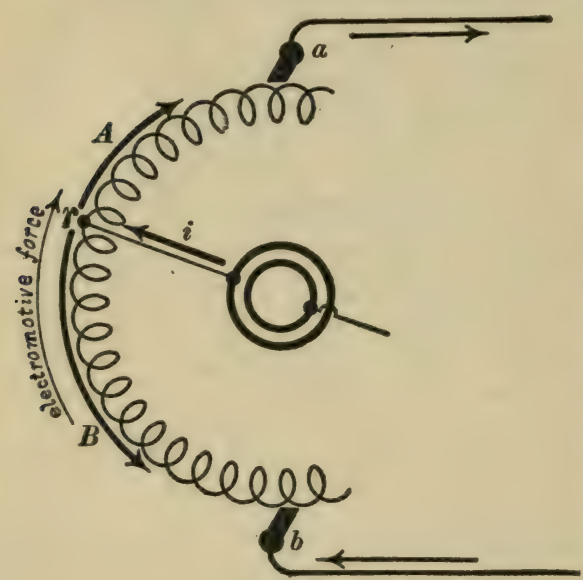

Fig. 149.

$b$ be chosen as the zero or reference point of potential so that the electromotive force between $b$ and any other given point may be spoken of as the potential at the given point.

Let the half-circle represent one side of the armature, and let $r$ be the position at a given instant of the point of attachment of one of the collector rings. At the given instant a definite current $i$ is entering the armature at $r$ from the alternator, which is driving the converter ; this current is entering at a potential which is less than the potential of the brush $a$, a portion $B$ of the current $i$ flows down hill (against the induced electromotive force in the windings, which electromotive force is represented by the fine line arrow) to brush $b$ and delivers power to the portion $r b$ of the armature in the form of motor action, and the remainder $A$ of the current $i$ flows up hill (with the induced electromotive force) to brush $a$ and receives power from the portion $r a$ of the armature in the form of generator action. That is to say at each instant certain portions of the armature of a synchronous converter have motor action and develop torque in the direction of the motion of the armature, and other portions of the armature have generator action and develop torque tending to oppose the motion of the armature. In the two-ring converter the motor action at times greatly exceeds the generator action, at other times the generator action greatly exceeds the motor action, and the armature is alternately accelerated and retarded. This must be so because the single-phase alternating current delivers power to a tworing converter in pulses. In a polyphase converter, however, the motor action is at all times very nearly equal to the generator action, being just enough in excess to supply the mechanical and magnetic friction losses in the motor.

* See a paper on "The energy transformations in the synchronous converter," by W. S. Franklin, Trans. Am. Inst. Elec. Eng., Vol. 22, pages 17-33, 1903. 
87. The starting of the synchronous converter. *- Synchronous converters are often used in sets, one or more of the machines being always in operation. When this is the case a converter not in use may be started as a direct-current motor, taking direct current from the other machines, and its speed may be adjusted until it is in exact synchronism with the alternating-current supply by which it is to be driven, and then, when its alternating electromotive force is opposite in phase to the supply voltage, the alternating current switches may be closed. The procedure is exactly the same as the starting of a synchronous motor as described in Art. 73 and the same synchronizing devices are used.

When direct current is not available for starting a converter, an induction motor is generally used to bring the machine up to speed. Any polyphase alternator however contains the essential features of an induction motor, and therefore a polyphase alternator or synchronous converter can be started by its own induction motor action. In some cases this method of starting is employed. See Art. 132.

88. The operation of the synchronous converter. - Not only in the matter of starting but also in certain matters of operation the synchronous converter is like a synchronous motor. Consider, for example, a four-ring converter. Such a converter is often called a two-phase converter because it is supplied with two alternating currents $90^{\circ}$ apart in phase, each alternating current being delivered to a pair of opposite collector rings, that is, to collector rings which are connected to opposite points on the armature of a two-pole machine. Let $B$ be the effective value of the total induced electromotive force between opposite collector rings, $R$ the resistance of the armature between opposite collector rings, $L$ the inductance of the armature between opposite collector rings plus any outside inductance that may be connected in series in each alternating-current circuit between the supply mains and the machine, and let $A$ be the effective value of the supply voltage (each phase) and $\omega / 2 \pi$ the frequency.

* See a series of short practical papers on this subject by A. Wagner, Electric Journal, Vol. II, pages 436, 494 and 572, Ig05. 
Lay off the line $O C$, Fig. I 50 , to represent $B$, describe a circle about $C$ of which the radius represents the value of $A$, draw the line of making with $O C$ the angle whose tangent is $\omega L / R$, and draw the line $s O s^{\prime}$ perpendicular to $e f$. Let us suppose for the sake of simplicity that the efficiency of the converter is Ioo per cent. so that the intake of power may be considered equal to the output. The figure shows the phase relation between $A$ and $B$ (each phase) for a direct-current load equal to 2 times $\overline{O Q}$ times $B / \sqrt{R^{2}+\omega^{2} L^{2}}$ watts, where $\overline{O Q}$ is expressed in volts (of course all lengths in Fig. 150 represent

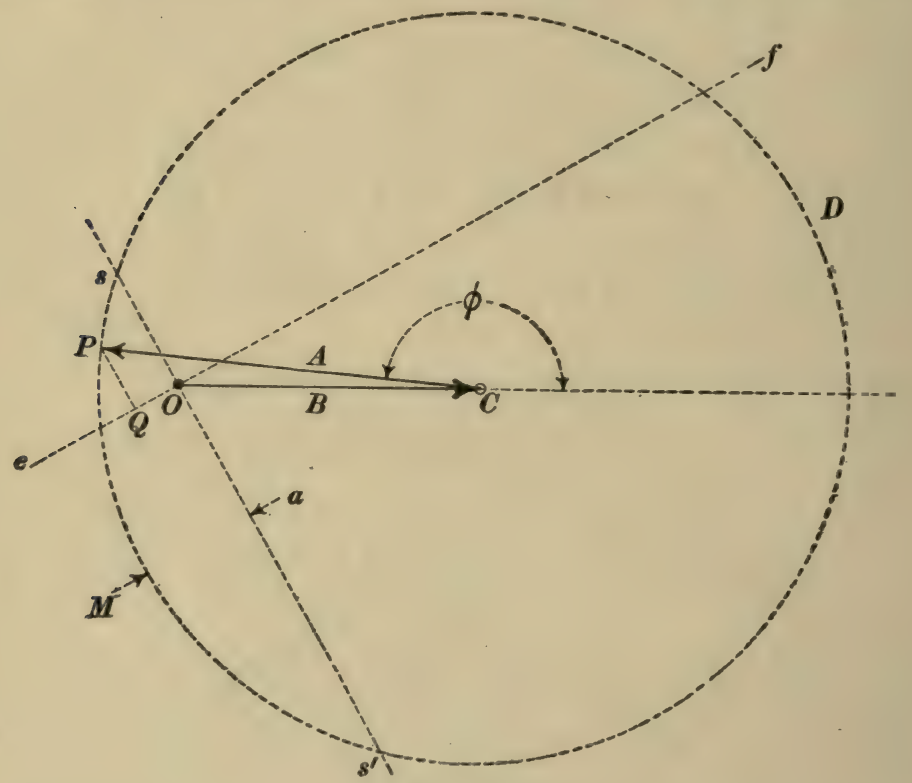

Fig. 150.

volts). This relation is explained in Art. 69, and the factor 2 is introduced because the expression given in Art. 69 gives the intake of one phase only.

If the direct-current output is decreased, the point $P$, Fig. I 50, moves towards $s$ causing $\overline{O Q}$ to decrease; if the direct-current output is increased the point $P$ moves towards $M$, causing $\overline{O Q}$ to increase ; and if the direct-current output is greatly increased 
the point $P$ is carried beyond the point $M$, and the machine falls out of synchronism and ceases to operate. The maximum intake of power is 2 times $\overline{M a}$ times $B / \sqrt{R^{2}+\omega^{2} L^{2}}$.

A synchronous motor can operate for a fairly wide range of values of voltage $B$, the supply voltage $A$ being given, provided there is considerable inductance in the circuit or, in case of a polyphase machine, in each circuit; but this possible variation of $B$ shows itself as a variation of the terminal voltage only when there is inductance (or resistance) between the supply mains and the synchronous motor. Therefore, if it is desired to control the voltage on the direct-current side of a synchronous converter by changing the field excitation, inductance (or resistance) must be placed in each circuit between the supply mains and the machine. Then a change of field excitation changes the terminal voltage of the machine on both sides. Inductance is always used rather than resistance when it is desired to control the voltage of a synchronous converter without changing the supply voltage.

The hunting action of the synchronous motor is described in Art. 72. The synchronous converter is especially prone to develop hunting oscillations as stated in Art. 72, and the methods for obviating this trouble are there described.

When the synchronous converter is driven as a direct-current (shunt) motor and loaded by delivering alternating current, it is called, usually, an inverted rotary, and when so operated the machine exhibits one peculiarity which should never be lost sight of, namely, the machine tends to run at an excessively high speed when it is overloaded. This rather paradoxical behavior is due to the weakening of the field by the demagnetizing action of the alternating current which is taken from the machine, and, like any shunt motor, its speed rises when its field is weakened, since there is but little mechanical opposition to the motion.

* What is here said concerning the maximum intake of power of a synchronous converter and its stoppage due to overload is based on the assumption that $B$ is constant, whereas, in fact, $B$ may be greatly reduced by armature reaction when the load on the machine is excessive. In fact, a synchronous converter can scarcely be stopped by overload. 
89. Armature reaction of the synchronous converter. - The magnetizing action of the current in the armature of a dynamo is called armature reaction, and armature reaction has in general two distinct effects, namely, (a) It crowds the flux to one side or the other of the pole pieces of the field magnet, and (b) it tends to weaken (or strengthen) the field. The first is called distortion and the second is called demagnetizing action. The armature reaction of a synchronous converter is generally quite small and therefore of but little practical importance.

In discussing the armature reaction of a synchronous converter it is permissible to consider that the alternating current which is delivered to the machine and the direct current which is delivered by the machine each flows through the armature windings as if the other did not exist.

Distortion. - The crowding of the flux to one side or the other of the pole pieces of a dynamo accompanies and is in a certain sense the cause of the torque with which the field magnet acts upon the armature. When a synchronous motor is running steadily there is only sufficient torque action to overcome mechanical and magnetic friction and the distortion of the field is negligibly small. When a synchronous converter hunts its speed pulsates and a very considerable torque acts upon the armature, first in one direction and then in the other, and the flux is crowded to one side and then to the other side of the pole pieces. This action is discussed in Art. 72.

Demagnetizing action. - Since there is no perceptible field distortion in a synchronous converter the axis of direct-current commutation is approximately at right angles to the axis of the field, so that the direct current in the armature has no perceptible demagnetizing action, positive or negative. See pages $\mathbf{I} \mathbf{I}$ to $\mathbf{I} 6 \mathbf{I}$ of the first volume of this text.

The demagnetizing action of the alternating current in the armature of a synchronous converter depends upon the phase difference between $B$ and $I$, these letters being used in the sense in which they are used in Chapter VIII. When $B$ and $I$ are exactly opposite to each other in phase, that is when the angle $(B I)$ is equal to $180^{\circ}$, there is no perceptible demagnetizing action, positive or negative, due to the alternating current in the converter armature. When the angle $(B I)$ differs from $180^{\circ}$ the demagnetizing action of the alternating current in the armature is proportional to the sine of this angle as explained in Art. 65 .

90. Electromotive force relations of the synchronous converter.There is a fairly definite relation between the value of the electromotive force between the direct-current brushes of a synchronous converter and the effective value of the alternating electromotive force between collector rings. The following discussion of these electromotive force relations is based on the assumption that the alternating electromotive force of the machine is harmonic and 
that the $R I$ losses of voltage in the armature windings are negligible.

Two-ring converter. - In the two-ring converter the two rings are connected to commutator bars, one of which comes under a positive direct-current brush at the instant that the other comes under a negative direct-current brush ; at this instant the electromotive force between the collector rings is at its maximum value and it is equal to the éectromotive force between the directcurrent brushes. Therefore the direct electromotive force $E_{0}$ of a two-ring converter is equal to the maximum value, $\sqrt{2} E_{2}$, of the harmonic alternating electromotive force between the two collector rings. Therefore

$$
E_{2}=\frac{E_{0}}{\sqrt{2}}
$$

in which $E_{2}$ is the effective value of the alternating electromotive force between the collector rings.

Converter with $n$ collector rings. - Consider all of the armature conductors which constiture one of the paths between the two rings of a two-ring converter. These conductors are evenly distributed
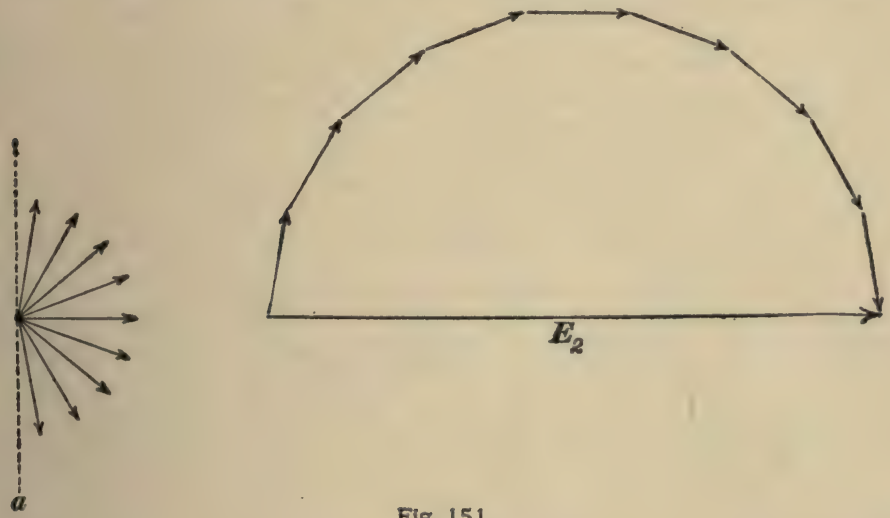

Fig. 151.

over one half of the armature, and the harmonic alternating electromotive forces which are induced in the respective conductors are all equal in value but they are distributed in phase throughout an angle of $180^{\circ}$ as shown by the vectors $a$, Fig. I 5 I. There- 
fore the effective electromotive force between the collector rings of a two-ring converter is represented by the diameter, $E_{2}$, Fig. I $5 \mathrm{I}$, of the polygon whose sides are equal and parallel to the vectors $a$ which represent the effective values of the electromotive forces in the individual armature conductors.

The electromotive force (effective) between two collector rings which are connected to the armature winding at points $\mathrm{I} 2 \mathrm{O}$ electrical degrees apart, that is between any two rings of a three-ring converter, is represented by that chord of the polygon, Fig. I 5 I, which subtends an angle of $120^{\circ}$; the effective electromotive force between two rings which are connected to the armature winding at points 90 electrical degrees apart, that is between any two adjacent rings of a four-ring converter, is represented by that chord of the polygon, Fig. I $5 \mathrm{I}$, which subtends an angle of $90^{\circ}$; and, in general, the effective electromotive force between adjacent rings of an $n$-ring converter is represented by that chord of the polygon, Fig. I $5 \mathrm{I}$, which subtends an angle of $360^{\circ} / n$. Therefore, treating the polygon of Fig. I 5 I as a circle, we have

$$
E_{n}=E_{2} \sin \frac{\pi}{n}
$$

or, using the value of $E_{2}$ from equation (i) we have

$$
E_{n}=\frac{E_{0}}{\sqrt{2}} \cdot \sin \frac{\pi}{n}
$$

which gives the following expressions for the effective values of the alternating electromotive forces between adjacent rings of a two-ring, a three-ring, a four-ring, and a six-ring converter in terms of the electromotive force $E_{0}$ between the direct-current brushes

$$
\begin{aligned}
& E_{2}=\frac{E_{0}}{\sqrt{2}}=0.707 E_{0} \\
& E_{8}=\frac{\sqrt{3} E_{0}}{2 \sqrt{2}}=0.612 E_{0}
\end{aligned}
$$




$$
\begin{gathered}
E_{4}=\frac{E_{0}}{2}=0.500 E_{0} \\
E_{6}=\frac{E_{0}}{2 \sqrt{2}}=0.354 E_{0}
\end{gathered}
$$

These electromotive force relations are shown geometrically in Fig. 152 .

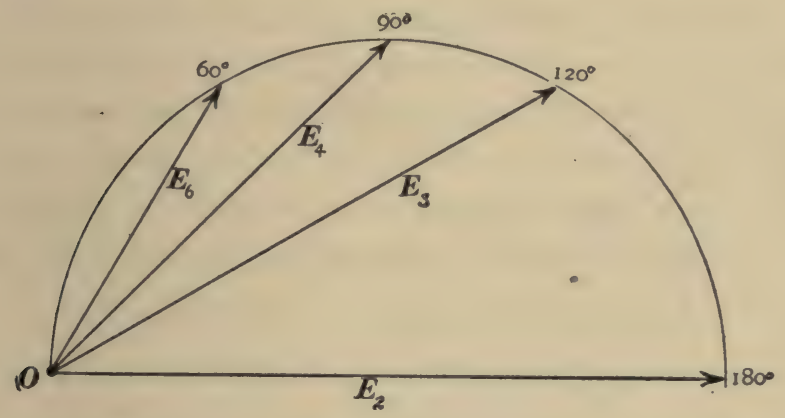

Fig. 152.

Actual electromotive force relations. - The relations given in equations (I 7), (I8), (I9) and (20) are not exactly realized in practice. The departure of an actual machine from these ideal ratios depends upon: Ist, the wave shape of the alternating electromotive force of supply; $2 \mathrm{~d}$, the wave shape of the alternating electromotive force of the converter itself, which depends chiefly upon the percentage of the armature surface that is covered by the pole faces; $3 \mathrm{~d}$, the resistance of the armature windings which causes losses of voltage when the machine is loaded; 4 th, the position of the direct-current brushes, and 5 th, the manner in which the machine is used, that is, whether it is used to convert from alternating to direct current or vice versa.

When the alternating electromotive force of supply is approximately harmonic, and the pole faces of the converter cover threequarters of the armature surface, and when the direct-current brushes are in the neutral position the actual voltage ratios are approximately as follows : 
TABLE.

Voltage Ratios of Two-Ring and Three-Ring Converters (550-volt type).

\begin{tabular}{|c|c|c|c|c|}
\hline & \multicolumn{3}{|c|}{ Actual Ratio $E_{n} / E_{0}$} & \multirow{2}{*}{$\begin{array}{l}\text { Ideal Ratio } \\
E_{n} / E_{0}\end{array}$} \\
\hline & Zero Load. & $\begin{array}{l}\text { Full Load; } \\
\text { (Straight) }\end{array}$ & $\begin{array}{l}\text { Full Load. } \\
\text { (Inverted.) }\end{array}$ & \\
\hline $\begin{array}{l}E_{2} \\
E_{3}\end{array}$ & $\begin{array}{l}0.7 \times 5 \\
0.610\end{array}$ & $\begin{array}{l}0.725 \\
0.620\end{array}$ & $\begin{array}{l}0.705 \\
0.600\end{array}$ & $\begin{array}{l}0.7071 \\
0.612\end{array}$ \\
\hline
\end{tabular}

The values of $E_{4}$ and $E_{6}$ are not included in this table inasmuch as the alternating voltage of a four-ring converter is usually specified as the effective voltage between opposite rings which is the same as $E_{2}$, and the alternating voltage of a six-ring converter is usually specified either as the voltage between opposite rings $\left(=E_{2}\right)$ or the voltage between a given ring and the next ring but one, between rings $I$ and 3 for example $\left(=E_{3}\right)$.

Voltage control of the synchronous converter. It is evident from the above table that the direct voltage $E_{0}$ of a synchronous converter is very nearly constant if the alternating supply voltage $E_{n}$ is constant. Therefore it is not possible to alter $E_{0}$ at will if $E_{n}$ is constant. If the field excitation of a synchronous converter is reduced, the machine takes lagging wattless current* from the alternating-current supply, and the effect of this wattless current in the armature is to help the field flux and make up almost completely for the decrease of direct-current field excitation; if the field excitation of the machine is increased, the machine takes the leading wattless current, the effect of which in the armature is to oppose the field flux and counteract almost completely the increase of direct-current field excitation. If, however, an inductance is connected in the alternating-current circuit outside of the machine, the lagging wattless current in the first instance causes a considerable drop of alternating voltage, or the leading wattless current in the second instance causes a considerable rise of alternating voltage, and the direct voltage of the converter is decreased or increased correspondingly. To be able to

* The statements here made are worded to apply to the single-phase converter. 
control the direct voltage of a synchronous converter by means of a field rheostat, an inductance must be connected in the alternating-current circuit.*

The compounding of the synchronous converter. If the field magnet of a synchronous converter is excited in part by a shunt winding and in part by a series winding, then an increase of direct-current output gives an increased field excitation, and, if $E_{n}$ is kept at a constant value, this increase of field excitation is counteracted by a leading wattless current in the armature, and $E_{0}$ remains sensibly unchanged in value. In order that a series field winding may produce an increase of $E_{0}$ with increase of direct-current output (over-compounded machine), an inductance must be connected in the alternating-current circuit.

91. Current relations of the synchronous converter. - It is evident from the preceding article that there is a nearly fixed ratio between $E_{0}$ and $E_{n}$ of a synchronous converter. The ratio of the direct-current output of a converter $\left(I_{0}\right)$ to the effective value of the alternating current entering at a collector ring $\left(I_{r}\right)$ is subject to greater variations than the voltage ratio. The voltage ratio must be known with considerable accuracy if one is to be able to design a transformer to deliver alternating current to a converter which in its turn is to deliver direct current at a specified voltage; an exact knowledge of the current ratio, on the other hand, is of no great importance. Indeed the current ratio is of importance chiefly in connection with the discussion of armature heating, which determines the power rating of the converter, and for this purpose an approximate value of the current ratio is sufficient.

In the discussion of the current relations of a synchronous converter we shall assume $(a)$ that the efficiency of the machine. is 100 per cent., that is, we shall assume that the intake of power is equal to the output of power, and $(b)$ that the alternating current $I_{n}$ flowing in the armature between two collector rings is

* See Appendix B for a description of the split-pole converter. 
exactly opposite in phase to the alternating electromotive force $B$ that is induced in that portion of the armature winding.

This second condition means that the synchronous converter is assumed to operate at unity power factor, and that the point $P$

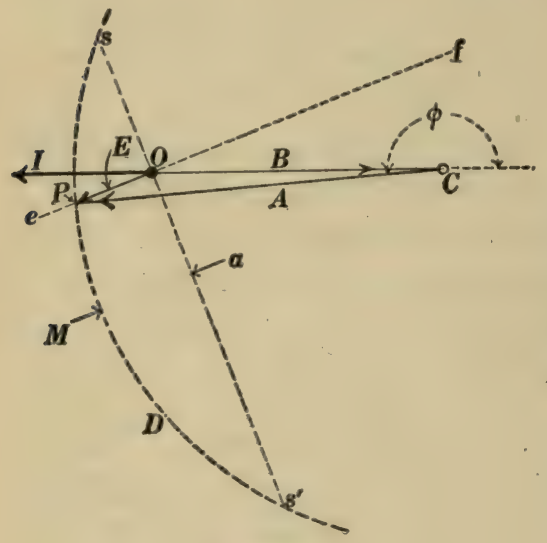

Fig. 153. of the clock diagram, Fig. I 50 , is located as shown in Fig. 153 , in which $B$ and $I$ are opposite in phase, so that, the angle $(B I)$ being equal to $180^{\circ}$, the power intake of the machine per phase is equal to $B I$. This second condition can in general be realized, for any given load on the converter, by adjusting the field excitation of the machine until the alternating current intake is a minimum as explained in section 2, of Art. 78 .

Current in each section of the converter armature. - Let $E_{0}$ be the electromotive force between direct-current brushes and $I_{0}$ the direct-current output of the machine. Then the power output is $E_{0} I_{0^{*}}$ Let $E_{n}$ be the effective value of the induced electromotive force in one section of the armature between adjacent collector rings and $I_{n}$ the effective value of the alternating current in the same section of the armature winding. Then, since $E_{n}$ and $I_{n}$ are assumed to be exactly opposite to each other in phase, the intake of power per phase is $E_{n} I_{n}$ and the total intake of power is $n E_{n} I_{n}$. Therefore, assuming Ioo per cent. efficiency, we have

$$
E_{0} I_{0}=n E_{n} I_{n}
$$

whence, substituting the value of $E_{n}$ in terms of $E_{0}$ from equation (ii) of Art. 90 and solving for $I_{n}$, we have

$$
I_{n}=\frac{\sqrt{2} I_{0}}{n \sin \frac{\pi}{n}}
$$


whence

$$
\begin{aligned}
& I_{2}=\frac{I_{0}}{\sqrt{2}}=0.707 I_{0} \\
& I_{3}=\frac{2 \sqrt{2}}{3 \sqrt{3}} \cdot I_{0}=0.544 I_{0} \\
& I_{4}=0.500 I_{0} \\
& I_{0}=0.471 I_{0}
\end{aligned}
$$

Current entering at each collector ring. - Figure 154 shows the points of attachment of collector rings to the armature winding of a synchronous converter, the two

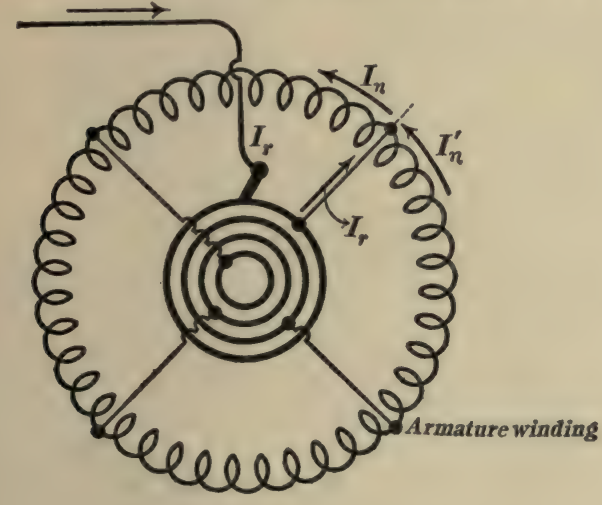

Fig. 154 .

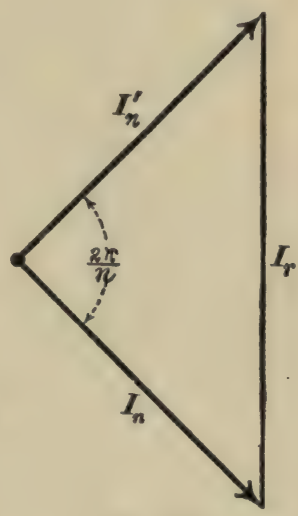

Flg. 155.

arrows $I_{n}$ and $I_{n}^{\prime}$ show the directions in which the currents in the two armature sections are considered as positive, and the arrow $I_{r}$ showes the positive direction of the current $I_{r}$. From this figure it is evident that $I_{r}=I_{n}-I_{n}^{\prime}$ (the vector difference of course). Therefore, since $I_{n}$ and $I_{n}{ }^{\prime}$ differ in phase by the angle $2 \pi / n$, as shown in Fig. 155, it is evident that the value of $I_{r}$ is

$$
I_{r}=2 I_{n} \sin \frac{\pi}{n}
$$

whence, using the value of $I_{n}$ from equation (i) above, we have

For a two-ring converter $I_{r}=1.414 I_{0}$

For a three-ring converter $I_{r}=0.942 I_{0}$

* This entire article applies to the two-pole machine (armature winding having two paths). In case of a multipolar machine having $p^{\prime}$ current paths through the armature, this equation becomes

$$
I_{n}=\frac{\sqrt{2} I_{0}}{p^{\prime} n \sin \frac{\pi}{n}}
$$


For a four-ring converter $I_{r}=0.707 I_{0}$

For a six-ring converter $I_{r}=0.471 I_{0}$

92. The armature heating of the synchronous converter. Synchronous converter ratings. - Inasmuch as the armature reaction of a synchronous converter is negligible, the power rating of such a machine is determined by armature heating. This power rating is always expressed in terms of the rating the same machine would have if used as an engine-driven direct-current generator, and the difference in rating of a machine when used as an engine-driven direct-current generator and when used as a synchronous converter is determined wholly by the $R I^{2}$ losses in the armature windings, inasmuch as the eddy current and hysteresis loss in the armature core is the same (nearly) in the two cases.

The following table gives the ratings of synchronous converters expressed in terms of the rating of the same machine when used as an engine-driven direct-current generator. It will be noted, for example, that the rating of a six-ring converter is nearly twice as great as the rating of the same machine when used as an engine driven direct-current generator, and it must be remembered that this increased rating refers to the increase of permissible direct-

TABLE.

POWER RATINGS OF SYNCHRONOUS CONVERTERS.

\begin{tabular}{c|c|c|c|c}
\hline $\begin{array}{c}\text { As engine driven } \\
\text { generator, }\end{array}$ & $\begin{array}{c}\text { As a two-ring } \\
\text { converter. }\end{array}$ & $\begin{array}{c}c \\
\text { As a three-ring } \\
\text { converter. }\end{array}$ & $\begin{array}{c}d \\
\text { As a four-ring } \\
\text { converter. }\end{array}$ & $\begin{array}{c}\text { As a six-ring } \\
\text { converter, }\end{array}$ \\
\cline { 1 - 2 } & 0.85 & $\mathbf{1 . 3 2}$ & $\mathbf{1 . 6 2}$ & $\mathbf{1 . 9 2}$ \\
\hline 1.00 & 0.62 &
\end{tabular}

current output, the voltage of the machine being fixed. Thus a six-ring converter rated as a IOOO ampere direct-current generator would be rated as a 1920 ampere synchronous converter and this increased current rating necessitates a much larger commutator so as to permit of a greatly increased contact surface between the direct-cuirrent brushes and the commutator. In fact a striking feature of the synchronous converter is the large size of 
its commutator as compared with the commutator of a directcurrent generator having the same armature and field magnet

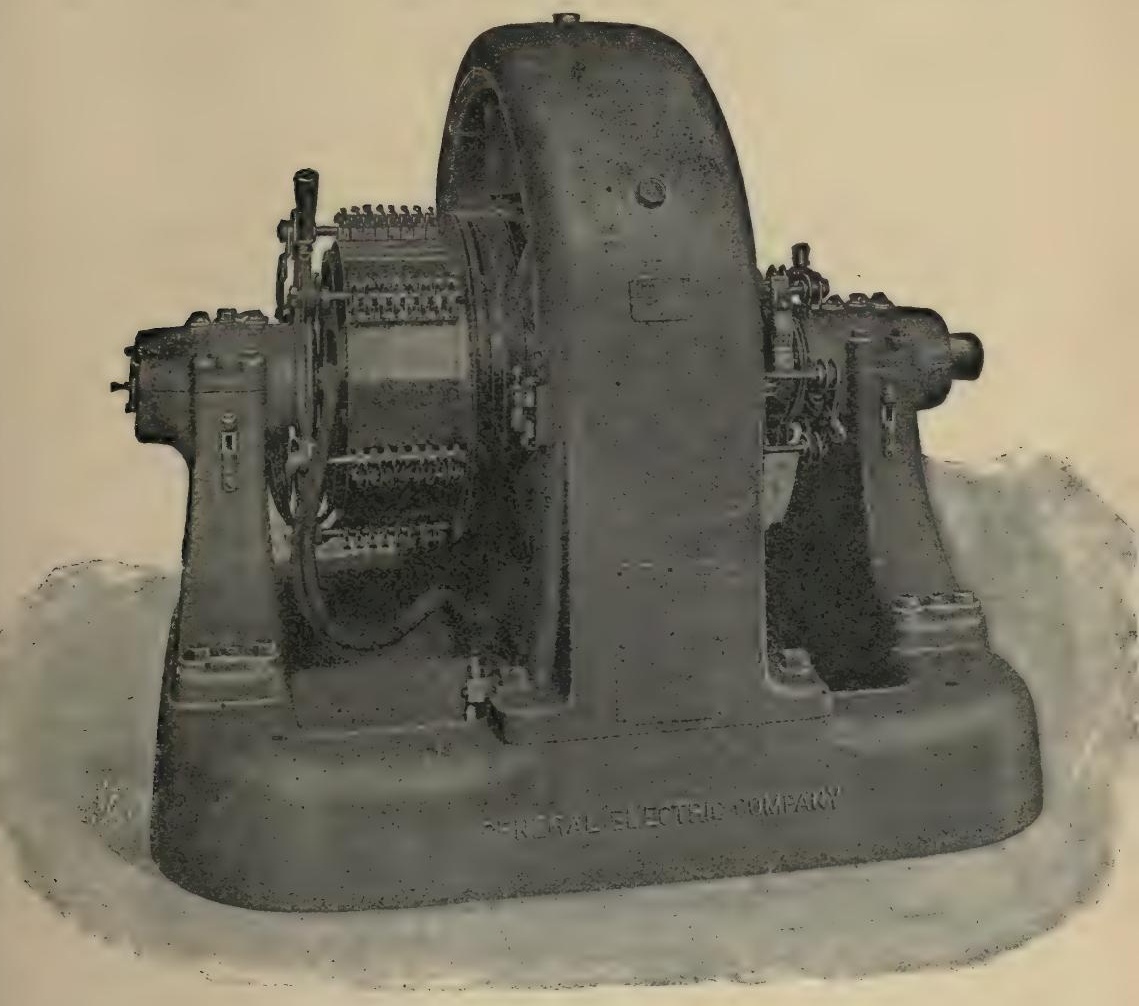

$300 \mathrm{kw}$. Three-phase Rotary Converter.

Fig. 156.

structure. Thus, Fig. I 56 shows a 300 kilowatt, three-ring, synchronous converter of the General Electric Company.

93. Instantaneous current in a given armature conductor of a rotary converter. - Let $r$ and $r^{\prime}$, Fig. 157, be the points of attachment of adjacent collecting rings of an $n$-ring converter, and let the line $O M$ bisect the arc $r r^{\prime}$. Consider an armature conductor $c$ between $r$ and $r^{\prime}$, and let the angle $c O M$ be represented by $\alpha$. The largest possible value of $\alpha$ is $\pi / n$ or one half of the angle between $r$ and $r^{\prime}$.

Let $\omega t$ be the angle between $O M$ and the axis of the field. Then the alternating current between the collecting rings $r$ and $r^{\prime}$, that is, the alternating current in the conductor $c$, is at its maximum value $\sqrt{2} I_{n}$ when $\omega t=0$. Therefore 
the expression for the instantaneous value of this current is $\sqrt{2} I_{n} \cos \omega t$.

When the conductor $c$ is at brush $b^{\prime}, \omega t=\left(90^{\circ}-\alpha\right)$, when the conductor $c$ reaches brush $b, \omega t=\left(270^{\circ}-\alpha\right)$, and when conductor $c$ reaches brush $b^{\prime}$ again $\omega t=\left(450^{\circ}-\alpha\right)$, and so on. Each time the conductor $c$ passes a brush the direct current $I_{0} / 2$ (discussion applies to a 2-pole machine) in conductor $c$ is reversed. Therefore the total current in conductor $c$ is

$$
i=\sqrt{2} I_{n} \cos \omega t \pm \frac{I_{0}}{2}
$$

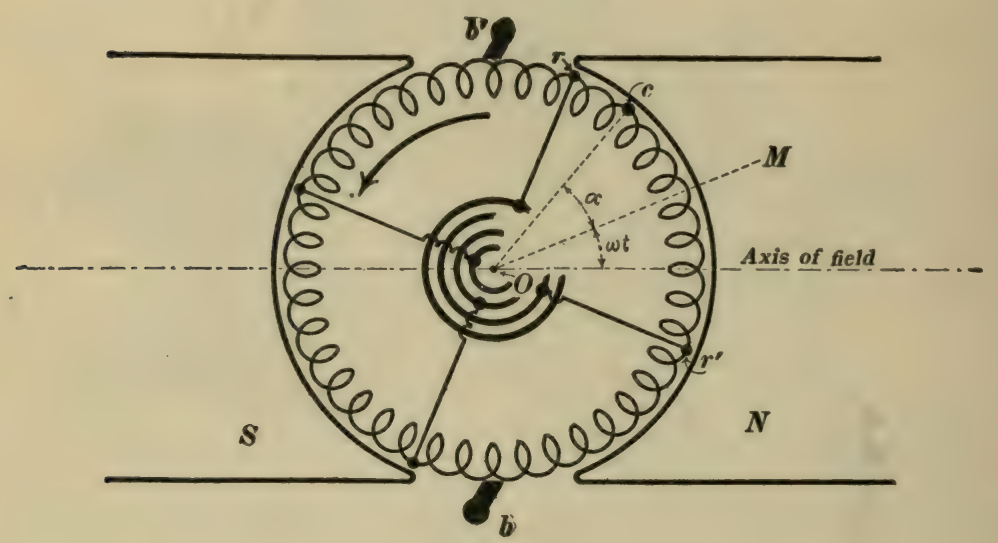

Fig. 157.

The + sign is to be taken between $\omega t=\left(90^{\circ}-\alpha\right)$ and $\omega t=\left(270^{\circ}-\alpha\right)$, the - sign is to be taken between $\omega t=\left(270^{\circ}-\alpha\right)$ and $\omega t=\left(450^{\circ}-\alpha\right)$, the + sign again between $\left(450^{\circ}-\alpha\right)$ and $\left(630^{\circ}-\alpha\right)$, and so on. The angle $\alpha$ determines simply the phase of the alternating current in the conductor at the instant that the direct current is reversed.

The meaning of equation (i) is made more clear perhaps by the curves shown in Figs. 158 and 159. The upper curves in each figure are the component current curves ; that is, the ordinates of the square-wave curve represent that part of the current in the given conductor which depends upon the direct-current output of the machine, and the ordinates of the sine curve represent that part of the current in the given conductor which depends upon the alternating-current intake of the machine. The alternating currents are assumed to be exactly opposite in phase to the alternating electromotive forces of the machine.

94. The heating of the armature conductors of a rotary converter. - Equation (i) Art. 93 expresses the instantaneous value of the actual current in a given armature conductor of a rotary converter during the time that this conductor is passing from one direct-current brush to the other; that is, from $\omega t=-\left(90^{\circ}+\alpha\right)$ to $\omega t=\left(90^{D}-\alpha\right)$. The current passes through a similar set of values during the next half revolution of the armature. The average rate at which heat is generated in the given conductor is proportional to the average value of $i^{2}$ [equation (i) Art. 93] during the time 


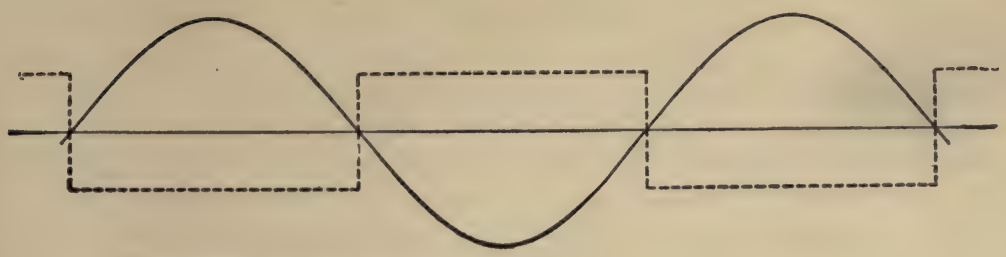

Component curves $n=2, \alpha=0$.

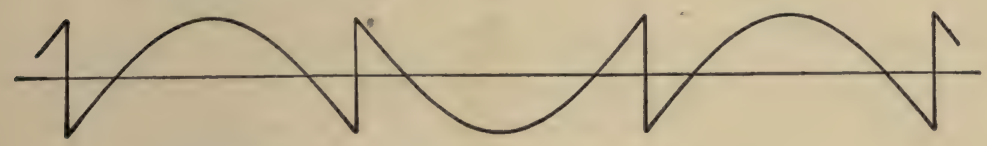

Resultant curve $n=2, \alpha=0$.

Fig. 158.

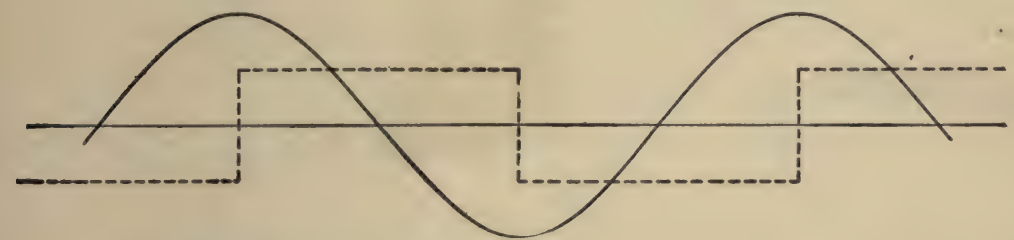

Component curves $n=2, \alpha=90^{\circ}$.

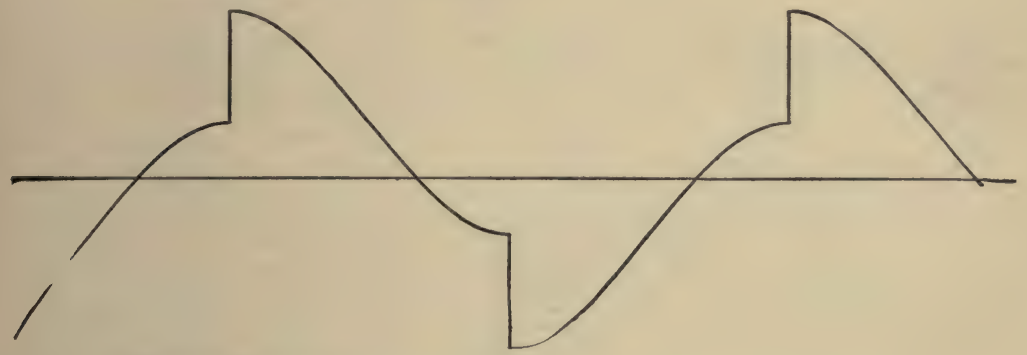

Resultant curve $n=2, \alpha=90^{\circ}$.

Fig. 159.

$\omega t=-\left(90^{\circ}+\alpha\right)$ to $\omega t=\left(90^{\circ}-\alpha\right)$. Therefore

The average rate of gener- $\frac{I_{0}^{2}}{4}\left(\mathrm{x}-\frac{\mathrm{I} 6 \cos \alpha}{\pi n \sin \pi / n}+\frac{8}{n^{2} \sin ^{2} \pi / n}\right)$ tor $c$ is proportional to :

The rate at which heat would be generated in a given conductor by the direct current alone would be proportional to $I_{0}{ }^{2} / 4$, and equation (ii) shows that the con. ductor $c$ has

$$
\left(\mathrm{r}-\frac{16 \cos \alpha}{\pi n \sin \pi / n}+\frac{8}{n^{2} \sin ^{2} \pi / n}\right)
$$


times as much heat generated in it as would be generated in it by the direct current alone.

The conductors midway between the points of attachment of the collector rings $(\alpha=0)$ are heated least, and the conductors near the points of attachment of the collector rings $(\alpha= \pm \pi / n)$ are heated most. For example, in a two-ring converter ( $n=2$ ) the conductors midway between the points of attachment of the collector rings $(\alpha=0)$ have only 0.453 as much heat generated in them as would be generated in them by the direct current alone, and the conductors near the points of attachment of the collector rings $\left(\alpha= \pm 90^{\circ}\right)$ have three times as much heat generated in them as would be generated in them by the direct current alone.

95. The average heating of the entire armature of a rotary converter. - The average heating over the entire armature is found by integrating the equation (ii) Art. 94, with respect to $\alpha$ from $\alpha=-\pi / n$ to $\alpha=+\pi / n$, and dividing the result by $2 \pi / n$. This gives:

Average heating of armature of
ring converter is proportional to :

The average heating is therefore $\left(1-\frac{16}{\pi^{2}}+\frac{8}{n^{2} \sin ^{2}} \frac{\pi / n}{\pi / n}\right) *$ times as great as the heating of the armature by the direct current alone. Therefore an $n$-ring converter can put out

$$
\frac{\mathrm{I}}{\sqrt{\mathrm{I}-\frac{\mathrm{I}}{\pi^{2}}+\frac{8}{n^{2} \sin ^{2} \pi / n}}}
$$

times as much direct current as the same machine can when used as a simple directcurrent generator, for the same total armature heating. The table of ratings given in Art. 92 is calculated in this way.

\section{The use of the double-current machine as a direct-current} three-wire generator. - When the synchronous converter is driven by an engine and used to deliver both alternating current and direct current, it is called a double-current generator. Such a machine can be used to deliver direct current to an Edison threewire distributing system as mentioned on page 273 of the first volume of this treatise. This use of the double-current generator is due to Dobrowolsky. The essential features of this arrangement are shown in Fig. I60 for a two-ring machine. The voltage between the direct-current brushes $a$ and $b$ is, say 220 volts, and it is desired to supply direct current to an Edison three-

* Generally less than unity. 
wire system and maintain I Io volts (direct) between the middle main and each outside main. To do this the current in the middle main must be delivered to the armature at a potential midway between the potentials of the direct-current brushes.

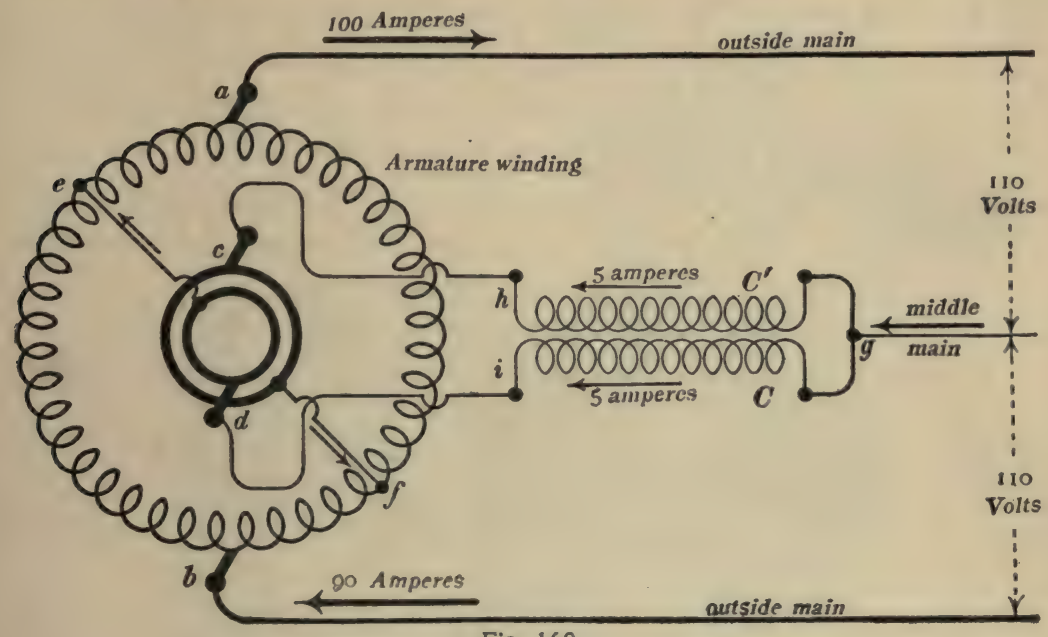

Fig. 160 .

The two coils $C$ and $C^{\prime}$ represent the two similar coils of a transformer so wound that the point $g$ is, as it were, the middle of one continuous winding the terminals of which, namely, $h$ and $i$, are connected to the two alternating-current brushes $c$ and $d$ of the machine. The terminals $h$ and $i$ are at the same potentials as the points $e$ and $f$ of the armature winding respectively. Half of the inflowing current in the middle main flows down hill, as it were, through coil $C^{\prime}$ to the point $f$ and delivers energy to coil $C^{\prime}$. This energy is transformed into coil $C$ and it causes the other half of the current in the middle main to flow up-hill, as it were, through coil $C$ to the point $e$.

In case the machine is engine-driven, all of the current entering at $e$ flows up-hill to brush $a$, and all of the current entering at $f$ flows up-hill to the brush $a$, because of the electromotive forces induced in the armature winding by its rotation. 


\section{CHAPTER $\mathrm{X}$.}

THE TRANSFORMER.

97. The transformer consists of a magnetic circuit of laminated iron linking with two separate and distinct coils of wire. Alternating current is supplied to one of these coils from an alternator or other source, this alternating current causes rapid reversals of magnetic flux through the magnetic circuit, and these reversals of flux induce an alternating electromotive force in the other coil

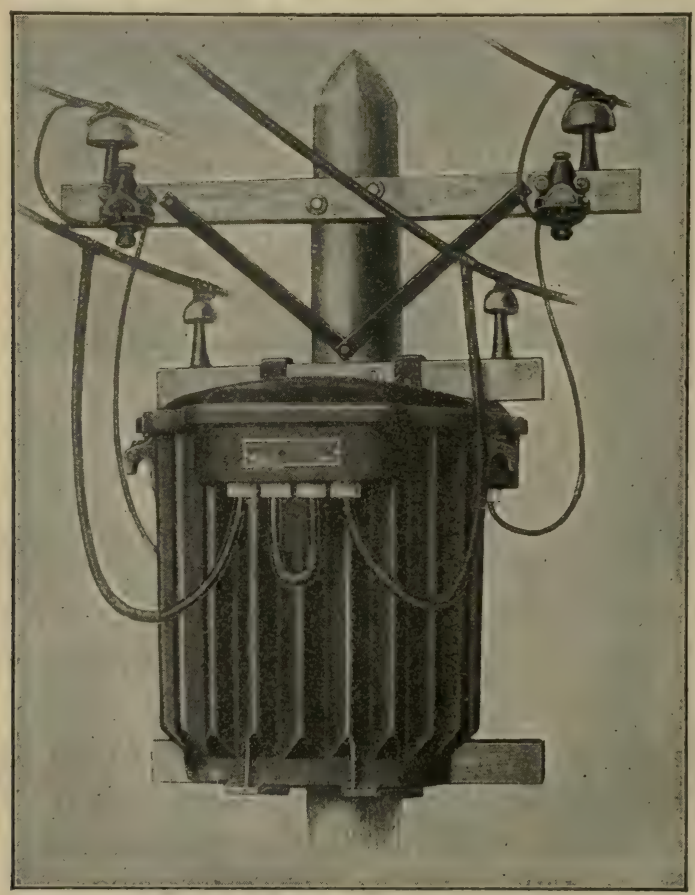

Fig. 161.

which delivers alternating current to a receiving circuit. The coil to which alternating current is supplied is called the primary coil, the coil which delivers current to a receiving circuit is called the secondary coil, and the laminated iron which forms the mag- 
netic circuit is called the transformer core, although it may have a shape more suggestive of a shell than of a core.

Step-up and step-down transformation. - Usually one coil of a transformer has many more turns of wire than the other. When the coil of few turns is the primary coil, the transformer takes large current at low electromotive force and delivers small current at high electromotive force. This is called step-up transformation.

When the coil of many turns is the primary, the transformer takes small current at high electromotive force and delivers large current at low electromotive force. This is called stepdown transformation. The object of step-up and step-down transformation is explained in Article 3.

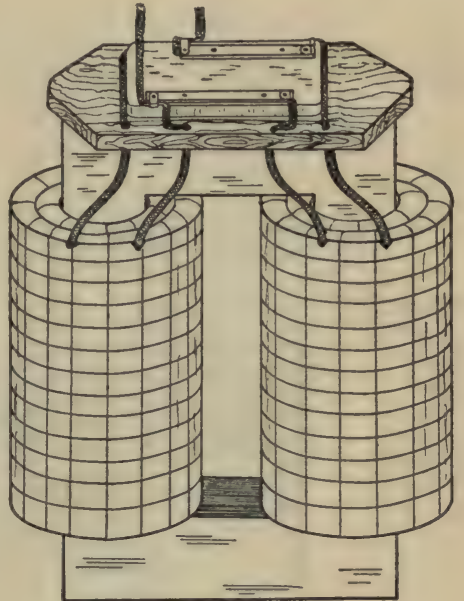

Fig. 162.

Mounting of transformers. - Transformers are usually enclosed

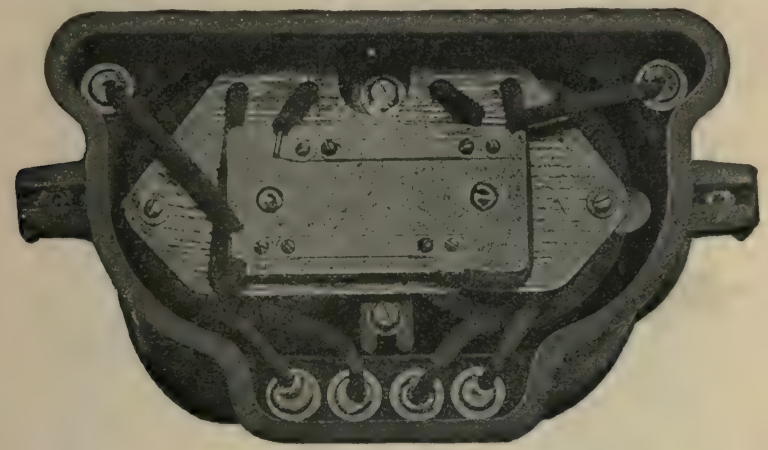

Fig. 163.

in metal cases, and the case is usually filled with oil; the purpose of the oil being partly to improve the insulation of the coils 


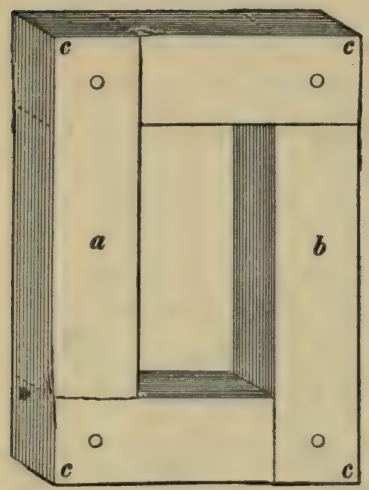

Fig. 164.

by impregnating the fibrous insulating material, and partly to facilitate cooling by carrying the heat from the core and coils to the containing case. Especial provision must be made for cooling very large transformers. For this purpose passage ways or ducts are left through the core and coils, and the oil circulates through these ducts and is itself cooled by a coil of water pipe placed in the top of the containing case. In some cases a blast of air is driven through the transformer by a fan-blower.

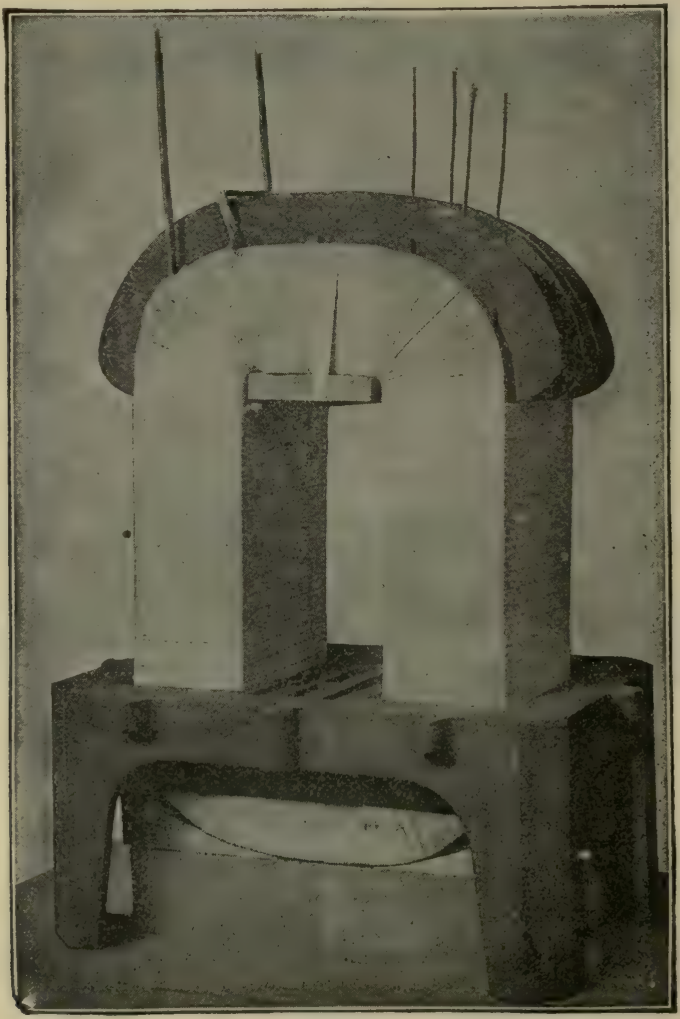

Fig. 165. 
Figure I6I shows a small transformer mounted on a pole, taking current from street mains (upper wires in the figure) at I, IOO volts, and delivering current to house mains (lower wires in the figure) at I Io volts. The two small cases attached to the upper cross-arm and through which the wires leading to the

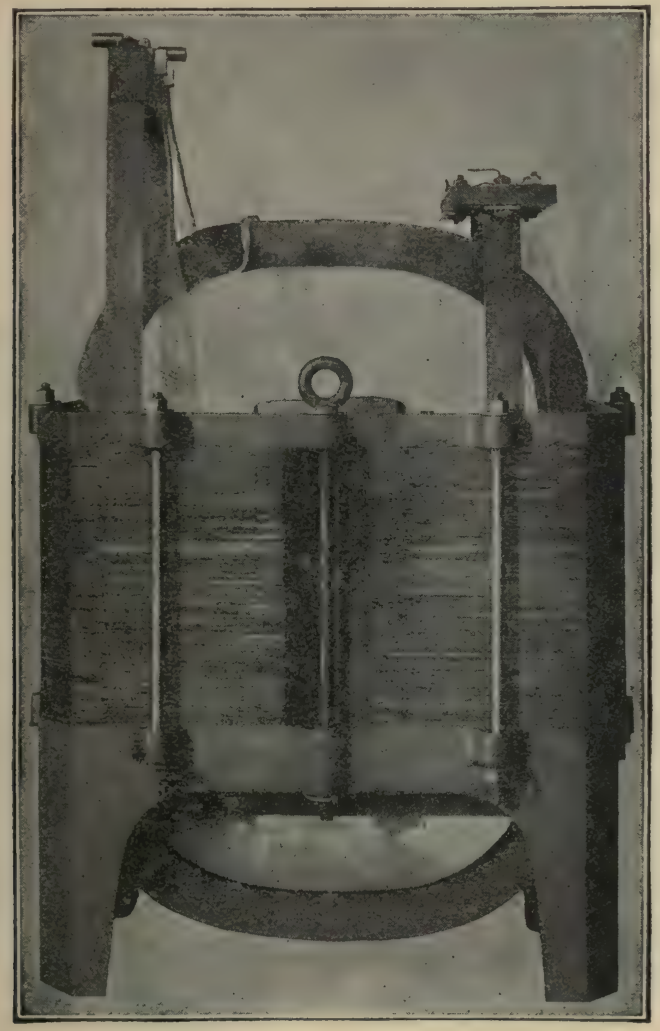

Fig. 166.

primary coil of the transformer pass, are hollow porcelain insulators with fusible cut-outs mounted inside. Figure 162 shows a small transformer with its connection board on top removed from its case, and Fig. 163 is a top view of the open case with the transformer in place. In this particular transformer the terminals of the fine wire (primary) coils, only, are attached to the connec- 
tion board (the figure shows the two primary coils connected in parallel with each other), and the four terminals of the two secondary coils pass out of the case as shown in the lower portion of Fig. 163 .

The core-type transformer. - A transformer in which the coils of wire surround a more or less elongated core of laminated iron

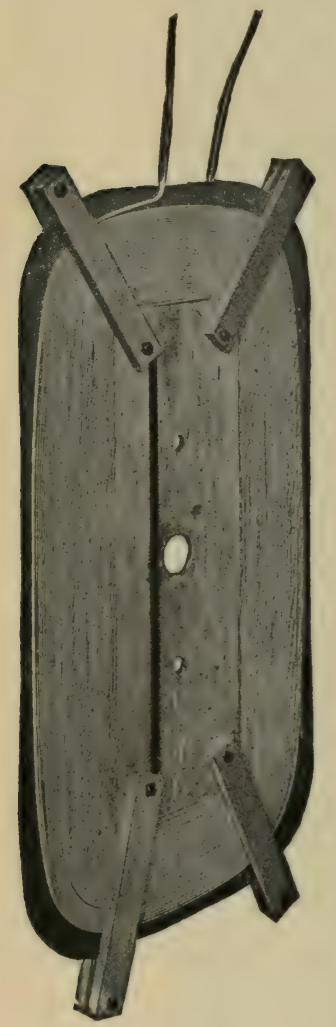

Fig. 167.

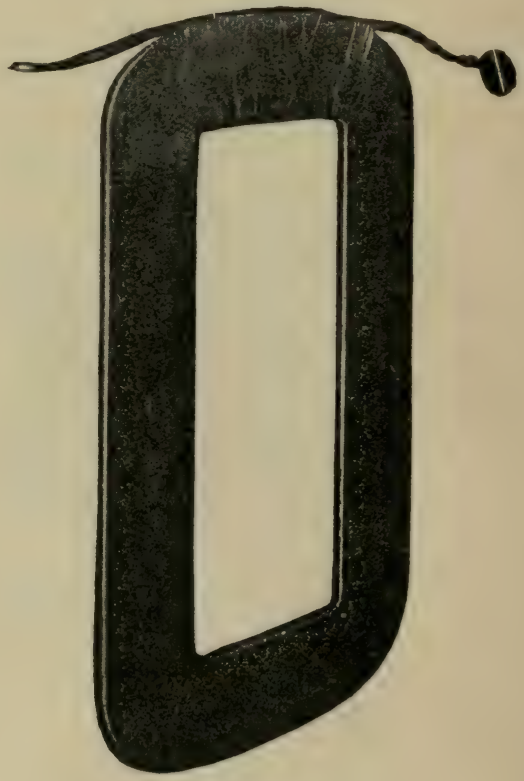

Fig. 168.

is called a core-type transformer. Figure 162 shows a core-type transformer of the Westinghouse Electric Company, and Fig. I64 shows the laminated iron core of this transformer. In this transformer half of the primary coil and half of the secondary coil are wound on each leg $a$ and $b$, Fig. 164, of the core, the 
fine wire (high voltage) coils being wound over the coarse wire (low voltage) coils. The core, Fig. I64, is built up of rectangular strips of sheet iron which interleave with each other at the corners $c c c c$.

The shell-type transformer. - A transformer in which the coils form an elongated structure shaped like Fig. I64, and are surrounded by laminated iron, is called a shell-type transformer.

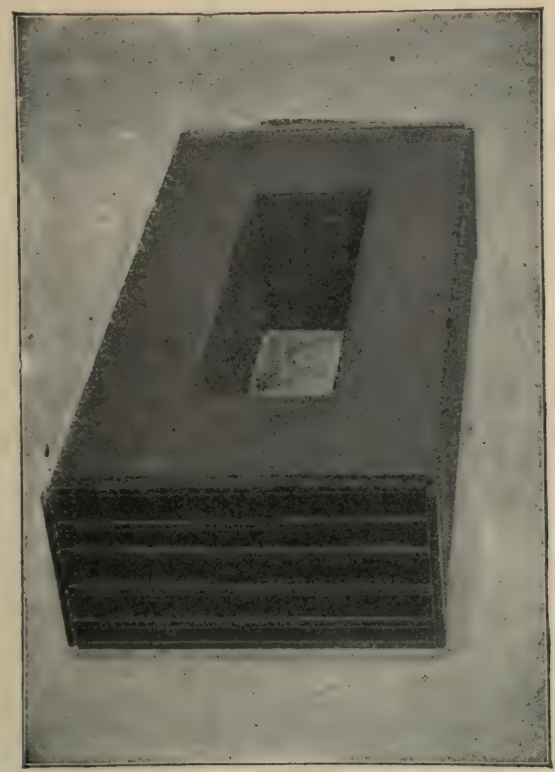

Finished coi's.

Fig. 169.

Figure 165 shows the assembled windings of a small shell-type transformer of the Allis-Chalmers Company, and Fig. I66 shows the coils surrounded by the laminations.

A somewhat clearer idea of the structure of the shell-type transformer may be obtained from Figs. 167, 168, I69 and 170, which represent a large shell-type transformer of the General Electric Company. Figure 167 shows one of the flat sections of the windings in the process of construction; two such coils 
are placed side by side with a thin sheet of insulating material between them, as shown in Fig. 16\%, they are connected together as a single coil or section leaving both the terminals on the outer rim, and wound with tape as shown in Fig. 168. A number of such sections are placed side by side, primary and secondary sections alternating, * with intervening spaces for the circulation of oil or air for cooling, and the whole is bound together as shown in Fig. I69. This complete coil structure is then mounted on a base and the laminations are built up around each leg, as shown in Fig. I 70 , the inner edges of the laminations being separated from the

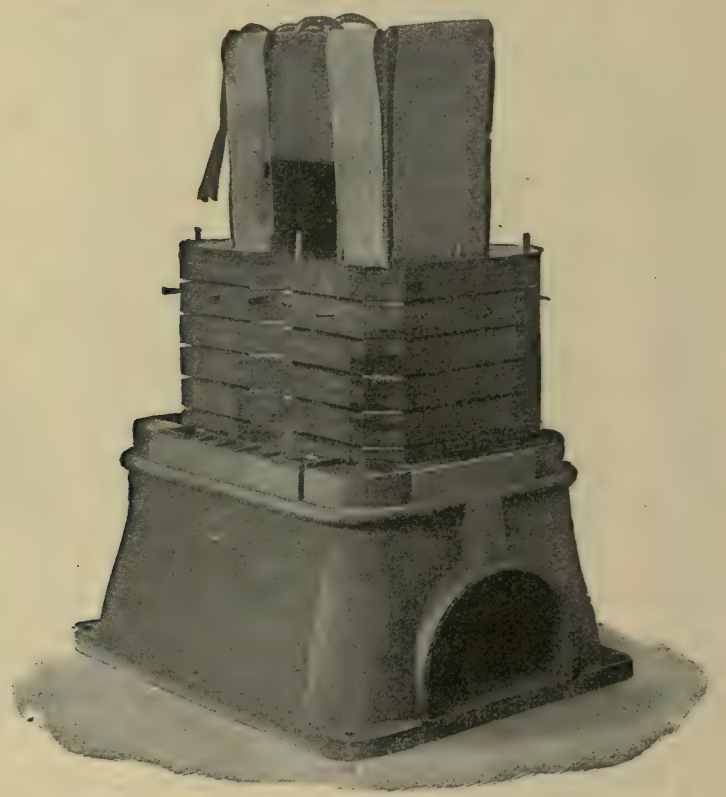

Fig. 170 .

coil structure by vertical strips of very tough insulating material, and spaces being left between the laminations at intervals, as shown, for the circulation of oil or air.

Figure I 7 I shows a large shell-type transtormer of the Westing-

* This detail of construction has for its object the reduction of magnetic leakage as explained in chapter XI. 
house Electric Company without the containing case, but with

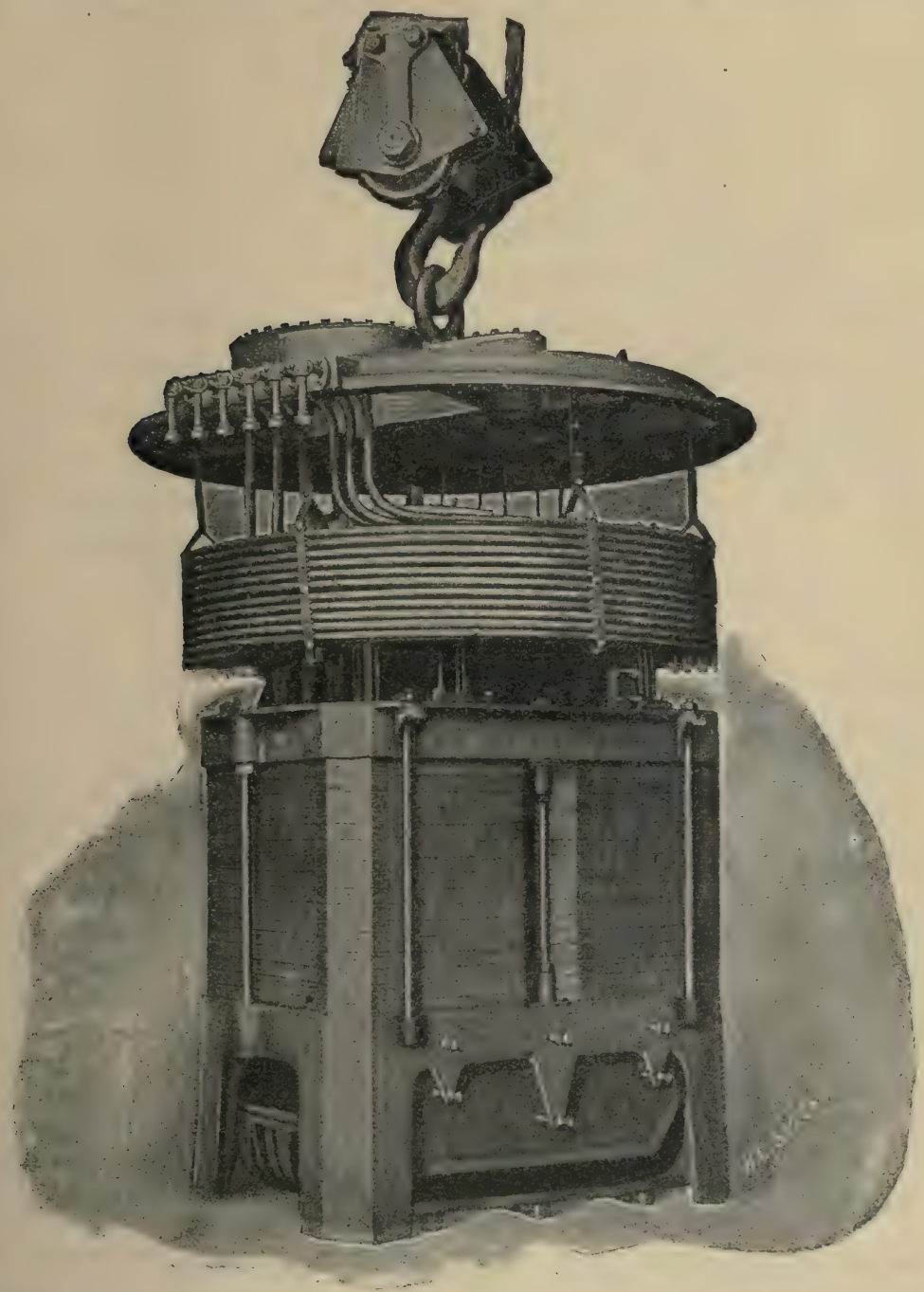

Large Transformer without Case - Showing Cover and Cooling Coils in Assembled Position.

Fig. 171.

the cover and the coils of water pipe for cooling. shown in the assembled position. 
98. The actual transformer and the ideal transformer. - The action of a transformer would be very simple $(a)$ if the coils of wire had zero resistance, $(b)$ if there were no magnetic leakage, that is, if all of the magnetic flux which links with one coil also linked with the other coil, and $(c)$ if the magnetic reluctance of the iron core were zero. A transformer satisfying these conditions would be called an ideal transformer. In an actual transformer, however, the coils always have more or less resistance ; some of the magnetic flux, the so-called leakage flux, links with one coil and does not link with the other; and a certain amount of magnetomotive force (ampere-turns) is necessary to force the magnetic flux through the transformer core. The effects of these things on the action of the transformer is quite complicated, but in many respects the behavior of the actual transformer approximates closely to the behavior of the ideal transformer. All operating engineers in dealing with transformers, make use of the very simple theory of the ideal transformer, whereas designing engineers find it necessary to take account of the effects of coil resistance, magnetic leakage, and core reluctance.

Transformer action. - In the following discussion $N^{\prime}$ represents the number of turns of wire in the primary coil and $N^{\prime \prime}$ represents the number of turns of wire in the secondary coil, the coils are assumed to have negligible resistance, and all of the flux which passes through one coil is assumed to pass through the other coil also. The transformer core, however, is not assumed to have zero reluctance.

Ratio of primary current to secondary current. - Aside from resistance, the only thing which opposes the flow of current through the primary coil is the reacting electromotive force induced in the primary coil by the reversals of magnetization of the core. The greater the range of this magnetization the greater the value of the reacting electromotive force. The combined magnetizing action of the primary and secondary coils is always such as to magnetize the core to that degree which will make the reacting electromotive force in the primary coil equal to the electromotive 
force of the alternator which is forcing current through the primary coil. Resistance of primary coil neglected.

When the secondary coil is on open circuit, just enough current flows through the primary coil to produce the degree of magnetization above specified. Let this value of the primary current, which is called the magnetizing current, be represented by $M$. When current $I^{\prime \prime}$ is taken from the secondary coil, additional current $I^{\prime}$, called the load current, flows through the primary coil. The current $M$ still suffices to magnetize the core, and the magnetizing action of $I^{\prime \prime}$ is exactly neutralized by the equal and opposite magnetizing action of $I^{\prime}$. The magnetizing action of $I^{\prime \prime}$ is measured by the product $N^{\prime \prime} I^{\prime \prime}$, and the magnetizing action of $I^{\prime}$ is measured by the product $N^{\prime} I^{\prime}$, so that, ignoring algebraic signs, we have

$$
\text { or } \quad \begin{aligned}
N^{\prime} I^{\prime} & =N^{\prime \prime} I^{\prime \prime} \\
\frac{I^{\prime}}{I^{\prime \prime}} & =\frac{N^{\prime \prime}}{N^{\prime}}
\end{aligned}
$$

Ratio of primary electromotive force to secondary electromotive force. - The rapid reversals of magnetization of the iron core induce a certain electromotive force $\alpha$ in each turn of wire surrounding the core. Therefore the total electromotive force induced in the primary coil is $N^{\prime} a$. This is the reacting electromotive force in the primary coil and it is equal and opposite, as pointed out above, to the electromotive force $E^{\prime}$ which is pushing current through the primary coil ; so that, ignoring signs, we have

$$
E^{\prime}=N^{\prime} a
$$

Similarly, the total electromotive force, $E^{\prime \prime}$, induced in the secondary coil is

Therefore

$$
E^{\prime \prime}=N^{\prime \prime} a
$$

$$
\overline{E^{\prime}}=\frac{N^{\prime}}{N^{\prime \prime}}
$$

* The above discussion should in strictness refer, primarily, to instantaneous values of $I^{\prime}$ and $I^{\prime \prime}$ and to instantaneous values of $E^{\prime}$ and $E^{\prime \prime}$. Thus $i^{\prime}$ and $i^{\prime \prime}$ 
Approximate equality of input and output of power. - The output of power by ąn ideal transformer would be exactly equal to the input of power. In an actual transformer the output of power is usually 97 per cent. or more of the power input when the transformer is fully loaded; that is the $R I^{2}$ losses in the coils and the eddy current and hysteresis losses in the core are together usually less than three per cent. of the full-load output of a good transformer. This high efficiency of the actual transformer shows how closely the actual transformer approximates to the ideal transformer in its action.

99. Particular cases of transformer action. (Harmonic electromotive forces and currents.) - The above discussion is not limited

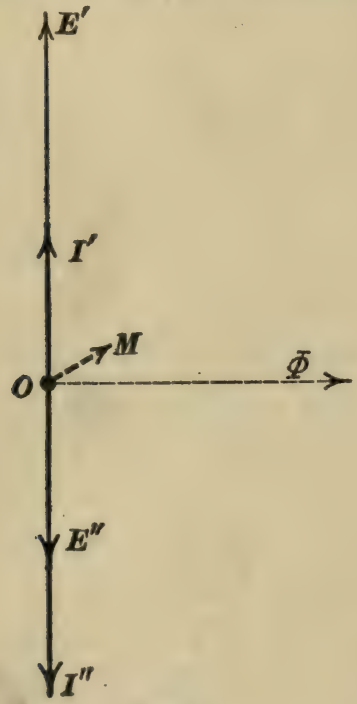

Fig. 172.

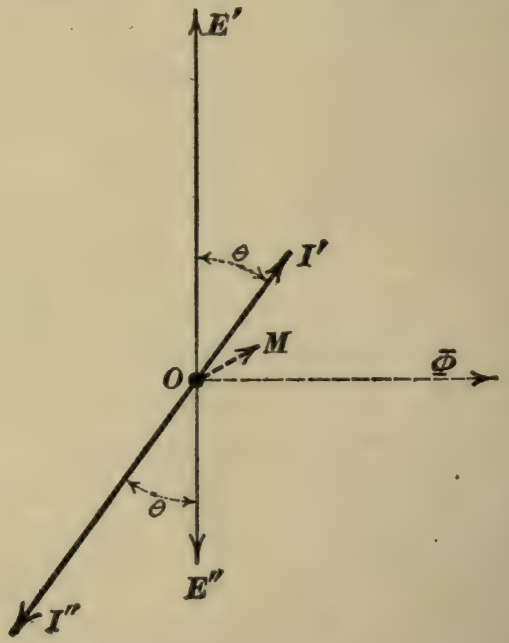

Fig. 173.

to any particular wave-form of electromotive force and current, but in order to show the voltage and current relations of a transformer in a clock diagram it is necessary to consider the case of are at each instant opposite to each other and in the ratio $N^{\prime \prime} / N^{\prime}$; and $e^{\prime}$ and $e^{\prime \prime}$ are at each instant opposite to each other and in the ratio $N^{\prime} / N^{\prime \prime}$. Therefore $I^{\prime}$ and $I^{\prime \prime}$ are opposite or $180^{\circ}$ apart in phase, and $E^{\prime}$ and $E^{\prime \prime}$ are opposite or $180^{\circ}$ apart in phase. 
harmonic electromotive forces and currents, inasmuch as nonharmonic electromotive forces and currents cannot be represented in a clock diagram.

The magnetizing current, $M$, of a transformer is not harmonic and it cannot, therefore, in strictness, be represented by a line in a clock diagram. In Figs. 172, I73 and I74, however, $M$ is represented by a line. The current so represented is called the " equivalent harmonic magnetizing current" (see Art. I I 5).

Case 1. Non-inductive receiving circuit. - In this case the current $I^{\prime \prime}$ produced in the receiving circuit is in phase with

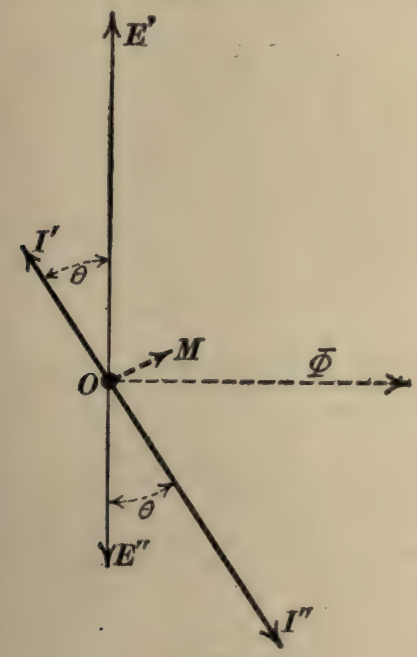

Fig. 174.

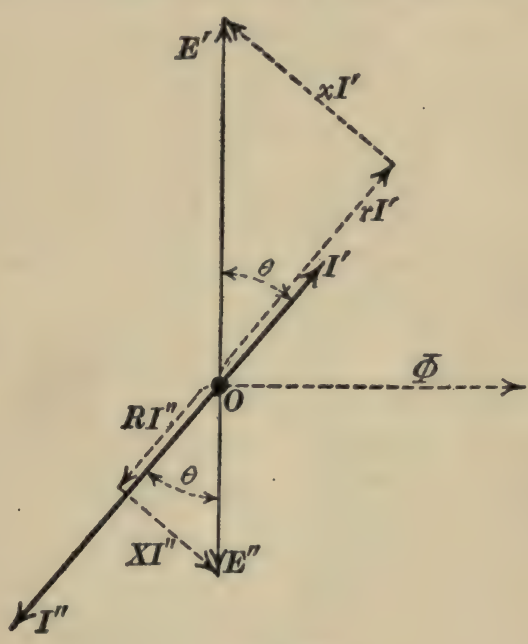

Flg. 175.

$E^{\prime \prime}$, as shown in Fig. I72, and, according to Art. 98, I' must, therefore, be in phase with $E^{\prime}$. The line $O M$ represents the magnetizing current in the primary coil, and the vector sum of $O M$ and $I^{\prime}$ represents the total primary current. The vector $O \Phi$ represents the harmonically varying core flux. Figures I 72 to 175 all represent a $2:$ I step-down transformation.

Case 2. Inductive receiving circuit. - In this case the current $I^{\prime \prime}$ produced in the receiving circuit lags behind $E^{\prime \prime}$ by an angle $\theta$ of which the tangent is equal to $X / R$, where $X$ is the reactance and $R$ is the resistance of the receiving circuit. 
The load current $I^{\prime}$ in the primary coil being opposite to $I^{\prime \prime}$ in phase is $\theta$ behind $E^{\prime}$, as shown in Fig. 173 .

Case 3. Receiving circuit containing a condenser. - In this case, which is shown in Fig. I74, $I^{\prime \prime}$ is ahead of $E^{\prime \prime}$ in phase and $I^{\prime}$ is ahead of $E^{\prime}$ in phase, as indicated in the figure.

100. Equivalent resistance and reactance of an ideal transformer feeding a given receiving circuit. - The primary of a transformer takes from the supply mains a definite current at a definite phase lag when the secondary of the transformer is delivering current to a given circuit. Consider a simple circuit, of resistance $r$ and reactance $x$, which, if connected directly to the supply mains, would take the same current that is delivered to the primary of the transformer as above and at the same phase lag. This simple circuit is equivalent to the transformer and the secondary receiving circuit, and $r$ and $x$ are called the equivalent primary resistance and the equivalent primary reactance, respectively, of the secondary receiving circuit.

In using the ideas of equivalent primary resistance and reactance, magnetizing current is always ignored.

Let $R$ be the actual resistance of the secondary receiving circuit, $X$ its actual reactance, $N^{\prime}$ the number of primary turns, and $N^{\prime \prime}$ the number of secondary turns; then

$$
r=\left(\frac{N^{\prime}}{N^{\prime \prime}}\right)^{2} R
$$

and

$$
x=\left(\frac{N^{\prime}}{N^{\prime \prime}}\right)^{2} X
$$

To derive these two equations, resolve the primary electromotive force $E^{\prime}$, Fig. I 75 , into components parallel to and perpendicular to $I^{\prime}$ as shown. The component parallel to $I^{\prime}$ is $r I^{\prime}$ and the component perpendicular to $I^{\prime}$ is $x I^{\prime}$. The triangle whose sides are $E^{\prime}, r I^{\prime}$ and $x I^{\prime}$ is similar to the triangle whose sides are $E^{\prime \prime}, R I^{\prime \prime}$ and $X I^{\prime \prime}$, therefore

$$
\frac{x I^{\prime}}{X I^{\prime \prime}}=\frac{E^{\prime}}{E^{\prime \prime}}
$$


THE TRANSFORMER.

and

But

and

$$
\frac{r I^{\prime}}{R I^{\prime \prime}}=\frac{E^{\prime}}{E^{\prime \prime}}
$$

$$
\frac{E^{\prime}}{E^{\prime \prime}}=\frac{N^{\prime}}{N^{\prime \prime}}
$$

$$
\frac{I^{\prime}}{I^{\prime \prime}}=\frac{N^{\prime \prime}}{N^{\prime}}
$$

so that

$$
\begin{aligned}
& r=\left(\frac{N^{\prime}}{N^{\prime \prime}}\right)^{2} R \\
& x=\left(\frac{N^{\prime}}{N^{\prime \prime}}\right)^{2} X
\end{aligned}
$$

Example of the use of the ideas of equivalent primary resistance and reactance. - A IO: I step-down transformer has a resistance of 150 ohms and a reactance of $100 \mathrm{ohms}$ connected in series with its primary coil which takes current from 2,000 volt supply mains, and the secondary coil of the transformer delivers current to a receiving circuit of which the resistance is $2.5 \mathrm{ohms}$ and the reactance is 2.0 ohms. Required the values of $I^{\prime}, I^{\prime \prime}, E^{\prime}, E^{\prime \prime}$, and all the phase angles. Multiplying $2.5 \mathrm{ohms}(=R)$ and $2.0 \mathrm{ohms}(=X)$ by $\left(\frac{10}{1}\right)^{2}$ we have the primary equivalent values, namely, $250 \mathrm{ohms}(=r)$ and $200 \mathrm{ohms}(=x)$, so that the primary current flows, as it were, through a circuit of which the resistance is $150 \mathrm{ohms}+250 \mathrm{ohms}$ and of which the reactance is $100 \mathrm{ohms}+200 \mathrm{ohms}$. That is, the impedance of this equivalent simple circuit is $\sqrt{(400)^{2}+(300)^{2}}=500 \mathrm{ohms}$, so that the current is 2,000 volts $\div 500$ ohms or 4 amperes. Taking this current as the reference axis, the components of the supply voltage are 4 amperes $\times 400 \mathrm{ohms}=\mathrm{r}, 600$ volts parallel to the current and 4 amperes $\times 300$ ohms $=1,200$ volts perpendicular to the current; the components of $E^{\prime}$ are 4 amperes $\times 250 \mathrm{ohms}=1,000$ volts parallel to the current and 4 amperes $\times 200$ ohms $=800$ volts perpendicular to the current ; 
the components of $E^{\prime \prime}$ are $\frac{1}{10} \times 1,000$ volts and $\frac{1}{10} \times 800$ volts parallel to and perpendicular to the current respectively ; and the value of $I^{\prime \prime}$ is of course 40 amperes. The student should represent these details in a clock diagram.

101. Relation between core flux and primary voltage. - The electromotive force in abvolts induced in each turn of wire of the primary coil of a transformer is at each instant equal to the rate of change of the core flux $\phi$, so that the total induced electromotive force in abvolts is equal to $N^{\prime} \cdot d \phi / d t$, and this induced electromotive force is equal and opposite* to the primary voltage $\epsilon^{\prime}$ at each instant (resistance of primary coil negligibly small), therefore

$$
e^{\prime}=N^{\prime} \frac{d \phi}{d t}
$$

where $e^{\prime}$ is expressed in abvolts.

Example of core flux for a particular non-harmonic electromotive force. - The relation between core flux and primary voltage is simplest for the case in which the electromotive force curve is rectangular like the full-line curve in Fig. 176. Suppose, for

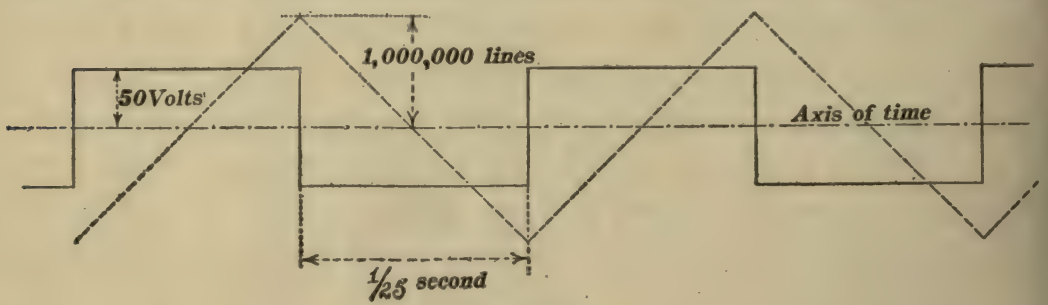

Fig. 176.

example, that a 50 -volt storage battery is connected through a reversing switch to the primary coil of a transformer, imagine that the switch is reversed 25 times per second, and let it be required to determine the curve of which the ordinates represent the varying value of the core flux, the primary coil having IOO turns of wire.

* In fact the induced electromotive force should properly be considered equal to $-N^{\prime} d \Phi / d t$ so that, placing $e^{\prime}$ equal and opposite to the induced voltage, we have equation (i). 
During each twenty-fifth of a second that the battery voltage continues to act steadily on the primary coil the core flux must change at the constant rate of fifty million lines per second according to equation (i) above, the resistance of the primary coil being ignored. Therefore $(a)$ the curve of core flux curve must be an inclined straight line during each twenty-fifth of a second, and (b) the total change of flux during each twenty-fifth of a second must be $\frac{1}{25} \times 50,000,000$ or two million lines. In every case, however, the core flux of a transformer pulsates between equal positive and negative maximum values, so that the total change of flux in a half-cycle is $2 \Phi$ where $\Phi$ is the maximum value of the core flux, positive or negative. Therefore, in the case here considered, the maximum value of the core flux is one million lines as shown by the dotted curve in Fig. I 76.

Example of core flux for harmonic electromotive force. - The relation between core flux and primary voltage in this case is most easily established by starting with a given harmonically varying core flux $\phi$ and finding the corresponding primary voltage. Therefore let

$$
\phi=\Phi \sin \omega t
$$

Differentiating this expression with respect to time we have

$$
\frac{d \phi}{d t}=\omega \Phi \cos \omega t
$$

and substituting this value of $d \phi / d t$ in equation (i) we have

$$
e^{\prime}=\omega N^{\prime} \Phi \cos \omega t
$$

from which it is evident: (a) That $e^{\prime}$ is harmonic; that is to say, a harmonically varying core flux induces a harmonic electromotive force in the primary coil, or a harmonic primary electromotive force always produces a harmonically varying core flux; (b) That the primary voltage is $90^{\circ}$ ahead of the harmonically varying core flux in phase, as shown in Figs. 172,173 and 174 ; and $(c)$ that the maximum value of $e^{\prime}$, namely $\mathbf{E}^{\prime}$, is equal to $\omega N^{\prime} \Phi$, that is :

$$
\mathbf{E}^{\prime}=\omega N^{\prime} \Phi
$$


or, substituting the effective value $E^{\prime}\left(=\mathrm{E}^{\prime} / \sqrt{2}\right)$, and solving for $\Phi$ we have

$$
\Phi=\frac{\sqrt{2} E^{\prime}}{\omega N^{\prime}}
$$

in which $E^{\prime}$ is the cffective value in abvolts of a harmonic voltage acting on the primary coil of a transformer, $N^{\prime}$ is the number of turns of wire in the coil, $\omega$ is the frequency in radians per second ( $=2 \pi$ times frequency in cycles per second), and $\Phi$ is the maximum positive and negative value between which the core flux pulsates.

The maximum flux density $\mathscr{B}$ reached in the transformer core is of course equal to $\Phi$ divided by the area of the crosssection of the core in square centimeters.

102. Curve of magnetizing current, primary voltage being harmonic. (a) On the assumption that the magnetic reluctance of the core is constant. - In this case the value $m$ of the magnetizing current at each instant would be proportional to the value $\Phi$ of the core flux at that instant, and therefore the magnetizing current would be a harmonic current $90^{\circ}$ behind the primary voltage in phase.

(b) When the hysteresis loop of the transformer core is given for that range of fux which is produced by the given primary voltage (eddy currents ignored). - Let ordinates of the dotted curve, Fig. 177, represent the values of core flux $\Phi$ and let

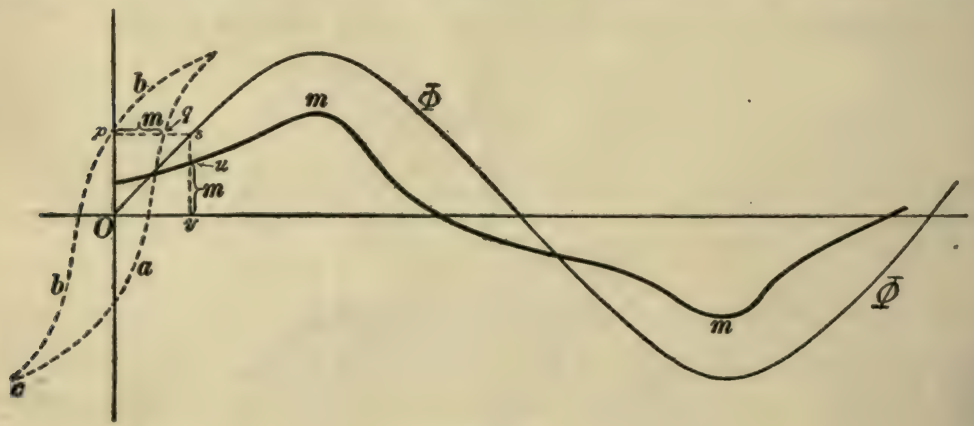

Fig. 177.

the abscissas of the dotted curve represent the corresponding values of steady current $m$ in the primary coil. Let the sine curve $\Phi$ represent the harmonically varying core flux corresponding to the given primary voltage. From a point $s$ on the flux-time curve draw the lines $s v$ and $s p$. Then lay off $v u$ equal to $p q$. Proceeding in this way the entire current-time curve may be constructed, it being remembered that the branch $a$ of the flux-current curve corresponds to increasing flux and branch $b$ to decreasing flux. 
The effect of eddy currents in the core is to cause a harmonic current in phase with $E^{\prime}$ to flow through the primary coil in addition to the current represented by the curve $m$ in Fig. 177 .

103. Loss of power in transformers. - The power output of a transformer is always less than its power intake because of the loss of power in the transformer. This loss of power in a transformer consists of two parts, namely, (a) The iron or core loss due to eddy currents and hysteresis; and (b) The copper loss due to the resistances of the primary and secondary coils.

The iron losses are practically the same in amount at all loads, and they depend upon the frequency, upon the range of the flux density $\mathscr{B}$, upon the quality and volume of the iron, and upon the thickness of the laminations.

The hysteresis loss in watts is

$$
P_{h}=a V f \mathfrak{B}^{1.6}
$$

where $f$ is the frequency in cycles per second, $\mathscr{B}$ is the maximum flux density in lines per square centimeter, $V$ is the volume of the iron in cubic centimeters, and $a$ is a constant depending upon the magnetic quality of the iron. For annealed refined wrought iron the value of $a$ is about $3 \times \mathrm{IO}^{-10}$.

The eddy current loss in watts is :

$$
P_{e}=b V f^{2} l^{2} \mathfrak{B}^{2}
$$

where $l$ is the thickness of the laminations in centimeters, and $b$ is a constant depending upon the specific electrical resistance of the iron. For ordinary iron the value of $b$ is about $1.6 \times 10^{-11}$. Insufficient insulation of laminations causes an excessive eddy current loss.

Equations (26) and (27) may be used for calculating the hysteresis and eddy current losses in any mass of laminated iron subjected to periodic reversals of magnetization, such as alternator armature cores and the rotor and stator iron in an induction motor.

The copper loss is :

$$
P_{c}=R^{\prime} I^{\prime 2}+R^{\prime \prime} I^{\prime \prime 2}
$$


This loss is nearly zero when the transformer is not loaded; it increases with the square of the current, and becomes excessive when the transformer is greatly overloaded.

104. Efficiency of transformers. - The ratio power output $\div$ power intake is called the efficiency of a transformer. The accompanying table shows the full-load efficiencies of various sized transformers of a recent type.

TABLE OF TRANSFORMER EFFICIENCIES.

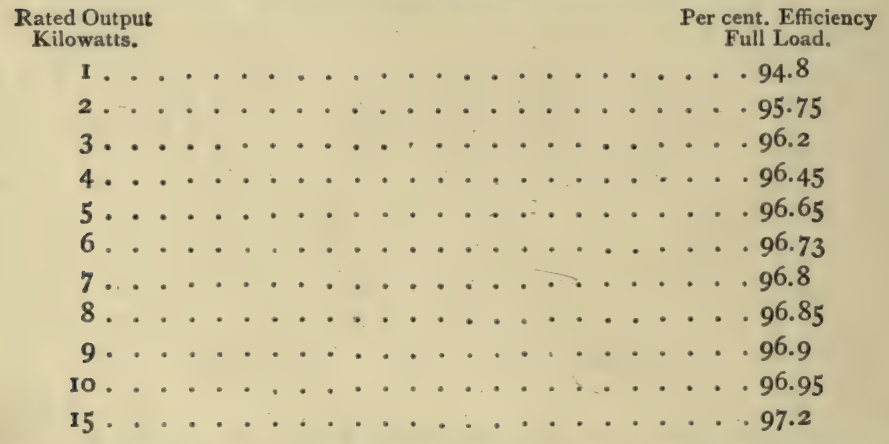

The efficiency of a given transformer is very low when the output is small; it increases as the output increases, reaches a maximum, and falls off again when the output is very great. This falling off of efficiency when the output is great is due to the great increase of copper losses. Figure I 78 shows the efficiency of a transformer at various loads.

All-day efficiency. - A transformer is usually connected to the mains continuously, although current may be delivered by the secondary for a few hours only each day. In such a case the iron loss is continuous and may represent a very considerable loss of energy each day. The copper loss on the other hand is not appreciable except when the transformers is vidadedns Theret fore a transformer which is ahwàys lcomected to thet mains idut which is loaded onlyi a oshortt timectach 9 daytoshoubdobesdesigned to have as small a power loss as possible in the iron, even thotogh the design may involve a considerable increase of the power loss in the copper.

"I" 
The all-day efficiency of a transformer (or of any machine) is defined as the quotient obtained by dividing the total energy delivered by the machine in a day by the total energy delivered to the machine in a day. For example, consider a transformer of which the iron loss is 300 watts and the copper loss at full load is also 300 watts, the full-load output of the transformer being ro kilowatts. The transformer is connected to the mains all day and the total energy lost in the core in a day is $24 \times 300$ watthours. The transformer is used at full load for $\mathrm{I} / 2$ hours each day, during which time the copper loss is $1 \mathrm{r} / 2 \times 300$ watt-hours,

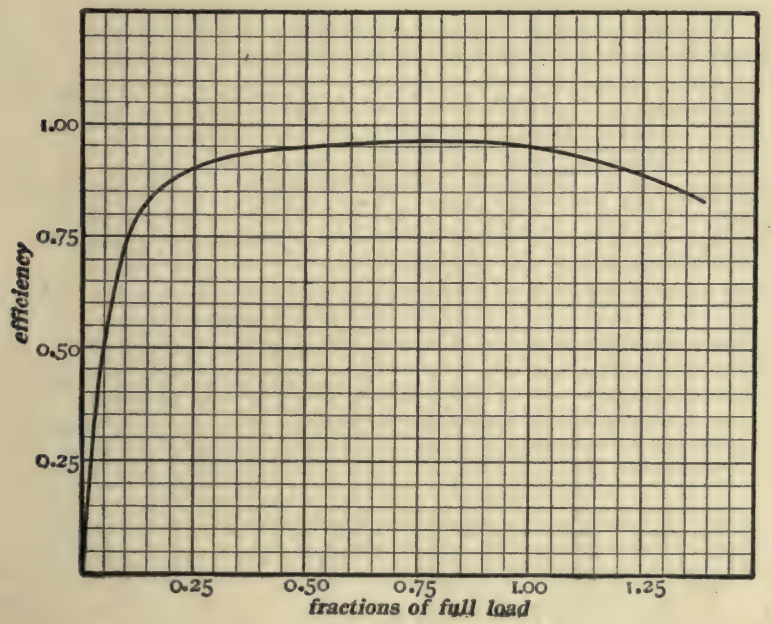

Fig. 178.

and the copper loss during the remaining $22 \mathrm{x} / 2$ hours is negligible. The total energy delivered by the transformer in a day is $10,000 \times 1 \mathrm{I} / 2$ watt-hours, and the total energy delivered to the transformer in a day is of course $[10,000 \times 1 \mathrm{I} / 2+24 \times 300$ $+1 \mathrm{I} / 2 \times 300]$ watt-hours, so that the all-day efficiency of the transformer under the given conditions is 15 , O0O watt-hours divided by 22,650 watt-hours, or 66.2 per cent.

Calculation of efficiency. - Given the details of design of a transformer to calculate its efficiency for given value and frequency of $E^{\prime}$ (or $E^{\prime \prime}$ ) and given current output $I^{\prime \prime}$. 
The power output is equal to $E^{\prime \prime} I^{\prime \prime}$ on non-inductive load. If the receiving circuit is inductive the power output is $E^{\prime \prime} I^{\prime \prime}$ multiplied by the power factor of the receiving circuit.

The power input is equal to the power output plus the losses, so that, if the losses can be calculated, the power input is known, and then the efficiency may be calculated by dividing output by input.

The copper loss may be easily found from the equation

$$
W_{\mathrm{e}}=R^{\prime} I^{\prime 2}+R^{\prime \prime} I^{\prime \prime 2} \text {. }
$$

In order to determine the iron loss, calculate $\Phi$ from equation (25) Art. IOI and divide by the sectional area of core to find the maximum flux density $\mathscr{B}$. Then, knowing volume of iron $V$, thickness of laminations $l$, frequency $f$, and the two empirical constants $a$ and $b$ in equations (26) and (27) of Art. 103, the values of $P_{h}$ and $P_{\circ}$ may be calculated.

105. Transformer regulation. - When a transformer is at zero load the ratio of primary to secondary voltage is almost exactly equal to the ratio $N^{\prime} / N^{\prime \prime}$, but with increasing load the secondary terminal voltage decreases, the supply voltage being constant, or, in other words, the secondary terminal voltage increases in value as the load on the transformer is decreased. The degree of regulation is usually specified by expressing the increase of secondary terminal voltage from full load to zero load in percent of full-load voltage. Thus a given transformer supplied from constant voltage mains gives a secondary terminal voltage of 108.5 volts at full load and I 2 volts at zero load and its percentage regulation is

$$
\frac{\text { I I } 2-108.5}{108.5} \times 100=3.22 \text { per cent. }
$$

The decrease of secondary terminal voltage of a transformer with increase of load is due to coil resistances and to magnetic leakage. When the receiving circuit is non-inductive the decrease of secondary terminal voltage is due almost entirely to coil resistances, when the receiving circuit is very highly inductive the 
decrease of secondary terminal voltage is due almost entirely to magnetic leakage, and when the receiving circuit is a condenser the effect of magnetic leakage is to cause the secondary terminal voltage to increase with increase of load. The theory of transformer regulation is developed in Chapter XI.

106. Transformer rating. - An increase of the current output of a transformer, with given value and frequency of $E^{\prime}$, causes a decrease of secondary terminal voltage and an increased generation of heat in the transformer coils. Increase of $E^{\prime}$ or decrease of frequency, on the other hand, causes an increase of $\Phi$ according to equation (25), Art. IOI, and this increase of $\Phi$ causes an increase of iron loss according to equations (26) and (27), Art. IO3.

The voltage rating, frequency rating, and current rating of a transformer are therefore determined by the allowable heating and by the required degree of constancy of secondary voltage, and usually the allowable heating is the determining factor.

A transformer is usually rated by specifying the value and frequency of the primary voltage with which it is intended to be used, and by specifying its ratio of transformation and the power it can deliver steadily (at rated voltage) to a non-inductive receiving circuit.

The rating of a transformer is by no means rigid and it is allowable to use a transformer under conditions differing very greatly from the conditions specified in its rating, but when so used a transformer should be watched so that it may be disconnected if its temperature becomes excessive.

107. The constant current transformer. - When a number of arc lamps (or other receiving units) are operated in series, individual lamps must be short-circuited to cut them out of service, and in order that the lamps may be independent of each other, the circuit must be supplied with a constant current. When alternating current is used to operate arc lamps in series, the constant current transformer is used. This transformer takes current from 
a constant voltage source and delivers constant current to the arc lamp circuit. Figure I 79 shows a constant current transformer

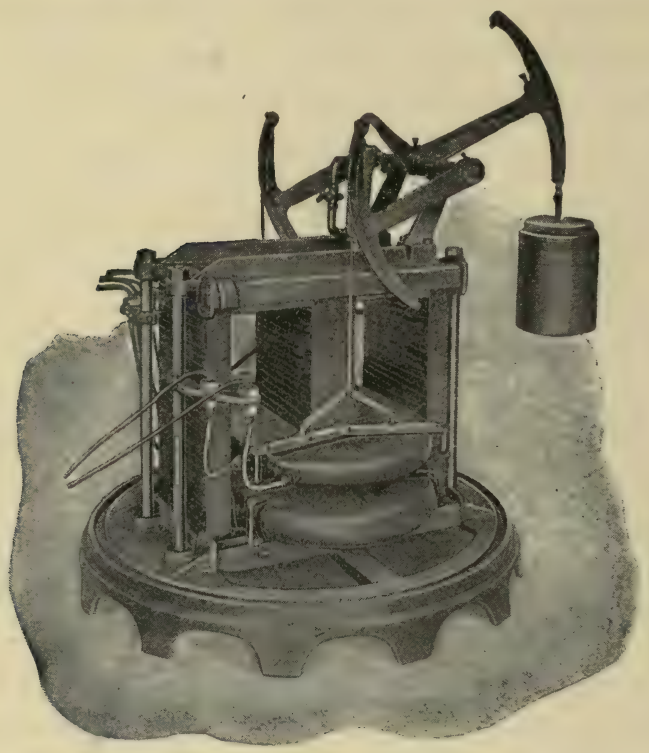

Fig. 179.

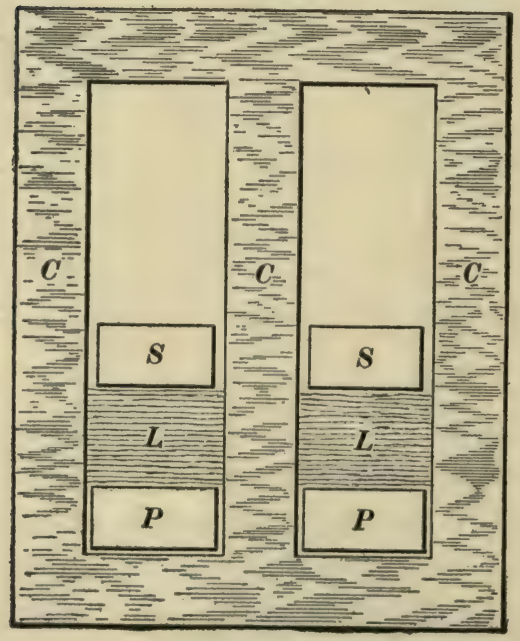

Fig. 180. 
of the General Electric Company. The essential features of this transformer are shown in Fig. I80, in which CCC is the laminated iron core, $P P$ is the stationary primary coil, and $S S$ is the secondary coil which is suspended from a counterpoised lever system so as to move up and down with great ease. When lamps are short-circuited, the slight increase ${ }^{*}$ of current causes an increased repulsion between $P$ and $S$, the coil $S$ moves upwards, and the increase of leakage flux across the regions $L L$ lessens the useful flux through the secondary coil $S$, which reduces the induced voltage in $S$, thus counteracting the tendency for the current to increase.

108. Simple tranformer connections. Connections in parallel. Connections in series. - When transformers are used to supply current to groups of lamps or motors at constant voltage, stepping down from constant voltage transmission lines, the primary

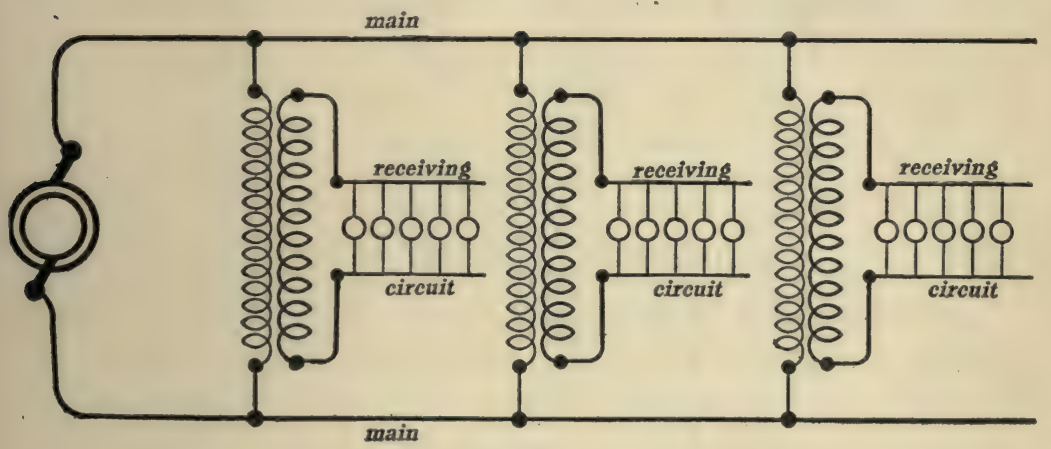

Fig. 181.

of each transformer is connected directly across the high voltage mains and each transformer delivers current to a separate group of lamps or motors as shown in Fig. I $8 \mathrm{I}$. This arrangement is called the parallel connection of transformers.

* An initial increase of current must be produced in order to start the moving coil upwards, but the lever system may be so designed as to keep the equilibrium value of the current almost exactly constant with decrease of resistance of receiving circuit, or even to cause the equilibrium value of the current to decrease with decrease of resistance of receiving circuit. 
The primary coils of several transformers may be connected in series with each other as shown in Fig. I 82. This arrangement, which is called the series connection of transformers, is seldom used.

Transformers with divided coils. - Alternators in moderately small lighting plants give usually I, IOO or 2,200 volts electromotive force, and the standard voltages for incandescent lamps are I I v volts or 220 volts. Small transformers for lighting plants are therefore usually made with two-part primary coils and two-part

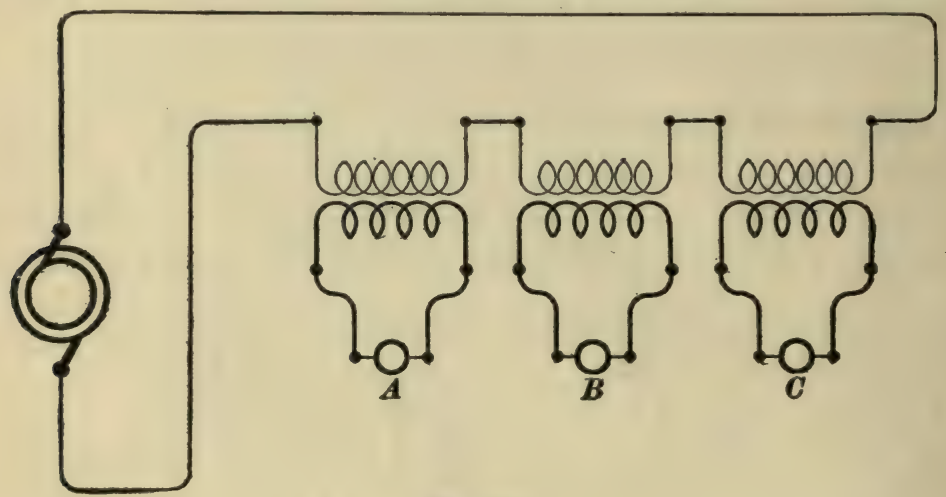

Fig. 182.

secondary coils, so that the two parts of the primary may be connected in series with each other for 2,200 volts or in parallel with each other for I, IOO volts, and so that the two parts of the secondary may be connected in series with each other for 220 . volts or in parallel with each other for r io volts.

In connecting the two parts of a transformer coil in parallel with each other the utmost care must be taken to connect the coils so that one coil does not act as a short-circuit for the other, and in connecting the two parts of the primary coil of a transformer in series with each other the utmost care must be taken to be sure that the two parts work together in opposing the primary impressed voltage. Failure in either case to make the proper connections may lead to serious damage to the transformer when it is connected to the mains. 
109. The autotransformer.* $\_$Consider the supply mains which bring current from an alternator, and the service mains which deliver current to a customer, as shown in Fig. 183. There are
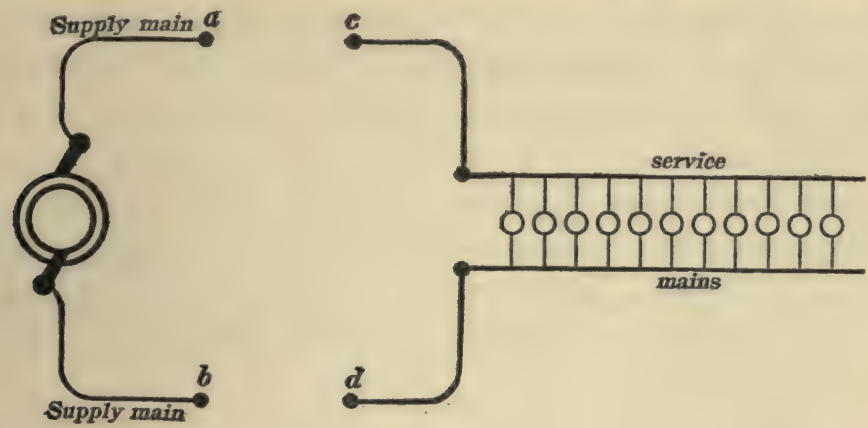

Fig. 183.

three methods for establishing communication from $a b$ to $c d$, as follows: (a) By conductive connections, that is by connecting wires from $a$ to $c$ and from $b$ to $d$ in Fig. $183,(b)$ by transformer connections, that is by connecting the primary of a transformer to $a b$ and the secondary to $c d$, and $(c)$ by a combination of conductive and transformer connections. Any transformer which is connected up for this combination method is called an autotransformer.

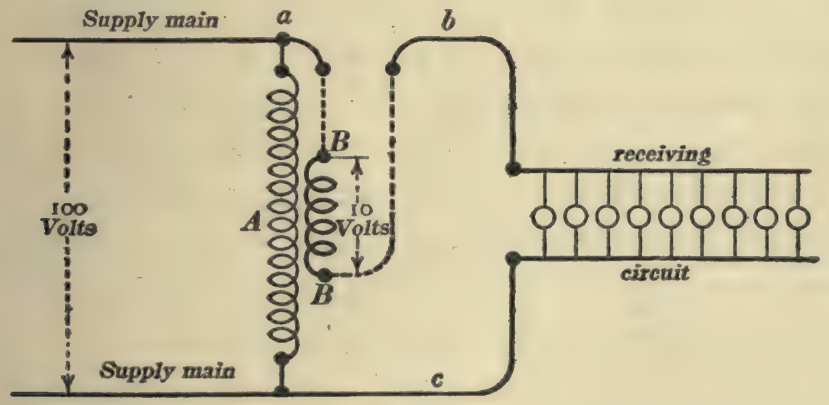

Fig. 184.

The action of the autotransformer may be brought out most clearly by considering a numerical example. Let the coils $A$

* It is misleading to speak of the autotransformer inasmuch as a so-called autotransformer is exactly the same thing as an ordinary transformer, and it acts in the same way. The name autotransformer refers to a scheme of connections. 
and $B$, Fig. 184 , represent the fine wire coil and the coarse wire coil respectively of an ordinary Io to I transformer. The fine wire coil is connected across 100 volt supply mains, and of course an electromotive force of 10 volts is induced in the coarse wire coil. One terminal of the receiving circuit is connected directly to one of the supply mains as shown, and the other terminal $b$ is connected through the coil $B B$ to the supply main $a$. This connection may be made so that the ro volts in $B B$ is added to the supply voltage thus giving I I o volts across $b c$, or it may be made so that the $I O$ volts in $B$ is subtracted from the supply voltage thus giving 90 volts across $b c$. In the first case we have

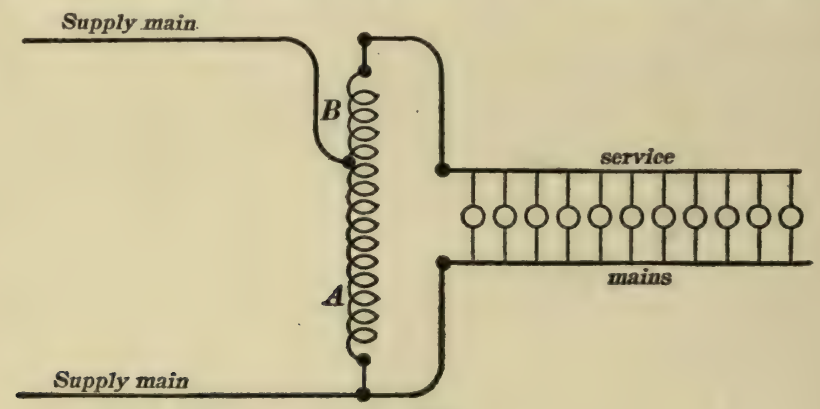

Fig. 185.

auto-step-up transformation, and in the other case we have autostep-down transformation. In the first case the coil $A$ acts as a primary, receiving power from the supply mains; and the coil $B B$ acts as a secondary, delivering power to the receiving circuit. In the second case the coil $B B$ acts as a primary, receiving power from the current which is forced through it in opposition to its voltage; and the coil $A$ acts as a secondary, delivering power back to the supply mains. In both cases the power which is actually transformed from $A$ to $B$ or from $B$ to $A$ is equal to Io volts multiplied by the current $I$ which is delivered to the receiving circuit, whereas the total amount of power delivered to the receiving circuit is $\mathrm{I} I 0 \times I$ in the first case, and $90 \times I$, in the second case. Therefore a ro to I transformer rated as a Ikilowatt transformer would suffice for the delivery of I I kilowatts 
to the receiving circuit in the first case, or 9 kilowatts in the second case. This illustrates the most important feature of the autotransformer when the supply voltage is to be only slightly raised or lowered, namely, that a small transformer suffices for the delivery of a large amount of power, and of course the losses of power are less than would be involved in a large transformer used in the ordinary way.

The action of the autotransformer in altering the supply voltage is most clearly represented as shown in Figs. I 85 and I 86 ;*

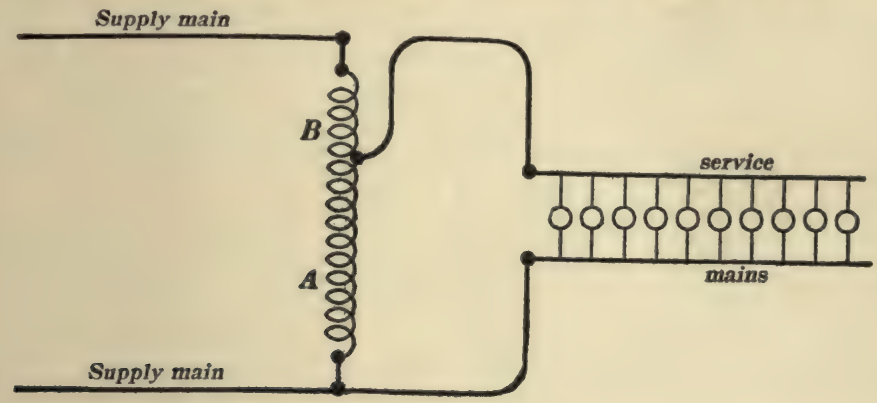

Fig. 186.

Fig. I 85 represents auto-step-up transformation, and Fig. I 86 represents auto-step-down transformation. In each of these figures the two coils $A$ and $B$ are related to each other as parts of one continuous winding, and the voltage of the whole winding ( $A$ and $B$ together) is to the voltage of any given part of it as the whole number of turns is to the number of turns in the given part.

110. The arrangement of transformers in polyphase systems. (Without changingathe number of plsesses.) - Step-up and stepdown transformation in polyphase_systems is arcompdished, in general, by using an independent totransformer for each phase, that is by transforming, each phase by its individual transformer пw When threer transfonmers are ased in a threetwire three-phase system, the primary colls of the three transformers may be $\Delta$ -

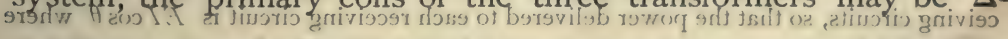

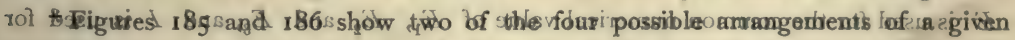

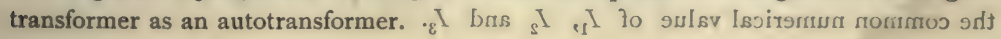


connected or $Y$-connected to the supply mains, and in either case the secondary coils may be $\Delta$-connected or $Y$-connected to the service mains, so that four arrangements are possible, namely, the $\Delta \Delta$-connection, the $\Delta Y$-connection, the $Y \Delta$-connection, and the $Y Y$-connection. The $\Delta \Delta$-connection is, however, the usual arrangement, for, in this case, the system remains operative when one of the transformers is disconnected; thus with the $\Delta \Delta$-connection the burning-out of one of the transformers need not render the system inoperative, although the two remaining transformers would operate at a disadvantage, not only because the two transformers would have to carry the load of three, but because the currents in the two transformers are increased in a greater ratio than $2: 3$ as explained in the following paragraph.

The use of two transformers for three-phase transformation. - Figure 187 shows the connections of two transformers $A a$ and $B b$ for three-phase transformation. It is at once evident that this arrangement maintains the proper voltage (proper, that is, in value and in phase) between service mains $I$ and 2 , and the proper voltage between service mains 2 and 3 ; but a proper voltage between $\mathbf{I}$ and 2 and a proper voltage between 2 and 3 gives necessarily a proper voltage between 3 and $\mathbf{I}$.

\section{Supply main ,}

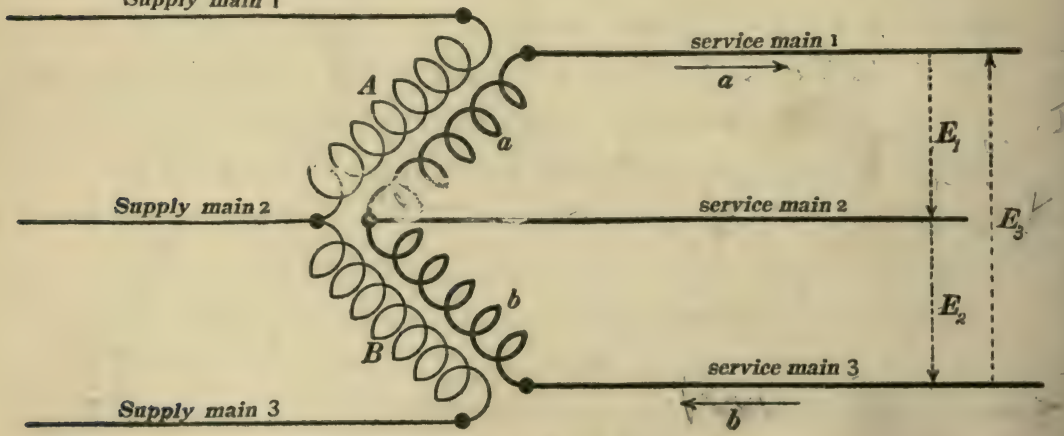

Fig. 187.

Suppose that the service mains are delivering current to three similar $\Delta$-connected receiving circuits, each current lagging $\theta^{\circ}$ behind the corresponding voltage, as shown in Fig. I88. The lines $I_{1}, I_{2}$ and $I_{3}$ represent the currents in the respective receiving circuits, so that the power delivered to each receiving circuit is $E I \cos \theta$ where $E$ is used for the common numerical value of $E_{1}, E_{2}$ and $E_{3}$, and $I$ is used for the common numerical value of $I_{1}, I_{2}$ and $I_{3}$. 
The current $a$ in service main $\mathrm{I}$ is the vector difference $I_{1}-I_{3}$, and the current $b$ in service main 3 is the vector difference $I_{2}-I_{3}$, as shown in Fig. 188. The arrows in Fig. 187 represent the directions which are chosen as positive. Of course the currents in the secondary coils $a$ and $b$ are precisely the same as the currents in mains 1 and 3 respectively. The voltage $E_{1}$ which is generated in coil a develops an amount of power equal to $E_{1}$ times $a$ times the cosine of the phase difference between $E_{1}$ and $a$, or $E_{1} a \cos \left(\theta+30^{\circ}\right)$; and the voltage $E_{2}$ which is gener-

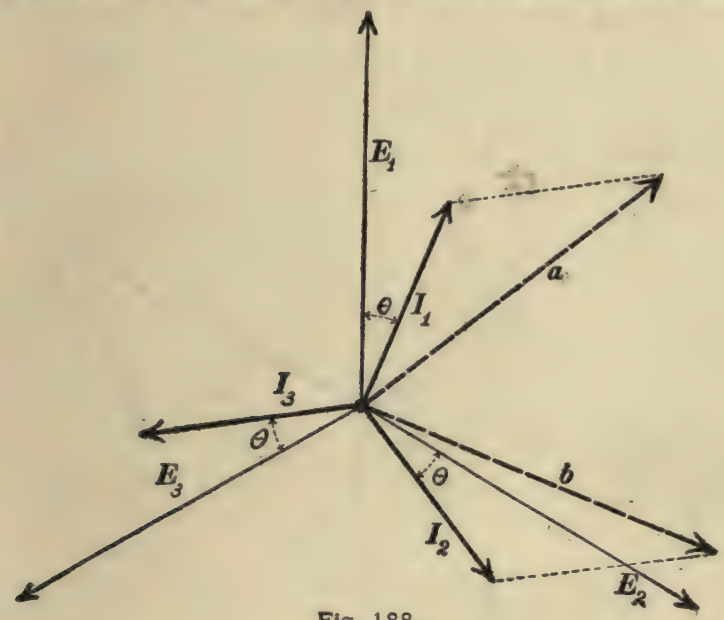

Fig. 188.

ated in coil $b$ develops an amount of power equal to $E_{2} b \cos \left(\theta-30^{\circ}\right)$. Using, therefore, $E$ for the common numerical value of $E_{1}$ and $E_{2}$, and $a$ for the common numerical value of $a$ and $b$, we have, as an expression for the total power delivered

or

$$
E a\left[\cos \left(\theta+30^{\circ}\right)+\cos \left(\theta-30^{\circ}\right)\right]
$$

$2 E a \cos \theta \cos 30^{\circ}$

or

$$
2 \times 0.866 E a \cos \theta
$$

Example. - Consider three $\Delta \Delta$-connected Ioo-kilowatt transformers delivering three-phase currents to balanced receiving circuits. These transformers can deliver 300 kilowatts if the receiving circuits are non-inductive or in general they can deliver $300 \cos \theta$ kilowatts. If one transformer is put out of service, the two remaining ing transformers will operate at full load, not on $200 \cos \theta$ kilowatts, but on $0.86 \times 200 \cos \theta$ kilowatts; that is, the two transformers have a full-load capacity of only 0.577 of the full-load rating of the three transformers.

111. Polyphase transformers. - It is possible to combine the magnetic circuits of transformers which are used for polyphase transformation. Thus Fig. I 89 shows what may be called a two-phase transformer. It consists of two similar transformers 
$A$ and $B$, the magnetic circuits of both being completed through the portion $c$ of the core. When used for two-phase step-up or step-down transformation, transformer $A$ is used for one phase and transformer $B$ for the other phase. In this case the magnetic flux in core $a$ as in phase-quadrature with the magnetic flux in core $b$, and the return flux in core $c$ is related to the fluxes in $a$ and $b$ as shown in the clock diagram in Fig. 190.

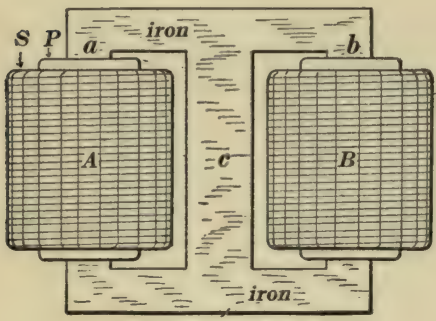

Fig. 189.

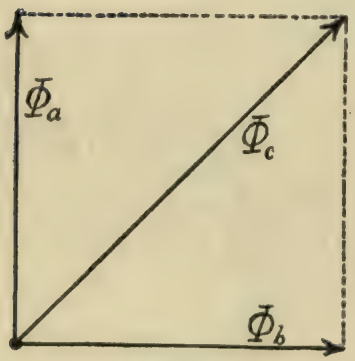

Fir. 190.

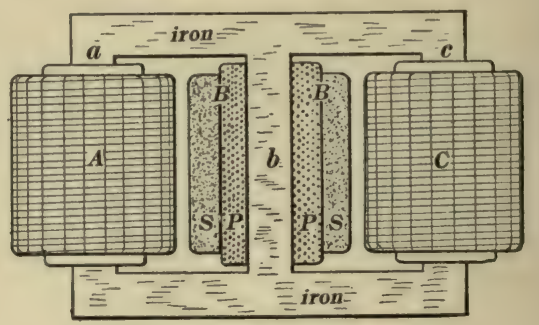

Fig. 191.

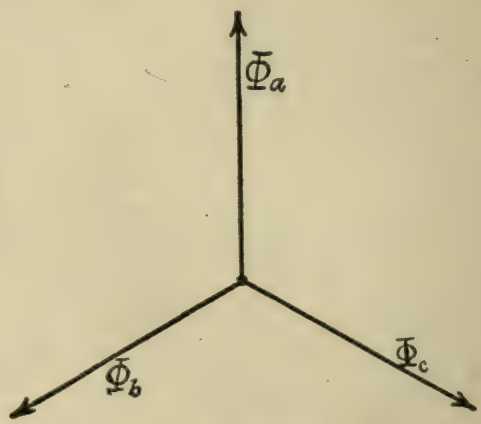

Fig. 192.

Figure I9I shows what may be called a three-phase transformer. It consists of three similar transformers $A, B$ and $C$, with a single core-structure as shown. When used for threephase step-up or step-down transformation, transformer $A$ is used for one phase, transformer $B$ for another phase, and trans-

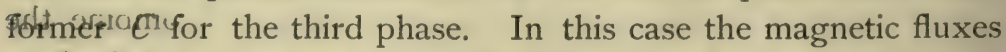
ninctores $q a, b$ and $c$ are $120^{\circ}$ apart in phase, as shown in the clock didgtafri of fluxes, Fig. 192, and each core may be looked upon as therreteluff path for the flux in the other two. 
112. Transformation of phases. - Transformers in polyphase systems are often arranged so as to change the number of phases and at the same time to produce step-up or step-down transformation. Thus at one of the large power stations at Niagara Falls, two-phase alternators are used, and the step-up transformers which supply the Buffalo transmission line are arranged to change from two-phase to three-phase so to take advantage of the fact that three-phase transmission requires less copper than two-phase transmission, for the same voltage between wires. Also, when

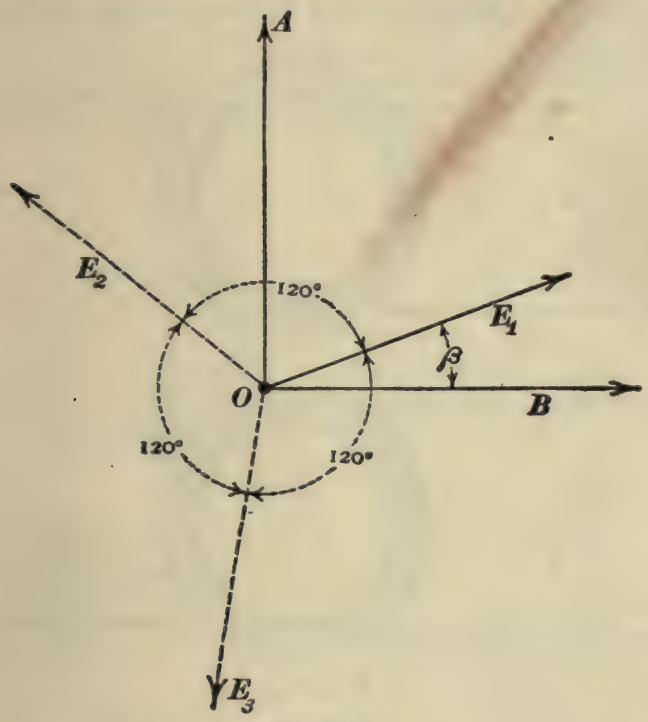

Fig. 193.

it is desired to operate, from an $n$-phase supply, a synchronous converter having $m$ collector rings, the step-down transformers must be arranged to change the number of independent phases.

It is impossible by means of transformers to convert a singlephase supply into a polyphase supply, because a transformer cannot convert the pulsating power delivered by a single-phase alternator into the steady flow of power required in the polyphase system. A transformer (that is, an ideal transformer) delivers energy at each instant at the same rate that it receives energy. 
The principle involved in the transformation of phases may be best brought out by considering the transformation from two phases to any number of phases, say, three; although any arrangement of transformers which will transform from two-phase to $n$-phase will serve to transform from $n$-phase to two-phase.

Let $A$ and $B$, Fig. 193, represent the two electromotive forces of a two-phase alternator, and let $E_{1}$ be any given electromotive force which it is desired to produce. The component of $E_{1}$ parallel to $A$ is $E_{1} \sin \beta$, and the component of $E_{1}$ parallel to $B$ is $E_{1} \cos \beta$; and to produce the desired electromo-

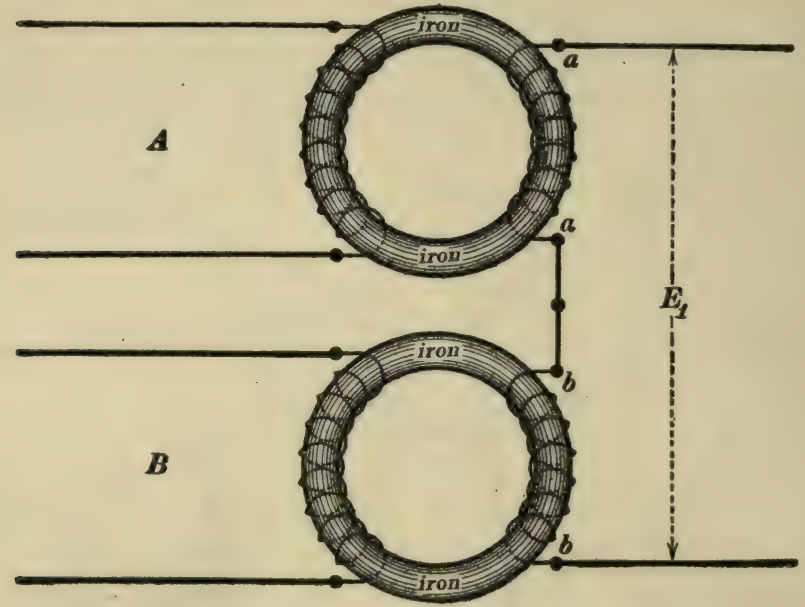

Fig. 194.

tive force $E_{1}$ it is only necessary to produce the components of $E_{1}$ in the respective secondaries of two transformers, of which the primaries are supplied from $A$ and $B$ respectively, and to connect these secondary coils in series. Thus Fig. 194 shows two separate transformers with their primary coils connected to phase $A$ and phase $B$, respectively, of the two-phase alternator, the secondary coil $a$ has a number of turns sufficient to produce the the electromotive force $E_{1} \sin \beta$, the secondary coil $b$ has a number of turns sufficient to produce the electromotive force $E_{1} \cos \beta$, and the two coils $a$ and $b$ connected in series give 
the required electromotive force $E_{1}$. Similarly, the electromotive forces $E_{2}$ and $E_{3}$, Fig. 193, may be produced each by a pair of properly proportioned secondary coils. Therefore, the two-phase three-phase transformer consists of two transformers with their primary coils supplied from the two-phase system, and having, in general, six secondary coils which are connected in pairs in series, each pair supplying one of the three-phase voltages. Such an arrangement can be used to change from two-phase to three-phase or to change from three-phase to two-phase.

The Scott transformer. - The general type of two-phase threephase transformer above described may be greatly simplified, and the simplest arrangement is that due to Chas. F. Scott.

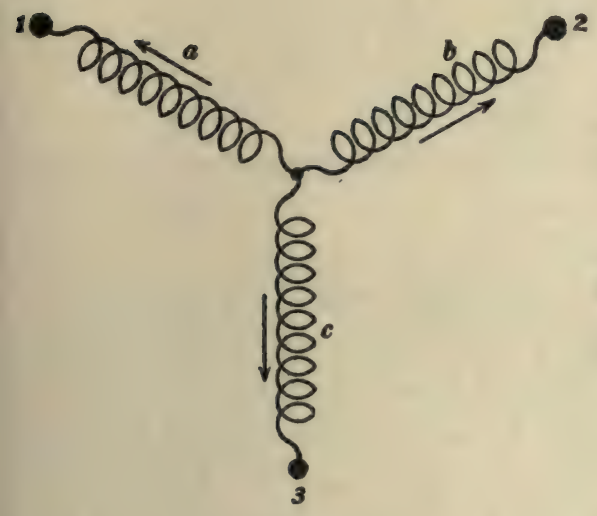

Fig. 195.

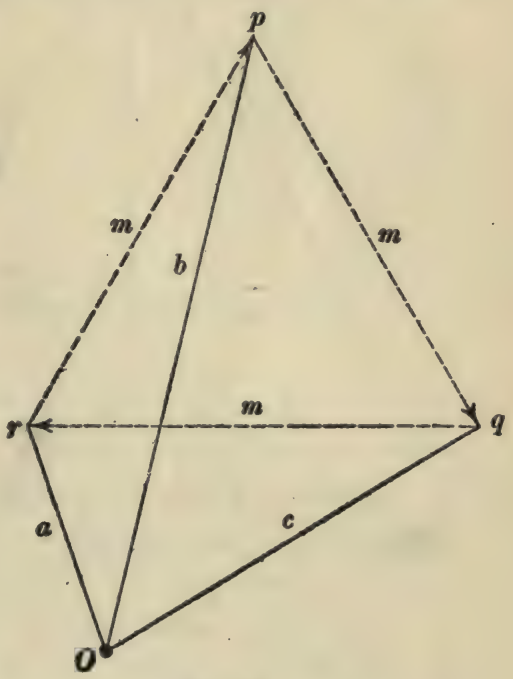

Fig. 196.

To understand the Scott transformer it is necessary to consider an even more general type of two-phase three-phase transformer than that described above, and to make use of the following proposition : Consider three units of any kind, alternators, or combinations of transformer secondaries, $a b$ and $c$, which are $\mathrm{Y}$-connected to three mains I, 2 and 3 , as shown in Fig. 195 ; if the lines $a, b$ and $c$, Fig. 196, which represent the electromotive 
forces generated in $a, b$ and c, Fig. 195, terminate at the apices of an equilateral triangle, then the $Y$-connected units give symmetrical three-phase voltages between the mains. This is evident when we consider that the voltage from main I to main 2, Fig. I95, is the vector difference $a-b$, that the voltage from main 2 to main 3 is the vector difference $b-c$, and that the voltage from main 3 to main $\mathrm{I}$ is the vector difference $c-a$.

In the Scott transformer the point $O$, Fig. 196, lies midway between the points $p$ and $r$ as shown in Fig. 197, so that the
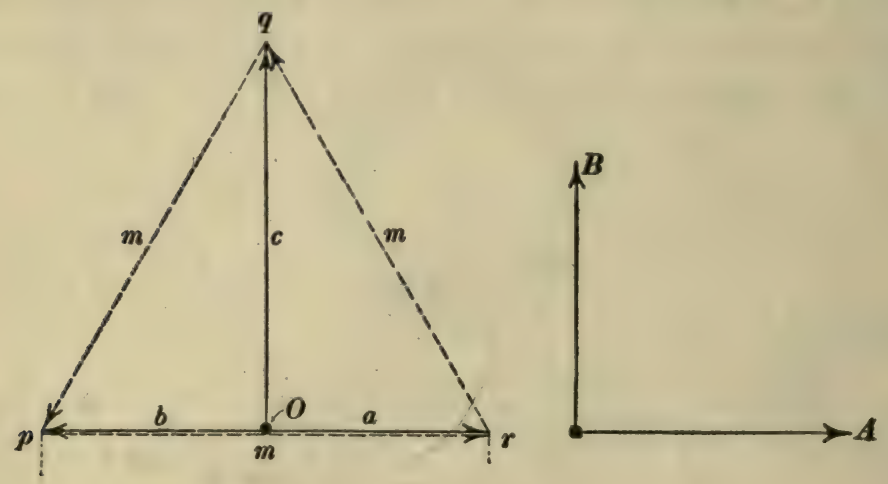

Fig. 197.

lines $a$ and $b$ are parallel to each other and at right angles to the line $c$. Then the voltage $c$ may be produced by the single secondary of a transformer supplied from phase $B$ of the twophase system, and the voltages $a$ and $b$ may be produced by two secondary coils (one for $a$ and one for $b$ ) of a transformer supplied from phase $A$ of the two-phase system, the three secondaries $a, b$ and $c$ being $Y$-connected or, as it is sometimes called, $T$-connected, to the three-phase mains as shown in Fig. 198. The one condition that is necessary is that the coils $a$ and $b$ be related to each other as halves of one continuous winding.

Example of a Scott transformer. - The similar primary coils of two transformers have $\mathrm{I}, 000$ turns of wire each and they are connected to the respective phases of a I,Ooo volt two-phase 
supply; and it is desired to deliver three-phase currents at 240 volts between mains. Each side of the equilateral triangle pqr, Fig. 197, therefore, must represent 240 volts, so that each of the secondary coils $a$ and $b$, Fig. I98, must generate 120 volts

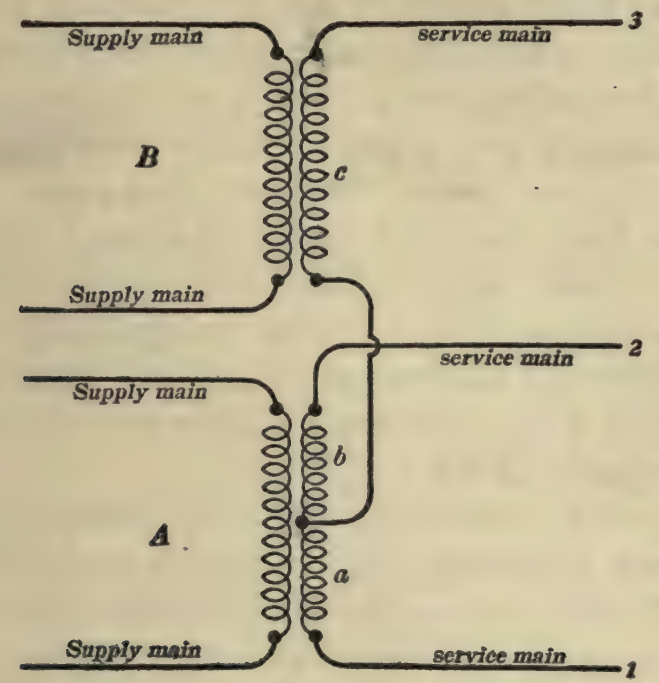

Fig. 198.

and have I 20 turns of wire; and the secondary coil c, Fig. I98, must generate $\sqrt{3} / 2$ times 240 volts or 208 volts and have 208 turns of wire. The coils $a$ and $b$ constitute one continuous winding of 240 turns of wire, with a lead coming out from its middle point.

Dephasing action of the Scott transformer. - When two transformers are used for three-phase transformation as described in Art. I Io, the currents in the transformer coils are not in phase with the induced voltages even when the receiving circuits are non-inductive. The mutual action of the two transformers which causes the currents $a$ and $b$ to differ in phase from the voltages $E_{1}$ and $E_{2}$, Fig. 188, and which lowers the power rating of the two transformers in the ratio of $I$ to 0.866 is called dephasing action. There is a somewhat similar dephasing action in one of the two transformers which together constitute a Scott transformer. Thus, when the Scott transformer delivers current to balanced non-inductive receiving circuits, the currents in the three coils $a, b$ and $c$, Fig. 198, are represented by lines drawn from the center of the equilateral triangle $p q r$, Fig. 197, towards the apices $r, p$ and $q$ respectively. That is, the current in coil $a$ is $30^{\circ}$ behind the induced voltage in $a$, and the current in coil $b$ is $30^{\circ}$ ahead of the induced voltage in $b$. 
113. Transformer connections for synchronous converters. - An $n$-ring converter must be supplied with alternating current from an $n$-wire system, the supply wires being connected to the rings in proper order. Thus a four-ring converter (sometimes called a two-phase converter) cannot be supplied from a threewire two-phase system, but the two phases must be entirely separate, each transmitted over a separate pair of wires.

Three-ring converter. - A three-ring converter is supplied with alternating currents from a three-wire three-phase system, the three phases being stepped down to the proper voltage, and the three service wires being connected to the three rings. The order of connections of service wires to rings depends on the direction in which the converter is to be driven. If the alternatingcurrent supply is two-phase it is necessary to transform to threephase as explained in Art. I 12.

The four-ring converter. - A four-ring converter must be supplied with alternating currents from a four-wire two-phase system. If the two-phase currents are transmitted over a threewire line, the step-down transformers must deliver currents to four service wires, two separate wires for each phase. The wires of phase $A$ are connected to two opposite * collectorrings, and the wires of phase $B$ are connected to the other two rings. The reversal of the connections of either phase will reverse the direction of driving.

If the alternating current supply is three-phase it is necessary to transform to two-phase as explained in Art. I I 2.

Six-ring converter. - Any converter having $2 n$ rings can be supplied with alternating currents from an $n$-phase system, provided the $n$ armature windings of the $n$-phase supply generator are not electrically connected with each other, each generator winding must have its owen separate pair of collector rings ( $2 n$ rings in all) and must deliver current over a separate pair of transmission wires. If the $n$-phase generator has $n$ collector rings,

\footnotetext{
* Opposite collector rings are those which are tapped into opposite points of a two-pole armature winding.
} 
the $n$-phases being transmitted over $n$ wires, then a $2 n$-ring converter may be supplied, if the $n$-phases be kept entively separate on the secondary side of the step-down transformers, that is, if each of the $n$ secondary coils delivers current to the converter over a separate pair of wires. This is exemplified by what is said above concerning the four-ring converter. It is also exemplified by the supply of currents to a six-ring converter from a three-phase system, the three phases being separate and each delivered to the

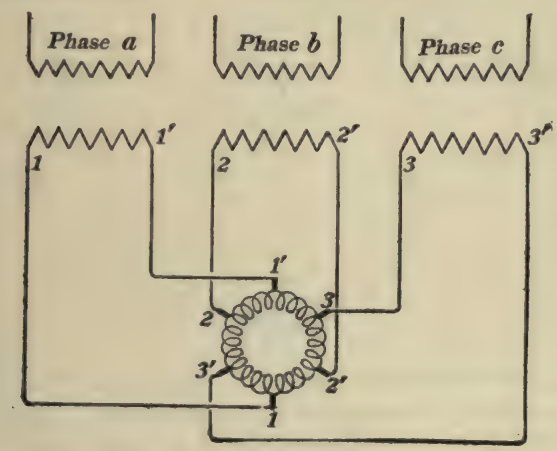

Fig. 199.

converter over two wires. To supply a six-ring converter from a two-phase system it is necessary to transform to three-phase.

The simplest method of connecting a six-ring converter to a three-phase (six-wire) system is to connect each of the three phases to opposite collector rings of the six-ring converter. This is called the diametrical connection and it is shown in Fig. 199.

There is some confusion as to the exact meaning of the term $\boldsymbol{n}$-phase system, as is evident from the above discussion which shows the possibility of operating a four-ring converter from a two-phase system and the possibility of operating a six-ring converter from a three-phase system. Indeed one should speak of an $n$-phase alternator as one which has $n$ armature windings displaced $\mathrm{I} / n$ of $\mathrm{r} 80$ electrical degrees* from each other. Thus, a two-phase alternator is an alternator which has two armature windings 90 electrical degrees apart, and a three-phase alternator is an alternator having three armature windings 60 electrical degrees apart; and the electromotive forces of two-phase and three-phase alternators may be most properly represented by the full

* One electrical degree is I/I80 of the angular distance between adjacent north and south field magnet poles center to center. 


\section{ELEMENTS OF ELECTRICAL ENGINEERING.}

lines in the clock diagrams of Fig. 200 and Fig. 20r. If the armature windings are not interconnected, then any phase may be connected in any way to any receiving cir-

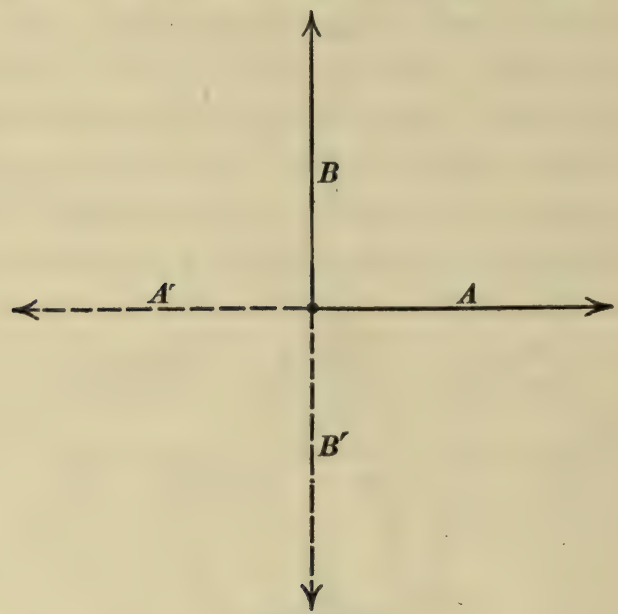

Fig. 200.

cuit, that is to say, the electromotive force $A$ may be reversed with respect to any receiving circuit by reversing its connections to that circuit. Therefore the two independent phases, $A$ and $B$, Fig. 200, constitute in a certain sense a four-phase system

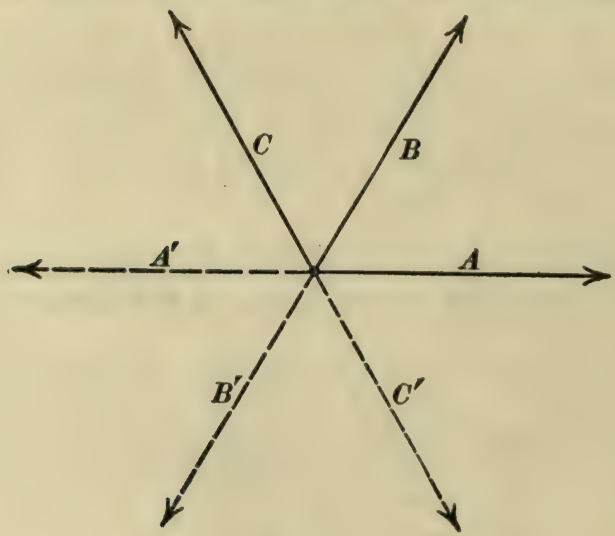

Fig. 201.

having the available voltages $A, B, A^{\prime}$ and $B^{\prime}$; and three independent phases, $A$ $B$ and $C$, Fig. 20I, constitute in a certain sense a six-phase system having the available voltages $A, B, C, A^{\prime}, B^{\prime}$ and $C^{\prime}$. 
CHAPTER XI.

\section{GENERAL THEORY OF THE TRANSFORMER.}

114. Effects of coil resistances, magnetic leakage, and core reluctance on the action of a transformer. - The discussion of the transformer which is given in Chapter $\mathrm{X}$ is based very largely upon ideal conditions as explained in Art. 98, and the present chapter is devoted to the discussion of the effects of coil resistances, magnetic leakage, and core reluctance. These effects are usually small, and their influence on each other (which is very small) is ignored. That is, to say, the effect of each is here considered on the assumption that the transformer is otherwise ideal, and the combined effect of all three is considered to be the sum of the effects of each by itself. These effects may be summarized in a general way as follows :

Coil resistances do not effect the ideal current relation, namely, $I^{\prime} / I^{\prime \prime}=N^{\prime \prime} / N^{\prime}$; but they do affect the ideal voltage relation, namely, $E^{\prime} / E^{\prime \prime}=N^{\prime} / N^{\prime \prime}$; and the extent to which the coil resistances affect the ideal voltage relation depends upon the transformer load, the greater the load the more pronounced the inequality $E^{\prime} / E^{\prime \prime}>N^{\prime} / N^{\prime \prime}$.

Magnetic leakage does not* affect the ideal current relation of a transformer, but it does affect the ideal voltage relation; and the greater the transformer load the more pronounced the inequality $E^{\prime} / E^{\prime \prime}>N^{\prime} / N^{\prime \prime}$, except when the receiving circuit is like a condenser when the inequality is reversed.

Core reluctance necessitates magnetizing current, and of course the existence of magnetizing causes the ratio of primary current to secondary current to differ from the inverse ratio of primary to secondary turns. The extent to which the ideal current rela-

* In the constant-current transformer, which is described in Art. 107, the magnetic leakage is very large when the load is small, and in this case the magnetic leakage does affec: the current relation perceptibly. 
tion is thus vitiated by magnetizing current decreases with increase of transformer load.

The so-called potential transformer. - When it is desired to operate a transformer to transform a voltage in an accurately known ratio so as to enable a low-reading voltmeter to measure a high voltage, the effect of coil resistances and the effect of magnetic leakage must be reduced to a minimum.

The so-called current transformer. - When it is desired to operate a transformer to transform a current in an accurately known ratio so that an ammeter need not be connected in the main circuit of an alternator, the effect of core reluctance (magnetizing current) must be reduced to a minimum.

A transformer which is used to supply a voltmeter should be fairly large so that the current delivered to the voltmeter may be a light load for the transformer.

A transformer which is used to supply an ammeter should be small so that the current delivered to the ammeter may be a heavy load for the transformer. In saying that the transformer should be small it is understood that it is to be designed to take a small magnetizing current, and mere smallness does not ensure the relization of this condition.

115. Effect of core reluctance. Magnetizing current.-The magnetizing current of a transformer is not harmonic, ${ }^{*}$ but it is assumed to be harmonic in all calculations of transformer action; and its power component $M_{p}$ and its wattless component $M_{w}$ may be calculated from the known details of design of the transformer as follows :

Power component $M_{p^{*}}$ - Knowing the thickness of laminations, kind of iron, volume of iron, and frequency of the alternating current used, the total core loss $P_{h}+P_{0}$ of the transformer may be calculated as explained in Art. IO3. This total core loss is

* In the induction motor the mágnetizing current is very nearly harmonic if the supply voltage is harmonic, because of the large and constant magnetic reluctance of the air gap. 
equal to $E^{\prime} M_{p}$, so that

$$
M_{p}=\frac{P_{h}+P_{s}}{E^{\prime}}
$$

Wattless component $M_{w}$. - Knowing the maximum flux density in the transformer core (see Art. IOI) the permeability of the iron may be found from permeability tables or curves and the magnetic reluctance $\mathscr{R}$ of the magnetic circuit of the transformer may be calculated from the known dimensions of the core. The product of this magnetic reluctance by the maximum core flux $\Phi$ gives the maximum magnetomotive force required to magnetize the core, and this is equal to $4 \pi /$ ro times $N^{\prime}$ times the maximum value of the wattless component $\left(\sqrt{2} M_{10}\right)$. That is

from which we have

$$
\Phi \mathscr{R}=\frac{4 \pi}{10} N^{\prime} \sqrt{2} M_{10}
$$

$$
M_{w}=\frac{10 \Phi \mathscr{R}}{4 \sqrt{2} \pi N^{\prime}}
$$

Admittance* corresponding to magnetizing current. - The value of the magnetizing current of a transformer is very nearly independent of the value of the load current $I^{\prime}$ which flows through the primary coil, very much as if the magnetizing current flowed through a circuit of definite admittance $g_{1}-j b_{1}$, connected in parallel with the primary coil of an ideal transformer. That is to say, a transformer which takes a definite magnetizing current is equivalent to an ideal transformer with a certain circuit shunted across its primary coil. The conductance $g_{1}$ is that factor which multiplied by $E^{\prime}$ gives the power component of the magnetizing current, and the susceptance $b_{1}$ is that factor which multiplied by $E^{\prime}$ gives the wattless component of the magnetizing current. That is

$$
M_{p}=g_{1} E^{\prime}
$$

* The shunt circuit which is imagined to be connected in parallel with the primary coil of an ideal transformer to represent the effect of core reluctance, could of course be specified in terms of impedance (resistance and reactance) instead of in terms of admittance (conductance and susceptance), but specification in terms of conductance and susceptance is to be preferred. See Arts. 29 and 46. 
and

$$
M_{w}=b_{1} E^{\prime}
$$

whence, using the values of $M_{p}$ and $M_{v}$ from equations (i) and (ii) we have:

$$
g_{1}=\frac{P_{h}+P_{e}}{E^{\prime 2}}
$$

and

$$
b_{1}=\frac{\operatorname{IO} \Phi \mathscr{R}}{4 \sqrt{2} \pi N^{\prime} E^{\prime}}
$$

116. Effect of coil resistances. - The clock diagram, Fig. 202, shows the general effect (greatly exaggerated) of the resistances of the transformer coils. The line $O \Phi$ represents the harmon-

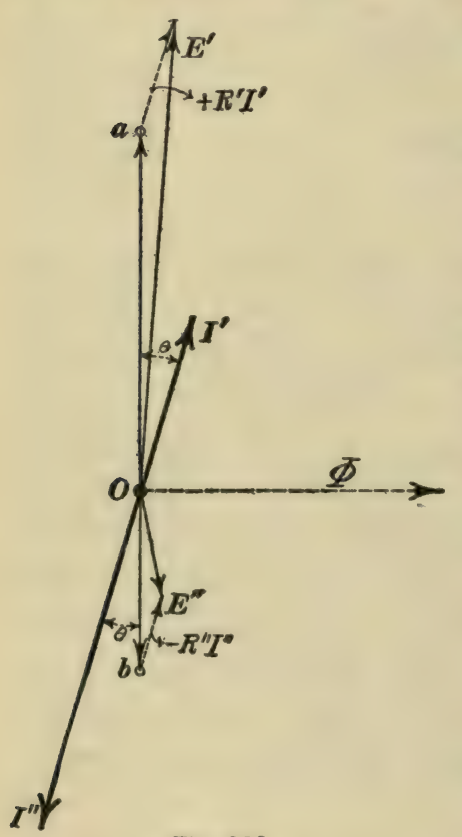

Fig. 202. ically varying flux in the transformer core; the line $\mathrm{Oa}$ represents the useful part of $E^{\prime}$, that is, the part which balances the electromotive force which is induced in the primary coil by the varying core flux; and the line $O b$ represents the total electromotive force induced in the secondary coil. The ratio of $O a$ to $O b$ is equal to $N^{\prime} / N^{\prime \prime}$, the magnetic leakage being zero. The line $O I^{\prime \prime}$ represents the secondary current, the secondary receiving circuit having a given power factor equal to $\cos \theta$, and the line $O I^{\prime}$ represents the primary current. The total primary voltage $E^{\prime}$ is the vector sum of $O a$ and $R^{\prime} I^{\prime}, R^{\prime} I^{\prime}$ being of course parallel to $I^{\prime}$; and the electromotive force $E^{\prime \prime}$ at the terminals of the secondary coil is the vector difference $O b-R^{\prime \prime} I^{\prime \prime}, R^{\prime \prime} I^{\prime \prime}$ being of course parallel to $I^{\prime \prime}$.

When the secondary receiving circuit has unity power factor, 
that is when the angle $\theta$ is zero, then $R^{\prime} I^{\prime}$ and $R^{\prime \prime} I^{\prime \prime}$ are parallel to $O a$ and $O b$ respectively. In this case $O a$ is equal numerically to $E^{\prime}-R^{\prime} I^{\prime}$, and $E^{\prime \prime}$ is equal numerically to $O b-R^{\prime \prime} \Gamma^{\prime}$. That is, coil resistances have their greatest effect in causing the secondary terminal voltage of a loaded transformer to fall below the ideal value $\left(E^{\prime \prime}=E^{\prime} \times N^{\prime \prime} / N^{\prime}\right)$ when the secondary receiving circuit has unit power factor.

When the secondary receiving circuit has a very low power factor, that is, when the angle $\theta$, Fig. 202 , is nearly $90^{\circ}$, then $R^{\prime} I^{\prime}$ and $R^{\prime \prime} I^{\prime \prime}$ are nearly at right angles to $O a$ and $O b$ respectively. In this case $O a$ is very nearly equal, numerically, to $E^{\prime}$, and $O b$ is very nearly equal, numerically, to $E^{\prime \prime}$, so that the ratio $E^{\prime} / E^{\prime \prime}$ is nearly equal to the ideal value $N^{\prime} / N^{\prime \prime}$.

Transformer regulation is mainly dependent upon coil resistances when the receiving circuit is non-inductive, inasmuch as we shall find that magnetic leakage has little effect on the voltage relation in this case. On the other hand, when the angle $\theta$, Fig. 202 , is nearly $90^{\circ}$, the regulation is not greatly affected by coil resistances.

117. Effect of magnetic leakage. - It is shown in the next article that a transformer having magnetic leakage (but of course ideal in other respects) is equivalent to an ideal transformer with a certain outside inductance, $P$, connected in series with its primary coil. This inductance is called the leakage inductance of the transformer, and the reactance value $\omega P$ of this inductance is called the leakage reactance of the transformer.

The clock diagrams, Figs. 203 and 204, show the general effect (greatly exaggerated) of the magnetic leakage of a transformer. The various lines have the same significance as in Fig. 202, except, of course, the line $\omega P I^{\prime}$, which represents the part of $E^{\prime}$ which is lost in overcoming the leakage reactance. The line $\omega P I^{\prime}$ is $90^{\circ}$ ahead of $I^{\prime}$ in phase. It is to be noted that the entire magnetic leakage is equivalent to the inductance $P$ in the primary circuit, so that the secondary coil is to be considered as 
having no inductance and therefore the total secondary induced voltage $O b$ is available as terminal voltage.

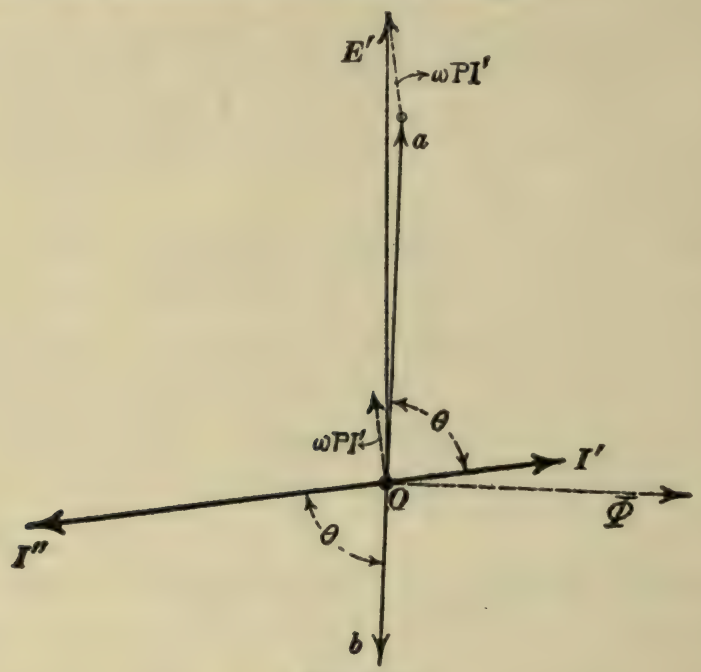

Fig. 203.

When the secondary receiving circuit is highly inductive, that is, when the secondary current $I^{\prime \prime}$ lags nearly $90^{\circ}$ behind $E^{\prime \prime}$,

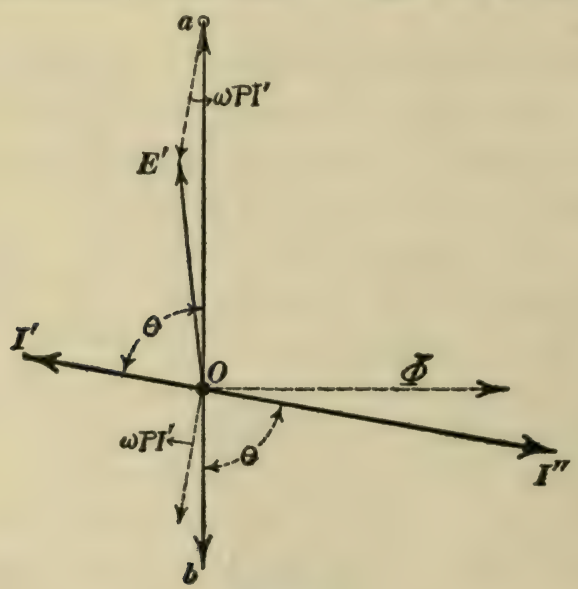

Fig. 204.

as shown in Fig. 203, then $\omega P I^{\prime}$ is nearly parallel to $O a$ and the useful part of the primary voltage, $O a$, is numerically equal 
to $E^{\prime}-\omega P I^{\prime}$. In this case the effect of magnetic leakage is to cause the secondary voltage of the loaded transformer to fall considerably below its ideal value.

When the secondary receiving circuit is non-inductive, that is, when the angle $\theta$ in Fig. 203 is zero, then $\omega P I^{\prime}$ is at right angles to $O a$. In this case $O a$ is very nearly equal numerically to $E^{\prime}$, so that the secondary voltage is nearly equal to its ideal value.

When the secondary receiving circuit is like a condenser, that is, when the secondary current $I^{\prime \prime}$ is nearly $90^{\circ}$ ahead of $E^{\prime \prime}$ in phase as shown in Fig. 204, then $\omega P I^{\prime}$ is nearly parallel but opposite in direction to $O a$ so that $O a$ is nearly equal numerically to $E^{\prime}+\omega P I^{\prime}$. In this case the effect of magnetic leakage is to cause the secondary voltage of the loaded transformer to exceed its ideal value.

118. Magnetic leakage is equivalent to an inductance in series with the primary coil. - To show that the magnetic leakage of a transformer is equivalent to an inductance in series with the primary coil (magnetizing current being ignored) it is necessary to consider the relation between the alternating current $i$ in any coil of wire and the harmonically varying flux produced by $i$. The flux is proportional to $i$, it is of course in phase with $i$, and it is the effects of such a flux that constitutes what is called the inductance of the coil. If, therefore, we can show that the total flux through the primary coil of a transformer can be resolved into two parts, one of which passes through the primary
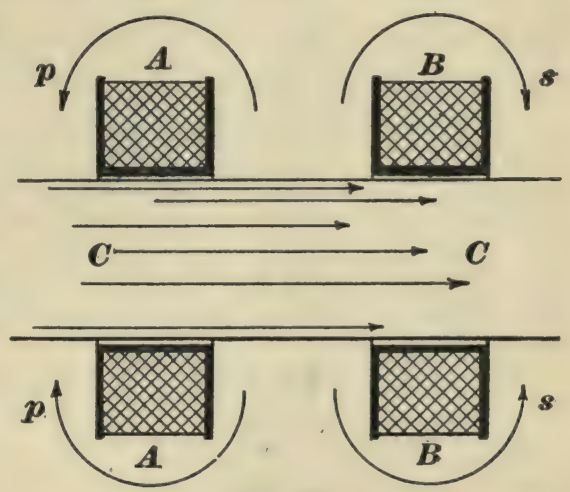

Fig. 205. coil only and is proportional to and in phase with the primary current, and the other of which passes through both the primary and the secondary coils, then our proposition will be established. 
Let $A$, Fig. 205, be the primary coil, $B$ the secondary coil, and $C$ the iron core of a transformer. As the (harmonic) alternating currents in coils $A$ and $B$ pulsate in value, harmonically varying fluxes are produced through the core and around each coil. Let the line $O C$ in the clock diagram, Fig. 206, represent

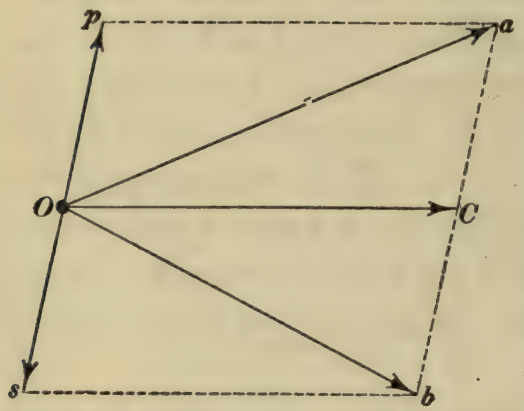

Flg. 206. the harmonically varying flux through the core, let $O p$ represent the harmonically varying flux which encircles coil $A$, and let $O s$ represent the harmonically varying flux which encircles coil $B$. The fluxes $O p$ and $O s$ are proportional to and in phase with $I^{\prime}$ and $I^{\prime \prime}$ respectively. But $I^{\prime \prime}$ is proportional to and opposite in phase to $I^{\prime}$, so that $p O s$, Fig. 206 , is a straight line and its length $p s$ is proportional to $I^{\prime}$ and of course in phase with $I^{\prime}$.

The total harmonically varying flux through coil $A$ is the vector sum of $O p$ and $O C$ which is $O a$, and the total harmonically varying flux through coil $B$ is the vector sum of $O s$ and $O C$ which is equal to $O b$, but $O a$ is equal to the vector sum of $O b$ and $b a$, and $b a$ is parallel and equal to $s p$. Therefore the flux $O a$ which passes through the primary coil can be considered to be made up of two parts, one of which $O b$ passes through both coils, and the other of which $b a$ passes through the primary coil only and is proportional to and in phase with $I^{\prime}$.*

* The student will find that many writers on alternating current theory represent the effects of magnetic leakage of transformers and induction motors as equivalent to a certain reactance in the primary circuit together with a certain reactance in the secondary circuit; but when the effects of magnetizing current are ignored (and all writers do ignore them because to take these effects into account in the discussion of magnetic leakage leads to great complications which do not seem to be justified by the requirements of practice) it is only possible to separate the effects of magnetic leakage into two such reactances arbitrarily and without any basis in fact. 
119. Calculation of leakage inductance $P$. - The value of the inductance-equivalent of the magnetic leakage of a transformer depends upon the size and shape of the primary and secondary coils, and it is very small when the windings are arranged in alternate sections of primary and secondary as described in Art. 97. The calculation of the inductance-equivalent of magnetic leakage would be very simple if the whole of the leakage flux, $p+s$ in Fig. 205, passed between the primary and secondary coils and no portion of it passed through the region occupied by the windings of wire; but in an actual transformer the two coils are always placed as near together as possible, and nearly the whole of the leakage flux passes across the region occupied by the windings. We shall, however, calculate the value of the leakage inductance $P$ on the basis of the idea that all of the leakage flux $p$, Fig. 205, encircles all of the primary turns, and that all of the leakage flux $s$, Fig. 205, encircles all of the secondary turns; and, in calculating the total leakage flux, $p+s$, on this basis, we shall count only I $/ n$th part of any portion of leakage flux which encircles $\mathrm{I} / n$th of either coil. Thus $\phi$ lines of flux encircling one half of one of the transformer coils is equivalent to $\phi / 2$ lines of flux encircling the whole coil.

When we shall have calculated the total leakage flux, $p+s$ (which is represented by the line $b a$, Fig. 206, and which is to be thought of as encircling the primary coil), due to a given value, $i^{\prime}$, of current in the primary coil and a corresponding current, $i^{\prime \prime}\left(=i^{\prime} \times N^{\prime} / N^{\prime \prime}\right)$, in the secondary coil, then the product of the leakage flux, $p+s$, times the number of primary turns $N^{\prime}$ is equal to the product of the inductance $P$ times the current $i^{\prime}$, as explained in Appendix $A$, so that

$$
P=\frac{(p+s) N^{\prime}}{i^{\prime}}
$$

In the following discussion it is convenient to consider the total leakage flux $p+s$ in three parts, namely, the part $\Phi^{\prime}$ which depends upon the flux which passes through the region 
occupied by the primary coil, the part $\Phi^{\prime \prime}$ which depends upon the flux which passes through the region occupied by the sec-

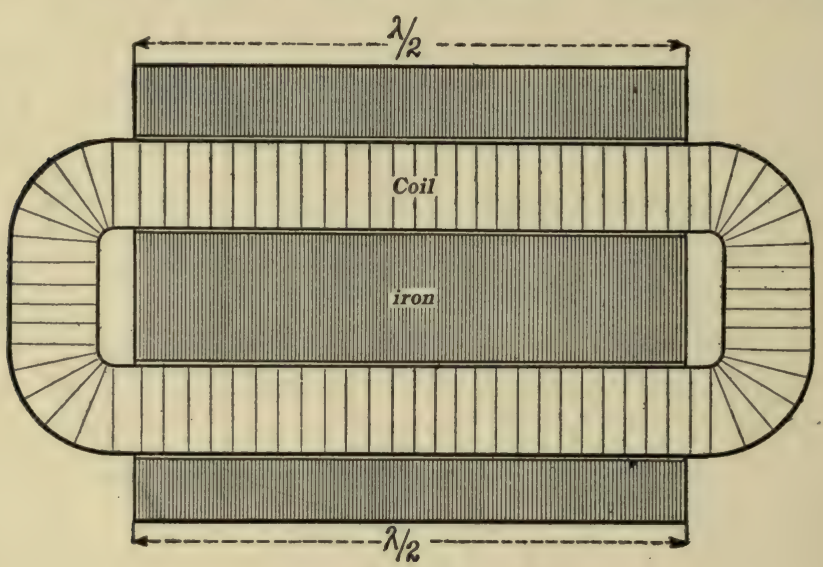

Fig. 207.

ondary coil, and $\Phi^{\prime \prime \prime}$ which is the actual flux passing between the coils so that

$$
p+s=\Phi^{\prime}+\Phi^{\prime \prime}+\Phi^{\prime \prime \prime}
$$

Figures 207 and 208 show side and end views of a shell-type transformer of which the primary and secondary coils are not in-

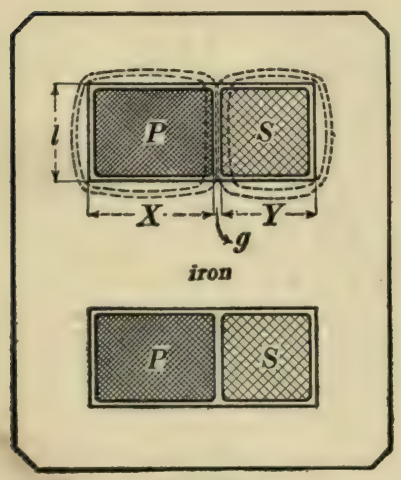

Fig. 208. terleaved. The trend of the leakage flux is shown by the dotted lines in the upper part of Fig. 208 (omitted from the lower part of the figure for the sake of clearness), and the dimensions $X, Y, g, \lambda$ and $l$ are to be expressed in centimeters. Fig. 209 is an enlargement of the upper part of Fig. 208.

Consider the flux, $d \phi$, which crosses the primary coil between the dotted lines $a a$, Fig. 209; the complete path of this flux, as indicated by the dotted line, encircles a fractional part $x / X$ of the primary turns $N^{\prime}$ of which the 
magnetomotive force $4 \pi i^{\prime} \times x / X \times N^{\prime *}$. Ignoring the reluctance of the iron, the magnetic reluctance of the path of the leakage flux $d \phi$ is the reluctance, $l /(\lambda d x)$, of the air gap $a a$, of which the sectional area is $\lambda d x$ (counting both legs of the coils each of length $\lambda / 2$ as shown in Fig. 207) and the length is $l$. Therefore, placing $d \phi$ equal to magnetomotive force divided by reluctance, we have

$$
d \phi=\frac{4 \pi i^{\prime} N^{\prime} \lambda}{l X} \cdot x d x
$$

but this flux $d \phi$ encircles the fractional part $x / X$ of the primary turns, therefore the fractional part $x / X$ of $d \phi$ should be

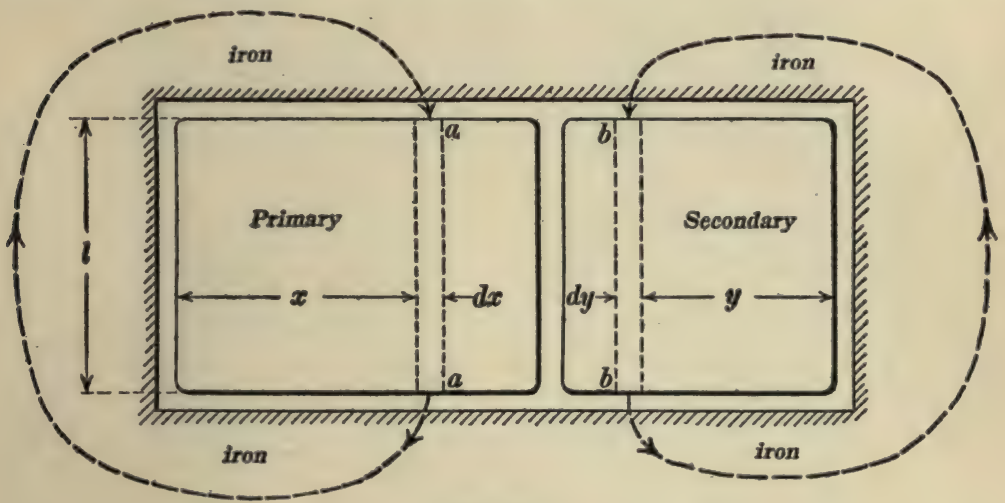

Fig. 209.

counted as encircling the whole primary coil. Representing by $d \Phi^{\prime}$ this flux, $x / X \times d \phi$, which is to be thought of as encircling the whole primary coil, we have

$$
d \Phi^{\prime}=\frac{4 \pi i^{\prime} N^{\prime} \lambda}{l X^{2}} \cdot x^{2} d x
$$

Therefore the value of $\Phi^{\prime}$ is

$$
\Phi^{\prime}=\frac{4 \pi i^{\prime} N^{\prime} \lambda}{l X^{2}}-\int_{x=0}^{x=x} x^{2} d x=\frac{4 \pi i^{\prime} N^{\prime} \lambda X}{3 l}
$$

Similarly, remembering that $N^{\prime} i^{\prime}$ equals $N^{\prime \prime} i^{\prime \prime}$, we may find

\footnotetext{
* All quantities in this article are understood to be expressed in c.g.s. units.
} 
244 ELEMENTS OF ELECTRICAL ENGINEERING.

$$
\Phi^{\prime \prime}=\frac{4 \pi i^{\prime} N^{\prime} \lambda Y}{3 l}
$$

The flux $\Phi^{\prime \prime \prime}$ which passes between the coils is produced by the full magnetomotive force $4 \pi N^{\prime} i^{\prime}$ (or $4 \pi N^{\prime \prime} i^{\prime \prime}$ which is the same thing), and the air part of the magnetic circuit through

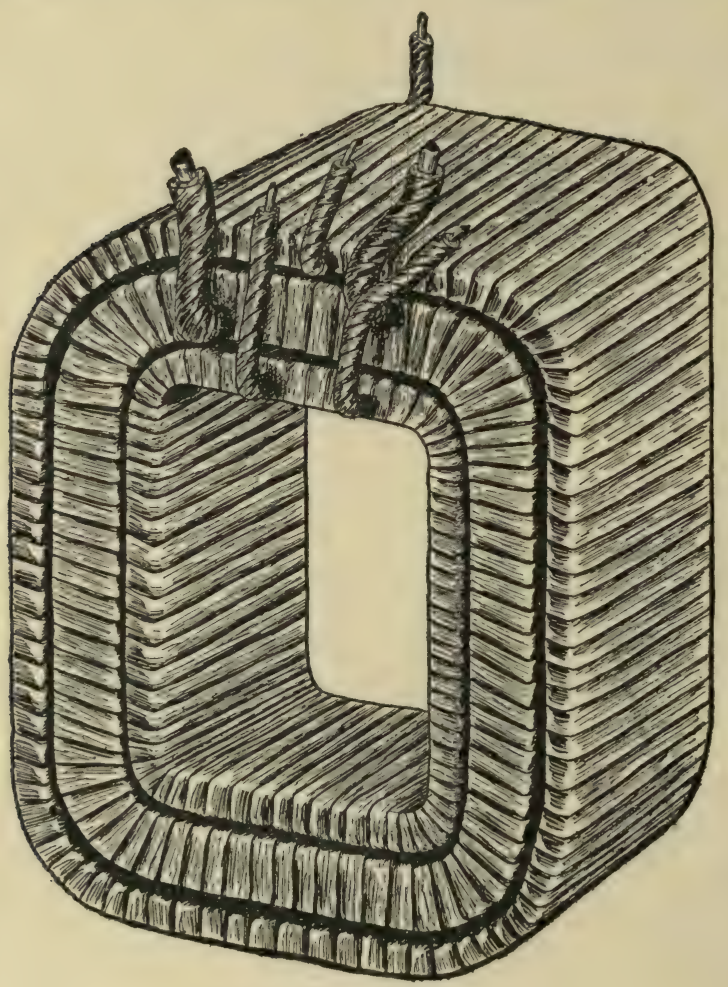

Fig. 210.

which $\Phi^{\prime \prime \prime}$ passes has a length $l$ and a sectional area $\lambda g$ (counting both legs of the coils). Therefore

$$
\Phi^{\prime \prime \prime}=\frac{4 \pi i^{\prime} N^{\prime} \lambda g}{l}
$$

The total leakage flux, counted as if it all passed between the coils, is $\Phi^{\prime}+\Phi^{\prime \prime}+\Phi^{\prime \prime \prime}$ which is equal to $p+s$ in equation 
(i). Therefore substituting the value of $\Phi^{\prime}+\Phi^{\prime \prime}+\Phi^{\prime \prime \prime}$ from equations (iii), (iv) and (v) for $p+s$ in equation (i) we have

$$
P=\frac{4 \pi N^{\prime 2} \lambda}{l}\left[\frac{X}{3}+\frac{Y}{3}+g\right]
$$

Some leakage flux passes between the transformer coils where they project beyond the shell-type iron core. This leakage flux has a longer air-path than $l$, say three times $l$. Therefore for $\lambda$ we should take the total length of the coils lessened by, say, $2 / 3$ of the length which is beyond the core.

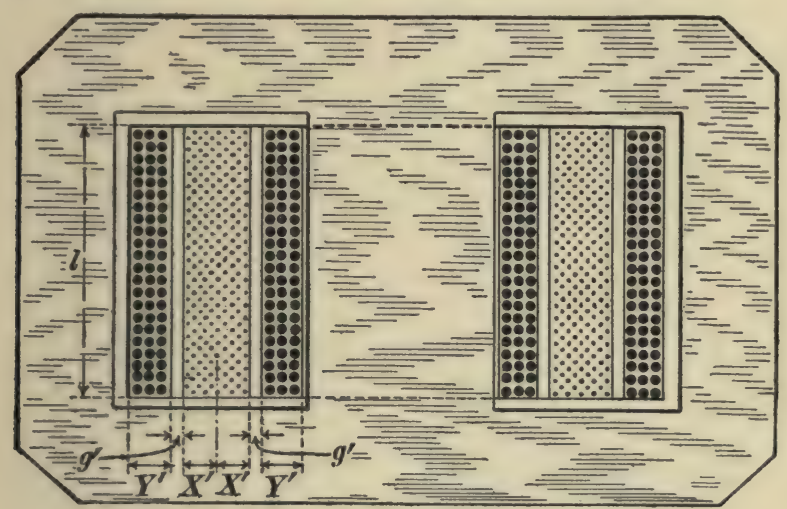

Fig. 211.

Equation (29) expresses the leakage inductance in centimeters, and the right-hand member of this equation must be divided by $10^{9}$ to give $P$ in henrys, all dimensions being in centimeters.

When the primary and secondary coils are wound in sections and interleaved, equation (29) must be modified accordingly. Thus Fig. 2 ro shows the primary coil placed between two halves of the secondary. In this case equation (29) becomes

$$
P=\frac{\pi N^{\prime 2} \lambda}{l}\left[\frac{X^{\prime}}{3}+\frac{Y^{\prime}}{3}+g^{\prime}\right]
$$

The dimensions $X^{\prime}, Y^{\prime}$ and $g^{\prime}$ are shown in Fig. $21 \mathrm{I}, \lambda$ being the length of the coils lessened by, say, $2 / 3$ of the length of the portions which project beyond the core. 
Figure 212 is a sketch of a core-type transformer showing the dimensions which are to be used in equation (30) to give the
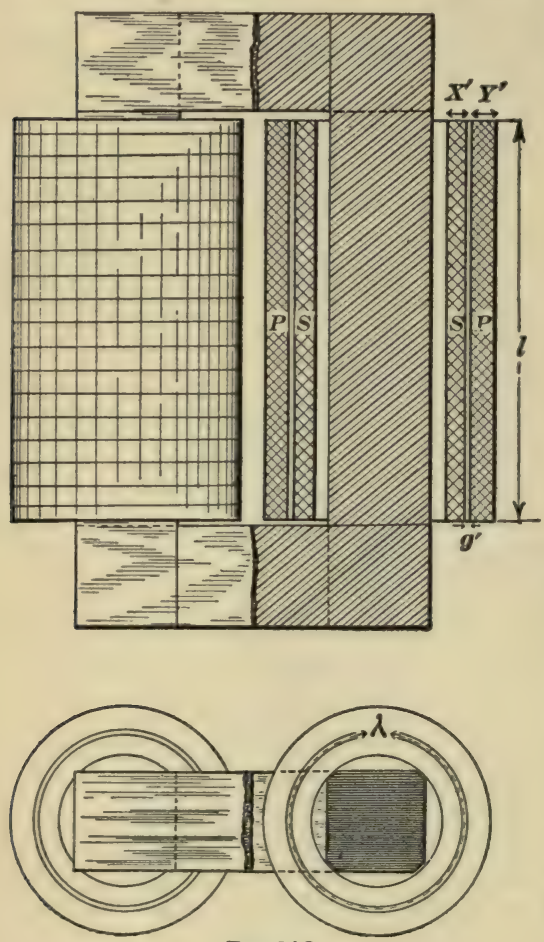

Fig. 212. leakage inductance, $N^{\prime}$ being the total number of turns in both parts of the primary coil, which parts are supposed to be connected in series with each other.

120. Combination of simple circuits which is equivalent to a transformer delivering current to a given secondary receiving circuit. - In Art. IOO it is shown that an ideal transformer delivering current to a receiving circuit of given resistance $R$ and given reactance $X$ is equivalent to a simple circuit of resistance $r$ and reactance $x$ connected directly to the supply mains, where $r=\left(N^{\prime} / N^{\prime \prime}\right)^{2} R$ and $x=\left(N^{\prime} / N^{\prime \prime}\right)^{2} X$. An actual transformer delivering current to a given receiving circuit is equivalent to an ideal transformer combined with certain simple circuits, and this combination in turn is equivalent to a combination of simple circuits from which all transformer action is eliminated.

Figure 2 I 3 represents an actual transformer delivering current to a given receiving circuit ; Fig. 2 I 4 represents a combination of simple circuits with an ideal transformer, which combination is equivalent to Fig. $2 \mathrm{I} 3$; and Fig. 2 I 5 represents a combination of simple circuits which is equivalent to Fig. 2I3. In these figures $\omega P$ represents the leakage reactance of the transformer, $R^{\prime}$ the resistance of the primary coil, $R^{\prime \prime}$ the resistance of the 


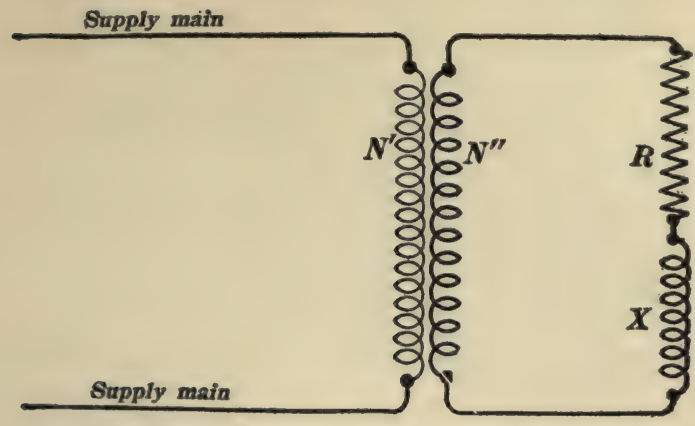

Actual transformer delivering current to a given receiving circuit.

Fig. 213.

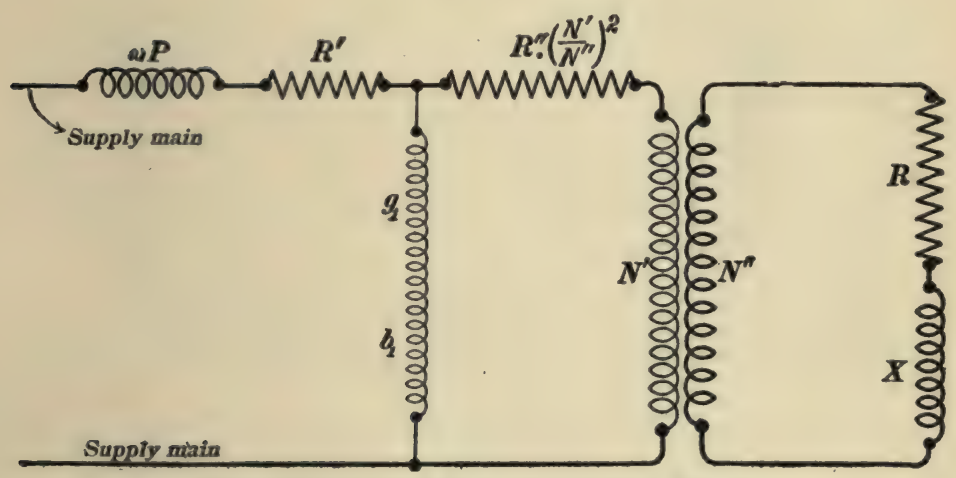

Ideal transformer arranged to be equivalent to Fig. 213.

Fig. 214.

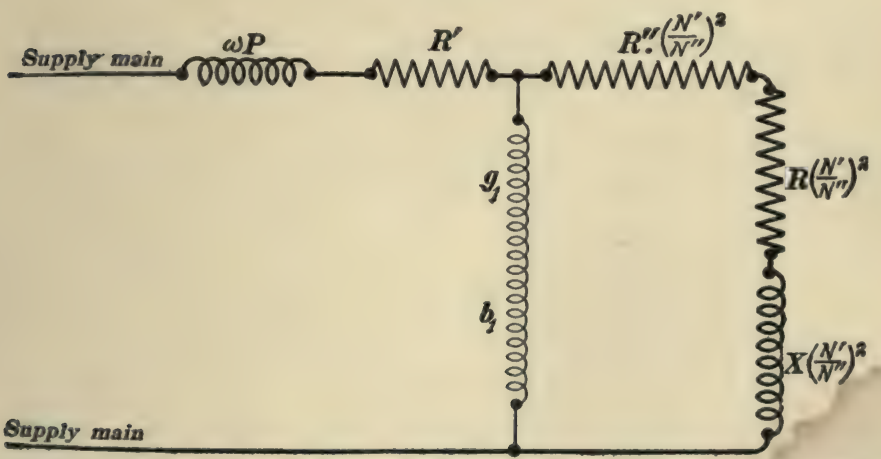

Combination of simple circuits which is equivalent to Fig. 213. Magnetizing current variable.

Fig. 215. 
secondary coil, $N^{\prime}$ the number of turns of wire in the primary coil, $N^{\prime \prime}$ the number of turns of wire in the secondary coil, $R$ the resistance of the secondary receiving circuit, $X$ the reactance of the secondary receiving circuit, and $g_{1} b_{1}$ is the circuit in which the magnetizing current $M$ may be thought of as flowing, $g_{1} E^{\prime}$.being the power component of $M$, and $b_{1} E^{\prime}$ being the wattless component of $M$. (See Art. I I 5).

When $R^{\prime}$ and $\omega P$ are small, the loss of voltage in $R^{\prime}$ and

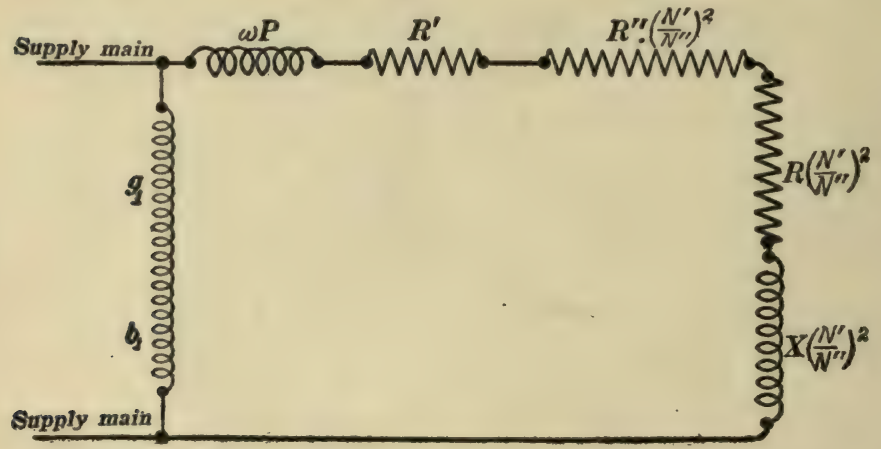

Combination of simple circuits which is approximately equivalent to Fig. 2r3. Magnetizing current assumed to be constant.

Fig. 216.

$\omega P$, Fig. 215 , is negligible, and the voltage across the circuit $g_{1} b_{1}$ is sensibly equal to the supply voltage, whatever the load on the transformer may be, so that, the supply voltage being constant, the magnetizing current $M$ is sensibly the same in value at all loads. In this case the combination shown in Fig. 216 reproduces the conditions of Fig. 2 I 3 with sufficient accuracy for most practical purposes, and in fact nearly all practical calculations of transformers and induction motors are based on Fig. 216.

121. The circle diagram of the transformer when the secondary receiving circuit is non-inductive. - It was shown in Art. 38 that the locus of the current produced by a constant voltage in a circuit of constant reactance and variable resistance is a circle of which the diameter is at right angles to (and $90^{\circ}$ behind) the voltage. To vary the resistance $R$ of the secondary receiving 
circuit (reactance $X$ of secondary receiving circuit being zero) is to change the resistance in the main circuit of Fig. 216 without changing its reactance $\omega P$. Therefore, for varying values of $R$, the locus of the load current $I^{\prime}$ in the primary coil of a transformer is a circle, and the total primary current is the vector sum of $I^{\prime}$ and the magnetizing current which flows through the shunt circuit $g_{1} b_{1}$. This current locus is shown in Fig. 2I7. The line $E^{\prime}$ represents the constant primary voltage, the line $M$ represents

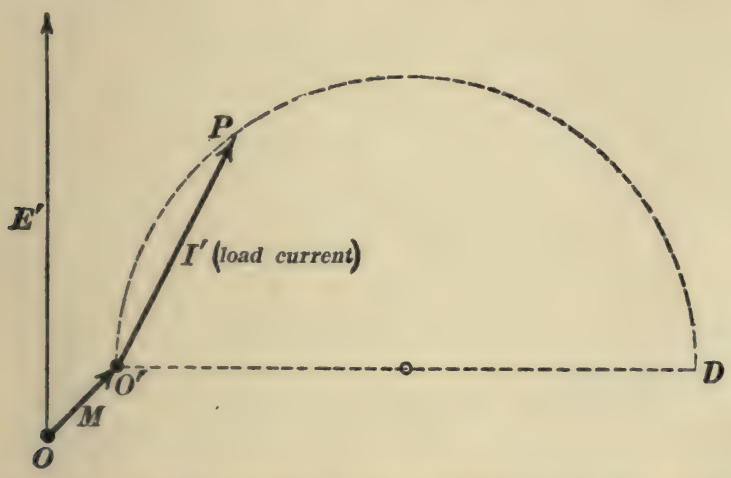

Circle diagram of transformer delivering current to non-inductive receiving circuit. Based on Fig. 216.

Fig. 217.

the constant magnetizing current, and the line $I^{\prime}$ drawn from the point $O^{\prime}$ to any point $P$ on the circular locus represents a possible value of the load current in the primary coil. The diameter $O^{\prime} D$ of the circle represents the current which $E^{\prime}$ would produce in a circuit of reactance $\omega P$ and of zero resistance, this diameter therefore represents $E^{\prime} / \omega P$ amperes. The total primary current is of course the vector sum of $M$ and $I^{\prime}$ and it is represented by a line drawn from $O$ to $P$. This circle diagram is especially useful in induction motor calculations.

122. Calculation of transformer regulation. - Given a transformer of which the leakage reactance $\omega P$, the coil resistances $R^{\prime}$ and $R^{\prime \prime}$, and the primary and secondary turns $N^{\prime}$ and $N^{\prime \prime}$ are known. The transformer is operated with a constant primary 
voltage $E^{\prime}$ of given value, and it is required to calculate the secondary terminal voltage when the transformer delivers current to a receiving circuit of given reactance $X$ and given resistance $R$. The effect of magnetizing current is entirely negligible, and to base the solution of this problem on Fig. 2 I 6 rather than upon Fig. 215 is merely to neglect the effect of magnetizing current. Solution. - Calculate $I^{\prime}$ by dividing $E^{\prime}$ by the impedance of the main circuit in Fig. 216 , remembering of course that the total resistance of the circuit is $R^{\prime}+\left(N^{\prime} / N^{\prime \prime}\right)^{2} R^{\prime \prime}+\left(N^{\prime} / N^{\prime \prime}\right)^{2} R$, and that the total reactance of the circuit is $\omega P+\left(N^{\prime} / N^{\prime \prime}\right)^{2} X$. Having thus found the value of $I^{\prime}$, calculate the reactance voltage $I^{\prime}\left(N^{\prime} / N^{\prime \prime}\right)^{2} X$ and the resistance voltage $I^{\prime}\left(N^{\prime} / N^{\prime \prime}\right)^{2} R$. The square root of the sum of the squares of these two voltages multiplied by $N^{\prime \prime} / N^{\prime}$ is the required secondary terminal voltage.

If the transformer load is reduced to zero the secondary te1minal voltage rises to the ideal value $\left(=E^{\prime} \cdot N^{\prime \prime} / N^{\prime}\right)$, and, by expressing the increase of secondary terminal voltage in per cent. of the value of the secondary terminal voltage under load, we have the regulation in per cent. 


\section{CHAPTER XII.}

\section{THE INDUCTION MOTOR.}

123. The induction motor. - The induction motor consists of a primary member, to the windings of which polyphase* alternating currents are supplied, and a secondary member with short-circuited windings. The primary member is generally stationary and it is called the stator, and the secondary member generally rotates and it is called the rotor. The stator windings are exactly like the windings of a polyphase alternator armature, $\uparrow$ the alternating currents in these windings produce a rotating state of magnetism in the laminated iron of the stator, this rotating magnetism induces currents in the short-circuited rotor windings, and the stator magnetism exerts force on these induced currents and causes the rotor to revolve.

The structure of the induction motor is shown in Figs. 218 to 222 , which show the details of a small motor manufactured by the Westinghouse Electric Company. Figure 218 shows the cast iron frame for holding the stator laminations, Fig. 2 I9 shows the stator lamina-

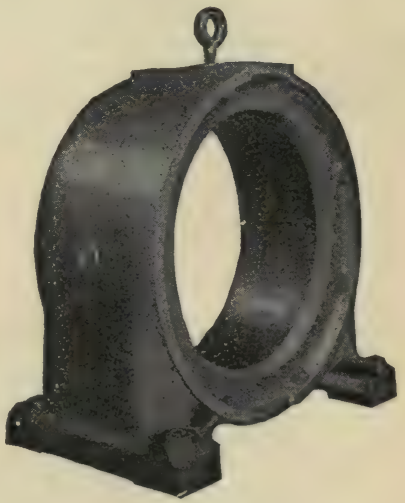

Fig. 218. tions assembled, Fig. 220 shows the stator complete with its windings, Fig. 22I shows the laminated core of the rotor mounted on its shaft, and Fig. 222 shows the rotor complete. The rotor shown in Fig. 222 is of the so-called squirrelcage type, and its windings consist of heavy copper rods placed in nearly closed slots and connected at both ends to massive

* The single-phase induction motor is described later.

fOr like the windings of a direct-current armature with connections like the alternating-current connections of a synchronous converter armature. 
short-circuiting rings of copper. Figure 223 shows a complete three-phase induction motor, the three wires for supplying the

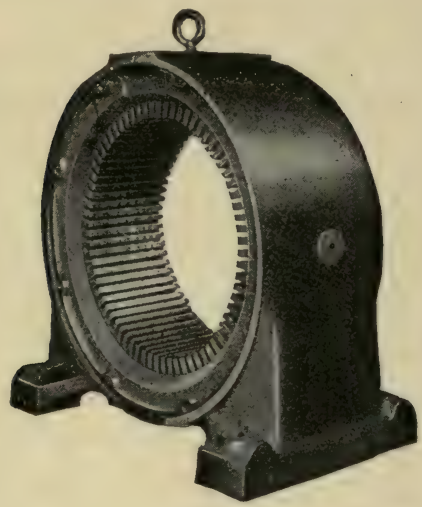

Fig. 219.

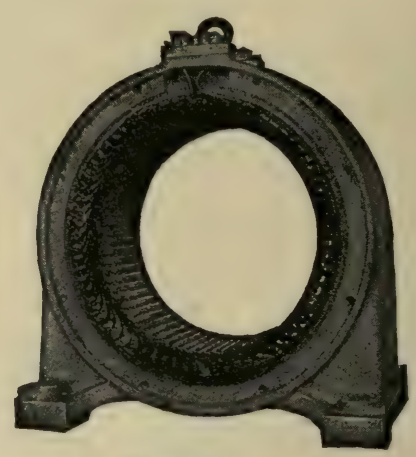

Fig. 220.

three-phase currents to the stator are shown at the lower lefthand side in the figure.

In some cases, especially for induction motors of large size, the rotor is wound like a polyphase alternator armature, and the winding is short-circuited through an adjustable resistance. Thus

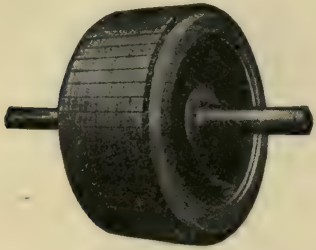

Fig. 221.

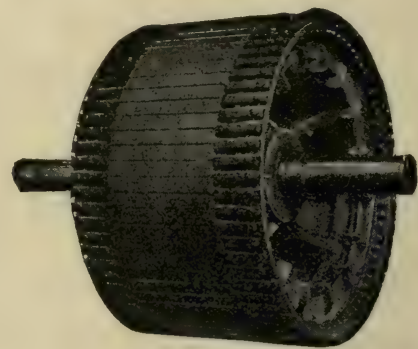

Fig. 222.

Fig. 224 shows a wire-wound rotor with a device in its interior for varying the short-circuit resistance of the windings ; the resistance is usually controlled by pushing on a knob at the end of the rotor shaft. In Fig. 224 the resistance is controlled by a lever (not shown in the figure) which engages the ring-like projection on the short sleeve. Figure 225 shows a wire-wound rotor with the terminals of the winding brought out to three collector rings through which the windings may be connected to an external 


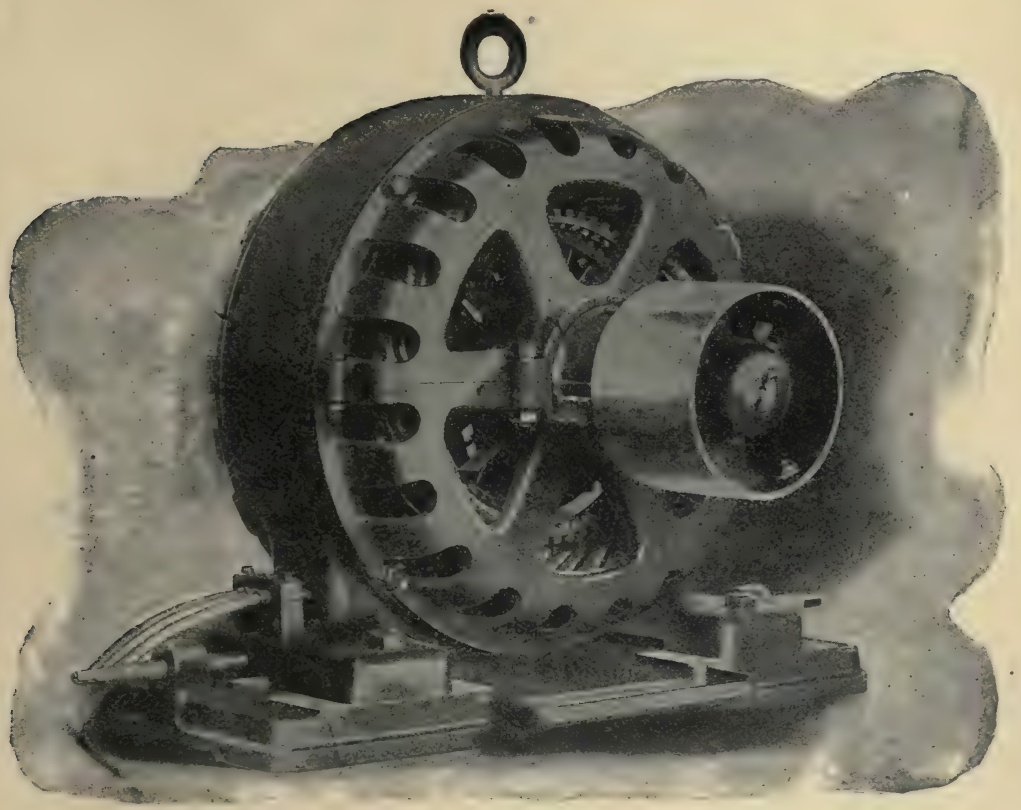

Westinghouse Induction Motor Complete.

Fig. 223.

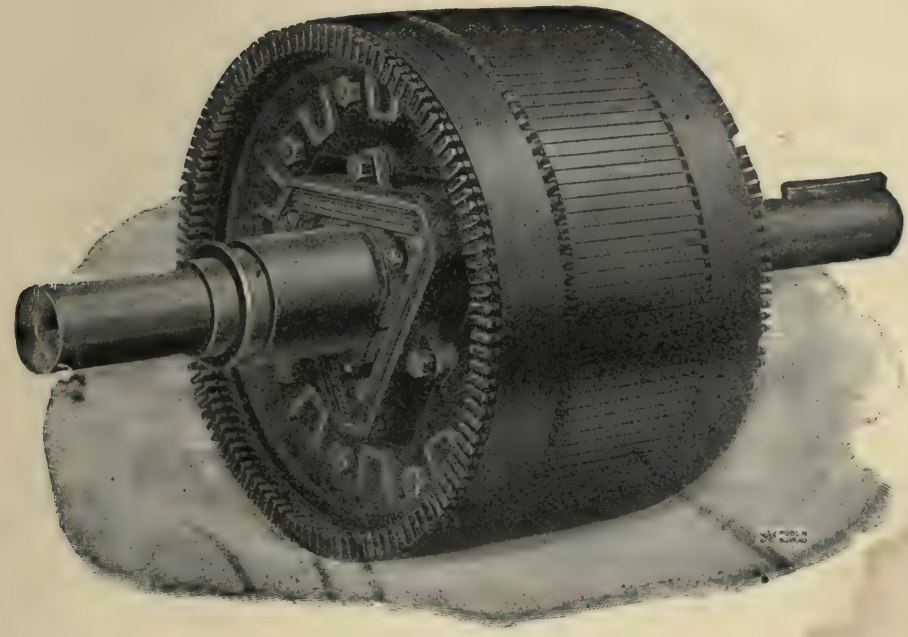

Fig. 224. 
adjustable resistance. Figures 224 and 225 are taken from the publications of the General Electric Company.

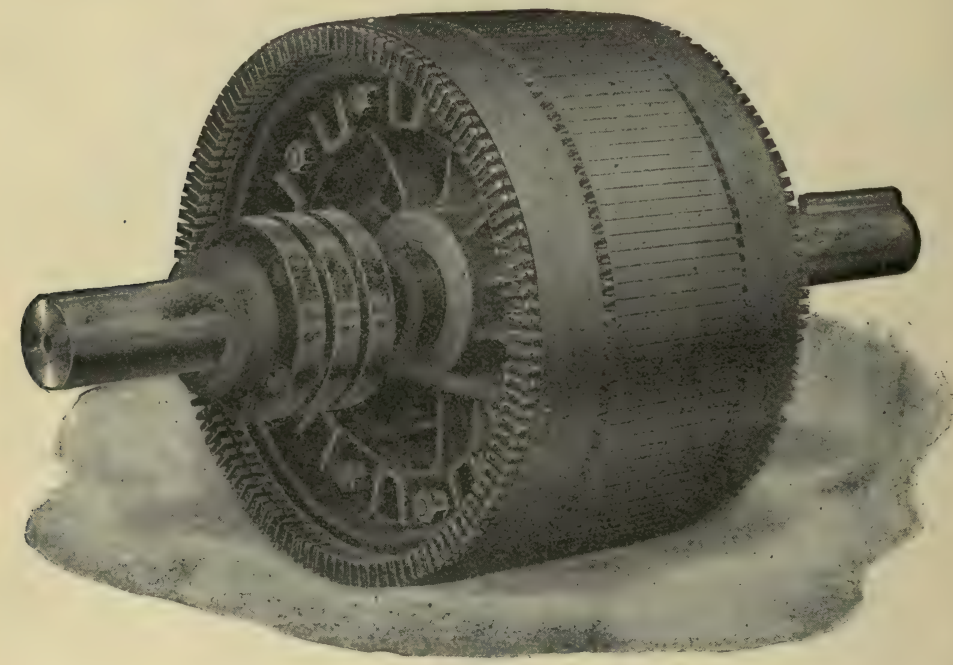

Fig. 225.

124. Action of the induction motor.* - The stator windings of the polyphase induction motor produce a rotating magnetism which acts upon the short-circuited rotor in the same way that the rotor would be acted upon by a mechanically rotated directcurrent-excited field magnet, as indicated in Fig. 226. The action of this mechanically rotated field magnet upon the short-circuited rotor, as indicated in Fig. 226, is as follows : Electromotive forces are induced in the rotor rods under the moving poles NSNS, these electromotive forces produce currents in the short-circuited rotor rods, and the field flux pushes sidewise on the rotor rods because of the currents in the rods, and thus exerts a dragging torque upon the rotor.

The production of rotating magnetism by the stator windings. As stated above the stator windings are arranged exactly like the armature windings of a polyphase alternator armature. Figure

* A very simple discussion of the action of the induction motor is given in Appendix B, Art. 15 . 


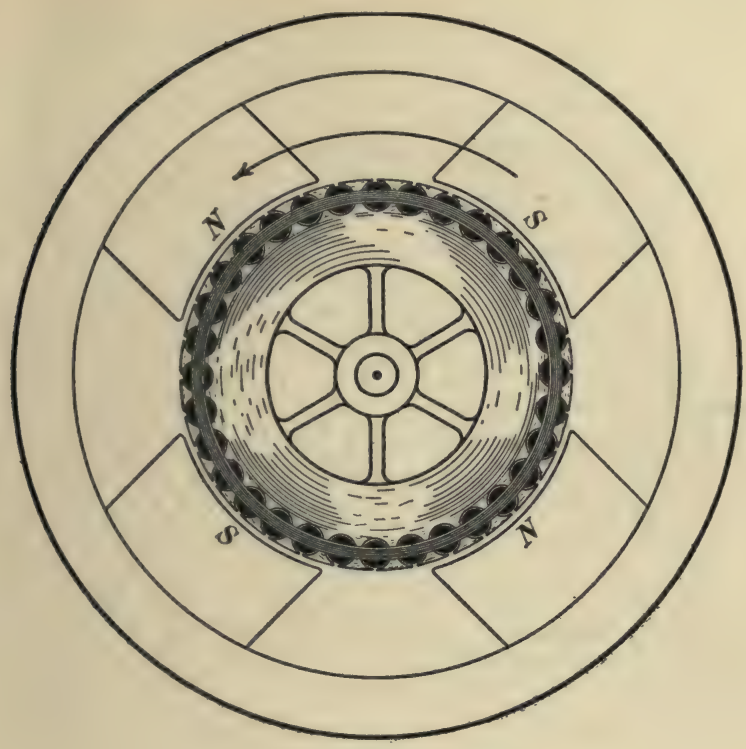

Fig. 226.

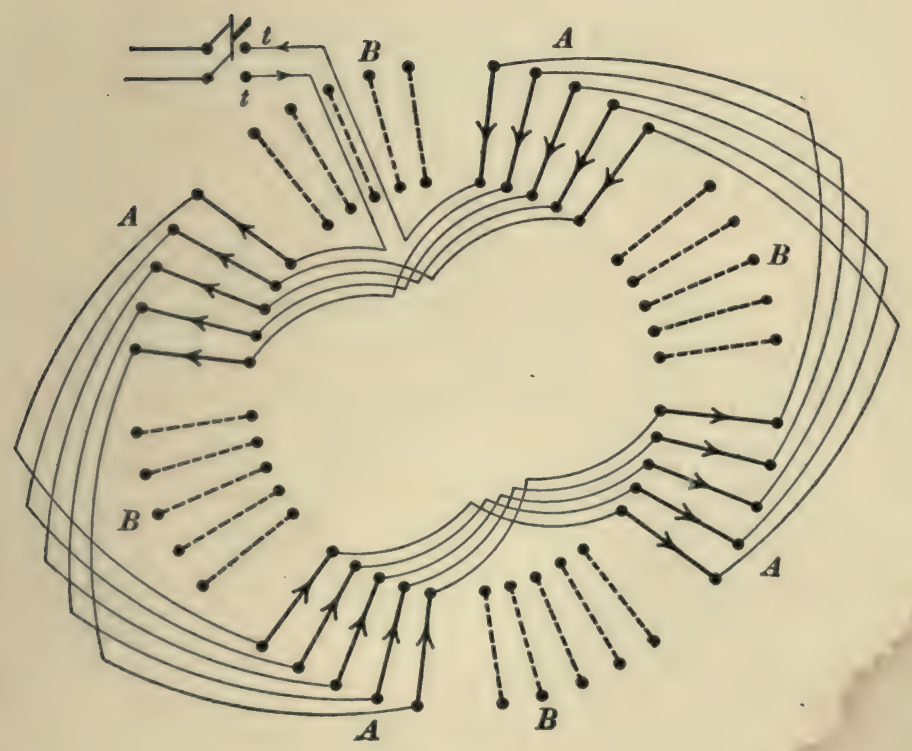

Fig. 227. 
227 is a diagram of a two-phase stator winding for the production of four rotating magnet poles, a four-pole winding as it is called, and Fig. 228 is a diagram of a three-phase stator winding for the production of four rotating magnet poles. In each of these diagrams the complete connections are shown for one phase only, namely the $A$ phase. The other phase, or phases, are exactly

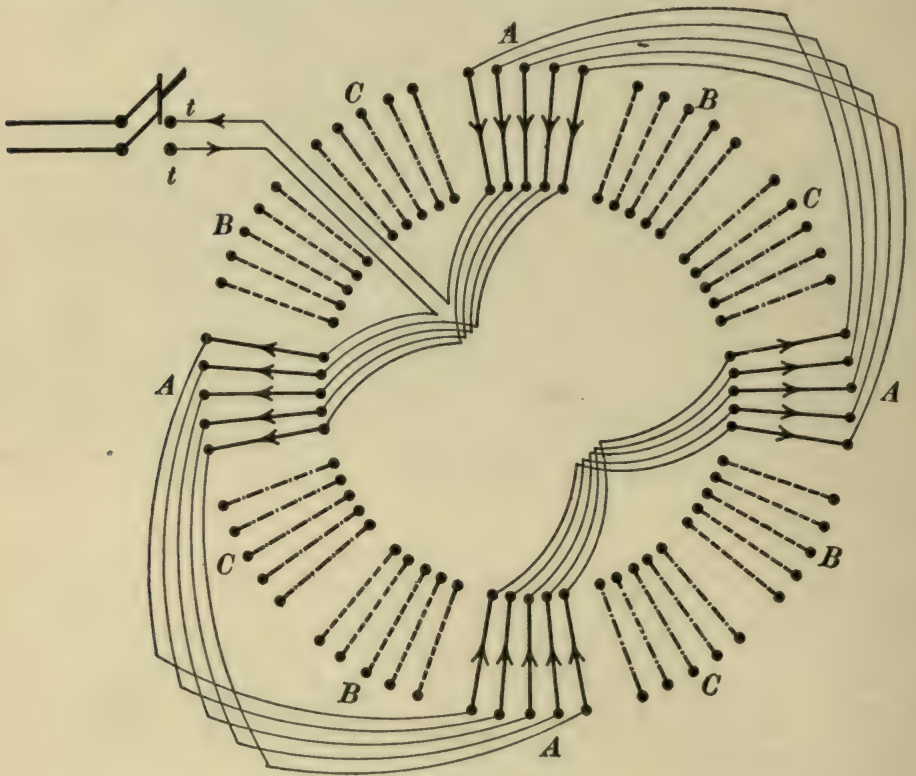

Fig. 228.

like the $A$ phase. The short radial lines in these diagrams represent the portions of the windings which lie in the slots of the stator iron, the curved lines outside of the radial lines represent the connections at one end, and the curved lines inside of the radial lines represent the connections at the other end of the stator. The following discussion of the action of the stator windings applies to the two-phase winding shown in Fig. 227.

From Fig. 227 it is evident that the stator conductors are arranged in groups or bands, that is, a band of conductors belonging to the $A$ phase, then a band of conductors belonging to the $B$ phase, and so on, and it is also evident that the current of one 
phase flows in opposite directions in adjacent bands of conductors belonging to that phase.

Figures 229, 230 and 23I represent an end view of a twophase induction motor; the rotor slots and conductors are omitted for the sake of clearness, and the bands of stator conductors are shown slightly separated from each other, so that they may be easily distinguished; before proceeding to the discussion of the action of the stator windings, however, it is necessary to consider the effect of current in a band of conductors between two masses
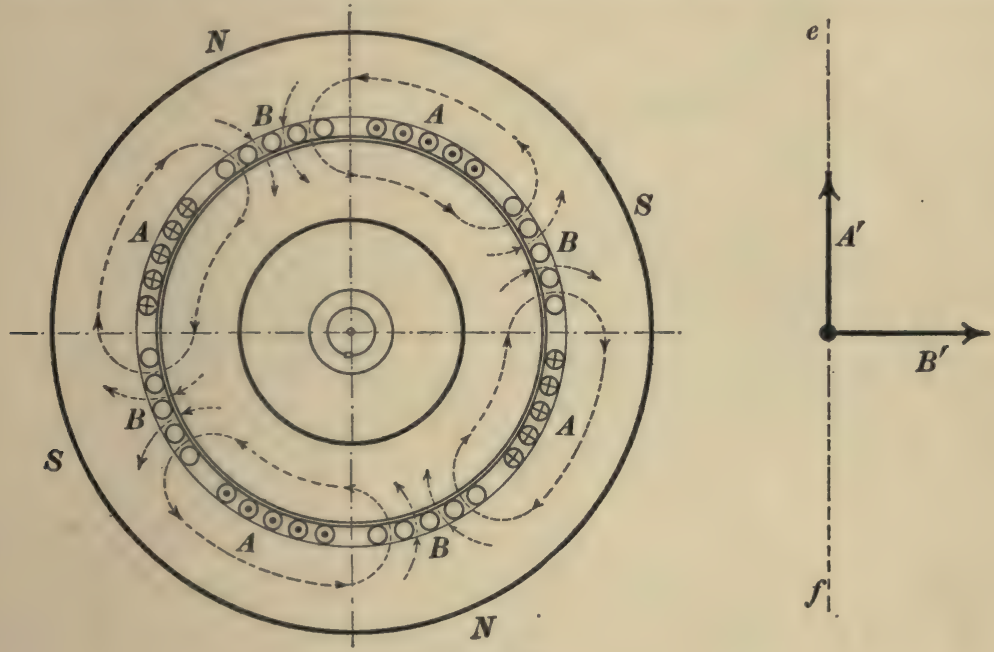

Fig. 229.

of iron. This effect is shown in Figs. 232 and 233. The small circles represent the sections of the conductors, conductors carrying up-flowing currents are marked with dots, and conductors carrying down-flowing currents are marked with crosses. The action of the currents in these bands is to produce magnetic flux along the dotted lines in the directions indicated by the arrows.

The lines $A^{\prime}$ and $B^{\prime}$ in Figs. 229, 230 and 23 I are clock-diagram lines imagined to be rotating, and their projections on the fixed line of represent at each instant the values of the currents which flow in the $A$ bands and $B$ bands of stator conductors respectively. 

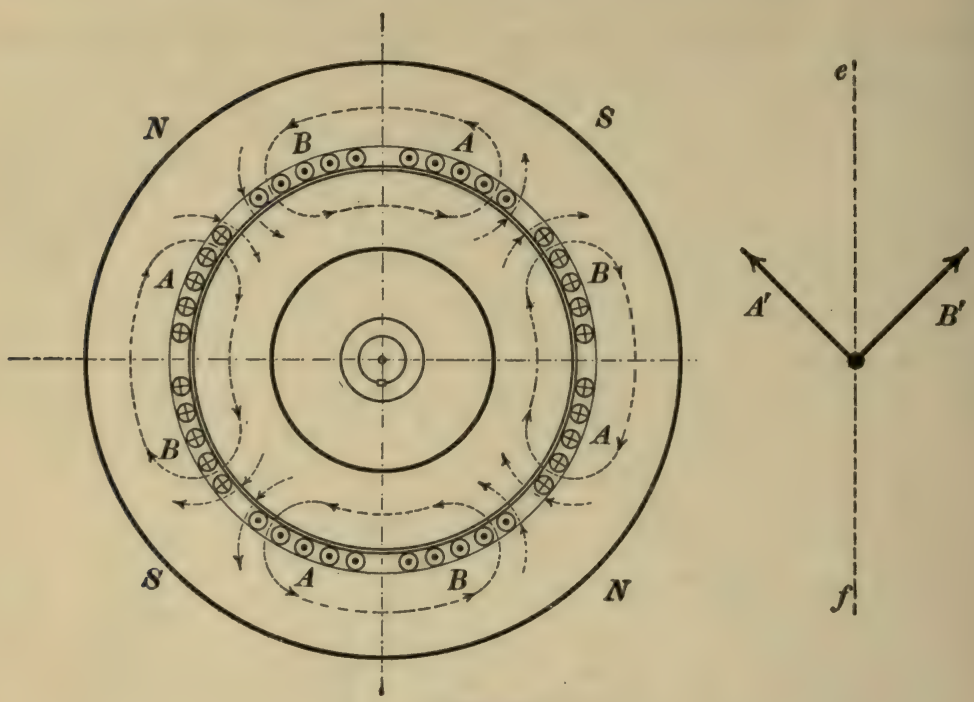

FIg. 230.

At the instant represented in Fig. 229 the current in the $A$ bands is at its maximum value, the current in the $B$ bands is

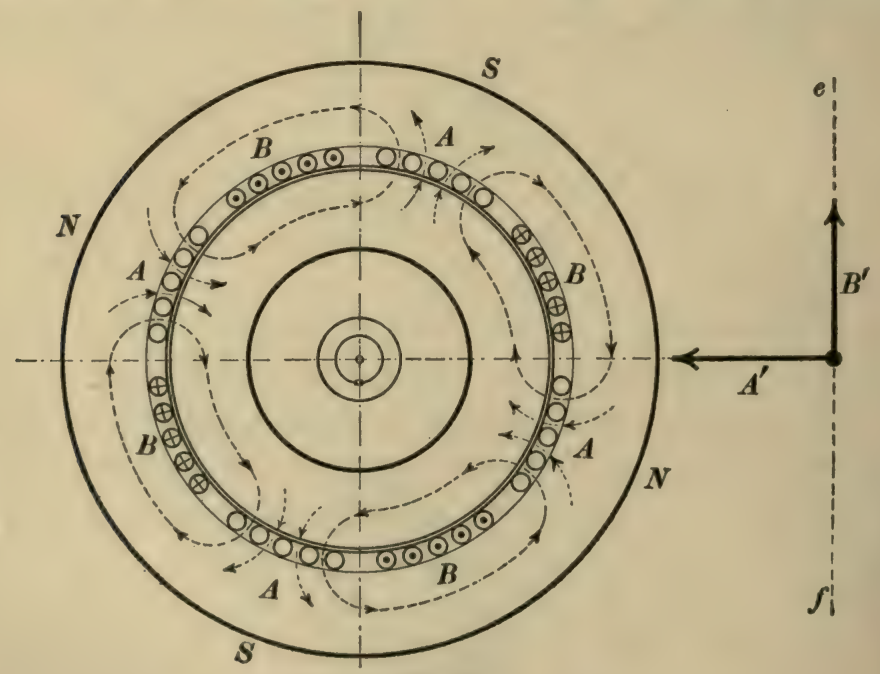

Fig. 231.

zero, and the magnetic flux due to the stator windings at this instant is represented by the dotted lines in Fig. 229. Where the 
flux passes from stator iron into rotor iron the stator iron has north magnetic polarity, and where the flux passes from rotor iron into stator iron the stator iron has south magnetic polarity. The location of these poles at the given instant is shown by the letters NSNS.

When the lines $A^{\prime}$ and $B^{\prime}$ have rotated $1 / 8$ of a revolution, as shown in Fig. 230, the current in the $A$ bands has decreased and the current in the $B$ bands has increased, the two currents have become equal in value, and the magnetic flux due to the stator windings has shifted, as indicated by the dotted lines in Fig. 230.

When the lines $A^{\prime}$ and $B^{\prime}$ have rotated $1 / 4$ of a revolution, as shown in Fig. 23I, the current in the $A$ bands has decreased

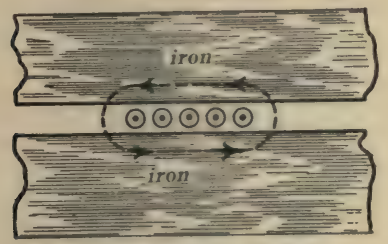

up flowing currents

Fig. 232.

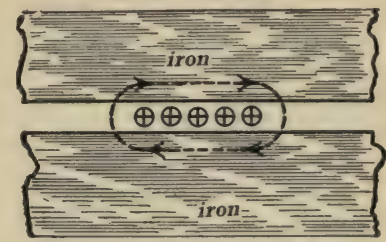

down flowing currents

Fig. 233.

to zero, the current in the $B$ bands has increased to its maximum value, and the magnetic flux due to the stator windings has shifted still farther, as indicated by the dotted lines in Fig. $23 \mathrm{I}$.

As a matter of fact, the magnetic flux due to the stator windings shifts continuously forwards, and by comparing Fig. 229 with Fig. 23 I it is seen that the forward shift of the polar regions of the stator during $1 / 4$ of a cycle ( $1 / 4$ of a revolution of the clock diagram $A^{\prime} B^{\prime}$ ) is equal to $1 / 2$ of the distance from $N$ to $S$ in Fig. 229. In a whole cycle, therefore, the forward shift of the polar regions is two times the distance from $N$ to $S$ in Fig. 229 which is equal to the distance from $N$ to $N$. That is to say, the stator magnetism makes a complete revolution in $p / 2$ cycles, where $p$ is the number of polar regions on the stator iron (NS NS in Fig. 229). Therefore, if the alternating cur- 
rents supplied to the stator windings have a frequency of $f$ cycles per second, we have :

$$
n=\frac{2 f}{p}
$$

in which $n$ is the speed of the stator magnetism in revolutions per second.

The number of polar regions, or the number of poles as it is usually expressed, is equal to the number of bands of stator conductors in each phase of the stator winding. Thus each phase has four bands of conductors in Figs. 227, 228, 229, 230 and $23 \mathrm{I}$, and the windings represented in these figures are therefore. called four-pole windings $(p=4)$.

\section{Relation between speed and torque of the polyphase induc-} tion motor. - When an induction motor is running at zero load, but little torque is required to drive it, and the rotor revolves at a speed but very little less than the speed of the stator magnetism (the speed of the stator magnetism is called the synchronous speed). The rotor cannot, ${ }^{*}$ however, reach the full synchronous speed, because at synchronous speed the rotor conductors would move along with the stator magnetism; there would be no electromotive force, and therefore no current induced in the rotor conductors; and consequently the stator magnetism could exert no driving force on the rotor conductors.

When an induction motor is running with a load, the driving torque must be large, so that the current in the rotor conductors must be large in order that the stator magnetism may exert the necessary driving force upon the rotor conductors; therefore the induced electromotive forces in the rotor conductors must be sufcient to produce the necessary rotor currents, and consequently the rotor must run appreciably below synchronism in order that

* The effect of magnetic hysteresis in the rotor iron is to produce a certain slight torque the value of which is independent of the relative speed of stator magnetism and rotor, and this hysteresis torque may, and in same cases does, bring the rotor of an induction motor up to full synchronous speed when the motor is without load. 
sufficient electromotive forces may be induced in the rotor conductors by the more rapidly moving stator magnetism.

It is at once evident from the foregoing statement that the speed of the rotor at full load will be but little less than synchronous speed if the short-circuited rotor windings have a very low resistance, for, in this case, but little electromotive force need be induced in the rotor conductors to produce sufficient current to enable the stator magnetism to exert the necessary driving torque. If, however, the resistance of the short-circuited rotor windings is large, then the rotor speed will fall off greatly with

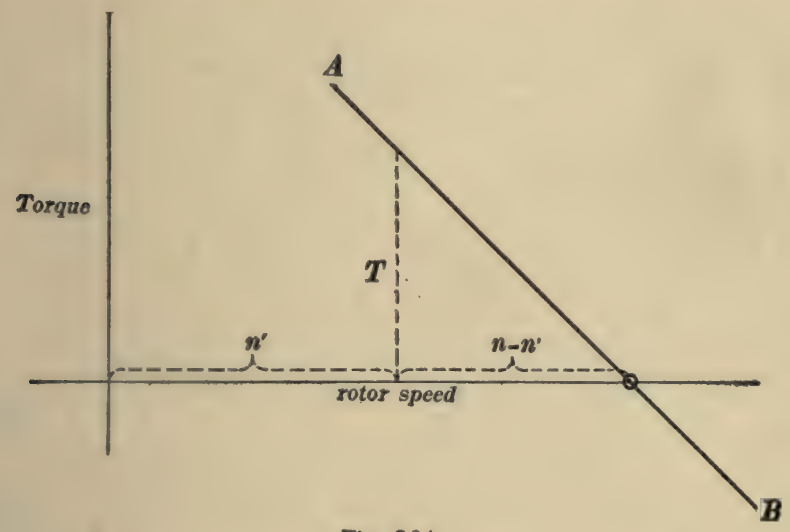

Fig. 234.

increase of load. That is, low rotor resistance is necessary for good speed regulation of an induction motor.

If the magnetic flux $\Phi$ which enters the rotor from one of the polar regions of the stator were constant in value, that is if it did not decrease in value with increase of load, then the difference between synchronous speed $n$ and rotor speed $n^{\prime}$ would be exactly proportional to the torque to be exerted on the rotor, that is to say the speed-torque curve of the induction motor would be a straight line $A B$, as shown in Fig. 234. This is evident if we consider that to double the value of $n-n^{\prime}$ would, if $\Phi$ were constant, double the induced electromotive forces in the rotor windings, this would double the rotor currents, and the 
doubled rotor currents would be acted upon by a doubled driving force.

As a matter of fact, however, the flux $\Phi$ decreases with increase of load because of the demagnetizing action of the rotor currents, and this decrease of $\Phi$ causes a two-fold decrease of torque inasmuch as $(a)$ it causes a decrease of induced electromotive forces and therefore a decrease of rotor currents at a given rotor speed, and $(b)$ these decreased rotor currents are acted upon by the reduced flux to produce a greatly reduced torque. A true speedtorque curve of an induction motor is shown in Fig. 235. The

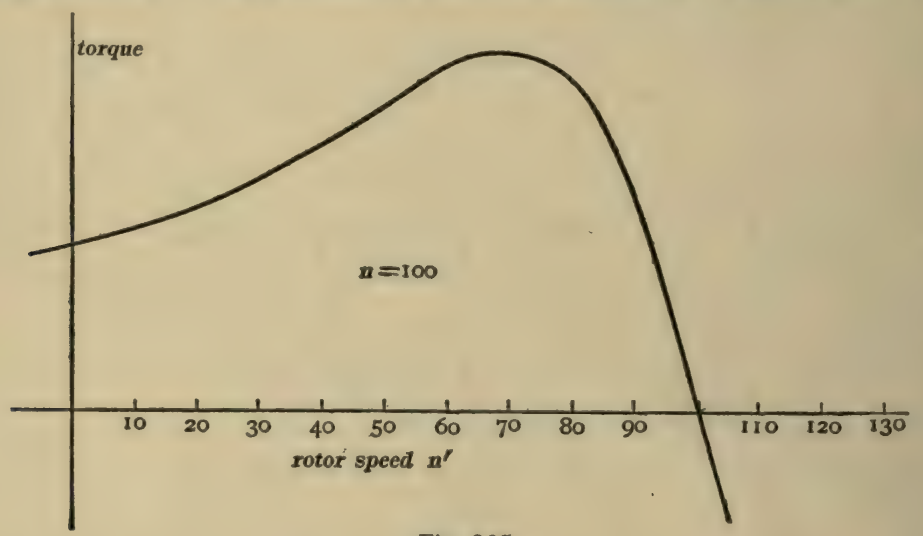

Fig. 235.

torque is zero at synchronous speed $\left(n^{\prime}=n\right)$, and the torque increases, reaches a maximum, and then falls off as $n^{\prime}$ is decreased.

Break-down of the induction motor. - If the load on an induction motor is increased until the motor develops its maximum torque, then any further increase of load will cause the motor to come to a standstill. This phenomenon is called the breaking down of the motor.

Use of a starting resistance in the rotor windings. - Imagine the rotor resistance of an induction motor to be doubled, then, if the relative speed, $n-n^{\prime}$, of the stator magnetism and rotor is doubled, the electromotive forces induced in the rotor windings are doubled, the rotor currents are the same as before, and the 
torque is the same as before. Therefore the rotor speed which corresponds to maximum torque depends upon the resistance of the rotor windings, and the value of the maximum torque is independent of the rotor resistance. This is shown by the curves I, 2, 3, 4 and 5 in Fig. 236. These curves show the relation between speed and torque of an induction motor whose rotor windings are short-circuited through an adjustable resistance; curve I shows the relation between speed and torque when the

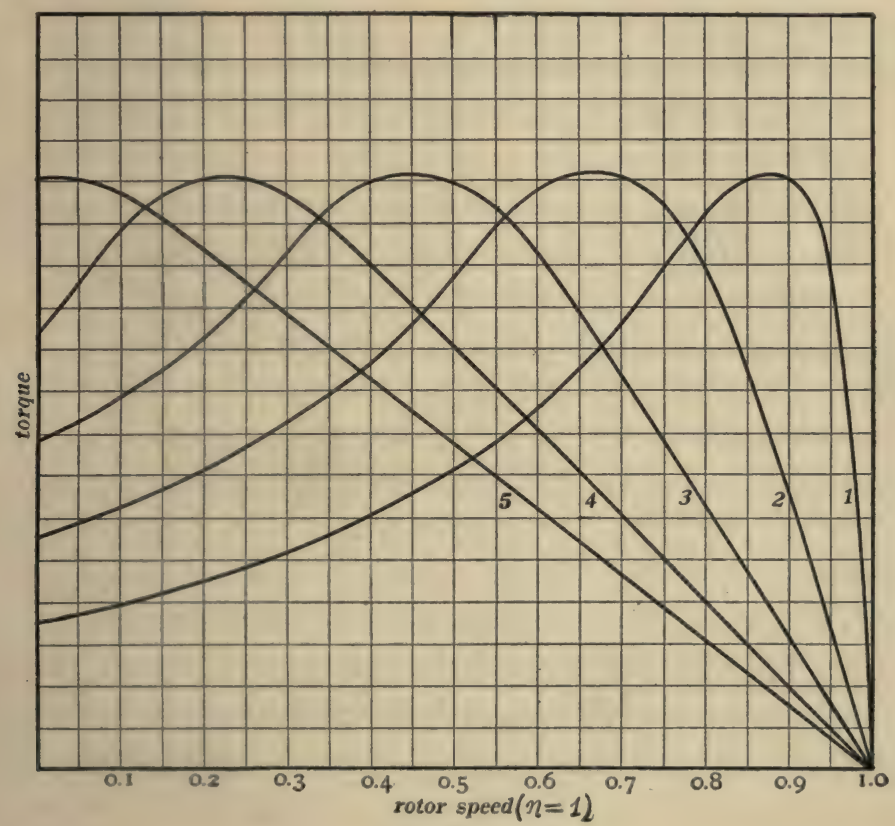

Fig. 236.

rotor resistance is very small, and curves 2, 3, 4 and 5 show the relations between speed and torque for greater and greater rotor resistance.

A certain value of rotor resistance gives the maximum torque at zero rotor speed, as shown by curve 5 in Fig. 236, and uhen an induction motor is started it is desirable to increase the rotor resistance so as to produce the maximum torque at starting; this is especially desirable if the motor starts under load. 


\section{Relation between speed and efficiency of an induction motor.}

- Of the total power delivered to an induction motor, part is lost in heating the stator windings and in heating the stator iron on account of eddy currents and hysteresis, and the remainder is delivered to the rotor; this power which is delivered to the rotor is partly lost in heating the rotor windings, and the remainder* appears as mechanical power in driving the rotor; and this mechanical power which drives the rotor is partly lost in mechanical friction in the motor bearings and in air friction, and the remainder is delivered by the motor as its mechanical output. Let us for the present ignore all of the losses of power except the power lost in heating the rotor windings.

Under this assumption all of the power delivered to the stator windings is to be thought of as being used to produce the rotating stator magnetism, exactly as if this delivered power were mechanical power used to revolve an ordinary field magnet as indicated in Fig. 226. Let $T$ be the torque which this revolving field magnet exerts on the rotor, then of course the rotor reacts and opposes the rotation of the field magnet with the same torque $T$. The power required to drive the field magnet at speed $n$ against this opposing torque $T$ is equal to $2 \pi n T, \dagger$ and the power developed by the torque $T$ in driving the rotor at speed $n^{\prime}$ is equal to $2 \pi n^{\prime} T$. That is, the power input is $2 \pi n T$ and the power output is $2 \pi n^{\prime} T$, so that (ignoring the various losses above specified, losses which are, however, by no means really negligible) the efficiency of the motor, output divided by input, is $n^{\prime} / n$.

Efficiency and rotor resistance. - According to Art. I25 an induction motor with a given load runs the more nearly at synchronous speed the less the resistance of the short-circuited rotor windings. Therefore the efficiency of the motor, which is approximately equal to $n^{\prime} / n$, is the greater the less the resistance of the short-circuited rotor windings; and therefore, when pro-

+Eddy current and hysteresis losses in the rotor iron are generally quite small and these losses are here ignored.

* If $T$ is expressed in pound-feet and $n$ in revolutions per second, then $2 \pi n T$ expresses the power in foot-pounds per second. 
vision is made for having a large rotor resistance at starting, as explained in Art. I 25, it is very desirable to arrange for the cutting out of this resistance when the rotor reaches nearly full speed, in order to give high efficiency, and also to lessen the tendency for the motor to change its speed with change of load.

Relation between the mechanical power developed in turning the rotor and the electrical power developed in the rotor windings. The power $2 \pi n T$ required to drive the "revolving field magnet" against the opposing torque $T$, as above explained, is all delivered to the rotor, but the mechanical power developed in the rotor is $2 \pi n^{\prime} T$ (less than $2 \pi n T$ ), and the remainder, $2 \pi n T-2 \pi n^{\prime} T$, is the electrical power developed in the rotor windings. Therefore, ignoring the losses above specified, the power $P$ delivered to the stator of an induction motor, the mechanical power $P_{m}$ developed in the rotor, and the electrical power $P_{e}$ developed in the rotor windings are proportional to $n, n^{\prime}$, and $n-n^{\prime}$ respectively, that is,

$$
P: P_{m}: P_{e} \text { as } n: n^{\prime}: n-n^{\prime}
$$

Analogy between the induction motor and the friction clutch. A friction clutch, the two parts of which continue to slip on each other so that the driving part $A$ runs continuously at speed $n$ and the driven part $B$ runs continuously at a slower speed $n^{\prime}$, is analogous in some respects to the induction motor. If we ignore the friction losses in the two bearings at either side of the clutch, the total power required to drive the part $A$ is $2 \pi n T$, and the mechanical power developed in part $B$ is $2 \pi n^{\prime} T$, where $T$ is the torque with which the driving part $A$ drags the driven part $B$; and the difference, $2 \pi n T-2 \pi n^{\prime} T$ is the energy developed as heat where the slipping occurs. The efficiency of transmission of such a clutch would evidently be equal to $n^{\prime} / n$.

127. Rating of induction motors. - An induction motor is always rated to carry a specified load in horse-power when supplied with a specified number of phases of alternating current at 
a specified frequency and a specified voltage per phase, and in addition, either the speed or the number of poles is given. Thus, one would speak of a ten horse-power, two-phase, four-pole, 60 cycle, 220-volt induction motor.

To overload an induction motor, frequency and voltage of supply being normal, causes the motor to take excessive current at a reduced power factor, and the motor runs at a slightly reduced speed. Excessive overload causes the motor to break down as described in Art. 125.

An induction motor may be operated quite satisfactorily at a frequency or voltage considerably different from normal. A change of frequency produces a proportional change of speed; and a change either of voltage or frequency causes a change in the value of the magnetic flux $\Phi$ which emanates from each polar region of the stator iron, a change in the magnetizing (zero-load) current, and a change in the eddy current and hysteresis losses which losses occur chiefly in the stator iron.*

The behavior of an induction motor when it is operated by an abnormally high or low voltage is especially important. In fact the torque that is developed by an induction motor at a given relative speed, $n-n^{\prime}$, of stator magnetism and rotor is approximately proportional to the square of the voltage. This is evident when we consider that to increase the voltage in the ratio of $I$ to $k$ causes the same ratio of increase of flux $\Phi$, of rotor electromotive forces, and of rotor currents (for given value of $\left.n-n^{\prime}\right)$; but, since the torque is proportional to the product of flux and rotor currents, it is evident that the torque is increased in the ratio of $\mathbf{I}$ to $k^{2}$.

128. The starting of the induction motor. - (a) Small induction motors with squirrel-cage rotors are nearly always started by connecting their stator windings directly to the polyphase supply mains.

(b) Large induction motors with wire wound rotors are usually

* See G. B. Werner, On the effect of voltage and frequency variations on induction motor performance, Electric Journal, Vol. III, page 400, July, 1906. 
started by inserting resistance in the rotor windings, as explained in Art. I 23 (Fig. 224). The advantages of using a high resistance in the rotor windings at starting are $(a)$ that the starting torque is thereby increased and $(b)$ the stator currents at starting are reduced in value.

(c) The starting compensator consists of two or three autotransformers (two for twophase supply and three for three-phase supply) with switches arranged for conveniently supplying the motor with low voltage polyphase currents at starting, which voltage is increased in steps as the motor speeds up, until finally the full supply-voltage is connected to the motor. The objection to the use of the starting compensator is that the starting torque is very small when the voltage is small; indeed, the starting compensator can scarcely be used in the case of a motor which is to be started under load. The advantage of the starting compensator is that the motor takes very much smaller currents from the supply mains at starting than it would if connected directly to the supply mains.

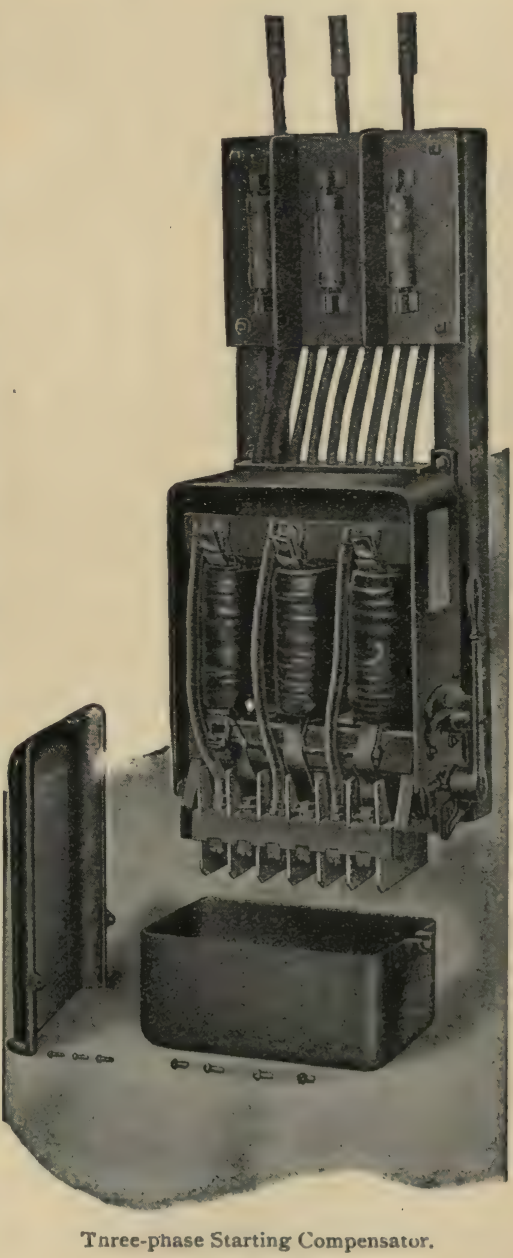

Fig. 237.

The starting compensator is nearly always used when it is desired to start a polyphase alternator or a polyphase synchronous 
converter by inherent induction motor action, as explained in Art. 132.

Figure 237 shows the interior of a three-phase starting com-

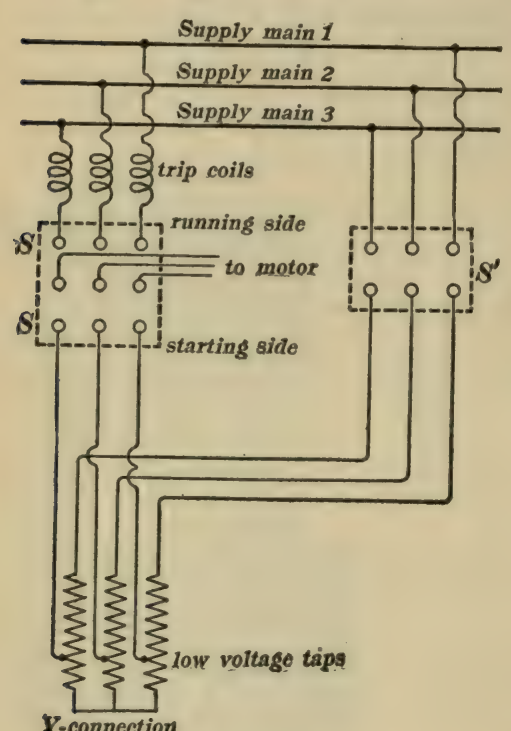

Fig. 238. pensator in the lower part of which is a cylinder switch for making the successive connections above mentioned. Figure 238 is a diagram of connections of a three-phase compensator in which one intermediate voltage is provided for. To start the motor, switch $S^{\prime}$ is closed, and then switch $S$ is closed on the starting side, thus connecting the low voltage taps of the compensator to the motor. When the motor reaches a moderate speed, switch $S$ is suddenly thrown to the running side, thus connecting the full supply voltage to the motor, and then switch $S^{\prime}$ is opened, leaving the compensator entirely disconnected. The trip coils actuate a trigger which opens a circuit breaker if the current exceeds a prescribed value.

129. Speed control of the induction motor. - The speed $n$ of the stator magnetism of an induction motor is fixed by the frequency $f$ of the alternating-current supply and by the arrangement of the stator windings (number of polar regions on stator). Therefore the zero-load speed of an induction motor, which is of course equal to the speed of the stator magnetism, is invariable, unless the frequency of supply or the arrangement of the stator windings is changed.

On the other hand, the rotor speed $n^{\prime}$ of an induction motor with given load may have any value between zero and normal full speed if the rotor resistance is increased to the proper value. 
There are therefore two feasable methods for controlling the speed of an induction motor which is supplied with alternating currents at a given frequency, namely, $(a)$ To arrange the stator windings so that they may be changed quickly from, say, a fourpole winding to an eight-pole winding by means of switches, and (b) To bring out the rotor windings to collector rings, as shown in Fig. 225 and short-circuit the rotor through external adjustable resistances.

The difficulty with the first method of speed control is that the arrangement of the stator windings becomes very complicated if more than two speeds are provided for; and the difficulties with the second method of speed control are $(a)$ that the motor is very inefficient at low speeds, and $(b)$ that the speed varies greatly with the load when the rotor resistance is great enough to give a low speed with a given load.

130. Use of the induction motor as a frequency changer.- If we consider that the effect of the stator windings of an induction motor is to produce a rotating state of magnetism which acts upon the rotor exactly as would a mechanically revolving field magnet, it is evident that the electromotive forces in the rotor windings are zero in value and zero in frequency when the rotor speed is equal to the speed of the stator magnetism, that the rotor electromotive forces increase in value and in frequency as the rotor speed decreases, that when the rotor stands still the frequency of the rotor electromotive forces is the same as the frequency of the alternating currents supplied to the stator windings, and that the rotor electromotive forces are still further increased both in value and in frequency if the rotor is driven backwards by an external source of power.

Under the ordinary operating conditions of an induction motor the rotor speed $n^{\prime}$ is nearly equal to the speed $n$ of the stator magnetism. In this case the rotor electromotive forces are small, their frequency is very low, and the amount of electrical power developed in the rotor windings is but a small fraction $\left[\left(n-n^{\prime}\right) / n^{\prime}\right]$ of the mechanical power developed in the rotor. 
When, however, $n$ and $n^{\prime}$ are very different, that is when the relative speed $n-n^{\prime}$ of stator magnetism and rotor is large, then the rotor electromotive forces are large, and if the rotor windings are brought out to collecting rings as shown in Fig. 225 , the rotor may deliver a very considerable amount of electrical power to external receiving circuits at any desired frequency. $\mathrm{W}^{\text {then }}$ an induction motor is used in this way it is called a frequency changer. As usually employed the frequency changer is used to change a low frequency alternating-current supply, say 25 cycles per second, to a higher frequency, say 60 cycles per second for operating electric lamps. When so used the rotor of the frequency changer is driven backwards by a synchronous motor or by an induction motor taking power from the low frequency supply mains.

When the rotor runs backwards the relative speed of stator magnetism and rotor is $n+n^{\prime}$ and the frequency of the rotor electromotive forces is $\left(n+n^{\prime}\right) / n$ times the primary frequency.

In order to determine the relation between the electrical power delivered to the stator of the frequency changer and the mechanical power required to drive its rotor backwards at speed $n^{\prime}$, let us consider the torque action $T$ between stator and rotor, and let us for a moment think of the stator as a mechanically revolved field magnet. Then, ignoring losses, $2 \pi n T$ is the power supplied electrically to the stator, $2 \pi n^{\prime} T$ is the power supplied mechanically to the rotor in driving it backwards at speed $n^{\prime}$, and $2 \pi$ $\left(n+n^{\prime}\right) T$ is the electrical power developed in the rotor windings.

Example. - A four-pole, three-phase induction motor has a $\Delta$-connected stator winding supplied from 25 cycle, 220-volt, three-phase mains. The rotor winding is also three-phase, it is $\Delta$-connected to the collector rings, and it has the same number of conductors as the stator winding, so that when the rotor is standing still the voltage between the collector rings is 220 volts. The speed of the stator magnetism is $12 \mathrm{x} / 2$ revolutions per second, the rotor is driven backwards at a speed of $17 \mathrm{I} / 2$ revolutions per second; and, therefore, the rotor frequency is 60 cycles per 
second, and the voltage between collector rings is 528 volts $\left[=\left(n+n^{\prime}\right) / n \times 220\right.$ volts $]$. Suppose that the rotor delivers a total electrical output of IOO kilowatts, then, since electrical output of rotor : electrical intake of stator: mechanical intake of rotor as $n+n^{\prime}: n: n^{\prime}$, it follows that $4 \mathrm{r} 2 / 3$ kilowatts of power is delivered directly to the stator windings from the 25 cycle mains, and that $58 \mathrm{r} / 3$ kilowatts of mechanical power is delivered to the rotor by the motor which drives it.

131. The induction generator. - When the rotor of an induction motor is driven at a speed $n^{\prime}$ which is greater than the speed $n$ of the stator magnetism, a torque $T$ is developed which opposes the motion of the rotor, so that mechanical power amounting to $2 \pi n^{\prime} T$ must be used to drive the rotor. A portion of this mechanical power appears as electrical power developed in the rotor windings, and the remainder $2 \pi n T$ is delivered to the stator, where, ignoring losses, it is delivered to the alternating-current supply mains in the form of alternating-current output from the stator windings. When used in this way the induction motor is called an induction generator. To operate as a generator the induction motor must be connected in parallel with an ordinary alternator.

132. The starting of the polyphase synchronous motor (or converter) by inherent induction motor action. - The windings of a polyphase alternator armature are like the stator windings of an induction motor, and the polyphase current in such an armature produces a rotating state of magnetism in the armature core exactly as the stator windings of an induction motor produce a rotating state of magnetism in the stator iron.

When an alternator is in operation either as a generator or as a synchronous motor or converter, the magnetism due to the armature currents rotates with reference to the armature structure in a direction opposite to that in which the armature rotates, ${ }^{*}$ and at the same speed as the armature. Therefore the armature mag-

* This statement is made to refer to the machine with stationary field and rotating armature for the sake of simplicity. 
netism due to armature currents is stationary in space (see Arts. 63 and 65).

If polyphase currents are connected to the armature of a polyphase alternator or synchronous converter at stand-still, the rotating magnetism produced in the armature core acts upon the field structure in the same way that the rotating stator magnetism of an induction motor acts upon the rotor. The torque action between the armature and field of the alternator under the above conditions sets the armature into motion, the armature increases in speed until it is nearly in synchronism, and the slight torque due to hysteresis causes the speed to come up to full synchronism.

This induction motor action is frequently used in the starting of polyphase synchronous motors and converters. The polyphase supply voltages are reduced to a fraction of the normal value by means of a starting compensator (see Art. I28), and connected to the armature terminals of the polyphase machine. The armature being entirely free to rotate, speeds up and reaches full synchronous speed as above explained, then the supply voltage is increased to its full value, and the field magnet of the polyphase machine is then excited by closing the field switch.

The rotating armature magnetism produces a pulsating flux through the field coils of a polyphase machine, which is being started in the manner here described, and this pulsating flux induces a very large electromotive force in the many turns of wire which constitute the field winding. To avoid the rupture of the insulation of the field winding by this large electromotive force, a multi-pole switch is arranged to break the field circuit at a number of points so as to avoid the accumulation of the total induced electromotive force at the terminals of the complete winding where it would endanger the insulation.

133. The single-phase induction motor. - The polyphase induction motor, once in operation, continues to run and carry a moderate load when all of the stator windings (phases) but one are disconnected from the supply mains. When so operated, the induction motor is called a single-phase induction motor. The 
curve $A$, Fig. 239, shows the relation between torque and speed of a two-phase induction motor with both phases connected to the supply mains, and curve $B$ shows the relation between torque and speed of the same motor with but one phase connected to the supply mains. This figure shows that when driven by single-phase current (at normal voltage), the motor runs at a slightly lower speed to develop a given torque, it shows that the

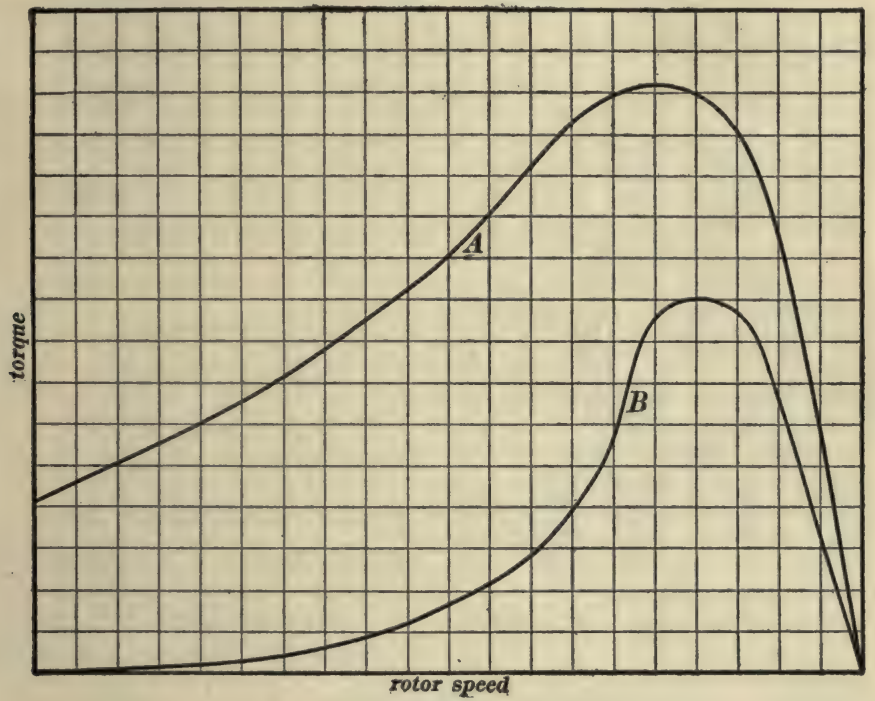

Fig. 239.

maximum or break-down torque occurs at a higher speed and is less in value than the break-down torque of the same motor when driven by two-phase currents, and it shows that the single-phase induction motor develops zero torque at stand-still. In fact, the single-phase motor must be started either as a polyphase induction motor or by other means as described in the next article.

134. Starting of the single-phase induction motor.

(a) Hand starting. - Small single-phase motors are sometimes intended to be started by closing the supply switch and giving a pull on the belt which connects the motor to the machine which it is intended to drive. 
(b) Split-phase starting.- Small single-phase induction motors are sometimes started as polyphase induction motors as follows : The stator of the motor is provided with a two-phase winding, and these two phases are connected in parallel to the single-phase supply mains with a resistance or a condenser in series with one of the phases. Under these conditions the currents in the two stator windings are out of phase with each other sufficiently to produce the desired starting torque, and when the machine reaches moderate speed, one of the stator windings is discon-

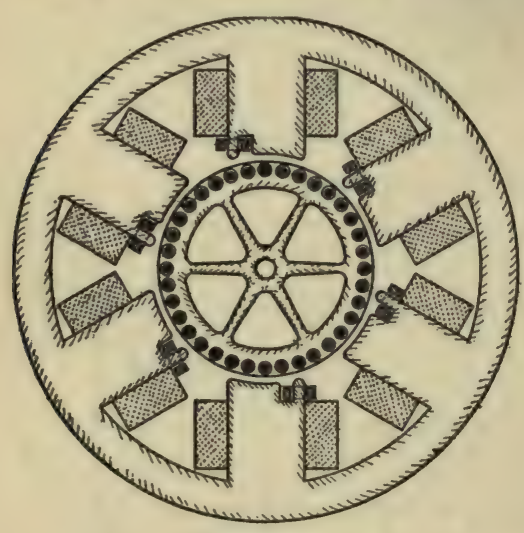

Flg. 240. nected, and the machine continues to operate as a singlephase motor. The split-phase method of starting is incorporated in many single-phase alternating-current fan-motors by means of a device called a shading coil. Figure 240 is an end view of such a singlephase fan-motor. The polar projections of the stator iron are wound with coils of wire which are connected in series and receive current from the single-phase supply mains, and one tip of each pole piece is surrounded by a short-circuited coil of wire or frame of copper, as indicated in the figure. This coil, or copper frame, is called a shading coil and it causes a phase difference between the pulsating flux that emanates from the main portion of each polar projection and the pulsating flux which emanates from the pole tip, thus introducing a two-phase action on the rotor which is sufficiently pronounced to start the motor.

(c) Repulsion motor starting. - The best method of starting single-phase induction motors is to design the motor so that it can be operated at starting as a repulsion motor and be quickly converted into an induction motor after it reaches approximately full speed. The single-phase induction motor of the Wagner Electric Company operates in this way. It is described in Chapter XIV. 


\section{CHAPTER XIII.}

GENERAL THEORY OF THE INDUCTION MOTOR.

135. The induction motor considered as a transformer. - When the rotor of an induction motor stands still, the stator and rotor windings constitute the primary and secondary coils of a transformer, differing in no essential particular from the ordinary transformer; when the rotor of an induction motor is running, however, the action of the motor is not like the action of an ordinary transformer, but all the details of action of an induction motor in operation can be described and calculated in terms of ordinary transformer action.

Magnetizing current. - When the rotor of an induction motor is running at synchronous speed $\left(n^{\prime}=n\right)$ there is no relative motion of stator magnetism and rotor, no electromotive forces are induced in the rotor windings, no rotor currents exist, and the currents in the stator windings are sufficient merely to produce that value of stator flux $\Phi$ which suffices to induce in the stator windings enough electromotive force to balance the supply voltage, exactly as in a transformer with its secondary coil on open circuit. The stator currents at synchronous rotor speed are therefore called the magnetizing currents of the machine; the magnetizing current in each phase of the stator winding is represented in the following discussion by the letter $M$.

The magnetizing current of the transformer supplies the core losses of the transformer as explained in Art. I I 5. In the induction motor the eddy current and hysteresis losses in the rotor iron are negligible because the frequency of magnetic reversals in the rotor iron is very low. The power components of the magnetizing currents of an induction motor supply the eddy-current and hysteresis losses in the stator iron.

Load current. - When an induction motor is loaded, the rotor runs at less than synchronous speed, the relative speed of stator 
magnetism and rotor induces electromotive forces in the rotor windings, these induced electromotive forces produce currents in the rotor, and the magnetizing action of these rotor currents is balanced by the equal and opposite magnetizing action of additional curvents in the stator windings, exactly as in a transformer of which the secondary delivers current to a receiving circuit; this additional current in each phase of the stator winding is called the load current and it is represented in the following discussion by the letter $I^{\prime}$.

Slip. - In considering the action of the induction motor under load, it is convenient to express the relative speed $n-n^{\prime}$ of stator magnetism and rotor in terms of the speed $n$ of stator magnetism (synchronous speed as it is called) taken as unity. When so expressed the relative speed is equal to $\left(n-n^{\prime}\right) / n$, it is called the slip of the motor, and it is represented in the following discussion by the letter $s$. It is well to think of slip always as signifying merely the relative speed of stator magnetism and rotor, and much confusion of thought may be avoided by remembering that the slip of an induction motor is usually less than unity, being zero when the rotor runs at synchronous speed and being equal to unity when the rotor stands still.

Energy transformations in the rotor of an induction motor. To reduce the action of an induction motor to an entirely equivalent transformer action, it is necessary to consider the energy transformations in the rotor; and in order to consider these transformations in the simplest possible way, let us return to the conception of the stator of an induction motor as a mechanically rotated field magnet. From this point of view, to assume that the rotating field magnet is without friction or other losses is to assume that the total power, $2 \pi n T$, required to drive it against the torque $T$ with which the stator and rotor act on each other, is the total power actually delivered to the rotor. Of this total power a portion $2 \pi n^{\prime} T$ appears as mechanical power driving the rotor, and the remainder $2 \pi n T-2 \pi n^{\prime} T\left[=2 \pi\left(n-n^{\prime}\right) T\right]$ 
appears as electrical power in the rotor windings.* Therefore the total power delivered to the rotor, the mechanical power developed in the rotor, and the electrical power developed in the rotor are to each other as $n: n^{\prime}: n-n^{\prime}$, or as $\mathrm{I}: \mathrm{I}-s: s$.

Equivalent transformer action of the induction motor. - If an induction motor is running at a given slip (with a given load), then the rotor electromotive forces, the rotor currents, and the magnetic flux $\Phi^{\prime}$ which enters the rotor from a polar region of the stator all have definite values, although the flux $\Phi^{\prime}$ is less than the flux $\Phi$ which leaves a stator pole, on account of magnetic leakage. However, the difference between $\Phi$ and $\Phi^{\prime}$ depends only upon the values of stator and rotor currents. This may be seen from Fig. 24I, inasmuch as the leakage flux $\Phi-\Phi^{\prime}$ which passes between the stator and rotor windings may be thought of as produced by the down-flowing currents in the $A$ conductors and the up-flowing currents in the $B$ conductors. The stator and rotor conductors are shown as covering only a portion of the periphery

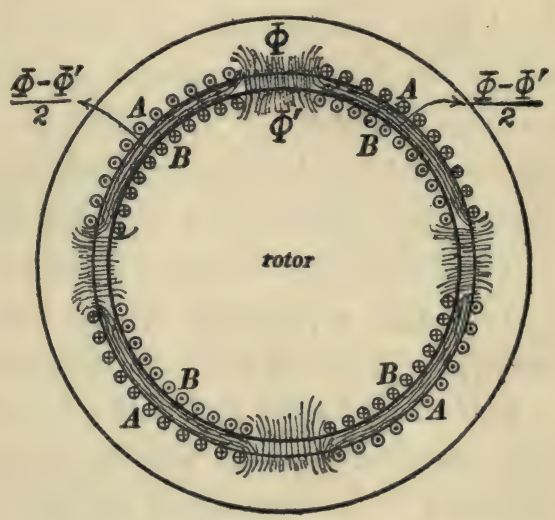

Fig. 241. in Fig. 24I for the sake of clearness.

Now, suppose that the rotor is brought to a standstill (slip = unity), and suppose that the flux $\Phi^{\prime}$ remains unchanged in value, then the electromotive forces induced in the rotor windings would be increased in proportion to the increase of slip $(s: \mathrm{I})$ and, if the resistance of the rotor windings were increased in the same ratio, the rotor currents would be the same as before, and $\Phi^{\prime}$ would in fact be unchanged.

* This ignores the eddy current and hysteresis losses in the rotor iron, and these losses are usually quite small because of the low frequency of the reversals of the magnetism of the rotor iron. 
Therefore every detail of action of an induction motor running at slip $s$ would be reproduced at standstill by increasing the rotor resistance in the ratio $\mathrm{I} / s$; except that while running, the power delivered to the rotor consists of mechanical power and electrical power in the ratio of $\mathrm{I}-s$ to $s$, whereas at standstill, although the same total power would be delivered to the rotor, it would all appear as electrical power in the increased resistance of the rotor windings. For example, the action of an induction motor running at $\frac{1}{20}$ slip would be reproduced as pure transformer action at standstill if the rotor resistance were increased twenty times, the action of an induction motor running at $\frac{1}{10}$ slip would be reproduced at standstill if the rotor resistance were increased ten times, and so on. That is, the larger the slip (the larger the load on the motor) the less the rotor resistance would have to be at standstill to reproduce the details of the induction motor action as pure transformer action, or, in other words, the heavier the motor load the heavier the load on the equivalent standstill transformer, the variations of load on the equivalent standstill transformer being due solely to changes of secondary (rotor) resistance.

\section{The application of the circle diagram to the induction} motor. - Since the action of an induction motor with varying load is equivalent to the action of a transformer (the equivalent standstill transformer) with varying secondary resistance, it is evident that the circle diagram of the transformer, which represents the action of a transformer with non-inductive secondary receiving circuit of variable resistance (see Art. I2I), may be used to represent the action of the induction motor.

Determination of the circle diagram by experimental tests on a given motor. - The magnetizing current $M$ in each phase of the stator winding may be determined by driving the motor at zero load, the effective value of $M$ being measured by a properly inserted ammeter, and the phase difference, $\theta_{m}$, between $E^{\prime}$ (of one phase) and $M$ being calculated from the measured values of $E^{\prime}$, and $M$, together with the power $E^{\prime} M \cos \theta_{m}$ as measured by a wattmeter. Lay off the line $O M$, Fig. 242, representing to scale 
the magnetizing current so determined, and we have one point $M$ on the circular locus of the stator current (per phase).

Another point on the circle may be determined by measuring the stator current and the power delivered (per phase) at any load or at standstill. Thus, if the stator current per phase and power per phase be measured at standstill, the line $O P^{\prime}$ representing the stator current per phase at standstill may be laid off in the same way as the line $O M$, as above explained.

Knowing the two points $M$ and $P^{\prime}$ on the circle, and knowing that the diameter of the circle is a line passing through $M$ at

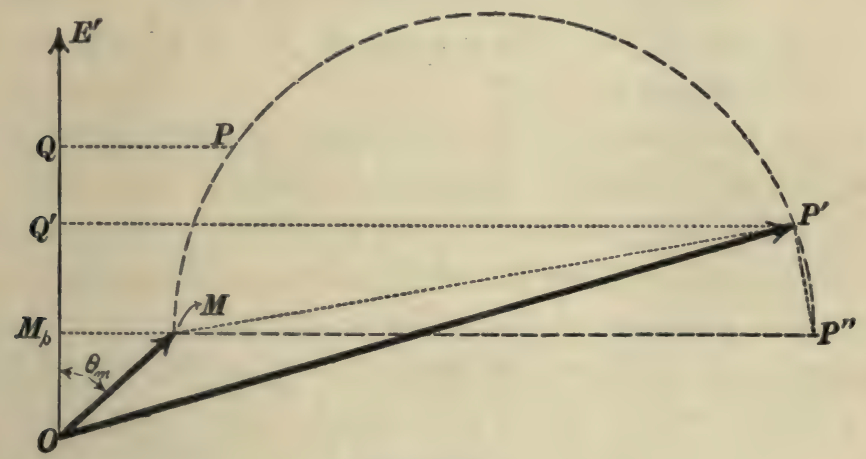

Fig. 242.

right angles to $O E^{\prime}$, a line $P^{\prime} P^{\prime \prime}$ may be drawn through $P^{\prime}$ at right angles to $M P^{\prime}$, and thus the diameter $M P^{\prime \prime}$ of the circle is determined. The circle may then be drawn.

In order to use the circle diagram for calculating the performance curves of an induction motor it is necessary to measure the resistance $R^{\prime}$ of each phase of the stator winding and to determine what is called the equivalent resistance $R^{\prime \prime}$ of the rotor per stator phase. If the rotor were wire-wound with the same number of conductors per phase as the stator winding, then $R^{\prime \prime}$ would be the actual resistance of each phase of the rotor winding; in this case the current in each rotor phase would be equal to the load current $I^{\prime}$ in each stator phase and the rotor $R I^{2}$ losses would be $q R^{\prime \prime} I^{\prime 2}, q$ being the number of phases. The equivalent rotor 
resistance $R^{\prime \prime}$ per stator phase is defined in any case by the relation: Actual rotor $R I^{2}$ losses $=q R^{\prime \prime} I^{\prime 2}$, in which $q$ is the number of stator phases and $I^{\prime}$ is the load current per phase in the stator.

The value of $R^{\prime \prime}$ may be calculated from the measured current and power input per stator phase at standstill as follows, $R^{\prime}$ having been measured. The core loss per phase at zero load equals $\overline{O M}_{p} \times E^{\prime}$ minus $R^{\prime} \times \overline{O M}^{2}$. The core loss is assumed to be the same at all loads. The measured power input per phase at stand-still minus the core loss per phase minus $R^{\prime} \times \overline{O P^{\prime}}$ (see Fig. 242) is equal to the rotor $R I^{2}$ loss per phase at stand-still, and this is equal to $R^{\prime \prime} I^{\prime 2}$ where $I^{\prime}$ is the current represented by $M P^{\prime}$.

Use of circle diagram for calculating the performance curves of an induction motor. - Having determined the values of $R^{\prime}$ and $R^{\prime \prime}$, the performance curves of the induction motor may be determined from the circle diagram as follows: Take a series of positions of the point $P$ on the circular current locus, and for each position make the following calculations: Motor intake equals $\overline{O Q} \times E^{\prime} \times q$ where $q$ is the number of phases. Power factor of motor equals the cosine of the angle $P O E^{\prime}$. The power $a$ delivered to the rotor equals $\overline{O Q} \times E^{\prime} \times q$ minus core loss per phase $\times q$ minus $R^{\prime} \times \overline{O P}^{2}$. Electrical power $b$ developed in rotor equals $R^{\prime \prime} \times \overline{M P}^{2} \times q$. Mechanical power developed in rotor equals difference between $a$ and $b$. Motor slip may be found from the relation $b=s a$, and motor speed $n^{\prime}$ is then known since synchronous speed $n$ is known. Efficiency equals $a-b$ divided by total motor intake. Torque may be found from the relation $2 \pi n T=a$; this gives the value of the torque in watts per unit angulas velocity, that is in watts per radians per second. The value of torque may be most conveniently expressed in terms of the power $a$ directly, which is the power in watts that the actual torque would develop if it were to drive the rotor at synchronous speed, that is to say, the value of $a$ expresses torque in "synchronous watts" so called. 
GENERAL THEORY OF THE INDUCTION MOTOR. 28I

Example. - The curves in Fig. 243 represent the results of the above calculations on a 5 -horse-power two-phase induction motor, the abscissas represent power output in watts, and the ordinates of the various curves represent the corresponding values of speed,

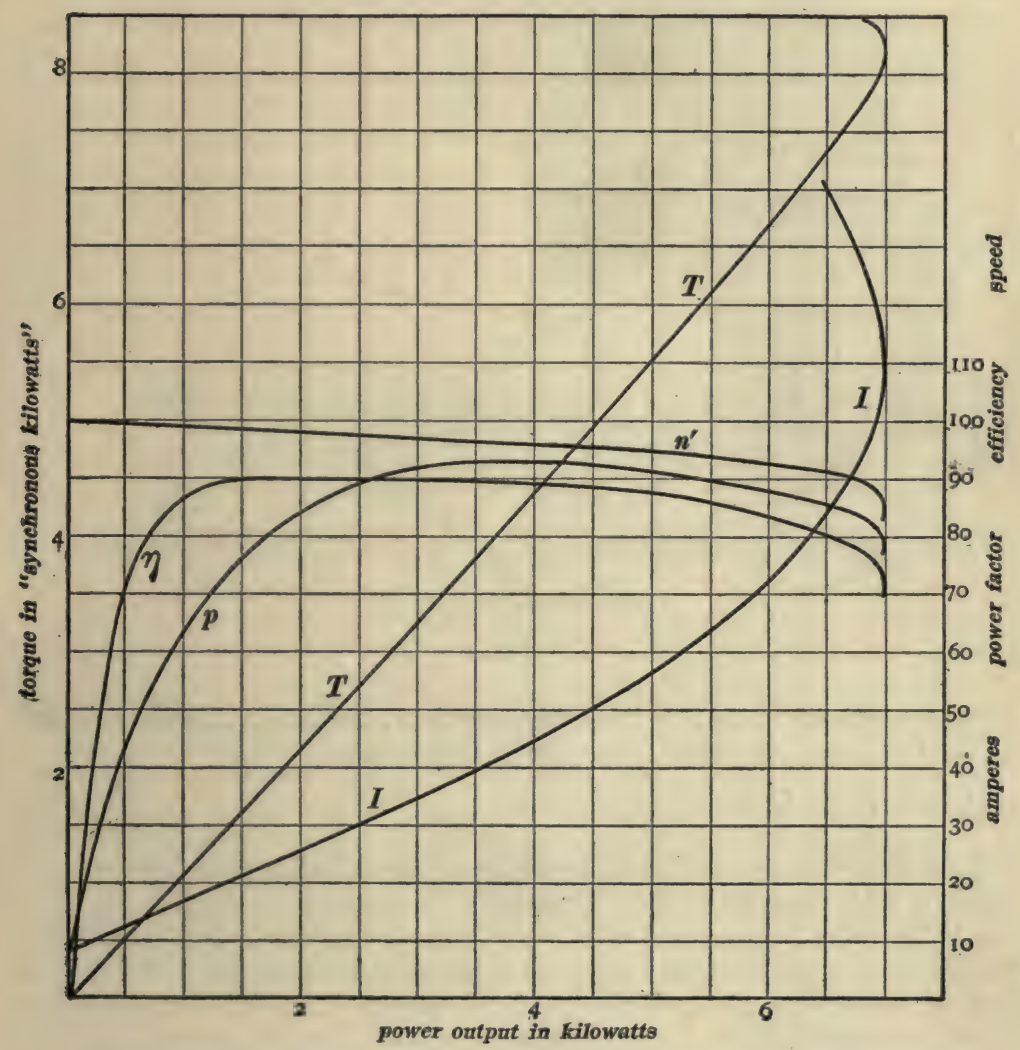

Torque $T$, stator current per phase $I$, rotor speed $\boldsymbol{n}^{\prime}$, power factor $\boldsymbol{p}$, and efficiency $\eta$.

Fig. 243.

torque, power factor, efficiency, and stator current in each phase of the stator winding. The speed of the motor is expressed in terms of synchronous speed taken as I00.

The results of the above calculations for speed, torque and power output are also shown in Fig. 244, in which the abscissas 
represent speed of rotor in per cent. of synchronous speed, and the ordinates represent power output in kilo-watts and torque in

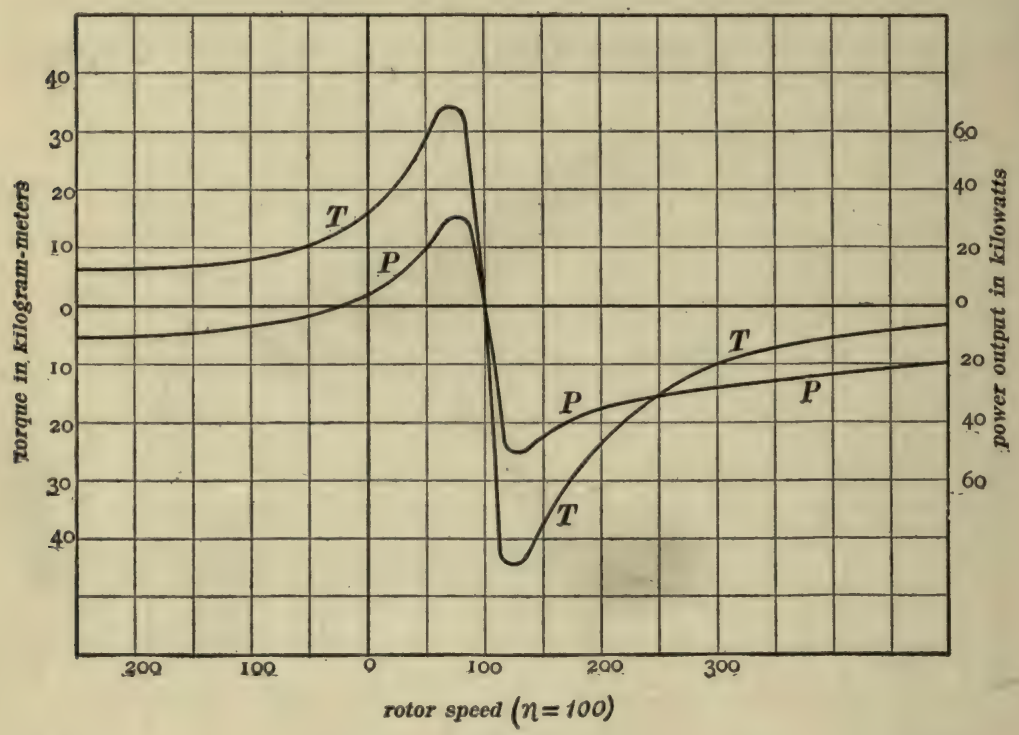

Fig. 244.

kilogram-meters. In this case, however, the calculations are extended above synchronous speed, where $n^{\prime}>n$; and below standstill, where $n^{\prime}$ is negative.

137. Determination of the circle diagram by calculation from the details of design of an induction motor. - In order to determine the circle diagram it is necessary to determine the phase and value of the magnetizing current, $M$, in each stator winding, and to determine the diameter of the circular current-locus. By referring to Arts. 120 and 121 it will be seen that the diameter of the circular current-locus is the current which would flow through each stator winding (the primary coil) if the resistance in the main circuit shown in Fig. 216 or in Fig. 251 were zero and only the reactance $X$ due to magnetic leakage were left. That is, the diameter of the circular locus is equal to $E^{\prime} \mid X$, and to determine it one must calculate the value of the magnetic leakage reactance $X$ of the stator windings per phase. When the circular current-locus has been determined, it is necessary to know the value of the resistance $R^{\prime}$ of each stator winding and the equivalent resistance $R^{\prime \prime}$ of the rotor per stator phase, before the circle diagram can be used for calculating the performance curves of the motor.

Calculation of magnetizing current per phase. - The calculation of the magnetizing current per phase consists of three steps, namely, $(a)$ The calculation of total 
flux $\Phi$ emanating from the polar region of the stator, and from this the calculation of the flux density in the gap space under that stator tooth which happens at a given instant to be in the center of a polar region, $(b)$ The calculation of the power component $M$ of the magnetizing current per phase, and (c) The calculation of the wattless component $M_{w}$ of the magnetizing current per phase.

In the calculation of the power component of the magnetizing current the resistance loss $M^{2} R^{\prime}$ in each stator winding will be ignored, since both $M$ and $R^{\prime}$ are fairly small ; to ignore the resistance loss is to assume that the only power delivered by the magnetizing current is the eddy current and the hysteresis loss in the stator iron.*

In the calculation of the wattless component of the magnetizing current the reluctance of the stator and rotor iron will be neglected on account of relatively large reluctance of the air gap.

(a) Calculation of flux $\Phi$. - When the rotor runs at synchronous speed the electromotive force induced in each stator winding by the rotating stator magnetism is sensibly equal to $E^{\prime}$, inasmuch as the electromotive force $M R^{\prime}$ required to force the

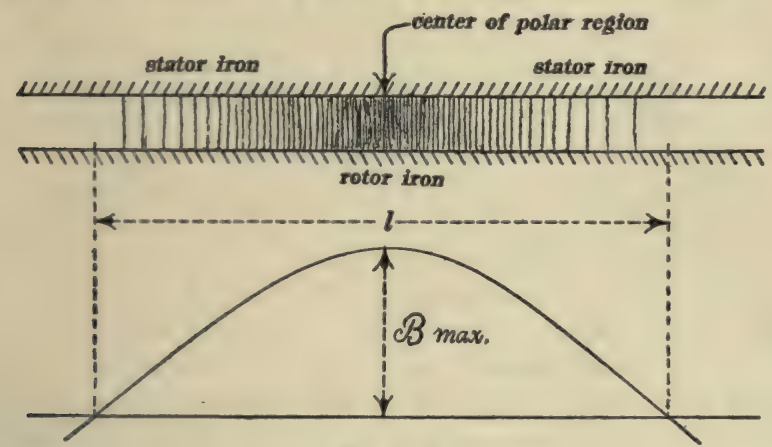

Fig. 245.

magnetizing current through the resistance of each stator winding is small. Now the flux $\Phi$ which emanates from a polar region of the stator iron is harmonically distributed (see Art. 6I) and the electromotive force induced in each stator winding by the rotation of the stator magnetism at speed $\boldsymbol{n}$ is

from which we have

$$
\begin{gathered}
E^{\prime}=k p \Phi Z n \div 10^{8}, \\
\Phi=\frac{E^{\prime} \times 10^{8}}{k p Z n}
\end{gathered}
$$

in which $p$ is the number of polar regions on the stator, $Z$ is the total number of conductors in one stator winding (one phase), $n$ is the speed of the stator magnetism in revolutions per second, and $k$ is the "phase constant" of one stator winding; ( $k=0.901$ for a two-phase stator winding, and $k=0.955$ for a three-phase stator winding see Art. 6r.)

* The eddy current and hysteresis loss in the rotor is very small under ordinary operating conditions of an induction motor on account of the low speed of the rotor relative to the stator magnetism. 
Consider one of the polar regions of the stator, as shown in Fig. 245, in which the stator and rotor surfaces are represented as being flat. The ordinates of the sine curve represent the flux densities (mean fux densities, the flux being considered as not concentrated in tufts under the stator teeth) in the gap space at various points in the polar region. Let $l$ be the breadth of the polar region as indicated in the figure, and let $\lambda *$ be the length of stator and rotor iron parallel to the motor shaft. The average flux density over the whole polar region is equal to two times the maximum flux density (maximum mean flux density is here referred to, tufts assumed not to exist) divided by $\pi$, inasmuch as the distribution is harmonic; but the average flux density over the whole polar area is $\Phi / \lambda l$, therefore the maximum flux density (irrespective of concentration of lines of force as tufts under stator teeth) is $\pi \Phi / 2 \lambda l$.

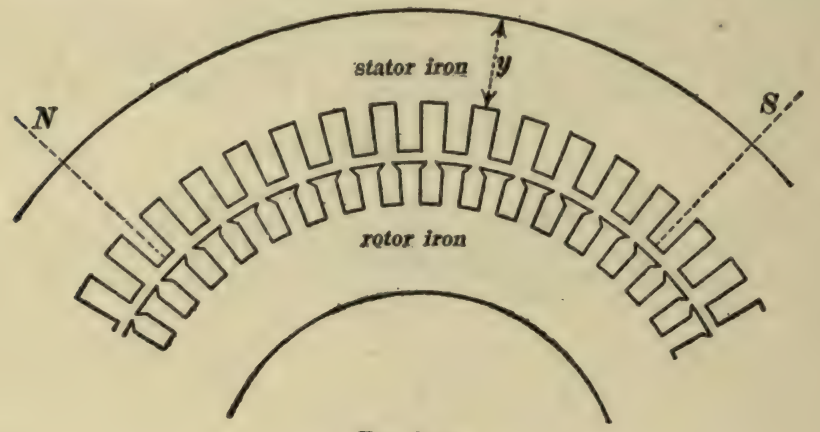

Fig. 246.

Figure 246 represents a portion of an induction motor, $N$ being the center of a north polar region and $S$ the center of a south polar region at a given instant. The mean flux density in the gap space at $N$ or $S$ is $\pi \Phi /(2 \lambda l), l$ being the distance from $N$ to $S$ measured along the gap space. The flux density in, or under, a stator tooth at $N$ or $S$ is $\pi \Phi /(2 \lambda l) \times(t+s) / t$, where $t$ is the width of a stator tooth and $s$ is the width of a stator slot. The flux density in the portion $y$ of the stator core is equal to $\frac{1}{2} \Phi$ divided by $\lambda y$.

(b) Calculation of power component of magnetizing current. - The product of the supply voltage by the power component of the magnetizing current per phase is equal to $\left(P_{e}+P_{h}\right) / q$ where $P_{e}+P_{h}$ is the total loss of power in the stator iron due to eddy currents and to hysteresis, and $q$ is the number of stator phases. Knowing the maximum flux density in the stator teeth, the volume of iron in the stator teeth, the thickness of the laminations, and the primary frequency $f$, the eddy-current and hysteresis losses in the stator teeth may be calculated as explained in Art. 103; and, knowing the maximum flux density in portion $y$ of the stator iron, the volume of this portion of iron, the thickness of the laminations, and the frequency, the total loss in the portion $y$ of the stator iron may be calculated. Then $M_{p}=\left(P_{\mathrm{e}}+P_{\mathrm{h}}\right) /\left(q E^{\prime}\right)$.

(c) Calculation of wattless component of the magnetizing current. - The wattless component of the magnetizing current is assumed to supply the magnetomotive

* All dimensions expressed in centimeters. 
force required to overcome the reluctance of the magnetic circuit. This reluctance is nearly all in the air gap, and the reluctance of the iron part of the magnetic circuit may be neglected. Therefore the total magnetomotive force due to the wattless component of the magnetizing currents in all of the stator phases is approximately equal to the magnetomotive force required to force the flux $\Phi$ twice across the air gap, and this is equal to twice the length $g$ of the gap space multiplied by the flux density under a stator tooth in the middle of a polar region, that is to $2 g \times \pi \Phi /(2 \lambda l) \times(t+s) / t$.

For the case of a two-phase stator winding let us consider the instant when the current $M_{v}$ is at its maximum value $\sqrt{2} M_{v}$ in one winding and at zero value in the other winding. This actual current $\sqrt{2} M_{w}$ in flowing through the $Z /(2 p)$ stator conductors in one band supplies the magnetomotive force $2 g \times \pi \Phi /(2 \lambda l) \times(t+s) / t$ so that

from which

$$
\frac{4 \pi}{10} \cdot \frac{Z}{2 p p} \cdot \sqrt{2} M_{w}=2 g \cdot \frac{\pi \phi}{2 \lambda l} \cdot \frac{t+s^{*}}{t}
$$

$$
M_{\mathrm{v}}=\frac{\operatorname{ropg\Phi }}{\sqrt{2} \lambda l Z} \cdot \frac{t+s}{t}
$$

in which $M_{v v}$ is the effective value of the wattless component of the magnetizing current in each phase of a two-phase stator winding, $Z$ is the total number of stator conductors, $p$ is the number of stator polar regions, $g$ is the radial length of the air gap, $\Phi$ is the total flux emanating from a polar region of the stator, $\lambda$ is the length of the stator and rotor iron parallel to the motor shaft, $l$ is the distance between adjacent polar regions ( $N$ and $S$ ) of the stator, $t$ is the breadth of a stator tooth, and $s$ is the breadth of a stator slot. This assumes that the rotor slots are nearly closed so that the effective area of the gap space is at all times sensibly equal to the area of the ends of the stator teeth.

For the case of a three-phase stator winding let us consider the instant when the magnetizing current $M_{w}$ is at its maximum value $\sqrt{2} M_{w}$ in one phase and at halfmaximum $M_{v 0} / \sqrt{2}$ in the other two phases. At this instant the three bands of stator conductors, each having $Z /(3 p)$ conductors, work together to supply the magnetomotive force $2 g \times \pi \Phi /(2 \lambda l) \times(t+s) / t$. The ampere-turns in the respective bands are $M_{v o} / \sqrt{2} \times Z /(3 p), \sqrt{2} M_{v} \times Z /(3 p)$, and $M_{v} / \sqrt{2} \times Z /(3 p)$, so that the aggregate is $2 \sqrt{2} M_{t v} Z /(3 p)$. Therefore we have

$$
\frac{4 \pi}{10} \cdot 2 \sqrt{2} M_{w} \cdot \frac{Z}{3 p}=2 g \cdot \frac{\pi \Phi}{2 \lambda l} \cdot \frac{t+s}{t}
$$

from which the value of $M_{w}$ may be calculated.

Calculation of magnetic leakage reactance per phase. - The magnetic leakage of an induction motor, that is, the passage of magnetic flux between the stator and

* The effect of a concentrated stator winding, or of a stator winding distributed in bands of prescribed width, in maintaining a harmonically distributed flux should in strictness be formulated in a manner involving a "phase constant" of distribution of the winding. The correct theory is very much like the theory of electromotive force which is developed in Art. 6r. It would be of no practical use, however, to develop the refined theory of magnetizing action. Equation (ii) gives an over-estimate of $M_{v}$, and equation (iii) gives an under-estimate of $M_{w}$. 
rotor windings, is equivalent in its effects to a certain amount of external inductance $P$ (or a certain reactance $\omega P$ ) connected in series with each of the stator windings, exactly as in the transformer as described in Arts. II 7 and 118.

Figure 247 shows a stator slot $S$ and a rotor slot $R$, the rotor being supposed to have the same number of slots as the stator, and the dotted lines $t$ and $t^{\prime}$ in Fig. 247 are the center lines of adjacent stator and rotor teeth. The magnetic reluctance of the iron is entirely negligible in comparison with the reluctance of the long air paths through which the leakage flux passes, and the only influence of the windings in adjacent stator slots (or in adjacent rotor slots) on each other, in so far as magnetic leakage is concerned, is the entirely negligible influence which may be stated as fol-

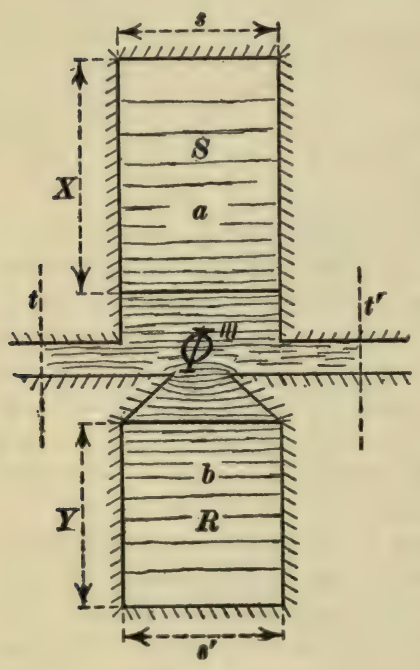

Flg. 247. lows: If one pair of slots alone is con idered, as shown in Fig. 247, then the leakage flux in the very narrow gap space comes out of the iron at $t$ and flows into the iron again at $t^{\prime}$, whereas, if a whole band of slots is considered simultaneously the leakage flux at $t$ and $t^{\prime}$ continues straight along the air gap, as shown in Fig. 247. Any flux crossing the gap space at $t$ and $t^{\prime}$ would encircle both slots $S$ and $R$ and would not be leakage flux. Therefore the discussion of magnetic leakage may be based upon the consideration of a single pair of slots $S$ and $R$, as shown in Fig. 247. If the number of rotor slots is greater (or less) than the number of stator slots, then the easiest method of procedure is to assume that the rotor slots are the same in number as the stator slots, and to consider each rotor slot as increased (or decreased) in width both at top and at bottom, in proportion to the assumed decrease (or increase) in the number of rotor slots.

Let $P_{s}$ be the inductance equivalent of magnetic leakage across one pair of stator and rotor slots. Then the inductance equivalent per phase is equal to $n P_{s}$ where $n$ is the number of stator slots per phase, all of the stator conductors of one phase being connected in series; and the reactance equivalent of magnetic leakage per phase is $\omega n P_{8}$.

The inductance equivalent of the magnetic leakage across one pair of stator and rotor slots is given by the equation :

$$
P^{s}=\frac{N^{\prime}\left(\Phi^{\prime}+\Phi^{\prime \prime}+\Phi^{\prime \prime \prime}\right)}{i^{\prime}}
$$

in which $N^{\prime}$ is the number of wires in one stator slot, $i^{\prime}$ is any assigned value of current in the stator winding, and $\Phi^{\prime}, \Phi^{\prime \prime}$ and $\Phi^{\prime \prime \prime}$ are three equivalent parts of the leakage flux corresponding to the current $i^{\prime}$ as explained below.

The actual leakage flux consists of three parts, namely, the flux $a$, Fig. 247, which crosses the stator slot through the winding of wire, the flux $b$ which crosses the rotor 
slot through the winding of wire, and the flux $\Phi^{\prime \prime \prime}$ which crosses between the stator and rotor windings. The flux $a$ is equivalent to a certain flux $\Phi^{\prime}$ crossing between the windings, and the flux $b$ is equivalent to a certain flux $\Phi^{\prime \prime}$ crossing between the windings exactly as described in Art. 119, and the values of $\Phi^{\prime}$ and $\Phi^{\prime \prime}$ are given by the equations of Art. 119, namely,

and

$$
\Phi^{\prime}=\frac{4 \pi i^{\prime} N^{\prime} \lambda X}{3^{s}}
$$

$$
\Phi^{\prime \prime}=\frac{4 \pi i^{\prime} N^{\prime} \lambda Y}{3^{s^{\prime}}} \frac{Y}{}
$$

in which $N^{\prime}$ is the number of conductors in one stator slot, $\lambda$ is the length of stator and rotor iron parallel to the motor shaft, and $X, Y, s$ and $s^{\prime}$ are the dimensions shown in Fig. 247, all being expressed in centimeters.

The value of the flux $\Phi^{\prime \prime \prime}$ which actually crosses between the coils may be roughly calculated from the length and section of each part of the air path through which $\Phi^{\prime \prime \prime \prime}$ passes, the magnetomotive force producing $\Phi^{\prime \prime \prime}$ being $4 \pi /$ I0 $\times N^{\prime} i^{\prime}$.

It is evident that any accurate estimate of $\Phi^{\prime \prime \prime}$ is impossible. In fact the value of $\Phi^{\prime \prime \prime}$ for a given value of $i^{\prime}$ varies considerably as the rotor slots sweep past the stator slots, for, at certain instants the stator teeth bridge across the openings of the rotor slots, and the broad ends of the rotor teeth bridge across the stator slots. This variation of $\Phi^{\prime \prime \prime}$ for a given current means that the inductance equivalent of magnetic leakage is a pulsating inductance, the frequency of the pulsations being equal to the product of the rotor speed times the number of rotor slots.

Calculation of equivalent resistance of the rotor per stator phase. - If the rotor were wire wound with the same number of phases and the same number of conductors per phase as the stator winding, and if each one of these rotor phases were short circuited on itself, then the short-circuit resistance $R^{\prime \prime}$ of one of these rotor phases would be the value of $R^{\prime \prime}$ to be used in the calculation of performance curves from the circle diagram. In practice, however, the rotor frequently has a squirrelcage winding, and in this case the value of $R^{\prime \prime}$ may be defined as that resistance which multiplied by the square of the load current $I^{\prime}$ in one stator phase will give $\mathbf{I} / q$ of the $R I^{2}$ losses in the rotor, where $q$ is the number of stator phases. To determine $R^{\prime \prime}$, therefore, it is necessary to find the rotor $R I^{2}$ losses for a given value of load current in the stator. For this purpose the rotor may be supposed to be stationary, with the given value of load current flowing in each stator phase. Under these conditions let us consider the rods of the rotor winding in groups or bands corresponding to the bands of the stator winding (see Figs. 227 and 228). In each of the rods of one of these bands the current is $I^{\prime \prime}=Z^{\prime \prime} \mid Z^{\prime} \times I^{\prime}$, where $Z^{\prime}$ is the total number of stator conductors, and $Z^{\prime \prime}$ is the number of rods in the squirrel-cage winding. * Therefore, knowing the length and sectional area of each rod, we can find its resistance and then calculate the total $R I^{2}$ loss in the rods. To determine the $R I^{2}$ loss in the shortcircuiting rings proceed as follows :

For the case of a two-phase stator winding. - Let $n$ be the number of rods in one of the above mentioned bands $\left(=Z^{\prime \prime} / 2 p\right)$, then $n I^{\prime \prime}$ is the total current entering an

* This assumes that the windings in each pair of slots, $S$ and $R$, Fig. 247, balance each other's magnetizing action. See Appendix B, Art. I7. 
end ring from the rods of one band. Let one side of the square, Fig. 248, represent the current entering an end-ring from one band of rotor rods, and let each side of the square be divided into $n$ equal parts (Fig. 248 is constructed for $n=6$ ), then the lines $a, b, c, d, e$, etc., drawn from the center of the square to the equidistant points on the sides of the square represent the values of current in the portions of the endrings between the points of attachment of the ends of the rods, and the average value of the square of the currents $a, b, c, d$, e, etc., is represented by the square of the radius of a circle $\left(=\left(2 n I^{\prime \prime}\right)^{2} / \pi\right)$ having the same area as the square in Fig. 248. Let $r$ be the resistance of one of the portions of the end-rings from center to center of adjacent rods, then $r \times\left(2 n I^{\prime \prime}\right)^{2} / \pi$ is the average power lost in each portion, and,

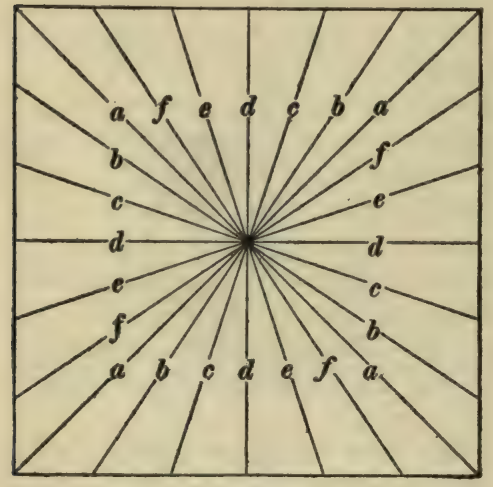

Fig. 248.

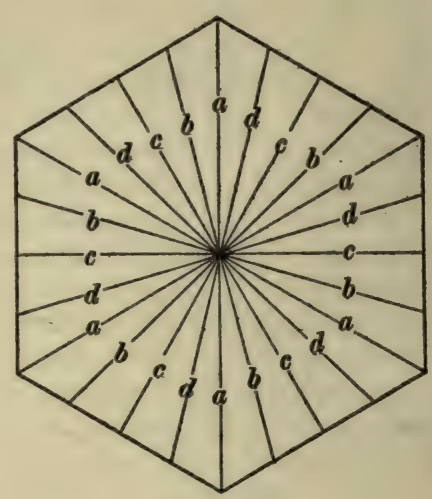

Fig. 249.

since there are $2 Z^{\prime \prime}$ portions, counting both end-rings, the total power lost in both end-rings is $2 Z^{\prime \prime} r$. $\left(2 n I^{\prime \prime}\right)^{2} / \pi$.

For the case of a three-phase stator winding. - As before let $n$ be the number of rods in each of the above mentioned bands $\left(=Z^{\prime \prime} / 3 p\right)$, then $n I^{\prime \prime}$ is the total current entering an end-ring from the rods of one band. Let one side of a regular hexagon, Fig. 249, represent the current $n I^{\prime \prime}$ entering an end-ring from a band of rotor rods, and let each side of the hexagon be divided into $n$ equal parts (Fig. 249 is constructed for $n=4$ ), then the lines, $a, b, c, d$, etc., drawn from the center of the hexagon to the equidistant points on the periphery represent the values of current in the portions of the end-rings between points of attachment of the ends of the rods, and the average value of the square of the currents, $a, b, c, d$, etc., is represented by the square of the radius of a circle $\left[=3 \sqrt{3}\left(n I^{\prime \prime}\right)^{2} /(2 \pi)\right]$ having the same area as the hexagon. Therefore the total power lost in both end-rings is $2 Z^{\prime \prime} r \cdot 3 \sqrt{3} / 2 \pi \cdot\left(n I^{\prime \prime}\right)^{2}$.

\section{The algebraic formulation of the problem of the induction} motor. - Figure 250 represents a combination of simple circuits which is equivalent to a given induction motor running at slip $s$, and Fig. $25 \mathrm{I}$ represents a combination of simple circuits which is approximately equivalent (magnetizing current assumed to be in- 
dependent of motor load) to a given induction motor running at slip s. Figure 250 is exactly like Fig. 215 , and Fig. $25 \mathrm{I}$ is

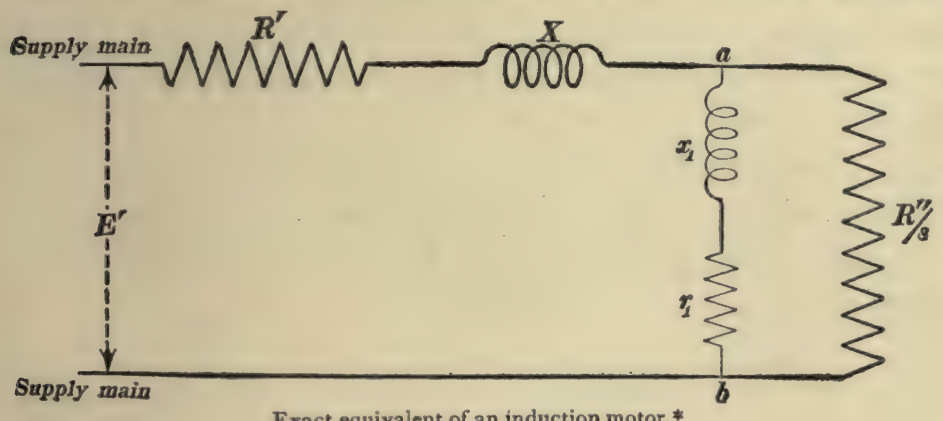

Exact equivalent of an induction motor.*

Fig. 250.

exactly like Fig. 216 (see Art. I20). The circle diagram of the induction motor, Fig. 242, is based on Fig. 251.

The algebraic formulation of the action of the induction motor

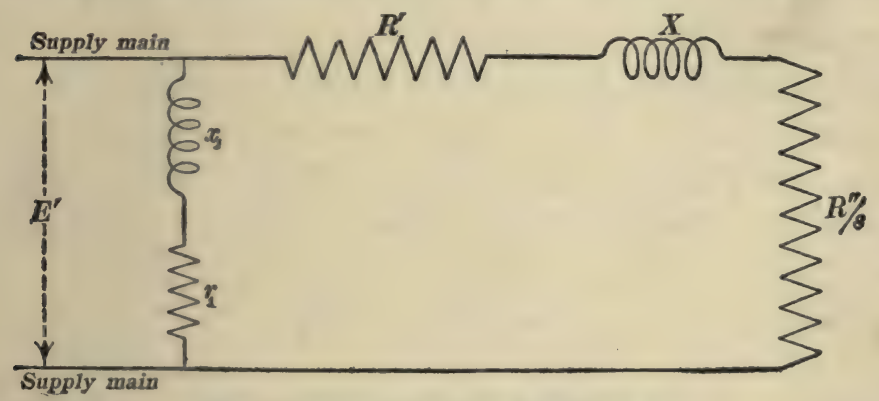

Approximate equivalent of an induction motor.

Fig. 251.

may be easily accomplished on the basis of either Fig. 250 or Fig. 25 I. On the basis of Fig. 250 the algebraic formulation of the induction motor is as follows: The reactance $X$ (equals $\omega P$ )

* A slightly more exact equivalent would be that in which part of the leakage reactance $X$ is on each side of the point $a$, inasmuch as the forcing of the leakage flux between stator and rotor windings by the load currents $I^{\prime}$ and $I^{\prime \prime}$ as shown in Fig. $24 \mathrm{I}$ makes it unnecessary for the magnetizing current to force this flux through the reluctance of the rotor iron. The placing of $X$ partly on each side of the point $a$ amounts to assigning a part of the magnetic leakage reactance to the secondary ; and in fact the only basis on which leakage reactance may be separated into primary leakage reactance and secondary leakage reactance is this basis of reluctance of rotor iron. See Art. 117 . 
represents the effect of magnetic leakage, $R^{\prime}$ is the resistance of one phase of the stator winding, $E^{\prime}$ is the voltage acting on one stator winding, the circuit $r_{1} x_{1}$ represents the effect of magnetizing current (the resistance $r_{1}$ and the reactance $x_{1}$ being such that the current in the circuit $r_{1} x_{1}$ is equal to the actual magnetizing current $M), R^{\prime \prime}$ is the equivalent resistance of the rotor per stator circuit. Let $A$ represent the voltage across $a b$, Fig. 250 , then from the figure we have, using complex-quantity notation,

$$
\begin{aligned}
& M=\frac{A}{r_{1}+j \bar{x}_{1}} \\
& I^{\prime}=\frac{s A}{R^{\prime \prime}} \\
& E^{\prime}=A+\left(M+I^{\prime}\right)\left(R^{\prime}+j X\right)
\end{aligned}
$$

Furthermore, the total power delivered to the rotor per stator phase is $\left(R^{\prime \prime} / s\right) I^{\prime 2}$, the electrical power developed in the rotor per stator phase is $R^{\prime \prime} I^{\prime 2}$, and the mechanical power developed in the rotor per stator phase is the difference between $\left(R^{\prime \prime} / s\right) I^{\prime 2}$ and $R^{\prime \prime} I^{\prime 2}$. Therefore the total mechanical power developed in the rotor is

$$
P=\frac{\mathrm{I}-s}{s} \cdot R^{\prime \prime} I^{\prime 2} q
$$

in which $q$ is the number of stator phases, and $P$ is the total mechanical power in watts developed by the motor. (The letter $P$ has been also used for magnetic leakage inductance.)

The torque $T$ which is developed by the motor produces the power $P$ (expressed, in watts of course) at the actual rotor speed $n^{\prime}$, and is convenient to express the value of the torque in terms of the power $(P \times n) / n^{\prime}$ which the same value of torque would produce at synchronous speed $n$. That is we may write

$$
T=\frac{n}{n^{\prime}} \cdot P=\frac{\mathrm{I}}{\mathrm{I}-\mathrm{s}} \cdot P
$$

This equation expresses torque in "synchronous watts." 
Data to be used in connection with equatious (i) to (v). - The values of $r_{1}$ and $x_{1}$ may be determined from the calculated components of the magnetizing current at zero load* as explained in Art. I 37, sections $(b)$ and $(c)$. Thus we have

$$
\begin{aligned}
& M_{p}=\frac{r_{1}}{r_{1}^{2}+x_{1}^{2}} \cdot E^{\prime} \\
& M_{v 0}=\frac{x_{1}}{r_{1}^{2}+x_{1}^{2}} \cdot E^{\prime}
\end{aligned}
$$

from which $r_{1}$ and $x_{1}$ may be calculated when $M_{p}$ and $M_{v 0}$ and $E^{\prime}$ are known.

The value of $R^{\prime \prime}$ may be determined as explained on page 287 under the heading "Calculation of equivalent resistance of the rotor per stator phase."

The value of $R^{\prime}$ may be determined by direct measurement of resistance of the stator windings, and the value of $X$ may be calculated as explained on page 285 under the heading "Calculation of magnetic leakage reactance per phase."

Calculation of performance curves from equations (i) to $(v)$. Knowing the values of $r_{1}, x_{1}, R^{\prime \prime}, E^{\prime}, R^{\prime}, X$, and $q$, the details of the behavior of the motor when running at any arbitrarily. assigned slip $s$ may be calculated as follows: Eliminating $A$ from equations (i), (ii) and (iii), we have two remaining equations (complex equations of course) which completely determine $M$, $I^{\prime}$, and $\left(M+I^{\prime}\right)$. The product of $E^{\prime}$ by the power component of $\left(M+I^{\prime}\right)$ gives the power input to the motor per phase, and the cosine of the angle between $E^{\prime}$ and $\left(M+I^{\prime}\right)$ gives the power factor of the motor when running at the given slip. Equation (iv) determines the mechanical output, ratio of output to input gives efficiency, and equation ( $v$ ) gives the motor torque.

Graphical solution of equations (i) to (v). - The algebraic solution of equations (i), (ii) (iii), (iv) and (v) is quite simple but very tedious, and the following graphical solution may be preferred.*

* It must be remembered that $M$ in equation $(i)$ is not assumed to be constant, it decreases with increasing load on the motor. 


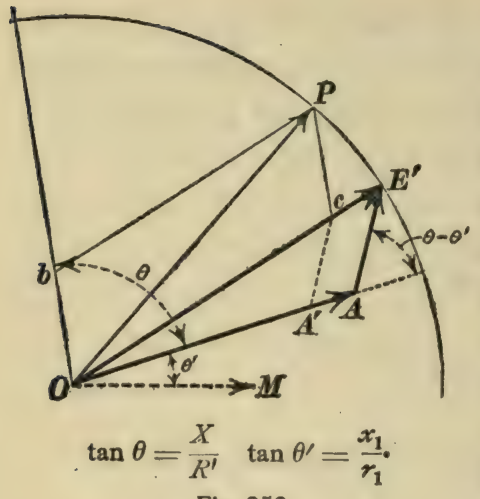

Flg. 252.

Choose the magnetizing current $O M$ as a reference axis. Lay off the line $O A$ representing the value of the electromotive force $A\left[=M\left(r_{1}+j x_{1}\right)\right]$, and lay off $A E^{\prime}$ equal to $M\left(R^{\prime}+j X\right)$, zeroload value of $M$ being used in each case. Then $O E^{\prime}$ is the zeroload position of the supply voltage of one of the stator windings. Draw about $O$ a circle of radius $E^{\prime}$, then a line drawn from $O$ to any point $P$ of this circle is a possible position of the supply-voltage vector with the motor running under load.

Evidently the voltage $A$ across $a b$, Fig. 250, decreases with increase of motor load (increase of current through $R^{\prime}$ and $X$ ) and of course any decrease of $A$ produces a proportional decrease of the magnetizing current and of the voltage drop in $R^{\prime}$ and $X$ due to the magnetizing current, so that, when the voltage across $a b$, Fig. 250 , is reduced to $O A^{\prime}$, Fig. 252, the voltage drop in $R^{\prime}$ and $X$ due to the reduced magnetizing current is $A^{\prime} c$ which is parallel to $A E^{\prime}$.

Now the total primary voltage (of one phase of course) $O P$ is equal to the vector sum of $O A^{\prime}, A^{\prime} c$, and the voltage drop $I^{\prime}\left(R^{\prime}+j X\right)$ in $R^{\prime}$ and $X$ due to the load current; but $I^{\prime}\left(R^{\prime}+j X\right)$ is in the direction $O b$ which is $\theta^{\circ}$ ahead of $I^{\prime}$ or $\theta^{\circ}$ ahead of $A$, since $A$ and $I^{\prime}$ are in phase with each . other. Therefore, having drawn the line $O b$, choose any point $P$, draw $P c$ parallel to $O b$, draw $P b$ parallel to $O E^{\prime}$, scale off the value of $O b$ in volts, and the value of $O A^{\prime}$ in volts. Dividing the value of $O b$ by $\sqrt{R^{\prime 2}+X^{2}}$ gives the value of $I^{\prime}$ in amperes, whence, from equation (ii) $s$ may be calculated, inasmuch as $R^{\prime \prime}$ is supposed to be given, and $A$ is known $\left(=O A^{\prime}\right)$.

Knowing $I^{\prime}$ and $s$, the mechanical power output of the motor may be calculated from equation (iv) for the chosen position of 
the point $P$ in Fig. 252, the motor torque may be calculated from equation (v), and the motor speed is known directly from the value of $s$.

If it is desired to find the power factor of the motor, the total power input, and the efficiency for the chosen position of the point $P$, Fig. 252, proceed as follows: Lay off the value of $I^{\prime}$ along the line $O A$, lay off the magnetizing current $\left(=O A^{\prime}\right.$ divided by $\left.\sqrt{r_{1}^{2}+x_{1}^{2}}\right)$ along the line $O M$, and find the resultant of the two. The cosine of the angle between $O P$ and this resultant current is the power factor of the motor, the product of this resultant current by $O P$ and by the power factor gives the power intake of the motor per phase, and the power output divided by the total power intake gives the efficiency.

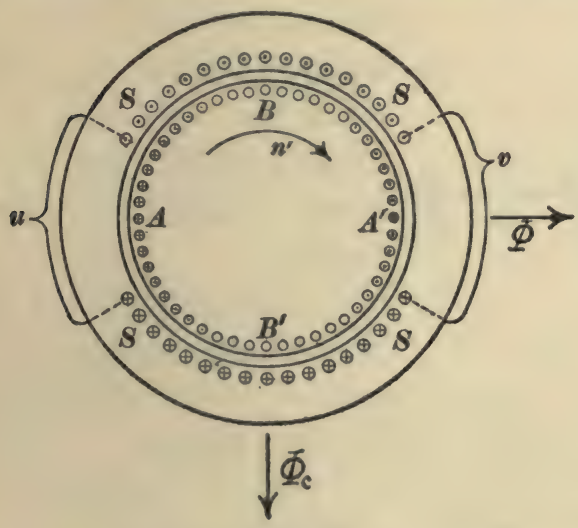

Fig. 253.

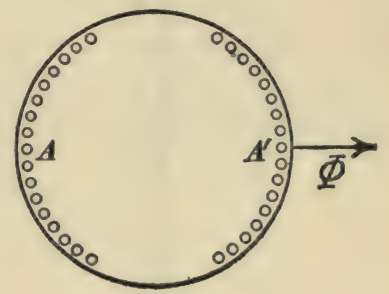

Fig. $253 a$.

139. The single-phase induction motor. - The internal action of the single-phase induction motor is very different from the internal action of the polyphase induction motor, and it will be described first on the basis of an ideal motor in which the resistance of the stator winding, the magnetic reluctance of iron and air gaps, and magnetic leakage are all negligibly small, and then these various things will be taken account of in applying the circle diagram to the single-phase machine. Briefly described, the action of the single-phase induction motor is as follows: $E^{\prime}$ 
is the supply voltage, $n$ is its frequency, and $n^{\prime}$ is the speed of the rotor in revolutions per second (two-pole model assumed).

(a) A pulsating flux $\Phi$, Fig. 253, is produced by the stator winding, and the electromotive force induced in the stator winding by this pulsating flux just balances the supply voltage $E^{\prime}$.

(b) The moving rotor rods cut the flux $\Phi$, and an* electromotive force, $A_{r}$, Fig. 254, is induced in the regions $A$ and $A^{\prime}$ of the rotor winding. This electromotive force $A_{r}$ has the same frequency as $\Phi$, it is in phase with $\Phi$, and it produces up-flowing currents at $A^{\prime}$ and down-flowing currents at $A$, Fig. 253. These currents are magnetizing currents $\left(M_{a}\right.$ in Fig. 254) and they produce a cross-flux $\Phi_{c}$ which pulsates at the pri-

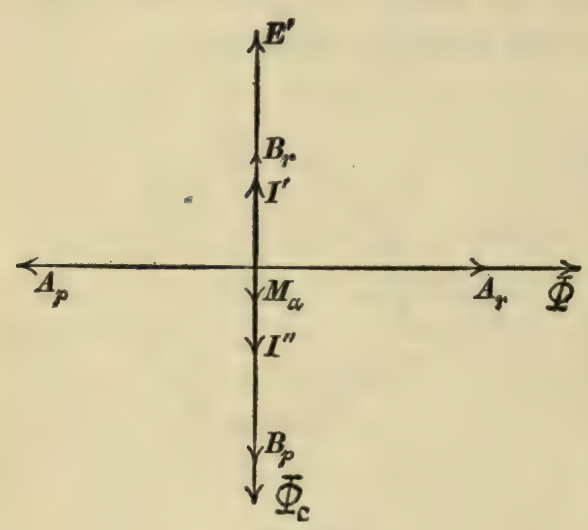

Fig. 254. mary frequency. The pulsations of this cross-flux induce an electromotive force, $A_{p}$, in the rotor rods at $A$ and $A^{\prime}$, and this electromotive force balances the electromotive force $A_{r}$.

(c) The pulsations of $\Phi$ produce an electromotive force $B_{p}$ in the rotor rods in the regions $B$ and $B^{\prime}$, Fig. 253, and the cutting of the cross-flux $\Phi_{c}$ induces an electromotive force $B_{r}$ in the same rods. When the rotor runs at synchronous speed ( $n^{\prime}$ equals $n$ ), then $B_{p}$ is exactly balanced by $B_{r}$, no currents flow in the rotor rods in the regions $B$ and $B^{\prime}$, and therefore nothing but magnetizing current flows in the stator. When the

*A squirrel-cage rotor presents a multiplicity of circuits, and to speak of the electromotive force or the current in the rotor, depends upon a scheme which is described in a subsequent part of this article. In speaking of an electromotive force or cur. rent in the rotor the electromotive force or current in a stationary group of the moving rotor rods is understood. New rods are, of course, coming into such a group continuously on one side and leaving the group continuously on the other side. An electromotive force induced by pulsation is indicated by a subscript $p$, and an electromotive force due to the cutting of lines of force by rotation is indicated by a subscript $r$. 
rotor speed is below synchronism ( $n^{\prime}$ less than $n$ ) then $B_{r}$ is less than $B_{p}$ the net electromotive force $\left(B_{p}-B_{r}\right)$ produces a load current $I^{\prime \prime}$ in the rotor rods in the regions $B$ and $B^{\prime}$, the magnetizing action of this load current is balanced by a corresponding load current $I^{\prime}$ in the stator winding, and the load currents in the rotor rods in the regions $B$ and $B^{\prime}$ are pushed sidewise by the cross-flux $\Phi_{\mathrm{c}}$ thus developing mechanical power.

It is evident that the flux $\Phi$ is independent of the rotor speed, inasmuch as it must by its pulsations induce sufficient electromotive force in the stator winding to balance the supply voltage $E^{\prime}$, and it is $90^{\circ}$ behind $E^{\prime}$ in phase. This flux is called the "transformer flux" for brevity.

The cross-flux $\Phi_{c}$ on the other hand is proportional to the rotor speed $n^{\prime}$, and it is equal numerically to $\Phi$ when $n^{\prime}$

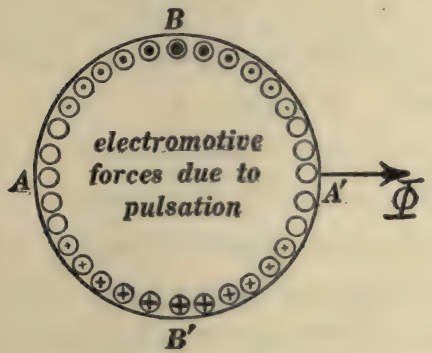

Flg. 255 .

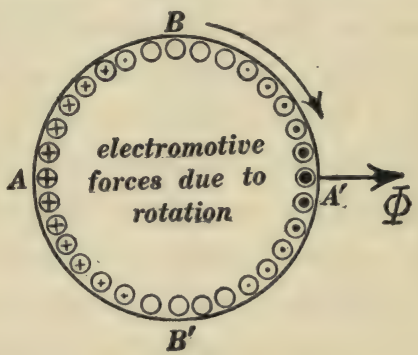

Fig. 256.

equals $n$. This is evident when we consider that the electromotive force $A_{r}$ due to cutting of the transformer flux $\Phi$ at speed $n^{\prime}$ must be balanced by the electromotive force $A_{p}$ due to the pulsations of the cross-flux $\Phi_{c}$.

It is interesting to note that at synchronous speed $\left(n^{\prime}=n\right)$ the magnetism of the single-phase induction motor is exactly like the magnetism of the polyphase induction motor, that is to say, the stator magnetism is constant in value and rotates at speed $n$. This is evident when we consider that the two fluxes $\Phi$ and $\Phi_{c}$ are equal in value when $n^{\prime}=n$, and that they are in time quadrature with each other.

In order to proceed further in the analysis of the action of the single-phase induction motor, it is necessary to consider the exact 
relation between the electromotive force induced in the rotor windings by the pulsation of a flux ( $\Phi$ or $\left.\Phi_{c}\right)$ and the electromotive force produced in the rotor windings by the cutting of a flux ( $\Phi$ or $\left.\Phi_{c}\right)$, as shown in Figs. 255 and 256 . Electromotive forces due to pulsation are in time quadrature with the alternating flux, and electromotive forces due to motion are in time phase with the alternating flux; electromotive forces due to pulsation are greatest in the rotor rods distant $90^{\circ}$ from the axis of the flux, whereas electromotive forces due to motion are greatest in the rotor rods which are near the axis of the flux, as shown in Figs. 255 and 256. Furthermore, a certain frequency of pulsation in Fig. 255 gives certain definite values of induced electromotive force in the rotor rods, the same frequency of rotation in Fig. 256 gives exactly the same values of induced electromotive force in the rotor rods, and a reduced frequency of rotation in Fig. 256 gives proportionally reduced values of induced electromotive force in the rotor rods. See Appendix B, Art. I 5 .

Generally the rotor is of the squirrel-cage type presenting a multiplicity of independent short-circuits, and it is necessary for the sake of brevity and clearness to devise a scheme whereby one may speak of the electromotive force induced in the rotor, of the rotor current, and of the rotor resistance $R^{\prime \prime}$, as if the rotor winding were a single circuit with respect to the induced electromotive force or current under consideration. To do this the rotor winding must be reduced to its primary or stator equivalent as follows:

Let $a$ be the number of conductors in one stator band and let $b$ be the number of rotor rods which for any given position of the rotor lie under the stator band. Then, for a given load current $I^{\prime}$ in the stator winding, the current in each of the $b$ rotor rods is $a I^{\prime} / b$, and, if $b$ is an even number, the currents in the successive portions of the short-circuiting end-rings are $a I^{\prime} / b, 2 a I / b, 3 a I^{\prime} / b \ldots$ up to $\frac{1}{2} a I^{\prime}$, counting from the center of a band (or $\frac{1}{2} a I^{\prime} / b, \frac{3}{2} a I^{\prime} / b, \frac{5}{2} a I^{\prime} / b \ldots$ up to $\frac{1}{2} a I^{\prime}$ if $b$ is an odd number). The current throughout the portions $u$ and $v$, 
Fig. 253, of the short-circuiting end-rings is $\frac{1}{2} a I^{\prime}$. Therefore, knowing the size and length of each rotor rod and the size and length of each portion of the short-circuiting end-rings, the rotor $R I^{2}$ loss may be easily calculated for an assumed value of $I^{\prime}$. The equivalent resistance $R^{\prime \prime}$ of the rotor is equal to this rotor $R I^{2}$ loss divided by $I^{\prime 2}$. Using the value of $R^{\prime \prime}$ so defined, the load current in the portions $B B^{\prime}$ of the rotor winding (see Fig. 253) may be thought of as equal to the load current in the stator; and insofar as the induced electromotive forces in the regions $B$ and $B^{\prime}$, Fig. 253 , are concerned, the rotor winding may be thought of as containing the same number of conductors as the stator winding, all connected in series and constituting a single circuit.

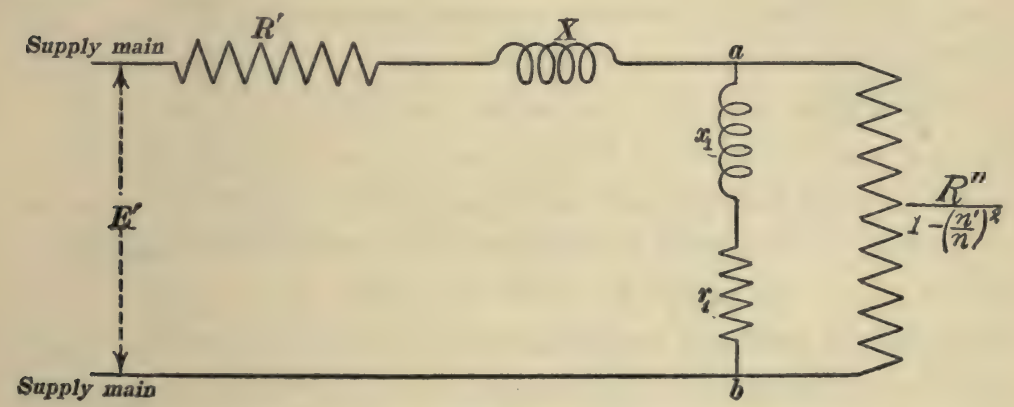

Fig. 257.

Furthermore, insofar as the induced electromotive forces $A_{p}$ and $A_{r}$ in the regions $A$ and $A^{\prime}$, Fig. 253, are concerned, the rotor winding may be thought of as consisting of $S$ conductors all connected in series and distributed exactly like the stator conductors but in the position shown in Fig. 253a, $S$ being the number of stator conductors.

On the basis of the above considerations the clock diagram, Fig. 254, may be understood in every detail. At synchronous speed $\left(n^{\prime}=n\right)$ we have $A_{r}=A_{p}=B_{r}=B_{p}=E^{\prime}$, and $I^{\prime}$ and and $I^{\prime \prime}$ are both zero, so that the motor takes in no power (except that represented by the stator magnetizing current) and the rotor develops no mechanical power. 
When the rotor speed is below synchronism ( $n^{\prime}$ less than $\left.n\right)$, then $A_{r}=n^{\prime} / n \cdot E^{\prime}=A_{p}$, also $\Phi_{c}=n^{\prime} / n \cdot \Phi$, and $B_{r}=\left(n^{\prime} / n\right)^{2} B_{p}$ [ $=\left(n^{\prime} / n\right)^{2} \cdot E^{\prime}$ since $B_{p}$ is always equal to $\left.E^{\prime}\right]$. Therefore $I^{\prime}=I^{\prime \prime}=\left(B_{p}-B_{r}\right) / R^{\prime \prime}=\left[\mathrm{I}-\left(n^{\prime} / n\right)^{2}\right] E^{\prime} / R^{\prime \prime}$, so that the intake of power $E^{\prime} I^{\prime}$ is equal to $\left[\mathrm{I}-\left(n^{\prime} / n\right)^{2}\right]{E^{\prime}}^{2} / R^{\prime \prime}$ all of which (in the ideal motor) is delivered to the rotor and of which the portion $R^{\prime \prime} I^{\prime \prime 2}=R^{\prime \prime} I^{\prime 2}=\left[I-\left(n^{\prime} / n\right)^{2}\right]^{2} E^{\prime 2} / R^{\prime \prime}$ is developed as electrical power in the rotor and the remainder is developed as mechanical power. Therefore the mechanical power is equal to $\left(s_{1}-s_{1}^{2}\right) E^{\prime 2} / R^{\prime \prime}$, where $s_{1}$ is written for $\left[\mathrm{I}-\left(n^{\prime} / n\right)^{2}\right]$, and the torque in synchronous watts is equal to $n / n^{\prime} \times\left(s_{1}-s_{1}^{2}\right){E^{\prime}}^{2} / R^{\prime \prime}$.

The action of the ideal single-phase induction motor running at speed $n^{\prime}$ would be reproduced as pure transformer action at stand-still if the rotor resistance were increased to $R^{\prime \prime} / s_{1}$. This is evident when we consider $(a)$ that the net electromotive force in the region $B B^{\prime}$ of the rotor (see Fig. 253) is equal to $B_{p}-B_{r}$ or to $\left[\mathrm{I}-\left(n^{\prime} / n\right)^{2}\right] E^{\prime}$ when the rotor is running at speed $n^{\prime},(b)$ that this net electromotive force becomes equal to $E^{\prime}$ when $n^{\prime}=0$, that is, this net electromotive force is multiplied I/ $\left[\mathrm{I}-\left(n^{\prime} / n\right)^{2}\right]$ times by changing from speed $n^{\prime}$ to zero, and $(c)$ that this increased electromotive force in the region $B B^{\prime}$ would produce an unchanged value of current in the rotor if the rotor resistance were increased in the same ratio as the net electromotive force.

Now it may be shown ( $a$ ) that the effect of magnetic leakage in an actual single-phase induction motor is the same as an outside reactance $X$ in the primary circuit of an ideal single-phase induction motor, $(b)$ that the effect of resistance of the stator winding is the same as an outside resistance $R^{\prime}$ in the primary circuit of an ideal single-phase induction motor, and $(c)$ that the effect of core reluctance (necessity of magnetizing current) in an actual single-phase induction motor is the same as a shunt circuit of proper resistance $r_{1}$ and reactance $x_{1}$ connected across the supply mains of an ideal motor. Therefore the combination of 
simple circuits shown in Fig. 257 represents the action of an actual single-phase induction motor, and the combination of simple circuits shown in Fig. 258 represents the action on the assumption that the magnetizing current in the stator winding is constant.*

The circle diagram may be applied to the single-phase induction motor on the basis of Fig. 258 as follows: Measure the magnetizing current and the power input of the motor at zero load. This determines the vector $O M$, Fig. 242. Measure the stator current and power input at stand-still, thus determining the

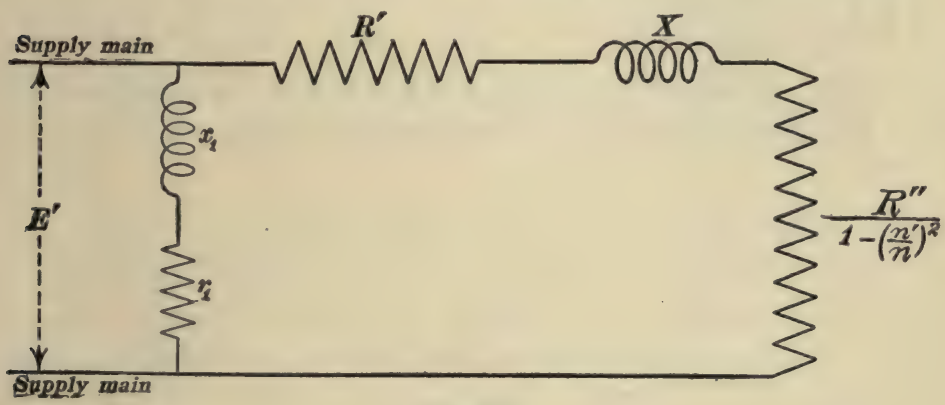

Fig. 258.

vector $O P^{\prime}$, Fig. 242. Draw the circle exactly as in the case of the polyphase motor. Measure the stator resistance $R^{\prime}$. The core loss at zero load is equal to $\overline{O M_{p}} \times E^{\prime}$ minus $R^{\prime} \times \overline{O M}^{2}$ (see Fig. 242). The core loss is assumed to be the same at all loads. Then $\overline{O Q} Q^{\prime} \times E^{\prime}$ minus core loss minus $R^{\prime} \times \overline{O P^{\prime}}$ is the power delivered to the rotor at stand-still, and this power divided by $I^{\prime 2}\left(=\bar{M} \bar{P}^{\prime 2}\right)$ gives the equivalent resistance, $R^{\prime \prime}$, of the rotor.

To calculate the performance curves of the single-phase induction motor from the circle diagram, choose a series of positions of the point $P$, Fig. 242, and for each position make the following calculations: $E^{\prime} \times \overline{O Q}$ is the total power input. The rotor current $I^{\prime \prime}\left(=I^{\prime}\right)$ is equal to $M P$, and the rotor $R I^{2}$

* This assumption is not nearly so accurate in the single-phase motor as it is in the polyphase motor because of the great reduction of the cross-flux $\Phi_{c}$ with decrease of speed. 
loss is $R^{\prime \prime} \times \overline{M P}^{2}$. The total power delivered to the rotor is $R^{\prime \prime} / s_{1} \times \overline{M P}^{2}$. The total power delivered to the rotor is also equal to $E^{\prime} \times \overline{O Q}$ minus core loss minus $R^{\prime} \times \overline{O P}^{2}$, whence the value of $s_{1}\left[=\mathrm{I}-\left(n^{\prime} / n\right)^{2}\right]$ is known and $n$ may be found since synchronous speed $n$ is given. Subtracting the $R I^{2}$ loss in the rotor from the total power delivered to the rotor gives the mechanical power developed, and multiplying the mechanical power by $n / n^{\prime}$ gives the torque in synchronous watts. The entire procedure is the same as in the case of the polyphase induction motor except that $s_{1}\left[=\mathrm{I}-\left(n^{\prime} / n\right)^{2}\right]$ is used instead of $s\left[=\left(n^{\prime}-n\right) / n\right] . *$

* The student is referred to the excellent treatise on Alternating Current Motors by A. S. McAllister (McGraw, N. Y.) for further developments of the theory of induction motors. 


\section{CHAPTER XIV.}

\section{THE SINGLE-PHASE SERIES MOTOR.*}

\section{Behavior of a direct-current motor when the supply voltage}

is suddenly reversed. - Any direct-current motor will continue to run in an unchanged direction when the supply voltage is reversed, because the reversal of the supply voltage reverses both the armature current and the field current. Therefore any directcurrent motor might be operated by a repeatedly reversed supply voltage, that is by an alternating supply voltage, if it were not for certain effects which are produced at the moment of voltage reversal.

In the case of a shunt motor a reversal of the supply voltage would be followed by a slow dying away of the previously existing field current and a slow growth of a reversed field current, because of the very large inductance of the field winding ; whereas the current in the armature would quickly drop to zero and an

* The following papers give a very complete discussion of the theoretical and practical aspects of the single-phase commutator motor.

Single-phase Motors, by Max Deri, Transactions of the International Electrical Congress, St. Louis, 1904, Vol. III, pages 129-148.

Alternating-current machines with commutators, by Marius Latour, Ibid., pages 149-154.

Transmission and distribution problems peculiar to the single-phase railway, by P. M. Lincoln, ibid., pages 186-193.

Alternating versus Direct-current traction, by F. Niethammer, ibid., pages 194-27I.

The Westinghouse single-phase railway system, by Clarence Renshaw, Electric Journal, Vol. I, pages 134-149.

The alternating-current series motor, by F. D. Newburg, Electric Journal, Vol. II, pages 135-140.

Single-phase railway motor control, by R. P. Jackson, Electric Journal, Vol. II, pages $525-532$, and $762-763$.

Alternating current for Railway service, by B. G. Lamme, Street Railway Journal, January 6, 1906.

Alternating Current Motors, by A. S. McAllister, published in 1906 by The McGraw Publishing Company, Chapters XIII to XVI on the repulsion motor and on the series motor. 
excessive momentary flow of reversed current would take place. This difference in the behavior of the field current and armature current of a shunt motor when the supply voltage is suddenly reversed would show itself as a very considerable phase difference between field current (field magnetism) and armature current if the attempt were made to operate a shunt motor from alternatingcurrent supply mains, and the motor would develop but little torque. The shunt motor cannot be operated satisfactorily from alternating-current supply mains.

In the case of a series motor a reversal of the supply voltage would not be followed by an excessive flow of current, because the armature and field windings are in series; and there can be no phase difference between armature current and field magnetism of a series motor which is operated from alternating-current supply mains.

Sparking. - The most serious difficulty that is encountered when the attempt is made to operate an ordinary series motor by alternating current is the tendency to excessive sparking at the

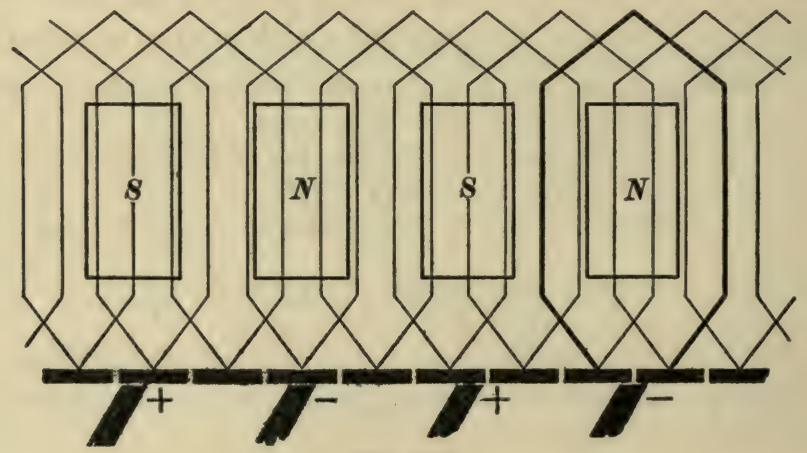

Fig. 259.

brushes. Whenever a brush touches two commutator bars, an armature coil or section is short-circuited; at the moment of this short-circuit the armature coil surrounds a field pole $*$ as shown by the heavy line in Fig. 259, so that the armature coil is related

* This particular statement applies to the lap drum winding, but the effect referred to exists in every variety of armature winding. 
to the field winding as the short-circuited secondary coil of a transformer of which the field winding is the primary coil. Therefore an excessive short-circuit current is produced through the armature coil and through the tip of the brush; and the result is an increased heating of the armature coils, commutator, and brushes, and an excessive sparking at the brush tips as the commutator bars pass from under the brushes.

Low power factor. - A less serious difficulty in the operation of a series motor by alternating current is that the inductance of the field and armature windings cause the current to lag behind the supply voltage in phase, so that the power factor of the series motor when driven by alternating current is always considerably less than unity.

The single-phase series motor.-The ordinary series motor when designed so as to operate satisfactorily with alternating current is called the single-phase commutator motor, or the single-phase series motor. The peculiar features of design and the special devices for reducing sparking and for increasing the power factor of such a motor are described in a subsequent article. Every part of the magnetic circuit of a single-phase series motor must be made of laminated iron.

141. The clock diagram of the single-phase series motor.-Let the line $O I$, Fig. 26o, represent the current flowing through the

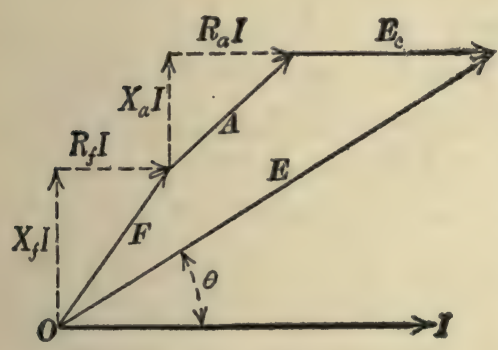

Fig. 260 . field winding and armature of a series motor, let $X_{f}$ be the reactance of the field winding, $X_{a}$ the reactance of the armature winding, $R_{f}$ the resistance of the field winding, and $R_{\alpha}$ the resistance of the armature winding. The components of the electromotive force $F$ which is required to force the current $I$ through the field winding are $X_{f} I$ and $R_{f} I$, as shown in Fig. 260, and the components of the electromotive force $A$ which is required 
to overcome the reactance and resistance of the armature winding are $X_{a} I$ and $R_{a} I$ as shown.

The effect of the current $I$ in flowing through the field winding is to produce an alternating flux $\Phi$ through the armature. This flux is in phase with the current and it induces in the armature windings an electromotive force $E_{c}$ which is equal to $\Phi Z^{\prime} n$, where $\Phi$ is the flux entering the armature from one field pole, $Z^{\prime}$ is equal to $p Z \mid p^{\prime} \div 10^{8}$, and $n$ is the speed of the armature in revolutions per second; $p$ is the number of field poles, $Z$ is the total number of armature conductors, and $p^{\prime}$ is the number of current paths between positive and negative brushes. ${ }^{*}$ Since $\Phi$ is an alternating flux which is in phase with the field current $I$, it is evident that $E_{c}$ (equals $\Phi Z^{\prime} n$ ) is an alternating electromotive force of the same frequency and in phase with $I$. Therefore since $E_{c}$ is a counter electromotive force, the portion of the supply voltage which overcomes it is equal to it and is represented by the line $E_{c}$ in Fig. 26o, and the total supply voltage is represented by the line $O E$ which is equal to the vector sum of $F$, $A$, and $E_{c}$.

The power lost in the field winding is $R_{f} I^{2}$, the power lost in the armature is $R_{a} I^{2}$, and the power which is used to force the current against the counter electromotive force $E_{c}$, namely, $E_{c} I$, appears as mechanical power. Eddy current and hysteresis losses are ignored in Fig. 260.

Design of single-phase series motor to give large power factor. It is evident from Fig. 260 that a large power factor (small value of the angle $\theta$ ) depends upon a large value of $E_{\mathrm{c}}$ in comparison with $X_{f} I$ and $X_{a} I$; of course $R_{f} I$ and $R_{a} I$ should both be as small as possible because they represent energy losses in the windings.

(a) In the first place the reactance $X_{\alpha}$ of the armature winding may be reduced nearly to zero by the use of the neutralizing winding as explained in the following article. A neutralizing winding is therefore an important feature of the single-phase series motor.

* See page 43 of the first volume of this text. 
(b) In the second place, it is to be noted that the reactance voltage $X_{f} I$ which is lost in the field winding is proportional to the product of the number of field turns and the maximum value of the alternating field flux $\Phi$, exactly as in the case of the primary coil of a transformer, and we may assume that we have a motor in which a specified maximum flux is to be produced; then to make the reactance voltage $X_{f} I$ a minimum, the machine must be designed so that the desired flux may be produced by the least possible number of field turns. That is to say, the reluctance of the magnetic circuit must be small, or in other words the gap space between the pole faces and the armature core must be short and the iron of the field magnet and armature core must be operated at a low degree of magnetic saturation. Of course there is no clearly defined value of magnetic saturation which gives the best results, and all that can be said in regard to this second point is that low degree of magnetic saturation and short air gaps are important features of the single-phase series motor.

(c) In the third place the value of $E_{c}$ at a given speed may be made fairly large, even though the value of $\Phi$ be rather small, by increasing the number of armature conductors. Therefore the use of a large amount of armature copper is an important feature of the single-phase series motor.

(d) In the fourth place, suppose that we have a single-phase series motor running at given speed with given value (effective) of current and given value (effective or maximum) of field flux. Under these conditions, the counter electromotive force is definite in value (effective), the mechanical power $E_{c} I$ is definite, and a reduction of the frequency of the supply voltage would produce a proportional reduction of the reactance voltages $X_{f} I$ and $X_{a} I$ without affecting the value of $E_{c}$; that is, a reduction of frequency would increase the power factor of the motor. Therefore it is important to use a low frequency alternating current for a singlephase series motor. A low frequency will be found to be desirable in order to reduce the tendency to spark at the brushes. 
142. The neutralizing of armature inductance. - A winding of wire on an iron core has considerable inductance, that is, a current in the winding magnetizes the iron core and causes a considerable amount of magnetic flux through the core. The inductance of such a winding may be annulled by anything which counteracts the magnetizing action of the winding on the iron core. Thus, Fig. 26I shows a layer of wire, $W W$, wound on an iron rod, the magnetic flux due to the current in the winding is indicated by fine lines; and Fig. 262 shows a second layer of wire, $a a$, wound over $W W$ and so connected in series with $W W$ that the current flows in opposite directions around the rod in $a a$ and in $W W$, as indicated by the dots and crosses. The magnetizing action of the winding $W W$ is neutralized by the opposite

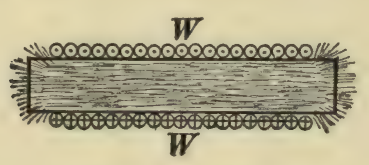

Fig. 261.

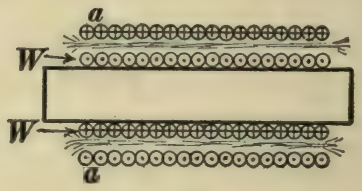

Fig. 262.

magnetizing action of the auxillary winding $a \alpha$, the only magnetic flux produced is the very small amount of flux between $W$ and $a$, and therefore the inductance of $W W$ and $a a$ combined is nearly zero.

If an alternating current is made to flow through the winding $W W$, Fig. 262, and if the auxiliary winding $a a$ is short-circuited, then the magnetizing action of the current in $W W$ will be very nearly.neutralized by the current which is induced in the short-circuited winding $a a$; in this case the number of turns in the auxiliary coil $a a$ need not be the same as the number of turns of wire in the winding $W W$.

The auxiliary winding $a a$, Fig. 262 , which is used to annul the inductance of the given winding $W W$ is called a neutralizing or compensating winding. When the auxiliary winding is connected in series with the given winding, we have what is called forced neutralization or forced compensation, and when the auxil- 


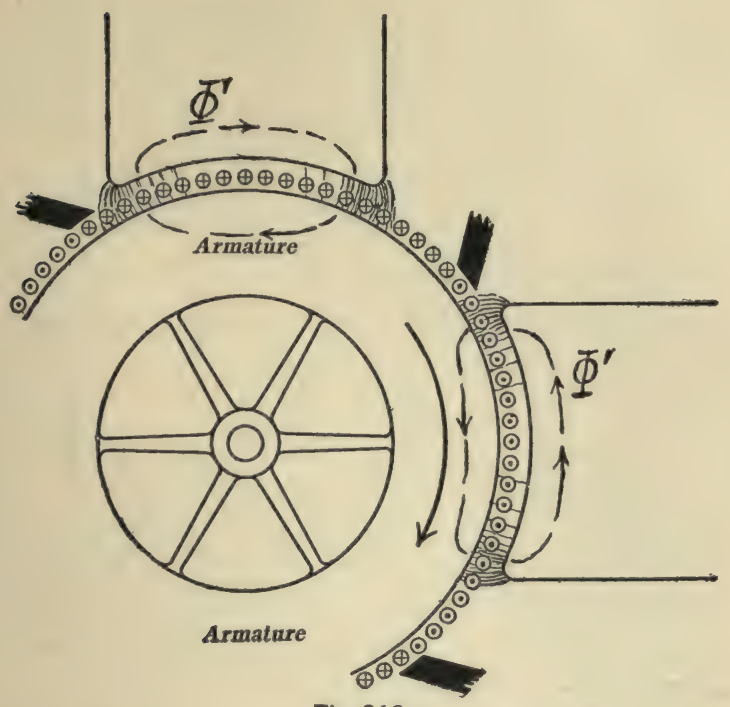

Fig. 263.

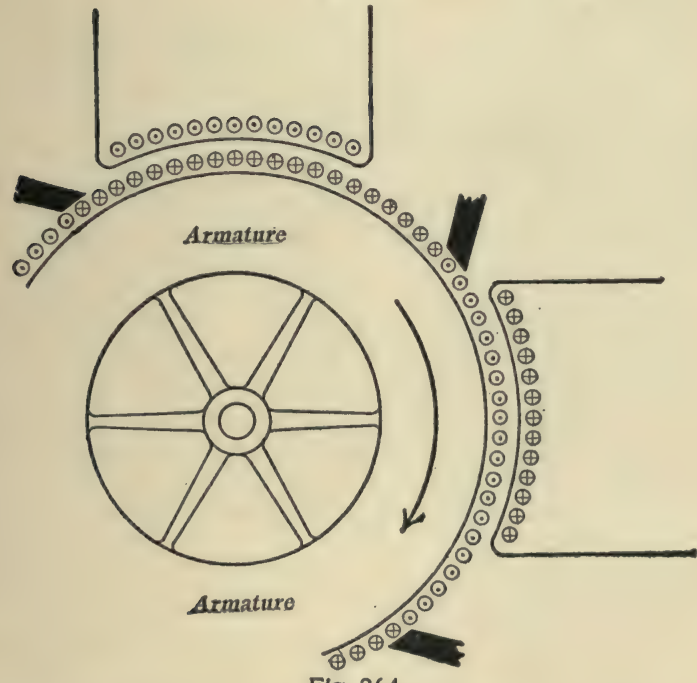

Fig. 264.

iary winding is short-circuited we have what is called inductive or transformer neutralization. Forced compensation can be used for both direct current and for alternating current, whereas transformer compensation can be used only for alternating current. 
The magnetizing action of the armature current of a directcurrent motor or of a single-phase series motor produces magnetic flux, as shown in Fig. $26_{3}$, the inductance of the armature

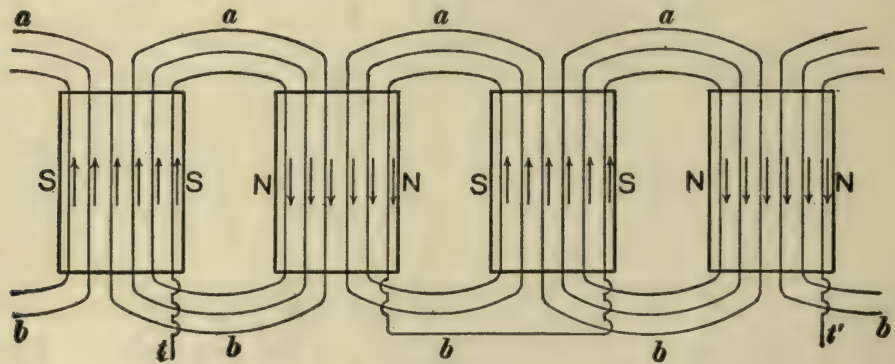

Fig. 265.

winding is due to this flux, and this inductance may be annulled by a neutralizing winding placed in slots in the pole faces, as shown in Fig. 264.* This neutralizing winding is connected,

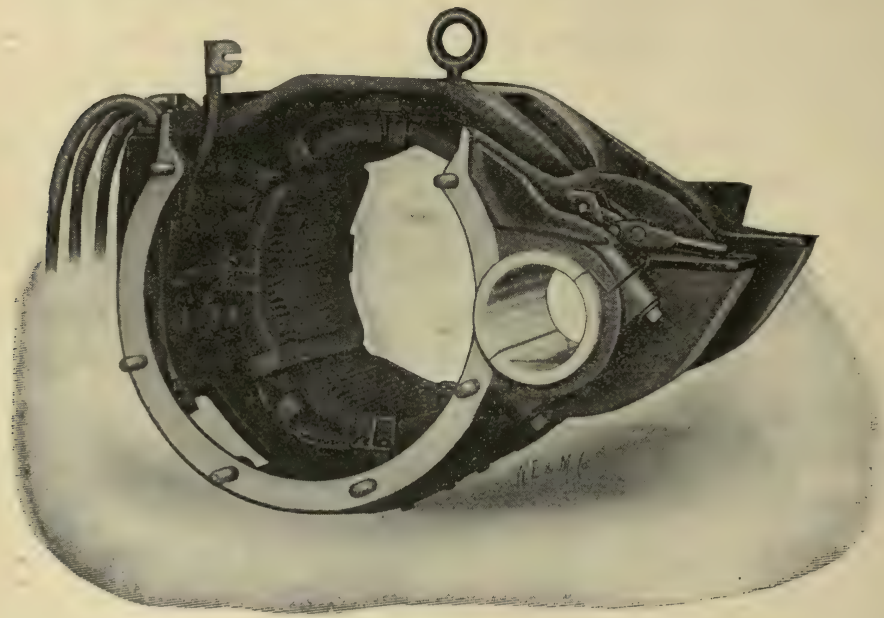

Fig. 266.

as shown in Fig. 265 , so that the current flows in one direction in all of the conductors which lie under north poles and in the opposite direction in all of the conductors which lie under south

* See pages $15^{1}$ to $15^{8}$ of the first volume of this text. 
poles as indicated by the dots and crosses in Fig. 264 and by the arrows in Fig. 265. In the single-phase series motor this neutralizing winding may be connected in series with the armature (forced compensation), or it may be short-circuited by connecting the terminals $t$ and $t^{\prime}$, Fig. 265, together (transformer compensation).

Figures 266 and 267 show the field member of a four-pole single-phase series motor of the Westinghouse Electric and

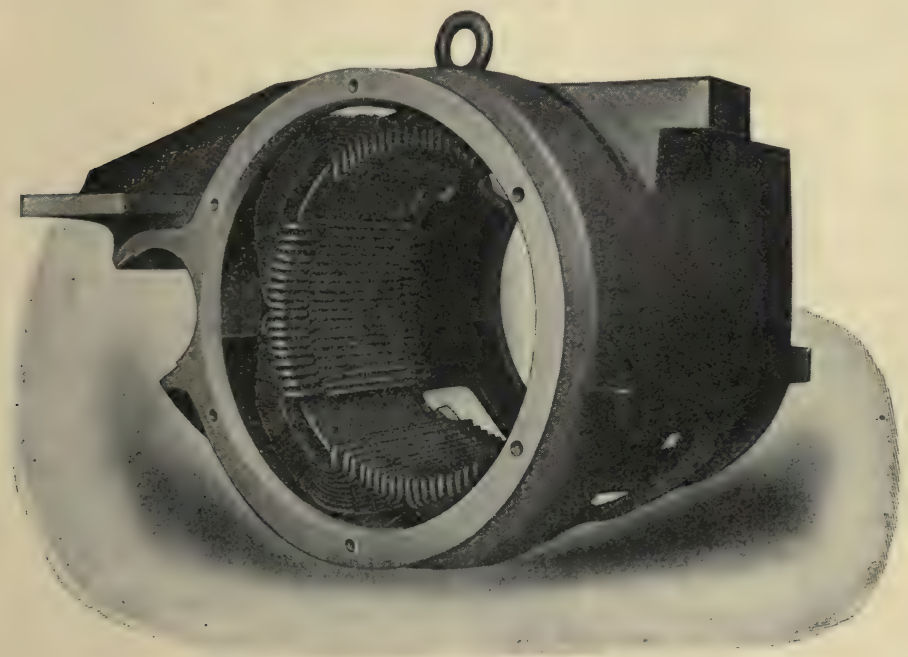

Fig. 267.

Manufacturing Company. The field windings and the neutralizing windings are both shown in position in Fig. 266, and in Fig. 267 the field coils are removed so as to expose the neutralizing windings to view. The neutralizing windings are placed in slots in the laminated pole pieces, and the portions $a a a a$ and $b b b b$, Fig. 265, of the neutralized windings are bent back in Figs. 266 and 267 to make room for the ends of the field coils.

143. Prevention of sparking in the single-phase series motor.A number of undesirable effects are included under the general term of sparking, as follows: (a) The effects that are presented 
in the direct-current dynamo* and which are due to the necessity of a sudden reversal of the current in a given armature section as the terminal bars (commutator bars) of the section pass under a brush; and $(b)$ the effects of the excessive current produced in each short-circuited section by the electromotive force which is caused by the pulsations of the field flux. The effects $(a)$ need not be considered because they are less serious than effects (b), and because anything which obviates the great difficulties due to effects (b) will likewise obviate the lesser difficulties due to effects $(a)$.

The effects of excessive short-circuit currents due to pulsations of the field flux are, to heat the armature winding, to heat the commutator, to heat the brushes, and to roughen the commutator by sparking, and these short-circuit currents represent a considerable loss of power. The cure for these undesirable effects is, of course, to prevent excessive short-circuit currents and to shorten their duration as much as possible.

The electromotive force induced in a short-circuited armature section by the pulsating field flux is proportional to the number of turns of wire in the section, proportional to the maximum value reached by the pulsating field flux, and proportional to the frequency of the pulsating flux. Therefore the short-circuited current may be reduced by reducing the number of turns of wire per armature section (the resistance of the short circuit not being reduced in proportion to the turns because of the relatively large resistance at the brush contacts), by reducing the maximum value of the pulsating flux, and by reducing the frequency. It is essential, therefore, in the design of the single-phase series motor to provide for few turns of wire per armature section (many commutator bars), to provide for small field flux per pole (many field poles), and to use alternating current of low frequency.

The duration of a short circuit of an armature section by a brush is proportional to the thickness of the brush, and therefore it is desirable to use thin brushes. If the armature winding is of the

* See pages $16 r$ to 164 of the first volume of this text. 
simplex type, the necessary thickness of the brushes is determined solely by the necessary area of brush contact to collect the given current. If the armature winding is of the multiplex type, the brush must never touch fewer than $n$ commutator bars, where $n$ is the number of constituent windings in the multiplex winding. In the multiplex winding, however, adjacent commutator bars do not form terminals of an armature section, and therefore the touching of two bars by a brush does not short-circuit the armature section of a multiplex winding; in fact the brush must touch $n+\mathrm{I}$ commutator bars of an $n$-plex winding to short-circuit an armature section.* Therefore thick brushes may be used with multiplex armature windings.

Special devices for preventing sparking. - The foregoing discussion refers to those general features of design which tend to reduce short-circuit currents in the armature sections of singlephase series motors. It does not seem to be possible, however, to produce a single-phase series motor which will operate satisfactorily without special devices for preventing excessive shortcircuit currents. The simplest device for this purpose is the insertion of a moderate amount of resistance in each commutator lead, and a number of special inductive devices have been proposed, of which, perhaps, the simplest is the balanced choke-coil arrangement of Mr. S. S. Seyfert.

The resistance leads. - The coils $b c d e f$, Fig. 268 , represent the sections of an armature winding, and rrrr represent resistances inserted in the commutator leads. The figure shows the armature section $d$ short-circuited by a brush, and the heavy arrow represents the electromotive force induced in the section $d$ by the pulsating field flux. The short-circuit current must flow

* Short circuits are produced in a multiplex winding by the simultaneous connecting of two of the constituent windings by two brushes. Some of these short circuits pass through one constituent winding from a positive to a negative brush and back to the positive brush through another constituent winding; some of them pass through one constituent winding from one positive (or negative) brush set to another positive (or negative) brush set and back through another constituent winding, and some of them pass through the winding from one positive (or negative) brush set to another positive (or negative) brush set and back through the brush leads. 
through two of the resistances $r$ in series, as shown by the short arrows, whereas the useful current which enters or leaves the armature through the brush must flow through two of the resistances $r$ in parallel. When the brush touches but one commutator bar there is no short circuit, and the whole of the useful current must flow through one of the resistances $r$. The resistances $r$ are entirely idle except

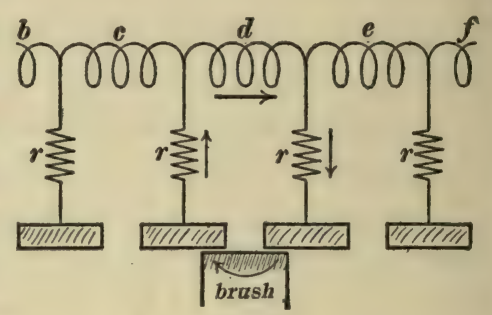

Fig. 268. at the moments when the bars to which they are connected pass under a brush.

Resistance leads are objectionable because they involve a loss of power, and because of the difficulty of finding room for them in a compact armature structure. While a motor is in actual operation the resistance leads come into service in rapid succession, and the heat is distributed among all of the leads; at starting, however, there is a possibility of the motor being stuck fast, so that a given resistance lead may be in circuit for an indef-

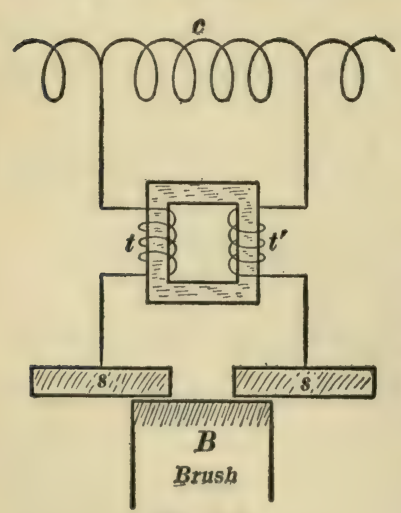

Fig. 269. inite time. In view of this possibility it is necessary to make the resistance leads of large current-carrying capacity, and to place the leads so that they can be easily repaired in case of their being burned out. The resistance leads are usually made of heavy strips of German silver thoroughly insulated and placed in the bottoms of the armature slots.

Balanced choke coils. - The essential features of the balanced choke coil arrangement for preventing sparking may be seen in Fig. 269 , in which $c$ is a section of the armature winding, ss are two commutator bars and $t t^{\prime}$ are two choke coils through which 
the commutator leads pass. The two coils $t t^{\prime}$ are wound on a small iron core, and they are so connected that their magnetizing actions on the iron core are equal and opposite when equal currents flow into (or out of) the armature winding through both coils; whereas their magnetizing actions work together when current tends to flow out of the armature winding through one coil and into the armature winding through the other coil. The consequence of this arrangement is that current can flow into or out of the armature through both of the coils $t t^{\prime}$ without being choked, whereas opposite currents in the coils $t$ and $t^{\prime}$ (the short-circuit current in the section) are greatly choked.

The complete arrangement of the balanced choke coils is shown in Fig. 270; each commutator lead is split into two

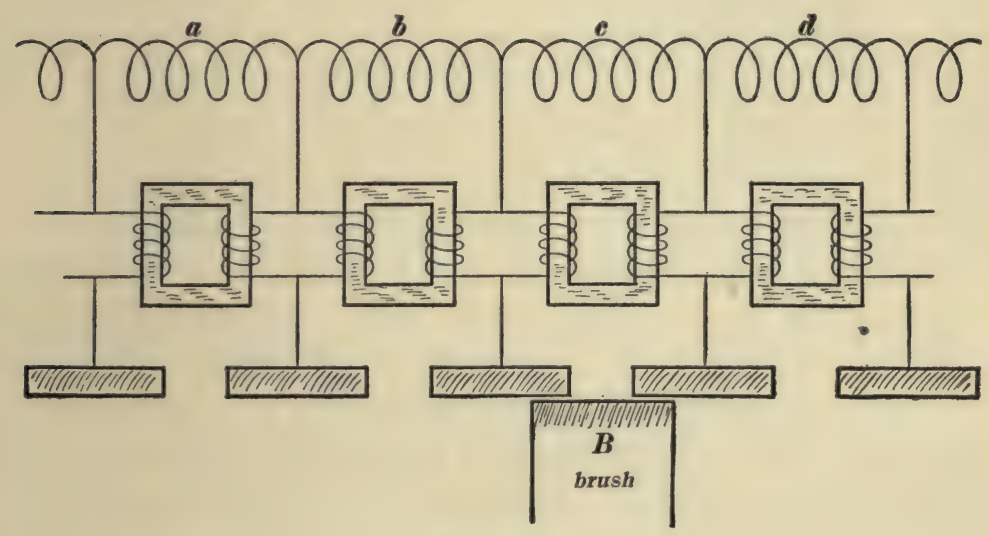

Fig. 270.

branches, and each branch contains a choke coil which is balanced against a choke coil in an adjacent commutator lead. With this arrangement a brush must never touch less than two commutator bars, because of the excessive choking of useful current when it has to enter the armature through the two choke coils in one lead; but if the brush never touches less than two bars, it must at certain instants touch three bars, and when this occurs, a short circuit is established which is unchoked except 
by the combined $*$ ohmic resistance of four of the choke coils in series.

144. Characteristics of the single-phase series motor. - The single-phase series motor is very much like the direct-current series motor in its speed and torque characteristics. The chief difference between the direct-current motor and the single-phase motor grows out of the fact that an alternating voltage of a given value gives less current than a direct voltage of the same value. $\dagger$

Advantages of single-phase series motor for railway service. The operation of a railway by electric motors involves two distinct problems, namely $(a)$ the problem of transmitting power from a central station to the motors and $(b)$ the problem of converting the electrical power into mechanical power. The first problem is a problem in long distance transmission when the railway exceeds a few miles in length and high voltage must be used. The second problem seems to admit of no other practical solution than by the use of the series motor which develops a large starting torque and operates at a fairly high efficiency throughout a wide range of speed. The series motor, however, is essentially a low voltage machine, and indeed the single-phase series motor cannot operate at as high a voltage as the directcurrent series motor on account of the greater tendency of the single-phase machine to spark. Therefore the operation of a railway by electric power requires high-voltage transmission and step-down transformation, and the choice may be said to lie between $(a)$ the series motor supplied with direct current at a voltage not exceeding about I,Ooo volts, and $(b)$ a series motor supplied with single-phase alternating current at a voltage not exceeding about 500 volts. If the direct-current motor is used, synchronous-converter sub-stations have to be placed a few miles apart all along the line of railway, and not only is the total cost

* This is due to transformer action as may be readily seen by tracing the shortcircuit current in two armature sections.

† A brief description of the performance diagram (clock diagram) of the singlephase series motor is given by V. Karapetoff, Trans. Am. Inst. Elec. Engineers, Vol. XXXIII, page 78,1904 . 
of the electrical equipment high, but the synchronous converters must operate a large portion of the time at light load so that the power losses must be great and the efficiency low. If the singlephase series motor is used, the trolley wire can itself be the high voltage transmission line, and a step-down transformer on the car or locomotive can deliver current at any desired voltage to the motors.

The most serious difficulty involved in the use of the singlephase series motor is its large size as compared with a directcurrent motor of the same power rating. Thus, the electric locomotives of the New York Central Railway Company have direct-current motors of sufficient capacity to slip the wheels, whereas the electric locomotives of the New York, New Haven and Hartford Railway Company, which are about the same weight as the New York Central locomotives and which are equipped with single-phase series motors, can exert a tractive effort not to exceed 50 per cent. of that which would correspond to slipping of wheels.

The series motor requires a very low voltage at starting, and the voltage across the motor terminals must be increased as the speed increases. When direct current is used, this variation of motor voltage can be accomplished only by the use of rheostat control, as explained in Art. 6I of the first volume of this text, but when alternating current is used, a number of taps can be brought out from the secondary coil of the step-down transformer so that the low voltage required at starting can be taken directly from a few secondary turns, and the voltage can be increased at will by bringing a larger and larger number of secondary turns into action as the speed increases. Rheostat control is therefore not necessary when alternating current is used and the loss of power in rheostats is obviated. Furthermore, the series-parallel system of direct-current motor control, the object of which is to reduce the range of rheostat control of voltage, is unnecessary when alternating current is used.

145. The repulsion motor. - The repulsion motor consists of a 
primary or stator member which is identical to the stator of the single-phase induction motor, and a secondary or rotor member which is like an ordinary direct-current armature with a commutator and brushes. The primary member is supplied with singlephase alternating current, and the secondary member is shortcircuited by connecting its brushes directly together. The essential features of the repulsion motor are shown in Fig. 27 I, which represents a two-pole model. The stator winding produces through the armature a pulsating flux in the direction of the arrow $\Phi$, and, in order to describe the action of the motor, this flux may be resolved into two component parts, one of which, $T$, is parallel to the line of the brushes $b b$, and the other of which, $F$, is perpendicular to $b b$. The stator winding may also be considered in two parts, namely, the part $t$ which produces the flux $T$ and the part $f$ which produces the flux $F$. It is

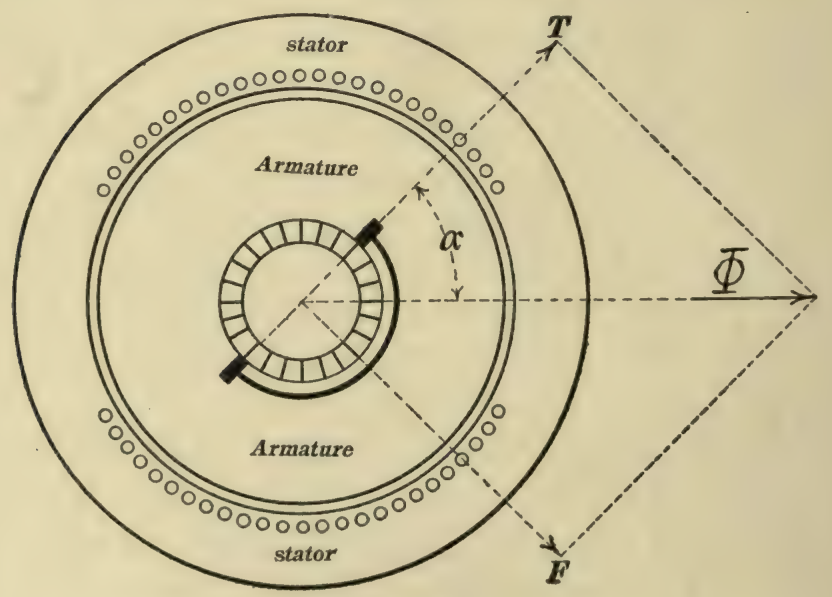

Fig. 271.

impossible to actually separate the stator winding shown in Fig. $27 \mathrm{I}$ into the two parts $t$ and $f$, because certain portions of the stator winding help to produce both $T$ and $F$. Figure 272, however, shows a more or less fanciful arrangement in which there are two separate stator windings $t$ and $f$ with their axes at right angles to each other. 
The repulsion motor may be shown to be fundamentally equivalent to the simple series motor in its action and in its torque-speed characteristics as follows: The stator winding $t$, Fig. 272, produces the pulsating flux $T$ and acts like the primary of a transformer of which the secondary is the short-circuited armature winding. Let us assume that this transformer action is ideal (zero reluctance for flux $T$ ) and let us assume that the ratio of transformation is $\mathrm{I}: \mathrm{I}$. Then the armature current is identical

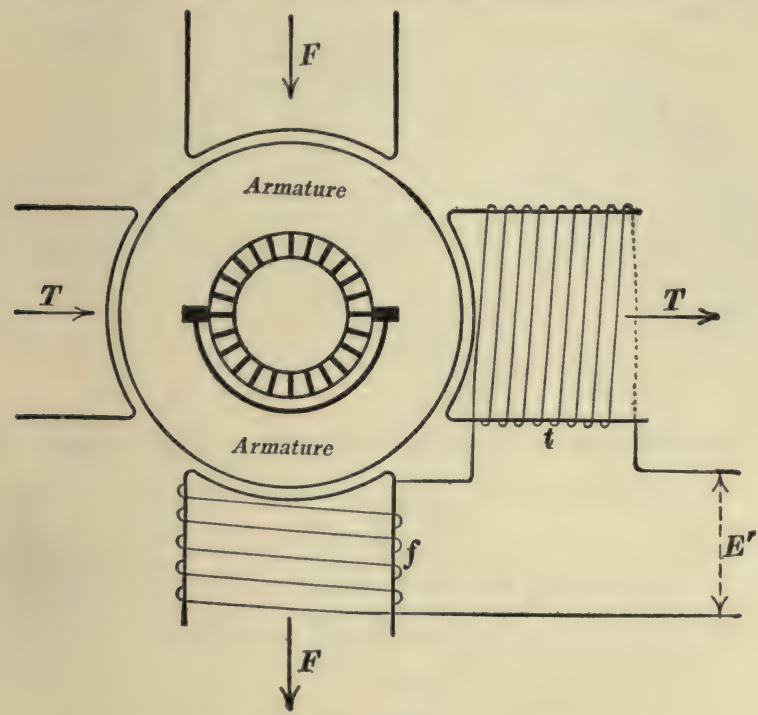

Fig. 272.

in value and in phase * to the current in the coil $t$ just as if the armature were included in the primary circuit.

The stator winding $f$ produces the pulsating flux $F$. The armature is an open circuit with respect to the electromotive forces induced by the pulsations of this flux, but the electromotive forces produced by the cutting of $F$ by the rotating armature act through or around the short-circuit formed by the brushes and these electromotive forces are reproduced in the

* The secondary current of a transformer is usually thought of as exactly opposite in phase to the primary current, but whether it be considered as opposite to or in phase with the primary current depends on a mere convention as to the choice of the direction in the secondary coil which is to be considered as the positive direction. 
stator winding $t$ by transformer action just as if the armature itself were included in the primary circuit.

The simple series motor is different from the repulsion motor in that the transformer flux $T$ does not exist in the simple series motor, and this transformer flux has an interesting effect on the rotating armature conductors as follows: The armature sections which are short-circuited under the brushes have electromotive forces induced in them by the pulsation of the field flux $F$ exactly as in the simple series motor, but these short-circuited sections are cutting the transformer flux $T$ at the time of their short circuit, and the electromotive force due to this cutting is opposed to the electromotive force produced by the pulsation of the field flux $F$. In fact, the electromotive forces due to cutting of the transformer flux $T$ are equal and opposite to the electromotive forces due to pulsation of the field flux $F$ at synchronous speed and therefore the repulsion motor has no great tendency to spark at synchronous speed.

146. Repulsion-motor-starting of the single-phase induction motor. - The most valuable feature of the repulsion motor is that it can be easily and quickly converted into a single-phase induction motor by bringing a metal ring into contact with all of its commutator bars, thus converting the armature into what is substantially a squirrel-cage rotor. The single-phase induction motor of the Wagner Electric Company, for example, is started and brought up to speed as a repulsion motor, and then converted into a single-phase induction motor.

A general view of a four-pole Wagner motor is shown in Fig. 273. The commutator is radial so that the brushes can be lifted off the commutator by a movement parallel to the armature shaft. At starting the brushes (which are connected by short heavy conductors) rest against the commutator and short-circuit the armature as above explained, and the machine acts as a repulsion motor. When a certain speed is reached the weights $V$, Fig. 274 , are thrown outwards by centrifugal action, and the connecting rods $U$ push the sleeve $I$ along the shaft, bringing the 
copper ring $K$ into contact with the inner ends of the commutator segments and lifting the brushes off the commutator. The machine then operates as a single-phase induction motor. The

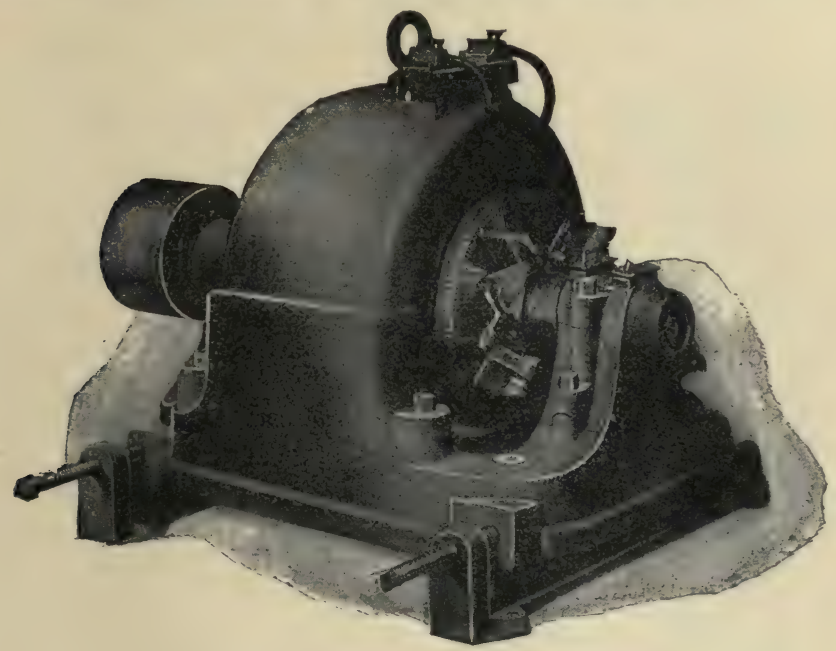

Fig. 273.

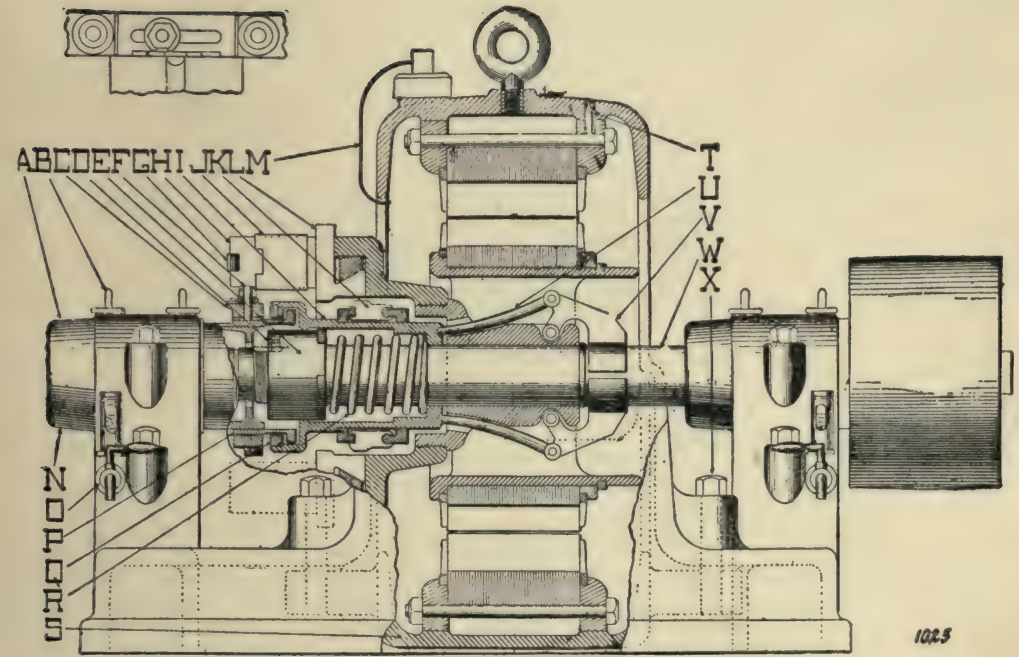

Fig. 274.

lifting of the brushes off the commutator is not necessary but desirable in order to reduce friction and wear. 


\section{CHAPTER XV.}

\section{SWITCHBOARDS AND SWITCHBOARD APPLIANCES.}

147. Recapitulation. - The preceding chapters are devoted chiefly to the discussion of individual machines such as the alternator, the transformer, the induction motor, and the rotary converter, and but little has been said of the combinations of machines into complete systems for the generation, transmission and utilization of power. This chapter refers to the general arrangement of apparatus in generating and receiving stations and Chapter XVI refers to the arrangement of transmission lines.

Most of the details of arrangement in generating and receiving stations refer to the accessory apparatus which must be used for controlling and operating the various machines. Thus, a group of alternating-current generators operating in parallel require the following accessory apparatus: One or more exciters with their field rheostats and switches, field regulators such as the Tirrill regulator which is briefly described on page 14 and more fully described in Art. I 55, ammeters, voltmeters, potential and current transformers (see pages 46 and 234), switches for opening and closing the main circuits of the generators, and synchronizing devices (see page 155 and also Art. I 5 I). Quick-acting circuit-breakers are preferably inserted in the various feeder circuits, because a quick-acting circuit-breaker in the main circuit of a generator throws the entire system out of operation in case of a short circuit.

A set of feeders, single-phase or polyphase, leading out from a generating station to a certain locality is generally provided with the following accessory apparatus in the generating station: switches, circuit breakers or fuses, ammeters, ground detectors, and lightning arresters.* A voltage regulator (and voltmeter) is

* See pages 210-220 of the first volume of this text. 
sometimes installed in connection with a set of feeders as explained in Art. I 50.

A synchronous motor is generally provided with switches and fuses, an ammeter, an exciter with its switches and rheostats, a synchronizer, and an arrangement for starting. In some cases, an auxiliary motor is used as a starter, and in other cases a synchronous motor is started by inherent induction motor action as explained on page $27 \mathrm{I}$, in which case a starting compensator is used as explained on page 267 .

A synchronous converter is generally provided with the same accessory apparatus as a synchronous motor with the addition of a complete direct-current switchboard equipment. *

A small induction motor is usually provided with a switch and fuses. Large induction motors, however, are usually provided with switches and fuses, ammeters, and starting compensators, as described on page 267 .

Wattmeters are frequently installed in connection with alternating-current generators in order that the station attendant may have a knowledge of the relative power outputs of the several generators. Sometimes watt-hour meters are installed in a generating station to record the total output of energy, and watt-hour meters are frequently installed in connection with synchronous motors, synchronous converters, and induction motors.

148. Switchboards for generating stations. - An alternatingcurrent generating station is usually equipped with a number of alternators, and one or more direct-current exciters; and such a station generally delivers current to a number of feeder circuits. Each alternating-current generator, each direct-current exciter, and each pair of feeders must be provided with accessory devices, as specified above, and it is the usual practice to group all of the accessory devices belonging to one machine, or to one pair of feeders, on one switchboard panel. Thus, we have what are called generator panels, exciter panels, and feeder panels.

When a station is equipped with several alternating-current

* See pages $184-199$ of the first volume of this text. 
generators which are to be operated in parallel, the main conductors, to which the generators are to be connected, run back of all the switchboard panels and these main conductors are called the main bus-bars.

An auxiliary set of bus-bars, called the synchronizing buses, connects all of the generator panels, and is so arranged that a single synchronizing device may be used when any one of the generators is to be put into operation and connected in parallel with the others.

Whenever it is desirable to have a ground connection at each switchboard, a single wire is installed back of all the switchboards and connected to ground. This wire is called the ground bus.

Generator Panels. - Figure 275 shows the complete connections of an alternating-current three-phase generator panel (A. T. G. panel) including the connections of the exciter. The generator is represented as a revolving field machine, the exciting current being delivered to the field windings through collector rings. The field switch is provided with what is called a discharge resistance to prevent excessive sparking and possible damage to the insulation of the field windings when the field circuit is opened. A rheostat is shown in the field circuit of the generator, and another rheostat is shown in the field circuit of the exciter. The three armature terminals of the three-phase generator are directly connected through the three ammeters and through a three-phase single-throw oil switch to the main buses.* Two "potential transformers" are shown connected to the three-phase mains (the use of two transformers for three-phase transformation is explained on page 222), and the low voltage coils of the potential transformers are connected to what is called the "potential receptacle" and the "synchronizing receptacle," as indicated. The potential receptacle is a form of plug switch into which the "potential plug" may be inserted to connect the voltmeter so as to

* In some cases, two separate sets of main buses are installed, and each of the main switches is double-throw so that any generator may be connected to either set of buses. 
measure the voltage of any one of the three phases, as may be seen by tracing the connections from the potential receptacle and potential plug to the voltmeter in Fig. 275. The synchronizing
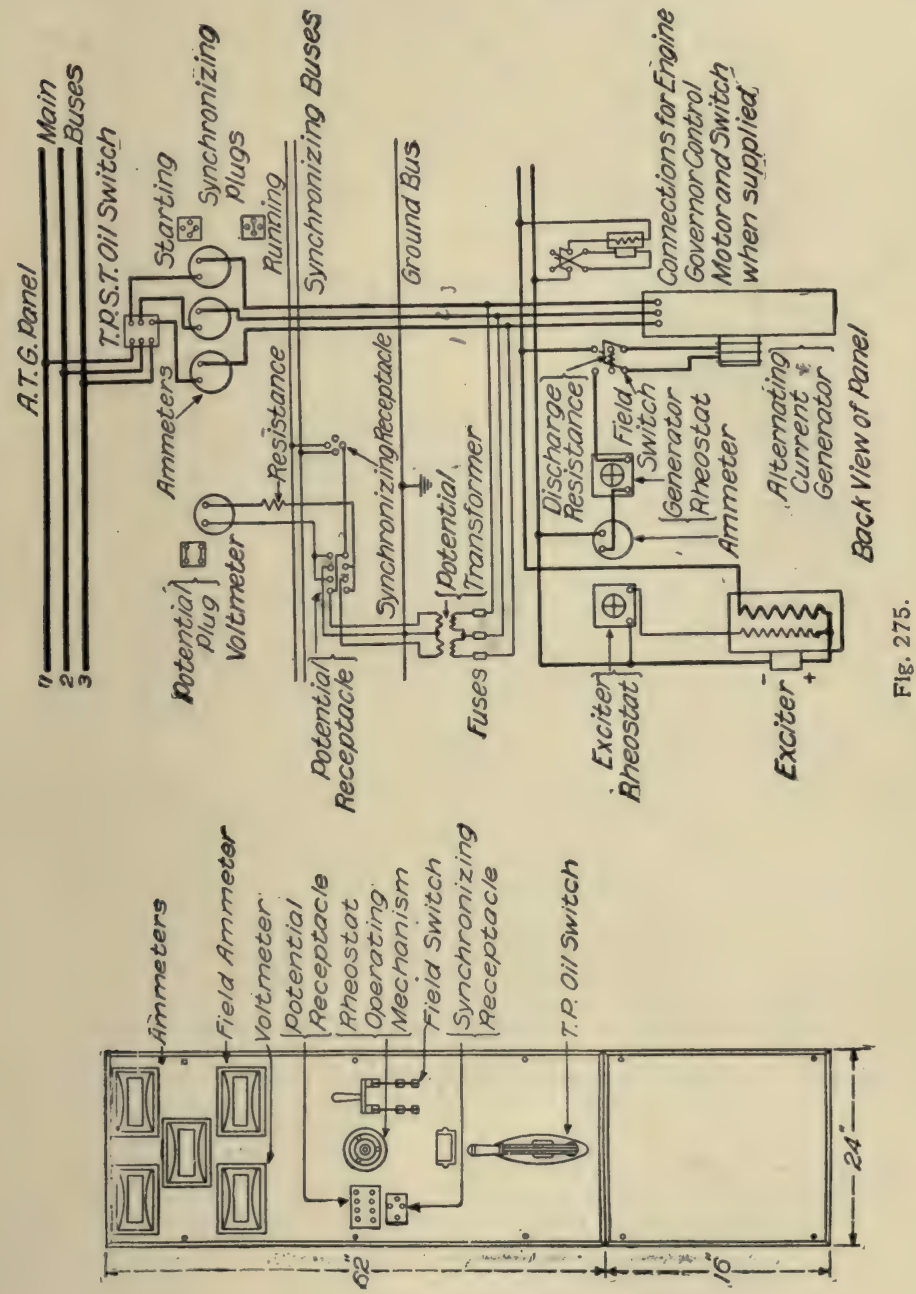

receptacle is a plug switch into which the two forms of "synchronizing plug" may be inserted, namely, the "starting plug" and the "running plug." 
Other generators being in operation and connected to the main buses, the generator which belongs to the panel shown in Fig. 275 is started as follows: The "starting plug" is inserted into the synchronizing receptacle ("running plugs" being in the synchronizing receptacles of all the machines which are already in operation), and the exciter is started and brought up to full speed. The new generator is then started and brought up to approximately full speed, its field rheostat is adjusted until the voltmeter shows that its voltage is approximately the same as the voltage across the main bus-bars, then the speed of the driving engine is carefully adjusted until the synchronizing device (either synchronizing lamps or a dial synchronizer) shows that the two necessary conditions which are explained on page I 55 are satisfied, and then the three-phase single-throw oil switch is closed so as to connect the alternating-current generator to the bus-bars. The generator is then in full operation and the "running plug" is put in place of the "starting plug" in the synchronizing receptacle.

In the case of direct-current generators operating in parallel, the ammeters indicate the relative loads on the two machines. In the case of alternating-current generators operating in parallel, however, the ammeters on two machines may give the same indication and still the power output of one machine may be much greater than that of the other. It is desirable, therefore, to install a wattmeter on each generator panel so that the approximate* power output of each generator may be indicated, and the field rheostats of the generators adjusted to make them share the station load equally.

In many cases the ammeters for indicating the alternating-current delivered by each phase of an alternating-current generator are connected through current transformers. In Fig. 275, however, the ammeters are shown inserted directly in the generator leads.

* A single wattmeter indicates the approximate value of the power delivered by a polyphase generator when the system is approximately balanced, as explained on page II 2 . 
It is not desirable to use automatic switches (circuit breakers) on alternating-current generator panels. Thus, the three-phase single-throw oil switch shown in Fig. 275 has no trip coil and it does not automatically disconnect the machine in case of overload or short-circuit.
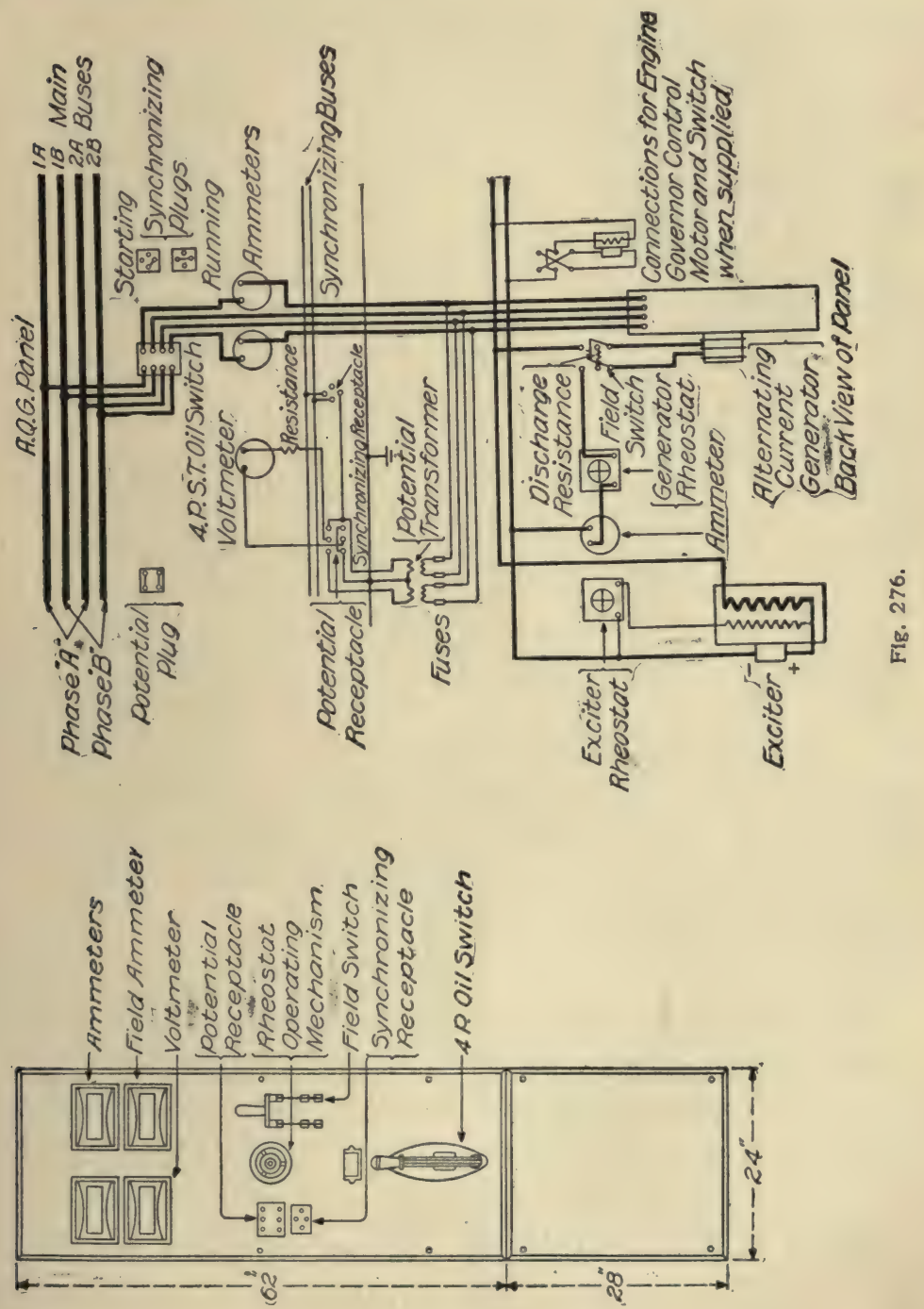
Figure 276 shows a quarter-phase* alternating-current generator panel (A.Q.G. panel) including exciter connections.

Feeder panels. - Single-phase circuits are frequently supplied from three-phase generating stations. When this is done, provision is usually made for connecting the single-phase feeder to one or another pair of main bus-bars at will. Thus, Fig. 277
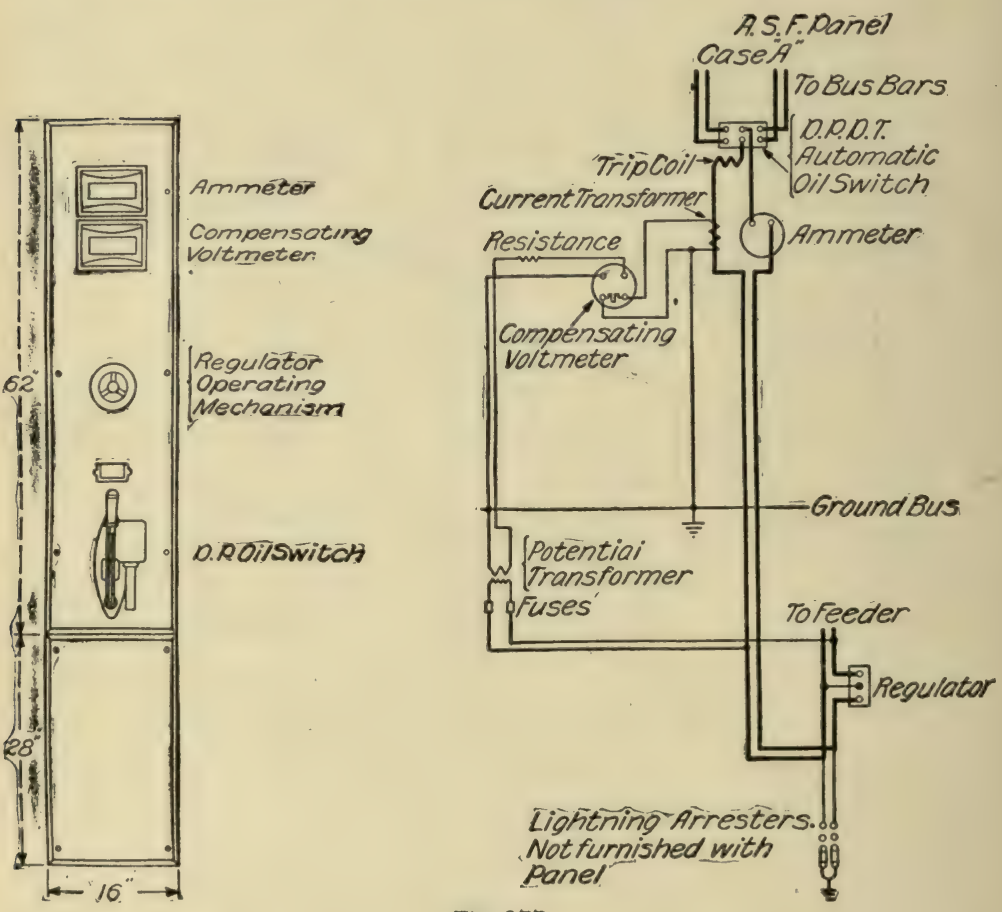

Fig. 277.

shows the front view of an alternating-current single-phase feeder panel (A.S.F. panel), and the connections on the back of the same. The voltmeter is represented as being compensated as explained in Art. I 52 ; the main switch is a double-pole doublethrow automatic oil switch arranged to connect the pair of feeders to one or another pair of buses; and the connections of a voltage

* The term quarter-phase is frequently used to designate a two-phase system. 
regulator (see Art. I 50) are indicated, the regulator being operated by the hand wheel shown on the front of the switchboard.

An alternating-current three-phase feeder panel (A.T.F. panel) and its diagram of connections are shown in Fig. 278. In this
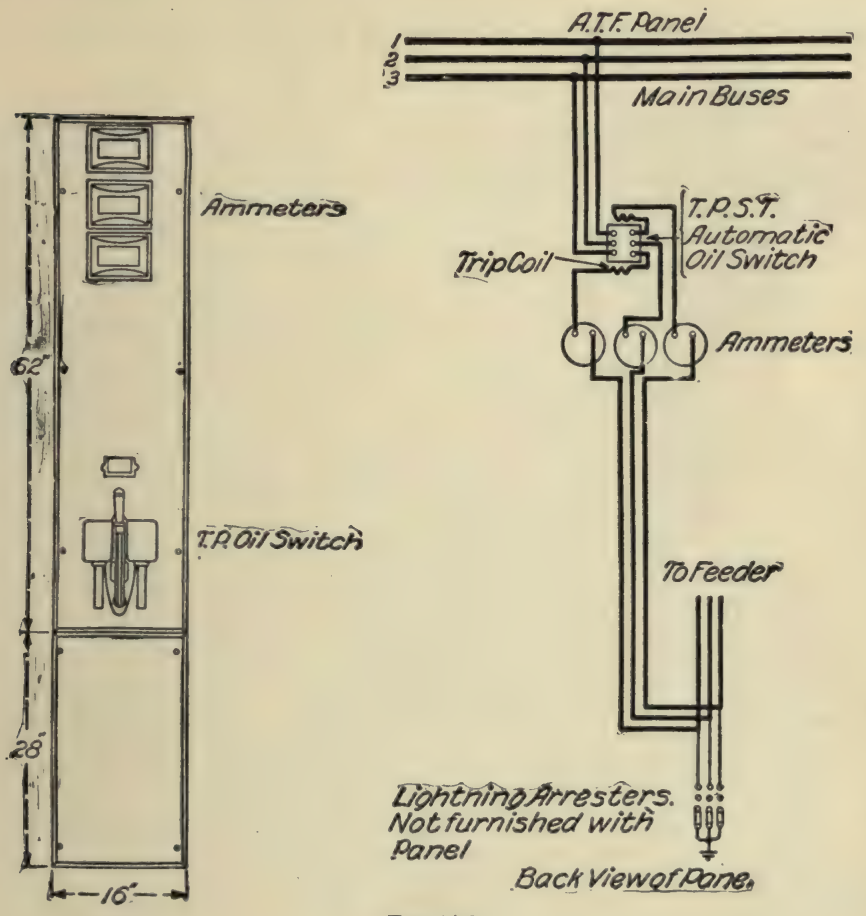

Fig. 278.

case, the main switch is a three-phase single-throw automatic oil switch so arranged that an excess of current in either one of two of the feeder wires may trip the switch.

Figure 279 is a front view of a three-phase switchboard consisting of two generator panels, and two three-phase feeder panels. A synchronism indicator of the dial form is shown mounted on a bracket at one side of the board. In this switchboard the exciter accessories (rheostat and field switch) are mounted on the generator panels. 
Complete wiring diagram of a large alternating-current generating station. - In very large alternating-current generating stations a synchronous motor or an induction motor is sometimes installed in preference to a small steam engine for driving auxiliary apparatus, and if direct current is needed for local service it is usually supplied by a rotary converter which is driven by the main alter-

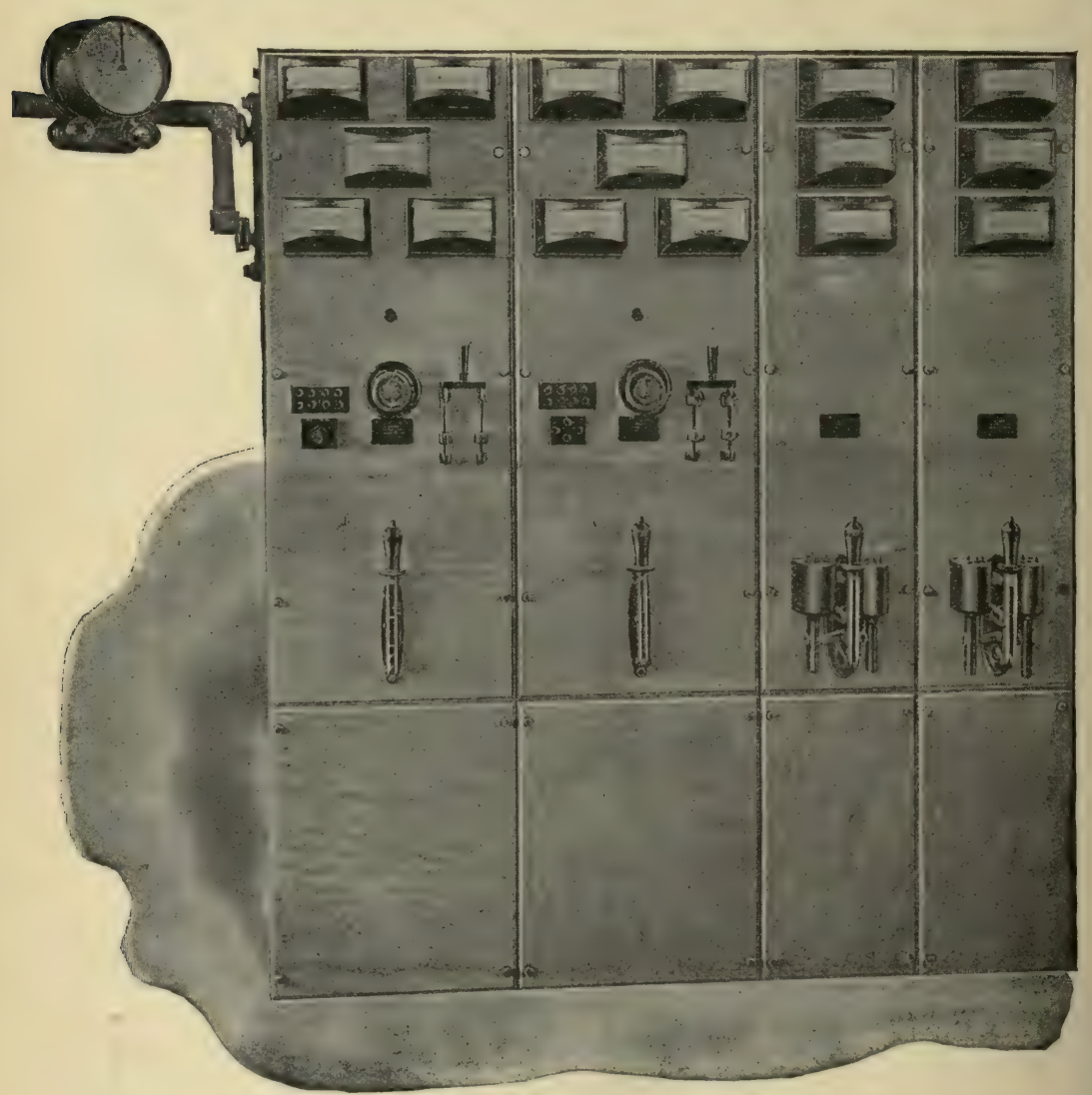

Fig. 279.

nating-current generators. Thus Fig. 280 is the complete wiring diagram of a power station consisting of a three-phase, alternatingcurrent, steam-turbine driven, 2,300-volt, 1,000-kilowatt generator 
SWITCHBOARDS AND SWITCHBOARD APPLIANCES. 329

(A.T.G. 2,300 V. I,000 K.W.), and a 50-kilowatt exciter driven by an alternating-current, three-phase, 2,300-volt, 75-horse-power induction motor (A.T.I. 2,300 V. 75 H.P.). The figure shows

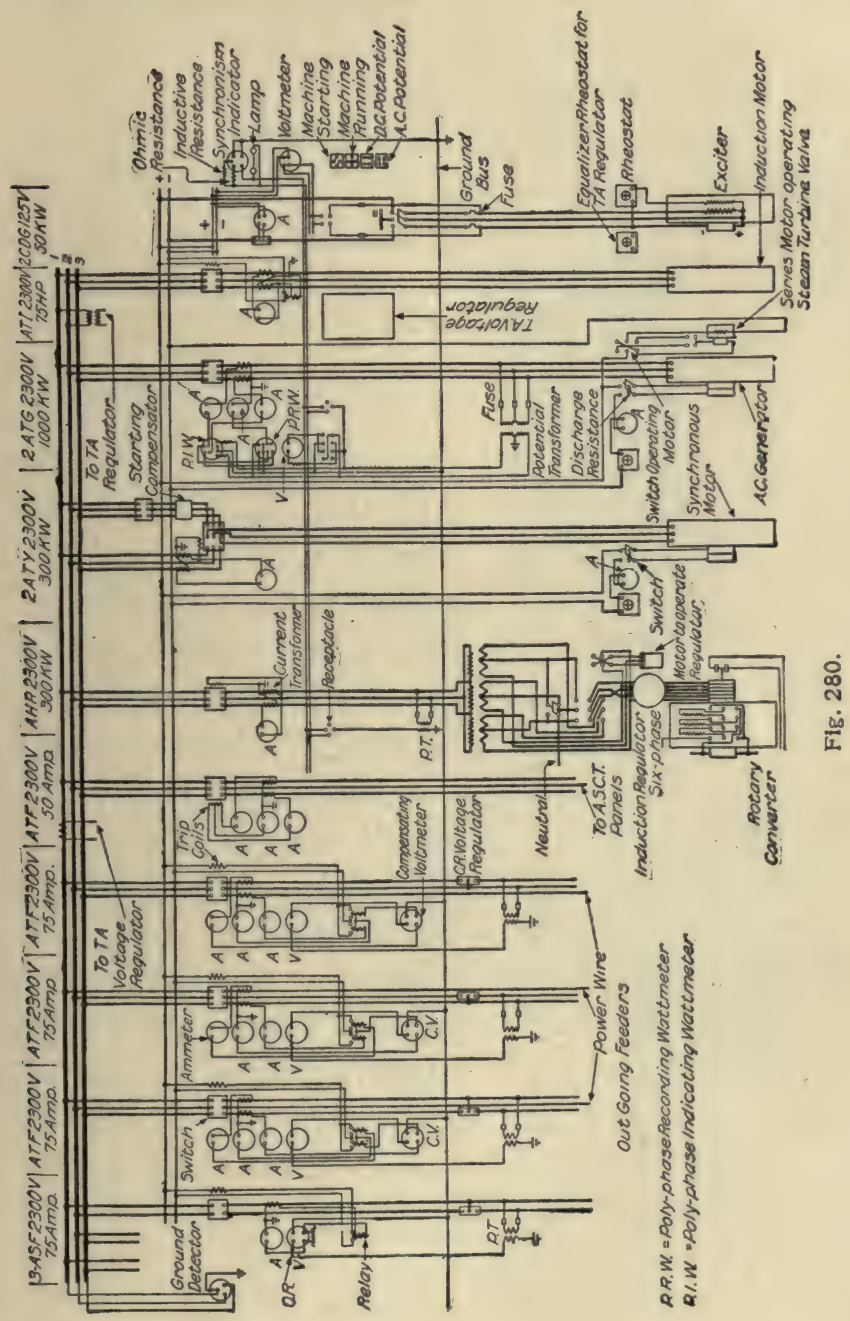

the complete connections of one alternating-current, single-phase feeder panel (A.S.F. 2,300 V. 75 Amp.), and of three alternating- 
current three-phase feeder panels (A.T.F. 2,300 V. 75 Amp.). The figure shows also the connections of a 300-kilowatt, threephase synchronous motor ( $2 A T Y 2,300 \mathrm{~V} .300 \mathrm{~K}$.W.), and the connections of a 300-kilowatt six-phase rotary converter (AHR 2,300 V. $300 \mathrm{~K} . \mathrm{W}$.) with its step-down transformer and synchronizing receptacle.

The "TA regulator" which is indicated in Fig. 280 is an accessory of the generator $(A T G 2,300 \mathrm{~V}$. I,000 K.W.). The

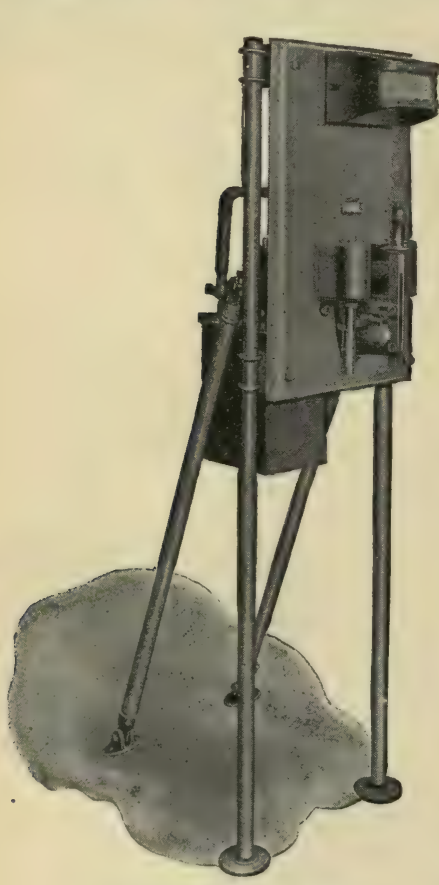

Induction Motor Panel. Fig. 281. connections of the " $T A$ regulator" are not fully shown in Fig. 280 but they are described in detail in Art. I 55. The oscillating armature of the voltage regulator short-circuits a resistance, as explained in Art. I 55. This resistance may be inserted permanently either in circuit with the field winding of the exciter or in circuit with the field winding of the alternating-current generator. This resistance is marked " equalizer rheostat for $T A$ regulator" in Fig. 280.

The device on each feeder panel marked "relay" in Fig. 280 serves to control the time which elapses between the instant of an overload or short-circuit on the pair of feeders and the instant of tripping of the circuit-breaker.

The essential features of the ground detector which is shown in Fig. 280 are described in Art. I 53. The dial synchronizer, or synchronism indicator, which is shown in Figs. 279 and 280, is described in Art. I $5 \mathrm{I}$.

149. Switchboards for receiving stations.

(a) Induction motor panels. - Small induction motors are generally provided with a 
switch for closing the circuit and a fuse for opening the circuit in case of excessive over-load or short-circuit, and these simple accessories are usually mounted on the wall conveniently near to the motor. Large induction motors are usually provided with an autotransformer for delivering current at reduced voltage at starting, as explained on page 267 , and it is usual to provide an automatic oil switch, or circuit-breaker, and an ammeter, and these accessories are usually mounted on a switchboard panel as shown in Fig. 28I. The diagram of connection for a three-phase induction motor and starting compensator is shown on page 268. Figure 282 shows front and side views of the panel shown in Fig. $28 \mathrm{I}$.

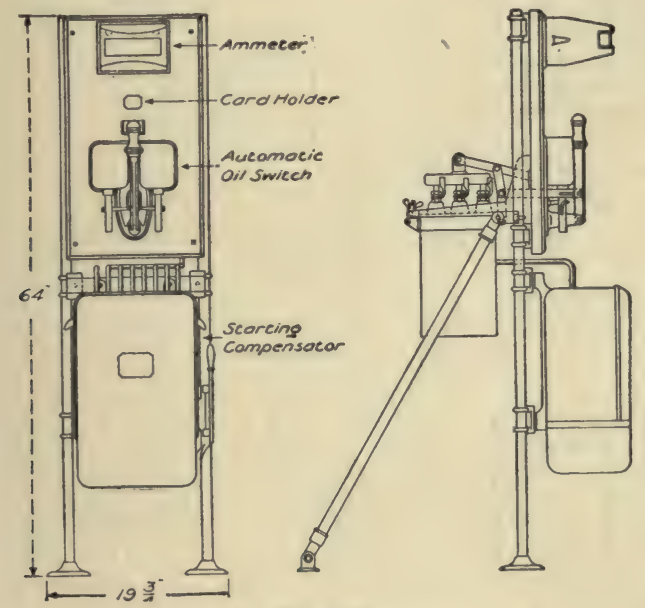

Fig. 282.

Synchronous converter panels. Figure 280 shows a six-phase synchronous converter (AHR 2,300 V $300 \mathrm{~K}$.W.) with an oil switch, current transformer and ammeter, synchronizing receptacle, potential transformer, and three step-down transformers which are delta-connected to the supply mains and which supply sixphase currents to the converter through an induction regulator. The regulator is operated by a small motor, and the field winding of the rotary converter is broken up into sections to avoid the 
accumulation of high voltage at starting, as explained on page 272 .

The essential connections of two three-phase rotary converters to the alternating-current supply and to the direct-current busbars are shown in Fig. 283, and one mode of connecting a sixphase converter through its step-down transformers to the alter-

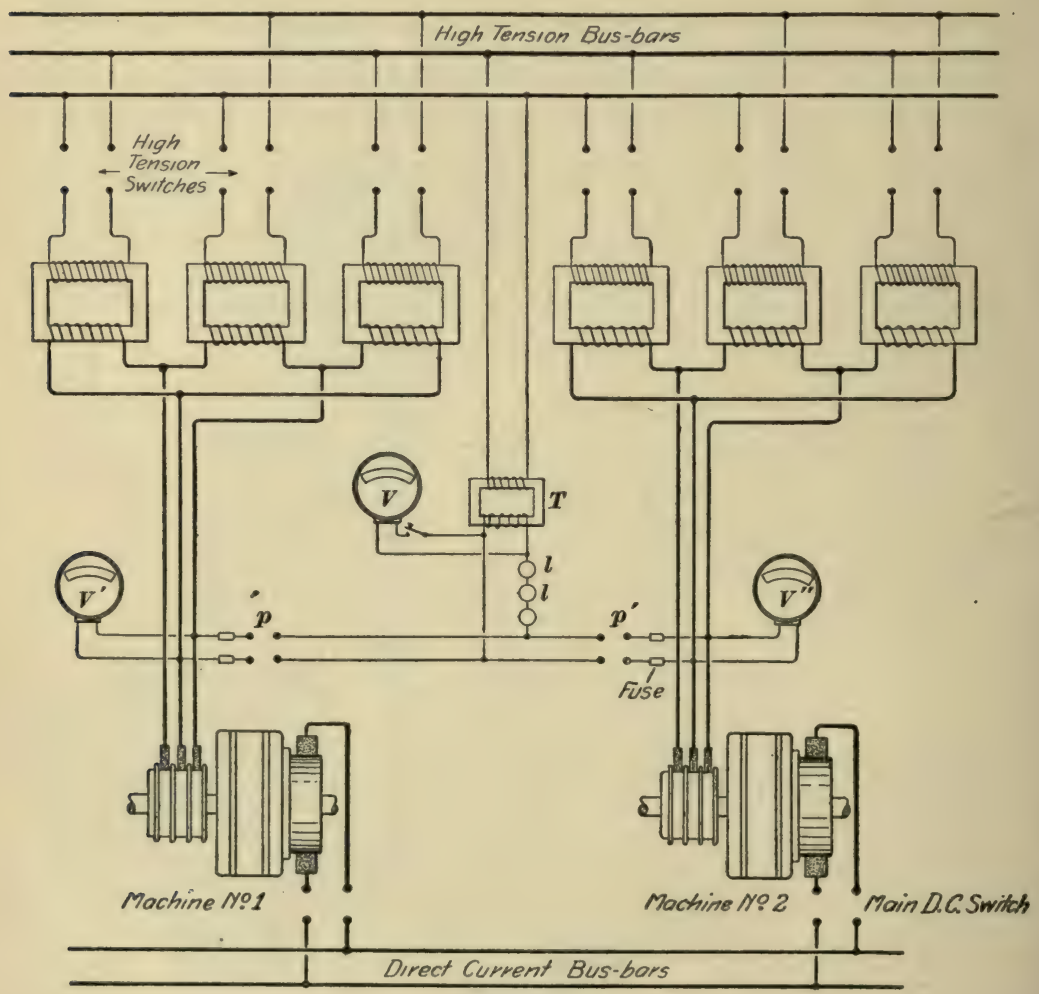

Fig. 283.

nating-current supply is shown in Fig. 284.* Figure 285 is a complete diagram of connections of a three-phase rotary converter arranged to deliver direct current to a street railway. No choke coils are shown in connection with the lightning arresters in Fig. 285. In fact, however, a choke coil is inserted in each of the

* See page 230. 
transmission lines between the point of attachment of the lightning arrester and the bus-bars in the station.

Synchronous motor panels. - A synchronous motor panel is essentially the same thing as the alternating-current panel of a synchronous converter, except that a synchronous motor panel usually includes the switches and rheostats of a small direct-cur-

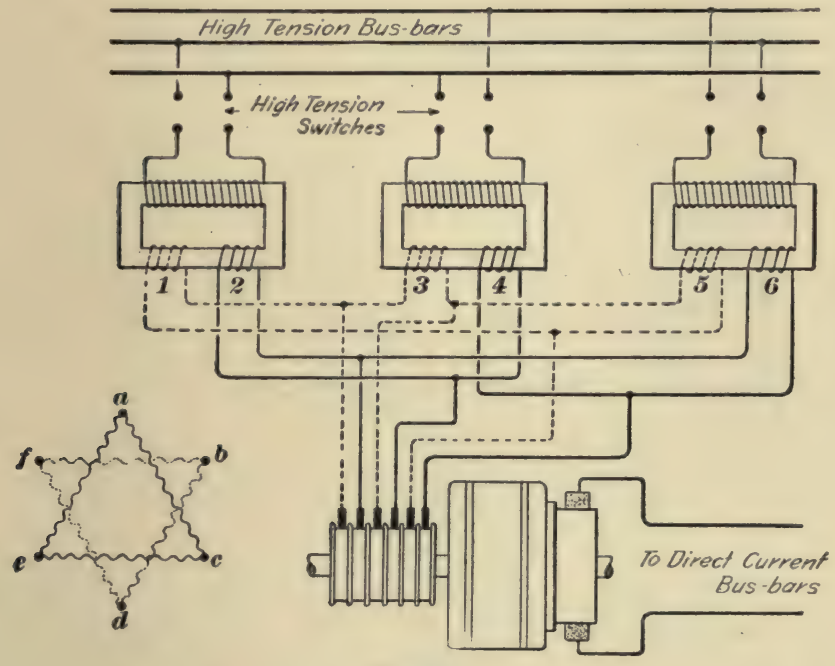

Fig. 284.

rent generator, the exciter. Thus the right-hand side of Fig. 285 shows the essential features of a three-phase synchronous motorpanel, except that the exciter accessories are absent. Figure 280 shows the connections of a three-phase synchronous motor ( $2 A T Y$ $2,300 \mathrm{~V} .300 \mathrm{~K}$.W.) with its field current supplied from the same exciter that supplies field current to the main generator. This synchronous motor is arranged to be started by inherent induction-motor action, as explained on page $27 \mathrm{I}$ and in this case no synchronizing device is used.

150. Voltage regulators. The simplest device for regulating the voltage of a pair of feeders in a generating station, or for regulating the voltage of alternating-current supply to a rotary 


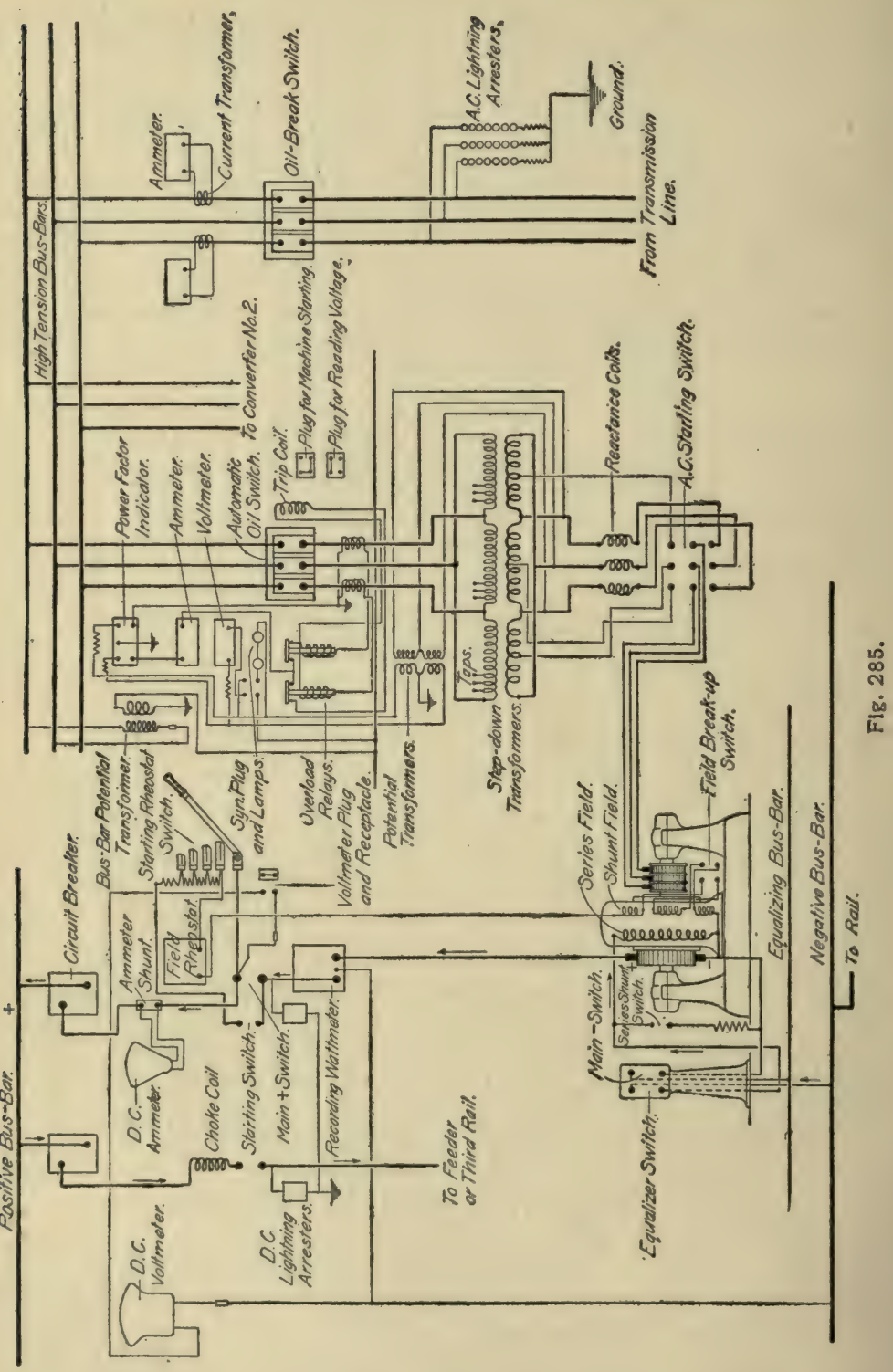

converter, is to bring out a number of taps from the primary or secondary coils of the step-up or step-down transformers and 
arrange a switch so that the number of turns of wire in the transformer coils may be altered at will, thus varying the ratio of transformation and changing the secondary voltage even though the primary voltage be constant. Thus, Fig. 285 shows three taps brought out from successive turns at one end of the primaries of each of the step-down transformers. This device for regulating the voltage delivered to a receiving circuit is usually called the dial regulator. The details of the dial regulator are shown in Fig. 286. The contact finger, which is shifted from one to

\section{supply main.}

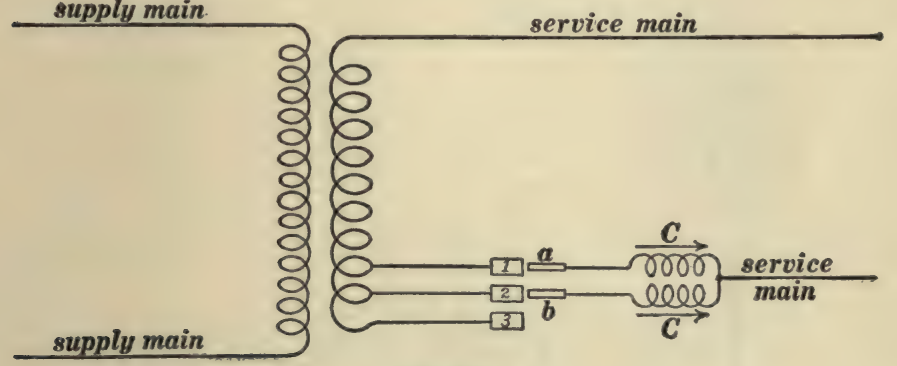

Fig. 286.

another of the contact points I, 2, and 3, consists of two pieces of metal $a$ and $b$ far enough apart to bridge across from one contact point to the next as shown. With this arrangement the circuit is never broken as the contact finger is shifted over the contact points, and the short-circuit of one turn of the transformer which takes place when the contact finger touches two contact points includes the two choke coils $C C$ which are so wound on an iron core that they permit current to flow outwards or inwards through both as indicated by the arrows, but do not permit it to flow outwards through one and inwards through the other.

Another form of voltage regulator is shown in section in Fig. 287. Imagine an iron carcase, similar to the carcase of an induction motor, to have a single-phase primary winding on its stator and a single-phase secondary winding on its rotor. Such a transformer could have its primary winding connected across the supply mains and its secondary winding connected in series 
with the circuit leading to the receiving circuit of which the voltage is to be regulated, and to turn the secondary member through half a revolution would be equivalent to reversing its connections so that the electromotive force generated in the secondary member could be added to or subtracted from the supply voltage by adjusting the position of the secondary member, thus making the receiver-circuit voltage greater or less than the supply voltage. This form of regulator is usually called the induction regulator.

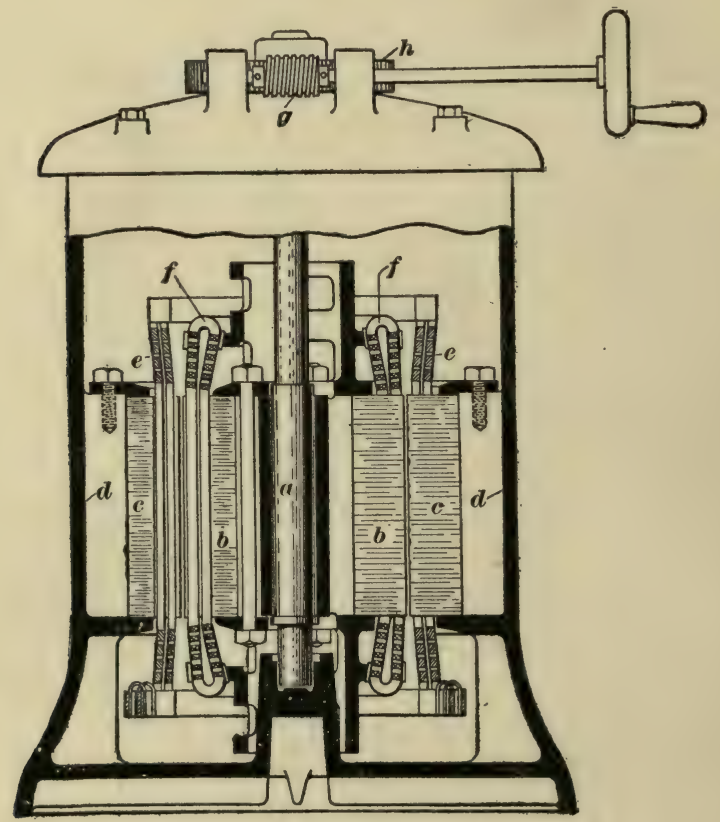

Fig. 287 .

It can be arranged to raise or lower the voltages of any number of phases simultaneously, by providing the primary and the secondary members both with polyphase windings. The secondary member in Fig. 287 is supported on the spindle $a$, and it may be turned into any position by means of the handwheel and worm-gear shown in the figure.

151. The dial synchronizer is a device for causing a pointer to 
rotate at a speed which is equal to the difference in frequency of two sources of alternating current. Figure 288 is an external view and Fig. 289 shows the essential features of the dial synchronizer of Mr. Paul M. Lincoln. Suppose that an alternator $A$ is to be started up, brought into synchronism with alternators

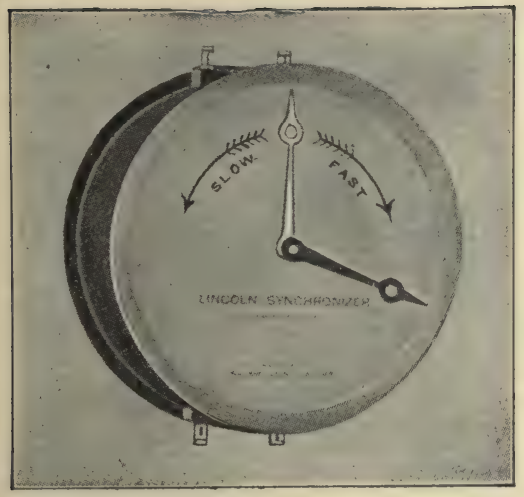

Fig. 288.

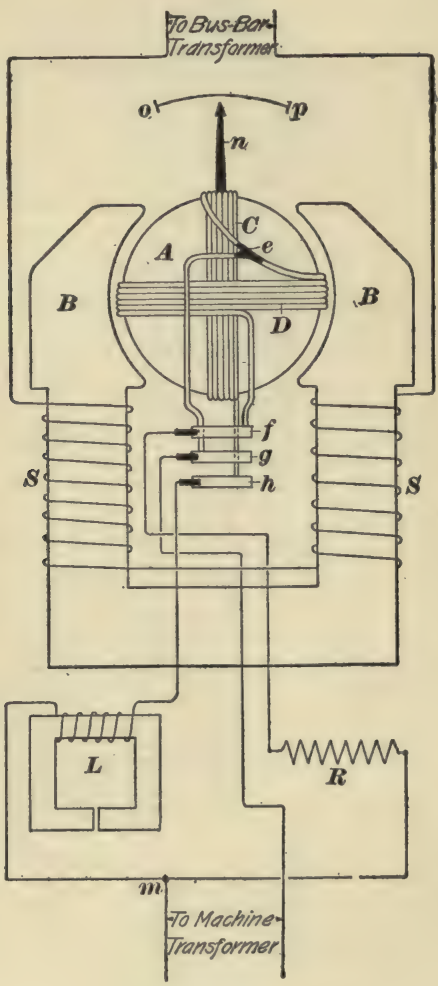

Fig. 289.

already in operation, and connected to the bus-bars in parallel with the other alternators. The synchronizer being connected as explained below, its pointer does little more than vibrate at first; but, when the frequency $a$ of the alternator $A$ is nearly as great as the bus-bar frequency $b$, the pointer begins to rotate at a speed equal to $b-a$, becomes stationary when $a=b$, and 
starts to rotate in a reverse direction when $a$ is greater than $b$. When the pointer rotates very slowly we have approximate synchronism, when the pointer is in a certain position the alternator $A$ has the proper phase relation to the bus-bar voltage, and when both of these conditions are realized the main switch which connects alternator $A$ to the bus-bars is closed.

The potential transformer which is connected to the bus-bars supplies current to the windings $S S$, Fig. 289, and the potential transformer which is connected to the machine which is being started supplies current to the two windings $C$ and $D$ of a rotating armature $A$. The collector ring $g$ is connected to one of the terminals of the "machine-transformer" and thence to the common junction $e$ of the coils $C$ and $D$, so that coil $C$ is connected between the terminals of the "machine-transformer" with a high inductance $L$ in series with it, and coil $D$ is connected between the terminals of the " machine-transformer" with a large non-inductive resistance $R$ connected in series with it. The core $A$ is mounted on a spindle which carries the pointer $n$. Under these conditions the pointer $n$ stands stationary when the bus-bar voltage and the machine voltage are in exact synchronism, and when the two voltages are not in synchronism the pointer $n$ rotates in one direction or the other at a speed which is equal to the difference of the frequencies of the two voltages.

152. The compensated voltmeter. - When a voltmeter is connected between the mains at a generating station, either directly or through a potential transformer, it of course indicates the voltage between the mains at the station, which is generally greater than the voltage between the mains at a distant receiving station. It is frequently desirable to control the voltage between a pair of feeders at a generating station so as to maintain a constant voltage at the distant receiving station which is supplied with current through the given pair of feeders, and, in order to do this, it is necessary to arrange a voltmeter in such a way that it will indicate the voltage across the mains at the distant receiving station. A voltmeter so arranged is called a compensated voltmeter, and 
the essential features of the compensating device are as follows : The voltmeter $V$ is connected to the mains through a potential transformer $P T$ and in series with an adjustable resistance $r$ and an adjustable reactance $x$ through which a current $i$ proportional to the line current $I$ is sent from a current transformer CT, as shown in Fig. 290. The relationship between

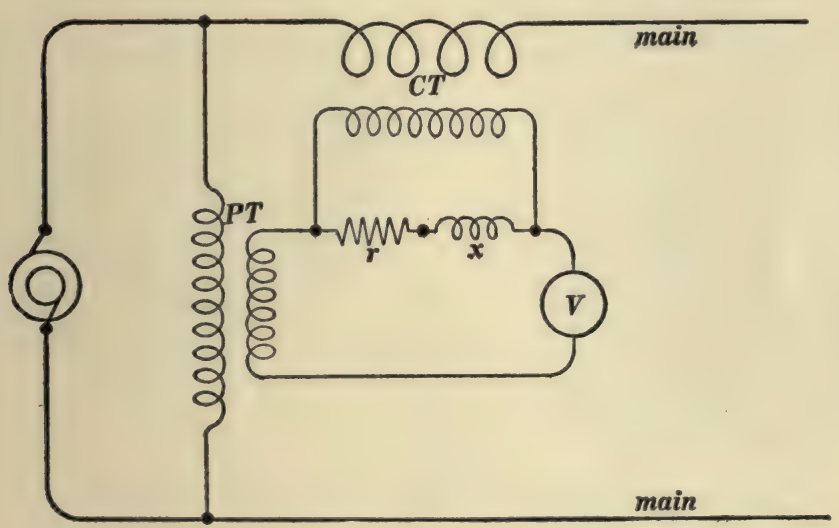

Fig. 290.

the voltage $E_{0}$ at the generating station, the voltage $E_{1}$ at the receiving station, the line current $I$, the line resistance $R$ and the line reactance $X$ is shown in Fig. 291. Let $e_{0}$, Fig. 292,

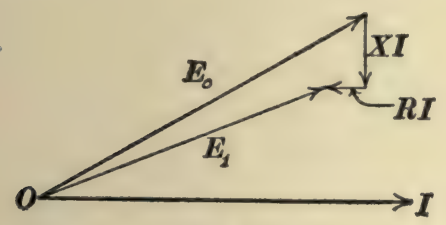

Fig. 291.

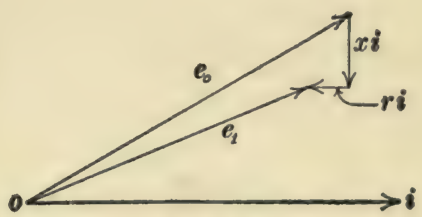

Fig. 292.

represent the secondary voltage of the potential transformer, and let $r i$ and $x i$ be the voltage drops across the resistance $r$ and the reactance $x$, of Fig. 290 , respectively, so that $e_{1}$ is the voltage which acts on the voltmeter. Then, since $i$ bears a fixed ratio to $I$ (the ratio of transformation of the current trans- 
former), it is possible to choose the values of $x$ and $r$ so that the clock diagram, Fig. 292, is exactly similar to the clock diagram, Fig. 29I. Under these conditions the voltage $e_{1}$ which acts on the voltmeter bears a fixed ratio to $E_{1}$ at the receiving station and the voltmeter scale is numbered so as to indicate the values of $E_{1}$ directly.

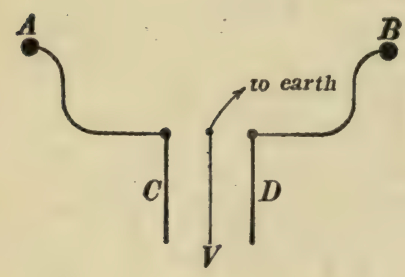

Fig. 293.

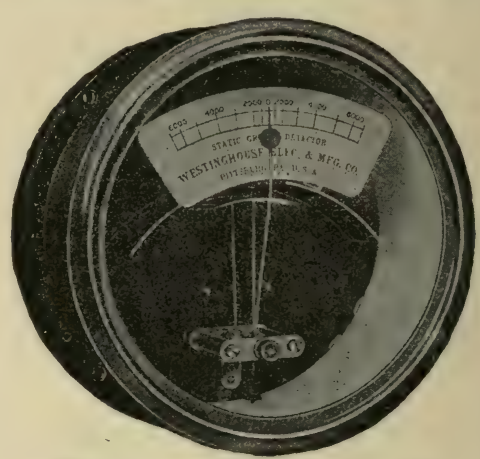

Westinghouse Static Ground Detector. Fig. 294.

153. Ground detectors.* The electrostatic ground detector is almost universally used for moderately high voltage alternatingcurrent feeders. The essential features of the electrostatic ground detector are shown in Fig. 293. Two insulated metal plates $C$ and $D$ are connected to the two feeders $A$ and $B$, and a light metal vane $V$ is suspended between $C$ and $D$ and connected to earth. The grounding of feeder $A$ establishes a connection from $A$ to $V$, so that the voltage between $C$ and $V$ is zero, whereas the voltage between $D$ and $V$ is large and the vane is attracted by $D$. The grounding of feeder $B$ in the same way causes the vane to be attracted by $C$. Fig. 294 shows the electrostatic ground detector of the Westinghouse Electric \& Manufacturing Company. In this instrument, the moving vane is arranged so that it can not be brought into contact with the stationary plates however far it may move, and additional provision is usually made to avoid possible short-

* The use of incandescent lamps as ground detectors is described on pages 209 and 2 ro of the first volume of this text. 
circuit through the ground detector, by inserting a condenser between $A$ and $C$ and another between $B$ and $D$ in Fig. 293, which represents the diagram of connections of the Westinghouse ground detector.

154. Oil switches. - Figures 295 and 296 show front and rear views of an automatic oil switch (circuitbreaker) of the Westinghouse Electric and Manufacturing Company. Figure 295 shows the tripping magnet which releases the lever of the switch and causes the switch to open, and Fig. 296 shows the essential parts of the switch. This particular switch is a threephase single-pole switch, that is to say, it is three separate single-pole switches, one for each phase of a three-phase system, and each switch breaks under oil. One of the oil

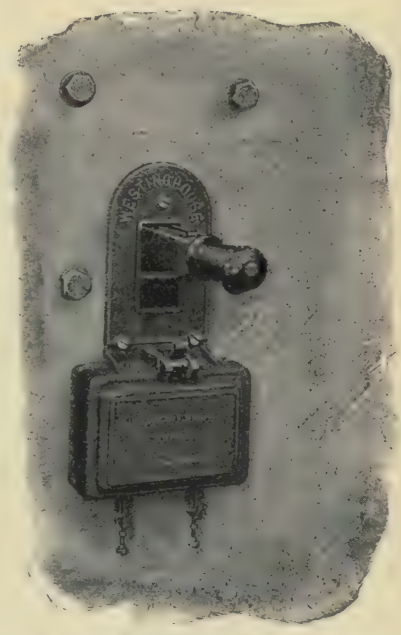

Type B Oil Circuit-breaker. (Switchboard Mounting-Front View) Fig. 295. vessels is shown removed in Fig. 296.

155. The Tirrill regulator. - The essential features of the Tirrill regulator as constructed by the General Electric Company are shown in Fig. 297.

The contact $C$ short circuits the field rheostat of the exciter; when the contact $C$ is closed the exciter voltage rises, the current in the field winding of the main generator grows, and the voltage of the main generator tends to increase.

The relay $R$, with a differential winding $A$ and $B$, actuates the contact $C$. When the points $T$ and $T^{\prime}$ are not in contact, current flows through coil $A$ only, the relay is excited, and the contact $C$ is opened; when the points $T$ and $T^{\prime}$ are in contact, the current which flows through coil $B$ neutralizes the magnetizing action of coil $A$, the relay is not excited, and the contact $C$ is closed. 
A solenoid, called the $D$. C. solenoid, is supplied with current from the exciter bus bars, and it acts upon a plunger $P$ which actuates the lever $L$. A set of springs (not shown in the figure) are attached to the lever $L$ and adjusted so that the lever moves through a certain definite range when the voltage of the exciter changes from its lowest value (amount required to give normal

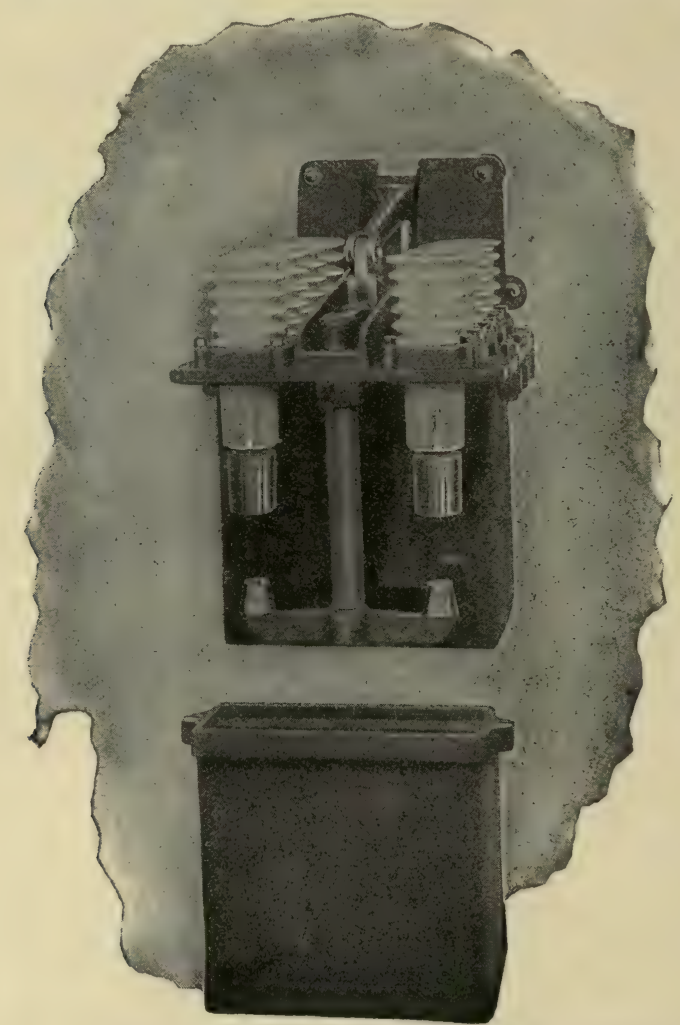

Type B Oil Circuit-breaker. (Switchboard Mounting-Rear View.)

Fig. 296.

voltage on the alternating-current generator at zero load) to its highest value (amount required to give normal voltage on the alternating-current generator at full load).

Suppose the contact point $T^{\prime}$ to stand in a given fixed posi- 
tion, then, if the points $T$ and $T^{\prime}$ do not touch, the relay is excited, the contact $C$ is open, and the exciter voltage decreases thus causing the plunger to rise until the points $T$ and $T^{\prime}$ come into contact. The moment this contact occurs, the relay

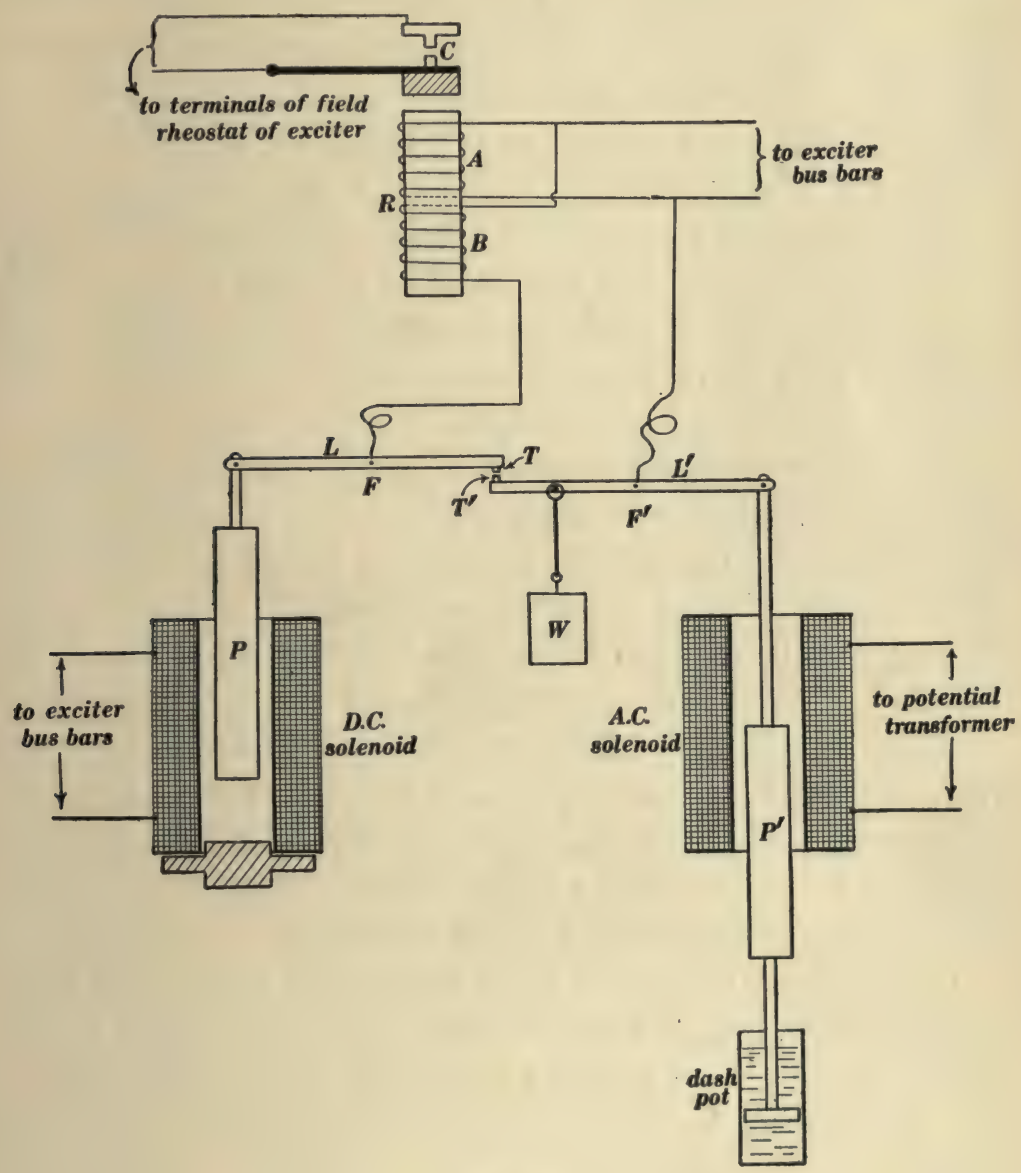

Fig. 297.

loses its excitation, the contact $C$ closes, and the exciter voltage rises very slightly and at once separates the points $T$ and $T^{\prime}$. This separation of the points $T$ and $T^{\prime}$ is followed by a slight drop of exciter voltage which quickly brings the points $T$ and 
$T^{\prime}$ into contact again, after which the exciter voltage rises slightly causing the points $T$ and $T^{\prime}$ to separate, and so on. The result is that the exciter roltage is kept oscillating very slightly above and below the value necessary to hold the lever $L$ in the position which barely gives contact between $T$ and $T^{\prime}$. If the terminal $T^{\prime}$ is permanently lowered, the exciter voltage is reduced until $T$ and $T^{\prime}$ come into contact and the abovedescribed oscillations begin. If the point $T^{\prime}$ is permanently raised, the exciter voltage is increased until the above-described oscillations begin. Therefore, all that is needed to vary the field excitation of the alternator is to cause the point $T^{\prime}$ to move up when the field excitation is to be increased, and to cause it to move down when the field excitation is to be decreased.

This movement of the terminal $T^{\prime}$ is accomplished by means of an A.C. solenoid and a plunger $P^{\prime}$. The plunger $P^{\prime}$ is counterpoised by the weight $W$, and the end of $P^{\prime}$ is near the middle of the solenoid so that an extremely slight change of the alternating voltage which acts on the solenoid will cause $P^{\prime}$ to move up or down through a considerable distance. Then any very slight reduction of the alternating voltage lowers $P^{\prime}$ and raises the point $T^{\prime}$ which causes an increased excitation of the alternator as above described; and any very slight increase of the alternating voltage raises $P^{\prime}$ and lowers the point $T^{\prime}$ which causes a decreased excitation of the alternator.

If it is desired to maintain a constant voltage between the alternating-current bus bars in the station, the A.C. solenoid is connected exactly as an ordinary voltmeter would be (through a potential transformer to the high-voltage alternating-current bus bars).

If it is desired to cause the station voltage to increase with increase of current output so as to compensate* for line drop of

\footnotetext{
* This compensation is not theoretically correct because a variation of the power factor of the receiving circuit with given value of current output of the station affects the line drop. See Art. 152 on the compensated voltmeter.
} 
SWITCHBOARDS AND SWITCHBOARD APPLIANCES. 345

voltage, the A.C. solenoid is compounded, that is, it is provided with two windings, one of which is connected to a potential transformer and the other is connected to a current transformer.

In practice, condensers are connected across the contacts $C$ and $T T^{\prime}$ to reduce the amount of sparking, and reversing switches are arranged to reverse the currents through $C$ and through $T T^{\prime}$ (once a day) so as to give equal wear on the contact points. 


\section{CHAPTER XVI.}

\section{TRANSMISSION LINES.*}

156. Direct-current and alternating-current line calculations compared. - A transmission line is generally designed to deliver a certain amount of current to a receiving circuit at a specified voltage, the difference between generator voltage and receiving circuit being given. In the case of direct-current transmission, this problem is solved by considering the resistance drop $R I$ in the line, as fully explained in Chapter IX of the first volume of this text. In the case of alternating current, an exact solution of the problem would require the effect of line resistance, of line inductance, and of line capacity to be taken into account. The effect of line capacity, however, is negligible on moderately short lines and it is ignored in this chapter. $\dagger$

In the case of alternating current, the product of line resistance by the effective value of the line current is called the resistance drop in the line, the product of the line reactance by the effective value of the line current is called the reactance drop in the line, the product of the line impedance by the effective value of the line current is called the impedance drop in the line, and the numerical difference between generator voltage and receiver voltage is called simply the line drop. The general relation between resistance drop, $r I$, reactance drop, $x I$, and the impedance drop, $z I$ is shown in Fig. 79, Chapter IV and it is evident that the line drop (difference in numerical values of $E_{0}$ and $E_{1}$ ) depends not only upon the value of the impedance drop $\left(I \sqrt{r^{2}+x^{2}}\right)$, but also upon the phase relations as shown in Fig. 79 .

157. Line resistance. - The resistance of a wire for alternating current is in nearly all practical cases equal to the resistance of

* See a paper by F. A. C. Perrine and F. G. Baum, Transactions Am. Inst. E. E. Vol. XVII, pages 345-377; also a paper by F. G. Baum, Trans. Int. Elec. Cong. Vol. II, pages $243-278$, St. Louis, 1904 . 
the same wire for direct current. When the wire is very large, however, or when the frequency is very high, the alternating current near the axis of a wire lags in phase behind the current near the surface of the wire, and the resistance of the wire is therefore larger for alternating current than for direct current. This effect is called the skin effect.*

158. Line reactance. - The reactance of a transmission line (outgoing and returning wires side by side) depends upon the size of the wires and upon their distance apart center to center, and it is proportional to the length of the line and to the frequency. $\dagger$ The following table gives the resistance and reactance per half-mile of transmission line:

Resistance and Reactance of One Mile of Wire ( $1 / 2$ Mile of Transmission Line) (Еmmet).

\begin{tabular}{|c|c|c|c|c|c|c|c|}
\hline \multirow{3}{*}{$\begin{array}{c}\text { Size of } \\
\text { wire B \& } \\
\text { S. gauge. }\end{array}$} & \multirow{3}{*}{$\begin{array}{l}\text { Resis- } \\
\text { tance in } \\
\text { ohms. }\end{array}$} & \multicolumn{6}{|c|}{ Reactance in Ohms. } \\
\hline & & \multicolumn{3}{|c|}{ At 60 cycles per sec. } & \multicolumn{3}{|c|}{ At 125 cycles per sec. } \\
\hline & & $\begin{array}{l}\text { Wires } \\
\text { I2 inches } \\
\text { apart. }\end{array}$ & $\begin{array}{l}\text { Wires } \\
x 8 \text { inches } \\
\text { apart. }\end{array}$ & $\begin{array}{c}\text { Wires } \\
24 \text { inches } \\
\text { apart. }\end{array}$ & $\begin{array}{l}\text { Wires } \\
\text { x2 inches } \\
\text { apart. }\end{array}$ & $\begin{array}{l}\text { Wires } \\
18 \text { inches } \\
\text { apart. }\end{array}$ & $\begin{array}{l}\text { Wires } \\
24 \text { inches } \\
\text { apart. }\end{array}$ \\
\hline 0000 & .259 & .508 & .557 & .591 & 1.06 & I. 17 & I. 23 \\
\hline 000 & .324 & .523 & .573 & .607 & 1.09 & 1.20 & 1.26 \\
\hline oo & .412 & .534 & .588 & .618 & -1. 12 & I. 23 & I. 29 \\
\hline 0 & .519 & .550 & .603 & .633 & I. 15 & I. 26 & I. 32 \\
\hline I & .655 & .565 & .614 & .648 & 1.18 & 1.28 & 1.35 \\
\hline 2 & .826 & .580 & .629 & .663 & I. $2 \mathrm{I}$ & I. 3 I & I. $3^{8}$ \\
\hline 3 & I. 041 & .591 & .644 & .674 & I. 24 & 1.34 & 1.41 \\
\hline 4 & I. $3^{1} 3$ & .606 & .656 & .690 & 1. 26 & 1.37 & I. 44 \\
\hline 5 & 1.656 & .620 & .670 & .704 & I. 30 & 1.40 & $\mathrm{I} .47$ \\
\hline 6 & 2.088 & .633 & .685 & .720 & $1 \cdot 3^{2}$ & 1.43 & 1.49 \\
\hline 7 & 2.633 & .647 & .700 & .730 & I. 35 & 1.46 & 1.52 \\
\hline 8 & 3.320 & .662 & .712 & .742 & 1. $3^{8}$ & 1.48 & 1. 55 \\
\hline 9 & 4. 186 & .677 & .727 & .761 & $1.4 \mathrm{I}$ & I. $5^{I}$ & I. $5^{8}$ \\
\hline ro & 5.280 & .688 & .742 & .776 & I. 44 & I. 54 & 1.62 \\
\hline
\end{tabular}

159. Calculations of a single-phase transmission line to give a specified line drop. $\$-\mathrm{A}$ single-phase transmission line is to deliver a prescribed amount of power $P$ at a prescribed electro-

* See Merritt, Physical Review, Vol. 5, page 47.

†The formula for the reactance of a line is developed in Appendix A, and so also is the formula for line capacity.

† See a paper by R. D. Mershon, American Electrician, June, 1897 . 
motive force $E_{1}$ to a receiving circuit of which the power factor, $\cos \theta$, is given ; the line drop, frequency, length of line, and distance apart of wires being given.

The generator voltage $E_{0}$ is equal to the sum (numerical sum) of $E_{1}$ and line drop.

The full load current $I$ is found from the relation $E_{1} I \cos \theta$ equals $P$.

The component of $E_{1}$ parallel to $I$ is $E_{1} \cos \theta$, and the component of $E_{1}$ perpendicular to $I$ is $E_{1} \sin \theta$.

By treating the problem first as a direct-current problem, the approximate resistance $r^{\prime}$ of the line is found from the relation $r^{\prime} I$ equals line drop. From this approximate resistance and the known length of the line, the approximate size of the wire and the line reactance $x$ may be found from the table; and since the line reactance varies but little with the size of the wire, the value of $x$ need not be further approximated.

The component of $E_{0}$ parallel to $I$ is $E_{1} \cos \theta+r I$, where $r$ is the true resistance of the line, and the component of $E_{0}$ perpendicular to $I$ is $E_{1} \sin \theta+x I$. Therefore

or

$$
E_{0}^{2}=\left(E_{1} \cos \theta+r I\right)^{2}+\left(E_{1} \sin \theta+x I\right)^{2}
$$

$$
r=\frac{\sqrt{E_{0}^{2}-\left(E_{1} \sin \theta+x I\right)^{2}}-E_{1} \cos \theta}{I}
$$

From this equation the true line resistance $r$ may be found and thence the correct size of wire.

Example:

$E_{1}=20,000$ volts.

$P=\mathrm{I}, 000$ kilowatts.

$\cos \theta=0.85=$ power factor of receiving circuit.

$E_{0}=23,000$ volts, or line drop $=3,000$ volts.

frequency $=60$ cycles per second.

distance $=30$ miles.

distance apart of wires $=$ I 8 inches. 
From these data we find :

$$
\begin{aligned}
I & =58.8 \text { amperes. } \\
r^{\prime} & =5 \mathrm{I} \text { ohms. }
\end{aligned}
$$

Therefore, from the table we find that, approximately, a No. 2 $\mathrm{B} \& \mathrm{~S}$. wire is required so that $x=37.7 \mathrm{ohms}$.

Furthermore,

$$
\begin{aligned}
& E_{1} \cos \theta=\mathrm{I} 7,000 \text { volts. } \\
& E_{1} \sin \theta+x I=\mathrm{I} 2,700 \text { volts. }
\end{aligned}
$$

and from equation (i) we find

$$
r=37.3 \mathrm{ohms} \text {. }
$$

from which the correct size of wire is found to be, approximately, a No. I B. \& S.

160. Calculation of double line for two-phase transmission (four wires). - In this case each line is calculated to deliver half the prescribed power. Thus, if it is desired to deliver I,O0O kilowatts at 20,000 volts two-phase, at a frequency of 60 , line drop of 3,000 volts, etc., then each line is calculated as a single-phase line to deliver 500 kilowatts at 3,000 volts line drop.

161. Calculation of a three-wire transmission line for threephase currents. - The calculation will be carried out for the case in which both the generator and the receiver are $\mathrm{Y}$-connected as

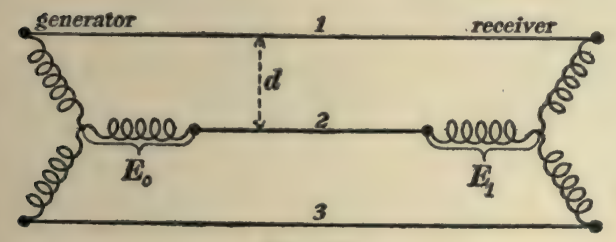

Fig. 298. shown in Fig. 298. If it is desired to state the problem by specifying the voltage between mains at generator and at receiver and current in each main, the specified voltage between mains may be divided by $\sqrt{3}$ to give the values of $E_{0}$ and $E_{1}$ (see Fig. 298).

Let $\cos \theta$ be the power factor of each receiving circuit, $P$ the total power to be delivered, $E_{1}$ the electromotive force between the terminals of each receiving circuit, and $E_{0}$ the electromotive force of each armature winding on the generator; all 
prescribed (see Fig. 298). Then

$$
P={ }_{3} E_{1} I \cos \theta
$$

from which the full-load line current $I$ may be calculated.

The numerical difference $E_{0}-E_{1}$ is the electromotive force drop in one wire. Therefore, looking upon the problem as one in direct currents, we have $E_{0}-E_{1}=r^{\prime} I$, where $r^{\prime}$ is the approximate resistance of one wire. From this the approximate size of the wire may be found from the table.

Consider one of the wires, say wire number 2. The other two wires together constitute the return circuit for this wire, and, the three wires being arranged as indicated in Fig. 299, the distance from wire number 2 to each of the other wires is equal to $d$, which is given. Find the reactance $x$ of a pair of wires at the prescribed distance apart center to center, from the above table.

The component of $E_{1}$ parallel to $I$ is $E_{1} \cos \theta$, and the component of $E_{1}$ perpendicular to $I$ is $E_{1} \sin \theta$.

The resistance drop in one main is $r I$ and the reactance drop in one main is $\frac{1}{2} x I$, the former being parallel to $I$

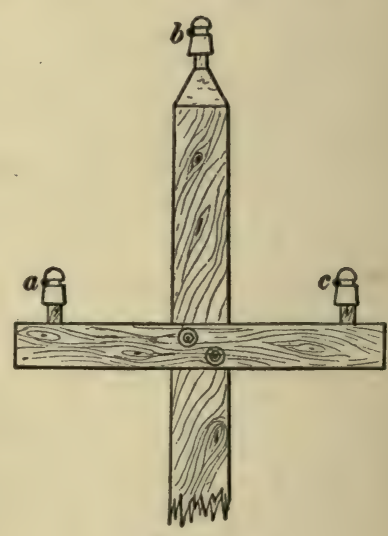

Fig. 299. and the latter being perpendicular to $I$. Then the components of $E_{0}$ are $E_{1} \cos \theta+r I$, and $E_{1} \sin \theta+\frac{1}{2} x I$, respectively, so that whence

$$
E_{0}^{2}=\left(E_{1} \cos \theta+r I\right)^{2}+\left(E_{1} \sin \theta+\frac{1}{2} x I\right)^{2}
$$

$$
r=\frac{\left.\sqrt{E_{0}^{2}-\left(E_{1} \sin \theta+\frac{1}{2} x I\right.}\right)^{2}-E_{1} \cos \theta}{I}
$$

which gives the true resistance $r$ of one wire from which the correct size of wire is easily found.

Example. - The electromotive force between mains at the receiving stations is to be 20,000 volts. Therefore, the electromotive force between terminals of $Y$-connected receiving circuits 
would be

$$
E_{1}=20,000 \div \sqrt{3}=\text { I I,550 volts (see Fig. 298). }
$$

The electromotive force between mains at the generating station is to be 23,000 volts. Therefore,

$$
E_{0}=23,000 \text { volts } \div \sqrt{3}=13,280 \text { volts (see Fig. 298). }
$$

Further specifications: $P=\mathrm{I}, 000$ kilowatts, $\cos \theta=0.85$, frequency $=60$ cycles per second, distance $=30$ miles, distance apart of wires $=2 \mathrm{I}$ inches.

From these data we find $I=34$ amperes, and $r^{\prime}=50.9 \mathrm{ohms}$. Therefore, approximately, a number 5 wire is required. The reactance $x$ of a 30-mile double line of number 5 wires, $2 \mathrm{I}$ inches apart center to center, at 60 cycles per second, is

$$
x=4 \text { I.2 ohms }
$$

which substituted in equation (i) gives

$$
r=46.5 \mathrm{ohms}
$$

so that a wire between number 4 and number 5 would give the prescribed line drop.

162. Interference of separate alternating-current transmission lines. - When a telephone line is near an alternating-current transmission line, an alternating current may be produced in the telephone line in three ways, as follows: (a) By magnetic induction, that is the alternating current line may act like the primary and the telephone line like the secondary of a transformer; $(b)$ By electrostatic induction. The alternating current transmission wires are repeatedly charged and discharged with the alternations of the electromotive force, and the telephone wires are repeatedly charged and discharged "by influence," that is, the positive charge on the alternating-current wires draws a negative charge into the telephone wires from the ground, and a negative charge on the alternating current wires draws a positive charge into the telephone wires from the ground. This charging and discharging of the telephone wires produces an alternating current in the telephone line; $(c)$ By leakage. If the alternating-current line 
is not thoroughly insulated from the telephone line, more or less current will leak across from one to the other.

What is here said of the effect of an alternating-current line upon a telephone line applies also to the effect of one alternatingcurrent transmission line upon an adjacent alternating-current

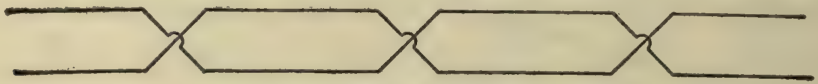

Fig. 300.

transmission line. These effects are especially troublesome, however, in telephone lines which are adjacent to alternating-current transmission lines, and they may be obviated * by thorough insulation, and by what is called transposition of wires of one of the lines. The essential features of transposition are shown in Fig. 300. At frequent intervals along the line the two wires of the line cross over or change places. Fig. 30I shows the method
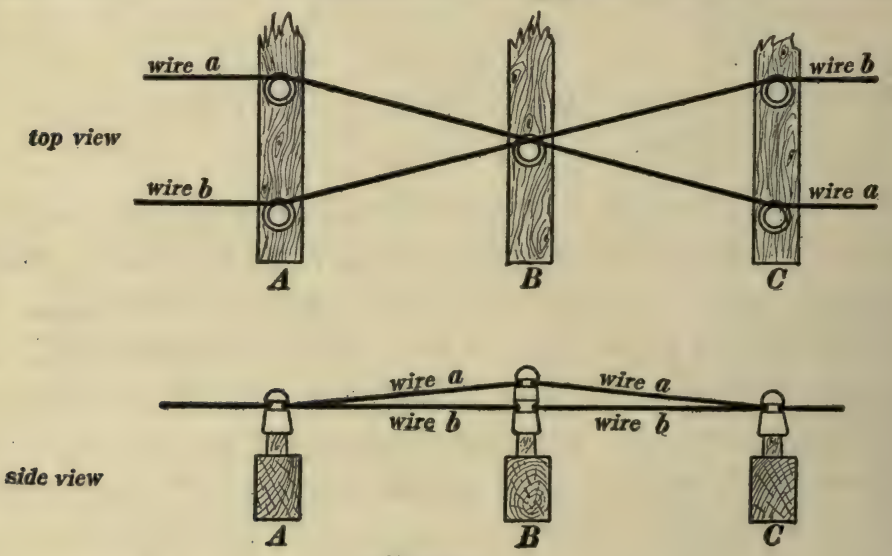

. Fig. 301.

of transposing telephone lines. Three cross-arms are attached to the pole at which the transposition is to be made, and upon the middle arm a "two story" insulator is placed so as to bring one of the wires above the other as indicated in the figure.

* See a paper by P. M. Lincoln, Transactions of the American Institute of Electrical Engineers, Vol. 21, pages 245 to 251 , and a paper by F. F. Fowle, Transactions of the American Institute of Electrical Engineers, Vol. 23, pages 659 to 689. The important part of this latter paper is included in pages 674 to 687 . 


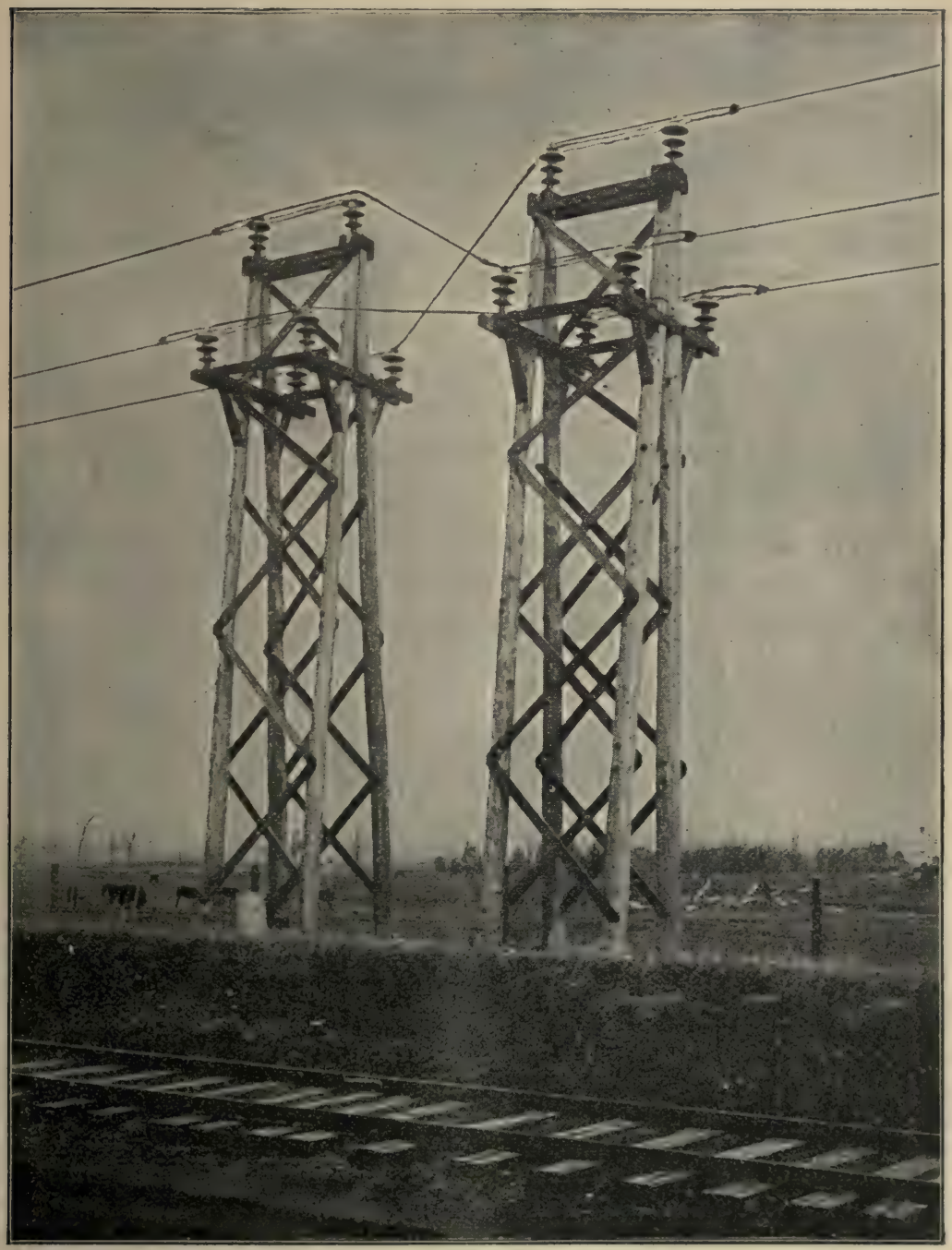

Fig. 302 .

Figure 302 shows a "cross-over" or "transposition" on a three-wire three-phase transmission line.*

* From a photograph loaned by Mr. R. D. Mershon. 


\section{APPENDIX A.}

\section{INDUCTANCE AND CAPACITY.}

1. A spark at break. - When an electric circuit is broken, the current continues to flow across the break for a short time, producing an electric arc or spark. This action of the electric current is suggestive of momentum, and, consequently, we shall speak of the momentum of the electric current. The intensity of the spark is a rough indication of the amount of this momentum.

The amount of momentum associated with a given current in a circuit made of a given length and size of wire depends upon the shape of the circuit and upon the presence of iron near the circuit. Thus, a current in a circuit $a$, Fig. I, has but little

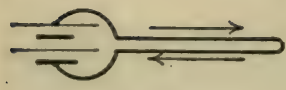

$a$

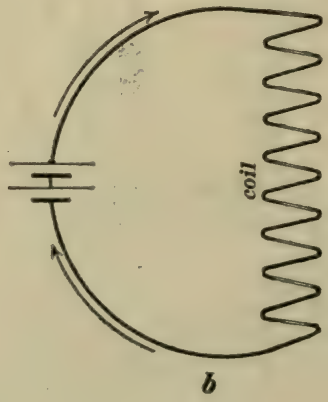

Fig. 1.

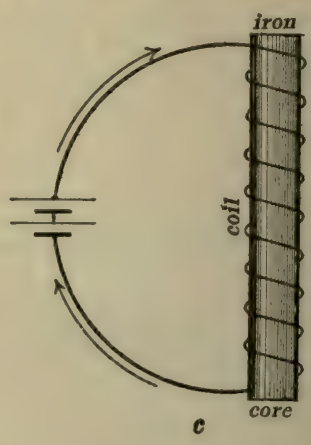

momentum; the same current in circuit $b$ has greater momentum; and the same current in circuit $c$ has very much greater momentum. Thus, when the circuit of an ordinary incandescent lamp is broken, a very slight spark is produced; the same amount of current flowing through a coil of wire produces a much more intense spark when the circuit is broken; and a spark several inches in length may be produced if the coil of wire surrounds a core consisting of a bundle of iron wires. 
The magnetic field which is produced by an electric current seems to be a state of motion of the ether, and the momentum of the electric current depends upon this magnetic field. Thus, a current in the circuit $a$, Fig. I, produces a very weak magnetic field, except in the small region between the wires, and the momentum of the current is small; the same current in circuit $b$ produces an intense magnetic field inside of the coil, and the momentum of the current is correspondingly great; and the magnetism of the iron core accounts for the very great momentum of the current in circuit $c$. In the following discussion the notion of kinetic energy is used in preference to the notion of momentum for the reason, mainly, that kinetic energy is expressed in mechanical units even when it is electrical kinetic energy.

2. The kinetic energy associated with a current in a circuit. Definition of inductance. - The ether motion which constitutes the magnetic field in the neighborhood of an electric circuit, represents kinetic energy, and the kinetic energy which is associated with an electric current is the total energy residing in the magnetic field produced by the current. We shall for convenience call this the kinetic energy of the current.

The amount of energy residing in a portion of a magnetic field is proportional to the square of the intensity of that portion of the field. This is analogous to the fact that the energy of a portion of a moving liquid is proportional to the square of the velocity of that portion. If the current in a circuit is doubled the field intensity is everywhere doubled, so that the energy of each portion of the field is quadrupled. Therefore the total energy of the field is quadrupled when the current is doubled, or in other words, the kinetic energy of a current in a given circuit is proportional to the square of the current, that is

$$
W=\frac{1}{2} L i^{2}
$$

in which $W$ is the kinetic energy of a current, $i$, in the given 
circuit, and $\left(\frac{1}{2} L\right)$ is the proportionality factor. The quantity $L$ is called the inductance of the given circuit.

Moment of inertia of a wheel. Analog of inductance. - The kinetic energy of a rotating wheel resides in the various moving particles of the wheel. If the angular velocity $\omega$ of the wheel is doubled, the linear velocity of every particle of the wheel is doubled, so that the kinetic energy of every particle is quadrupled. Therefore, the total kinetic energy of a wheel is quadrupled when the angular velocity is doubled, so that the kinetic energy of a given wheel is proportional to the square of the angular velocity, that is

$$
W=\frac{1}{2} K \omega^{2}
$$

in which $W$ is the kinetic energy of a rotating wheel, $\omega$ is the angular velocity of the wheel, and $\left(\frac{1}{2} K\right)$ is the proportionality factor. The quantity $K$ is called the moment of inertia of the wheel.

Units of inductance. - If $W$ in equation (I) is expressed in joules and $i$ in amperes, then $L$ is expressed in terms of a unit of inductance which is called a henry. That is to say, a circuit has an inductance of one henry when the kinetic energy of one ampere of current in the circuit is one half of a joule.

If $W$ in equation (I) is expressed in ergs, and $i$ in abamperes, then $L$ is expressed in terms of the c.g.s. unit of inductance. This c.g.s. unit of inductance is usually called the centimeter for the reason that the square of a current must be multiplied by a length to give energy or work; that is, inductance is expressed as a length and the unit of inductance is of course the unit of length.

Inductance of a coil. - Strictly, one cannot speak of the inductance of anything but an entire circuit, inasmuch as every portion of a circuit contributes its share to the magnetic field at each and every point ; it is, however, allowable to speak of the inductance of a coil when the terminals of the coil are near together, and when the remainder of the electric circuit does not produce any perceptible magnetic field in the region occupied by the coil. 
Non-inductive circuits. - A circuit is said to be non-inductive under given conditions when the inductance of the circuit is negligible, that is, when the electromotive force $L \times d i / d t^{*}$ is negligibly small, as compared with the electromotive force $R i$, which overcomes the resistance of the circuit. Thus, a given circuit might be considered to be non-inductive under conditions involving slow changes of current, whereas the same circuit could not be considered to be non-inductive under conditions involving very rapid changes of current. When a circuit consists of outgoing and returning wires side by side, its inductance is small and it may usually be considered to be non-inductive. The wires used in resistance boxes are usually arranged non-inductively. This may be done by doubling the wire back on itself, and winding the doubled wire on a spool. In this case the electromotive force between adjacent wires may be great, and the resistance coil may have considerable electrostatic capacity. In order to make a non-inductive resistance coil without this defect, the wire may be wound in one layer on a thin paper cylinder so as to bring the terminals as far apart as possible, and this cylindrical coil may then be flattened so as to reduce the region (inside) in which the magnetic field is intense.

Measurement of inductance. - The most accurate method for determining the inductance of a coil is by calculation from measured dimensions. This calculation can be carried out only when the coil is simple in shape, and even then the calculation is in most cases quite complicated. The simplest case is given in Art. 7. The inductance of an irregularly shaped coil may be determined by various electrical methods. $\dagger$

\section{Electromotive force required to cause a current to increase or} decrease. - To keep a rotating wheel in uniform motion, a torque sufficient to balance the drag of friction must act upon the wheel.

* See the next article.

† See Absolute Measurements by A. Gray, Vol. 2, Part 2, pages 438 to 509.

See also a series of articles in the Bulletin of the United States Bureau of Standards, by E. B. Rosa, Vol. I, page 125, and page 29I ; Vol. 2, page 87, page 16I, and page 359 ; Vol. 3 ; page $\mathbf{I}$. 
If the torque which acts upon the wheel is greater than the drag of friction, the wheel gains angular velocity, and if the torque which acts upon the wheel is less than the drag due to friction, the angular velocity of the wheel decreases.

To maintain a constant current in a circuit, an electromotive force equal to $R i$ must act upon the circuit to overcome the resistance of the circuit. If the electromotive force which acts upon the circuit is greater than $R i$, the current increases in value, and if the electromotive force which acts upon the circuit is less than $R i$, the current decreases in value. Let the electromotive force which acts upon a circuit exceed $R i$ by the amount $e$, then we have:

$$
e=L \frac{d i}{d t}
$$

in which $L$ is the inductance of the circuit, and $d i / d t$ is the rate at which current increases. When $e$ is negative (electromotive force less than $R i$ ) then $d i / d t$ is negative, that is, the current decreases.

Mechanical analog of equation (2).-Whenever an unbalanced force $e$ acts upon a body of which the mass is $L$, the body gains velocity at a rate $d i / d t$ such that

$$
e=L \frac{d i}{d t} \text { (force equals mass times acceleration) }
$$

Starting from the fact that force equals mass times acceleration, it can be shown that the kinetic energy of a moving body is equal to one half its mass times its velocity squared. The same argument reversed would show that force must be equal to mass times acceleration if kinetic energy is equal to one half mass times velocity squared; and an exactly similar argument would establish equation (2) on the basis of equation (I).

\section{Self-induced electromotive force. Reaction of a changing} current.-When one pushes on a wheel, causing its speed to increase, the wheel reacts and pushes back on the hand. This reacting torque is equal and opposite to the acting torque which is 
causing the increase of speed. When the speed of a wheel is increasing, the reaction of the wheel is a torque in a direction opposite to the motion, when the speed of a wheel is decreasing the reaction is a torque in the direction of the motion. Similarly when an electromotive force acts upon a circuit and causes a current to increase or decrease, the changing current reacts; the reacting electromomotive force is equal and opposite to the acting electromotive force, $L \times d i / d t$, which is causing the current to change; when the current is increasing, the reaction of the current is an electromotive force opposed to the current; and when the current is decreasing, the reaction is an electromotive force in the direction of the current. The reaction of a changing current is called self-induced clectromotive force.

\section{Differential equations of growing and decaying currents. -} A constant electromotive force $\mathcal{E}$ is at a given instant connected to a circuit of which the resistance is $R$ and the inductance is $L$; after the current becomes fully established it is equal to $\mathcal{E} / R$ according to Ohm's law, but during the time that the current is being established a portion of $\mathcal{E}$ is used to overcome the resistance of the circuit and a portion of $\mathcal{E}$ is causing the current to increase. The portion used to overcome resistance is equal to $R i$, and the portion used to cause the current to increase is equal to $L d i / d t$, and therefore we have

$$
\mathcal{E}=R i+L \frac{d i}{d t}
$$

in which $i$ is the value of the growing current at a given instant, and $d i / d t$ is its rate of increase.

When a circuit in which a given current is flowing, is shortcircuited and left to itself without any electromotive force acting to maintain the current, the current dies away; in this case equation (3) becomes

$$
\mathrm{o}=R i+L \frac{d i}{d t}
$$


6. Definition of flux-turns. Flux-turns due to a current in a coil. - The induced electromotive force in a coil of wire is equal to $Z \times d \Phi / d t$, where $Z$ is the number of turns of wire in the coil, and $\Phi$ is the flux through the coil (strictly, the flux through a mean turn of the coil). But $Z$ times the rate of change of $\Phi$ is equal to the rate of change of the product $Z \Phi$. Therefore $Z \Phi$ is a quantity whose rate of change is equal to the electromotive force induced in a coil. This quantity $Z \Phi$ is called the flux-turns.

When a current flows through a coil the region surrounding the coil and inside of the coil becomes a magnetic field, and the lines of force of this magnetic field pass through the coil. That is, the current in the coil produces magnetic flux through the opening of the coil. Let $\Phi$ be the flux through the opening of a coil due to a given current $i$ in the coil, and let $Z$ be the number of turns of wire in the coil, and $L$ the inductance of the coil (everything being expressed in c.g.s. units). Then

$$
Z \Phi=L i
$$

This is evident when we consider that the self-induced electromotive force in a coil is equal to $L \times d i / d t$, according to Art. 4 , and that $L \times d i / d t$ is equal to the rate of change of $L i$; but the self-induced electromotive force is also equal to the rate of change of $Z \Phi$, so that $Z \Phi$ must be equal to $L i$ :*

\section{Calculation of inductance in terms of flux-turns per unit} current. $\uparrow$ - According to equation (5) the inductance of a coil is equal to the quotient $Z \Phi / i$, where $Z \Phi$ is the flux-turns through the coil due to current $i$ in the coil. There are important cases in which the flux through the coil due to a given current may be easily calculated so that the inductance of such a coil may be easily determined.

* In this argument algebraic signs are ignored and $\Phi$ is assumed to be equal to zero when $i$ is equal to zero.

† Everything in this article is expressed in c.g.s. units except where expressly stated to the contrary. 
Case I. Long solenoid. - Consider a coil of wire wound in a thin layer on a long cylindrical rod of non-magnetic material, wood, for example. Let $r$ be the mean radius of the windings, $l$ the length of the coil, and $Z$ the number of turns of wire. The field intensity in the coil is $\mathscr{H}=4 \pi Z i / l, *$ and the area of the opening of the coil is $\pi r^{2}$, so that the flux through the opening is $4 \pi^{2} r^{2} Z i / l$, and for the flux turns, we have the value $4 \pi^{2} r^{2} Z^{2} i / l$, which, divided by $i$, gives the value of $L$ as above explained. Therefore

$$
L=\frac{4 \pi^{2} r^{2} Z^{2}}{l}
$$

in which the inductance $L$ is expressed in centimeters. To reduce to henrys, the right-hand member of equation (6) must be divided by $10^{9}$.

Case II. Coil wound on an iron core. - A coil of $Z$ turns of wire is wound on an iron ring $l$ centimeters in circumference (mean), and $s$ square centimeters in sectional area, as shown in Fig. 2. The coil produces through the ring a magnetic flux which is equal to the magneto-motive force of the winding divided by the magnetic reluctance of the ring. $t$ The magnetomotive force of the winding is $4 \pi Z i$, and the magnetic reluctance

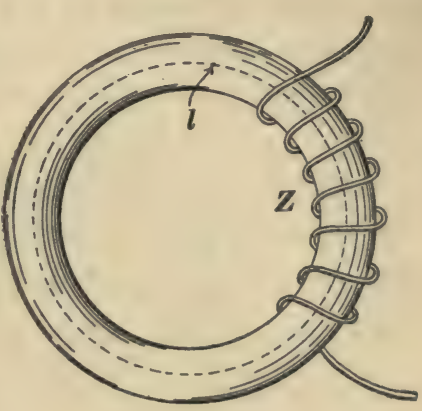

Fig. 2. of the ring is $l / \mu s, i$ being a given value of current in the coil, and $\mu$ being the permeability of the iron. Therefore

$$
\Phi=\frac{4 \pi Z i}{\frac{l}{\mu s}}, \quad \text { or } \quad \Phi Z=\frac{4 \pi \mu s Z^{2} i}{l}=L i
$$

* A proof of this equation may be found in Nichols and Franklin's Elements of Physics, Vol. 2, p. I 19.

+ See Appendix A of the first volume of this text. 
so that

$$
L=\frac{4 \pi \mu s Z^{2}}{l}
$$

It is to be particularly noted that the inductance of a coil of wire wound on an iron core varies with degree of saturation of the iron, inasmuch as the permeability of the iron is variable.

Case III. - The inductance of a transmission line is given by the equation

$$
L=0.0017 \times \log _{10} \frac{d}{R} \times l
$$

in which $L$ is the inductance of the line in henrys, $l$ is the length of the line (actual length of row of poles) in miles, $d$ is the distance apart of the two wires center to center, and $R$ is the radius of the wires, $d$ and $R$ being both expressed in the same units.

8. Electric charge. - A current of water through a pipe is a transfer of water along the pipe, and if $q$ be the amount of water which during $t$ seconds flows past a given point in the pipe, the quotient $q / t$ is the rate of flow of water through the pipe, and this rate of flow may be spoken of as the strength, $i$, of the water current. Suppose the strength $i$ of the water current to be given (rate of flow of water) then the amount of water flowing past a given point of the pipe in $t$ seconds is given by the equation

$$
q=i t
$$

Similarly, an electric current in a wire may be looked upon as a transfer of electricity along the wire, and the quantity $q$ of electricity which flows past a point on the wire during $t$ seconds may be defined as the product of the strength of the current and the time. That is

$$
q=i t
$$

If the strength of the electric current is variable, equation (9) must be written in the form

$$
\Delta q=i \cdot \Delta t
$$


in which $\Delta q$ is the small quantity of electricity which flows past a given point on the wire during the short interval of time $\Delta t$. Quantity of electricity is usually spoken of as electric charge or simply as charge.

Quantity of water is the fundamental and easily measured thing in hydraulics, and water current is most conveniently defined as quantity of water per second. On the other hand, in the case of electricity, the fundamental and easily measured thing is electric current and quantity of electricity is most conveniently defined as the product of electric current and time.

Units of electric charge. - The quantity of electricity transferred in one second by one ampere is called a coulomb; $q$ in equation (9) is expressed in coulombs when $i$ is expressed in amperes and $t$ in seconds.

9. Electrically charged bodies. - Consider two metal bodies, $A$ and $B$, Fig. 3 , which at a given instant are connected, as shown, to the terminals of a battery, or to any source of electromotive force. When the wire is connected a momentary pulse of current flows through the wire out of one body and into the other, and the bodies $A$ and $B$ are said to become charged with electricity. The body into which the momentary current flows is said to become positively charged, and the body out of which the momentary current flows is said to become negatively charged, that is, the

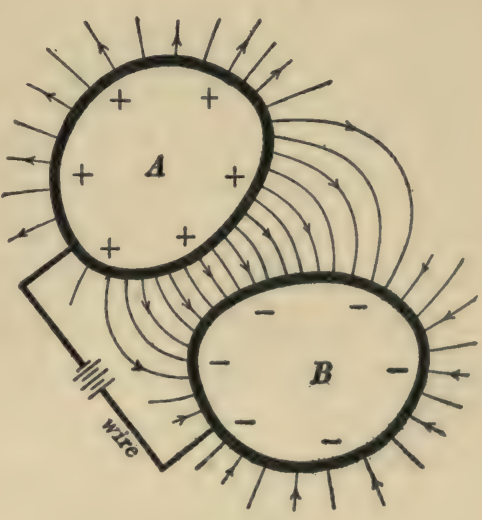

Fig. 3. charge on one body is $+q$ and the charge on the other body is $-q$. Electrically charged bodies always occur thus in pairs, the positive charge on one body being always associated with an equal negative charge on some other body or bodies. 
The electric field. The dielectric. - The region between the two bodies $A$ and $B$, Fig. 3, is of course filled with some electrical insulator such as air, or oil, or glass. An insulator between two charged bodies is called a diclectric. This dielectric is the seat of a peculiar stress called the electric field which is similar in many respects to the magnetic field. The lines of force of this electric field trend somewhat as shown in the figure, touching the surfaces of $A$ and $B$ at right angles. These lines of force are thought of as going out from the positively charged body and coming in towards the negatively charged body.

Electrostatic attraction. - The charged bodies $A$ and $B$, Fig. 3 , attract each other. This attraction, which is called electrostatic attraction, shows that the lines of force of an electric field are in a state of tension and have a tendency to shorten. This tension of the lines of force pulls outwards on the surface of $A$ and of $B$ at each point. This outward pull on the surface of a charged body is very strikingly shown by pouring a viscid liquid over the sharp lip of a charged metal ladle. The liquid is pulled into fine jets by the lines of force which emanate from the liquid as it passes over the lip. When melted rosin is used in this way, the jets congeal into very fine fibers which float about in the air.

If a small charged body such as a pith ball be suspended anywhere in the region between $A$ and $B$, it will be found to be acted upon by a force in the direction of the electric field, that is in the direction of the lines of force at the point where the pith ball is placed.

Need of large electromotive forces and good insulation. - The phenomena described above and, in fact, most of the phenomena of electrostatics are easily perceptible only when the bodies are charged by electromotive forces of many thousands of volts. The most convenient means for producing these large electromotive forces is the Holtz or Wimshurst electrical machine. When such a machine is used, however, the bodies $A$ and $B$ must be well insulated, because such electrical machines cannot supply charge at a rapid rate, that is such a machine can deliver only a very small current. 
Dielectric strength. - When the electromotive force $E$ acting to charge two bodies $A$ and $B$, Fig. 3 , is increased, a value is eventually reached which breaks down, or ruptures, the dielectric and allows the charge on the bodies to pass in the form of an electric spark. When the bodies $A$ and $B$ are flat, as shown in Fig. 4, the electromotive force required to break down the intervening dielectric is, for a given dielectric, proportional to the distance $x$ between the plates, and the quotient $E / x$ is called the electric strength

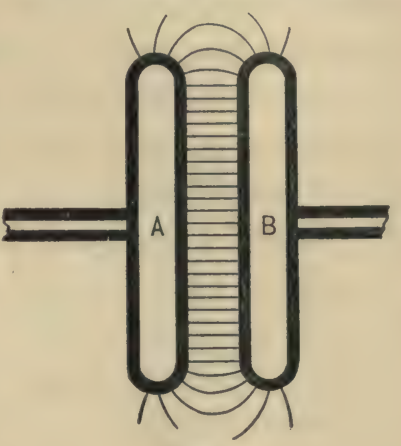

Fig. 4. of the dielectric. The following table gives the strengths of various dielectrics.

\section{TABLE.}

Dielectric STRENGTHS.

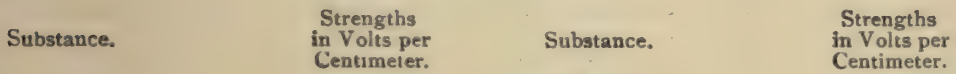

Oil of turpentine . . . . . 94,000| Paraffined paper . . . . . 360,000

Paraffine oil ...... 87,000 Beeswaxed paper .... . 540,000

Olive oil . . . . . 82,000 Air (thickness $5 \mathrm{~cm}$ ) . . . 23,800

Paraffine (melted) . . . . 56,000 $\mathrm{CO}_{2}$ " " . . . 22,700

Kerosene oil ...... . . 50,000 O . . . 22, 200

Paraffine (solid) . ..... I30,000 $\mathrm{H}$. " ". . . . 15, 100

10. The condenser.* Electrostatic capacity. - The amount of charge $q$ which flows out of $B$ into $A$, Fig. 3 , when the battery is connected, is proportional to the electromotive force of the battery. Therefore we may write

$$
q=C E
$$

in which $q$ is the charge that is drawn out of $B$ and forced into $A$ in Fig. 3 by a battery of which the electromotive force is $E$, and $C$ is a constant depending upon the size and shape of $A$ and $B$, and upon the intervening dielectric. This quantity $C$ is called the electrostatic capacity or simply the capacity of the pair of

* See pages 19 to 23 for a description of the Hydraulic Analogue of the Condenser. 
bodies $A$ and $B$. If the bodies $A$ and $B$ are in the form of metal plates separated by a thin layer of dielectric, their electrostatic capacity is large. Such an arrangement is called a condenser. Condensers of large capacity are made by sheets of tin foil separated by sheets of waxed paper or mica. The Leyden jar is a condenser made by coating the inside and outside of a glass jar with tin foil.

Units of capacity. - A condenser is said to have a capacity of one farad when one coulomb of charge is drawn out of one plate and forced into the other plate by an electromotive force of one volt; $C$ in equation (I I) is expressed in farads, when $q$ is expressed in coulombs and $E$ in volts. The farad is an enormously large capacity as compared with capacities ordinarily met with in practice, and the microfarad (one-millionth of a farad) is frequently used as a unit.

11. Inductivity of a dielectric. - The capacity of a condenser with plates of given size and at a given distance apart depends upon the dielectric. The quotient: capacity of condenser with given dielectric divided by the capacity of the same condenser with air between its plates is called the inductivity of the dielectric. For example, the inductivity of petroleum is about 2.04, that is, the capacity of a given condenser is about 2.04 times as great when the dielectric is petroleum as it is when the dielectric is air. A condenser is called an air condenser, a mica condenser, or a paraffine condenser, according to the dielectric used between the plates. The following table gives the inductivity of a few dielectrics :

\section{TABLE.}

INDUCTIVITIES OF VARIOUS SUBSTANCES.
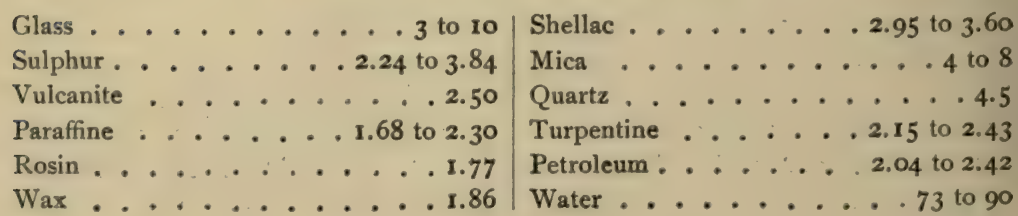
12. Dependence of the capacity of a condenser upon the size and distance apart of its plates. - The capacity $C$ of an air condenser is proportional to the area $a$ of its plates, and inversely proportional to the distance $x$ between its plates, that is $C$ is proportional to $a / x$, or the capacity of a condenser with a given dielectric is proportional to $k a / x$, where $k$ is the inductivity of the dielectric. Indeed the capacity of a condenser may be calculated by the equation

$$
C=885 \times 10^{-16} \times \frac{k a}{x}
$$

in which $k$ is the inductivity of the dielectric, $x$ is the thickness of the dielectric in centimeters, and $a$ is the area in square centimeters of the dielectric layer between the plates. This equation gives the value of $C$ in farads.

The capacity of a transmission line, the two wires being considered as the two "plates" of a condenser, is given by the formula

$$
C=\frac{1.943 \times l}{10^{8} \times \log _{10} \frac{d}{R}}
$$

in which $C$ is expressed in farads, and $l, d$ and $R$ have the same significance as in equation (8), that is, $l$ is the length of the line in miles.

13. Mechanical and electrical analogies. - The analogy between moment of inertia and inductance as pointed out in the discussion of inductance is but a small part of an extended analogy between pure mechanics and electricity. This extended analogy is here briefly outlined.

$$
x=v t \quad \text { (1) } \mid \quad \phi=\omega t \quad \text { (2) } \quad q=i t
$$

in which $x$ is the distance in which $\phi$ is the angle in which $q$ is the electric traveled in $t$ seconds by a turned in $t$ seconds by a charge which in $t$ seconds body moving at velocity $v$. body turning at angular flows through a circuit carvelocity $\omega^{\text {. }}$

$$
W=F x \quad(4) \quad W=7 \phi
$$
rying a current $i$.

in which $W$ is the work in which $W$ is the work in which $W$ is the work done by a force $F$ in pull- done by a torque $T$ in turn- done by an electromotive 
ing a body through the distance $x$.

$$
P=F v
$$

in which $P$ is the power developed by a force $F$ acting upon a body moving at velocity $\gamma$.

$$
W=\frac{1}{2} m v^{2}
$$

in which $W$ is the kinetic energy of a mass $m$ moving at velucity $v$.

$$
F=m \frac{d v}{d l}
$$

in which $F$ is the force required to cause the velocity of a body of mass $m$ to increase at the rate $\frac{d v}{d t}$

$$
\begin{gathered}
x=a F \\
\frac{4 \pi^{2} m}{\tau^{2}}=\frac{\mathbf{I}}{a}
\end{gathered}
$$

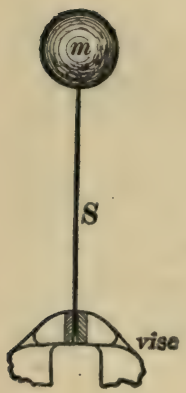

Fig. a. angle $\phi$.

$$
\text { . }
$$

in which $P$ is the power developed by a torque $T$ acting on a body turning at angular velocity $\omega$.

$$
W=\frac{1}{2} K \omega^{2}
$$

in which $W$ is the kinetic energy of a wheel of moment of inertia $K$ turning at angular velocity $\omega$.

$$
T=K \frac{d \omega}{d t}
$$

in which $T$ is the torque required to cause the angular velocity of a wheel of moment of inertia $K$ to increase at the rate $\frac{d \omega}{d t}$

$$
\begin{gathered}
\phi=b T \\
\frac{4 \pi^{2} K}{\tau^{2}}=\frac{\mathbf{I}}{b}
\end{gathered}
$$

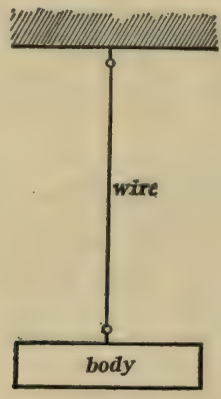

Fig. $b$, force $E$ in pushing a charge $q$ through a circuit.

$$
P=E i
$$

in which $P$ is the power developed by an electromotive force $E$ in pushing a current $i$ through a circuit.

$$
W=\frac{1}{2} L i^{2}
$$

in which $W$ is the kinetic energy of a coil of inductance $L$ carrying a current $i$.

$$
E=L \frac{d i}{d t}
$$

in which $E$ is the electromotive force required to cause a current in a coil of inductance $L$ to increase at the rate $\frac{d i}{d t}$

$$
\begin{gathered}
q=C E \\
\frac{4 \pi^{2} L}{\tau^{2}}=\frac{\mathbf{I}}{C} .
\end{gathered}
$$

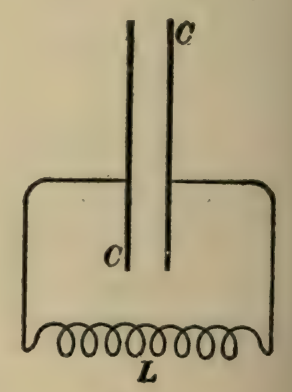

Fig. c.
A body of mass $m$ is supported by a flat spring $S$, clamped in a vise as shown in Fig. a. A force $F$ push. ing sidewise on $m$ moves it: a distance $x$, which is pro-
A body of moment of inertia $K$ is hung by a wire as shown in Fig. b. A torque $T$ acting on the body will turn the body and twist the wire through an angle
A condenser $C$ is connected to the terminals of a coil of inductance $L$ as shown in Fig. c. An electromotive force $E$ acting anywhere in the circuit 
portional to $F$, according $\mid \phi$, which is proportional to $\mid$ pushes into the condenser to equation (16). When $T$, according to equation a charge $q$, which is prostarted the body $m$ will con- (I7). When started, the portional to $E$, according tinue to vibrate back and body will vibrate about the to equation (18). When forth and the period $\tau$ of its wire as an axis and the started the electric charge vibrations is determined by equation (19). period $\tau$ of its vibrations is determined by equation (20). will surge back and forth through the coil, constituting what is called an oscillatory current and the period of one oscillation is determined by equation (2I). 


\section{APPENDIX B.}

14. The split-pole rotary converter.* - The discussion of the electromotive force relations of the synchronous converter in Art. 90 of Chapter IX is based on the assumption that the directcurrent brushes of the machine are fixed permanently in the neutral axis. Under these conditions, the voltage ratio of a rotary converter is very nearly fixed in value as shown in the table on page I84. A possible method for controlling the voltage between the direct-current brushes of a rotary converter (with unvarying voltage between the alternating-current terminals of the machine) would be to shift the direct-current brushes from the neutral axis. In this way the voltage between the direct-current brushes could be reduced from its normal value, as given in the table on page 184 , to zero. This method of voltage control is, however, impracticable because of the sparking which is produced at the direct-current brushes when the brushes are shifted from the neutral axis. To overcome this difficulty Mr. J. L. Woodbridge has proposed the split-pole converter in which each field pole is split into two (or three) parts. One of these parts is permanently excited and it produces the fringe of field which is necessary for sparkless reversal, as explained on page 162 of the first volume of this text. The other portion (or portions) of each pole piece is arranged so that its excitation may be increased or decreased at will, thus shifting the resultant axis of the field with respect to the stationary direct-current brushes. The result is equivalent to the shifting of the direct-current brushes in so far as the voltage relations of the converter are concerned. This type of rotary converter is manufactured by the General Electric Company.

* See paper by Comfort A. Adams, Proceedings of Am. Inst. Elec. Eng., June, I908, pp. 899-925. 
15. A simple representation of the action of the polyphase induction motor. - A clear idea of the action of the induction motor may be obtained with the least difficulty by considering an induction motor of which the stator is supplied with an indefinitely large number of phases of alternating current, each phase, of course, being supplied by a separate source. Figure 4 shows a scheme

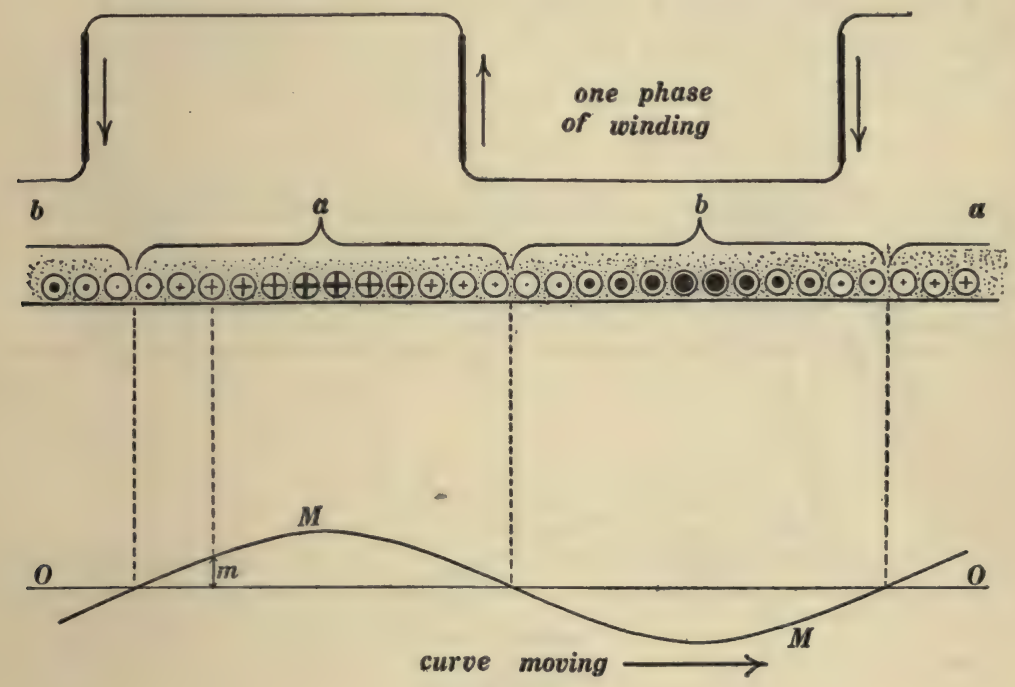

Fig. 4.

for representing the flow of currents in the stator conductors of such a multiphase induction motor. The small circles with dots and crosses represent the stator conductors (end view), and one phase of the winding is indicated in the upper part of the figure. Consider the sine curve $M M$, and imagine it to travel in the direction of the arrow along the axis 00 . Then the ordinate $m$ of this curve at any point represents the value of the current at the given instant in the stator conductor which is directly above $m$. Thus, the crosses of different sizes in the group $a$ of stator conductors represent currents of different values flowing away from the reader; and the dots of different sizes in the group $b$ represent currents of different values flowing towards the reader. 
The group $a$ of stator conductors is a group in which currents flow away from the reader, the group $b$ of stator conductors is a group in which currents flow towards the reader, and these two groups travel along with the curve $M M$ as the curve moves to the right, although, of course, the stator conductors themselves remain stationary.

Figure 5 represents a portion of the stator and rotor of a multiphase induction motor, and the ordinates of the moving curve $M M$ represent the values of the current in the stator conductors

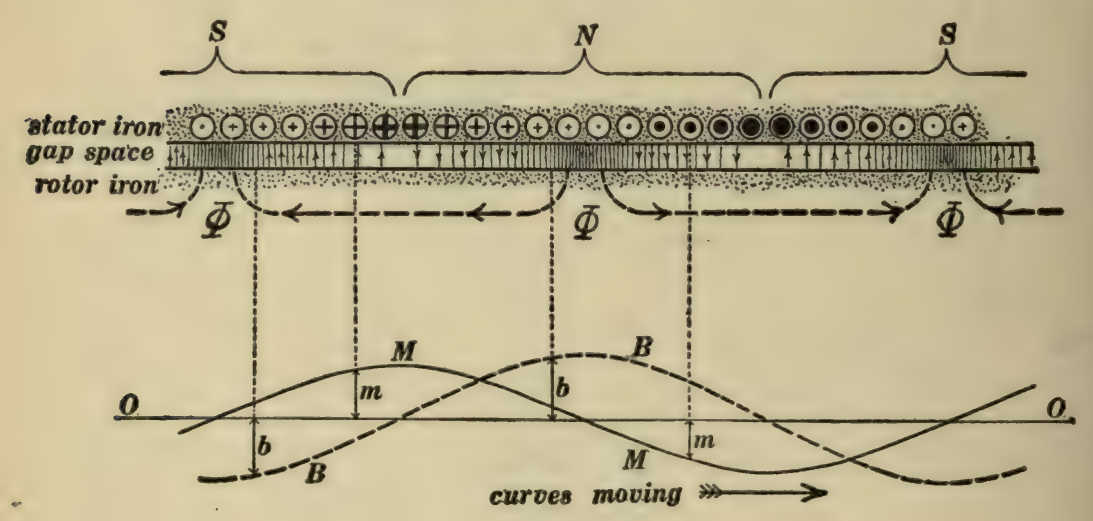

Fig. 5.

as in Fig. 4. A careful study of the figure will show that the groups $a$ and $b$ of Fig. 4 produce magnetic flux which crosses the gap space as indicated in Fig. 5, the regions $S, N$, $S$, etc., being regions of south polarity, north polarity, and south polarity, respectively, of the stator iron, and these polar regions travel along with the moving curve $M M$. The flux density at any point in the gap space is represented by the ordinate $b$ of the curve $B B$. The currents in the stator conductors, which are represented by the dots and crosses in Fig. 5, constitute what are called the magnetizing currents of the motor, inasmuch as their sole effect is to produce the moving state of magnetism which is represented by the moving curve $B B$. 
Figure 6 shows both the stator and rotor conductors. The rotor is understood to travel at a slower speed than the magnetism $B B$ of Fig. 5, and therefore the rotor conductors are being continually cut by the traveling flux so that electromotive forces are induced in the rotor conductors. These electromotive forces produce currents in the rotor conductors as represented by the dots and crosses in Fig. 6. The magnetizing action of these rotor currents is balanced by the magnetizing action of

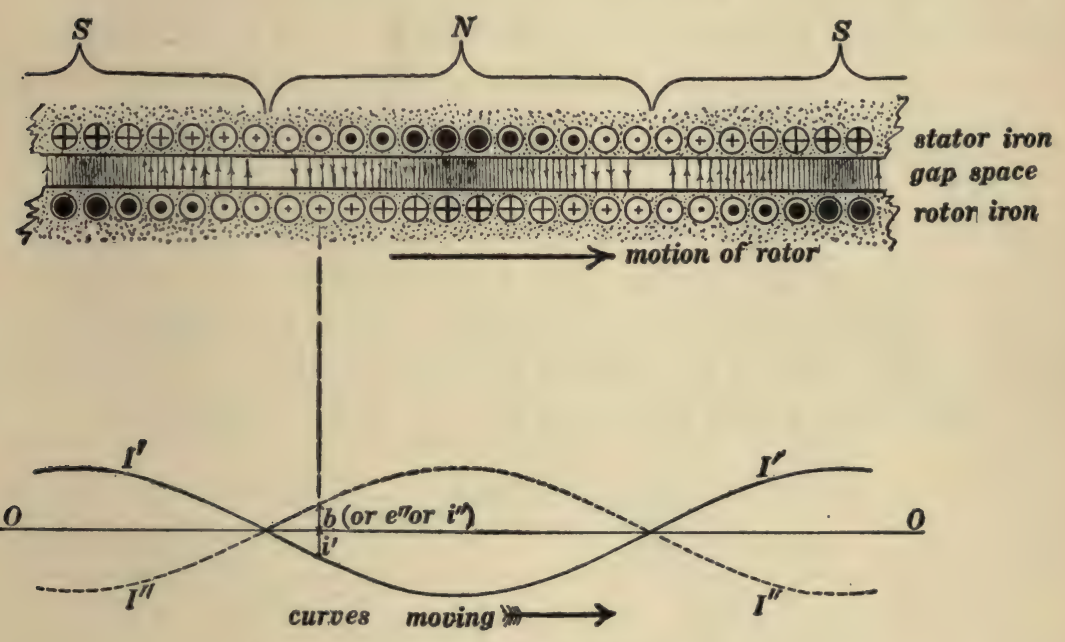

Fig. 6.

additional currents in the stator conductors. These additional currents in the stator conductors are called the load currents of the motor to distinguish them from the currents which are represented in Figs. 4 and 5 (magnetizing currents). A careful study of Figs. 5 and 6 will enable one to understand the time-phase relations of all the electromotive forces and currents involved in the action of the motor, and it also will show the interesting space relations between the magnetizing currents and the load currents in the stator winding. Thus, the magnetizing currents in the stator windings are at their maximum values in the regions between adjacent polar areas as shown in Fig. 5, whereas the load currents 
in the stator windings are at their maximum values in the regions in the middle of the polar areas. It will be noticed, furthermore, that the currents in the rotor conductors are at their maximum values in the regions where the flux density in the gap space has its maximum values. The torque which acts upon the rotor is due to the side forces with which the flux pushes on the rotor conductors.

\section{Induced electromotive forces in the rotor of a single-phase} induction motor. - The theory of the single-phase induction motor as developed in Art. I 39, Chapter XIII, is based upon a proposition concerning the relationship between the electromotive forces which are induced in the rotor rods by a pulsating flux, and the electromotive forces which are induced in the rotor rods by the cutting of the same flux. Consider, for example, the flux $\Phi$ in Fig. 253 of Chapter XIII. Let $n$ be the frequency of the singlephase alternating-current supply, let $n^{\prime}$ be the speed of the rotor, and suppose, for the sake of simplicity, that the motor is of the two-pole model as shown in Fig. 253. Consider (a) the

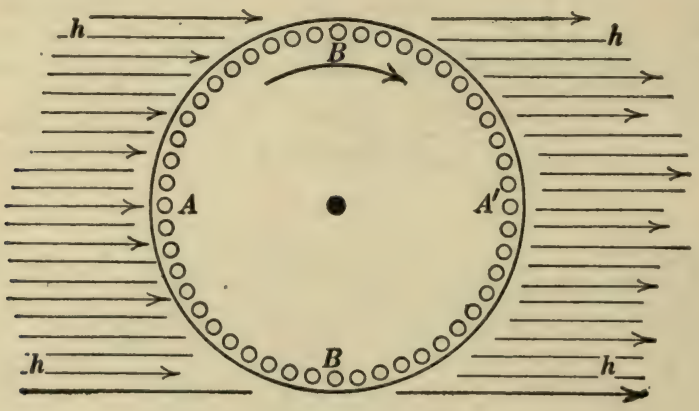

Fig. 7.

electromotive forces induced in the rotor rods by the cutting of $\Phi$ and $(b)$ the electromotive forces induced in the rotor rods by the pulsation of $\Phi$. The electromotive forces $(a)$ are in time quadrature with the electromotive forces $(b)$, the electromotive forces (a) are distributed about the axis $A A^{\prime}$ in exactly the same way that the electromotive forces $(b)$ are distributed about the axis $B B^{\prime}$, 
and the ratio of the electromotive forces (a) to the electromotive forces $(b)$ is equal to the ratio of $n$ to $n^{\prime}$. In order to establish these propositions, consider the arrangement shown in Fig. 7 , namely, a squirrel-cage rotor placed in a uniform magnetic field $h$. The radial component of this uniform field at the surface of the rotor is equivalent to a harmonically distributed flux. Furthermore, suppose that the uniform field $h$ pulsates in value in accordance with the equation

$$
h=H \sin 2 \pi n t .
$$

The motion of the rotor induces electromotive forces in the regions $A A^{\prime}$, and the pulsation of the field produces electromotive forces in the regions $B B^{\prime}$. Consider two pairs of opposite rotor rods $p p$ and $q q$, as shown in Fig. 8 , the angle $\beta$ having any given value. The above propositions are established if we can show that the electromotive forces induced in the rods $p p$ by pulsation are $n / n^{\prime}$ times as great in

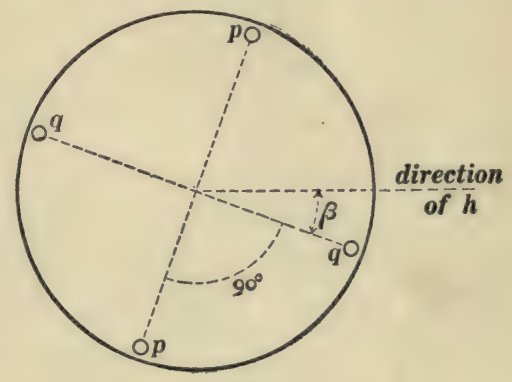

Fig. 8. value and in time quadrature with the electromotive forces which are induced in the rods $q q$ by motion. Let $r$ be the radius of the rotor and $l$ its length parallel to the motor shaft.

The radial component of $h$ at the rotor rods $q q$ is equal to $h \cos \beta$, and the velocity of the rods $q q$ is equal to $2 \pi n^{\prime} r$. Therefore the electromotive force which is induced in each of the rods $q$ by motion is equal to $2 \pi n^{\prime} r \times l \times h \cos \beta$, or, substituting the value of $h$ from equation (i), we have

$$
e_{q}=2 \pi n^{\prime} r l H \cos \beta \sin 2 \pi n t \text {. }
$$

The component of $h$ which is perpendicular to the plane of the turn of wire which is formed by the rotor rods $p p$ is equal 
to $h \cos \beta$, so that the total flux through this turn is equal to $2 r l h \cos \beta$, that

$$
\Phi_{p}=2 r l H \cos \beta \sin 2 \pi n t
$$

but, $d \Phi_{p} / d t(=4 n r l H \cos \beta \cos 2 \pi n t)$ is the electromotive force which is induced in the turn of wire $p p$ by pulsation, so that the electromotive force which is induced in one of the rods $p$ is

$$
e_{v}=2 \pi n r l H \cos \beta \cos 2 \pi n t .
$$

The above propositions are at once evident from equations (ii) and (iii).

A clear understanding of the theory of the single-phase induction motor as developed in Art. I 39 of Chapter XIII depends on a clear knowledge of the relationship between the two fluxes $\Phi$ and $\Phi_{c}$ in Fig. 253. The flux $\Phi$, which is assumed to be harmonically distributed, induces electromotive forces in the rotor rods in the regions $A A^{\prime}$ because of the motion of the rotor, and these electromotive forces due to the cutting of $\Phi$ are balanced by the electromotive forces which are induced in the regions $A A^{\prime}$ by the pulsation of the flux $\Phi_{c^{*}}$. A careful consideration of the above propositions will show, therefore, that the flux $\Phi_{c}$ is in time quadrature with the flux $\Phi$, that it pulsates at the same frequency as $\Phi$, and that its maximum value is equal to $n^{\prime} / n$ times the maximum value of $\Phi$.

The clock diagram of the single-phase induction motor as shown in Fig. 254 of Chapter XIII, involves a very interesting consideration of the question as to positive directions around the rotor (along a turn of the rotor winding) in the regions $A A^{\prime}$ and $B B^{\prime}$ respectively. To ignore this question of proper choice of positive directions makes it appear that the load current $I^{\prime}$ in Fig. 254 should be opposite in phase to the impressed primary voltage $E^{\prime}$, which, of course, is incorrect.

17. Rotor currents of the two-phase induction motor. - The discussion of equivalent resistance of the rotor per stator phase which is given on page 287 is based on the assumption that the 
magnetizing action of the "load current" in each " rod" of the stator winding is balanced by the magnetizing action of the current in the rotor rod which is immediately beneath it. This condition is approximately realized on account of the low magnetic reluctance of the local magnetic circuit which surrounds a stator slot and a rotor slot as shown in Fig. 247. Figure 9 represents

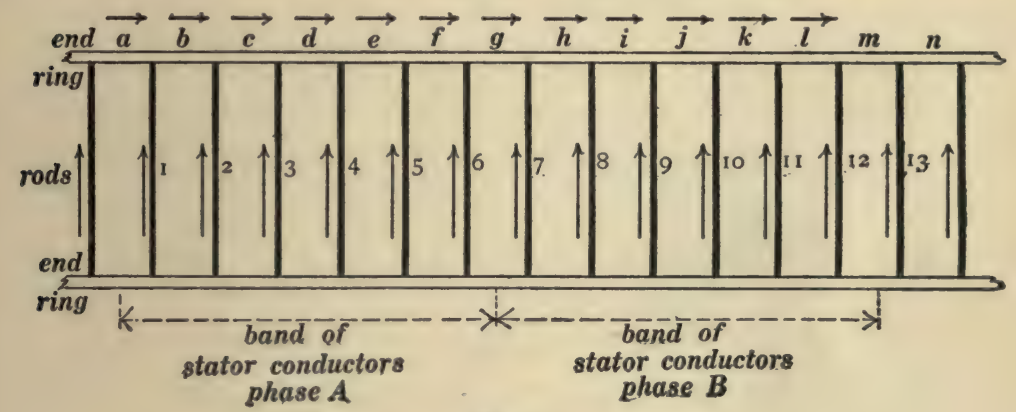

Fig. 9.

a surface view of the rotor rods and end-rings of a two-phase induction-motor rotor, and it shows the positions of two adjacent bands of stator conductors of phase $A$ and phase $B$, respectively. The arrows in Fig. 9 represent arbitrarily chosen positive directions. All of the rotor rods which lie under a stator band of phase $A$ carry equal currents which are in phase with each other, namely, the currents which are represented by the vectors I, 2, $3,4,5$ and 6 in Fig. IO; all of the rotor rods which lie under a stator band of phase $B$ carry equal currents which are in phase with each other, namely, the currents which are represented by the vectors $7,8,9$, IO, I I and I 2 of Fig. IO. It is evident that these two sets of currents must be in quadrature with each other because they balance the magnetizing actions of "load currents" in phase $A$ and phase $B$, respectively. . The currents which flow through the sections $a, b, c$, etc., of the end-rings cannot be completely determined from the vector diagram of currents*

* The determination of currents in a network of conductors depends upon the application of two principles, namely, Kirchhoff's first law together with the condition of minimum dissipation of power as $R I^{2}$ loss in the network. 
as will appear later. Let the vector $a$, Fig. IO, drawn from any chosen point $O$, represent the current in section $a$ of the upper end-ring in Fig. 9. It is evident from the choice of signs in Fig. 9 that the current in section $b$ is the vector sum of the currents $a$ and I, the current in section $c$ is equal to the vector sum of $b$ and 2 , etc. Therefore, in so far as the vector diagram

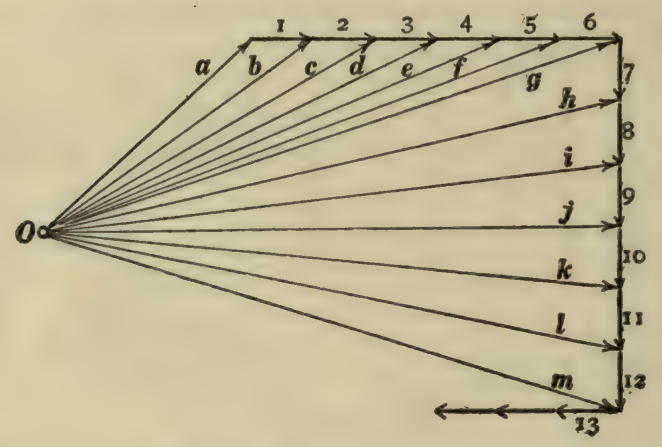

Fig. 10.

of currents is concerned, the currents in the respective sections of the end-rings may be represented by vectors drawn from any point $O$ to equidistant points on the sides of a square. A further condition, however, must be satisfied by the current distribution in the rotor, namely, that the $R I^{2}$ loss be a minimum. This condition is satisfied by choosing the point $O$ in Fig. 10 at the center of the square as shown in Fig. 248 of Chapter XIII. 


\section{APPENDIX C.}

\section{PROBLEMS.}

\section{Chapter I. The Alternator.}

1. A rheostat of 6 ohms resistance, in a circuit in which a steady current of IO amperes is flowing, is short-circuited 25 times per second, each time for $\mathrm{I} / \mathrm{I} 00$ second. What is the equivalent constant resistance of the rheostat? Ans. $4.5 \mathrm{ohms}$.

Note. - It is important that the physical argument of this problem be given by the student.

2. (a) At what speed must a 40-pole alternator like Figs. Io and I I be run to give a frequency of 25 cycles per second? (b) At what speed would the field magnet shown in Fig. I 2 have to be driven in a suitable stationary armature to give a frequency of 25 cycles per second? Ans. (a) 75 revolutions per minute, (b) 500 revolutions per minute.

3. An alternator is run at a speed of 3,000 revolutions per minute. (a) How many poles must its field magnet have to give a frequency of 10,000 cycles per second? (b) If the machine is constructed according to the design shown in Fig. I3, how many polar projections will be required on each end of the inductor? Ans. (a) 400 poles, (b) 200 poles.

Note. - See a description of a I0,000 cycles per second alternator by B. G. Lamme, Trans. Am. Inst. Elec., Vol. 23, pages 417 to 428 , 1904 .

4. An electromotive force of Ioo volts acts on a circuit containing resistance only, and the electromotive force is reversed every 0.01 second. The resistance of the circuit is Io ohms. Plot the electromotive force curve and plot the current curve; and find the average current during half a cycle and the effective current. Ans. Average current Io amperes, effective current ro amperes. 
5. The above repeatedly reversed electromotive force acts on a circuit containing inductance only. The inductance of the circuit is O. I henry. Plot the electromotive force curve and the current curve, and find $(a)$ the average current during half a cycle and (b) the effective current. Ans. (a) 2.5 amperes, (b) 2.89 amperes.

Note. - The value 2.89 is found by dividing the half-cycle into ro equal parts and measuring the middle ordinate of each part.

The current curve which is required in this problem is strictly indeterminate. $\mathrm{Al}_{1}$ that is known from the given data is that the current increases (or decreases) by the amount of ro amperes during the time that the electromotive force acts in a given direction. Plot the current curve on the assumption that the maximum positive and negative values of the current are equal to each other.

6. An electromotive force which is represented by the curve in Fig. $6 p$, acts on a circuit which contains a condenser only.

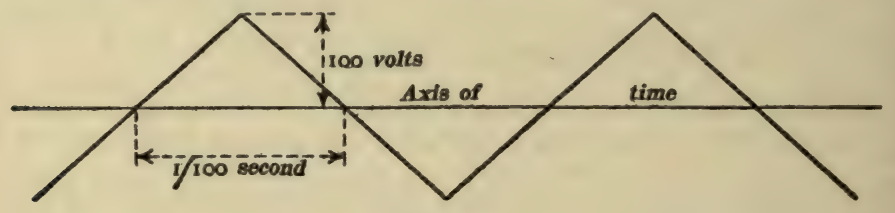

Fig. $6 p$.

The capacity of the condenser is Io microfarads. Plot the current curve and find $(a)$ the average current during half a cycle and $(b)$ the effective current. Ans. (a) 0.2 amperes, $(b) 0.2$ amperes.

Note. - The current is in this case at each instant equal to the product of the capacity in farads times the rate of change of the electromotive force.

7. If the frequency of the electromotive force were doubled in problems 5 and $\mathbf{6}$, how would the effective current be changed in each case? Ans. In problem 5 the effective current would be reduced to one half, and in problem 6 the effective current would be doubled.

Note. - It is important that the physical argument of this problem be given by the student.

8. A two-pole alternator, having one conductor on its armature, has pole pieces shaped as shown in Fig. $8 p$. The total flux emanating from the $N$ pole is I,000,000 lines, and the armature 
makes 25 revolutions per second. Plot the electromotive force curve, neglecting fringes of field at corners of pole pieces, and determine average value and effective value of the electromotive force during each half cycle. The long and short air gaps under the $180^{\circ}$ pole face are as $2: 1$. Ans. Average value is 0.5 volt during each half-cycle; effective value is 0.707 volt in the half-

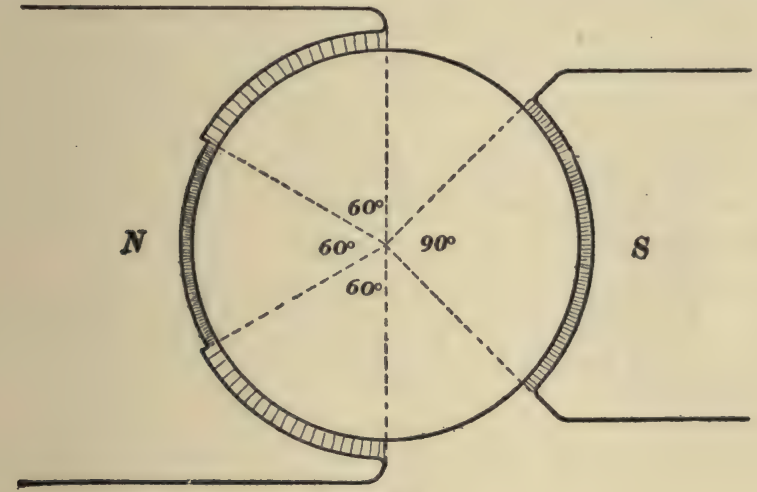

Fig. 8p.

cycle corresponding to the $90^{\circ}$ pole face, and 0.5303 volt in the half-cycle corresponding to the $180^{\circ}$ pole face.

9. Plot the curve of current which would be produced by the electromotive force of the alternator of problem 8 , in a circuit containing inductance only, the inductance of the circuit being O. I henry.

Note. - The current curve here required is strictly indeterminate, as explained in the note to problem 5. Determine the current curve on the basis of the assumption specified in the note to problem 5 .

10. Plot the curve of current which would be produced by the electromotive force of the alternator of problem 8 in a circuit containing a condenser but having a negligible resistance. If it is impossible to plot the curve state what assumptions in problem 8 and in this problem make the plotting impossible.

11. The following are instantaneous values, in volts, of the electromotive force of an alternator, taken at equal intervals during an entire circle: $0,30,60,80,90,95,90,80,60,30,0,-30$, $-60,-80,-90,-95,-90,-80,-60,-30$ and o. The 
corresponding values in amperes of the current are : $-65,-45$, $-25,0,25,45,65,75,78,75,65,45,25,0,-25,-45,-65$, $-75,-78,-75$, and -65 . Find the instantaneous values of the power, plot the curves of electromotive force, of current and of power, and find the average power. Ans. Average power is $2 \mathrm{I} 38.6$ watts.

12. Plot the curves of instantaneous power in problems $4,5,6$, and 9, and find the average power in each case. Ans. Average power is $\mathrm{I}, \mathrm{OOO}$ watts in problem 4 , zero in problems 5,6 and 9 .

13. Plot the electromotive force curve of the alternator specified in problem 8 on the assumption that it has 100 armature conductors spread out in one band $40^{\circ}$ wide.

Note. When a band of conductors is just beginning to enter the space under a pole face the electromotive force is zero (fringes of field ignored), and the electromotive force rises at a constant rate until the whole band is under the pole face.

\section{Chapter II. Alternating Current Measurements.}

14. The spring of a Siemens electrodynamometer is twisted through an angle of $220^{\circ}$ to balance the force action of a current of 18.8 amperes. What current will require a twist of $165^{\circ}$ ? Ans. 16.3 amperes.

15. Given a direct-reading ammeter (or voltmeter) of the directcurrent type of which the scale divisions are of equal length so that an observer can read, say, to $\frac{1}{10}$ of an ampere anywhere on the scale. (a) Find the percentage error due to the error of reading when the reading is I ampere; $(b)$ find ditto when the reading is Io amperes. Ans. (a) Io per cent.; (b) I per cent.

16. An electrodynamometer-ammeter (a Siemens instrument) has a scale of equal parts. The position of the pointer can be read to, say, $\frac{1}{2}$ of a division anywhere on the scale. (a) Find how many times as great the error in amperes (due to the given error in the reading) is when the reading of the pointer is 25 divisions than when the reading of the pointer is 250 divisions; $(b)$ find the percentage error in each case. Ans. (a) 3.16; (b) I per cent., $\frac{1}{10}$ per cent. 
17. Given a direct-reading ammeter (or voltmeter) of the alternating-current type of which the actual length of a scale division under the pointer is proportional to the reading of the pointer; a scale division being supposed to correspond to, say, o. I ampere over the entire scale. The position of the pointer can be read to a certain fraction of a degree of angle, so that the error in amperes due to an error of reading is inversely proportional to the length of the scale division under the pointer, that is, inversely proportional to the reading of the pointer. (a) Find how many times as great the error in amperes (due to an error in reading) is when the instrument reads I ampere than when the instrument reads Io amperes. (b) Find how many times as great the percentage error is in the first case than in the second case. Ans. (a) Io times as great; (b) IOO times as great.

Note. - Ammeters and voltmeters which are suitable for direct-current circuits, only, always give a deflection which is approximately proportional to the current or voltage. Therefore the scales of direct-reading instruments of this type are scales of approximately equal parts.

Ammeters and voltmeters which are suitable for both alternating and direct-current circuits always give a deflection which is nearly proportional to the square of the current or voltage. Let $y$ be the deflection of such an instrument read on a scale of equal parts, or, say in degrees, let $d y$ be one of the divisions of this scale, and let $x$ be the current or voltage corresponding to the deflection $y$. Then

$$
y=k x^{2} \text { and } d y=2 k x d x
$$

where $k$ is a constant. Now, in a direct-reading instrument each scale division corresponds to a certain constant fraction part of an ampere or volt which may be represented by $d x$. Then the length of a scale division of such a direct-reading instrument expressed in degrees is given by the value of $d y$, which, according to the above equation, is proportional to $x$, where $x$ is the reading in amperes or volts of the directreading instrument.

Problems 15, 16, and 17 touch upon a matter of very great practical importance, and two simple examples covering the entire ground may be worth while. A standardized direct-current ammeter (or voltmeter) which indicates 100 amperes (or volts) with a certain degree of precision will indicate 50 amperes (or volts) with the same actual error or with twice the percentage error. A standardized alternating-current ammeter (or voltmeter) which indicates Ioo amperes (or volts) with a certain degree of precision will indicate 50 amperes (or volts) with twice as large an actual error or with four times as large a percentage error.

18. An ammeter indicates 6.2 amperes of alternating current flowing through an arc lamp, a voltmeter indicates 82 volts across 
the arc, and a wattmeter indicates 440 watts delivered to the arc. What is the power factor of the arc? Ans. 0.865 .

19. A coil of wire which is connected across IIO-volt alternating current mains, takes Io amperes of current. The resistance of the coil is 2 ohms. What is the power factor of the coil ? Ans. 0.182.

20. A wattmeter is connected as shown in Fig. 20p. The resistance of the coil $B$ is 5 ohms. The wattmeter indicates 55 watts when used to measure the power delivered to a single lamp which takes one ampere of current. What is the true power delivered to the lamp? Ans. 50 watts.

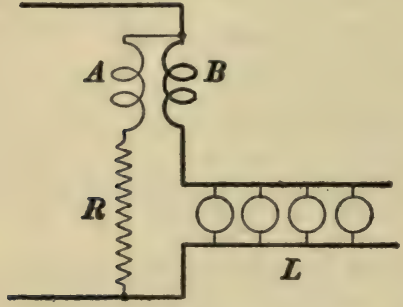

Fig. $20 p$.

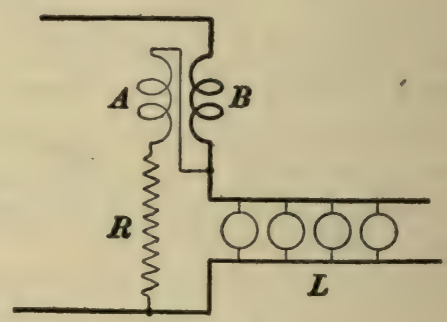

Fig. 21p.

21. A wattmeter is connected as shown in Fig. $2 \mathrm{I} p$. The resistance of the circuit $A R$ is 1,200 ohms. The wattmeter indicates 60 watts when used to measure the power delivered to a I ro-volt glow lamp. What is the true power delivered to the lamp? Ans. 49.92 watts.

Chapter III. Harmonic Electromotive Force and Current.

22. Two alternators $A$ and $B$ are connected in series. The electromotive force of $A$ is I, IOO volts, and the electromotive force of $B$ is 1,200 volts. The electromotive force of $A$ is $90^{\circ}$ ahead of the electromotive force of $B$ in phase. (a) What is their combined electromotive force? The two alternators give a current of 125 amperes which lags $30^{\circ}$ behind their resultant electromotive force in phase.

(b) What is the power output of 
each alternator? Ans. (a) I,628 volts; (b) output of $A 29,780$ watts, output of $B \quad 146,470$ watts.

23. The electromotive force of alternator $A$, problem 22 , is I $35^{\circ}$ ahead of the electromotive force of alternator $B$ in phase. A current of $\mathrm{I} 2 \mathrm{O}$ amperes flows through both alternators, lagging $30^{\circ}$ behind their resultant electromotive force. What is the output of each alternator? Ans. Output of $A 30,800$ watts negative, output of $B \quad 122,770$ watts positive. .

Note. - Negative output means intake as a motor.

24. Two coils $A$ and $B$ are connected in series across I IOvolt alternating-current mains. The voltage across $A$ is 76 volts and the voltage across $B$ is 80 volts. What is the phase difference between the voltages across $A$ and $B$, assuming all of the voltages to be harmonic? Ans. $89^{\circ} 39^{\prime}$.

Note. - It is impossible to determine from the data of this problem which of the voltages, $A$ or $B$, is ahead of the other in phase.

25. A non-inductive rheostat of which the resistance is 60 ohms is connected in series with an alternating-current fan motor to I IO-volt alternating supply mains. The voltage across the $60-0 h m$ rheostat is $62 \frac{1}{2}$ volts, and the voltage across the fan motor is 64 volts. Find $(a)$ the current flowing through the circuit, $(b)$ the phase difference between the current and the voltage across the fan motor, and $(c)$ the power delivered to the fan motor. Ans. (a) 1.04 amperes, (b) $59^{\circ} 10^{\prime},(c) 34.17$ watts.

Note. - It is impossible from the data of this problem to tell whether the voltage across the $60-o h m$ rheostat is ahead of or behind the voltage across the fan-motor in phase. As a matter of fact, the voltage across the $60-$ ohm rheostat is in phase with the current, and the voltage across the fan-motor is ahead of the current in phase.

26. An alternator delivers 200 amperes of current to glow lamps, and 75 amperes to start an induction motor. The power factor of the motor while starting is 0.3 . Find the total current. Ans. 233.7 amperes.

27. A harmonic alternating current of which the effective value is 15 amperes has a frequency of 133 cycles per second. Find the rate at which the current changes at the instant it passes through zero value, and $(b)$ find what value the current 
would reach if it were to continue changing at this rate for $\frac{1}{4}$ of a cycle. Ans. (a) I 7,725 amperes per second, (b) 33.3 amperes.

28. A 60 cycle alternator giving a harmonic electromotive force of which the effective value is I I v volts acts upon a circuit of which the inductance is 0.02 henry and of which the resistance is negligible. Find the effective value of the current produced. Ans. I4.6 amperes.

29. An alternator having a harmonic electromotive force of 140 volts (effective) delivers 200 amperes (effective) to a circuit of which the power factor is 0.70 . Find the maximum positive and maximum negative values of ei. Ans. 47,600 watts, $-8,400$ watts.

Note. - The algebraic expression for $e$ is $e=\mathbf{E} \sin \omega t$ where $\mathbf{E}=\sqrt{2} \times 140$ volts, and the algebraic expression for $i$ is $i=\mathbf{I} \sin (\omega t-\theta)$, where $\mathbf{I}=\sqrt{2} \times 200$ amperes, and $\theta$ is the angle whose cosine is 0.70 . Therefore

$$
e i=\mathbf{E I} \sin \omega t \sin (\omega t-\theta),
$$

and it is required to find the maximum and minimum values of this function.

30. (a) Find the power component and the wattless component of the current in the fan motor specified in problem 25, these components being taken with reference to the voltage across the terminals of the motor. (b) Find the power component and the wattless component of the voltage across the terminals of the fan motor in problem 25. Ans. (a) Power component 0.533 amperes, wattless component 0.893 amperes; $(b)$ power component 32.8 volts, wattless component 54.95 volts.

\section{Chapter IV, Fundamental Problems.}

31. A harmonic alternating current, maximum value 100 amperes, frequency 60 cycles per second, flows through a circuit consisting of a non-inductive resistance of $2 \mathrm{ohms}$, a resistanceless inductance of 0.003 henry, and a condenser of which the capacity is 0.00006 farad, all connected in series. Draw a clock diagram representing the phases of the electromotive forces across the resistance, across the inductance and across the condenser, respectively, and calculate the effective value of each. Ans. I4 I.4 volts across the resistance, 79.97 volts across the inductance, and 3,126 volts across the condenser. 
32. A circuit has an inductance of 0.2 henry and a resistance of 6 ohms. Calculate the effective value of the current produced by an electromotive force of which the effective value is Ioo volts and of which the frequency is 60 cycles per second. Calculate the phase difference between electromotive force and current. Ans. Current 1.32 amperes, phase difference $85^{\circ} 27^{\prime}$.

33. (a) Calculate the reactance value of 0.12 henry at 60 cycles per second. (b) Calculate the reactance value of a 20 microfarad condenser at 60 cycles per second. (c) Calculate the reactance of both in series. (d) At what frequency is the reactance value of both in series equal to zero? Ans. (a) $.45 .2 \mathrm{ohms,}$ (b) - I 32.6 ohms, (c) - 87.39 ohms, (d) IO2.7 cycles per second.

34. A harmonic electromotive force of I, IOO volts (effective) produces a harmonic current of 100 amperes (effective) in a given circuit, the current being $20^{\circ}$ behind the electromotive force in phase. Calculate the resistance, reactance, and impedance of the circuit. Ans. Resistance $10.34 \mathrm{ohms}$, reactance $3.76 \mathrm{ohms,}$ impedance II ohms.

35. A harmonic electromotive force of I IO volts produces a current of 15 amperes in a receiving circuit of which the power factor is equal to 0.7. Find the values of resistance, reactance, and impedance. Ans. Resistance 5.13 ohms, reactance \pm 5.24 ohms, impedance 7.33 ohms.

Note. - From the data of this problem it is impossible to determine whether the current is ahead of or behind the electromotive force in phase. It is desirable always in specifying the value of a power factor of a receiving circuit to state whether the current leads or lags; thus one would speak of a 70 per cent. leading power factor or a 70 per cent. lagging power factor, as the case may be.

36. Alternating-current mains deliver 100 amperes of current to a non-inductive circuit, to glow lamps for example. An inductive circuit of negligible resistance is then connected to the mains and it takes Io amperes. What is the total current delivered by the mains? Ans. 100.5 amperes.

37. From the data of problem 34, find the conductance, susceptance and admittance of the circuit. Ans. (a) 0.0854 recipro- 
cal ohms, (b) 0.03I I reciprocal ohms, (c) 0.0909 reciprocal ohms.

Note. - The values of conductance, susceptance, and admittance are expressed in terms of a unit which is the reciprocal of the ohm, just as the conductivity (reciprocal of the resistance of a circuit) is expressed in terms of the reciprocal of the ohm.

38. An inductance of 0.08 henry and a condenser of 25 microfarads capacity are connected in series, the resistance of the circuit being 7 ohms. (a) What is the critical frequency of the circuit, that is to say, at what frequency does resonance occur? (b) What is the effective value of the electromotive force across the condenser terminals when an electromotive force of I IO volts at the critical frequency acts upon the circuit? Ans. (a) I I 2.5 cycles per second, (b) 888.9 volts.

39. An inductance of O. I 2 henry and a condenser of which the capacity is 25 microfarads are connected in parallel. The resistance of the inductive circuit is $6 \mathrm{ohms}$ and the resistance of the condenser circuit is I ohm. What is the critical frequency at which resonance occurs? Ans. 9I.9 cycles per second.

40. An inductance of $\mathrm{O}$. I henry and a resistance $R$ are connected in series to I ro-volt 60-cycle mains. Calculate the current values when $R$ equals zero, when $R$ equals one ohm, when $R$ equals 5 ohms, when $R$ equals ro ohms, when $R$ equals $20 \mathrm{ohms}$, when $R$ equals $50 \mathrm{ohms}$, and when $R$ equals IOO ohms, and plot a curve of which the abscissas represent $R$ and the ordinates represent corresponding values of the current. Ans. 2.9I 8 amperes, 2.9I7 amperes, 2.893 amperes, 2.82 I amperes, 2.578 amperes, I.757 amperes, and 1.029 amperes.

41. A non-inductive resistance of $20 \mathrm{ohms}$, a resistance-less inductance of 0.06 henry, and a condenser of 105 microfarads capacity are connected in series to I Io-volt 60-cycle mains. Find the electromotive force, $(a)$ between the terminals of the resistance, $(b)$ between the terminals of the inductance, and $(c)$ between the terminals of the condenser. Ans. (a) I09.o volts, $(b)$ I 23.3 volts, $(c)$ I 37.8 volts.

42. A transmission line of which the resistance is $4 \mathrm{ohms}$ and 
the reactance is $3 \mathrm{ohms}$ delivers 100 amperes to a non-inductive receiving circuit, the electromotive force across the terminals of the receiving circuit being 10,000 volts. (a) What is the value of the generator voltage, and $(b)$ what is the phase difference between generator voltage and the voltage across the receiving circuit? Ans. (a) 10,404.3 volts, (b) $\mathbf{I}^{\circ} 39^{\prime}$.

43. A sensibly non-inductive transmission line having a resistance of 4 ohms delivers 100 amperes of current to a receiving circuit. The phase difference between voltage and current in the receiving circuit being sensibly $90^{\circ}$, the voltage across the terminals of the receiving circuit is 10,000 volts. What is the value of the generator voltage? Ans. 10,008 volts.

44. If the transmission line specified in problem 42 were to deliver 100 amperes of current at 10,000 volts to a condenser, what would be the value of the generator voltage? Ans. 9,703. I volts.

45. The transmission line specified in problem 42 delivers 100 amperes of current at 10,000 volts to a receiving circuit of which the power factor is 0.7 . What is the value of the generator voltage? Ans. 10,494.6 volts.

46. Two condensers, each of negligible resistance, have capacities of 0.5 and 0.05 microfarad respectively. The two condensers are connected in series to I, IOO-volt alternating-current mains. What is the electromotive force between the terminals of each condenser? Ans. IOO volts, and I,000 volts respectively.

Note. - The student should give the physical argument of this problem.

47. An electrostatic voltmeter has a capacity of 0.0006 microfarad when its deflection is $a$, and 0.0008 microfarad when its deflection is $b$. The electromotive force between the terminals of the instrument is 75 volts when its deflection is $a$, and 125 volts when its deflection is $b$. An auxiliary condenser of 0.007 microfarad capacity is connected in series with the instrument. Find the electromotive forces necessary to produce deflections $a$ and $b$. Ans. 81.4 volts, and $\mathrm{I} 39.3$ volts, respectively.

48. A direct-reading electrostatic voltmeter having 0.0006 
microfarad capacity is connected through 50,000 ohms noninductive resistance to 6o-cycle mains. Find the percentage error* of the readings of the instrument, and state whether it indicates too high or too low. Ans. 0.0064 per cent. too low.

49. The above electrostatic voltmeter is connected in series with an inductance of 2 henrys, resistance negligible. Find the percentage error of the voltmeter readings when a frequency of 60 cycles is used, and state whether it indicates too high or too low. Ans. 0.001 7 per cent. too high.

50. An electrodynamometer having an inductance of 0.02 henry and a resistance of I,500 ohms gives the same deflection for a certain 60-cycle electromotive force as it does for 127.5 volts direct electromotive force. What is the effective value of the 6o-cycle electromotive force? Ans. 127.5016 volts.

51. An electrodynamometer has a resistance of $500 \mathrm{ohms}$. When used as an alternating voltmeter at a frequency of 60 cycles per second its percentage error is $\frac{1}{10}$ per cent. What is its inductance? Ans. 0.0593 henry.

52. An alternator delivers current to two receiving circuits in parallel. One receiving circuit takes 20 amperes and its power factor is 0.9 , and the other receiving circuit takes 25 amperes and its power factor is 0.7 . What is the power factor of the combination? Ans. o.801.

53. An alternator delivers 100 amperes at 1,100 volts and 60 cycles per second to a receiving circuit of which the power factor is 0.85 . What capacity condenser would be required to compensate for lagging current? What number of leaves of paraffined paper 22 by 27 centimeters would be required for this condenser, thickness of paraffined paper being 0.08 centimeter, allowing I centimeter margin beyond the tinfoil ? Take inductivity of paraffined paper equal to 2. Ans. 127.0 microfarads, I 4,800 leaves.

* In this and subsequent problems the percentage error of an instrument is understood to mean the difference between the instrument reading and the true value of the quantity measured, divided by the true value of the quantity. 
Note.-See Arts. IO, II, and 12 of Appendix $A$ for a discussion of the condenser.

\section{Chapter V. The Use of Complex Quantity.}

54. An alternating current of 100 amperes flows in a circuit. Choosing the current vector as a reference axis, the two components of $E$ are 700 volts and 200 volts respectively; that is, $E=700$ volts $+j \times 200$ volts. Find the resistance and the reactance of the circuit. Ans. 7 ohms resistance and 2 ohms reactance.

55. An alternating electromotive force of 1,000 volts acts on a circuit. Choosing the electromotive force as the reference axis, the components of $I$ are 75 amperes and 20 amperes; that is, $I=75$ amperes $+j \times 20$ amperes. Find the resistance, reactance, impedance, conductance, susceptance, and admittance of the circuit. Ans. 12.45 ohms resistance, $-3.32 \mathrm{ohms}$ reactance, $12.88 \mathrm{ohms}$ impedance, 0.075 reciprocal ohms conductance, 0.02 reciprocal ohms susceptance, 0.0776 reciprocal ohms admittance.

56. The components of the current specified in problem 54 with reference to an arbitrary axis are 80 amperes and 60 amperes respectively; that is, $I=80$ amperes $+j \times 60$ amperes. The resistance of the circuit is 7 ohms and the reactance of the circuit is 2 ohms. Find the components of $E$ with reference to the same arbitrary axis. Ans. $x$-component 440 volts, $y$ component 580 volts.

57. Given $E=100$ volts $+j \times 60$ volts and $I=3$ amperes $-j \times 2$ amperes. Find $(a)$ effective values of $E$ and $I,(b)$ power delivered to the circuit, $(c)$ resistance of the circuit, $(d)$ reactance of the circuit, $(e)$ impedance of the circuit, $(f)$ conductance of the circuit, $(g)$ susceptance of the circuit, and $(h)$ admittance of the circuit. Ans. (a) 116.6 volts and 3.61 amperes, $(b) 180$ watts, $(c) 13.85$ ohms, $(d) 29.23$ ohms, $(e) 32.35$ ohms, $(f) 0.01324$ reciprocal ohms, $(g) 0.02794$ reciprocal ohms, and $(h) 0.03091$ reciprocal ohms.

Note. - It is instructive in calculating power to use the formula on Art. 45, page 95, and also to use the formula $P=R I^{2}$.

58. An electromotive force of which the components are 50 volts and 75 volts respectively; that is, $E=50$ volts $+j \times 75$ volts, produces a current through a circuit of which the resistance is 10 ohms and the reactance is -6 ohms. Find the components of the current and find the power delivered to the circuit. Ans. $x$-component of current 0.368 amperes, $y$-component of current 7.72 amperes, power 597.5 watts.

59. Separate the components of the complex expression

$$
\left(\frac{a+j b}{c+j d}\right)(e+j f) \text {. }
$$

Ans. Real component $\frac{e(a c+b d)+f(a d-b c)}{c^{2}+d^{2}}$,

$$
j \text {-component } \frac{f(a c+b d)+e(b c-a d)}{c^{2}+d^{2}} .
$$

60. Separate the components of the complex expression

$$
E_{1}=r_{1} I_{1}+j \omega L_{1} I_{1}+\frac{\omega^{2} M^{2} I_{1}}{r_{2}+j \omega L_{2}}
$$


Note. - See page 97 .

61. Show that $E(\cos \theta+j \sin \theta)$ is $\theta^{\circ}$ ahead of $E$ in phase and that its numerical value is equal to the numerical value of $E$.

62. Find the expression for $\sin 4 x$ in terms of $\sin x$ and $\cos x$, using De Moivre's theorem.

63. Find the expression for $\sin (x+y+8)$ in terms of sines and cosines of $x$, $y$, and 2.

64. A transformer (without iron) consists of two long cylindrical coils each having Io turns of wire per centimeter of length (one layer). The coils are each 50 centimeters long and their radii are 2 centimeters and 3 centimeters respectively, the smaller coil being inside the larger. Calculate the value and phase of the electromotive force required to maintain a current of ro amperes at 60 cycles per second in the outer coil ; calculate the current in the inner coil, and calculate the true and apparent reactance, and apparent resistance of the outer coil. The outer coil has 2 ohms resistance and the inner coil has $1 \mathrm{I} / 2$ ohms resistance and its terminals are short-circuited. Ans. 21.61 volts, $17^{\circ} 45^{\prime}$ phase difference, 1.944 amperes, 0.6697 ohms true reactance, $0.6584 \mathrm{ohms}$ apparent reactance, $2.0568 \mathrm{ohms}$ resistance.

Note.-See Appendix A for formula, for calculating the inductance of a long coil.

The mutual inductance, in henrys, of two coaxial solenoids is, approximately,

$$
M=4 \pi^{2} r^{\prime \prime 2} z^{\prime} z^{\prime \prime l} \div 10^{9}
$$

in which $z^{\prime}$ and $z^{\prime \prime}$ are the turns of wire per unit length on the respective coils, $r \prime \prime$ is the radius of the inside coil, and $l$ is the length of the coils.

65. The expression (iii) page 97 may be written

$$
\left[E_{1}=\left[\left(r_{1}+R\right)+j\left(x_{1}-X\right)\right] I_{1}\right.
$$

where $R$ is the apparent change of resistance of the primary coil due to the presence of the secondary coil, and $X$ is the apparent change of reactance of the primary coil due to the presence of the secondary coil. The primary coil has in circuit with it an adjustable non-inductive resistance so that $r_{1}$ may be varied at will. Assume that $R$ and $X$ are small compared with $r_{1}$ and $x_{1}$ so that $R^{2}$ and $X^{2}$ are negligible. Show that the impedance (numerical value) of the primary circuit is not altered by the presence of the secondary when $r_{1} / x_{1}=X / R$. How is the impedance (numerical value) of the primary circuit affected by the presence of the secondary when $r_{1}$ is less than $x_{1} X / R$; when $r_{1}$ is greater than $x_{1} X / R$ ?

\section{Chapter VI. The Polyphase System.}

66. The distance from center to center of adjacent poles of an alternator measured on the surface of the armature is 10.4 inches. How far apart must two armature conductors be placed so that there may be a phase difference of $55^{\circ}$ in the electromotive forces induced in the respective conductors? Ans. 3.18 inches.

67. A common return wire is used for the two currents of a two-phase system. The system is balanced and each current is 
equal to IOO amperes. What is the current in the common return wire? Ans. 141.4 amperes.

68. The electromotive force of each phase, problem 67 , is 500 volts. What is the electromotive force between the outside wires? Ans. 707 volts.

69. A three-phase alternator is provided with 4 collecting rings and 4 mains as shown in Fig. 94. Three similar receiving circuits are connected as follows: One from main I to main 4, one from main 2 to main 4 , and one from main 3 to main 4 . Each receiving circuit takes $\mathrm{I} 50$ amperes. When the armature windings are properly connected to the collector rings the current in main 4 is zero. What is the current in main 4 when one armature winding has its connections reversed? Draw a diagram showing the phase relations between the currents in mains I, 2, 3 , and 4 when the armature winding $A$, Fig. 94, is reversed. In constructing this diagram consider directions outwards from machine as the positive direction in each main. Ans. 300 amperes.

70. Three similar receiving circuits are $\Delta$-connected to 3-phase mains, the electromotive force between each pair of mains being I Io volts. The power delivered to the three circuits is I5O kilowatts and the power factor of each circuit is 0.90. What is the current in each circuit and in each main? Ans. 505 amperes, 875 amperes.

71. Three similar receiving circuits are $\mathrm{Y}$-connected to the 3phase mains, problem $7 \mathrm{I}$; the total power delivered is I 50 kilowatts and the power factor of each circuit is .90. What is the current in each circuit and in each main, and what is the electromotive force between the terminals of each circuit? Ans. 875 amperes, 875 amperes, 63.5 volts.

72. A given 3-phase alternator is provided with six collector rings so that it may be connected, $\Delta$ or $\mathrm{Y}$, for experimental purposes. The electromotive force developed by each winding is IOO volts. Find $(a)$ the current in each receiving circuit, $(b)$ the current in each main, $(c)$ the voltage between mains and $(d)$ the 
current in each armature winding, when the alternator is $\Delta$-connected to the mains, and the mains are $\mathrm{Y}$-connected to three similar receiving circuits, each one of which has a resistance of 4 ohms and a reactance of 3 ohms. Ans. (a) I I.55 amperes, (b) same as $(a),(c)$ IOo volts, $(d) 6.67$ amperes.

73. Find $(a)$ the current in each receiving circuit, $(b)$ the current in each main, $(c)$ the voltage between mains, and $(d)$ the current in each armature winding, when the generator specified in problem 72 is $\mathrm{Y}$-connected to the mains and the mains are $\Delta$-connected to the given receiving circuits. Ans. (a) 34.64 amperes, $(b) 60$ amperes, $(c)$ I 73.2 I volts, $(d) 60$ amperes.

74. The electromotive force developed in each winding of a 2phase alternator is 100 volts. What is the electromotive force given by the two windings connected in series? Ans. 14I volts.

75. The electromotive force developed in each winding of a 3 -phase alternator is IOO volts. What is the electromotive force developed by the three windings in series? Ans. 200 volts, or zero.

76. Two watt-meters are arranged as shown in Fig. I05 to measure the power delivered to three similar receiving circuits each having a power factor equal to 0.75 . The total power delivered to the three circuits is 20 kilowatts. Find the reading of each watt-meter. Draw a vector diagram similar to Fig. 106 representing the relationship between voltages and current.

Note. - This problem is based on the assumption that the electromotive forces and currents are harmonic.

Chapter ViI. Voltage and Voltage Regulation of Alternators.

77. The alternator represented in Fig. 107, Chapter VII, is a 4-pole single-phase machine having 8 conductors in the 8 slots, as specified on page $\mathrm{I} 2 \mathrm{O}$. The speed of the machine is 30 revolutions per second and the flux emanating from each pole is $\mathrm{I}, 000,000$ lines. Calculate the effective value of the electromotive force generated by the machine. Ans. I I.03 volts.

78. Assuming the field flux of an alternator to be harmonically distributed, as explained on page I $2 \mathrm{I}$, calculate the phase con- 
stant of an armature winding in which the conductors lie on the surface of a smooth armature core as bands, the width of each band being $\frac{3}{5}$ of the distance from center to center of adjacent field poles.

Note. - The data of this problem are purposely chosen so that the answer to the problem may not be given in the table on page 124. Assume each band to have an indefinitely large number of conductors.

79. Using the data in Figs. I I 7 any I I 8, referring to a given 2,000 kilowatt delta-connected 3-phase alternator, calculate the half-load regulation of the machine for the case in which the receiving circuits have a power factor equal to 0.6 ; $(a)$ by the electromotive force method, and $(b)$ by the magnetomotive force method.

Note. - The specifications in this problem are purposely chosen so that the answer to the problem may not be included in the examples given on pages $134-137$.

80. A ten-pole alternator having 720 armature conductors supplies 30 amperes to a receiving circuit of which the power factor is 0.866 . Calculate the average demagnetizing ampere-turns due to the armature current. Ans. 763 ampere-turns.

\section{Chapter ViII. The Synchronous Motor.}

81. Plot curves showing the values of $P^{\prime}, P^{\prime \prime}$ and $R I^{2}$ for two synchronous alternators in series, the alternator $A$ having an electromotive force of $\mathrm{I}, 200$ volts, the other machine $B$ an electromotive force of 800 volts, the resistance of the circuit being I ohm and the reactance 2 ohms. Result similar to Fig. I 27.

82. An alternator $A$ has an electromotive force of 1, I OO volts, a resistance of $\mathrm{I} \mathrm{ohm}$, and a reactance of $0.58 \mathrm{ohm}$. The machine is driven as a synchronous motor with zero load, from $\mathbf{I}, 000$-volt mains. (a) What is the value of the current? (b) What is the component of this current which is $90^{\circ}$ ahead of the supply electromotive force in phase? (c) What capacity of condensers would take the same amount of leading current from the $1,000-$ volt mains at a frequency of 60 cycles per second? Ans. (a) 21 I.4 amperes, (b) 206.6 amperes, (c) 548 microfarads.

83. Two governed engines drive two alternators which are connected in multiple and feed one pair of mains. Engine $A$ drops from 150 revolutions per minute at zero load to 145 revolutions per minute at 100 horse power. Engine $B$ drops from 150 
revolutions per minute at zero load to $\mathbf{1 4 7}$ revolutions per minute at full load of 75 horse power. The station output is such as to require a total of 125 horse power to be delivered by the engines. What power is delivered by each engine, and what is their common speed? Ans. (a) 55.6 H. P., (b) 69.4 H. P., (c) 147.22 r. p. m.

84. An alternator $B$, of which the electromotive force is 1,000 volts, takes 75 kilowatts from alternator $A$. To what value must the electromotive force of $A$ be adjusted, so that the efficiency of transmission may be maximum, and what is the corresponding value of the current, resistance of circuit being $\mathbf{I} \mathrm{ohm}$ and reactance of circuit being $0.5^{8} \mathrm{ohm}$ ? Ans. 1,076 volts, 75 amperes.

85. The electromotive force of alternator $A$ in problem 84 is 1,076 volts. To what value must the electromotive force of alternator $B$ be adjusted to give maximum efficiency of transmission, and what is the corresponding value of the current, intake of $B$ being 75 kilowatts? Ans. 1,002 volts, 74.9 amperes.

86. The electromotive force of alternator $A$ is $\mathbf{I}, 100$ volts, and that of alternator $B$ is $\mathbf{I}, 000$ volts, resistance of circut is $\mathrm{I}$ ohm and reactance of circuit $0.5^{8} \mathrm{ohm}$. What is the angular lag of $B$ behind $A$, for which power is most efficiently transmitted from $A$ to $B$, what is the intake of $B$ under these conditions, what is the output of $A$, and what is the efficiency? Ans. $181.8^{\circ}, P^{\prime \prime}=-92$ kilowatts, $P^{\prime}=$ I0r. 8 kilowatts, efficiency 91.3 per cent.

87. What is the angular lag $\phi$ of $B$ behind $A$, in problem $8 \mathrm{I}$ for which power is most efficiently transmitted from $B$ to $A$; what is the intake of $A$ under these conditions, what is the output of $B$, and what is the efficiency? Ans. 159.47 $P^{\prime}=-36.4$ kilowatts, $P^{\prime \prime}=148$ kilowatts, efficiency 23.2 per cent.

88. The electromotive force of $A$ is 900 volts, that of $B$ is 800 volts, resistance of circuit is I ohm, and reactance of circuit is I ohm. What is the maximum intake of machine $B$ as a synchronous motor? Ans. I 89. I kilowatts.

89. What is the greatest value of $B(A, \omega L$, and $R$ being as in problem 84 ) which will permit machine $B$ to act as a synchronous motor. Ans. I,243.9 volts.

90. What value must $B$ have in order that the maximum intake of machine $B$ (problem 89) may be the greatest possible, and what is this intake? Ans. 621.9 volts, 289.4 kilowatts.

91. Given $A=800$ volts, $R=\mathbf{I} \mathrm{ohm}, \omega L=\mathbf{I} \mathrm{ohm}, P^{\prime \prime}=30$ kilowatts ; plot a curve showing different values of $I$ corresponding to different values of $B$. Result similar to Fig. 143.

92. An alternator of which the electromotive force is $I 50$ volts is to be run as a motor from IIO-volt mains. The resistance of the alternator armature is $\mathbf{I}$ ohm. What is the minimum amount of inductance required in the circuit, the frequency being 60 cycles per second? Ans. 0.00246 henry.

93. Suppose a condenser were to be connected in series with two alternators $A$ and $B$ so that the total reactance of the ciruit will be $-0.5^{8} \mathrm{ohm}$; the resistance of the circuit being I ohm, the electromotive force of machine $A$ I, 100 volts, and the electromotive force of machine $B$ I, 000 . Plot curves showing the values of $P^{\prime}$ and $P^{\prime \prime}$, and $R L^{2}$ for various values of $\phi$. 


\section{Chapter IX. The Synchronous Converter.}

94. The plain multicircuit ring-wound armature of a six-pole direct-current dynamo has 360 conductors on its face. These conductors are numbered from I to 360 .

(a) One collecting ring of a rotary converter is connected to conductor No. I. To what other conductors must this ring be connected? Ans. $A$ to $\mathrm{I} 2 \mathrm{I}$ and $24 \mathrm{I}$.

$(b)$ To what conductors must a second ring $(B)$ be connected to give a two-ring converter? Ans, $B$ to $6 \mathrm{I}, \mathrm{I} 8 \mathrm{I}$ and 301 .

(c) To what conductors must two additional rings $B$ and $C$ be connected to give a three-ring converter? Ans. $B$ to $4 \mathrm{I}, \mathrm{I} 6 \mathrm{I}$ and $28 \mathrm{I}, C$ to $8 \mathrm{I}, 2 \mathrm{I}$ and $32 \mathrm{I}$.

(d) To what conductors must three additional rings $B, C$ and $D$ be connected to give a four-ringed? Ans. $B$ to $3 \mathrm{I}, \mathrm{I} 5 \mathrm{I}$ and 27I, $C$ to $6 \mathrm{I}, \mathrm{I} 8 \mathrm{I}$ and $30 \mathrm{I}, D$ to $9 \mathrm{I}, 2 \mathrm{II}$ and $33 \mathrm{I}$.

(e) To what conductors must four additional rings $B, C, D$ and $E$ be connected to give a five-ring converter? Ans. $B$ to 25, 145 and $265, C$ to 49,169 and $289, D$ to 73,193 and $313, E$ to 97,217 and 337 .

95. A four-pole, two-circuit, single drum winding has 102 conductors numbered consecutively from I to 102 . The conductors are connected as follows: $1-26-5$ I-76-IOI . . 28-53-78 and back to $\mathrm{I}$. To what conductors must the three rings $A, B$ and $C$, of a three-ring converter be connected? Ans. $A$ to No. I, $B$ to No. 35 , and $C$ to No. 69 .

96. Make a diagram of the following winding and show three collecting rings connected to conductors I, 19 and 37 . The winding is a four-pole, two-circuit, single drum winding with 54 conductors connected up as follows : I-I 4-27-40-53 . . 3-1629-42 and back to I.

97. A 50-kilowatt direct-current dynamo is to be used as an $n$-ring converter. What is its capacity rating when $n=2$, when $n=3$, when $n=4$, and when $n=6$ ? Ans. $42.5,66,8 \mathrm{I}$, and 96 kilowatts. 
98. A two-ring converter with negligible armature resistance and reactance takes current over a $0.2-\mathrm{ohm}$ line through a reaction coil having $0.3 \mathrm{ohm}$ reactance. The effective alternating electromotive force at the generator terminals is kept constant at I 20 volts. The excitation of the converter can be varied at will. Find greatest value of alternating electromotive force of converter for which it can run as a synchronous motor, and find the corresponding value of the direct electromotive force of the converter. Ans. 216.25 volts and 306 volts, respectively.

99. Plot the curve of which the ordinates represent the instantaneous values of the current in an armature conductor of a four-pole, three-ring converter with multicircuit winding, the value of the direct current output of the machine being 250 amperes: (a) When the conductor is adjacent to the connection of a ring, and $(b)$ when the conductor is midway between the connections of two rings.

Note. - It is to be remembered that the discussion given in Arts. 91 to 95 of Chapter IX refers explicitly to the two-pole machine with two current-paths between its direct-current brushes. The necessary modifications of the formula given in Arts. 91 to 95 are explained in the footnoote on page 186.

100. If a 100-kilowatt direct current generator were to be provided with five collector rings forming a five-ring converter, what would its power rating be as based on average armature heating?

Note. - The specifications in this problem are purposely chosen so that the answer to the problem will not be given in the table on page 188 .

\section{Chapter X. The Transformer.}

101. An electromotive force of I Io volts, from a battery for example, is applied during $\frac{1}{1} \frac{1}{0}$ second intervals in reversed directions to a coil of 100 turns of wire wound on an iron core. Plot the curve representing the above electromotive force and, neglecting the resistance of the coil, plot the curve representing the core flux produced. The iron core is supposed to have a constant magnetic reluctance of 0.00014 . Plot the curve representing the magnetizing current.

A secondary coil of 50 turns is wound on the above core. This secondary coil supplies current to a non-inductive receiving circuit having $200 \mathrm{ohms}$ resistance. Plot curves representing (a) secondary electromotive force, $(b)$ secondary current, and $(c)$ 
total primary current. Resistance of secondary coil is to be neglected.

102. A transformer has $I, 300$ turns of wire in its primary coil and I 30 turns in its secondary coil. The primary coil takes current from I, IOO-volt mains and the secondary coil delivers 200 amperes to a receiving circuit of which the power factor is 0.85 . What is the equivalent primary resistance and reactance of the secondary receiving circuit? Ans. $r=46.75 \mathrm{ohms}, x=28.98 \mathrm{ohms}$.

103. A IO: I transformer has its primary coil connected to I, IOO-volt mains through a non-inductive resistance of $200 \mathrm{ohms}$ and its secondary arranged to deliver current to a non-inductive resistance of 3 ohms. Find : (a) the resistance of a simple circuit which is equivalent to the whole arrangement here specified; $(b)$ the current delivered to the primary circuit of the transformer; (c) the secondary current; $(d)$ the secondary terminal voltage; (e) primary terminal voltage. Ans. (a) 500 ohms, (b) 2.2 amperes, $(c) 22$ amperes, $(d) 66$ volts, $(e) 660$ volts.

104. The transformer specified in problem 103 is connected as specified, with the exception that the secondary receiving circuit has a reactance of 3 ohms and a negligible resistance. Find $(a)$ the resistance and reactance of the simple circuit which is equivalent to the whole arrangement; $(b)$ the primary current; $(c)$ the secondary current ; $(d)$ the secondary terminal voltage; and $(e)$ the primary terminal voltage. Draw a clock diagram showing the phase relations of the various electromotive forces and currents. Ans. (a) $200 \mathrm{ohms}$ resistance and $300 \mathrm{ohms}$ reactance, (b) 3.05 amperes, $(c) 30.5$ amperes, $(d) 91.5$ volts, $(e) 915$ volts.

105. A transformer has its primary in two sections which can be thrown in series or in parallel at will. This primary takes current through a rheostat of resistance $R^{\prime}$, and the secondary supplies current to a non-inductive receiving circuit of resistance $R^{\prime \prime}$. Find the relation between $R^{\prime}, R^{\prime \prime}, N^{\prime}$ (total), and $N^{\prime \prime}$ for which the secondary current is the same whether the primary coils are in series or in parallel. Ans. $R^{\prime}=\frac{1}{2}\left(N^{\prime} / N^{\prime \prime}\right)^{2} R^{\prime \prime}$.

106. A bundle of iron wires of which the total sectional area is 
12.6 square centimeters is to be magnetized by alternating current taken from I33-cycle I IO-volt mains so that the maximum flux density in the core may be 4,500 lines per square centimeter. (a) How many turns of wire are required? (b) How many turns of wire would be required at 60 cycles per second, other things remaining the same? Ans. (a) 328 turns, (b) 728 turns.

107. A transformer has 60 turns of wire in its primary coil, and it takes 750 amperes from an alternator at I IO volts and steps up to 20,000 volts. (a) How many turns of wire are there in the secondary coil ? A usual allowance in transformers is 500 circular mils of sectional area of wire for each ampere of current. (b) Find size of primary wire and of secondary wire of the above transformer. Ans. (a) 10,909 turns, (b) 375,000 circular mils, 2,062 circular mils.

108. The sectional area of the core of the transformer in problem IO7 is I6I square centimeters. Find the maximum core flux and maximum flux density at a frequency of 60 cycles per second. Ans. $\Phi=687,700, \mathscr{B}=4,272$.

109. A transformer has 800 turns of wire on the primary coil, 40 mils in diameter, and 80 turns on the secondary coil, secondary wire being ten times as large in sectional area. The sectional area of the magnetic circuit of the iron core is 12 square inches. Allowing 500 circular mils of conductor per ampere, and allowing a maximum flux density of 4,000 lines per square centimeter, calculate the electromotive force, current and power ratings of the transformer at 60 cycles per second. Ans. 660.4 volts, 3.2 amperes, 2. I 3 kilowatts.

110. A given transformer is rated at 5 kilowatts and is designed to take current from I, IOO-volt mains at a frequency of 60 cycles per second. Under these conditions $P_{h}, P_{e}$, and $P_{c}$ will be called normal.

(a) The transformer gives 6 kilowatts output at the rated electromotive force and frequency. Find $P_{o}$ in terms of normal.

(b) The transformer is used at rated electromotive force but at a frequency of 75 cycles per second. Find $P_{h}$ and $P_{e}$ each, in terms of normal. 
(c) The transformer is used at rated frequency but with primary electromotive force of 1,500 volts. Find $P_{h}$ and $P_{\sigma}$ each in terms of normal.

(d) The transformer is used on a primary electromotive force of 1,500 volts. Find $f$ for which $P_{h}$ is normal. Show that $P_{e}$ is independent of frequency with given $E^{\prime}$.

(e) With primary electromotive force of $\mathrm{I}, 650$ volts what load would give normal $P_{c}$ ?

Ans. (a) $\mathrm{I} .44 \times$ normal $P_{c} ;(b) 0.87 \times$ normal $P_{h}$, no change in $P_{0} ;(c) \mathrm{I} .64 \times$ normal $P_{h}, \mathrm{I} .86 \times$ normal $P_{\bullet} ;(d)$ I 37.2 cycles per second; $(e) 7.5$ kilowatts.

111. Following are the data for a shell type transformer (see Figs. 207 and 208).

A fine wire coil consists of 560 turns of No. I 7 B. \& S. copper wire. Mean length of turn $29 \frac{1}{2}$ inches.

The coarse wire coil consists of 28 turns of two No. 7 wires in parallel. Mean length of turn $29 \frac{1}{2}$ inches.

Section of magnetic circuit is $10 \frac{1}{2}{ }^{\prime \prime} \times 1 \frac{13}{16}{ }^{\prime \prime}$, and $\frac{8}{10}$ of this section is iron. Volume of iron $24 \frac{1}{2}$ sq. in. $\times 10^{1 \prime \prime} \times \frac{8}{10}$. Thickness of lamination 14 mils.

(a) Find the electromotive force, current, and power ratings of this transformer at a frequency of 60 cycles per second, allowing 500 circular mils per ampere, and allowing a maximum flux density of 5,000 lines per square centimeter.

(b) Find the copper loss and iron loss at full load.

(c) Find the efficiency of the transformer at full load.

(d) Find the all-day efficiency of the transformer when it is operated at full load for three hours each day, and at zero load for the remainder of the time.

112. The losses in a direct-current shunt motor when it is running at zero load are equal to 125 watts and the losses in the motor when it is running at full load equal to 175 watts. The full-load output of the motor is one horse-power. Find the allday efficiency of the motor when it is operated at full load for one minute of time every ro minutes during the day. 
113. A IO-kilowatt I, IOO: I IO-volt transformer is connected as an autotransformer to step up from I, IOO volts to I, 2 IO volts. (a) Find the amount of power that can be delivered to a noninductive receiving circuit at 1,2 IO volts without exceeding the rating of the transformer; $(b)$ find the current in each coil of the transformer; and $(c)$ specify the amount of power delivered to or delivered by each coil.

114. A IO-kilowatt I,IOO: I rO-volt transformer is connected as an autotransformer to step down from I, IOO volts to 990 volts. Find (a) the amount of power that can be delivered to a noninductive receiving circuit at 990 volts without exceeding the rating of the transformer; (b) find the current in each coil of the transformer; and $(c)$ specify the amount of power delivered to or by each coil.

115a. A transformer has two coils $A B$ and $C D$ arranged as shown in Fig. I $5 p$. The coil $A B$ is connected to I lo-volt mains, point $A$ being connected to main number one, and point $B$ being connected to main number two. Specify the exact manner in which the coil $C D$ must be connected in order that auto-step-up transformation may be accomplished and find the value of the voltage across the service mains.

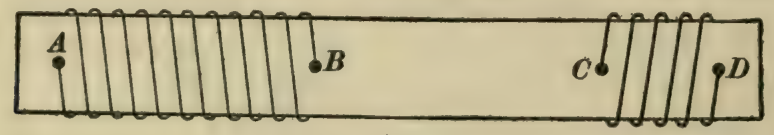

Fig. $115 p$.

115b. Specify the exact manner in which the coil $C D$, Fig. I I $5 p$, must be connected in order that auto-step-down transformation may be accomplished, with the coil $A B$ connected to I ro-volt supply mains, and find the value of the voltage across the service mains.

115c. The service mains in problem i $5 b$ deliver current to a non-inductive receiving circuit of which the resistance is $2 \mathrm{ohms}$. Find $(a)$ the current and power delivered to the receiving circuit; (b) the power developed in coil $C D$ (specify whether this coil 
gives out power or receives power); (c) the current flowing in coil $A B$ and the power developed in coil $A B$ (specify whether this coil gives out power or receives power), and $(d)$ the total current delivered by the supply mains.

116. Three similar $\mathrm{I}, \mathrm{OOO}$ to IOO-volt transformers have their I,OoO-volt coils delta-connected to three-phase I,ooo-volt mains, and the secondaries are $\mathrm{Y}$-connected to service mains. Give a diagram of the connections and find the electromotive force between the pairs of service mains. Ans. 173 volts.

117. The primaries of the transformers in problem 116 are $\mathrm{Y}$ connected to the 3-phase supply mains and the secondaries are delta-connected to the service mains. Find the electromotive force between each pair of service mains.

118. Two Ioo-kilowatt transformers are arranged as shown in Fig. 187 , Chapter $\mathrm{X}$ for the step-down transformation of a 3-phase supply. Find the power which can be delivered to balance receiving circuits of which the power factor is 0.85 without exceeding the voltage and current ratings of the two transformers.

119. Two transformers are connected as shown in Fig. I87, Chapter $\mathrm{X}$, and they deliver current to three similar receiving circuits of which the power factor is 0.80 . The total power delivered is 50 kilowatts, find the power delivered by each transformer. Ans. 14. 8 kilowatts, 35.82 kilowatts.

120. Find the power delivered by each transformer specified in problem I I9 if the power factor of the similar receiving circuits is 0.30 . Ans. 70.9 kilowatts, -20.9 kilowatts.

121. The primary coils of two transformers have each 560 turns of wire and they are connected to two-phase mains, the electromotive force of each phase being 800 volts. Calculate the turns of wire in each of two secondary coils (one on each transformer) so that these coils when connected in series may give an electromotive force of 400 volts, $30^{\circ}$ ahead of one of the twophase electromotive forces. Ans. I 40 turns, 242.5 turns.

122. A Scott transformer is to transform from 1,000 volts, 60 
cycles, two-phase, to I IO volts three-phase. The cross-section of each iron core is 75 square centimeters. Find $(a)$ the number of turns of wire in each primary coil, and $(b)$ the number of turns of wire in each secondary coil, allowing a maximum flux density of 3,500 lines per square centimeter. Ans. (a) $N^{\prime}=1,429$ turns, $(b)$ number of turns in coils $a, b$ and $c$, Fig. I98 are 79 , 79, I36, respectively.

123. A three-ring converter is to supply direct current at 500 volts to a street-car line. At what voltage must the alternating currents be delivered to the machine? The step-down transformation is accomplished by three similar transformers with theis primaries delta-connected to the high voltage mains and their secondaries $\mathrm{Y}$-connected to the three rings of the converter. What is the ratio of transformation of each transformer, the line voltage being 10,000 volts? Ans. 306 volts. $N^{\prime \prime} / N^{\prime}=0.0177$.

\section{Chapter XI. General Theory of the Transformer.}

Specification of a shell-type transformer. - Following are the data for a shell-type transformer (see Fig. 207, 208, and 209):

The fine wire coil consists of 560 turns of number I7 B. \& S. copper wire. Mean length of turn $29 \frac{1}{2}$ inches.

The coarse wire coil consists of 28 turns of two number seven (B. \& S.) wires in parallel. Mean length of turn $29 \frac{1}{2}$ inches.

$l=\mathrm{I} \frac{3}{4}$ inches, $g=\frac{1}{8}$ inch, $Y=\mathrm{I} \frac{1}{4}$ inches (coarse wire coil), $X=\mathrm{I} \frac{1}{2}$ inches (fine wire coil), and $\lambda / 2=10 \frac{1}{2}$ inches.

At each end of the core $4 \frac{1}{2}$ inches of length of coils are exposed. Section of magnetic circuit $=10 \frac{1}{2} \times I_{1} \frac{1}{16}$ square inches, and $\frac{8}{10}$ of this is iron. Volume of iron $=24 \frac{1}{2} \times 10 \frac{1}{2}$ cubic inches $\times \frac{8}{10}$. Thickness of laminations I4 mils.

Specification of a core-type transformer. - Following are the data for a core-type transformer (see Fig. 2 I2):

Each fine wire coil, $P$, Fig. 212, consists of I,000 turns of number I7 B. \& S. copper wire. Length of mean turn $=11 \frac{3}{4}$ inches. 
Each coarse wire coil, $S$, consists of I 00 turns of two number seven wires in parallel (B. \& S.). Length of mean turn $9 \frac{1}{2}$ inches.

$l=5$ inches, $g^{\prime}=\frac{1}{8}$ inch, $Y^{\prime}=\frac{5}{8}$ inch (fine wire coil), $X^{\prime}=\frac{3}{4}$ inch (coarse wire coil), and $\lambda=10 \frac{5}{8}$ inches.

The net sectional area of the iron core is 3.7 square inches, the mean length of the magnetic circuit is 22 inches, and the volume of the iron is $8 \mathrm{I} .4$ cubic inches. Thickness of laminations 14 mils.

124. Find the two components $M_{p}$ and $M_{w}$ of the magnetizing current of each of the above transformers, $E^{\prime}$ being I, IOO volts at I 33 cycles per second. Parts of coils in core-type transformer being connected in series $\left(N^{\prime}=2,000, N^{\prime \prime}=200\right)$.

125. Calculate the leakage inductance of each of the above transformers, parts of coils of core-type transformer being connected in series.

126. Determine the regulation of the above shell-type transformer when it takes current from I, IOO volt I 33 cycle mains and delivers current to a receiving circuit of which the resistance is $\mathrm{I} .25 \mathrm{ohms}$ and the reactance is $\mathrm{I} .3 \mathrm{ohms}$.

127. Determine the regulation of the above core-type transformer when it takes current from I, IOO volt I33 cycle mains and delivers current to a receiving circuit of which the resistance is $1.25 \mathrm{ohms}$ and the reactance is $\mathrm{I} .3 \mathrm{ohms}$.

\section{Chapter XII. The Induction Motor.}

128. The winding of an ordinary ring-wound direct-current armature has 24 sections. These sections are disconnected from the commutator and numbered in order from I to 24. Specify the manner in which these 24 coils are to be connected to twophase mains to produce in the ring a rotating state of magnetism with four poles, with six poles, and with twelve poles. Specify the connections of the 24 coils to three-phase mains to produce in the ring a rotating state of magnetism with two poles, with four poles, and with eight poles. State the speed of the mag- 
netism in each case in revolutions per second, the frequency of the polyphase currents being 60 cycles per second.

Sample answer for 4-pole 3-phase connections. - Phase $A$ is connected as follows: Positive main to $+($ section $\mathrm{I})-$ to $+(\mathrm{sec}-$ tion 2$)-$ to $-(\operatorname{section} 7)+$ to $-($ section 8$)+$ to $+($ section 13$)$ - to $+($ section 14$)-$ to $-($ section 19$)+$ to $-($ section 20$)$ + to negative main.

Phase $B$ is connected as follows : Positive main to $+($ section 3$)$ - to + (section 4$)-$ to $-($ section 9$)+$ to $-($ section 10$)+$ to $+($ section 15$)-$ to $+($ section 16$)-$ to $-(\operatorname{section} 2 \mathrm{I})+$ to - (section 22) + to negative main.

Phase $C$ is connected as follows: Positive main to + (section 5) - to $+($ section 6$)-$ to $-($ section II $)+$ to $-($ section 12$)$ + to $+($ section 17$)-$ to $+($ section 18$)-$ to $-($ section 23$)+$ to - (section 24) + to negative main.

Speed: Two-phase, four poles 30 revolutions per second; six poles 20 revolutions per second; twelve poles 10 revolutions per second. Three-phase, two poles 60 revolutions per second; four poles 30 revolutions per second; eight poles I 5 revolutions per second.

129a. The armature of an ordinary direct-current dynamo is short circuited between the brushes, and the resistance of the armature between brushes is $0.036 \mathrm{ohm}$. The number of armature conductors is 260 , and the flux through the armature is I,500,000 lines, which is assumed to be invariable. The field magnet is set rotating about the shaft as an axis at a speed of 25 revolutions per second, carrying the direct-current brushes with it. Calculate the torque dragging upon the armature when its speed is 24 revolutions per second and when its speed is 23 revolutions per second. What portion of the power expended in driving the field is available at the armature belt, and what portion is lost in heating the armature, friction losses being ignored?

Ans. (a) Torque $=6.86 \mathrm{~kg}$.-meters, 96 per cent. of the power is available at the armature belt and 4 per cent. is lost in heating the armature. 


\section{PROBLEMS.}

(b) Torque I $3.72 \mathrm{~kg}$.-meters, 92 per cent. of the power is available at the armature belt and 8 per cent. is lost in heating the armature.

Note. - This problem may be solved by the use of the ordinary equations of the direct current dynamo, namely :

$$
\begin{aligned}
& \mathcal{E}=\phi Z^{\prime} n, \\
& \mathscr{J}=\mathcal{E} / R, \\
& T=\mathrm{I} \cdot 4 \mathrm{I} \Phi Z^{\prime} \mathfrak{I} \text { pound inches, }
\end{aligned}
$$

where $Z^{\prime}$ in the present case is $260 \div 10^{8}$, and $n$ is the relative speed.

129b. The synchronous speed $n$ of an induction motor is Ioo. The motor develops its maximum torque when the rotor speed is 80 . How many times must the rotor resistance be increased in order that the motor may develop its maximum torque at stand-still? Explain.

130. An ideal three-phase induction motor takes 5 amperes of current into each phase of its delta-connected primary member at 200 volts and 60 cycles per second. The rotor, which has a three-phase winding, delta-connected to collecting rings, supplies 25 amperes of current to each of three similar circuits, each having a power factor equal to 0.75 . The rotor runs at $\frac{2}{3}$ synchronous speed. (a) What is the ratio of the stator to the rotor turns? (b) What is the rotor terminal voltage? (c) What is the total intake of power? (d) What is the electrical output of power? (e) What is the mechanical output of power? Ans. (a) Ratio of turns is $5: \mathrm{I}$. (b) Rotor terminal voltage is $\frac{1}{3} \times \frac{1}{5} \times 200$ volts. (c) Total intake of power is 2,250 watts. (d) Total electrical output is 750 watts. (e) Total mechanical output is $I, 500$ watts.

131. A 3-phase 4-pole induction motor has its stator windings delta-connected to I, IOO volt 25 cycle 3 -phase mains. The rotor has a 3 -phase winding which is $\mathrm{Y}$-connected to collector rings, the number of conductors in the rotor winding being $\frac{1}{4}$ as great as the number in the stator winding. The rotor is driven backwards at a speed of $\mathrm{I}, 050$ revolutions per minute. Neglect- 
ing the effects of magnetizing current, coil resistance, and magnetic leakage, $(a)$ find the frequency and value of the voltage between each pair of rotor collector rings.

The rotor collector rings are $\mathrm{Y}$-connected to three similar receiving circuits of which the power factor is 0.85 and of which the impedance is Io ohms. Find $(b)$ the total electrical power delivered by the rotor; $(c)$ the total electrical power delivered to the stator and $(d)$ the total mechanical power required to drive the rotor.

\section{Chapter XiII. The General Theory of the INDUCTION MOTOR.}

132. A 2-phase 6-pole induction motor takes current from 2phase supply mains across each pair of which the electromotive force is I, IOO volts, frequency 60 cycles per second. When the motor is run at zero load, an ammeter indicates 5.2 amperes in each phase of the stator winding, and a wattmeter indicates a delivery of 1,200 watts to each phase of the stator. At standstill the current delivered to each phase is observed to be 75 amperes and the power delivered to each phase is observed to be I 4,000 watts. The resistance of each stator winding as determined by direct-current ammeter and voltmeter is I.I ohms. From these data construct a circular diagram of the induction motor, and plot performance curves similar to Fig. 243.

133. One stator winding only in the above induction motor is to be used, thus making it a single-phase motor. When the motor is driven at zero load, an ammeter indicates 10.6 amperes delivered to the stator, and a wattmeter indicates 2,450 watts. At standstill the current delivered to the stator winding is observed to be 75 amperes and the power as measured by a wattmeter is found to be 14,500 watts. From these data construct a circular diagram for the motor (single-phase) and plot performance curves somewhat similar to Fig. 243. 


\section{INDEX.}

Abhenry, definition of, 356

Admittance of an alternating-current circuit, definition of, $70,9 x$

Alternating current, 2 advantages of, 14 and direct current calculations contrasted, 19

curves, 24, 35

lighting, 16

measurements, 38

motors, 16,17

electromotive force, 2

average values and effective values of, 30

curves, 24, 35

Alternation current, conversion of, into direct current, $\mathbf{I} 7$

Alternator, electromotive force of, $\mathbf{I} 7$

field excitation of, ro

power delivered by, 32

saturation curve of, ${ }_{132}$

synchronous reactance curve of, I33

the, I

the composite, 12

the single-phase, 98

the three-phase, Ior

the two-phase, 98

voltage regulation of, $\mathbf{I} \mathbf{1 7}$

Alternators in parallel, I4I, I59

power rating of, 139

single-phase and polyphase, 8

Ammeters for alternating currents, $3^{8}$

Armature reaction of the alternator, 137 windings, concentrated, 9 distributed, 9

Autotransformer, the, 219

Average values and effective values of harmonic electromotive forces and currents, 60

of alternating electromotive forces and currents, 30
Balanced polyphase system, I ro

Bedell and Crehore, Alternating Currents, 73

Bus-bars, 322

Buses, synchronizing, 322

Capacity and inductance, 354

concentrated, $2 \mathbf{r}$

distributed, 2 I

electrostatic, 365

units of, 366

Charge, electric, definition of, 362

Choice of signs in complicated networks, I04

Circle diagram of the induction motor, 278 of the transformer, 248

Coils in parallel, 86, 95

in series, 93

Compensated voltmeter, the, $33^{8}$

Compensator, the starting, 267

Complex quantity, use of, 88

Composite alternator, the, 12

Composition and resolution of harmonic electromotive forces and currents, 55

Commutator rectifier, the, 12

Concentrated armature windings, 9 capacity and distributed capacity, 2 I

Condenser, the, 365

Conductance of an alternating-current circuit, definition of, 70, 9I

Constant current transformer, the, 215

Conversion of alternating current into direct current, 17,170

Copper economy of two-phase and threephase transmission lines, I 15

loss in transformers, 2 I I

Core losses in transformers, 2 II

Core-type transformers, 198

Current and electromotive force curves, examples of, 79, 8 I

curves, 24, 35 
Current, harmonic, rate of change of, 57 loci, 82

locus of the transformer, 248

transformer, the, 46, 234

Curves of current, 24, 35

of electromotive force, 24, 35 and current, examples of, $79,8 \mathrm{I}$

Decay and growth of current in an inductive circuit, 7 I

DeMoivre's theorem, 9 r

Dial synchronizer, the, 336

Direct-current and alternating-currentcalculations contrasted, 19

Distributed armature windings, 9

capacity and concentrated capacity, 2 I

Dobrowolsky's double-current generator, 193

Double-current generator, the, 192

Drop of voltage in transmission lines, 84

Effective values and average values of harmonic electromotive forces and currents, óo

of alternating electromotive forces and currents, 30

Efficiency, all-day, 212

Emmet's table of line reactances, 347

Electric charge, definition of, 362 momentum, 354

Electrical and mechanical analogies, 367

Electrodynamometer, the, 39

Electromotive force and current curves, examples of, 79, 8x

curves, 24, 35

of the alternator, 117

self-induced, $35^{8}$

Electrostatic capacity, 365

voltmeter, $4^{\mathrm{I}}$

Equivalent reactance of transformer and receiving circuit, 206

resistance of transformer and receiving circuit, 206

Farad, definition of, 366

Field excitation of the alternator, 10
Flux turns, definition of, 360

Frequencies, standard, 140

Frequency and speed, 23

changer, the, 269

Generator, the alternating-current, I the double-current, 192

Ground detector, 340

Growth and decay of current in an induc. tive circuit, $7 \mathbf{I}$

Harmonic current, rate of change of, 57 electromotive force and current, 52

Henry, definition of, 356

Heyland diagram of the induction motor, 278

Hot-wire ammeter, the, 39 voltmeter, the, 39

Hunting of synchronous motor, 150

Impedance as an algebraic operator, 89 of an alternating-current circuit, definition of, 69

Inclined coil ammeters and voltmeters, 43

Inductance and capacity, 354

calculation of, 360

measurement of, 357

mechanical analogue of, 356

of a coil, 356

on an iron core, 361

of a long solenoid, $36 \mathrm{r}$

of a transmission line, 362

units of, 356

Induction generator, the, $27 \mathrm{I}$ motor, action of, 254, $37 \mathrm{I}$ algebraic formation of, $\mathbf{2 8 8}$ analogy to the friction clutch, $26_{5}$ as a frequency changer, 269 as a generator, $27 \mathrm{I}$ break-down of, 262 circle-diagram of, 278 electrical power developed in rotor of, 265

energy transformations in the rotor of, 276

equivalent transformer action of, 277 
Irduction motor, general theory of, 275

load current of, 275

magnetizing current of, 275

mechanical power developed by,

265

performance curves of, 280,282

rotor resistance and efficiency of, 264

single-phase, 292

induced electromotive

forces in, 374

starting of, 273

slip, 276

speed and efficiency of, 264

control of, 268

starting compensator for, 267 of, 266

of the polyphase synchronous motor, $27 \mathrm{I}$

the single-phase, 272

stator windings of, 254

the, $25 \mathrm{I}$

torque and speed relations of, 260

two-phase, rotor currents in, 376

use of starting resistance for, 262

motors, rating of, 265

Inductivities, table of, 366

Inductivity of a dielectric, 366

Iron losses in transformers, see core losses

Kinetic energy of the electric current, 355

Lagging current, definition of, 54 currents, compensation for, 86

Leading current, definition of, 54

Leakage reactance of transformer, 237, 24 I

Lincoln synchronizer, the, 336

Load current of the induction motor, 275

Magnetic leakage of transformer, 233 effect of, 237

Magnetizing current of the induction motor, 275

of the transformer, 234
Magnetizing current of transformers, 203, 2 10

Measurement of alternating current, $3^{8}$ electromotive force, 38

of power in alternating-current circuits, 46

in polyphase systems, 112

Measurements in alternating currents, 38

Mechanical and electrical analogies, 367

Mercury-arc rectifier, 172

Methods in alternating currents, 88

Microfarad, definition of, 366

Momentum of the electric current, 354

Motor-generator, the, I7 I

Motor, the induction, see the induction motor

the repulsion, see repulsion motor series, see series motor

the synchronous, see synchronous motor

Non-inductive circuits, 357

Oil switches, 34I

Oscillatory current, the, 73

Parallel operation of alternators, 141, 159

Phase constant of an armature winding, definition of, 124

constants of armature windings, table of, 124

difference, definition of, 53

transformation, 225

Plunger-type ammeters and voltmeters, 42

Polyphase induction motor, action of, 37 I the, 251

power, measurement of, 112

system, balanced, I ro

the, 98

unbalanced, I ro

transformers, 223

Potential transformer, the, 46, 234

Power component of current, 64 of electromotive force, 64

delivered by alternator, 32

expression of, in case of harmonic electromotive forces and currents, 63 
Power, factor, definition of, 47

in polyphase systems, I IO

loss in transformers, 211

measurement of in alternating-current circuits, 46

in polyphase systems, 112

rating of alternators, $\mathbf{I} 39$

of induction motors, 265

of synchronous converters, 188 of transformers, 215

Problems, fundamental, of alternating currents, 66

Rating of alternators, 139

- induction motors, 265

synchronous converters, 188

transformers, 215

Reactance of an alternating-current circuit, definition of, 69 of transmission line, 347

Reactance, synchronous, definition of, 13 I

Rectifier, the, I 70

the commutator, 12

the mercury-arc, $\mathbf{I 7 2}$

Rectifying commutator, the, ryo

Regulation of the alternator, II 7

of transformers, 214

transformer, calculation of, 249

Regulator, the Tirrell, 34I

Repulsion motor, the, 315

starting of the single-phase induction motor, 274,318

Resistance of an alternating-current circuit, definition of, 69 . of transmission line, 346

Resolution and composition of harmonic electromotive forces and currents, 55

Resonance, electric, 74

mechanical, 76

multiplication of current by, 78

of electromotive force by, 77

Rotary converter, see synchronous converter

Scott transformer, the, 227

Self-induced electromotive force, $35^{8}$

Series motor, behavior of, when driven

by alternating current, 302
Series motor, the single-phase, $30 \mathrm{I}$

characteristics of, 314

clock diagram of, 303

design of, to give large power factor, 304

neutralizing of armature

inductance of, 306

prevention of sparking in, 309

Seyfert, S. S., spark preventing device of, 3 I I

Single-phase induction motor, 272, 292 induced electromotive forces in, 374

series motor, characteristics of, $3 \mathbf{I} 4$ clock diagram of, 303

design of, to give large power factor, 304

neutralizing armature inductance of, 306

prevention of sparking in, 309

the, 301

system, the, 98

transmission line, calculation of, 347 series motor, use of resistance leads in, 311

Shell-type transformers, 199

Shunt motor, behavior of, when driven

by alternating current, 3 or

Slip of induction motor, 276

Spark gauge, the, 43

Sparking distances, table of, 44

Speed and frequency, 23

Split-phase starting of the single-phase induction motor, 274

Split-pole rotary converter, the, 370

Starting compensator for the induction motor, 267

Susceptance of an alternating-current circuit, definition of, 70, 9 I

Synchronism, definition of, 24

Synchronizer, the, 155

the dial, 336

Synchronizing buses, 322

Synchronous converter, the, 157, 172, I74 armature heating of, $\mathbf{1} 88$ 
Synchronous converter armature, reaction

$$
\text { of, } 180
$$

compounding of, 185

current relations of, 185

electromotive force relations of, 180

internal action of, 175

operation of, 177

rating of, 188

table, 189

split-pole, the, 370

starting of, 177

by induction motor action, 271

voltage control of, 184

converters, transformer connections

for, 230

motor efficiency of, $\mathbf{r} 6 \mathbf{I}$

excitation characteristics of, 167

hunting of, $x_{50}$

maximum electromotive force of, 166

intake of, 165

over-excited, 157

polyphase, 157

stability of running of, 149

starting of, 155

by induction motor action,

27 I

the, 14 I

reactance, definition of, $\mathrm{I}_{3} \mathrm{I}$

Switchboard appliances, 320

Switchboards, 320

feeder panel, 326

for generating stations, $32 \mathrm{I}$

for receiving stations, 330

generating panel, 322

induction motor panel, 330

synchronous converter panel, 33 I

motor panel, 333

Table of inductivities of various dielec-

trics, 366

of phase constants, 124

of sparking distances, 44

synchronous converter ratings, 189
Three-phase alternator, the, ror

and two-phase transmission lines, copper economy of, II 5

electromotive forces and currents, IO4-I IO

transmission line, calculation of, 349

Tirrill field regulator, the, 14, 34I

Transformation of phases, 225

step-up and step-down, 195

Transformer action, 202

particular cases of, 204

and receiving circuit, equivalent resistance and reactance of, 206

connections, 217

core flut of, 208

current relations of, 202

connections for synchronous converters, 230

efficiency, calculation of, 213

flux and voltage relations of, 208

general theory of, 233

leakage reactance of, $237,24 \mathrm{I}$

magnetizing current of, 203, 210,234

multipliers for alternating-current instruments, 45

rating, 215

reduction of, to simple circuit equiv-

alents, 246

regulation, 214

calculation of, 249

the, 96, 194

circle diagram of, 248

constant current, 215

Scott, 227

Scott, dephasing action of, 229

voltage relations of, 202

Transformers, arrangement of in polyphase systems, 22I

core-type, 198

efficiency of, 212

in parallel, 217

in series, 217

polyphase, 223

power loss in, $2 \mathbf{I I}$

shell-type, 199

with divided coils, 218 
Transmission line calculations, 346 inductance of, 362

reactance, 347

reactances, table of, 347 resistance, 346

single-phase, calculation of, 347

three-phase, calculation of, 349

transposition of, $35^{2}$

two-phase, calculation of, 349

Transmission lines, 346

direct-current and alternatingcurrent, compared, 346

interference of, 35 I

two-phase and three-phase, copper economy of, 115

voltage drop in, 84

Two-phase alternator, the, 98

and three-phase transmission lines, copper economy of, II 5

electromotive forces and currents, Ioo
Two-phase induction motor, rotor currents in, 376

transmission line, calculation of, 349

Unbalanced polyphase system, I 10

Voltage drop in transmission lines, 84 regulation of the alternator, $\mathrm{II} 7$ of the alternator, calculation of, I3I

of the alternator, theory of, 127 regulators, 333

Voltmeter, the compensated, $33^{8}$

Voltmeters for alternating electromotive force, 38

Watt-hour meter, the, 49

Wattless component of current, $\mathbf{6 4}$ of electromotive force, 64

Wattmeter, inductance error of, 49 the, 47 




$$
5)^{x^{22^{0}}}
$$




\section{PLEASE DO NOT REMOVE CARDS OR SLIPS FROM THIS POCKET}

\section{UNIVERSITY OF TORONTO LIBRARY}

\section{S8M A 220}


